\title{
57th Annual Conference of Indian Society of Hematology and Blood Transfusion 2016, Jaipur
}

\section{Topic: Abstracts of JC Patel Award Papers}

\section{JCP 1}

Intracellular Iron Overload Leading to DNA Damage of Lymphocytes and Immune Dysfunction in Thalassaemia Major Patients

Jyoti Shaw", Ayan Chakraborty, Arijit Nag, Arnab Chattopadyay, Anjan K. R. Dasgupta, Maitreyee Bhattacharyya

Email ID for Correspondence: jyotishaw.mvk@gmail.com

Department of Biochemistry and Center for Excellence in Systems Biology and Biomedical Engineering, University of Calcutta and Institute of Haematology and Transfusion Medicine, Medical College, Kolkata

Abstract: Immune dysfunction in thalassemia is a major concern worldwide. Complications of thalassemia such as iron overload, transfusion therapy, role of spleen and the disease itself is considered as the fundamental cause of immune dysfunction. Iron overload is a major complication in both thalassemias intermedia and major. We demonstrated that intracellular iron overload in thalassemia causes oxidative damage of lymphocyte DNA as exhibited by DNA damage assay. The inference is further confirmed by partial inhibition of such damage by chelation of iron and the concurrent lowering of the ROS level in presence of chelators like deferasirox. Notably this effect is exclusively observed for thalassemia patient and not for normal subjects. The obvious hint in this case is that in thalassemia patients the import of iron from the extracellular pool is enhanced and may be the underlying cause of DNA breakage. Lower import of iron is known to cause lymphocyte dysfunction as reported in the case of severe immunodeficiency (SID), excess import of iron also causes DNA fragmentation of lymphocytes which will ultimately lead to lymphocyte dysfunction. This may contribute to immune dysfunction and increased susceptibility to infection in thalassemia patients.

Keywords Nucleated red blood cells, Lymphocytes, Iron overloading, Oxidative stress, Iron chelator

\section{JCP 2}

Analysis of Expression of Neutrophil CD 64 \& Monocyte HLA-DR as Diagnostic Markers for Early Onset Neonatal Sepsis

S. Gulati, N. Sharma ${ }^{\#}$, R. Jain, S. Sitaraman, P. Jain

Email ID for Correspondence: nidhi11112222@gmail.com

SMS Medical College, Jaipur

Introduction: Neonatal sepsis is a major cause of neonatal mortality and morbidity. Early onset sepsis presents with inconspicuous signs and symptoms and its diagnosis is challenging. Aims and Objectives: This study was undertaken to assess expression of neutrophil CD64 (nCD64), monocyte HLA-DR (mHLA-DR) and their ratio as diagnostic markers in early onset neonatal sepsis and to compare it with others sepsis markers. Materials and Method: This study was carried out in neonatal units of Department of Pediatrics, SMS Medical College, Jaipur from June 2014 to May 2015. 100 neonates having antenatal risk factors for sepsis and signs and symptoms suggestive of sepsis were selected. They were subjected to sepsis screening tests and subsequently assigned into infected $(\mathrm{n}=48)$ and non-infected $(\mathrm{n}=52)$ groups. nCD64 and mHLA-DR expression was determined using flow cytometry technology. Results: nCD64 expression was significantly enhanced in the infected group. This test had a sensitivity and NPV value of $98 \%$ each which was higher than the commonly used haematological and biochemical markers. The specificity and PPV was about $85 \%$. mHLA-DR expression was lowered in infected group. It had a sensitivity and specificity of $60 \%$ and $73 \%$ respectively. Sepsis index was calculated on the basis of ratio of nCD64 and mHLA-DR which had sensitivity and specificity of $77 \%$ and $90 \%$ respectively. Combination of CRP and nCD64 raised the sensitivity and NPV to $100 \%$. Conclusion: Endorsement of flow cytometric testing of cell surface markers for early detection of neonatal sepsis appears to be promising approach. Addition of CRP further improves the efficiency. Results can be made available quickly and with a minimal blood volume.

Keywords Flow cytometry, Neutrophil CD64, Monocyte HLA-DR 


\section{JCP 3}

Identification of Phenotypic and Genotypic Profile of Pediatric Cases with Iron Refractory Iron Deficiency Anemia (IRIDA): Need for a Systematic Investigational Approach in an Iron Deficient Endemic Population

\section{Prateek Bhatia", Aditya Singh, Avani Hegde, Richa Jain, Deepak Bansal}

Email ID for Correspondence: prateekbhatia@rediffmail.com

Post Graduate Institute of Medical Education and Research, Chandigarh, India

Background and aims: Prevalence of IDA is very high in our population $(60 \%)$ and since most of children with microcytic hypochromic anemia are put on oral iron trial, partial or complete refractoriness to oral iron therapy is now increasingly being recognised. Recently, mutations in TMPRSS6 gene have also been described to affect iron metabolism. Hence, aim of study was to establish a screening model to investigate refractory cases and defining their phenotypic and genotypic profile. Methods: Children (0-12 years) with microcytic hypochromic anemia were screened and those with very low RBC indices and refractoriness to 4-6 weeks of oral iron were selected and labelled as iron refractory. HbA2, CRP, ESR and TTg were performed to rule out secondary causes. Plasma biomarkers like ferritin and Hepcidin were then performed by Chemiluminescence and ELISA assay to identify cases with IRIDA phenotype. TMPRSS6 gene analysis in cases with IRIDA phenotype was then performed by multiplex PCR followed by Sanger sequencing of all 18 exons of the gene. Data was analyzed using in house bioinformatics pipeline (AMAP) and polyphen scoring software to score adverse effects of mutations noted. NCBI dsSNP database was searched and mapped to note any novel or rare polymorphisms. Results: Out of 550 cases screened, $60(11 \%)$ were iron refractory. The mean age of cohort was 2.06 years. 26/60 (43\%) had mild anemia, 22 $(37 \%)$ moderate and $12(20 \%)$ severe anaemia. 53/60 (88\%) had partial response to oral iron and $7(12 \%)$ had no response. Based on biomarker levels, IRIDA phenotype was noted in 23/60 (38.3\%) cases. Normal to high ferritin and hepcidin levels were noted in $25 / 60$ $(41.6 \%)$ and $47 / 60(78.3 \%)$ respectively. PCR and sequencing data was available in $20 / 23$ cases due to non consent in others. The data revealed 15 exonic and 49 intronic mutations (total 64 variations in 20 cases). Out of 15 exonic mutations, 11 were synonymous (i.e. non functional without amino acid change). However, 2 were non synonymous exonic polymorphisms (P555S and V736A), seen in three cases (P555S-1 case; V736A-1 case and both together in 1 case). Out of 49 intronic mutations, only 20 were effective or functional as per RNAreg software analysis. These functional intronic mutations involved transcriptional regulatory motifs in 10 cases ( 1 of them common in 4 cases-g.23404T $>$ A), UTR binding element loss in 4 cases (g.25335T $>C$ common in all 4 cases), C-to U RNA editing site generation in 5 cases (g.35000C $>\mathrm{T}$ common in all 5 cases), Exon splicing silencer insertion in 3 cases (g.11184A $>$ G common in all 3 cases) and splice site shift and cryptic splice site generation in 1 case each. Conclusion: Though $11 \%$ of microcytic hypochromic anemia cases screened by us over 1 year had oral iron refractoriness, but only $38 \%$ (23/60 cases) of them had IRIDA phenotype. Of the 20 cases which could be sequenced, only 3 had effective exonic SNPs while 11 cases had functional mutations in non coding or intronic regions of TMPRSS6 gene. The intronic mutation induced variants are known to act synergistically in a case and likely to precipitate iron-refractoriness as has also been shown by Athiyarath $\mathrm{R}$ et al. in their study. However, large prospective studies are needed to better assess and define their functional significance in relation to iron-refractoriness. The common exonic SNPs (P555S and V736A) are known to cause a drop in $\mathrm{Hb}$ of $0.2-0.4 \mathrm{gm} / \mathrm{dl}$ in various large GWAS studies. The same could be responsible for precipitation of mild IRIDA phenotype in our cases too, but role of other iron-metabolism related genes cannot be ruled out. As per the screening model followed our study could genetically define and predict IRIDA phenotype in nearly $11 / 20$ cases (55\% cases). Thus it needs to be stressed that other causes or reasons for improving refractoriness to iron (thorough compliance, $H$. pylori infection, change in oral iron supplement or prolonged oral iron challenge) still need to be addressed before genetic studies for TMPRSS6 gene be undertaken.

Keywords IRIDA, Mutations, Oral iron, Phenotype, TMPRSS6 gene

\section{JCP 4}

Osteoblast Differentiation of Stromal Cells Induced by Leukemic Cells: Implication in Drug Resistance and Quiescence?

Saravanan Ganesan", Hamenth Kumar Palani,

Nithya Balasundaram, Ansu Abu Alex, Biju George,

Poonkuzhali Balasubramanian, Vikram Mathews

Email ID for Correspondence: saravanang@cmcvellore.ac.in

Christian Medical College, Vellore, India

Abstract: There are numerous reports on bone marrow micro-environment mediated drug resistance (EM-DR) in cancer. We had previously reported that there was significant EM-DR to ATO in APL. We had also shown that the effect of cross talk between leukemic cells and stromal cells leads to increased activation of NF-kB pathway in the leukemic cells. There are limited data available on the effect of leukemic cell interaction on the stromal cells. We undertook a study to evaluate the changes induced by leukemic cells (NB4 cell line) on the HS-5 stromal cell line. In a gene expression profiling on HS-5 cells upon co-culture, we observed an enrichment of Wnt signaling, BMP signaling pathway and a significant enrichment of osteoblast differentiation genes which was further validated. Similar differentiation effects were seen with other malignant leukemic cells (U937, U266 and Jurkat) suggesting that this effect is common with different subtypes of leukemia. We did not observe any effect of PBMNC/ normal cord blood CD34+ cells on HS-5 cells in inducing osteoblast differentiation. Further, miRNA analysis revealed 21 miRNA were differentially regulated in the HS-5 cells co-cultured with leukemic cells. Consistent with the gene expression profile, we observed that most of the miRNA differentially regulated were involved in the regulation of osteoblast differentiation (such as hsa-miR-33b-5p, hsamiR-210-3p and hsa-miR-222-5p).

This study demonstrates a possible role of leukemic cells in establishing quiescent leukemic niche in the bone marrow by inducing osteoblast differentiation of stromal cells. The role of this leukemic cell induced osteoblast differentiation of stroma in drug resistance against various chemotherapeutic agents needs further evaluation.

\section{JCP 5}

Prognostic Significance of End-Induction Day 29 Minimal Residual Disease Measured by 6 Color Flow Cytometry in Indian Adult B-Lineage Acute Lymphoblastic Leukemia Patients

Rahul Kumar Sharma\#, Manoranjan Mahapatra, Sudha Sazawal, Tulika Seth, Pravas Mishra, Renu Saxena

Email ID for Correspondence: rahulsharmabio@gmail.com

All India Institute of Medical Sciences, New Delhi

Introduction: Prognostic significance of minimal residual disease (MRD) although extensively studied in pediatric ALL but very less in adults and nil from Indian cohort. Present study is aimed to unravel 
prognostic role of day +29 end-induction immunophenotypic MRD over a comprehensive pre-therapeutic risk parameters in Indian adult B-ALL patients. Materials and Methods: Total 104 adult B-ALL (15-60 yr) patients were recruited. MRD assessment was carried out by applying 2 uniform and 1 optional 6 color tube by taking cut-off of $\geq 0.01 \%$. Along with MRD, various baseline risk parameters including age, sex, WBC, cytogenetics, CNS status, bcr-abl (RTPCR) status, NCI-risk group, day-7 BM response, BMI status, CD45 MFI of blasts, individual and dual co-expression of CD13 and CD33, Expression of CD34 and LSC $\left(\mathrm{CD} 34^{+} \mathrm{CD} 38^{\mathrm{dim} /-} \mathrm{CD} 45^{\mathrm{dim} /-}\right)$ in the blasts were assessed to find its association with MRD positivity, 3 year event free survival (EFS) and overall survival (OS). Results: Total $60(57.6 \%)$ out of 104 patients in day +29 clinical remission bone marrow were detected MRD positive $(\geq 0.01 \%)$. MRD positivity was found significantly associated with patients of $\geq 25$ year age $(p=0.037)$ and bcr-abl positive by RT-PCR $(p=0.004)$. EFS was significantly low in ber-abl positive $(10.2 \pm 6.7 \%$ vs. $46.1 \pm 7.1 \%$, $\mathrm{p}=0.000$ ), CNS positive ( $0 \%$ vs. $39.5 \pm 6 \%, \mathrm{p}=0.009)$, NCI high risk category $(21.9 \pm 8.3 \%$ vs. $48.4 \pm 7.4 \%, p=0.017)$, dual coexpression of CD13 and CD33 $(23 \pm 8.2 \%$ vs. $42.8 \pm 7.5 \%$, $\mathrm{p}=0.019)$ and MRD positive $(17.8 \pm 5.4 \%$, vs. $74.2 \pm 8.1 \%$, $\mathrm{p}=0.000)$ patents. OS was significantly low in CNS positive (0\% vs. $46.7 \pm 5.8 \%, \quad \mathrm{p}=0.003)$, bcr-abl positive $(11.8 \pm 7.7 \%$ vs. $53.6 \pm 6.4 \%, \mathrm{p}=0.000)$ and MRD positive $(24 \pm 6.2 \%$ vs. $74.2 \pm 8.1 \%, \mathrm{p}=0.000)$ patients. In the standard risk group, MRD positivity was the only significant parameter to predict EFS and OS. Conclusion: End-induction day +29 MRD measured by 6 color flow cytometry plays a significant prognostic role in total as well as standard risk adult B-ALL patients. Its implementation in routine clinical care to decide about earlier treatment intensification requirement in high risk patients is the need of hour.

\section{JCP 6}

Evaluation of a 54 Gene Targeted Next Generation Sequencing Panel for Acute Myeloid Leukemia

Rohan Kodgule", Goutham Raval, P. G. Subramanian, Shraddha Kadechkar, Pratibha Aamre-Kadam, Dhanashree Shetty, Gaurav Chatterjee, Nikhil Rabade, Shruti Chaudhary, Swapnali Joshi, Russel Mascarenhas, Prashant Tembhare, Sumeet Gujral, Nikhil Patkar

Email ID for Correspondence: rohan_kodgule@yahoo.co.in

\section{Tata Memorial Centre, Mumbai}

Introduction: Recent whole genome and exome sequencing studies have identified key driver mutations that are harboured by myeloid malignancies. Amongst these, acute myeloid leukemia (AML) is perhaps the most biologically heterogeneous entity comprising of chromosomal abnormalities and genetic mutations. It is important to recognize these mutations as some of them may be of prognostic and therapeutic value. Here, we identify somatic mutations in AML using a 54 gene panel using targeted NGS on an Illumina MiSeq platform. Methods: 54 patients of AML (WHO2008 criteria) were sequenced. FISH and conventional karyotyping was done to risk stratify the patients. Approximately $50 \mathrm{ng}$ of genomic DNA was used as a template. Sequencing oligonucleotides were hybridized to target regions of the genomic DNA, followed by extension and ligation. Barcode indices and sequencing adapters were added followed by PCR based amplification. The products were then purified, normalized and pooled. Sequencing was done on an Illumina MiSeq instrument using the V3 chemistry kit. Base calling was done by the MiSeq Reporter software. Sequence alignment and generation of VCF files was done using Base space and analyzed using Variant Studio v2.2.1 and ITDseek. Results: 25 patients were of favourable risk, 24 were of intermediate risk and 05 were poor risk, (mean age: 33.77 years; $\mathrm{M}: \mathrm{F}=2.6: 1)$. Analysis of data quality indicated that all of the samples met the predefined acceptance criteria ( $Q$ score $>30$ ). The average amplicon mean coverage depth was $4294 \times$. An average of 2.3 mutations was detected per sample. Most common mutations amongst favourable cytogenetic risk were KIT (29\%), ASXL1 (19\%), NRAS (14\%), TET2 (12\%). In Intermediate cytogenetic risk group NPM1 (19\%), DNMT3A (12\%), ASXL1 (10\%), NRAS and IDH1 (9 \% each). Conclusion: Gene panel testing by NGS approach was applicable for detection of various known gene mutations of AML in the clinical laboratory set up. These initial results indicate importance of rapid comprehensive molecular profiling of patient samples in the era of personalised medicine.

\section{Topic: Benign Haematology—Clinical}

\section{BHC 1}

Cutaneous Thrombosis in Paroxysmal Nocturnal Haemoglobinuria: A Rare Complication

\section{Gurmeet Singh", Rashee Varshney, Raju Bhaisare, Manisha Kango, M. Ravindranath, M. Bhalla}

Email ID for Correspondence: vicky.gurmeet@gmail.com Jawaharlal Nehru Hospital and Research Centre, Bhilai

Introduction and Background: Paroxysmal nocturnal haemoglobinuria $(\mathrm{PNH})$ is a rare, acquired hematological disorder characterized by haemolysis, bone marrow dysfunction and thrombosis. Recent researches have dramatically demonstrated its complex pathophysiology, that is of, close integration and influence of the complement and coagulation systems over each other upto the extent that thrombin itself has been shown to activate the alternate pathway of complement. By far, the most feared complication and significant cause of mortality is thrombosis amongst which cutaneous thrombosis is quite uncommon. Patient/Material and Methods: A 39 year old female, known case of $\mathrm{PNH}$, presented with erythematous and violaceous plaques, mainly located over the face and trunk. Skin biopsy was done. H\&E stained section showed multiple dermal vessels showing features suggestive of small vessel thrombosis. The patient was treated with heparin therapy. The patient has presented with multiple episodes of similar episodes in last two years. Results: Based on the history and histopathology, diagnosis of recurrent cutaneous thrombosis related to PNH was made. Conclusions: Recurrent cutaneous thrombosis in $\mathrm{PNH}$ is a rare complication and its correct and timely diagnosis should be done.

\section{BHC 2}

Safety of Turoctocog Alfa for Prophylaxis and Treatment of Bleeding Episodes in Patients with Severe Haemophilia A: Results from the Guardian ${ }^{\mathrm{TM}_{2}}$ Trial

\section{R. Kolla\#, S. Lejniece, M. Martin-Salces, I. Matytsina, I. Rageliene, A. Rosholm, E. Santagostino}

Email ID for Correspondence: roko@novonordisk.com Novo Nordisk India Private Limited

Introduction and Background: The long-term safety and efficacy of turoctocog alfa, a B-domain-truncated recombinant factor VIII product for the prevention and treatment of bleeds in previously treated patients with severe haemophilia $\mathrm{A}$, is currently being investigated in the guardian ${ }^{\mathrm{TM}_{2}}$ trial. This open-label, non-controlled, international clinical trial is an extension to a pivotal trial in adolescents or adults 
(12-65 years; guardian $\left.{ }^{\mathrm{TM}} 1\right)$ and in children ( $<12$ years; guardian $^{\mathrm{TM}} 3$ ). Patients from pharmacokinetics trials could also enter guardian ${ }^{\mathrm{TM}} 2$. This analysis evaluated new long-term safety data from guardian $^{\mathrm{TM}} 2$ including 14 patients enrolled into an on-demand subtrial. Patient/Material and Methods: Patients with severe haemophilia A without inhibitors to FVIII $(<0.6$ Bethesda units [BU] $)$ were enrolled and received turoctocog alfa prophylactically (20-50 IU kg-1 every second day or $20-60 \mathrm{IU} \mathrm{kg}^{-1}$ three times weekly $\mathrm{n}=199)$ or on-demand $\left(20-200 \mathrm{IU} \mathrm{kg}^{-1} ; \mathrm{n}=14\right)$. The primary endpoint was inhibitor development ( $\geq 0.6 \mathrm{BU})$. All adverse events (AEs) were reported. Results: This analysis involved 213 patients [mean (SD) patient age was 23.6 (14.6) years] with a total exposure of 95,972 days. No patient developed FVIII inhibitors $(\geq 0.6$ $\mathrm{BU})$ during this extension period. Adverse events were reported in $84 \%$ of patients (1.8 AEs per patient year of exposure). In $14.6 \%$ of patients, there were 38 serious AEs, including two deaths (subdural haemorrhage and hepatic cancer), all of which were judged unlikely to be related to turoctocog alfa. Eight AEs (all mild or moderate) in five $(2.3 \%)$ patients were considered possibly/probably related to turoctocog alfa ( 0.013 per patient year of exposure). No hypersensitivity or allergic reactions to turoctocog alfa and no other safety concerns were reported. Conclusions: This analysis from the guar$\operatorname{dian}^{\mathrm{TM}} 2$ trial supports the long term (over 5 years for some patients) use of turoctocog alfa in previously treated patients with severe haemophilia A. No inhibitor development and no other safety concerns were observed.

\section{BHC 3}

Successful Use of rFVIII, Turoctocog Alfa, During Orthopedic and Non-orthopedic Surgery in Patients with Severe Hemophilia A

S. Shankar", L. Solimeno, S. R. Lentz, I. Matytsina, N. Zeuthen, F. A. Karim, E. Santagostino

Email ID for Correspondence: ssqq@novonordisk.com

Novo Nordisk India Private Limited

Introduction and Background: Turoctocog alfa was found to be safe and effective in a limited number of patients with hemophilia A undergoing surgery. This analysis reports new data on the safety and efficacy of turoctocog alfa in patients with severe hemophilia A undergoing surgery in the ongoing guardian ${ }^{\mathrm{TM}} 2$ clinical trial (interim cut-off date, 31 December 2013). Hemostatic outcomes were assessed according to a predefined 4-point scale: excellent, good, moderate, none. Patient/Material and Methods: A total of 57 procedures were performed in 40 patients aged 2-57 years with severe hemophilia A and no history of inhibitors. Before, during, and after surgery, turoctocog alfa dosing was at the investigators' discretion. Only 1 patient received continuous infusion during surgery. In 12 major ( 9 orthopedic) and 3 minor surgeries on 15 patients (aged 6-56 years) data on hemostatic outcomes were collected during and after surgery ( 5 of the major surgeries have been presented previously). In 42 other surgical procedures (those requiring $<7$ days of surgery-related treatment with turoctocog alfa) on 31 patients (aged 2-57 years), data were collected during the procedure only. Results: Turoctocog alfa provided successful (defined as 'excellent' or 'good' hemostatic outcome) coverage in 15/15 (100\%) major or minor surgeries. Successful outcomes were reported in 41/42 (98\%) other surgical procedures; 1 endoscopy procedure was not provided with a rating. None of the patients developed FVIII inhibitors during the surgery period, and no safety concerns were observed when reviewing adverse events, consumption of turoctocog alfa, blood loss, hemoglobin levels, or use of bloodproduct transfusions. Conclusions: The success rate for hemostatic response during both major and minor surgeries was $100 \%$. No inhibitors or other safety concerns were identified. Turoctocog alfa has a favorable efficacy and safety profile that makes it suitable for use in patients with severe hemophilia A undergoing surgery.

\section{BHC 4}

\section{Diltiazem Induced Pancytopenia}

\section{Saurabh Nigam", Abhishek Agrawal, Prof. Ravi Tandon}

Email ID for Correspondence: hazard107.sn@gmail.com IMS, BHU, Varanasi

Introduction and Background: Diltiazem is a non-dihydropyridine calcium channel blocker used in the treatment of hypertension, angina pectoris and some types of arrhythmias. Common side effects are headache, dizziness, peripheral edema etc. Rare ones are photosensitivity, thrombocytopenia, haemolytic anemia etc. Pancytopenia is found among people $(<1 \%)$ who take Diltiazem Hydrochloride, especially for the elderly people ( $>60 \mathrm{yrs}$ ) predominantly in males, and is considered to be the rare side effect. The presenting patient is female who was consuming Diltiazem $(180 \mathrm{mg} / \mathrm{day})$ for the 6 month period and presented as a case of pancytopenia. Patient/Material and Methods: 45 Year old Hindu female presented to us with the chief complaints of multiple ecchymotic patches all over the body for the past 10 days associated with generalized weakness and easy fatigability for past 2 months. The patient was taking Diltiazem $90 \mathrm{mg}$ twice daily for 6 months for the treatment of Atrial fibrillation. On examination-pallor + , multiple ecchymotic patches, no organomegaly. On investigations-CBC-TLC-2400, $\mathrm{Hb}-7 \mathrm{gm} \%$, platelet10,000 cells/dl, Retic count-1\%. Bone marrow examination-Trilineage depression. Patient was managed conservatively and Diltiazem was stopped. Investigation review after 6 weeks revealed complete. Bone marrow recovery with normal peripheral counts. Results: Various case series show that Diltiazem causes pancytopenia in $<1 \%$ cases. Our patient presented with this rare side effect. Pancytopenia was judged to be due to Diltiazem after ruling out other possibilities. She got improved after stopping the drug for 6 weeks in terms of counts, bone marrow picture and clinical perspective. Conclusions: Diltiazem can cause Pancytopenia through Bone Marrow Suppression. It is a rare side effect. Drug discontinuation usually leads to complete recovery. Henceforth it is advised all the patients going under treatment with Diltiazem should be haematologically monitored periodically.

\section{BHC 5}

Rituximab in Chronic ITP: Experience at a Tertiary Care Centre in Eastern India

\section{Sumit Mitra ${ }^{\#}$, Maitreyee Bhattacharyya}

Email ID for Correspondence: sumitsummit@rediffmail.com Institute of Hematology and Transfusion Medicine

Introduction and Background: Immune thrombocytopenic purpura is thought to be mediated by autoantibodies against platelet surface glycoproteins. Rituximab, a chimeric anti-CD20 monoclonal antibody, is useful in the treatment of these patients. Patient/Material and Methods: We report the result of Rituximab treatment in twenty four adult patients of refractory and relapsed disease during the period of August 2015 through July 2016. All patients have been treated with one to five different regimens and three had undergone splenectomy. Rituximab was administered at a standard dose of $375 \mathrm{mg} / \mathrm{m}^{2}$ once weekly for four infusions. Results: The overall response rate was $37.5 \%$. Five patients $(20.9 \%)$ achieved CR (Platelet $\left.>100 \times 10^{9} / \mathrm{l}\right)$ 
and four patients $(16.6 \%)$ had a partial response $\left(>50 \times 10^{9} / 1\right) .15$ patients did not respond. The median time to response was seven weeks (range 4-8 weeks) after first infusion. Two patients who had undergone splenectomy responded to Rituximab. Four patients $(16.7 \%)$ have remained in remission. This includes two CR and two PRs after three to ten months of follow up. Conclusions: Rituximab has low efficacy and short duration of remission in Indian population. This needs confirmation by a larger study.

\section{BHC 6}

\section{A Rare Case of Disseminated Histoplasmosis Presenting as Pancytopenia in an Immunocompetent Individual}

\section{Abhishek Purohit, Shashi Bansal, Arpita Mathur", Upendra Sharma}

Email ID for Correspondence: arpitamathur30@gmail.com Bhagwaan Mahaveer Cancer Hospital and Research Centre

Introduction and Background: Histoplasma capsulatum is a systemic fungal infection and usually seen in immunocompromised patients. The presence of disseminated histoplasmosis in an immunocompetent adult is rare. We present here a case of disseminated histoplasmosis, diagnosed incidentally on bone marrow examination. Patient/Material and Methods: A 52 year male patient presented with history of recurrent fever, anorexia and weight loss since two and a half months. On general physical examination, hepatosplenomegaly was found. Complete blood count revealed pancytopenia. When subjected to bone marrow aspiration, smear showed many intracellular and extracellular yeast like organism with pericellular halo. Perl's stain on bone marrow aspiration smear and PAS stained bone marrow biopsy confirmed the diagnosis. Results: Bone marrow aspirate showed intracellular and extracellular oval globose yeast like cells measuring approximately $3-4 \mu \mathrm{m}$ in size suggestive of Histoplasma capsulatum. These cells gave a positive Prussian blue reaction on Perl's staining. PAS stain done on bone marrow biopsy revealed these yeast-like cells as bright eosinophilic structures. Hence, the diagnosis of disseminated histoplasmosis was established. The patient was thus treated with Amphotericin B and eventually responded to the treatment. Conclusions: Disseminated histoplasmosis is a less common manifestation and mainly infect immunocompromised individuals. A high index of clinical suspicion is required to diagnose disseminated histoplasmosis in an immunocompetent patient. Timely diagnosis is important because it is associated with a high mortality.

\section{BHC 7}

\section{Etiological Spectrum of Anemia in People Aged 60 Years and Above}

\section{Rajat Jain", Mrinalini Kotru, Ashish Goel, Meera Sikka, Satendra Sharma}

Email ID for Correspondence: drrajatjain1985@gmail.com University College of Medical Sciences

Introduction and Background: Anemia in the elderly is currently being widely studied. According to various studies the prevalence of anemia in the elderly more than 65 years is around $10 \%$ and rises to $50 \%$ in those above 80 years of age $(2,3)$. Studies on Indian pop- ulation is not available. This study was done with the aim to determine the frequency of different types of anemia in people aged $60 \mathrm{yrs}$ and above. Patient/Material and Methods: Prospective study conducted on 75 patients visiting Department of Medicine UCMS and GTBH. Informed consent with detailed clinical data was taken and patients were subjected to $\mathrm{CBC}$ with peripheral smear, serum iron studies, folic acid and vitamin B12 levels. Results: Median age of the study group was 70 years. $45(60 \%)$ were men and $30(40 \%)$ women. Patients were clinically worked up and had varied clinical presentation such as infectious diseases $35(46.6 \%)$, acute coronary syndrome $9(12.2 \%)$, chronic lung disease $7(9.5 \%)$, anemia 5 $(6.8 \%)$ and non-hematological malignancy $2(5.7 \%)$. Anemia was also incidentally detected in the follow up of patients with various comorbid diseases like DM, HT and CAD. Miscellaneous category consisted of patients with chronic liver and kidney disease and gastrointestinal disorders. Nutritional anemia $(50.6 \%)$ was the most common cause followed by anemia of chronic disease $(48 \%)$ and unexplained anemia $(21.3 \%) .1 .3 \%$ patient had anemia of kidney disease. Amongst the nutritional anemia, folic acid deficiency emerged as the commonest cause present in $25.3 \%$ of our patients. Studies in west found that nutritional anemia, anemia of chronic disease and unexplained anemia were all present in equal 1/3rd proportion in their population in contrary our study shows nutritional anemia to be more prevalent. Conclusions: To conclude nutritional anemia was the most common cause. Folic acid deficiency was the most common cause of nutritional anemia.

\section{BHC 8}

Low Dose Escalating Factor Concentrate Prophylaxis in Patients with Severe Hemophilia in a Comprehensive Hemophilia Care Centre of Western India

Aniket B. Mohite", S. Chandrakala, W. Nilesh, Farah Jijina, P. Vinod

Email ID for Correspondence: lifelineaniket@gmail.com Dr. J C Patel Department of Hematology, KEM Hospital, Mumbai

Introduction and Background: In absence of any epidemiological studies, it is expected that there are at least 1 lakh PWH in our country. ${ }^{1-5}$ Due to lack of awareness, diagnostic as well as treatment facilities, many of the patients are on cryoprecipitate/FC, as episodic treatment only. Patient/Material and Methods: All severe hemophiliacs between 1-6 years were screened for inclusion and exclusion criteria and then enrolled in study after informed consent from parents. All patients were assessed for bleeding history, HJHS, FISH and CHAQ at baseline and every 3 monthly. At each visit patients were observed for break-through bleeding, treatment taken. Parents were trained for infusion training. If on prophylaxis, patient had $\geq 1$ spontaneous breakthrough bleeds at any time period, dose was increased to 15 unit $/ \mathrm{kg}$ twice weekly and if patient still had bleed then it was escalated till maximum of $30 \mathrm{unit} / \mathrm{kg}$ twice weekly. Results: $11.1 \%$ on secondary prophylaxis and $88.9 \%$ on primary prophylaxis. Three patients developed breakthrough bleed. $77.97 \%$ reduction in AJBR, $70.8 \%$ in ABR, $80 \%$ in school absenteeism and CHAQ$83 \%$ improvement. $50 \%$ patients on home-therapy. The study had $100 \%$ compliance. The average dose of factor required 13.29 unit/ $\mathrm{kg} /$ patient/dose and 1382.96 unit/kg/patient/year. Conclusions: Prophylaxis with low dose $\mathrm{FC}$ with escalation protocol can decrease the frequency of joint and other hemorrhages, reduce school absenteeism and prevent joint damage. 


\section{BHC 9}

\section{Sickle Cell Crisis Mimicking Tumour Lysis Syndrome}

\section{Satvik Khaddar ${ }^{\#}$, Prashant Chhabra}

Email ID for Correspondence: satvikkhaddar@hotmail.com Institute of Medical Sciences, Banaras Hindu University

Introduction and Background: Homozygous sickle cell anemia usually presents as sickle cell crisis at young age, usually around $2-10$ years and very rarely in adulthood. Sickle cell trait is largely asymptomatic, rarely painless hematuria may be the only symptom. We present a case of a 19 year old boy who presented for the first time with sickle crisis with his clinical profile mimicking tumor lysis syndrome. Patient/Material and Methods: A 19 year boy presented with fever and generalized body pain for 15 days, yellowish discoloration of eyes and urine for 5 days, decreased urine output for 3 days and breathlessness and altered sensorium for last 2 days. There was no history of bleeding, previous similar episodes or familial predisposition. There was no lymphadenopathy, liver and spleen were mildly enlarged. His $\mathrm{Hb}$ was $4.3 \mathrm{~g} / \mathrm{dl}$, TLC $-1.38 \mathrm{lacs} / \mathrm{mm}^{3}$ with many precursors along with deranged LFT, RFT, hyperkalemia, hyperuricemia and hypocalcaemia with negative paracheck, leptocheck and viral screen. Results: The initial clinical picture was suggestive of acute leukaemia with tumour lysis syndrome. However, his GBP and bone marrow analysis, done few days after stabilization, revealed multiple sickle shaped cells with nucleated RBCs, high reticulocyte count and hypercellular marrow with erythroid hyperplasia respectively. Thus, a HPLC was performed which showed sickle cell trait pattern. Meanwhile patient was managed with haemodialysis, blood support and other conservative measures. Conclusions: Sickle cell syndromes are remarkable for their clinical heterogeneity. Some patients remain virtually asymptomatic into or even through adult life, while others suffer repeated crises requiring hospitalization from early childhood. Sickle cell trait is virtually asymptomatic that is why adult age, very high leukocyte counts with lab parameters of tumour lysis were misleading points in our case. However, GBP and Bone marrow aspiration after patient stabilization helped to reach correct diagnosis.

\section{BHC 10}

Outcome of Acquired Aplastic Anemia Treated with Indigenous Equine Antithymocyte Globulin (Thymogam) Along with Cyclosporine at Gujarat Cancer Research Institute (GCRI): A Regional Centre Experience

Preetam Kumar Jain", Harsha P. Panchal, Asha S. Anand, Apurva A. Patel, Sonia K. Parikh, Kalpesh Prajapathi, Gaurang Modi, Kamlesh Shah, Kinnari A. Patel, Sandip A. Shah

Email ID for Correspondence: pkgurjar@gmail.com Gujarat Cancer Research Institute, Ahmedabad, India

Introduction and Background: Acquired Aplastic anemia (AA) is a life threatening stem cell disorder. Hematopoietic stem cell transplant (HSCT) and immune suppressive (IST) are the primary modalities of treatment for AA. HSCT is the treatment of choice in young adults, but unfortunately in India, is limited due to the cost, lack of donor and limited resources availability. IST plays a major role in the treatment of AA who are not transplant candidates. Studies have shown encouraging results with Anti thymocyte globulin (ATG) and cyclosporine, improving the quality of life and survival. We report a retrospective study and the outcome of indigenous equine Anti
Thymocyte globulin (e ATG) with cyclosporine from January 2012 to December 2015. Patient/Material and Methods: A retrospective analysis of 91 subjects (adults and paediatrics) was done at GCRI who received IST. The e-ATG (Thymogam) is manufactured by Bharat serum and vaccines Ltd. All the standard investigations were done. Subjects with Fanconis anemia, history of cancer, pregnant women, HIV positive were not included in the study. The subjects received e ATG at a dose of $40 \mathrm{mg} / \mathrm{kg} / \mathrm{day}$ for 4 days and Cyclosporine $(5 \mathrm{mg} /$ $\mathrm{kg} / \mathrm{day}$ ) and methyprednisolone. Results: Out of 91 (68 adults, 23 children) subjects, 60 were male and 31 were female. The median age of adults was 31 years and children was 9 years. The number of very severe AA, severe AA, non severe AA were 30,57 and 4 , respectively. Total 63 (46 adults and 17 children) were alive, 28 (22 adults and 6 children) died. The overall response rate was $68.1 \%$ out of 91 subjects with 25, 37 and 1 subjects had CR, PR and NR respectively. 17 ( $9 \mathrm{CR}, 7 \mathrm{PR}$ and $1 \mathrm{NR}$ ) out of 23 childrens were alive after 2 years. 62 subjects enjoyed the transfusion independent life. The median response time was 6 months. The most common cause for deaths were intracerebral bleed, pneumonia and sepsis. One patient died of renal failure. Two patients had clonal evolution into acute leukemia. Conclusions: The indigenous e-ATG is safe and equally comparable with the innovator drug as a first line therapy in acquired AA who are not candidates for HSCT. It is cost effective, especially for the Indian population.

\section{BHC 11}

Mean Platelet Volume in Patients with Acute Coronary Syndromes: A Supportive Diagnostic Predictor

\section{Randheer Pal, Rajeev Bagarhatta, Sandhya Gulati,} Nidhi Sharma, Monika Rathore, Sarjana ${ }^{\#}$

Email ID for Correspondence: sarjana.soni@gmail.com SMS Medical College

Introduction and Background: Acute coronary syndrome is one of the leading cause of the morbidity and mortality. The diagnostic efficiency of cardiac troponins within 2 to $4 \mathrm{hr}$ of the symptom onset is limited so other laboratory tests that reduce delays in provision of immediate care for cardiac pain are required in conjunction with established markers. Platelets and their activity have a crucial role in acute coronary events. Larger platelets are enzymatically and metabolically more active and have a higher potential thrombotic ability as compared with smaller platelets. The aim of this study is to investigate whether there is an association between mean platelet volume (MPV) measurement and cardiac Troponin I (cTn I) in patients admitted with a suspected diagnosis of ACS and to assess the potential diagnostic efficiency of MPV in the diagnostic workup for ACS. Patient/Material and Methods: After thorough evaluation of 215 eligible patients, $3 \mathrm{ml}$ Venous blood collected using Becton, Dickinson and company vacutainer and MPV measured within 1-2 hr of sample collection. Sample for cTn I collected at $6 \mathrm{hr}$ and at $12 \mathrm{hrs,}$ if required and level measured using Biosite analyzer. Results: Mean platelet volume (MPV) was found to be higher among ACS patients as compared to non ACS, $11.44 \pm 1.23$ vs. $9.91 \pm 1.27 \mathrm{fl}$ (pvalue $<0.001)$. The NPV of MPV in the diagnostic workup of chest pain suggestive of ACS within 6 hours of presentation were found to be $82.53 \%$. Conclusions: In this study the MPV is significantly higher in patients with ACS than in those with chest pain of noncardiac origin and its negative predictive value of $82.53 \%$, it might be useful as an assisting rule-out test in conjunction with other markers in the early prediction of the risk of ACS. 


\section{BHC 12}

Evaluation of Knee Joint Damage in Hemophilia A Using Ultrasonography and Its Correlation with Clinical Parameters

\section{Arijit Nag\#, Debanjali Sinha, Sumantro Mondal, Siddhartha Shankar Roy, Maitreyee Bhattacharyya}

Email ID for Correspondence: coolwriju@gmail.com Institute of Hematology and Transfusion Medicine, Kolkata

Introduction and Background: India has the second highest global burden of Hemophilia A. $90 \%$ of severe Hemophilia A patients have arthropathy. Ultrasonography has emerged as a novel imaging modality in the assessment of Hemophilic arthropathy. Our study aims to evaluate knee joint damage in Hemophilia patients by Ultrasonography and correlate with clinical parameters. Patient/ Material and Methods: The study included 27 patients of Hemophilia A (Moderate and Severe). Patients with an acute bleed in the past 1 month were excluded. Relevant clinical parameters were collected. Ultrasonography was done of both their knees. Cartilage (average of medial, central and lateral compartments) and synovial thickness were measured and presence of any vascularity or intra-articular collection noted. Similar parameters in 27 age-matched male controls were compared. Correlation study was done between cartilage thickness and the above clinical parameters. Results: Of the Hemophilia A patients, 9 had moderate and 18 had severe disease. The mean age of the patients was 16.57 years. The mean cumulative bleeds in the Right and Left knee joints were 47.44 and 33.56 respectively. There was significant increase in synovial thickness and decrease in mean cartilage thickness in the patients across all age bands (Table 1). There was no significant co-relation between either the Cartilage thickness and, the age, disease duration, severity of haemophilia, the total number of clinically-evident joint bleeds or treatment received. $22.22 \%(6 /$ 27) patients had evidence of intra-articular blood collection despite being asymptomatic. Conclusions: Premature knee cartilage degeneration occurs in Hemophilic patients, irrespective of the age, severity, number of bleeds or treatment. Ultrasonography can also detect subclinical bleeds that may affect articular outcome in Hemophilia.

\section{BHC 13}

Bone Marrow Transplant for Recessive Dystrophic Epidermolysis Bullosa: A Case Report

Vipin Khandelwal", Dharma Choudhary, Gaurav Kharya, Sanjeev Sharma, Divya Doval, Bharti Sharma, Rasika Setia, Anil Handoo

Email ID for Correspondence: khandelwal_vipin@yahoo.com BLK Superspeciality Hospital

Introduction and Background: To analyze the effectiveness of Bone Marrow Transplant (BMT) for Recessive Dystrophic Epidermolysis Bullosa (EB). Patient/Material and Methods: A 2-year-old girl from Iraq a case of recessive dystrophic EB underwent matched sibling donor BMT after written consent from parents. On admission she had bullous lesions all over body and multiple ulcers in the mouth causing difficulty in swallowing food. Pretransplant work up done for donor and patient was normal. Conditioning chemotherapy was started after inserting hickman catheter and Percutaneous endoscopic gastrostomy (PEG) tube. It included busulfan $(0.8 \mathrm{mg} / \mathrm{kg}$ intravenously every 6 hours on days -9 to -6$)$, Fludarabine $\left(25 \mathrm{mg} / \mathrm{m}^{2} /\right.$ day, given intravenously on days -5 to -3 ), and cyclophosphamide $(50 \mathrm{mg} /$ $\mathrm{kg} / \mathrm{day}$, given intravenously on days -5 to -2 ). Graft versus host disease (GVHD) prophylaxis consisted of cyclosporine on day 3 before transplantation to day 100 after transplantation, with the dose tapered by $10 \%$ each week thereafter) and Mycophenolate Mofetil $10 \mathrm{mg} / \mathrm{kg} /$ dose three times day on day -3 to day 45 after transplantation. Results: She received Bone Marrow harvested stem cells from her sister with CD34 positive cells dose $2.32 \times 106 / \mathrm{kg} / \mathrm{L}$. Polymorph nuclear cell and platelet engraftment were seen on D+17 and D+19 respectively. She required intensive skin dressings every 3rd day and her nutrition was supported by PEG tube feeding regularly. She was discharged on day +26 with stable vitals on oral medications. Her disease status was assessed by detailed clinical examination at regular interval and number of bandages used per week for dressing. She had increased wound healing and decreased mucocutaneous blistering by day +40 . The percentage of body surface area affected was reduced according to parents and by clinical observation, with more objective evidence provided by documented reductions in bandage use. Her whole blood chimerism on day +21

Table 1

\begin{tabular}{llllll}
\hline & $\begin{array}{l}\text { Mean synovial } \\
\text { thickness }( \pm \text { SD })\end{array}$ & P-value & $\begin{array}{l}\text { Rt. Mean cartilage } \\
\text { thickness }( \pm \text { SD })\end{array}$ & P-value & $\begin{array}{c}\text { Lt. Mean cartilage } \\
\text { thickness }( \pm \text { SD })\end{array}$ \\
\hline $\begin{array}{l}\text { Patients } \\
0-10 \mathrm{y}\end{array}$ & $0.27( \pm 0.13)$ & 0.0379 & $0.20( \pm 0.08)$ & 0.0006 & $0.19( \pm 0.08)$ \\
$\begin{array}{l}\text { Control } \\
0-10 \mathrm{y}\end{array}$ & $0.12( \pm 0.02)$ & & $0.45( \pm 0.06)$ & $0.46( \pm 0.07)$ \\
$\begin{array}{l}\text { Patients } \\
11-20 \mathrm{y}\end{array}$ & $0.25( \pm 0.09)$ & 0.0383 & $0.24( \pm 0.12)$ & 0.0005 & $0.26( \pm 0.14)$ \\
$\begin{array}{l}\text { Control } \\
11-20 \mathrm{y}\end{array}$ & $0.18( \pm 0.04)$ & & $0.41( \pm 0.05)$ & $0.36( \pm 0.09)$ \\
$\begin{array}{l}\text { Patients } \\
>20 \mathrm{y}\end{array}$ & $0.33( \pm 0.08)$ & 0.0022 & $0.21( \pm 0.12)$ & 0.0159 & $0.22( \pm 0.05)$ \\
$\begin{array}{l}\text { Control } \\
>20 \mathrm{y}\end{array}$ & $0.20( \pm 0.02)$ & & $0.32( \pm 0.04)$ & & $0.32( \pm 0.04)$ \\
\hline
\end{tabular}

[All measurements in $\mathrm{cm}$ ] 
and day +50 showed $99.35 \%$ and $98.86 \%$ of donor cells respectively. However she had Grade II gut GVHD on day +68 which was managed with intravenous methylprednisolone. After day +100 her improvement in skin lesions was static. Presently, she is day +267 post transplant not completely cured but the frequency of eruption of bullous lesion has decreased and healing of these lesions had significantly improved. Conclusions: Allogeneic BMT can partially correct the collagen type VII deficiency and improve skin and mucosal integrity in patients with recessive dystrophic EB.

\section{BHC 14}

\section{Incidental Detection of Hereditary Persistence of Fetal} Hemoglobin (HPFH) in Father of $\beta$-Thalassemia Major Patient

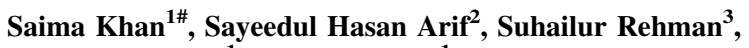 \\ Asim Israr Khan ${ }^{1}$, Aeman Khalid ${ }^{1}$ \\ Email ID for Correspondence: saimakhan.1501@gmail.com \\ ${ }^{1}$ Junior Resident, ${ }^{2}$ Professor, ${ }^{3}$ Senior Resident, Department of \\ Pathology, Jawaharlal Nehru Medical College, Aligarh Muslim \\ University, Aligarh
}

Introduction and Background: Hereditary persistence of fetal hemoglobin (HPFH) is a clinical term applied to conditions which are associated with elevated amounts of synthesis of $\mathrm{HbF}$ in adults in the absence of other hematologic abnormalities. The level of fetal hemoglobin $(\mathrm{HbF})$ in heterozygotes may vary from some $5 \%$ to nearly $40 \%$, while homozygotes might either have only $\mathrm{HbF}$ or less with $20 \%$ to $25 \%$ hemoglobin being the adult $\mathrm{Hb}$. They are derived from point mutations in $\mathrm{A} \gamma$ and $\mathrm{G} \gamma$ promoters. When these mutations are found with $\beta$-thalassemia mutations, they have an ameliorating effect in the disease because of increased production of gamma chains. Patient/Material and Methods: Parents and Siblings of $\beta$ thalassemia major were screened from the OPD and IPD of J.N. Medical College and Hospital, Aligarh. Whole blood samples were taken in EDTA vacutainer. Diagnosis was based on hematological investigations and levels of $\mathrm{HbF}$ were quantified by Alkaline Hemoglobin Electrophoresis pH 8.6. Results: Out of the total 36 cases of $\beta$-thalassemia, all the parents were $\beta$-thalassemia trait carriers. Siblings of 4 patients were thalassemic. In one case, father was detected as HPFH and on alkaline electrophoresis, Hb F level was $20.4 \%$, whereas the mother was a $\beta$-thalassemia trait. Conclusions: As HPFH has deletions in the $\beta$-globin locus, if such a person marries a thalassemia trait, the resultant offspring has higher chance of developing $\beta$ thalassemia major. Hence electrophoresis of father, mother and siblings of such patients are advised for detection of HPFH and thalassemia trait. Although HPFH is a benign, clinically asymptomatic condition it cannot be ignored and should be dealt same as that of Thalassemia trait for marriage and genetic counselling.

\section{BHC 15}

Quantification of Liver Iron Overload: Correlation of MRI Versus Liver Tissue Biopsy in Thalassemia Patients Undergoing Bone Marrow Transplant

\section{Varun Bafna", B. Chandrashekhar, K. S. Nataraj, B. Shobha, Sunil Bhat, Vimal Raj, Sharath Damodar}

Email ID for Correspondence: drvarunbafna6@gmail.com Narayana Hospital, Bangalore

Introduction and Background: The simplest methods available for assessment of body iron levels are biochemical measurements of the serum ferritin concentration. However, serum biochemical tests can be confounded by factors such as infection, inflammation, and malignancy and do not accurately reflect tissue iron levels. The reference method for evaluating the magnitude of body iron load in systemic iron overload is measurement of the liver iron concentration (LIC). The most direct clinical method of measuring LIC is through chemical analysis of needle biopsy specimens ${ }^{6}$. The biopsy sample can also be used for detection of liver fibrosis and cirrhosis, which have important prognostic implications for survival and risk of hepatocellular carcinoma. Here we will report a noninvasive method for the measurement of LICs, computed T2* using clinical magnetic resonance imaging (MRI) instruments. Patient/Material and Methods: We performed a prospective longitudinal study in Thalassemia patients undergoing bone marrow transplant. All the cases underwent MRI T2* by 1.5 Tesla machine of Liver and Heart followed by Liver biopsy for liver iron concentration by atomic absorption spectrometry. Also serum ferritin levels were done by immune-enzymetric assay around that period. And the data analysis was done by the STATA 11.2 software. Results: We studied 23 thalassemia patients who underwent transplant on maintenance hemodialysis of which male were $11(46 \%)$ and female were 12 $(54 \%)$ with mean age was 8.3 years with range $2-18$. There was a significant correlation relationship between the Liver Biopsy LIC with MRI T2* LIC with correlation of 0.768 with significant $\mathrm{P}$ value $<0.001$. Also there was significant correlation of serum ferritin with MRI R2* of 0.5647 with significant $\mathrm{P}$ value 0.005 and with LIC by liver tissue biopsy of 0.66 with significant $\mathrm{P}$ value $<0.001$ but at an individual level-high variance in SF value for a given LIC. There was no correlation between Hepatic Iron overload with cardiac iron overload by MRI. There was more cost effectiveness of MRI T2* as compared to Liver tissue biopsy for LIC with no any complication. Conclusions: There is strong correlation between LIC by non-invasive method and liver tissue biopsy. Serum Ferritin can estimate Iron overload but less reliably than quantitative MRI. In Thalassemia patients transfusion load is more accurate than ferritin. Also there is positive correlation between SF and LIC but at an individual patient level, SF is not good at predicting LIC accurately. Also there is costeffectiveness and safety profile with non-invasive method. In Thalassemia patients, hepatic iron overload is common but Cardiac iron overload is rare and does not correlate with hepatic iron overload.

\section{BHC 16}

Hematopoietic Stem Cell Transplant for Hurler's Disease: A Case Report

Anamika Bakliwal ${ }^{\#}$, Gaurav Kharya, Vipin Khandelwal, Divya Doval, Sanjeev Kumar Sharma, Dharma Chaudhary, Anil Handoo, Rasika Setia

Email ID for Correspondence: anamikabakliwal@yahoo.in Department of Hematoncology and Bone Marrow Transplant, BLK Super Specialty, Hospital, New Delhi

Introduction and Background: Mucopolysaccharidosis (MPS I) is a lysosomal storage disorder inherited as an autosomal recessive condition, caused due to deficiency of the lysosomal enzyme alphaiduronidase. Patients affected with MPS I are unable to degrade the glycosaminoglycan's, dermatan sulphate and heparin sulphate, which provide structural support to the extracellular matrix and cartilaginous structures such as joints and valves. Patient/Materials and Methods: Two year old infant diagnosed as a Hurlers disease at 14 months of age was taken up for mismatched unrelated donor (A locus mismatch) in the absence of suitable matched family donor. He received enzyme replacement therapy at weekly interval, starting 4 weeks prior to HSCT till the molecular engraftment ( 7 doses). He was conditioned with thymoglobulin@ $2.5 \mathrm{mg} / \mathrm{kg} /$ day; Fludarabine @ $40 \mathrm{mg} / \mathrm{m}^{2} / \mathrm{day}$; 
and Busulfan $3.2 \mathrm{mg} / \mathrm{kg} /$ day. GVHD prophylaxis was given as cyclosporine @ $3 \mathrm{mg} / \mathrm{kg}$ in two divided doses staring from day-3 and methotrexate $15 \mathrm{mg} / \mathrm{m}^{2}$ on day $+1,10 \mathrm{mg} / \mathrm{m}^{2}$ on day $+3,+6$ and +11 . Mycophenolate mofetil was added from day +15 onwards in view of single antigen mismatch. He received unmanipulated non-cryopreserved GCSF mobilized peripheral blood stem cell with CD34+ cell dose of 10 million cells $/ \mathrm{kg}$. Results: He had a robust engraftment with neutrophil count exceeding $>500$ on day +11 and platelet engraftment on day +12 . He was successfully discharged on day +14 . His post BMT donor chimerism is $99.76 \%$ and his alpha-iduronidase activity has gone up to $41.54 \mathrm{mmol} / \mathrm{hr} / \mathrm{mg}(26.07 \mathrm{mmol} / \mathrm{hr} / \mathrm{mg})$. Clinically majority of his signs and symptoms pertaining to Hurlers are resolving. His post BMT course was complicated by acute skin GVHD appearing on day +21 which was managed by steroids and CMV reactivation $(8978 \mathrm{cp} / \mathrm{ml})$ on day +27 and was treated with IV Ganciclovir followed by oral valganciclovir. Conclusion: HSCT is the only curative treatment for Hurlers disease. Pre transplant disease burden and post-transplant alpha-iduronidase activity are important predictors to decide long term outcome in hurlers disease.

\section{BHC 17}

Hereditary Haemolytic Anaemia Caused by Red Cell Pyrimidine 5 ' Nucleotidase Deficiency Association with Gilbert's Syndrome: A Clinico-genetic Study

\section{Prashant Warang\#, Prabhakar Kedar, Rati Devendra, Ashish Chiddarwar, Vinod Gupta, Malay Mukherjee}

Email ID for Correspondence: prashantw97@ rediffmail.com National Institute of Immunohaematology (ICMR), 13th Floor, New M.S.Bldg. K.E.M Hospital Campus, Parel, Mumbai 400012

Introduction and Background: Hereditary Pyrimidine $5^{\prime}$-nucleotidase deficiency is a rare autosomal recessive disorder of red blood cell nucleotide metabolism that causes hereditary non-spherocytic haemolytic anemia (HNSHA). The disease is usually characterized by mild to severe haemolytic anemia, reticulocytosis and hyperbilirubinemia. Here we presented three cases of HNSHA caused by red cell $\mathrm{P} 5 \mathrm{~N}-1$ deficiency and correlated this with the clinical profile, molecular and structural-functional abnormality. Patient/Material and Methods: We investigated 3 cases of HNSHA with recurrent Jaundice in the age group of 20 yrs to $63 \mathrm{yrs}$. All of them had anemia $(\mathrm{Hb} 6.7 \mathrm{~g} / \mathrm{dl}$ to $11.0 \mathrm{~g} / \mathrm{dl})$, reticulocytosis $(4.5 \%$ to $14 \%)$, indirect hyperbilirubinemia $(2.64$ to $6.89 \mathrm{mg} / \mathrm{dl})$, and splenomegaly. Their LDH levels were high with significantly low heptoglobin level. Of these, two cases had a history of blood transfusions and one of them had received more than 30 unit of blood and had liver transplantation. Standard methods were used to investigate the cause of hereditary hemolytic anemia and hyperbilirubinemia. Results: Peripheral blood smear showed the presence of marked basophilic stippling in all the three cases. Haemoglobin analysis by HPLC showed no evidence of hemoglobinopathies. Flow cytometric analysis of red cell membrane protein using eosin-5-maleimide dye showed the absence of red cell membrane protein defect. DCT and ICT were negative while PNH was normal. TA repeats promoter polymorphism in UGT1A1 gene showed the presence of Gilbert's syndrome in heterozygous state. G6PD deficiency was found in one case and molecular characterization showed the presence of G6PD Ashahi (202G- $\rightarrow$ A, Val68 $\rightarrow$ Met) mutation. Other red cell enzymes such as Pyruvate Kinase (PK) and Glucose phosphoisomerase (GPI) activity were normal. Purine/ Pyrimidine nucleotidase ratio was significantly reduced $(1.31 \pm 0.18)$ as compared with normal $(3.0 \pm 0.52)$ suggest the presence of $\mathrm{P}^{\prime} \mathrm{N}-1$ deficiency in all three cases. Molecular analysis showed the presence of two novel mutations, Glutamine 226- $\rightarrow$ Stop codon and Lysine $143 \rightarrow$ Arginine splice site mutation. Molecular modelling suggests that, this stop codon and splice site mutation have a significant effect on the protein structure and stability leading to severe clinical presentation. Conclusions: $\mathrm{P}^{\prime} \mathrm{N}-1$ deficiency is not uncommon and important cause of chronic haemolytic anaemia and needs to be investigated in detail including the molecular basis of this enzyme deficiency in Indian population.

\section{BHC 18}

Thrombotic Thrombocytopenic Purpura: A Case Series from a Tertiary Care Centre in Northern India

Sanjeev Kumar Sharma", Dharma Choudhary, Rasika Setia, Satyam Arora, Gaurav Kharya, Vipin Khandelwal, Anil Handoo, Divya Doval, Kirti Pessi, Tina Dadu, Anamika Bakliwal, Gaurav Dhamija

Email ID for Correspondence: sksanjeev13@yahoo.com BLK Superspeciality Hospital, New Delhi, India

Introduction and Background: Thrombotic thrombocytopenic purpura (TTP) is a disorder characterized by microangiopathic hemolytic anemia and thrombocytopenia associated with fever, renal dysfunction and neurological manifestations. Patient/Material and Methods: We report here ten cases of TTP who were treated at our hospital in last 4 years. Six patients developed features of TTP post allogenic stem cell transplantation. Case 1: A 48 years-old woman presented to the medical emergency with altered sensorium for 1 day and on evaluation, was found to have thrombocytopenia and schistocytes in peripheral blood. Case 2: A 56 years-old woman was being treated as immune thrombocytopenia elsewhere and was referred to our centre as she was not recovering. She had received intravenous methylprednisolone there for 3 days but platelets were less than $10 \times 10^{9} / 1$. Case 3: A patient on evaluation for fever was diagnosed to have brucellosis and was treated for that. His fever subsided and he remained afebrile for 3 weeks when again he developed fever with decreased vision (retinal hemorrhages). Case 4: A 25 years-old male was diagnosed as a case of ankylosing spondylitis (HLA B27 positive) four years back. He had received steroids off and on during exacerbation of backache. Cases 5 to 10: Six patients developed TTP following allogenic hematopoietic stem cell transplantation. Results: All patients with classic TTP recovered with plasma exchange and/or rituximab. Post-transplant TTP carries a poorer prognosis as five out of six post-transplant TTP patients died. Conclusions: Diagnosis of TTP requires a high index of suspicion and prompt treatment with plasma exchange, which results in a high cure rate. Rituximab is useful in patients relapsing or showing partial recovery. Post-transplant TTP may be due to interplay of multiple factors following transplant including conditioning chemotherapy induced endothelial injury, GVHD, calcineurin inhibitors and infections. It carries poor prognosis compared to TTP in general population. PEX has not been reported to be effective in post-transplant TTP.

\section{BHC 19}

\section{Coagulation Profile in Liver Diseases}

Gautam Bhatia", Rajnish Kumar, Seema Acharya, Sanjeev Kishore, Brijesh Thakur, Amit Varma

Email ID for Correspondence: jstmee6@gmail.com

Sri Guru Ram Rai Institute of Medical and Health Sciences, Dehradun

Introduction and Background: To study the alteration in coagulation profile in various liver diseases which helps to evaluate the risk of bleeding in patients with liver disease and to study the association of 
coagulation abnormality with the extent of liver disease. Patient/ Material and Methods: The study was conducted on 300 patients with liver diseases attending Internal Medicine clinics in a teaching hospital in Dehradun. The patients were divided in three groups: (1) 160 cases of cirrhosis, (2) 70 cases of hepatitis, (3) 70 cases of other liver diseases. Following six coagulation tests were performedProthrombin time (PT), International Normalized ratio (INR), Activated partial thromboplastin time (APTT), Platelet count, Bleeding time and Clotting time. Results: PT was prolonged (mean $=21.83 \pm 4.82 \mathrm{sec})$ in 126 cases out of 300 total cases. APTT was altered (mean $=40.6 \pm 5.34 \mathrm{sec})$ in 156 cases. INR was increased $($ mean $=2.56 \pm 1.2$ ) in 115 cases. Bleeding time and clotting time were deranged in 82 cases. The sex distribution of liver diseases in present study was $73 \%$ males and $27 \%$ females. Conclusions: The study concluded the fact that the coagulation profile was significantly altered in cases of cirrhosis. The alteration of coagulation profile was not significant in cases of hepatitis and other liver diseases. The risk of bleeding was more in cirrhosis with altered coagulation profile. Coagulation abnormalities were significantly associated with the severity of liver disease.

\section{BHC 20}

Allogeneic Hematopoietic Stem Cell Transplantation for $\beta$-Thalassemia Major in the Developing World: Experience from a Center in Western India

H. Balar", B. C. Mahadeva, D. Vaghasiya, A. Raiyani, N. Chaturvedi, D. Patel, H. Parekh, A. Joshi, S. Melinkeri, S. Kannan, R. Phatale, S. Apte

Email ID for Correspondence: hasmukhbalar@gmail.com Sahyadri Speciality Hospital, Pune

Introduction and Background: $\beta$-Thalassaemia major is an important cause of morbidity and premature death in young adults worldwide. The outcome of allogeneic Hematopoietic Stem Cell Transplant (HSCT) compares favorably with medical treatment and suggests that it remains an important treatment and the only cure for $\beta$-thalassaemia major. We report the results of 55 consecutive family related allogeneic-HSCT for children with $\beta$-thalassaemia major performed in our center. Patient/Material and Methods: In this retrospective study data from patients with $\beta$-thalassaemia major, who underwent allogeneic-HSCT at our center from 1999-to-2016 was reviewed under following headings.

1. Patient (Age/sex, Pesaro class)

2. Donor (HLA-match)

3. Transplant (conditioning)

4. GvHD (acute/chronic, grade/treatment/outcome)

5. Complications [infections/Veno-Occlusive-Disaese (VOD)/ CMV]

6. Follow up and overall outcome

Results: Data of 55 patients was analyzed.

- Overall-Survival (OS) was $78.18 \%$ at median 6.6 years of follow-up.

- 12 patients died. 11 due to aGVHD+sepsis and 1 due to VOD.

- OS in patients who had received Bu16+Cy200+HorseATG90 and Flu+Treosulphan+Thiotepa were $76.59 \%$ and $87.50 \%$ respectively.
- OS in patients who had Pesaro class I, II and III was $94.73 \%$, $80.00 \%$ and $61.90 \%$ respectively.

- 32 patients had GVHD, out of them 20 had aGVHD with 7 had Grade IV and 12 had cGVHD with 4 had extensive.

Conclusions: Majority of children with $\beta$-thalassaemia major and HLA-identical family donor, HSCT offer the only chance of cure with the return of life expectancy and quality of life to normal. Analysis of our center data shows a definite improvement in OS of patients who undergo HSCT, parallel to the global trends. OS is better in Flu+Treosulphan+Thiotepa arm. HSCT is however associated with a significant mortality/morbidity that has to be discussed with patients/families before the decision to proceed with HSCT is taken. For middle class socio-economic-status family medical management is far more expensive than allogeneic HSCT.

\section{BHC 21}

Uncommonness of a Common Infection

Vikas Ratnaparkhe ${ }^{1}$, Ravi Patwadkar', Digvijay Gajanan Ekbote ${ }^{3 \#}$, Ajinkya Patil ${ }^{3}$

Email ID for Correspondence: digja259@yahoo.co.in

${ }^{1}$ Professor and Unit Head, Department of General Medicine,

${ }^{2}$ Professor and Unit Head, Department of Pathology,

${ }^{3} \mathrm{Sr}$ Resident, Department of General Medicine,

Dr Hedgewar Rugnalaya, Auranganabad, Maharashtra

A 63 year old male, presented with fever along with chills, dry cough and dyspnea since 5 days. There was no history of rash, severe headache, altered sensorium, seizures or urinary complaints. On examination he was febrile, hemodynamically stable with mild pallor and icterus. There were no palpable lymph nodes, his oxygen saturation was $92 \%$ on room air. On systemic examination, he had bilateral basal coarse crepitations and soft, non-tender hepatosplenomegaly. On laboratory examination, his haemoglobin was $12.1 \mathrm{gm} \%$, leucocyte count of 3000 and platelet count of 59,000. His peripheral smear showed schizonts of $P$. vivax. He had mildly elevated liver enzymes with hyperbilirubinemia of $2.3 \mathrm{gm} / \mathrm{dl}$. His creatinine was $2.1 \mathrm{gm} / \mathrm{dl}$. His chest X-ray showed bilateral basal opacities. He was started on chloroquine and amoxicillin+clavulinic acid. He had persistent fever on day 2 and developed a petechial rash over trunk. Repeat hemogram showed thrombocytopenia of 7000 , TLC of 1300 and Hemoglobin $11 \mathrm{gm} \%$. Dengue IgM and IgG were negative. He was then transfused with single donor platelets. He continued to have fever on day 3, primaquine was started and antibiotic was changed to piperacillin+tazobactam. His rash progressed to lower limbs and thrombocytopenia persisted (6000) with leucopenia (1200) and anaemia (9.5 gm\%). Bone marrow aspiration and biopsy was taken which showed normocellular marrow with proliferation of macrophage cell system and phagocytosis with depleted megakaryocytes; suggestive of hemophagocytic lymphohistiocytosis. His fasting triglyceride levels $(323 \mathrm{mg} / \mathrm{dl})$ and ferritin levels $(1656 \mu \mathrm{g} / \mathrm{l})$ were markedly elevated. He was started with dexamethasone pulse therapy and $\operatorname{IvIg}$ for 3 days with meropenem for pneumonia. After 3 days his platelets were 70,000, TLC-5800 and haemoglobin was $13.8 \mathrm{gm} \%$. We would like to report this rare case of infection induced hemophagocytic lymphohistiocytosis. This serves as a rare diagnosis. Bone marrow histopathology photograph showing haemophagocytes: 


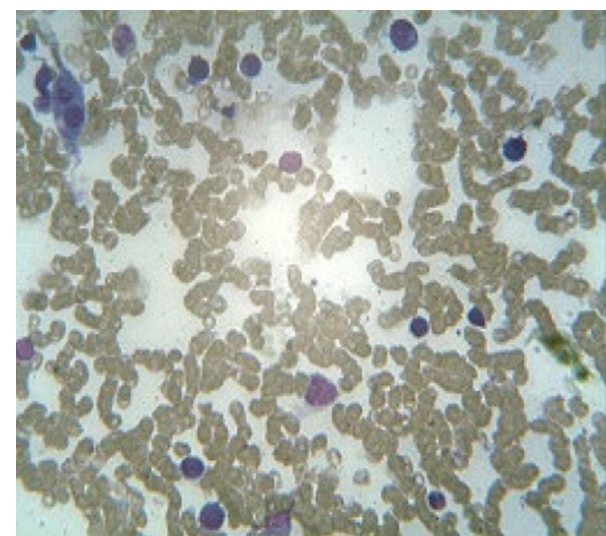

BHC 22

A Prospective Study to Evaluate the Efficacy, Safety and Response Duration of Newly Diagnosed Adult ITP Patients Treated with Low Dose Rituximab and High Dose of Dexamethasone

\section{Soumya Mukherjee", T. K. Dolai, P. K. Mandal, R. De,} P. Chakrabarti

Email ID for Correspondence: doctormukherjee80@gmail.com NRS Medical College and Hospital, Kolkata

Introduction and Background: Primary immune thrombocytopenia (ITP) is an acquired autoimmune disorder that involves antibody and cell mediated destruction of platelets as well as suppression of their production. Corticosteroids are initial standard therapy in adults. Initially Rituximab $\left(375 \mathrm{mg} / \mathrm{m}^{2}\right)$ was approved as one of the important second line therapy in chronic ITP. In the present prospective study we have evaluated the efficacy, safety and response duration of low dose Rituximab (100 mg) and high dose Dexamethasone as a front line therapy in new onset adult ITP cases. Patient/Material and Methods: Inj. Rituximab at a fixed dose of $100 \mathrm{mg}$ iv infusion for four consecutive doses (day 1, 8, 15, 22) and Dexamethasone tablet $40 \mathrm{mg}$ PO for four consecutive days in a fifteen days interval (day 1-4 and day 15-18). Response rate evaluated according to published guidelines. Results: Total no. of cases enrolled 22 (female-14). Age range 18 to 62 years. Median age 36 years. Course completed in all patients. Median follow up of the patients 9 month. CR achieved after 1 st dose of rituximab in 6 cases, 3 after 2 nd dose and 4 after 4 th dose of Rituximab. Partial response achieved in 2 cases after completion of therapy and no response seen in 7 cases. 9 cases achieved CR with sustained response after 9 months of completion of therapy. 4 cases who achieved CR initially reverted to partial remission (PR) after 6 month with dropping of platelet count but without any evidence of bleeding. Minor side effects like GI intolerance, nausea, vomiting occurred in 3 patients, High blood sugar level during treatment seen in 2 patients and severe adverse effects like pneumonia and intestinal obstruction seen in 2 patients who needed hospitalization. We did not observe any infusion-related reaction. Conclusions: Overall response rate seen $68 \%$ (CR-59\%). $69.23 \%$ Patients achieving CR shows sustained response after 9 months. Low dose Rituximab and high dose Dexamethasone is a useful treatment option in adult acute ITP patients.

\section{BHC 23}

Autoimmune Haemolytic Anemia Following H1N1 Virus Infection: A Case Report

Garima Goel ${ }^{\#}$, Devyani Pendharkar, Rajnish Joshi, Deepti Joshi, Neelkamal Kapoor

Email ID for Correspondence: drgarima_80@yahoo.co.in All India Institute of Medical Sciences, Bhopal

Introduction and Background: Autoimmune haemolytic anaemia (AIHA) is caused by haemolysis of red blood cells (RBCs) induced by reaction of autoantibodies. Various haematological disorders caused by immune mechanisms are associated with viral infections. However, H1N1 virus infection induced AIHA is very rare. Patient/Material and Methods: A 55 year old female presented with gradually worsening anaemia for 2 months with the haemoglobin levels going as low as $3.2 \mathrm{~g} / \mathrm{dl}$. Three months prior to the development of anaemia the patient suffered from H1N1 virus infection and was admitted for the same. Prior to the infection and during the hospital stay the haemoglobin levels were normal. On further investigations reticulocyte count was $12 \%$, serum bilirubin was increased to $2 \mathrm{mg} / \mathrm{dl}$, bone marrow examination showed erythroid hyperplasia and adequate iron stores and osmotic fragility was increased. However, direct Coomb's test and PNH screen was negative. Other investigations including upper gastrointestinal endoscopy, colonoscopy, gastric biopsy, haemoglobin electrophoresis, serum iron, serum ferritin and vitamin B12 levels were within normal limits. The serology for dsDNA and anti CCP antibody was negative. A diagnosis of AIHA was considered and the patient was administered Prednisolone. At the start of the treatment her haemoglobin was $7.2 \mathrm{~g} / \mathrm{dl}$, but 4 weeks later it improved to $9.8 \mathrm{~g} / \mathrm{dl}$. Results: In the present case the patient suffered from a Coombs negative AIHA following H1N1 virus infection. In literature Coombs negative AIHA have been reported in patients following either infection by influenza virus A or administration of influenza vaccine. Conclusions: To conclude influenza virus may lead to Coombs negative AIHA which can achieve remission following steroid therapy.

\section{BHC 24}

Hydroxyurea Therapy in Severe Thalassemia Management in the Indian Context

Reshma Srinivas ${ }^{\text {\#\#, Rajat Kumar } \text { Agarwal }^{2} \text {, Lawrence Faulkner }}{ }^{3}$

Email ID for Correspondence: reshmasrinivas15@gmail.com

${ }^{1}$ Rashrottana Parishath, Bangalore, India; ${ }^{2}$ Sankalp India Foundation, Bangalore, India; ${ }^{3}$ Cure2Children Foundation, Florence, Italy

Introduction and Background: Hydroxyurea $(\mathrm{Hu})$, also known as hydroxycarbamide, has the potential to decrease transfusion requirements, extramedullary erythropoiesis, ferritin and organomegaly. We assessed the impact of $\mathrm{Hu}$ in our severe thalassemia patient population. Patient/Material and Methods: Eighteen patients aged 3.8-16 (median 8.5 years) with severe thalassemia and hepato- and/or spleno-megaly ( $>2 \mathrm{~cm}$ from costal margin) or serum ferritins $>1.000 \mathrm{ng} / \mathrm{mL}$ were treated with $\mathrm{Hu}$ starting at $20 \mathrm{mg} / \mathrm{kg} / \mathrm{day}$ with dose escalation up to $50 \mathrm{mg} / \mathrm{kg} / \mathrm{day}$ based on hematological effect aiming at absolute neutrophil count between 1000 and $1500 / \mu \mathrm{L}$ and 
platelet count between $100,000-150,000 / \mu \mathrm{L}$ for at least 15 months. Data was collected using BMTPlus and was compared for each quarter using one tailed Student's $t$ test for matched pairs and using the first quarter as the baseline. Results: Significant reduction in the liver size was observed after 2 quarters of Hu therapy for the whole group $(\mathrm{p}=0.03)$. Below age of 9 years $(\mathrm{n}=11)$ significant response was seen in second $(p=0.07)$, third $(p=0.04)$ and fourth $(p=0.02)$ quarters. Liver size for children $>9$ years did not change significantly. Spleen size reduction was prominent in the second and third quarter for age $<9$ years $(p=0.06$ and $p=0.04$ respectively) after which it did not improve further. Children with age $>9$ years did not seem to respond to $\mathrm{Hu}$. The drop in serum ferritin was significant from the third quarter $(\mathrm{p}=0.018)$ and continue to improve till the fifth quarter $(p=0.0014)$. This effect was more pronounce in age $<9(0.000011)$ as opposed to those above 9 (0.176). Blood requirement significantly reduced from 47 to $36 \mathrm{ml} / \mathrm{kg} /$ quarter in the 4 th quarter $(\mathrm{p}=0.01)$ for both age groups. Conclusions: Hydroxyurea therapy might effectively contribute to reducing liver, spleen, blood transfusions and serum ferritin. This approach might be considered in ST patient with increased liver or spleen size, especially in children $<9$ years.

\section{BHC 25}

T-regulatory Cells in Donor Grafts May Predict the Severity of Acute Graft Versus Host Disease After Allogenic Hematopoiectic Stem Cell Transplantation

\section{Dinesh Chandra\#, Jasdeep Singh, M. Mahapatra, Pravas Mishra, Tulika Seth, Seema Tyagi, Lalit Kumar, Renu Saxena, H. P. Pati}

Email ID for Correspondence: dinesh3224@gmail.com All India Institute of Medical Sciences, New Delhi

Introduction: Acute Graft-Versus-Host Disease (aGVHD) and relapse are major problems for patients undergoing allogenic Hematopoietic Stem Cell Transplantation (allo-HSCT). T-regulatory (Treg) cells in the donor graft are negatively correlated with the incidence of aGVHD without any difference in incidence of relapse. We conducted this study to determine the association of Treg cells with aGVHD in allo-HSCT patients. Patient/Material and Methods: This is a prospective study of 32 patients with malignant and non-malignant hematological disorders, who underwent allo-HSCT. All patients received the graft from matched sibling donors. Sixteen patients received myeloablating (Acute myeloid leukemia-7, Mixed phenotypic acute leukemia-1, Chronic myeloid leukemia-blast crisis-2, Myelodysplastic syndrome-1, Chronic myelomonocytic leukemia-1 and Juvenile myelomonocytic leukemia-1) and 13 patients (Aplastic anemia-11 and Thalassemia major-2) received non-myeloablating conditioning regimen. Twenty nine patients who achieved engraftment were enrolled. Treg cells were quantified in donor graft by flowcytometry and assessed their association with aGVHD and other clinical outcome. Results: Fifteen of 29 patients developed aGVHD. According to the occurrence and severity of aGVHD, patients were divided into two groups: 20 (68.9\%) patients with grade $0-\mathrm{I}$ aGVHD and $9(31.1 \%)$ patients with grade II-IV aGVHD. Treg/CD4 ratio was significantly higher in the grade $0-I$ aGVHD group than in grade II-IV aGVHD group $(p=0.0002)$. The proportion of Treg cells dose was higher in grade 0-I aGVHD than grade II-IV aGVHD; however it fell short of statistical significance $(\mathrm{p}=0.13)$. There was no correlation between CMV activation or relapse with Treg cells dose and Treg/CD4 ratio. We could not find association CD34 $(\mathrm{p}=0.55)$ dose or CD3 dose $(\mathrm{p}=0.57)$ with the severity of aGVHD. Conclusions: Higher Treg/CD4 ratio in donor graft was associated with less severe aGVHD. Though more studies are needed, Treg/CD4 ratio may be used as a predictive marker for severity of aGVHD in post allo-HSCT and warrant a close follow up.

\section{BHC 26}

Aplastic Anemia: Response to Indigenous Anti-thymocyte Globulin-A Single Tertiary Care Centre Experience

\section{Saikat Datta ${ }^{\#}$, Siddhesh Kalantri, U. Akshatha Nayak, Sambit Samanta, Prof. Siddhartha Sankar Ray, Prof. Maitreyee Bhattacharyya}

Email ID for Correspondence: drsaikatdatta@gmail.com Institute of Haematology and Transfusion Medicine, Kolkata

Introduction and Background: Immunosupressive therapy (IST) with horse anti-thymocyte globulin (ATG) and cyclosporine (CSA) is the standard treatment option in severe aplastic anemia patients more than 35-50 years of age and younger patients without matched sibling donor. In India stem cell transplant is often not feasible due to financial constrain. Most of the patients receive IST. Published literature on efficacy of IST is mostly with ATGAM, original research product from multinational pharmaceutical house. However, we have much cheaper indigenous brand Thymogam. Only few studies have used this brand and reported the efficacy. Patient/Material and Methods: This was a single centre prospective analysis of total 27 patients with aplastic anemia who were treated with ATG and cyclosporine between 15th May, 2015 and 15th February 2016. Indigenous horse anti-thymocyte globulin (Thymogam) was used at a dose of $40 \mathrm{mg} /$ $\mathrm{kg} / \mathrm{day}$, given on 4 consecutive days. Cyclosporine was started along with ATG at a dose of $5 \mathrm{mg} / \mathrm{kg} /$ day. Patients were followed up monthly with complete haemogram. Cyclosporine trough blood level was maintained between 150-250 $\mu \mathrm{g} / \mathrm{L}$. Haematological response was assessed 6 month after ATG administration. Results: Among 27 patients, 22 had severe aplastic anemia, three patients had very severe and another two patient had non-severe aplastic anemia. Patients were aged between 12 to 60 years $($ mean $=42.1$ years). Seventeen patients $(63 \%)$ were male and $10(37 \%)$ were female. Over a follow up period of 6 months only 12 patients $(44.4 \%)$ achieved partial response and no patient achieved complete response. High baseline absolute reticulocyte count $(\mathrm{p}=0.002)$, low pre-treatment platelet transfusion $(\mathrm{p}=0.005)$ and high baseline platelet count $(\mathrm{p}=0.04)$ showed statistically significant association with haematological response. Conclusions: Haematological response rate with indigenous horse ATG combined with CSA was significantly lower than that was reported in international literature.

\section{BHC 27}

Total Knee Arthroplasty in Patients with Hemophilia: Single Center Experience in Western India

B. C. Mahadeva ${ }^{1 \#}$, H. Balar ${ }^{1}$, D. Vaghasiya ${ }^{1}$, A. Raiyani, N. Chaturvedi ${ }^{1}$, D. Patel, H. Parekh ${ }^{1}$, A. Joshi ${ }^{5}$, S. Melinkeri ${ }^{3}$, S. Kannan ${ }^{3}$, R. Phatale ${ }^{4}$, N. Likhate, R. Dange ${ }^{7}$, S. Apte ${ }^{2}$

Email ID for Correspondence: drdevbc@gmail.com

${ }^{1}$ DNB Resident, Hematology Department, Sahyadri Speciality Hospital, Pune; ${ }^{2}$ Head of Department and Consultant Hematologist, Department of Haematology and Bone Marrow Transplant Unit, Sahyadri Speciality Hospital, Pune; ${ }^{3}$ Consultant Hematologist, Sahyadri Speciality Hospital, Pune; ${ }^{4}$ Consultant Hematopathologist, Sahyadri Speciality Laboratory, Pune; ${ }^{5}$ Clinical Assistant, Hematology Department, Sahyadri Speciality Hospital, Pune; ${ }^{6}$ Consulting Orthopedic Surgeon, Sahyadri Speciality Hospital, Pune; ${ }^{7}$ Consultant Physiotherapist, Sahyadri Speciality Hospital, Pune

Introduction and Background: The Patients with Hemophilia (PWH) bleed spontaneously into the joints. The joints most frequently involved are the knees, ankles, and elbows. The consequences of recurrent bleeding into a joint leads to chronic hemophilic arthropathy. With the 
advent of the availability of Clotting Factor Concentrates (CFC), Total Knee Arthroplasty (TKA) can now be performed safely. Patient/Material and Methods: We retrospectively studied data from patients with Hemophilia, who underwent TKA at our center from 2005 to 2016 and was reviewed under following headings.

1. Patient-Age

2. Clinical outcome measured with Range-Of-Motion (ROM) and Flexion contractures which are compared with pre-operative.

3. CFC used (type \& dosage)

4. Complications.

5. Follow-up and overall outcome.

Results: Data of 26 patients was analyzed. All patients were severe Hemophilia-A. Mean patient age was 45.5 years. All patients were negative for inhibitors. 51 TKA procedures were done. One patient underwent femur pseudo tumor excision and TKA in same setting. 25 patients underwent simultaneous bilateral TKA in same setting. Average CFC used per patient of plasma derived/Recombinant type for $20 \mathrm{PWH}$ was $750 \mathrm{IU} / \mathrm{Kg}$-body-weight and CFC of long acting type for $6 \mathrm{PWH}$ was $650 \mathrm{IU} / \mathrm{Kg}$-body-weight. All patients received thrombo-prophylaxis with Low-molecular-weight-Heparin (LMWH) which has not caused any excessive bleeding. Mean follow-up was 6.5 years. ROM improved and flexion contractures were reduced significantly. Complications like prosthetic joint infection and aseptic loosening were not reported. Conclusions:

1. Simultaneous bilateral TKA is feasible in PWH and has significantly reduced CFC usage \& cost.

2. Reduced CFC usage compared with western protocol and with advent of long acting CFC there is further reduction.

3. Thrombo-prophylaxis with LMWH can be done safely.

4. The benefits of pain relief and improved ROM, reduced flexion contractures provided by TKA has reduced annual bleed rates in other joints with reduction in CFC usage and reduced disabilityadjusted life year (DALY).

5. Pre-operative \& post-operative physiotherapy aids functional recovery after TKA.

\section{BHC 28}

Evaluation of Clinical Course and Complications in NTDT-Hb E $\beta$ Thalassemia Patients in a Tertiary Care Center in Eastern India

\section{Vinodhini", S. Dutta, R. De, P. Chakrabarti, T. K. Dolai, P. K. Mandal}

Email ID for Correspondence: drvino97@yahoo.in Department of Haematology, NRS Medical College and Hospital, Kolkata

Introduction and Background: Non Transfusion Dependent Thalassemia patients often develop iron overload that requires chelation to levels below the threshold associated with complications. Cardiopulmonary disease, including Pulmonary Hypertension are due to Microvascular injury, Thromboembolism and Hemolysis-induced Nitric Oxide deficiency. Pulmonary hypertension is a major cardiac complication in non-transfusion-dependent thalassemia (NTDT). Several clinical and laboratory parameters, including iron overload, have been shown to have a positive correlation with the incidence of pulmonary hypertension and hepatic fibrosis. Patient/Material and Methods: A Cross-sectional Observation study is conducted in 50 NTDT-Hemoglobin E/ $\beta$ Thalassemia patients from Jan 2015 to August 2016. Pulmonary Hypertension was defined as increase in MPAP $>25 \mathrm{~mm}$ at rest or as peak Tricuspid Regurgitation Velocity $\geq 2.9 \mathrm{~m} / \mathrm{s}$ measured using 2D-Echo.
1. To study the clinical course \& complications in NTDT- $-\mathrm{Hb}$ E beta Thalassemia Patients.

2. To assess the correlation of various parameters such as Iron overload, Pulmonary Hypertension and Hepatic fibrosis.

Results: Out of 50 NTDT patients, M:F ratio is 1.3:1. Average transfusion requirement was 2-3 units/lifetime. S. ferritin $>800 \mathrm{ng} / \mathrm{ml}$ was seen in $24 \%$ patients, pulmonary HTN was seen in $42 \%$ patients. Majority of the patients had Grade I pulmonary Hypertension. Less than $50 \%$ patients had Grade II and III disease. Hepatic fibrosis detected by Fibroscan was seen in $5 \%$ patients. Leg ulcers were not noted in our study. None of the patients underwent splenectomy. Increased S. ferritin showed a positive association with pulmonary hypertension and hepatic fibrosis. Conclusions: Increasing age, minimal transfusion requirements, High S. Ferritin levels associated with iron overload increase the severity of Pulmonary Hypertension and hepatic fibrosis.

\section{BHC 29}

Clinical Profile of Patients with Auto Immune Haemolytic Anemia and Serological Characterisation of Different Subtypes

\section{Hitha", T. K. Dutta, K. V. Vinod, B. Vishnu Bhat, R. G. Kulkarni}

Email ID for Correspondence: b.hitha@gmail.com JIPMER, Puducherry

Introduction and Background: Autoimmune haemolytic anemia is caused by autoimmune destruction of RBCs by autoantibodies with various properties and target specificities. The clinical course of the disease as well as treatment decisions are influenced by the type of antibody involved. This study analyses RBC bound auto antibodies in auto immune haemolytic anemia patients with respect to grading of direct antiglobulin test (DAT) and antibody class and assess their association with severity of hemolysis. Patient/Material and Methods: The study group included patients of all age groups with a diagnosis of auto immune haemolytic anemia (AIHA) based on direct antiglobulin test (DAT) positivity between august 2014 and July 2016. The patients with $\mathrm{Hb}$ $<9 \mathrm{~g} / \mathrm{dl}$, DAT + ve, reticulocyte count $>2 \%$, serum bilirubin $>2 \mathrm{mg} / \mathrm{dl}$, serum LDH $>500 \mathrm{IU} / \mathrm{dl}$ were included. Severe haemolytic anemia was defined by $\mathrm{Hb}<7 \mathrm{~g} / \mathrm{dl}$. Polyspecific DAT was done using poly specific antihuman globulin reagent and the strength graded as grade 1,2,3 and 4. The samples were again analysed with gel card containing monospecific antihuman globulin (anti $\mathrm{IgG}, \operatorname{IgM}, \operatorname{IgA}$, anti C3c and $\mathrm{C} 3 \mathrm{~d}$ ) and Ig sub class was typed with gel card impregnated with monospecific IgG1 and IgG3. The patients were evaluated for secondary causes of AIHA. The patients were followed up to assess treatment response at 3 months and 6 months. $\mathrm{Hb}>10 \mathrm{~g} / \mathrm{dl}$ was regarded as adequate treatment response. Results: Data of 54 patients ( 50 adults and 4 children) with a diagnosis of AIHA were analysed. Median age of presentation was 33 years (range 4-82 years). Male:Female ratio was 1:2.8. Mean $\mathrm{Hb}$ at presentation was $5.03 \mathrm{~g} / \mathrm{dl}$. In 17 cases ( $31.4 \%$ ) secondary cause for AIHA was identified. 50 cases of warm antibody AIHA and 5 cases of cold antibody disease were identified. There were 2 cases of Evan's syndrome. IgG was the solitary antibody in 7 cases, $\mathrm{C} 3 \mathrm{~d}$ alone in 5 cases and multiple anti IgG and anti compliment antibody coated in 42 cases. 50 patients received prednisolone, response rate was $62.2 \%$ at 3 months \& $63 \%$ at 6 months. Six patients later received other immunosuppressive drugs; 5 patients underwent splenectomy. The mean $\mathrm{Hb}$ in patients with strong DAT reaction was $4.6 \mathrm{~g} / \mathrm{dl}$ and that in patients with mild DAT reaction was $7.1 \mathrm{~g} / \mathrm{dl}$ and the difference was found to be statistically significant ( $\mathrm{p}<0.001)$. Conclusions: The gel card technology helps us to identify RBC autoantibodies in AIHA, serologically characterize the disease and to decide treatment. The severity of haemolysis in AIHA depends on the 
strength of DAT reaction; patients with strong DAT reaction had more severe hemolysis. The response rate to treatment with corticosteroids at 6 months was found to be $63 \%$ in this study.

\section{BHC 30}

\section{Pancytopenia: A Clinico-hematological Study with Follow Up}

\section{Shruti Semwal $^{\#}$, Deepti Joshi, Garima Goel, Akriti Khare, Radhika Rai, Neelkamal Kapoor}

Email ID for Correspondence: shrutisemwal29@gmail.com Department of Pathology and Laboratory Medicine, All India Institute of Medical Sciences, Bhopal

Introduction and Background: Pancytopenia is presence of anemia, leukopenia and thrombocytopenia simultaneously. It is generally due to decrease in hematopoietic cell production in the marrow resulting from infections, toxins, malignant cell infiltration, chemotherapy and radiation. Patient with pancytopenia presents with different clinical features. In cases of pancytopenia marrow cellularity and composition differ in relationship to underlying pathological condition. The aim of the present study was to perform clinico-hematological correlation of all patients presenting with pancytopenia. Patient/Material and Methods: Total 30 cases constituted the study group. Inclusion criteria was presence of all 3 of the following: haemoglobin $<\mathrm{I} 0 \mathrm{~g} / \mathrm{dl}$, total leukocyte count $<4000 / \mu \mathrm{L}$, platelet count $<100,000 / \mu \mathrm{L}$. Peripheral blood smear examination, bone marrow aspiration and bone marrow biopsy were done in all the cases. Reticulocyte count, pearl's stain and Reticulin staining was also done. Results: Out of the 30 cases, I5 cases were male and 15 were females. The mean corpuscular volume (MCV) was normal in $60 \%$ of the total cases, decreased in $20 \%$ of cases and was found to be increased in $20 \%$ of the cases. On bone marrow biopsy, $44 \%$ cases were diagnosed as Hypoplastic marrow, I4 \% as Normocellular, $7 \%$ as Megaloblastic Change and $7 \%$ as Micronormoblastic. Hemophagocytosis, Acute leukemia, small round cell tumor, Metastatic carcinoma, Reactive Marrow, Granulomatous Lesion, Multiple Myeloma, Leishmania and dyserythropoeitic marrow constituted $3 \%$ each. Follow up data of the patients is being collected. Conclusions: With the help of detailed clinical history, physical examination, and hematological investigations, pancytopenia can be diagnosed and the causes can be ascertained.

\section{BHC 31}

Assessment of Hematological Parameters of Neonatal Cord Blood in Preeclamptic Females and Females with Normal Pregnancy

Samreen Farooqui", Sushma Gupta,

Hem Prabha Gupta, Syed Riaz Mehdi,

Abdussalam, Eqbal Anwer, Nitin Pandey

Email ID for Correspondence: ms_farooqui92@yahoo.com

Era'S Lucknow Medical College and Hospital

Abstract: The aim of this study was to assess the changes in haematological parameters in neonates using cord blood from preeclamptic and normal pregnancies. Material and Methods: This is a cross sectional study based on Umbilical Cord Blood (UCB) samples taken from 30 preeclamptic and 30 normal pregnancies. $3 \mathrm{ml}$ cord blood was collected immediately after delivery and transferred into an EDTA vial for CBC analysis and reticulocyte count was done using brilliant cresyl blue. The data entry and analysis was done on Microsoft Office Excel 2007. In all statistical analysis a p value $<0.05$ was considered significant. Results: RBC, Hb, Red cell indices were found to be higher in neonatal cord blood from preeclamptic females than those from normal pregnancies. Total leukocyte count and neutrophils were found to be lower in cord blood from preeclamptic females while lymphocytes were found to be higher in cord blood from preeclamptic females than from those from normal pregnancies. Reticulocyte count was found to be higher in neonatal cord blood from preeclamptic pregnancies. Also platelet count was lower in UCB from preeclamptic females than those from normal pregnancies. Conclusion: Newborns of preeclamptic mothers should be carefully monitored in terms of hematologic abnormalities. Thus, suspecting a deranged haematological profile early in the course of disease will be of great help in the preemptive management strategies that have proven to be playing a crucial role time and again in reducing the morbidities and mortalities associated with Preeclampsia.

Keywords RBC, Haemoglobin, Total leukocyte count, Platelets, MPV, Reticulocyte count

\section{BHC 32}

\section{A Rare Case of Hypotransferrinemia}

H. R. Bohra", S. Khera, D. Vedant, A. Dubey, P. Gautam, G. Singh, N. Gupta, A. Purohit, P. Elhence, S. Bhattacharya

Email ID for Correspondence: dr.harishbohra@gmail.com All India Institute of Medical Sciences, Jodhpur, Rajasthan

Introduction and Background: Hypotransferrinemia is a rare disorder, but it may be more common than is clinically diagnosed and may complicate the diagnosis of patients with anaemia and other coexisting disorders. Patient/Material and Methods: Here we report a 24 months old male child, case of hypotransferrinemia, who had anaemia since birth. He was operated for congenital diaphragmatic hernia and has been requiring regular blood transfusion every three months. On investigations, patient had haemoglobin value of $6.4 \mathrm{gm} /$ dL with hypochromic microcytic red cell picture. On examination, patient had severe pallor and hepatosplenomegaly. Patient was investigated thoroughly with estimation of serum ferritin levels, serum iron studies, haemoglobin HPLC, bone marrow examination and serum transferrin levels. Results: Patient was eventually was found to be a case of hypotransferrinemia. Conclusions: Hypotransferrinemia can present as non-responding childhood anaemia.

\section{BHC 33}

Clinical and Laboratory Predictors of Chronicity in Childhood Immune Thrombocytopenia (ITP)

Tusti Ganguly", Prof. Prantar Chakrabarti, Prof. Tuphan Kanti Dolai, Rajib De, Prakas Kumar Mandal

Email ID for Correspondence: tustidoc12@gmail.com Nil Ratan Sircar Medical College and Hospital, Kolkata

Introduction and Background: Age, gender, duration of symptoms, and associated viral illness are often cited as clinical factors which play a role in the development of chronic ITP in children. In this study, we assess the clinical and laboratory parameters in newly diagnosed cases of childhood ITP to determine which factors, if any, are associated with increased risk of developing chronic ITP. Patient/Material and Methods: This prospective observational study was carried out among patients aged $0-18$ years with newly diagnosed ITP in NRS Medical College between January 2015 to December 2015. Patients with secondary causes of thrombocytopenia and those previously treated for ITP were excluded. Relevant demographic, clinical and laboratory data were collected at diagnosis and the patients were followed up for a 
period of at least 1 year after diagnosis for development of chronicity. Results: 31 patients of newly diagnosed ITP were included in the study. The most common presenting features were skin bleeds, gum bleeding and epistaxis. Menorrhagia was common among postmenarchal females. About $50 \%$ of patients presented with preceding viral illness or past $\mathrm{h} / \mathrm{o}$ bleeding. After 1 year of follow up, $38.7 \%$ patients had no response $\left(<30,000 / \mathrm{mm}^{3}\right), 32.2 \%$ demonstrated complete response $(>100,000 /$ $\left.\mathrm{mm}^{3}\right)$, while $19.3 \%$ achieved partial response $\left(30,000-100,000 / \mathrm{mm}^{3}\right)$ with no overt bleeding episodes. Abrupt onset on bleeding manifestations, mucosal bleeding of moderate to severe nature, $\mathrm{h} / \mathrm{o}$ preceding viral infection and early recovery ( $\leq 3$ months) of platelet counts correlated with lower risk of chronicity. Distribution of age, sex, mean Hb, TLC and platelet counts at diagnosis were similar among the groups of patients. Conclusions: Insidious onset of bleeding, mild bleeding symptoms, absence of mucosal bleeding with no history of antecedent viral infection and failure to recover normal platelet counts within 3 months of diagnosis are associated with increased risk of chronicity in childhood ITP. transmitted by the bite of trombiculid mite. The infection is characterized by flu-like symptoms of fever, headache and myalgia lasting for over a week. In some, the illness turns fatal involving multi organ dysfunction and ultimately causing death. We describe a case of HLH developed secondary to ricketssial infection of $O$. tsutsugamush $i$ which is a rare presentation of the syndrome. Patient/Material and Methods: 36 year old male, non-alcoholic, non-smoker presenting with complaints of high grade fever $\left(100-102^{\circ} \mathrm{F}\right)$, continuous and associated with a pruiritic macular rash on the abdomen developed on 3rd day of fever. Associated symptoms were difficulty in breathing, swelling over limbs and yellowish discoloration of whole body for 5 days. IgG \& IgM serology for scrub typhus was found to be positive. Results: Patient fulfilled the essential diagnostic criteria ${ }^{(2009)}$ for HLH with fever, splenomegaly, cytopenia and hepatitis along with hyperferritinemia and hypertriglyceridemia. Haemophagocytosis was seen on bone marrow aspiration studies secondary to ricketssial infection.

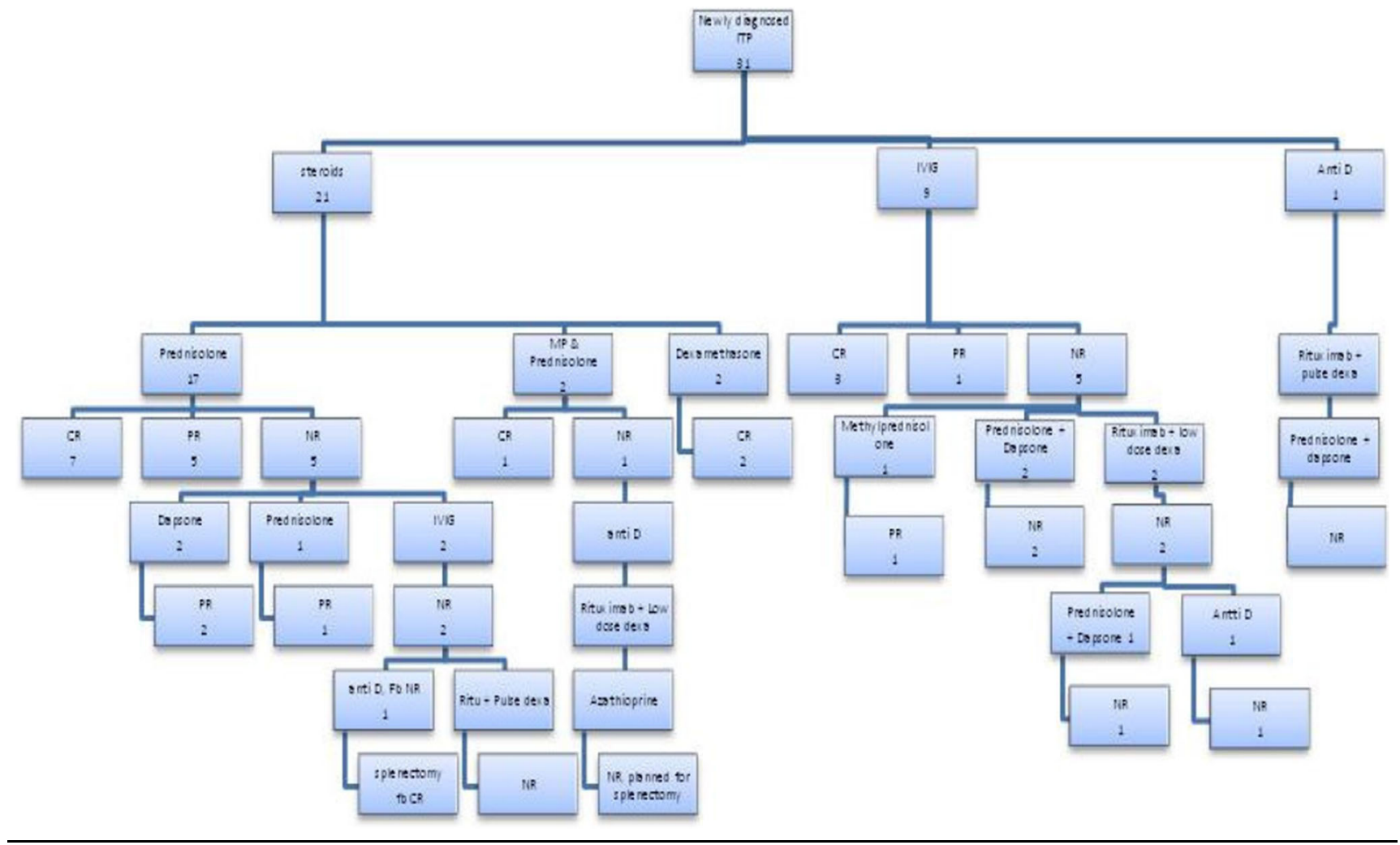

\section{BHC 34}

Hemophagocytic Lymphohistiocytosis (HLH) Associated with Scrub Typhus: Case Report from a Tertiary Care Centre

\section{Apoorva Gomber", Zeba Choudhary, Garima Baweja Madan, Anjali Sharma, Vijay Kumar, Sadhna Marwah, A. S. Nigam}

Email ID for Correspondence: drapoorvagomber@gmail.com PGIMER, Dr. RML Hospital, New Delhi

Introduction and Background: HLH is a fatal hyperinflammatory syndrome resulting from prolonged and excessive activation of antigen presenting cells and $\mathrm{CD} 8^{+} \mathrm{T}$ cells. It may be inherited or secondary to infections/malignancy at any age. The diagnosis is established by the Proposed HLH diagnostic criteria, 2009. Scrub typhus infection is an important etiology of acute undifferentiated fever in India. It is a zoonotic rickettsial illness caused by Oriental tsutsugamushi,
Conclusions: HLH should be considered in severe cases of rickettsial diseases especially associated with pancytopenia. Patients with scrub typhus usually have an excellent response to treatment, therefore early diagnosis and prompt treatment can prevent serious complications.

\section{BHC 35}

Clinico-hematological Profile of Systemic Onset Juvenile Idiopathic Arthritis (SOJIA): Review from Tertiary Care Centre

Shruti Dogra", Zeba Choudhary, Garima Baweja, Anjali Sharma, Vijay Kumar, Sadhna Marwah, A. S. Nigam

Email ID for Correspondence: d.shrutidogra@gmail.com PGIMER, Dr RML Hospital, New Delhi

Introduction and Background: Systemic onset juvenile idiopathic arthritis (SOJIA) is one of the rarest form of juvenile rheumatoid 
arthritis (JRA) accounting for just 10\%-20\% of all JRA cases. This condition is associated with high grade spiking fever, rash, and joint pain. The age of onset is between 5 to 10 years. It is a potentially life threatening paediatric condition. The objective of this study was to describe the clinical profile and hematological findings of SOJIA at a tertiary care center in North India. Patient/Material and Methods: In the present retrospective study, clinicohematological profile including bone marrow findings of clinically diagnosed cases of SOJIA were retrieved from archives of department of pathology PGIMER Dr RML Hospital during the period of Jan 2013-Aug 2016. Results: A total of 20 clinically diagnosed cases of SOJIA were reviewed with the age ranging from 6-14 years out of which 12 were males and 8 were females. Chief clinical and hematological manifestations included fever and joint pain in all cases and hemophagocytosis in $90 \%$ cases. The other clinical findings, haematological profile and bone marrow aspiration and biopsy observation will also be discussed. Conclusions: SOJIA is one of the important rheumatological condition of paediatric age group. The clinical profile and hematological findings including bone marrow smear examination findings are pertinent for the diagnosis of the disease. Hemophagocytosis is one of the key findings in the bone marrow smear examination of the patients.

\section{BHC 36}

Bone Marrow Study in Adults with Pancytopenia in North Bihar: No Paradigm Shift for Kala Azar

\section{Rajani Anand}

Email ID for Correspondence: dr.amrendra07@gmail.com Darbhanga Medical College

Introduction and Background: Bone marrow examination is extremely helpful in evaluation of pancytopenia. This study highlights the spectrum of causes of pancytopenia and also bone marrow morphology in cases of pancytopenia. The objectives of the study are:

1. To study the spectrum of causes of pancytopenia in adults in the age group of 18-70 years.

2. To study the bone marrow morphology in cases of pancytopenia.

Patient/Material and Methods: Fifty patients with a haematological diagnosis of pancytopenia were included. Demographic profile, clinical history recording and examination along with hemogram, peripheral smear and bone marrow aspiration were done in all the patients to identify the etiology. Results: Out of 50 cases, 33 were males and 17 were females, maximum from age group of 11-20 years $(28 \%)$. The sex distribution shows male preponderance. Haemoglobin varied from $2.9 \mathrm{gm} \%$ to $9.3 \mathrm{gm} \%$, total leukocyte count was in the range of $900-3700$ cells/cu $\mathrm{mm}$ and platelet count varied from 4000-147,000 cells/cu mm. The reticulocyte count varied from $0.3-2.5 \%$. Bone marrow showed the following types of cellularity.
a. Normocellular-54\%
b. Hypercellular-30\%
c. Hypocellular-16\%

Most common cause of pancytopenia was Kala-azar followed by megaloblasticanaemia (18\%), aplastic anaemia (16\%), AML and ALL (8\%), nutritional anaemia (8\%), malaria (6\%), MDS (2\%) and dengue fever $(2 \%)$. Peripheral blood smear in Kala azar showed pancytopenia with normocytic normochromic erythrocytes and relative increase in lymphocytes. Bone marrow were normocellular in all cases with erythroid hyperplasia. Amastigote form (LD body) was identified in 6 cases. Myeloid cells and megakaryocytes were normal. Megaloblastic anaemia was present in $18 \%$ of cases. Peripheral blood smear picture sowed macroovalocytes and anisopoikilocytosis. Mean corpuscular volume was more than $100 \mathrm{fl}$ with hypersegmented neutrophils and reduced platelets. Bone marrow picture was hypercellular with reduction of fat cells, erythroid hyperplasia with megaloblastic maturation and reversal of M:E ratio. Conclusions: The commonest cause of pancytopenia, throughout the world has been aplastic anemia however in India megaloblastic anemia is the most common cause. But in our study we found kala azar as the leading cause of pancytopenia. Kala azar is still has high prevalence in North Bihar and this study is the first study of pancytopenia from this region highlighting the impact of Kala azar even after effective treatment available for many years.

\section{BHC 37}

Pancytopenia-A Clinico Hematological Analysis of 210 Cases

Megha Sharma", Manika Khare, Garima Baweja Madan, Anjali Sharma, Vijay Kumar, Sadhana Marwah, A. S. Nigam

Email ID for Correspondence: meghambbs10@gmail.com PGIMER, Dr. Ram Manohar Lohia Hospital, New Delhi

Introduction and Background: Pancytopenia is characterized by simultaneous presence of anemia, leukopenia and thrombocytopenia resulting from a number of disease processes. It should be suspected when the patient presents with clinical symptoms like pallor, generalised weakness, recurrent prolonged fever etc. The clinical profile, peripheral blood and bone marrow findings of the patients presenting with pancytopenia were studied to identify the etiological factors. Patient/Material and Methods: The present retrospective study was carried out from April 2014 to April 2016 at Department of Pathology, PGIMER, Dr. RML Hospital, New Delhi. A total number of 210 patients of pancytopenia with $\mathrm{Hb}<9 \mathrm{gm} / \mathrm{dl}, \mathrm{WBC}$ count $<4000 / \mu \mathrm{l}$ and platelet count $<100,000 / \mu \mathrm{l}$ were included in the study. A detailed history, complete clinical examination, haematological profile and bone marrow examination were done. Results: Out of 210 patients, 136 patients were adults and 74 patients were children. Male preponderance was observed with a male:female ratio of 2.7:1. The mean age group of the patients was 42 years. Pallor (100\%), fatigue $(71 \%)$, fever $(48.8 \%)$, weakness $(67.8 \%)$ and breathlessness (44\%) were among the most common presenting symptoms. Splenomegaly and hepatomegaly were noted in $43.9 \%$ and $33.8 \%$ cases respectively. The haematological parameters revealed a mean haemoglobin level of $5.6 \mathrm{gm} / \mathrm{dl}$ with the leucocyte count ranging between $750-3800 / \mu \mathrm{l}$ and platelet count between 10,000-100,000/ $\mu$ l. Megaloblastic anemia $(62.5 \%)$ was found to be the most common cause of pancytopenia followed by aplastic anemia $(9.5 \%)$. Conclusions: Pancytopenia is a common haematological disorder. This study reveals that megaloblastic anemia and aplastic anemia are among the most common causes of pancytopenia.

\section{BHC 38}

Profile of Infections in Patients Receiving Immunosuppressive Therapy with Antithymocyte Globulin and Cyclosporine for Aplastic Anemia

Sharon Lionel Ninan", Anu Korula, Anup J. Devasia, P. N. Nisham, N. A. Fouzia, M. L. Kavitha, Aby Abraham, Alok Srivastava, Vikram Mathews, Biju George

Email ID for Correspondence: sharonlionel@gmail.com Department of Hematology, Christian Medical College, Vellore, Tamil Nadu

Introduction and Background: Immunosuppressive therapy with a combination of antithymocyte globulin (ATG) and cyclosporine is the treatment of choice for a patient with aplastic anemia (AA) lacking a HLA matched donor. Response rates with ATG range between $60-70 \%$ but the time to improvement of blood counts can take 
between 3-6 months. During this period, complications of infections and bleeding remain both related to the disease and ensuing neutropenia and the ongoing immunosuppression. This analysis was done to study the incidence of infectious complications in the first 3 months following administration of ATG. Patient/Material and Methods: This is a retrospective chart based review of all patients who were treated with ATG/ALG in the department of Haematology, CMC Vellore between 1995 and 2016. Data was collected from individual medical records and databases maintained in the department. Results: Six hundred and thirty-seven patients (including 409 males and 228 females) received ATG or ALG. The median age of the group was $33.7 \pm 17.9$ years) and $17.7 \%$ were children ( $<15$ years old). Very severe aplastic anemia (VSAA), severe aplastic anemia (SAA) and Non severe aplastic anemia (NSAA) accounted for $18.4 \%, 57.9 \%$ and $23.7 \%$ respectively. Documented infections were seen in $38.1 \%$. The prevalence of bacterial, viral and fungal infections was $22.3 \%, 5.3 \%$ and $10.5 \%$ respectively. Multi-drug resistant bacterial infections were seen in $5.8 \%$. In fungal disease, only $3.3 \%$ were "proven" (culture), $0.6 \%$ were "probable" (galactomannan) and $6.6 \%$ were "possible" (Imaging). E. coli and Klebsiella were the most common bacterial pathogens, while Herpes was the commonest viral infection. There was no difference in the infection rates among pediatric and adult populations $(p=0.263)$. However, disease severity had significant association with infection ( $\mathrm{p} \leq 0.001)$. Conclusions: One-third of patients receiving ATG have documented infections with it predominantly being bacterial infections with only $5.8 \%$ having infection with a multidrug resistant organism. Fungal infections accounted for $10 \%$ of which the majority was only possible infections. The utility of antibacterial and antifungal prophylaxis needs to be studied in this setting.

\section{BHC 39}

\section{Study of Hemoglobinopathies in Ajmer Region (Rajasthan)}

\section{Geeta Pachori, Supriya Singh Toor ${ }^{\#}$, Neena Kasliwal, Rashmi Sharma}

Email ID for Correspondence: drsupriyatoor@yahoo.com J.L.N. Medical College, Ajmer

Introduction and Background: Hemoglobinopathy is an inherited condition with alteration in the structure or quantity of hemoglobin, which reduces the oxygen carrying capacity of blood, manifesting as anemia that is unresponsive to conventional iron therapy. Patient/ Material and Methods: Data of 190 patients was collected for 6 months, retrospectively, who were screened by using High Performance Liquid Chromatography, HPLC (Bio Rad Variant II), red cell indices, peripheral blood film (PBF) and sickle cell test. Patients with severe anemia were included in the study. Results: A total of 44 $(23.15 \%)$ cases of various abnormal hemoglobins were detected using combination of red cell indices and HPLC technique. Most common hemoglobinopathy detected was $26(13.68 \%)$ cases of $\beta$ thalassemia minor, $7(3.68 \%)$ cases of delta-beta thalassemia, 5 $(2.63 \%)$ cases of $\beta$-thalassemia major, $2(1.05 \%)$ cases each of homozygous hemoglobin-D disease and hemoglobin-Q disease, 1 $(0.52 \%)$ case each of hemoglobin $\mathrm{E}$ disease and HbS-beta thalassemia. Out of total cases detected, $23(52.27 \%)$ cases were female patients, and $21(47.72 \%)$ cases were males. The age group of patients ranged from 6 months to 80 years, with most cases of $\beta$ thalassemia minor detected under 5 years of age. Conclusions: HPLC is an accurate and reliable tool to screen patients for hemoglobinopathies, especially targeting patients with refractory anemia. Screening and identification of these conditions has special importance in our national goal of achieving $12 \mathrm{gm} \%$ hemoglobin by the age of 12 in all children in the country.

\section{BHC 40}

A Study of Incidence of Bleeding in Children with Severe Hemophilia A on Low Dose Secondary Prophylaxis

\section{S. Gulshan", P. Chakrabarti, P. K. Mandal, R. De, T. K. Dolai}

Email ID for Correspondence: shzgulshan@gmail.com NRS Medical College and Hospital

Introduction and Background: Hemophilia $\mathrm{A}$ is an $\mathrm{X}$-linked inherited disease. $90 \%$ of the patients present with joint and muscle bleed. Once joint damage has occurred, it progresses over lifetime. Primary prophylaxis is the gold standard treatment. Secondary prophylaxis ( $\geq 2$ joint bleed and before onset of joint disease) is a more feasible option in developing countries. This study aims to determine the effect of low dose clotting factor concentrate (CFC) in terms of lowering bleeding episodes and hospital admission rate per year. Patient/Material and Methods: A prospective interventional study was conducted from January 2016 to August 2016 in Hematology Department of NRS Medical College, Kolkata. 20 children with severe Hemophilia A were given low dose factor VIII prophylaxis $20 \mathrm{IU} / \mathrm{kg}$ twice weekly for 3 months. All the patients were previously receiving on-demand f VIII or FFP. Patients were evaluated every week and data collected. Outcome was measured in terms of annual bleeding rate (ABR), number of days of school absenteeism per month and hospital admission rate (HAR) per year. Results: In our study median ABR (on demand) was 11 and median ABR (on prophylaxis) was 4 . The HAR when on demand was $3.2 /$ year and for when on prophylaxis was $0.2 /$ year. The mean school absenteeism (days/month) while on demand is 19.4 and while on prophylaxis is 15.8 .

\begin{tabular}{lll}
\hline & $\begin{array}{l}\text { Before } \\
\text { prophylaxis }\end{array}$ & $\begin{array}{l}\text { After } \\
\text { prophylaxis }\end{array}$ \\
\hline $\begin{array}{l}\text { Median annual bleeding rate } \\
\begin{array}{l}\text { Mean hospital admission rate } \\
\text { (no./year) }\end{array}\end{array}$ & 11 & 4 \\
$\begin{array}{l}\text { Mean school absenteeism (days/month) } \\
\text { Mean }\end{array}$ & 19.4 & 0.2 \\
\hline
\end{tabular}

Conclusions: Thus the present study indicates that children with severe Hemophilia A receiving low dose prophylaxis have a significant decrease in median ABR, HAR and moderate decrease in school absenteeism. The apprehension of trauma and bleed especially in rural areas also adds to the days of school absenteeism.

\section{BHC 41}

\section{Spectrum of Nutritional Deficiency Anemia in Pediatric Age} Group: A 3 Year Study

\section{Gunjan Nain", Richa Gupta}

Email ID for Correspondence: gunjan90nain@gmail.com Department of Pathology, Maulana Azad Medical College and Associated Hospitals

Introduction: Nutritional deficiency anemia is a rampant problem in pediatric age group. Nutritional deficiency in these children can lead to developmental delay, stunted growth and poor immunity. Published data regarding the spectrum of nutritional deficiency anemia in pediatric age group is scant. Materials and methods: A retrospective study was done to assess the type of anemia and its clinical manifestations in pediatric age group. All peripheral smears in the past 3 years were retrieved and correlated with the available clinical data. Observation: Over a period of 3 years, 3121 cases of pediatric anemia were studied out of which 600 cases were infants ( 28 days to 
12 months), 40 were neonates (1-28 days) and rest (2481 cases) were 1-12 yrs old. The most common type of anemia was microcytic hypochromic anemia $(41.7 \%)$ followed by dimorphic anemia. Microcytic hypochromic anemia most commonly presented with fever followed by fatigue and lethargy. Dimorphic anemia-38.3\% (1196 cases) mainly presented with fever and failure to thrive. $43.8 \%$ cases had hepatosplenomegaly. In macrocytic anemia $(38.3 \%)$ the most common presentation in was pneumonia, cough and coryza. 504 cases under went HPLC on the basis of coulter and smear findings out of which 35 cases were diagnosed as Beta thalassemia (31 cases- $\beta$ thalassemia trait, 2 case- $\beta$ thalassemia, 1 case of $\mathrm{HbE}$ thalassemia ana Hbs thalassemia each). Pancytopenia was seen in $29.5 \%$ of cases. It was most commonly associated with dimorphic anemia $(15.1 \%, 472$ cases), followed by macrocytic anemia (10.1\%, 317 cases) Pancytopenia was also observed in 132 cases of microcytic hypochromic anemia (4.2\%). Conclusion: Despite of various public health measures, nutritional deficiency anemia remains a rampant problem in children. Most of the children present with non specific complaints and fever secondary to infections as a result of reduced immunity. The most common type of anemia is microcytic hypochromic followed by dimorphic anemia. A large percentage of these children may also present with pancytopenia which is seen not only with macrocytic anemia but also with Microcytic hypochromic anemia secondary to Iron deficiency.

\section{BHC 42}

\section{Polycythemia Vera: An Unusual Presentation}

\section{Shubham Prasad ${ }^{\#}$, Priyanka Singh, Anand,} Prof. I. S. Gambhir, Prof. Vijay Tilak

Email ID for Correspondence: shubham769@gmail.com Institute of Medical Sciences, Banaras Hindu University, Varanasi

Introduction: Polycythemia Vera (PV) diagnosis is currently based on the 2008 WHO diagnostic criteria with evidence of erythrocytosis $(\mathrm{Hb}>18.5 \mathrm{~g} / \mathrm{dL}$ in men and $>16.5 \mathrm{~g} / \mathrm{dL}$ in women) being the first major and mandatory criterion. Here is a rare case report of a patient with final diagnosis of PV at $\mathrm{Hb}$ level of $15.4 \mathrm{~g} / \mathrm{dl}$. Patient presented with seizures and showed persistant toxic granules in neutrophils (mimicking sepsis) which is again very rare in PV. Case Report: A $55 \mathrm{yr}$ old male, farmer, presented with distal paresthesia for 6 months and 2 episodes of seizure in last 1 day. Examination revealed hepatomegaly and massive splenomegaly without fever, pallor, lymphadenopathy and bleeding manifestations. ABG parameters and blood sugar were normal. His peripheral blood counts over past 4 months were: $\mathrm{Hb} 15.4 \mathrm{~g} / \mathrm{dL}$, RBC $6,890,000 / \mathrm{mm}^{3}$ (MCV $68.0 \mathrm{fl}$, RDW $23.5 \%$, HCT $52 \%$ ), WBC $65,700 / \mathrm{mm}^{3}$ (neutrophils $84 \%$, lymphocyte $04 \%$, basophils $02 \%$, eosinophils $01 \%$ ) and platelets $674,000 / \mathrm{mm}^{3}$ with no blast cells. Majority of neutrophils were having toxic granules which failed to improve with broad spectrum antibiotics. Investigations did not reveal any source of infection. MRI brain with Venography and EEG were normal. Serum LDH-1009. CECT abdomen showed a large splenic infarct. BCR-ABL gene transcript was negative with positive JAKV617F EXON 14 mutation and low serum erythropoietin. Bone marrow biopsy showed trilineage hyperplasia with negative reticulin and absent iron on pearl stain. He was managed successfully with aspirin, hydroxyurea, allopurinol and weekly phlebotomy till hot below 45. Conclusions: True extent of $\mathrm{Hb}$ level is masked by corresponding increase in plasma volume and iron deficient state induced by PV per se. Since tests like red cell mass estimation and endogenous erythroid colony formation largely stands futile in routine clinical practice, the current WHO diagnostic criteria requires a revision with emphasis on a lower $\mathrm{Hb}$ threshold for accurate diagnosis and prompt treatment of masked PV.

\section{BHC 43}

Changes in Haematological Parameters Associated with Malarial Parasite Infection

\author{
Suman Kapuriya", Qadir Fatima, Mukesh Kumar, \\ Sonam Dubey
}

Email ID for Correspondence: mukeshrulaniya008@gmail.com S.P. Medical College, Bikaner

Introduction and Background: Malaria is a major mosquito-borne public health problem in Rajasthan. Various haematological changes occur with malarial infection. Early detection and proper treatment can prevent morbidity and mortality. For Detection of malaria complete blood count and peripheral blood smear are the earliest and most important test. Patient/Material and Methods: Haematological parameters of 150 patient present with fever and chills admitted in P.B.M. Hospital Bikaner, in which 70 patient are malaria infected and 80 are non malaria infected. Thick and Thin smear was made from EDTA blood sample and staining with Giemsa's staning for malaria parasite detection. Other haematological parameter test done by semi-automated haematological analyzer from the sample collected in EDTA. Platelet count and White blood cell count also done Manually by microscope. Results: Out of 70 patient $68 \%$ patient have decrease platelet count, $45 \%$ patient have absolute monocytosis and $40 \%$ patient have leukocytopenia. Conclusions: The study demonstrate that malaria is major health problem and associated with major illness and complications. Thrombocytopenia is one of the haematological abnormality observed in patient with malaria parasite infection. Other haematological changes are monocytosis and leukocytopenia.

\section{BHC 44}

\section{Haemophagocytic Syndrome with Cytophagic Histiocytic Panniculitis-A Rare Case Report}

\section{Deepak Vedant ${ }^{\#}$, Anupama Bains, Apoorvi Dubey, Parul Gautam, Neeraj Gupta, Abhishek Purohit, Shilajit Bhattacharya}

Email ID for Correspondence: dr.tomchi@gmail.com All India Institute of Medical Sciences, Jodhpur

Abstract: Haemophagocytic syndrome (HPS) is a potentially life threatening disorder characterized by proliferation of histiocytic cells throughout the reticuloendothelial system. Cytophagic histiocytic panniculitis (CHP) is a rare and fatal type of panniculitis which can occur in isolation or rarely as a cutaneous manifestation of HPS. We present a case of 19-year old female who presented with two-year history of moderate to high grade fever, joint pain and hepatosplenomegaly. Further, patient had developed hyperpigmented plaques for the 3 months. On investigations, she had bicytopenia. Histopathological examination of skin biopsy revealed fat lobules showing foamy histiocytes with engulfed red blood cells, lymphocytes and karyorrhectic debris giving a characteristic 'bean bag' appearance. Bone marrow aspirations and biopsy revealed prominence of histiocytic cells with evidence of haemophagocytosis. We present this case due to its rarity. 


\section{BHC 45}

Therapeutic Plasma Exchange (TPE) in Gullian Barre Syndrome a Study in SMS in Blood Transfusion Unit in SMS Hospital Jaipur

Kamal Kumar Sharma, Linu Hooda", Sarita Sharma, Ashalata, Sunita Bundas, Rachna Narayan, Ruchi, Prakash Shekhawat, Nippun

Email ID for Correspondence: linu321456@gmail.com SMS Hospital, Jaipur

Introduction and Background: In plasma Exchange the Pathological plasma containing the plasma antibodies is separated, Remove and is replaced with replacement. Resolution like colloid (albumen and fresh Frozen plasma Guillian barre syndrome is an acute poly radiculoneuropathy which is of rapid on set and this is treated by therapeutic plasma exchange or intravenous immunoglobulins therapy. Patient/ Material and Methods: The study was conducted in SMS Hospital patients from 2010 to 2016 the test was performed on GBS patients therapeutic plasma exchange was conducted in economically weak and BPL patients who could not afford the expensive intravenous immunoglobulins IgG. Results: Therapeutic plasma exchange was performed on GBS patients on three consecutive days and more then five procedures were conducted using Haemonetics Mcs+ on each occasion $2500 \mathrm{ml}$ plasma was extracted and was replaced with $100 \%$ replacement fluid of fresh frozen plasma and albumen the cost of therapeutic plasma exchange was about 35,000 which was of the kit of hemonotics MCS+ only' which was quite cheaper then intravenous immunoglobulins of about 2 lacs used for treating for GBS. Conclusions: Therapeutic plasma exchange has been proven to be a better and less costly treatment in guillian barre syndrome in comparison to intravenous immunoglobulin's and patients responded to therapeutic plasma exchange.

\section{BHC 46}

\section{Experience of a Stand Alone Low Resource BMT Set Up}

\section{Priya Marwah", Rajpreet Soni, Seema Bhattacharyya,} Lawrence Faulkner

Email ID for Correspondence: priyamarwah21@gmail.com South East Asia Institute for Thalassemia, Jaipur, India

Introduction and Background: Severe thalassemia syndromes (ST) are the most common non-infectious life threatening disease of children in South-East Asia and one of the main indications for BMT. ST is highly curable by BMT. Patient/Material and Methods: We analyzed a total of 50 consecutive first bone marrow transplants carried out between February 2012 and July 2016. G-CSF primed bone marrow was the source of hematopoetic stem cells in all cases. All patients were considered low or intermediate risk. All treatment and investigations were done at reasonably low cost. Results: Overall BMT actuarial outcomes in these 50 patients are $8 \%$ transplant related mortality (TRM), $18 \%$ rejection, $76 \%$ thalassemia free survival (TFS) and $90 \%$ overall survival (OS). In the 39 patients with low risk features based on liver size $<2 \mathrm{~cm}$ from costal margin and age less than 12 years outcomes are $5 \%$ TRM, $8 \%$ rejection, $87 \%$ TFS and $92 \%$ OS. These results compare very favourably to international standards. Moreover these BMTs have been performed on non profit basis, in the INR 800,000 lakhs range (12,000 USD). Conclusions: Bone marrow Transplantation in a low resource setting with results comparable to international standards is a possibility and should be considered as an opportunity for cure for thalassemia major children from middle and low socio economic backgrounds.

\section{BHC 47}

Burden of Haemophilia in Valley of Kashmir: Way Forward

Arooj Rashid", Ruby Reshi, Aleem Jan, Arshid Sidiqiue

Email ID for Correspondence: aleem_maleem@yahoo.co.in Sheri Kashmir Institute of Medical Science, Soura Srinagar J\&K 190011

Introduction and Background: Hemophilia, a disease of royals of England with destabilizing impact on Czars of Russia and a wrath in current times for people afflicted in underprivileged South East Asia. Aims and Objectives: To find the magnitude of Hemophilia in Kashmir valley and make suggestions to improve care in these patients. Patient/Material and Methods: One year study was conducted at Departments of Hematology, Blood Transfusion and Immunohematology of SKIMS and GMC Srinagar, clinical details, coaguloram, Factor VIII and IX assays type of treatment, risk factors for TTIs. All patients were tested for HBsAg, Anti HCV and HIV1/2 antibodies using third generation ELISA method. Results: 227 patients registered, majority $186(81.9 \%)$ had Hemophilia A and the remaining 41 (18.1\%) had Hemophilia B. $68.7 \%$ (156) patients had a significant family history. District Srinagar (22\%), district Baramulla (13.2\%) Anantnag $(12.8 \%)$ while less than $10 \%$ patients were registered from remaining seven districts. $21 \%, 36 \%$ and $30 \%$ patients were in first, second and third decades of life with only $13 \%$ patients above $30 \mathrm{yrs}$ of age. Oldest one being 60 year old from Bandipore with arthropathy. All patients received on-demand treatment mainly based on FFP and Cryoprecipitate. HCV infection positive in 48 (21.1\%). Mean age of anti-HCV reactive patients was $22.8 \pm 9.8$ years, perhaps because screening of anti-HCV prior to 2001 was not routinely done. Two tested positive for HBV infection $(0.4 \%)$. Knee being commonly affected target joint. Conclusions: Hemophilia is under diagnosed disease, need to improve coagulation laboratories. Treatment based mostly on FFP and Cryoprecipitate, exposes these patients to transfusion transmitted diseases, need for more sensitive screening methods, viral inactivation of products. Subsidized factors, on demand treatment susceptible to severe arthropathy necessitating the need for comprehensive care of these patients.

\section{BHC 48}

A Prospective Bone Marrow Study to Classify Anaemias Etiologically and Morphologically in Elderly Anaemic Patients

\section{A. S. Bansal ${ }^{\#}$, N. S. Mani, A. R. Nisal, A. J. Kelkar}

Email ID for Correspondence: ayushi13031303@gmail.com Bharati Vidyapeeth University Medical College, Pune 411043

Introduction and Background: Anaemia is a concern in elderly patients because of more severe complications, distinct aetiologies and effect on quality of life than in younger adults. NHANES-III (WHO) study revealed prevalence of anaemia as $11 \%$ in men and $10.2 \%$ in women aged 65 years and older. Rectification of aetiology significantly improves quality of life. Patient/Material and Methods: Bone marrow in 43 cases of anaemias in elderly $>65 \mathrm{yrs}$ as defined by the WHO were studied in 12 months. Clinical History, Biochemical, Radiological investigations, Haematological parameters using LH750 and Peripheral blood smears were recorded and correlated. These anaemias were classified etiologically and morphologically in correlation with their bone marrow findings. Results: Bone 
marrow studies in 43 cases of anaemic elderly were studied. There were 7 cases of haemophagocytosis, 6 cases of plasma cell dyscrasias, 5 cases of megaloblastic anaemia and 4 cases of aplastic anaemia. The remaining cases comprised a variety of diagnoses such as iron deficiency, acute leukaemias, myelodysplasias, lymphoproliferative disorders, immune thrombocytopenia etc. These findings were correlated with the etiological and morphological classification of these anaemias. Conclusions: The most common finding in bone marrow examination in our study was haemophagocytosis and was seen in a major portion of unexplained anaemias and anaemias due to chronic diseases (etiological classification) and normocytic normochromic anaemias (morphological classification). Bone marrow was found to be an essential requirement of the workup of elderly patients with anaemias in view of the variety of findings seen.

\section{BHC 49}

Phenotypic Heterogeneity of Haemoglobin E $\beta$ Thalassemia and Its Determinants-A Study from Tertiary Thalassemia Care Centre

\section{Kalantri Siddhesh", Soumita Choudhuri, Rudra Ray, Sambit Samanta, Siddhartha Sankar Ray, Maitreyee Bhattacharya}

Email ID for Correspondence: sidkalantri@gmail.com Institute of Hematology and Transfusion Medicine, Medical College Kolkata

Abstract: HbE Beta thalassemia is most common symptomatic hemoglobinopathy in many Asian countries. This is a very diverse disease with phenotypic variation from those who require regular transfusion to those who do not require any transfusion. Many environmental and genetic factors are identified for such a diverse nature of this disease. We studied 245 cases of E Beta thalassemia to document clinical heterogeneity in this group of patients and to identify various genotypic and environmental factors responsible for such heterogeneity. We did PCR (the most sensitive method) for detection of malarial infection in 90 patients. We documented asymptomatic malarial infection in $35.55 \%$ of these 90 cases. Xmn 1 polymorphism, alpha deletion and heterozygous HPFH gene were significantly more common in NTDT arm. Hemoglobin (E+A2) percentage and $\mathrm{Hb} F$ were significantly higher in NTDT arm. Still many factors are not unidentified to explain how just a small difference of haemoglobin (about 1-2 gm/dl) cause large variation in phenotypic presentation in these patients.

Keywords NTDT, TDT, HbE beta thalassemia, Malaria, Genotype, Phenotype

\section{BHC 50}

Evaluation of Macrocytosis in Routine Haematological Investigations in a Tertiary Health Set Up

Aeman Khalid ${ }^{1 \#}$, Noora Saeed ${ }^{2}$, Saima Khan ${ }^{1}$, Ayasa Parveen ${ }^{3}$, Sayeedul Hasan Arif ${ }^{4}$

Email ID for Correspondence: aemankhalid@gmail.com

${ }^{1}$ Junior Resident, ${ }^{2}$ Senior Resident, ${ }^{4}$ Professor, Department of Pathology, Jawahar Lal Nehru Medical College, Aligarh Muslim University, Aligarh; ${ }^{3}$ Junior Resident, Department of Medicine, Jawahar Lal Nehru Medical College, Aligarh Muslim University, Aligarh

Introduction and Background: Macrocytosis is a condition in which erythrocytes are larger in size than normal. This is seen as increase in mean corpuscular volume (MCV $>100 \mathrm{fl})$. This study aims at identifying macrocytosis in routine hemograms, to find the underlying etiology and identify the associated hematological features. Patient/ Material and Methods: This study included 56 patients whose detailed medical history was recorded. A complete blood count and peripheral smear examination was done on all patients. Subsequently, Vitamin B12 assay, folate assay, bone marrow aspiration, liver function test and thyroid function test were performed wherever needed. Results: Vitamin B12 deficiency was identified as the commonest etiological factor in 34 cases $(61 \%)$, alcoholism in 10 cases $(18 \%)$, drug intake in 6 cases $(10 \%)$. These three conditions accounted for $89 \%$ of macrocytosis. Other causes identified were chronic liver disease, chronic renal failure, aplastic anemia, myelodysplastic syndrome, folate deficiency and hypothyroidism. Anemia was observed in 52 cases (93\%), seen most commonly associated in Vitamin B12 deficiency. Remaining 4 cases showed isolated macrocytosis without anemia. Megaloblastic conditions showed hypersegmented neutrophils, macro-ovalocytes and increased red cell distribution width (RDW). In non-megaloblastic conditions uniform macrocytes were seen with normal RDW. Conclusions: Macrocytosis on routine hemograms should be evaluated even in absence of anaemia. Careful analysis of haemograms and peripheral blood smears assist in identifying the underlying cause and guiding further management.

\section{Topic: Benign Haematology—Laboratory}

\section{BHL 1}

Detection of a Rare Unstable Hemoglobin Variant by Gene Sequencing of Beta Globin Gene

\section{Nidhi Sharma", Reena Das, Sandhya Gulati, Jasbir Hira}

Email ID for Correspondence: nidhi11112222@gmail.com SMS Medical College, Jaipur

Introduction and Background: A 32 year old male was referred to the advanced hematology and HLA lab with complaints of repeated history of chest pain and brownish discoloration of urine. Investigations revealed an unusual pattern of hemoglobins on HPLC and further investigations led to a diagnosis of unusual hemoglobin. This case is being presented for its rarity. Patient/Material and Methods: Complete automated blood cell counts, reticulocyte counts, peripheral smear examination, hemoglobin electrophoresis at alkaline $\mathrm{pH}$ and HPLC of the hemolysate were done. Automated DNA sequencing was carried out of the beta globin gene in the Department of Hematology at PGIMER, Chandigarh. Results: The automated blood cell counts showed $\mathrm{Hb}-12.4 \mathrm{~g} / \mathrm{dL}, \mathrm{MCV}-93.7 \mathrm{fl}, \mathrm{MCH}-30.2 \mathrm{\rho g}, \mathrm{MCHC}-$ $32.2 \mathrm{~g} / \mathrm{dL}, \mathrm{RBC}-4.11 \times 10^{6} / \mu \mathrm{l}$ and RDW $16.3 \%$. He showed reticulocytosis with a reticulocyte count and percent being $322.6 \times 10^{9} / 1$ and $7.85 \%$. The peripheral blood film revealed schistocytes and presence of nRBCs (12/100 WBCs). HPLC on Variant revealed $\mathrm{HbF}: 0.6 \%, \mathrm{HbA}_{2}: 3.8 \%$, $\mathrm{HbD}: 3.2 \%$ with RT 4.1 mins and an Unknown peak at RT 4.83 mins: $21 \%$. Hemoglobin electrophoresis showed a normal pattern which brought in a suspicion of the presence of an unstable hemoglobin variant due to rapid degradation of the hemoglobin. Sequencing revealed that the patient had heterozygous beta 107 (G9) Gly>Arg (GGC-CGC; c.322G>C) which is Hemoglobin Burke which is an unstable hemoglobin. Tests for unstable $\mathrm{Hb}$ could not be done since a fresh blood sample was not available. The patient had a brother whose HPLC also revealed similar findings. Conclusions: Hemoglobin Burke is a rare hemoglobin variant and till date this has been reported as sporadic cases in two members of a Caucasian family and two unrelated Japanese. We report the presence of this rare variant of hemoglobin for the first time in India and the presence in two members of a family shows an autosomal dominant mode of inheritance. 


\section{BHL 2}

Detection of Hemoglobinopathies and Thalassaemia in Delhi, Punjab and Haryana Using CE-HPLC: Study on 8240 Subjects

\section{Naresh Kumar Kuchhal ${ }^{\#}$, Sachin Aggarwal, Vijay Jha \\ Email ID for Correspondence: nkkuchhal@gmail.com Bio-Diagnostics, Rohini}

Introduction and Background: Hereditary disorders of $\mathrm{Hb}$ mainly includes hemoglobinopathies and thalassaemia. Early and accurate detection of these disorders are immensely important both epidemiology and economically. These disorders can be prevented simply by population screening using Cation exchange High performance liquid chromatography (CE-HPLC), a technique introduced for the accurate and quick screening of hemoglobinopathies and thalassaemias. Worldwide, approximately 250 million people constituting $4.5 \%$ of the world population carry beta thalassemic gene and in India alone, the number is approximately 30 million with $50 \%$ in South East Asia. Similarly the burden of hemoglobinopathies in India is high with nearly 12,000 infants being born every year with severe disorders. These numbers imply that every hour 1 child is born, who may suffer with this genetic disorder. The present study was undertaken to evaluate the spectrum and frequency of various hemoglobinopathies and thalassaemia in the main city Delhi and adjoining states of Haryana and Punjab from where we have been receiving the maximum number of blood samples for Hemoglobinopathies screening. Patient/Material and Methods: The present single centre (Bio Diagnostics, Rohini), retrospective cross-sectional study was carried out on 8240 subjects which included 7860 antenatal mothers and 380 males The Study centre is a private lab specialized in doing Immuno-Assays, HPLC and Molecular Biology since 1991 in Delhi at Rohini. The lab is approved by BARC for doing RIA. The whole blood samples were received in K3 EDTA vacutainers and were analysed on the same day in a batch of 10-20 sample after receiving. CBC's were done on KX-21, Sysmex corporation and HPLC were done on D1O Bio-Rad fully automated Analyzer. The period of study was from April 2013 to Feb 2016. Patients with a recent history of blood transfusion were excluded from this study. Results and Discussion: Out of 8240 subjects were screened during the period of approximately three years, of which $7860(95.5 \%)$ were antenatal mothers and $380(4.5 \%)$ males. Almost all the subjects were Hindu. Overall 7560 were normal $(91.74 \%)$, while $680(8.26 \%)$ were abnormal of which $640(7.77 \%)$ were females and 40 $(0.49 \%)$ were males. Among the abnormal cases we have detected, 440 cases (400 female and 40 male) $(5.33 \%)$ of beta thalassemia minor (BTT), 90 cases of HbD Punjab (1.09\%) and 34 cases $(0.48 \%)$ of $\mathrm{Hb}$ E heterozygous $32(0.38 \%)$ cases of $\mathrm{Hb} \mathrm{J}$ Meerut, $12(0.14 \%)$ case of $\mathrm{Hb} \mathrm{Q}$ India and 4 case $(0.04 \%)$ of HbD Iran 8 cases of beta thalassaemia major $(0.09 \%), 11$ cases of sickle cell disease $(0.13 \%) 24$ cases of double heterozygous of beta thal with sickle $(0.29 \%)$ and 25 cases $\mathrm{HbE}$ with beta thal $(0.30 \%)$. CE-HPLC is a sensitive quick and accurate method for screening hemoglobinopathies. Conclusion: The study indicates that there is high prevalence of BTT and HbD Punjab in the state of Delhi, Punjab and Haryana.

\section{BHL 3}

Abstract has been retracted.
BHL 4

Performance of a New System for Image Based Analysis of Peripheral Blood Smears

Preethi S. Chari ${ }^{\#}$, Bharath Cheluvaraju, Kumudini Kakwani, Manish Kaushal, Apurv Anand, Rohit Kumar Pandey, Tathagato Rai Dastidar, Sujay Prasad

Email ID for Correspondence: preethichari@gmail.com Anand Diagnostic Laboratory, Bangalore

Introduction and Background: In this study, we evaluate a new system for image analysis based automated review of blood smears, SigTuple Shonit ${ }^{\mathrm{TM}}$ and analyze by (a) comparing numerical results with an automated haematology analyzer (Sysmex $\mathrm{XN} 3000^{\mathrm{TM}}$ ) and (b) manual verification of morphology analysis by qualified pathologists. Patient/Material and Methods:

(1) The study was carried out over 410 samples (100 with normal counts/no flags on cell counter, and 310 from patients with one or more quantitative or qualitative flagging).

(2) All slides were smeared and stained using May Grunwald Geimsa stain by Sysmex XN 3000-SP 10 autostainer.

(3) The smears were scanned and analyzed Shonit ${ }^{\mathrm{TM}}$. The parameters analyzed included WBC differential count, RBC and platelet morphology, $\mathrm{RBC}$ and platelet indices (the indices computation being still under validation).

(4) WBC morphology analysis was verified manually by three different pathologists.

(5) Quantitative parameters were analyzed by computing the correlation of the values with Sysmex XN3000 ${ }^{\mathrm{TM}}$.

Results: WBC morphology analysis by Shonit ${ }^{\mathrm{TM}}$ (WBC classification): Specificity $>99 \%$ and sensitivity $>98 \%$ for all normal WBC classes (neutrophil, lymphocyte, monocyte, eosinophil, basophil). RBC morphology analysis (size and shape): Specificity $>90 \%$ and sensitivity $>90 \%$ for all poikilocytes considered-normocyte, macrocyte, microcyte, elliptocyte, ovalocyte, teardrop, target, acanthocyte, fragmented, nucleated RBC. Schistocytes, sickles and spherocytes are still under validation. WBC differential count: Correlation between the differential counts generated by Shonit ${ }^{\mathrm{TM}}$ and Sysmex $\mathrm{XN} 3000^{\mathrm{TM}}$ was found to be within the known interanalyzer variability. Conclusions: Shonit ${ }^{\mathrm{TM}}$ has been found to be a helpful system for analyzing peripheral blood smears. The accuracy of WBC morphology analysis is within limits of inter-observer variability, while the WBC differential count correlates well with a standard five part hematology analyzer. Analysis of the poikilocytes with respective percentages, identification of platelet clumps and giant platelets and evaluating other RBC and platelet indices were found to be very helpful.

\section{BHL 5}

Comprehensive Evaluation of Iron Status in Patients of Chronic Kidney Disease-A Study to Assess the Best Indicators Including Serum Transferrin Receptor Assay

Surg Capt V. Manu\#, Maj Shilpi Saxena, Col Arun Kumar

Email ID for Correspondence: vmanu70@gmail.com Armed Forces Medical College, Pune 
Introduction and Background: Anaemia is an early complication of chronic kidney disease (CKD) and Iron deficiency is an important component in our setting. Functional iron deficiency can also occur in spite of normal indices. Routine standalone iron tests do not accurately reflect the actual status in the complex environment of CKD. Effective management requires identification, close monitoring and effective treatment of iron deficiency especially during treatment with recombinant erythropoietin (rEPO). False assessment of iron status may lead to inaccurate management of patients. Patient/Material and Methods: Serum Iron, ferritin, Total Iron Binding Capacity (TIBC) and transferrin Receptor (sTFR) levels were estimated in 68 newly diagnosed CRF cases and 100 primary IDA patients (controls). The values of percentage saturation (per_sat or TSAT), Log of ferritin (log_ferritin) and the ration of sTFR to $\log$ ferritin (sTFRlog_f) were calculated. The test group was divided based on ferritin levels (cut offs at 30, 50, 70 and $100 \mathrm{ng} / \mathrm{ml}$ ) and statistically analysed including ROC and AUC, to choose the best fit diagnostic test/parameters for accurate assessment of iron status in CKD cases. Results: Mean serum ferritin was $11.34 \mathrm{ng} / \mathrm{ml}$, $28.70 \mathrm{ng} / \mathrm{dl}$ and $281.81 \mathrm{ng} / \mathrm{ml}$ and mean sTFR was $2.34 \mu \mathrm{g} / \mathrm{ml}$, $1.82 \mu \mathrm{g} / \mathrm{ml}$ and $1.28 \mu \mathrm{g} / \mathrm{ml}$ in groups 1,2 and 3 . Among all groups the difference was found to be significant for S. iron, $\log _{-}$ferritin and sTFRlog_f, sTFR and sTFRlog_f showed good discrimination between pure IDA and ACD in the CKD cases. STFR_f gave the best discrimination at all the cut off levels of ferritin. At ferritin cut off of $50 \mathrm{ng} / \mathrm{ml}$, the sTFRlog_f value of 0.83 gave a sensitivity of $93.5 \%$ and specificity of $95.45 \%$ and at $100 \mathrm{ng} / \mathrm{dl}$, the same value of 0.83 gave a sensitivity of $95.5 \%$ and specificity of $86.5 \%$. Conclusions: Ratio of sTFR to log ferritin was the best indicator for assessing iron status in CKD and can be used effectively.

\section{BHL 6}

\section{S-Beta Thalassemia Leading to Avascular Necrosis of Left Hip} Joint in a Young Male-A Rare Case Report

\section{Sonia Agarwal", Shubhi Saxena, R. M. Jaiswal,} H. B. Saxena, B. P. Nag

Email ID for Correspondence: drsoniaagarwal@gmail.com; shoobheee@gmail.com

Mahatma Gandhi University of Medical Science and Technology, Sitapura, Jaipur

Introduction and Background: Sickle beta thalassemia is a disorder which represents the double heterozygous state for the $\mathrm{Hb}-\mathrm{S}$ and the $\beta$-thalassemia genes. The clinical and hematological manifestations of sickle beta thalassemia are highly variable due to existence of two types of genes, beta ${ }^{0}$ thalassemia gene and beta ${ }^{+}$gene. Beta ${ }^{0}$ gene leads to complete absence of $\mathrm{Hb}-\mathrm{A}$ levels, whereas beta ${ }^{+}$gene leads to production of $\mathrm{Hb}-\mathrm{A}$ levels $10-30 \%$. This disorder is diagnosed by levels of $\mathrm{HbS}, \mathrm{HbA} 2$ and $\mathrm{HbF}$ in Hemoglobin Electrophoresis. Patient: A young male patient presented with anemia, fatigue and joint pain with characteristic features of a vascular necrosis of left hip joint in X-ray and MRI. Results: For the etiological diagnosis further investigation in the form of capillary haemoglobin electrophoresis and for final confirmation genetic analysis by PCR is done. The patient was diagnosed as Sickle $\mathrm{Beta}^{+}$thalassemia. Conclusions: Hemoglobinopathies are a group of genetic disorders of hemoglobin in which there is abnormal production or structure of the hemoglobin molecule. These hereditary disorders are major public health problem in many parts of the world including India. The capillary haemoglobin electrophoresis and genetic study confirms the patient is compound heterozygous for Beta thalassemia and sickle cell and is likely to suffer from disease.

\section{BHL 7}

Utility of Raw Data in Flaged Differential Count (NE) in $A^{c} t 5 d i f f$ Cell Counter

Dinesh A. Rathod ${ }^{\#}$, Nita Ninama, Ms. Meenakshi Agarwal, Ms. Nisha Bhindora

Email ID for Correspondence: rathodpathology09@gmail.com Shraddhadeep Greencross Pathology Laboratory, Gandhinagar, Gujarat

Introduction and Background: The Raw data are parameters generated during analysis of sample by cell counter and from this data final results are derived. The present study aims to judge utility of Raw data of eosiniphils and to evaluate sensitivity and specificity of Raw data of eosinophil for absolute eosinophil count. Patient/Material and Methods: Total 114 cell counter CBC (Complete blood count) and Raw data were included. The 38 normal healthy individuals with no flag and values within reference range were considered as "normal". The 55 individual with AEC $>500 / \mathrm{cmm}$ were considered as "eosinophilia". The 22 CBC result with no value on differential count of neutrophils and eosiniphils is marked as "NE". Results: The Raw data mean show significant difference in AEC $>500 / \mathrm{cmm}$ and AEC $<500 / \mathrm{cmm}$ group $(\mathrm{P}<0.0001)$. ROC analysis shows specificity $(88 \%)$ and sensitivity $(100 \%)$ for detection of eosinophilia at Raw data value $>295$. Conclusions: Raw data of eosinophils is useful when differential count not given in "NE" flag as it gives insight view before peripheral smear examination.

\section{BHL 8}

Serum Transferrin Receptor in the Diagnosis of Iron Deficiency Anemia (IDA) Abhishek Pathre, Ruchika Sodhi", Meera Sikka, Sunil Gomber,
Satendra Sharma, Mrinalini Kotru

Email ID for Correspondence: ruchika.sodhi@gmail.com University College of Medical Sciences and GTB Hospital, Delhi

Introduction and Background: IDA constitutes a major public health problem in developing and developed countries. It adversely affects cognitive function, delays neurologic development and is associated with poor scholastic performance among children and adolescents. It is thus imperative to diagnose children with IDA and treat them early. Patient/Material and Methods: This cross sectional study was conducted in the Departments of Pathology and Pediatrics, UCMS and GTB Hospital, Delhi on 90 patients of age $\leq 12$ years. Iron profile and serum ferritin (SF) were done in all patients. Sixtytwo patients with IDA ( $\mathrm{SF}<16 \mu \mathrm{g} / \mathrm{L}$ and/or \% Transferrin saturation $(\% \mathrm{TS})<16 \%$ ) were finally included. Thirty five non anemic children were studied as controls. Serum transferrin receptor (sTfR) levels were measured (ELISA) to study it's utility as a diagnostic test for IDA. Results: Mean \pm SD sTfR was significantly $(\mathrm{p}<0.001)$ higher in patients $(6.0 \pm 2.9 \mu \mathrm{g} / \mathrm{ml})$ as compared to controls $(1.2 \pm 0.6 \mu \mathrm{g} /$ $\mathrm{ml})$. sTfR was elevated $(>1.8 \mu \mathrm{g} / \mathrm{ml})$ in $59(95.1 \%)$ patients and in 4 $(11.4 \%)$ controls. ROC curves were constructed. The AUC of sTfR was 0.985 indicating it's high clinical value as a diagnostic test for IDA. sTfR $\geq 2.25 \mu \mathrm{g} / \mathrm{ml}$ had a sensitivity and specificity of $90.3 \%$ and $94.1 \%$ respectively in diagnosis of IDA. Ratio of sTfR and $\log$ ferritin (sTfR-F index) was also significantly $(<0.001)$ higher in patients $(84.5 \pm 87.6)$ as compared to controls $(0.7 \pm 0.4)$. Conclusions: Raised receptor levels are a useful indicator of a functionally significant iron deficit. sTfR levels are not affected by an active acute phase reactant, thus it's more advantageous and specific than serum 
iron. Measurement of sTfR and sTfR-F index may be used as a non invasive tool for early diagnosis of IDA.

\section{BHL 9}

Early Detection of Disseminated Histoplasmosis on Peripheral Blood Smear in a Patient with Hepatitis B Infection

\section{Mayurakshi Das", Sandip Kumar \\ Email ID for Correspondence: mayurakshi4@gmail.com Institute of Medical Sciences, Banaras Hindu University, Varanasi, Uttar Pradesh}

Introduction and Background: Disseminated histoplasmosis is a progressive and often fatal haematogenous extra-pulmonary infection. Histoplasma capsulatum, an intracellular fungus of the reticuloendothelial system, is the pathogen responsible for histoplasmosis. Dissemination occurs in approximately 1 in 2000, mainly in immunecompromised patients and accounts for high morbidity and mortality. Patient: A 28 year old male patient presented with a history of low grade fever associated with chills and abdominal distension for 3 months. Physical examination revealed tender hepatomegaly and massive splenomegaly. There were no skin lesions or peripheral lymphadenopathy. Respiratory system, cardiovascular system and central nervous system were unremarkable on examination. A routine hemogram revealed pancytopenia. Total bilirubin and liver enzymes were mildly elevated. Serology for Hepatitis $B$ surface antigen and IgM antibody for Salmonella Typhi were positive. ELISA for HIV antibody detection was negative. Abdominal sonogram revealed hepatomegaly and splenomegaly with dilated portal vein and ascites. Peripheral blood smear revealed features of pancytopenia and occasional monocytes filled with numerous intracellular, PAS-positive, round to oval organisms of approximately $2-3 \mu$ size, and with eccentric nuclei, thickened wall and a clear halo suggestive of Histoplasma capsulatum. Bone marrow aspiration smears showed macrophages containing intracellular organisms of similar morphology. Fungal culture in Sabouraud's agar yielded colonies of Histoplasma capsulatum and confirmed the diagnosis. Results: We report a very rare case of disseminated histoplasmosis, initially diagnosed on peripheral blood smear, along with serological evidence of hepatitis B virus infection. The patient was not undergoing any immunosuppressive treatment nor had evidence of HIV infection. Conclusions: Recognition of disseminated histoplasmosis is extremely important as it is fatal without treatment. This case emphasizes the importance of meticulous microscopic examination of peripheral blood films as an early, rapid, simple and inexpensive tool for diagnosis of disseminated histoplasmosis in suspicious or immune-compromised patients.

\section{BHL 10}

\section{Heparin-Induced Thrombocytopenia: Correlation of Clinical} and Laboratory Parameters

\section{Narender Kumar", Varun Uppal, Jasmina Ahluwalia, Pankaj Malhotra, Neelam Varma}

Email ID for Correspondence: narenderkalson@gmail.com Postgraduate Institute of Medical Education and Research, Chandigarh

Introduction and Background: Heparin-induced thrombocytopenia (HIT) is an adverse drug reaction caused by heparin. HIT occurs in $0.5 \%$ to $5 \%$ patients; within 7 to 14 days following treatment with heparin. HIT occurs due to IgG antibodies directed against heparinbound platelet factor 4 (PF4). The aim of the study was to assess the ability of two commercially available immunoassays for detection of anti-PF4/heparin antibodies and to evaluate the result of the testing with the $4 \mathrm{~T}$ score to predict the pre-test clinical probability for HIT. Patient/Material and Methods: This was a prospective study carried out over a period of 2 years (2014-2016). A total of 17 patients with a suspected diagnosis of HIT were enrolled. The $4 \mathrm{~T}$ scoring of each case was performed. Testing for HIT was carried out by ID PaGia Heparin/PF4 antibody test and by STic Expert HIT 20. Results: Nine patients were on unfractionated heparin (UFH) while eight were receiving low molecular weight heparin (LMWH). Testing by STic Expert HIT test demonstrated positivity in three cases (no. 13, 14, and 16) while testing by ID PaGia Heparin/PF4 antibody test kit revealed four positive cases (no. 13, 14, 16, and 17). Among cases with a positive anti-PF4 antibody, three were on UFH and one on LMWH. The mean $4 \mathrm{~T}$ score in these cases is 6.25 as compared to 4.7 in PF4 antibody negative cases. Fifty percent (4/8) of cases with High $4 \mathrm{~T}$ score showed anti-PF4 antibody while none with the cases of low and intermediate $4 \mathrm{~T}$ score. Conclusions: The incidence of HIT is $23 \%$ in the present study of selected patients. The patients with high $4 \mathrm{~T}$ score have higher chances of HIT, however such cases should be tested for anti-PF4 antibody for confirmation.

\section{BHL 11}

Clinical Utility of Thrombin Generation Test and aPTT Clot Waveform Parameters in the Evaluation of Bleeding Phenotype in Patients with Bleeding Disorders

\section{Rutvi Gautam Dave ${ }^{\#}$, Sukesh Chandran Nair, Joy John Mammen, Tulasi Geevar, Ms. V. Ramya}

Email ID for Correspondence: rutvidave87@gmail.com Christian Medical College, Vellore

Introduction and Background: Thrombin is the central enzyme in the coagulation cascade. Estimation of one's potential to generate thrombin may correlate closely with clinical heterogeneity of bleeding severity. The clot based tests measure clotting time when only $5 \%$ of the total thrombin is generated and thus may not supply enough information to diagnose and treat patients according to their phenotype. Global haemostasis tests like thrombin generation test (TGT) and clot waveform analysis (CWA) might improve this circumstance. So, we correlated questionnaire based International Society of Thrombosis and Haemostasis-Bleeding Assessment Tool (ISTH-BAT score), parameters of TGT and CWA with factor levels and clinical bleeding severity in patients with clotting factor deficiency and VWD to assess the utility of TGT, CWA and ISTH-BAT in evaluation of bleeding phenotype. Patient/Material and Method: 147 patients with bleeding symptoms with deficiencies of factor VIII, IX, XI, II, V, VII, X or combination, VWD type 1 and 3 or no haemostatic defect diagnosed at CMC, Vellore were enrolled. We performed TGT by calibrated automated thrombogram, CWA by Sysmex CS2000i coagulometer and factor levels by one stage clot based assays. Patients were grouped according to clinical severity of bleeding and ISTH-BAT score. Results: All the parameters of TGT and CWA showed statistically significant difference between non-severe and severe bleeders. On ROC analysis in Haemophilia A, the peak thrombin (AUC 0.82) was a better discriminator than factor levels (AUC 0.77) to distinguish severe and nonsevere bleeders. The ISTH-BAT score had a sensitivity of $89.2 \%$ to identify bleeders. Conclusions: ISTH-BAT score appears to be sensitive screening tool to identify bleeders, while parameters of global haemostasis tests like CWA and TGT can more accurately predict the bleeding severity than conventional PT, APTT or factor assays in patients with factor deficiencies. Thus, a combined approach using these tools can improve the prediction of bleeding phenotype and management of patients with bleeding disorders. 


\section{BHL 12}

\section{Study of Coagulation Profile in Hepatic Disorders in a Tertiary} Care Centre

\section{Takutila M. Ozukum ${ }^{\#}$, Sandhya Gulati, Nidhi Sharma, Gaurav Gupta}

Email ID for Correspondence: scarlett.ozukum@gmail.com SMS Medical College, Jaipur

Introduction and Background: Liver is one of the vital organs in the body carrying out the functions of metabolism, synthetic, detoxification, etc. Liver diseases cause a deleterious effect on all these functions. Coagulopathy is one of the most commonly observed and routinely measured parameters in patients with liver disease. The aim of the study was to find the proportion of altered coagulation profile among patients with hepatic disorder. Patient/Material and Methods: A total of 170 cases of liver disease were taken. PT, APTT and platelet count were the parameters evaluated. PT was measured by Neoplastine Cl Plus kit, APTT by kit Cephascreen and platelet count by automated hematology analyser (Sysmex XT 4000i). Results: The proportion of patients with altered coagulation profile was found to be $90 \%$. The proportion of patients with altered PT, APTT and platelet count was found to be $80 \%, 82.5 \%$ and $52.5 \%$ respectively. Conclusions: Coagulation profile was found to be significantly altered in patients with liver disease but more studies are required in this field to evaluate the thrombotic events seen in patients with liver disease.

\section{BHL 13}

\section{A Comparison of Tests Used for Identification of Lupus Anticoagulants}

\section{Meera Sikka\#, Akanksha Rawat, Ruchika Sodhi, Usha Rusia, Kiran Guleria}

Email ID for Correspondence: meerasikka55@gmail.com University College of Medical Sciences and GTB Hospital, Delhi

Introduction and Background: Lupus anticoagulants (LA) are acquired antiphospholipid antibodies seen frequently in women with recurrent pregnancy loss. Treatment of these women increases the chances of a subsequent successful pregnancy. Their identification is hence important. This study compared the performance of four tests used in the identification of Las. Patient/Material and Methods: PT, APTT, LA sensitive APTT, KCT, dRVVT screening test were done in 100 women with recurrent fetal loss and 50 women with no obstetric complaints followed by dRVVT confirmatory test using commercially kits. Results: APTT and LA sensitive APTT were prolonged in $8 \%$ and $21 \%$ patients respectively. Mixing studies confirmed the presence of an inhibitor in all. A prolonged dRVVT screening time was detected in 16 women, 15 of whom showed a positive confirmatory test. LAs were detected in $15 \%$ women. dRVVT had the highest sensitivity $(98.8 \%)$ and specificity $(100 \%)$ followed by LA sensitive APTT. Conclusions: LAs are present in a significant proportion of women with RPL and can be identified using dRVVT screening test and LA sensitive APTT. Treatment of these women will improve their chances of a subsequent successful pregnancy.

\section{BHL 14}

Clinico-hematological Features and Spleen Histopathology in Patients of Genetic Hemolytic Anemias

\section{Murari", K. C. Pani, S. Sharma}

Email ID for Correspondence: mmurari@sgpgi.ac.in

Department of Pathology, SGPGIMS, Lucknow

Introduction and Background: Genetic hemolytic anemias are broad category of hematological disorders that includes the hemoglobinopathies, enzymopathies and membrane disorders. Splenectomy plays a very important role in management of genetic hemolytic anemias, it reduces transfusion requirements, relieves cytopenias consequent to hypersplenism and ameliorates risk of acute splenic sequestration crisis. It also eliminates the discomfort from mechanical pressure of enlarged spleen. Patient/Material and Methods: We studied 155 cases of various genetic hemolytic anemias over a twoyear period. Splenectomy was done as part of clinical management in twelve of these cases. The operative procedure was carried out in the department of surgical gastroenterology and paediatric super speciality. The indication of splenectomy was hypersplenism in most of the cases. In one patient partial splenectomy was done. Results: The twelve patients included 8 males and 4 females. Age ranged from 9 to 38 years. The spectrum included cases of hereditary spherocytosis, $\beta$ Thalassemia E $\beta$ Thalassemia, Sickle cell anemia, Sickle $\beta$ Thalassemia. Most of the thalassemia cases had intermedia phenotype. In one patient of sickle cell anemia open cholecystectomy was performed and small sized spleen was also removed. Gross examination revealed moderate to marked splenomegaly with either focal or diffuse areas of congestion. The histopathogical findings included prominent congestion of splenic cords with relative sparing of splenic sinus in patients with hereditary spherocytosis. Other changes included extramedullary haematopoiesis, focal hemophagocytosis, increased deposition of hemosiderin, presence of pseudogaucher cells and entrapped sickle cells in the congested sinusoids were also observed. Conclusions: Spleen histopathology revealed interesting observations that are infrequently reported in case series of genetic hemolytic anemias. The hematological profile improved in most of the cases post-splenectomy.

\section{BHL 15}

Serum Hepcidin in Multi-transfused Patients with $\beta$-Thalassemia Major and Its Correlation with Other Parameters

\section{Neha Niharika", Meera Sikka, Sunil Gomber, Satendra Sharma, Mrinalini Kotru}

Email ID for Correspondence: nehaniharika90@gmail.com University College of Medical Sciences and GTB Hospital, Delhi

Introduction and Background: $\beta$-Thalassemia major (BTM) is associated with significant morbidity and mortality due to iron overload resulting from ineffective erythropoiesis and repeated blood transfusions. Anemia and iron overload, both of which are present in children with BTM have opposing influence on hepcidin expression. This study aimed to assess the influence of iron overload and enhanced erythropoiesis as measured by sTfR on the levels of serum hepcidin in multitransfused patients with BTM. Patient/Material and Methods: Complete blood counts, serum iron (SI), total iron binding capacity (TIBC), percent transferrin saturation (\% TS), serum ferritin (SF), serum transferrin receptors (sTfR) and serum hepcidin were measured in 52 patients with BTM and 35 controls. Results: SF and sTfR were significantly $(\mathrm{p}<0.001)$ higher in patients as compared to controls. Serum hepcidin was significantly $(\mathrm{p}<0.001)$ higher in patients $(28.3 \pm 3.2 \mathrm{ng} / \mathrm{ml})$ as compared to controls $(8.3 \pm 4.6 \mathrm{ng} / \mathrm{ml})$. A negative correlation was seen between serum hepcidin and SI, TIBC and \%TS. No correlation was observed between hepcidin and SF as also sTfR. The hepcidin/ferritin ratio was significantly $(\mathrm{p}<0.001)$ lower in patients as compared to controls. Conclusions: A significantly higher level of hepcidin was seen in 
BTM patients. Hepcidin showed no correlation with SF and sTfR, suggesting that the stores and erythropoietic activity may not play a role in hepcidin expression. Low hepcidin/ferritin ratio indicated a suppression of hepcidin relative to degree of iron overload.

\section{BHL 16}

Hemoglobinopathy: Spectrum and Effects of Coexisting Nutritional Deficiency

\section{Shivani Nayar $\#$, Seema Acharya, Sanjeev Kishore, Rajeev Acharya (Professor, Obstetrics and Gynaecology)}

Email ID for Correspondence: drshivaninayar@yahoo.co.in Shri Guru Ram Rai Institute of Medical and Health Sciences

Introduction and Background: India is a significant reservoir of $\beta$ thalassemia and allelomorphs of $\mathrm{Hb} \beta^{\mathrm{A}}$ with variable geographical distribution. This study was undertaken to find out the spectrum of hemoglobinopathies by CE HPLC in local population, to evaluate the haematological parameters in these cases and to evaluate effects of nutritional deficiency on $\mathrm{HbA}_{2}$ levels. Patient/Material and Methods: In this prospective study conducted on 2197 samples, RBC indices were obtained by Sysmex XP 100 and CE HPLC was performed on BIORAD D10. The variant hemoglobins were identified on the basis of their percentage, retention times and peak characteristics. $\mathrm{PBF}$, reticulocyte count, $\mathrm{HbH}$ inclusion and sickling test were done in selected cases. Serum ferritin was assayed in 139 cases by chemiluminescence. Megaloblastic anemia was diagnosed by $\mathrm{CBC}$ and $\mathrm{PBF}$ with bone marrow examination/vitamin B12 levels in a few cases. Statistical analysis was done using Student $t$ test. Results: Hemoglobinopathies was seen in $5.28 \%$ cases. Heterozygous and homozygous $\beta$ thalassemia comprised $3.27 \%$ and $0.18 \%$ of total cases respectively. $2 \%$ variant hemoglobins (46/2197) included HbD trait (21), $\mathrm{HbE}$ trait and disease (12), HbS trait (06), HbQ India (02), $\mathrm{HbD}$ Iran (02), $\mathrm{HbJ}$ Meerut (01) and $\mathrm{HbH}(02)$. Four cases were compound heterozygous. A presumptive diagnosis of $\alpha$ thalassemia was made in $49.2 \%(32 / 65)$ of cases. Coexisting iron and vitamin B12 deficiency was seen in $27.27 \%(6 / 22)$ and $8.3 \%(6 / 72)$ of $\beta$ thalassemia traits respectively. Mean $\mathrm{HbA}_{2}$ levels was higher in megaloblastic anemia and lower in iron deficiency anemia in cases with normal HPLC pattern $(\mathrm{p}<0.05)$. Conclusion: Antenatal screening for hemoglobinopathies is recommended using CE HPLC. Coexisting nutritional deficiency alters $\mathrm{HbA}_{2}$ level significantly.

\section{BHL 17}

\section{Study of Endothelial Activation Markers in Polytransfused Children with Beta Thalassemia}

\section{T. Pallewar" ${ }^{\#}$ S. Sharma, J. Chandra, A. Nangia}

Email ID for Correspondence: tusharpallewar87@gmail.com Department of Pathology, Lady Hardinge Medical College, New Delhi

Introduction and Background: Patients of thalassemia have a chronic hypercoagulable state with an increased incidence of thromboembolic events. The etiopathogenesis of hypercoagulable states in these patients is multifactorial. Endothelial activation is one of the contributing factor. The present study was aimed at assessing the serum levels of Endothelial activation markers i.e. soluble Intercellular adhesion molecule (sICAM-1), E-selectin and their association with serum ferritin in polytransfused children with $\beta$-Thalassemia Major (TM). Patient/Material and Methods: In a cross-sectional study over a period of two years, 62 polytransfused $\beta$-TM children along with 26 age and sex matched controls were analysed for Complete Blood Count (CBC), Serum Ferritin, Liver Function Tests, Renal Function Tests, Prothrombin Time (PT), Activated Partial Thromboplastin Time (APTT), D-dimer, Intercellular adhesion molecule-1 and E-selectin. Results: The patients had mean age of $10.69 \pm 3.06$ years with male to female ratio of $1: 1$. The mean PT and APTT were within normal range, although found to be significantly higher $(\mathrm{p}<0.05)$ in patients than controls. The mean D-Dimer level was also significantly high in cases $(0.29 \pm 0.06 \mu \mathrm{g} / \mathrm{ml} \mathrm{FEU}$, $\mathrm{p}<0.001)$. The mean serum ICAM-1 and E-selectin levels were $731.34 \pm 343.97 \mathrm{ng} / \mathrm{ml}$ and $111.75 \pm 40.13 \mathrm{ng} / \mathrm{ml}$ respectively which were significantly higher than controls $(\mathrm{p}<0.001$ for both) however, no significant correlation was found between ICAM-1, E-selectin, S. ferritin and any of the coagulation parameters. Conclusions: The significantly high level of endothelial activation markers in thalassemia patients confirms that endothelial activation and dysfunction contributes to the subclinical hypercoagulable state in these patients.

\section{BHL 18}

Enhancing the Interpretational Skills of Complete Blood Count Reports by Second Year Medical Students

\section{Renu G'Boy Varghese", M. Niraimathi, M. Anand}

Email ID for Correspondence: renugboy@gmail.com Pondicherry Institute of Medical Sciences

Introduction: Medical professionals rely on laboratory reports to make their diagnoses and assess the efficacy of treatment. So it is absolutely essential that interpretation of laboratory reports form a part of medical curriculum. Medical council of India has recommended that we follow a competency based curriculum. Here we evaluated if teaching the medical students the skill of interpreting lab reports will help them to develop this competency. Methods: After obtaining permission from the institute's research committee and ethics committee, the fourth semester students were assessed on the knowledge they already had about interpretation of complete blood count (CBC) reports. After being divided into two groups, Group A (the study group) was given a training on interpretation of $\mathrm{CBC}$ reports. Both groups were again assessed on their ability to interpret various $\mathrm{CBC}$ reports. Group B (the control group) was given the same training on another day. Both groups were again assessed 1 month later. Results: The study group had 43 participants and the control group had 65 participants. Repeated measures ANOVA with Greenhouse-Geisser correction determined the mean score differed statistically significant over time points $(\mathrm{p}<0.001)$. Post hoc tests using Bonferroni correction revealed that in the study group, the score from pre training to post training (13.0 \pm 9.3 vs. $45.4 \pm 3.6 ; \mathrm{p}<0.001)$, posttraining to one month later $(45.4 \pm 3.6$ vs. $35.5 \pm 11.6 ; \mathrm{p}<0.001)$ were significant, while in the control group, the pretraining to one month later $(28.6 \pm 9.4$ vs. $37.2 \pm 10.7, \mathrm{p}<0.001$ was only significant. Between the study and control group, the score at the end of one month $(35.5 \pm 11.6$ vs. $37.2 \pm 10.7$ ) was not statistically significant, proving that both groups equally benefitted from the session on CBC interpretation. Conclusions: It is imperative that $\mathrm{CBC}$ interpretation be introduced as a part of the curriculum, as it aids students to make decisions based on the reports later in life. 


\section{BHL 19}

Serum Hepcidin in Children with Iron Deficiency Anemia (IDA)

\section{Abhishek Pathre", Meera Sikka, Sunil Gomber, Satendra Sharma, Mrinalini Kotru}

Email ID for Correspondence: pathrepatho.uc@gmail.com University College of Medical Sciences and GTB Hospital, Delhi

Introduction and Background: Anemia is a global public health problem affecting mainly preschool children, pregnant and nonpregnant women. Deficiency of iron is the most frequent cause of anemia, producing cognitive dysfunction, delayed neurologic development and behavioural disturbances in children. Its early diagnosis is hence important. The currently available tests, all have certain limitations. Hepcidin is a key regulator of iron homeostasis. This study aimed to measure the sensitivity and specificity of serum hepcidin as a diagnostic test for IDA in children. Patient/Material and Methods: Ninety children with anemia were recruited and complete blood counts and iron parameters (serum iron, serum ferritin, TIBC, \% TS) were measured. Sixty-two children (age $\leq 12$ yrs) with IDA (SF $\leq 16 \mu \mathrm{g} / \mathrm{ml}$ and $\% \mathrm{TS} \leq 16 \%)$ were studied. Thirty-five non-anemic children (age $\leq 12 \mathrm{yrs}$ ) with normal iron parameters were included as controls. Serum hepcidin was measured in all patients and controls. Results: Serum hepcidin was significantly $(\mathrm{p}<0.001)$ lower in patients $(1.8 \pm 1.8 \mathrm{ng} / \mathrm{ml})$ as compared to controls $(8.3 \pm 4.6 \mathrm{ng} /$ $\mathrm{ml})$. Serum hepcidin showed a significant $(<0.001)$ positive correlation with Hemoglobin $(r=0.337)$ and TIBC $(r=0.348)$. The AUC of serum hepcidin was 0.910 . Serum hepcidin $\leq 3.35 \mathrm{ng} / \mathrm{ml}$ had a sensitivity of $88.7 \%$ and specificity of $94.1 \%$ for diagnosis of IDA. Conclusions: Serum hepcidin levels are significantly reduced in children with IDA. The significant positive correlation with haemoglobin and TIBC suggests that serum hepcidin has potential utility as a diagnostic test for IDA in children. The $A U C^{R O C}$ for serum hepcidin indicates good diagnostic ability for IDA in children.

\section{BHL 20}

Diversity and Complexity of Immediate Acting Inhibitors in Congenital Haemophilia Patients

\section{Rucha Patil ${ }^{1 \#}$, Shrimati Shetty ${ }^{1}$, Kanjaksha Ghosh ${ }^{2}$,} S. Chandrakala ${ }^{3}$, Anita Parihar ${ }^{1}$, Aniket Mohite ${ }^{3}$

Email ID for Correspondence: rucha_colours@hotmail.com ${ }^{1}$ National Institute of Immunohaematology, ICMR, Mumbai; ${ }^{2}$ Surat Raktadan Kendra and Research Centre Regional Blood Transfusion Centre, Surat; ${ }^{3}$ Department of Haematology, Seth GS Medical College and KEM Hospital, Mumbai

Introduction and Background: With the exception of acquired haemophilia, factor VIII inhibitors are known to be time-temperature dependent. We present the diagnostic challenges, diversity and complexity of immediate acting inhibitors (IAIs) in 53 congenital haemophiliacs. Patients/Material and Methods: From 2012-2015, out of 4900 congenital haemophiliacs screened for inhibitors, 53 were found to have IAIs $(1.1 \%)$. Laboratory tests included one-stage factor assay, incubated mix inhibitor screening, Nijmegen modified Bethesda, lupus anticoagulant (LA) by dRVVT and FVIII ELISA. Results: Out of 53 patients, $27(51 \%)$ were found to have $<1 \%$ FVIII and FIX levels. This was the first major issue. In new cases, it is difficult to provide a diagnosis. Also a huge mistake of only carrying out FVIII assay and not going ahead with FIX assay is possible in any laboratory. 4 were identified as HB and 16 as HA based on their early reports and diagnosis in family members; 7 patients could not be given diagnosis. A strong LA or/and a high titer inhibitors was seen in these patients. 41 were diagnosed as HA; $58.5 \%$ were negative for incubated mixing tests, $53.6 \%$ had high FVIII Bethesda titer; $44 \%$ were positive for FIX Bethesda and $44 \%$ had low FIX levels $(<1-40 \%)$. LA and ELISA were done in 18 patients. 2 were LA negative and positive by ELISA: having only FVIII inhibitors. 2 patients were found to be inhibitor negative by ELISA: being only LA positive. Non linearity in 1:10 and 1:20 dilutions was seen in LA positive patients. Thus these patients were classified as follows:

\begin{tabular}{|l|c|c|c|c|c|c|}
\hline & $\begin{array}{c}\text { Only LA (no } \\
\text { F8I }\end{array}$ & $\begin{array}{c}\text { Only } \\
\text { Immediate } \\
\text { Acting F8I }\end{array}$ & $\begin{array}{c}\text { LA+ } \\
\text { Immediate } \\
\text { Acting F8I }\end{array}$ & $\begin{array}{c}\text { LA+ slow } \\
\text { acting F8I }\end{array}$ & $\begin{array}{c}\text { LA+ slow } \\
\text { acting F8I+ } \\
\text { Immediate } \\
\text { Acting F8I }\end{array}$ & $\begin{array}{c}\text { LA+ Immediate } \\
\text { Acting F8I/ LA/ } \\
\text { Immediate } \\
\text { Acting F8I }\end{array}$ \\
\hline & $11 \%$ & $11 \%$ & $-39 \%$ & & & \\
\hline LA & + & - & + & + & + & ND \\
\hline F8 ELISA & - & + & + & + & + & ND \\
\hline F8 Bethesda & + tnon-linear & + +non-linear & + +non-linear & + & + & + \\
\hline $\begin{array}{l}\text { 2hr incubated mix } \\
\text { screening }\end{array}$ & - & - & - & + & $+(1 \mathrm{hr}>$ 2hr) & - \\
\hline Factor IX levels & RED & RED & RED & RED & $+<1 \%$ & $-/+$ \\
\hline F9 Bethesda & - & - & $-/+$ & $-/+$ & + & $-/+$ \\
\hline
\end{tabular}

Out of 53 patients, 5 were HB, these patients had FIX Bethesda ranging from $4-32 \mathrm{BU} / \mathrm{ml}$ and FVIII $<1-2.5 \%$. These patients definitely may have a mixture of FIX inhibitors and LA or only strong LA as strong LA are known to give false positive results in Bethesda. Conclusions: These findings accentuate the diversity and complexity of rare IAIs in congenital haemophilia, which can result in misdiagnosis. Alternate options are ELISA and chromogenic assays, though these are cost prohibitive. Another issue is to define the titer of these IAIs due to the complex kinetics where the pattern of inactivation is non-linear and may lead to under- or over-estimation of inhibitors.

BHL 21

\section{Quality Indicators in Hematology Laboratory}

Pratik Poladia ${ }^{\#}$ R. Mohite*, U. Gavhane, B. Pillai, M. Tiwari, S. Pal, A. Karmore, U. Gosavi, S. Vaykar, A. Kumari, P. Dalvi, T. Mestry, S. Kamble. V. Bhatt, C. Naresh, P. Chavan

Email ID for Correspondence: pratikpoladia@rediffmail.com ACTREC, Tata Memorial Centre, Kharghar, Navi Mumbai 410210

Introduction and Background: Quality indicators (QI) are measurable, objective, quantitative measures of key system performance. They can indicate the quality of the key, strategic and support processes. It is important that quality indicators address all three key processes in the laboratory: pre-analytical, analytical and post analytical. Besides self-evaluation, quality indicators can also be used for benchmarking. It helps to capture information about quality indicators in the haematology laboratory and to evaluate laboratory quality performance over time as a strategy for continuous quality improvement effort. Patient/Material and Methods: A retrospective analysis of the following quality indicators for the period of March 2015-March 2016 was carried out: (a) Sample Rejection, (b) Equipment Breakdown, (c) Critical Alerts Reporting, (d) Turn-around-Time (TAT), (e) External Quality Assurance Scheme (EQAS) and (f) Internal Quality Assurance Scheme (IQAS). Results: A total of 41,222 haematology samples were received. 33 samples were rejected based on criteria clotted, mismatch, unlabeled etc. Equipment breakdown occurred 27 times, $99.9 \%$ critical alerts were informed telephonically; in addition system generated short message service (sms) was 
sent to patients. $2 \%$ reports were beyond the given TAT. Out of 14 CBC EQAS challenges comprising 112 tests 10 were outliers and of 16 Coagulation EQAS challenges comprising 32 tests 2 were outliers. Necessary corrective actions were taken. Internal quality control was performed routinely; results were within the laboratory defined ranges. Conclusions: Use of quality indicators to assess and monitor the quality system of the clinical laboratory services is extremely valuable tool in keeping the total testing process under control in a systematic and transparent way.

\section{BHL 22}

\section{Study of Anemia in Rheumatoid Arthritis}

\section{Manju Kumari", Sadhana Marwah, Vivek Arya \\ Email ID for Correspondence: manju.yadav126@gmail.com PGIMER, Dr. R.M.L Hospital, New Delhi}

Introduction and Background: Rheumatoid arthritis (RA) is a chronic inflammatory systemic disease of unknown etiology with a variety of extraarticular manifestations including fatigue, subcutaneous nodules, lung involvement, pericarditis, peripheral neuropathy, vasculitis, and hematologic abnormalities including anaemia. Anemia in rheumatoid arthritis is common and multifactorial. We studied the prevalence, type of anemia and its correlation with disease activity in Indian patients in a tertiary care hospital. Patient/Material and Methods: Fifty patients (45 female and 5 males) with recent onset ( $<5$ years) RA were included in the study. Complete blood counts, iron profile, serum ferritin and serum vitamin B12 and folate levels were performed. Anemia was defined as hemoglobin $<12 \mathrm{~g} / \mathrm{dl}$ in females and $<13 \mathrm{~g} / \mathrm{dl}$ in males. The anemic patients were evaluated for the type of anemia and serum ferritin $\leq 50 \mu \mathrm{g} / \mathrm{l}$ was taken as cut off for defining iron deficiency anemia. Disease activity assessment was done using DAS-28 score and functional disability assessment by using modified health assessment questionnaires (MHAQ) in all the patients. Results: Forty two patients (84\%) out of fifty were found to be anemic with 19 patients $(45.2 \%)$ having iron deficiency anemia and $23(54.8 \%)$ having anemia of chronic disease. None of the patients had megaloblastic anemia. In anemic group DAS-28 score and MHAQ were found to be $4.95 \pm 1.21 \& 1.07 \pm 0.58$ respectively and in non-anemic group $3.41 \pm 0.60 \& 0.40 \pm 0.36$ respectively. The disease activity was higher in patients with iron deficiency anemia than those with anemia of chronic disease, but was not statistically significant. Conclusions: Anemia is a common extraarticular manifestation in patients with RA and significantly correlates with higher disease activity. Anemia of chronic disease is the most common type of anemia in these patients. However, further studies including a larger number of patients with long term follow up are needed for conclusive results.

\section{BHL 23}

Role of Red Blood Cell Fragment (RBCF) and EMA Dye Binding Test in the Diagnosis of Hereditary Pyropoikilocytosis

\section{Shiwangi Chauhan ${ }^{\#}$, Pronati Gupta, Amitava Pahari, Shilp}

Email ID for Correspondence: Shiwangichauhan84@gmail.com Apollo Gleneagles Hospital, Kolkata
Introduction and Background: Hereditary Pyropoikilocytosis (HPP) is a subgroup of Hereditary Elliptocytosis Syndrome, it is a severe autosomal recessive haemolytic anemia with extreme poikilocytosis, microspherocytosis, microelliptocytosis on peripheral blood smear with MCV extremely low, normal MCHC and increased Osmotic fragility. Genetics of HPP has revealed that HPP probands have inherited structural mutations in both $\alpha$-spectrin allels from HE parents in a homozygous or compound heterozygous manner. The affected individuals present in infancy or early childhood with moderate-to-severe haemolytic anemia and unlike HE, the haemolytic anemia is lifelong rather than transient. Patient/Material and Methods: A 14 day old baby was admitted to Apollo Gleneagles Hospital, with unconjugated hyperbilirubinemia, which started from day 2 of birth but did not subside inspite of getting phototherapy outside. At admission baby was started on double surface phototherapy, examination revealed pallor and palpable spleen tip. There was no encephalopathy. Results: Liver function test showed Unconjugated Hyperbilirubenemia, with other parameters being unremarkable. Direct Coomb's test was -negative. There was no $\mathrm{ABO}$ and $\mathrm{Rh}$ incompatibility (Mother and baby both had blood group O Positive). G6PD assay was normal. Peripheral smear showed Microcytic Hypochromic RBC, showing gross anisopoikilocytosis, elliptocytosis, pyropoikilocytosis. RBCF was high. HPLC was normal. Osmotic fragility was increased. A Hereditary haemolytic anemia was suspected. Hemogram of mother showed anemia and microcytic hypochromic picture with moderate anisopoikilocytosis, plenty of elliptocytes and few fragmented cells. Her RBCF was also high. Fathers complete hemogram was normal. A diagnosis of Hereditary Pyropoikilocytosis was suspected and an EMA dye binding test was performed. Conclusions: EMA dye binding test was abnormal in the baby and strongly supportive of HPP. This example illustrates the importance of RBCF and EMA dye binding test in evaluation of Hereditary Hemolytic anemia, where Hereditary Pyropoikilocytosis and Heredtitary Elliptocytosis is a possibility.

\section{BHL 24}

One Year Study of Clinicopathological Features of Patients with Platelet Related Qualitative and/or Quantitative Abnormalities Coming for Complete Coagulation Workup

\section{Arpan Mehta ${ }^{\#}$, V. Ramya, G. Surendar Singh,} Joy Mammen, Sukesh Nair

Email ID for Correspondence: mehtaarpan@yahoo.com Christian Medical College, Vellore

Introduction and Background: Sizeable number of bleeding disorders have platelet related qualitative and/or quantitative abnormalities which usually lead to distinct bleeding patterns and laboratory abnormalities. It is prudent for pathologists to be aware of the same for a definitive diagnosis so that appropriate treatment can be administered to the patient. Patient/Material and Methods: We have reviewed all consecutive patients who presented to Christian Medical College for evaluation of disorders of haemostasis over a one year period ending June 2016. Demographic, clinical and laboratory parameters were recorded using Standard data sheet in Microsoft Excel 2013. Results of laboratory testing including screening tests, confirmatory coagulation factor assays as well as platelet function tests were analysed. In addition, immunophenotyping was performed where indicated in patients with platelet function defects. Results: 
145 patients were concluded as having platelet related qualitative and/ or quantitative defects.

\begin{tabular}{lc}
\hline Disorder & Number of patients \\
\hline Glanzmann thrombasthenia (GT) & 55 \\
Bernard Soulier syndrome (BSS) & 11 \\
$\begin{array}{l}\text { Acquired platelet dysfunction with } \\
\quad \text { eosinophilia (APDE) }\end{array}$ & 30 \\
$\begin{array}{l}\text { Other platelet related qualitative and/or } \\
\quad \text { quantitative defects }\end{array}$ & 49 \\
Total & 145 \\
\hline
\end{tabular}

Mean age of the patients was 12 years (range: 9 months-71 years). There was slight female predilection ( $\mathrm{M}: \mathrm{F}=1: 1.2)$. Family history was positive in 46/145 (31.7\%) patients and 57/143 (39.9\%) were born of consanguineous marriage. Bleeding time was prolonged in $72.6 \%$ and closure time by Siemens PFA-200 was prolonged in $92 \%$ of patients examined. There were no significant abnormalities in plasma coagulation tests like PT, APTT and TT in majority of patients. Platelet function tests performed by platelet aggregometry showed significant abnormalities in $91.2 \%$ patients examined. Immunophenotyping helped in confirming diagnosis in GT and BSS. Conclusions: Platelet function defects continue to be a significant clinical problem in our country which may be due to persistence of consanguinity in the population. Detailed clinical history and laboratory evaluation are essential to classify and confirm diagnosis.

\section{BHL 25}

\section{Assessment of Myeloperoxidase (MPO) Gene Polymorphism in Patients with Chronic Kidney Disease (CKD)}

Sunil Debadwar", Satendra Sharma, Meera Sikka, O. P. Kalra, Neelam Wadhwa

Email ID for Correspondence: sunildebadwar@gmail.com Department of Pathology, UCMS \& GTB Hospital, Dilshad Garden, Delhi, India

Introduction and Background: $\mathrm{CKD}$ has estimated worldwide prevalence of $8-16 \%$. Analysis of molecular genes may help in understanding the pathogenesis of CKD. MPO gene has been implicated in underlying pathology of CKD. MPO has role in inflammation and endothelial dysfunction through MPO- $\mathrm{H}_{2} \mathrm{O}_{2}$-halide system and interaction with nitric oxide (NO) respectively. Hence MPO gene polymorphism may prove to be an early marker for identification of disease progression and its prevention. Patient/Material and Methods: MPO 463 G/A gene polymorphism studied in 50 patients with CKD (stages 3 to 5) and 50 healthy controls by Polymerase Chain Reaction Restriction Fragment Length Polymorphism (PCR-RFLP) technique. Serum MPO levels were measured by double sandwich Enzyme Linked Immunosorbent Assay (ELISA). Results: Serum MPO levels were significantly higher in patients with CKD than controls $(\mathrm{p}<0.001)$. Distribution of MPO genotypes GG, GA and AA genotype in patients was found to be $74 \%(37 / 50), 24 \%(12 / 50)$ and $2 \%$ (1/50) respectively. In controls GG, GA and AA genotypes were seen in $72 \%(36 / 50), 26 \%(13 / 50)$ and $2 \%(1 / 50)$ respectively. No significant difference was found in distribution of MPO genotypes in patients with CKD compared to controls $(\mathrm{p}>0.05)$. No significant correlation was found between MPO 463G/A polymorphism and serum MPO levels. Conclusions: Serum MPO levels were significantly higher in patients with CKD as compared to controls. This study had shown the role of MPO in CKD by virtue of its role in inflammation. Difference in distribution of MPO genotypes in patients with CKD and healthy controls was not found to be statistically significant. Similarly no significant difference found in serum MPO levels across MPO genotypes. However further larger studies are warranted to elucidate if there exists any significant association between MPO polymorphism and CKD and with serum MPO levels.

\section{BHL 26}

Incidences of Spurious High Platelet Count by Modern Six Part Hematology Analyzers-Hematologist's Dilemma!

\section{Rateesh Sareen", Rohit Jain, Menka Kapil, G. N. Gupta}

Email ID for Correspondence: drrateeshsareen@yahoo.co.in Santokba Durlabhji Memorial Hospital \& Cum Medical Research Institute

Introduction and Background: The use of automated analyzer in hematology has become a rule rather than an exception. With the lack of proper legislation many laboratories rely heavily on automated analyzers and reports are given to patients without examination of peripheral blood smear by Haematologist/Pathologist. There is no doubt that these instruments have improved the accuracy of results with optimal quality control measures in laboratory. There are wide varieties of conditions where automated parameters are fictitious and thus require careful attention as these dictate clinical management of patients. Patient/Material and Methods: We present a case of arte factual rise in platelet count on three consecutive occasions by six part automated hematology analyzers. Spurious elevation of platelet count are seen in patients with extensive burn, extreme microcytosis, cryoglobunemia, infections like bacterial, fungal \& yeast. In our case all possible causes of spurious elevation of platelet counts were ruled out except microcytosis. This poses a risk of under evaluation of true thrombocytopenia as patient's reported platelet count could be spuriously normal or high. Result and Conclusions: The present trend in modern day Hematology is to lessen the need to review blood smears but at the same time it is important that samples with unexpected blood counts in relation to clinical settings or those with specific flags require careful review of blood smear. Although computers are magnificent tools for realization of our dreams but no machine can replace the human spark of understanding, compassion and spirit.

\section{BHL 27}

Pharmacological Induction of Foetal Haemoglobin and Its Correlation with F-Cells in Severe HbE-Beta Thalassaemia

Sujana Biswas", Rudra Ray, Arijit Nag, Kaushik Roy, Kanjaksha Ghosh, Maitreyee Bhattacharyya

Email ID for Correspondence: sujana.sujana@gmail.com Institute of Haematology and Transfusion Medicine, Medical College, Kolkata

Introduction and Background: HbE-beta thalassemia is a common disorder in eastern India with varying clinical presentation. Pharmacological induction of foetal haemoglobin is shown to reduce disease severity in sickle cell disease. The efficacy of hydroxyurea in induction of foetal haemoglobin in severe HbE-beta thalassaemia and its correlation with F-cells was evaluated. Patient/Material and Methods: 60 patients with severe HbE-beta thalassaemia were started on Hydroxyurea $(10 \mathrm{mg} / \mathrm{kg} /$ day $)$. Their clinical and laboratory 
parameters were observed at the baseline level and every month during the follow-up period. All patients were followed up prospectively for transfusion requirement, drug effectiveness and toxicity. Their HbF was measured by HPLC and their number of F-cells was measured by Flow Cytometry. One normal adult and one cord-blood sample were used as control to detect the number of F-cells by Flow Cytometry. The mean proportion of F-cells in normal persons is $2.7 \% \pm 1.4 \%$, with a range of $0.5 \%-7.0 \%$ (Blood. 1975 Nov; 46(5):671-682). Results: With median follow-up of 18 months, out of the 60 patients, 47 showed an increase in $\mathrm{HbF}$ level and their transfusion requirements reduced significantly. Their $\mathrm{HbF}$ level increased from $12.5 \pm 0.19$ (mean $\pm \mathrm{SD}$ ) to $30.5 \pm 0.21$ (mean $\pm \mathrm{SD}$ ), and maintained the same value with significantly reduced requirements of transfusion; proving the efficacy of the drug. The number of F-cells measured by Flow Cytometry was correlated with the HbF level by HPLC. It was observed in most cases the rise of $\mathrm{HbF}$ is associated with increase of F-cells, while in some the above pattern was not observed. The number of F-cells of a normal healthy adult was observed to be $0.8 \%$ and the number of F-cells in cord blood was observed to be $92 \%$, which were taken as controls. Conclusions: Pharmacological induction of $\mathrm{HbF}$ lead to reduction of transfusion requirements in some patients with severe HbE-beta thalassaemia. By flow cytometry, the number of F-cells can be determined, which are actually responsible for the production of $\mathrm{HbF}$ (measured by HPLC). But it has been observed that rise of $\mathrm{HbF}$ is not always associated with proportionate increase of F-cells.

\section{BHL 28}

Reliability of Different RBC Indices and Formulae in Discriminating Between Various Hemoglobinopathies and Iron Deficiency Anemia in Antenatal Patients

Reema Bhushan", Shailaja Shukla, Divyanshu Singh, Deepshika Dhiman, Sunita Sharma

Email ID for Correspondence: drreems25@gmail.com Lady Hardinge Medical College, New Delhi

Introduction and Background: Thalassemia and related hemoglobinopathies affect the quantity and quality of haemoglobin. High performance liquid chromatography (HPLC) is considered the gold standard for diagnosis of hemoglobiopathies. This test is not available at many peripheral centres. Many formulae or indices based on complete blood counts are available that can aid in the diagnosis. The aim of the study was to analyse the reliability of these indices in distinguishing between the most common causes of microcytic hypochromic anemia i.e. iron deficiency anemia (IDA) and hemoglobinopathies. Patient/Material and Methods: A retrospective study was performed on 2000 women who underwent antenatal screening for $\beta$ thalassemia trait $(\beta-\mathrm{TT})$ and related hemoglobinopathies. Out of these, 63 were detected to have hemoglobinopathies, diagnosed on HPLC and 1194 were found to have iron deficiency anemia. Nine indices were calculated among both the groups using complete blood count parameters. Sensitivity and specificity of these indices to detect $\beta$-TT versus IDA was compared. Likelihood ratio, accuracy and youden's index for these indices were calculated. Results: Sensitivity of Shine and Lal index was highest $(98.4 \%)$ followed by Kerman 1 index $(66.7 \%)$. Mentzer Index, England and Fraser Index, RDW index and Ehsani index each showed high specificity $(99.7 \%)$. Kerman 1 index could point towards the correct diagnosis in $97.2 \%$ of the patients. Accuracy was highest for RDW index $(97.8 \%)$ followed by Kerman 1 index $(97.2 \%)$. Youden index was highest for Kerman 1 index (65.4) followed by RDW index (63.1). None of the index was $100 \%$ sensitive or specific. Conclusions: None of the indices for screening of hemoglobinopathies have $100 \%$ diagnostic accuracy. However, in the present study, Kerman 1 index and RDW index were found to be most useful in discriminating between hemoglobinopathies and IDA.

\section{BHL 29}

Clot Wave Form Analysis of Activated Partial Thromboplastin Time: An Early Marker of Sepsis?

\section{Febe Renjitha Suman\#, Ms. Umalakshmi, Mr. Ananda Kumar}

Email ID for Correspondence: febemd@gmail.com Sri Ramachandra Medical College and Research Institute, Chennai

Introduction and Background: Sepsis is a common cause of mortality and morbidity in any hospital. It is important to distinguish systemic inflammatory response syndrome (SIRS) and sepsis at an early stage using a cost effective early marker. It has been postulated that calcium dependent complexes are formed between very low density lipoprotein and $\mathrm{C}$ reactive protein which causes changes in the clot wave (CW) of activated partial thromboplastin time (APTT). The formation of precipitate and coincident turbidly is responsible for the early fall in light transmittance and is determined by continuous measurement during the performance of APTT. A biphasic wave form is noted in disseminated intravascular coagulation due to sepsis. Patient/Material and Methods: This study was done at the clinical pathology division of central laboratory services for a period of 3 months. Normal controls (n: 40), suspected sepsis (n: 40) and proven sepsis $(n=40)$ were enrolled. Only adult patients were included. The equipment used was C5 2400, Sysmex corporation, Kobe Japan. The APTT done on the day of culture requests were observed for the clot wave $(\mathrm{CW})$ as the instrument raw data file (dat file). The $\mathrm{CW}$ was analyzed by version 3.0/for windows software. For significant differences Welch t two sample $t$ test is used. The analysis was done for maximum coagulation velocity (Min 1), maximum coagulation acceleration (Min 2) and maximum coagulation deceleration (Max 2). Results: Max 2, Min 2/Min 1 and Max 2/Min 1 showed significant differences $(\mathrm{p}<0.05)$ between suspected sepsis and sepsis. Min 1 and Min 2 showed no significant difference between the groups. Conclusions: CWA of APTT may be a cost effective promising marker to predict sepsis at an early stage with diagnostic, therapeutic and prognostic value. However expertise is needed. As the CWA is still a research parameter, this pilot study needs further analysis.

\section{BHL 30}

Study of Anaemia in Type 2 Diabetes Mellitus and Its Correlation with Albuminuria and eGFR

\section{Neha Sharma", Sadhana Marwah, Harish Kumar Gupta}

Email ID for Correspondence: neha.llrm.kriti@gmail.com PGIMER \& Dr. RML Hospital, New Delhi

Introduction: Diabetes mellitus (DM) is a common metabolic disease. Anaemia is a common accompaniment to diabetes, particularly in patients with albuminuria or reduced renal function. Anaemia is considered as a key indicator of chronic kidney disease and an important cardiovascular risk factor in diabetes. Anaemia is more frequent and more severe at any level of glomerular filtration rate (GFR) in diabetics compared to nondiabetic patients. The pathogenesis of anemia in diabetes includes deficiency in erythropoietin synthesis and release, systemic inflammation, iron deficiency and 
probably iatrogenic factors, e.g., angiotensin converting enzyme (ACE) inhibitors. Materials and Methods: One hundred patients with type 2 Diabetes Mellitus were included in the study. Complete blood count, Serum Iron profile, Ferritin, Vitamin B12 and Folate, Kidney function tests and urine albumin creatinine ratio were assessed for these patients. Estimated Glomerular Filtration Rate (eGFR) was calculated with MDRD 4-variable formula. Results: Fifty four patients $(54 \%)$ were found to be anaemic. Serum ferritin $<50 \mu \mathrm{g} / \mathrm{l}$ was taken as cut off for defining Iron deficiency anaemia. 21 patients had iron deficiency anaemia (IDA) and 33 had anaemia of chronic disease (ACD). Serum vitamin B12 and folate levels were within normal limits in all patients. There was a rise in the prevalence of anaemia from $25.9 \%$ in patients with a normal ACR to $59.2 \%$ in those with microalbuminuria and to $75 \%$ in macroalbuminuria. There was increase in the prevalence of anaemia with an increase in the stage of renal disease which was classified by eGFR. Conclusions: Anaemia is a prevalent finding in type 2 diabetic patients. Any degree of renal impairment and albumiuria are the risk factors for anaemia in these patients. Hence screening, characterization and treatment of anaemia in type $2 \mathrm{DM}$ may be helpful in management of these patients.

\section{BHL 31}

\section{Utility of Bone Marrow Biopsy in Bony Lesions-An Underexplored Modality}

\section{Sarika Singh $^{1 \#, ~ A r u n a ~ C h h i k a r a ~}{ }^{2}$, Deeksha Singh ${ }^{2}$,} Anamika Kashyap ${ }^{2}$, Sunita Sharma ${ }^{2}$, Jagdish Chandra ${ }^{3}$

Email ID for Correspondence: sarikasingh97b@gmail.com ${ }^{1}$ Department of Pathology, Maulana Azad Medical College; ${ }^{2}$ Department of Pathology, Lady Hardinge Medical College; ${ }^{3}$ Department of Paediatrics, Lady Hardinge Medical College

Introduction: Bony lesions are generally diagnosed on the basis of radiological and biochemical findings. Bone biopsy is indicated to arrive at the final diagnosis in malignancies. However, utility of bone marrow examination is underutilized and underexplored in benign lesions of bone. Case History: Case-1: A 3 month old female presented with leukocytosis, thrombocytopenia and $5 \%$ blasts in peripheral smear. Her bone marrow aspirates on repeated occasions failed to reveal any significant findings. In view of these findings a possibility of hematolymphoid malignancy was suggested and bone marrow biopsy was advised. Her biopsy showed hypocellular bone marrow fragments with suppressed erythroid, myeloid and megakaryocytic series. Bone marrow also showed new bone formation at various stages of ossification and presence of $5 \%$ blasts demonstrated by immunohistochemistry. A final diagnosis of osteopetrosis was made. Case-2: A 8 month old female, who presented with anaemia, thrombocytopenia, failure to thrive and delayed milestones. Her bone marrow aspirate smears were diluted, while bone marrow biopsy showed replacement of hematopoietic elements by fibroblasts. Only a few erythroid, myeloid precursors and megakaryocytes were seen. The reticulin stain showed significant fibrosis consistent with myelofibrosis. Based on bone marrow biopsy findings and vitamin D levels a final diagnosis of rickets was made. Conclusion: Bony lesions can present with cytopenia of variable grades. In such cases, besides considering the usual hematological disorders, bony lesions should also be kept in mind. Complete clinical, radiological, biochemical, and pathological workup can help to arrive at an early and correct diagnosis of treatable conditions.
BHL 32

Impaired Fibrinolysis as a Cause of Venous Thrombosis in Indian Patients: A Comprehensive Study

\section{Aniket Prabhudesai", Shrimati Shetty, Kanjaksha Ghosh, Bipin Kulkarni}

Email ID for Correspondence: prabhudesai.aniket@gmail.com National Institute of Immunohaematology

Introduction and Background: Conventional thrombophilia markers Protein C, Protein S, Antithrombin, and Factor V Leiden are being routinely studied in Indian patients with venous thrombosis. About $2 / 3$ rd of these cases are found to be negative for these markers, though many of them have strong history of recurrent thrombosis and thrombosis in unusual sites. Impaired fibrinolytic function is increasingly being reported to be the cause of several kinds of thrombotic diseases. There is no data on comprehensive study of the fibrinolytic defects as causative factors of venous thrombosis in Indian patients. Patient/Material and Methods: 224 venous thrombosis patients were studied for conventional thrombophilia markers along with Fibrinolytic markers Plasminogen, Tissue Plasminogen Activator (TPA), Plasminogen Activator Inhibitor (PAI-1), Thrombin activatable fibrinolysis inhibitor (TAFI), Alpha-2-Antiplasmin, Thrombomodulin and Tissue factor pathway inhibitor (TFPI). Factor V Leiden mutation and PAI-1 promoter $4 \mathrm{G} / 5 \mathrm{G}$ polymorphism were also studied. Results: $17.9 \%$ (40 cases) of venous thrombosis were accounted by conventional thrombophilia screening of which $7.6 \%$ (17 cases) had Factor V Leiden mutation, $5.8 \%$ (13 cases) were Protein C deficient, $3.1 \%$ (7 cases) Protein S deficient and $1.3 \%$ (3 cases) Antithrombin deficient. However, a comprehensive study including fibrinolytic parameters could explain $33.48 \%$ (75 cases) of venous thrombosis. Of all the fibrinolytic markers studied, $16.1 \%$ (36 cases) had grossly elevated PAI-1 level, $4 \%$ (9 cases) had reduced TPA level, $2.7 \%$ (6 cases) had high Antiplasmin level, $1.3 \%$ (3 cases) had reduced plasminogen level, $0.9 \%$ (2 cases) had low thrombomodulin level and $0.9 \%$ high TAFI levels and $0.4 \%$ (1 case) had low TFPI level. $21.8 \%$ cases had 4G/4G genotype of the PAI-1 promoter polymorphism associated with high PAI-1 levels. Conclusions: Data shows that testing for markers of both the anti-coagulant system and the fibrinolytic pathway will facilitate in a comprehensive explanation of the cause of venous thrombosis.

\section{BHL 33}

\section{Congenital Methemoglobinemia: A Rare Entity}

Satendra Sharma ${ }^{1 \#}$, Priyanka Gogoi ${ }^{1}$, Preeti Diwakar ${ }^{1}$, Asha Sharma $^{2}$, Mrinalini Kotru ${ }^{1}$, Meera Sikka ${ }^{1}$, Rajesh Pathak ${ }^{1}$

Email ID for Correspondence: drsatendrasharma@gmail.com ${ }^{1}$ Department of Pathology, University College of Medical Sciences and Guru Teg Bahadur Hospital, Delhi; ${ }^{2}$ Department of Obstetrics and Gynaecology, St. Stephens Hospital, Delhi

Introduction and Background: Methemoglobin is an altered state of hemoglobin with heme iron in ferric $\left(\mathrm{Fe}^{+++}\right)$form. In methemoglobinemia, tissue hypoxia results in cyanosis. Congenital methemoglobinemia is associated with NADH methemoglobin reductase deficiency. It is of two types: Type I (affects RBCs, normal life expectancy) and Type II (affect all cell types, neurological complications, reduced life expectancy). Here, we report a case of 
congenital methemoglobinemia Type I which is a relatively rare clinical disorder characterized by life-long cyanosis. Patient: A $23 \mathrm{yr}$ old female at $38+$ weeks gestation presented with cyanosis and pedal oedema. Clinical examination revealed cyanosis of skin, palms, nails and tongue. $\mathrm{CBC}$ revealed $\mathrm{Hb}-11.9 \mathrm{~g} / \mathrm{dl}$, macrocytosis and reticulocytosis. G-6-PD enzyme screening showed normal activity. Hb electrophoresis on alkaline starch agarose showed normal pattern. $\mathrm{Hb}$ absorbance pattern showed an abnormal peak at $630 \mathrm{~nm}$. Methemoglobin level was $12.9 \%$. Methemoglobin reductase screening test showed persisting fluorescence. Spouse had no abnormality. Family screening revealed that eight members over three generations from the maternal side had asymptomatic cyanosis. A diagnosis of Congenital Methemoglobinemia, Type I was made. Patient delivered a healthy female child with $\mathrm{PAO}_{2}-86 \%$. Patient and baby were discharged on request. Conclusions: Congenital methemoglobinemia is a rare which should be considered as differential diagnosis in patients presenting with peripheral cyanosis. All possible investigations should be carried out along with family screening to reach at a diagnosis.

\section{BHL 34}

Acquired Platelet Dysfunction with Eosinophilia: A Descriptive Study

Mansi Kala\#, Sukesh C. Nair, Tulasi Geever, Joy John Mammen, Kevin Manuel, Ms. Ramya

Email ID for Correspondence: drmansikala@gmail.com CMC, Vellore

Introduction and Background: Acquired platelet dysfunction with eosinophilia (APDE) is a bleeding disorder with abnormal platelet function studies in patients with eosinophilia which improves after treatment. Predominantly a disease of South East Asia with few cases reported in western world and India. Patient/Material and Method: This was a retrospective study conducted at the department of Transfusion Medicine and Immunohematology, CMC Vellore. All cases who were evaluated for bleeding symptoms during 2011 to 2016 and diagnosed with APDE were included. Complete blood count, peripheral smear examination, screening coagulation test and platelet function studies were done. Results: There were a total of 53 cases with a median age of 25 (range: 3-47 years) and a male to female ratio 1.2:1. Most cases were from Kerala. Most common presentation was ecchymoses seen in $64 \%$ cases and most patients presented with a short duration of symptoms (6 months). Bleeding assessment tool (BAT) score was calculated for all patients which was significant in $50 \%$ of cases. Thrombocytopenia was seen in $20 \%$ and abnormal platelet morphology was observed in all cases. Prolonged bleeding time ( $>6$ mins) was noted in $65 \%$ cases. PFA 200 using ADP/Collagen and Epinephrine/Collagen was prolonged in $70 \%$ and $83 \%$ respectively. Platelet aggregometry using ADP, Epinephrine, Collagen, Arachidonic acid and ristocetin was abnormal in $73 \%, 64 \%$, $50 \%, 30 \%$ and $9 \%$ respectively. Five cases were followed up, of which four cases recovered clinically after treatment. Conclusions: APDE is a common bleeding disorder in children but is not uncommon in adults. It is more common in the coastal regions of India. It does not present with severe bleeding manifestation and rarely requires transfusion. Ecchymotic patches which develop over a short history ( $<6$ months) is the most frequent presentation. ADP and Epinephrine are the most sensitive agonists to detect the abnormality. There was no correlation between the degree of eosinophilia and bleeding.
BHL 35

Study of Megakaryocytic Morphology by Digital Morphometry in Bone Marrow Biopsy Specimens in Hematological Diseases

\section{Rajat Bajaj", D. K. Raman, S. Venkatesan, S. Sharma, R. Bharadwaj}

Email ID for Correspondence: drrajatrbajaj@gmail.com Armed Forces Medical College, Pune

Introduction and Background: Megakaryocytes are unique and dynamic cells which produce platelets by cytoplasmic fragmentation. They are affected in a variety of hematological conditions. A defect in any stage of megakaryocytopoesis can lead to dysmegakaryocytes or thrombocytopenia. This mandates the need to assess them qualitatively and quantitatively. Digital morphometric analysis can be used to precisely quantify the megakaryocytic morphology with respect to area, nuclear size, nuclear cytoplasmic ratio, nuclear roundness factor, nuclear contour ratio. Patient/Material and Methods: Hematoxylin \& Eosin (H\&E), Immunohistochemistry (IHC) stained sections of bone marrow biopsies were evaluated for megakaryocyte morphology. High resolution photomicrographs were taken for all cases and a minimum of 10 megakaryocytes were evaluated for each case. The cytoplasmic and nuclear delineation was done manually and precise measurements of cell area, perimeter, nuclear size, shape, nucleus to cytoplasm ratio and important indices will be evaluated by computer assisted digital morphometry and correlated. Results: 100 Bone marrow biopsies were studied which included myeloproliferative neoplasms (MPN) namely Chronic myeloid leukemia, Polycythemia vera, Essential thrombocytosis and Myelofibrosis; Idiopathic thrombocytopenic purpura, Myelodysplastic syndrome, Acute leukemia; Bone marrows as part of PUO investigation, HIV, plasma cell neoplasms and remission marrows post chemotherapy. Statistically significant morphological differences were seen in various haematological groups with regards to cell count, morphology, N:C ratio, nuclear and cytoplasmic perimeter, nuclear and cytoplasmic roundness. IHC was useful in highlighting the megakaryocytes which were missed on H\&E especially in MPN's. Conclusion: Megakaryocytes show significant quantitative and qualitative variations in various haematological disorders, especially myeloproliferative neoplasms. The morphometric parameters need to be reinforced and validated by a larger study to objectively classify the megakaryocytes.

\section{BHL 36}

\section{Rare Benign Haemoglobin Variants Identified During $\mathrm{HbA}_{1} \mathrm{C}$} Screening in Indian Population

\section{G. Senthil Kumar ${ }^{1 \#, ~ S . ~ J a n a n i ~ I s w a r y a ~}{ }^{2}$, S. Vanitha ${ }^{2}$, S. Gopinath ${ }^{1}$, R. V. Shaji ${ }^{1}$, Eunice S. Edison ${ }^{1}$}

Email ID for Correspondence: sen_cmc@yahoo.co.uk Christian Medical College, Vellore

Introduction: More than 1000 naturally occurring human haemoglobin variants with single amino acid substitutions in the globin chains have been described by laboratory findings. These $\mathrm{Hb}$ variants elute at different retention times in HPLC and they pose a diagnostic challenge during $\mathrm{HbA}_{1} \mathrm{C}$ screening for diabetes. Here, we report the clinical and molecular features of four rare $\mathrm{Hb}$ variants that were incidentally detected during $\mathrm{HbA}_{1} \mathrm{C}$ screening. Methods: Haemoglobin variants were analysed by VARIANT II HPLC System (Bio-Rad, Hercules, CA). DNA was extracted and the molecular 
analysis was performed by direct DNA sequencing of $\beta$ (HBB) and $\alpha$ globin (HBA1 and HBA2) genes. Results: Four patients with abnormal peaks during $\mathrm{HbA}_{1} \mathrm{C}$ were referred to our department and the samples were analyzed for further characterization. All the four patients were diagnosed cases of type 2 diabetes mellitus and had normal peripheral blood picture and haematological indices. The findings are tabulated below. Hb Tsukumi was reported earlier in Japanese and Moroccan populations, Hb Le-Lamentin in English, French, West Indian, Japanese and Spanish populations and Hb South Florida and $\mathrm{Hb} \mathrm{J}$-Rajappen in Caucasian and Indian populations. These $\mathrm{Hb}$ variants have normal stability and have not been reported to result in any decrease in the haemoglobin synthesis levels.

\begin{tabular}{|c|c|c|c|c|c|c|}
\hline UPN & $\begin{array}{l}\mathrm{Hb} \\
(\mathrm{g} / \\
\mathrm{dL})\end{array}$ & $\begin{array}{l}\text { MCV } \\
\text { (fl) }\end{array}$ & $\begin{array}{l}\mathrm{HbF} \\
(\%)\end{array}$ & $\begin{array}{l}\mathrm{Hb} \mathrm{A} \mathrm{A}_{2} \\
(\%)\end{array}$ & $\begin{array}{l}\text { Unknown } \\
\text { variant }(\%)\end{array}$ & $\begin{array}{l}\text { Genetic variant } \\
\text { identified }\end{array}$ \\
\hline 1 & 14.5 & $*$ & 4.2 & 2.8 & $32.8 \%$ & $\begin{array}{l}\text { HBB:c. } 352 C>T \\
\text { HbTsukumi }\end{array}$ \\
\hline 2 & 11.8 & 85.2 & 0.9 & 1.9 & $21.6 \%$ & $\begin{array}{l}\text { HBA1:c272A }>C \\
\text { Hb J-Rajappen }\end{array}$ \\
\hline 3 & 12 & $*$ & 0.9 & 2.0 & $34.2 \%$ & $\begin{array}{l}\text { HBA2.c.63 C>G } \\
\text { Hb Le-Lamentin }\end{array}$ \\
\hline 4 & 10.9 & 79.6 & 0.7 & 2.8 & $9.7 \%$ & $\begin{array}{l}\text { HBB:c. } 4 \mathrm{G}>\mathrm{A} \\
\text { Hb South } \\
\text { Florida }\end{array}$ \\
\hline
\end{tabular}

* Not available

Conclusion: In India, haemoglobinopathies is a common disorder and the predominant haemoglobin variants found in this population are $\mathrm{Hb}-\mathrm{E}$ and $\mathrm{Hb}-\mathrm{S}$. However, this study shows that there are several rare haemoglobin variants in this population and they pose a diagnostic challenge during $\mathrm{HbA}_{1} \mathrm{C}$ screening. Co-inheritance of these variants along with other haemoglobin variants may modulate the phenotype and therefore documentation of such mutations is important.

\section{BHL 37}

Clinical and Molecular Evaluation of Thrombotic Microangiopathies in Indian Patients

\section{Sneha S. Yadav", Shrimati Shetty, Bipin Kulkarni}

Email ID for Correspondence: snehayadav69@yahoo.in National Institute of Immunohaematology, ICMR, Parel, Mumbai

Introduction and Background: This is a preliminary study done to diagnose and classify TTP-HUS cases. Thrombotic microangiopathy (TMA) shows presence of thrombocytopenia and haemolytic-anemia leading to small blood vessel thrombosis, accompanied by varying degrees of organ dysfunction. Two typical, rare, yet life threatening phenotypes of TMAs are Hemolytic Uremic Syndrome (HUS) and Thrombotic Thrombocytopenic Purpura (TTP). TTP is caused due to deficiency of vWF cleaving protease ADAMTS13, which could be genetic, or acquired resulting from autoimmune production of inhibitory anti-ADAMTS13 antibodies. Disorders of the alternate complement pathway regulation, due to mutations in the genes encoding the complement regulatory proteins that alone, or in combination may lead to aHUS. In India, there are hardly any centres where comprehensive evaluation of inherited and acquired Thrombotic microangiopathies is done. Establishing National level facilities for comprehensive evaluation of TTP-HUS will certainly have a direct impact on the systematic diagnosis and management of these patients from all across India. Identification of genetic variations in affected patients would be of immense significance to patient families to offer prenatal and carrier diagnosis. Patient/Material and Methods: 14 patients suspected of HUS-TTP exhibiting complete or partial classical pentad were included in this study. Samples were screened for ADAMTS13 Activity and plasma concentration of ADAMTS13 Auto Antibody, Complement factor $\mathrm{H}(\mathrm{CFH})$ and Complement Factor B (CFB) was quantified using immunofluorescence and ELISA. Samples that showed abnormal levels of plasma ADAMTS13 and complement protein were further analysed by Sanger sequencing to find variations in the respective genes. Results: Out of 14 patients, 6 (42.85\%) patients had ADAMTS13 activity less than $60 \%$ which were further screened for presence of ADAMTS13 Auto Antibody. 5 of $6(83.33 \%)$ patients showed elevated levels of plasma ADAMTS13 Auto Antibody. 1 of 6 patients having less ADAMTS13 activity had mutation at c. $1342 \mathrm{C}>\mathrm{G}$ leading to $\mathrm{p} . \mathrm{Q} 448 \mathrm{E}$ which is a known ADAMTS13 mutation. 1 patient had decreased complement factor $\mathrm{H}$ level in plasma. Conclusions: In this preliminary study, 5 Acquired TTP, 1 Congenital TTP and 1 aHUS cases were diagnosed. This is first of its kind of comprehensive study of HUS-TTP form India.

\section{BHL 38}

Role of Volume, Conductivity, Scatter Changes of Neutrophils and Monocytes in Diagnosis of Megaloblastic Anemia

\section{S. A. Deepa Adiga", Debarshi Saha, R. G. Karthick, Vishnupriya Mathivanan, K. S. Pooja, Anupama Hegde, H. B. Sridevi}

Email ID for Correspondence: deepa.adiga@gmail.com Kasturba Medical College, Mangalore, Manipal University

Introduction and Background: Megaloblastic anemias are a group of disorders characterized by: the presence of distinctive morphologic appearances of the developing red cells in the bone marrow usually due to deficiency of either cobalamine (vitamin B12) or folate. The deficiency affects all labile cells including WBCs. This is reflected in the peripheral smear as macro-ovalocytes and macropolycyte. Megaloblastic anemia is typically a macrocytic normochromic anemia. The MCV should be $100 \mathrm{fl}-140 \mathrm{fl}$. However increased MCV is not specific for megaloblastic anemia. We undertook this study to evaluate the possible role of coulter derived (Volume, Conductivity and Scatter) VCS parameters as an indicator of megaloblastic anemia. Patient/Material and Methods: We performed a case control study including 25 patients with low serum vitamin B12 or folate levels and 25 healthy volunteers respectively. Comparison of the coulter derived Volume, Conductivity and Scatter parameters for neutrophils and monocytes of cases and control were done. Results: The mean neutrophil volume of cases (MNV: $158.37 \pm 18.13$ ) was significantly higher $(\mathrm{p}=0.0001)$ as compared to controls (MNV: $141.26 \pm 4.22$ ). Similarly mean monocyte volume of cases (MMV: $183.34 \pm 16.90$ ) was significantly $(\mathrm{p}=0.0001)$ higher as compared to controls (MMV: $166.55 \pm 8.66$ ). The mean conductivity for neutrophils for cases (MNC: $138.58 \pm 4.67)$ was significantly $(\mathrm{p}=0.43)$ lower as compared to controls (MNC: $141.35 \pm 4.73$ ). Similarly the mean conductivity for monocytes for cases (MMC: $114.66 \pm 4.79$ ) was significantly $(\mathrm{p}=0.43)$ lower as compared to controls (MMC: $117.62 \pm 4.93)$. Conclusions: Our study suggest that analysis of coulter derived VCS parameters for neutrophils and monocytes was a simple and objective screening method that indicate the existence of subclinical deficiency of vitamin B 12 and folate. 


\section{BHL 39}

Prevalence of Low Prothrombin Time Pt/Inr Following Mechanical Heart Valve Surgeries

\section{Aniya Antony", Mark Christopher, Somanath Padhi, Renu G'Boy Varghese}

Email ID for Correspondence: aniya.antony@gmail.com Pondicherry Institute of Medical Sciences

Introduction and Background: Oral anticoagulant therapy is given to patients with mechanical heart valve prostheses to prevent thromboembolic complication. Measurement of PT/INR is essential for patients started on anticoagulation. American college of chest, cardiology and heart physicians recommends an INR of 2.5-3.5 following mechanical heart valve surgery. Fluctuations in the INR can be due to change in diet, poor compliance, effect of drugs etc. Our study was aimed to study the prevalence and etiological factors associated with low PT/INR. Patient/Material and Methods: We included 100 cases in whom INR was monitored among patients who had undergone mechanical heart valve surgeries on oral anticoagulants between October 2014 to July 2016. Patient's INR was collected and history was collected regarding their dietary habits, drug intake, compliance to the drug, other concomitant diseases or any other causes which could be associated with low PT/INR. Results: 100 patients included had 52 males and 48 females with majority of them falling in their second to fourth decade of life. 80/100 cases had combination of two valvular heart diseases with single valve replacement being done in $80 \%$ of the cases. Low INR was found to be in 37 cases $(37 \%)$ of the study subjects. There is a strong association between non-compliance and low INR, found in 14 cases who had occasionally missed doses and 7 cases who were not compliant at all. There was also significant association between vitamin $\mathrm{K}$ rich intake and low INR in $16(57.1 \%)$ cases. Other parameters like drugs and co-morbidities had no significant association with low INR. Conclusions: It is mandatory to keep good control of anticoagulant therapy in cases of valve replacement. Non-compliance and intake of vitamin $\mathrm{K}$ rich diet are the most common causes of low INR.

BHL 40

\section{Study of Hematological Profile in Malarial Infections}

\section{Anupreet Kaur", S. Kishore, Sheenam,}

\section{S. Kaushik, Aradhna, Deepti}

Email ID for Correspondence: anupreetkaur90@gmail.com Sri Guru Ram Rai Institute of Medical and Health Sciences, Dehradun

Introduction and Background: This study was undertaken to assess and correlate the hematological parameters, thrombocytopenia and parasitic index of 184 cases diagnosed with malaria. Patient/Material and Methods: This prospective study was conducted on 184 proven cases of malaria. 64 cases again presented with relapse after 12-16 days of initial treatment. RBC indices were obtained by Sysmex XP100, platelet and differential leukocyte count was done manually. Peripheral blood smears were examined for diagnosis and identification of malarial parasite and calculating parasitemia. Continuous variables are expressed as mean $\pm \mathrm{SD}$, while categorical variables are expressed as percentage. Results: Total 184 diagnosed cases of malaria comprised 163 cases $(88.6 \%)$ of $P$. vivax and 20 cases $(10.81 \%)$ of $P$. falciparum with average parasitic index of $5.47 \pm 5.27$ and $6.9 \pm 4.96$ respectively. Among P. vivax infected cases; $20(12.2 \%)$ had mild anaemia, $28(17.17 \%)$ had moderate anaemia, $22(13.4 \%)$ had severe anaemia whereas $93(57.05 \%)$ had no anaemia. 78 cases $(47.8 \%)$ presented with Grade 1 thrombocytopenia, 35 (21.4\%) Grade 2, 25 (15.3\%) Grade 3, $4(2.4 \%)$ had Grade 4 thrombocytopenia whereas 21 cases $(12.8 \%)$ had no thrombocytopenia. Among P. falciparum infected cases; 3 cases $(15 \%)$ had mild anaemia, $5(25 \%)$ moderate anaemia, $7(35 \%)$ severe anaemia whereas $5(25 \%)$ had no anaemia. $7(35 \%)$ cases had grade 1 thrombocytopenia, $2(10 \%)$ grade $2,6(30 \%)$ grade 3,1 $(5 \%)$ grade 4 and $4(20 \%)$ had no thrombocytopenia. 1 case $(0.54 \%)$ of mixed infection showed no anaemia and Grade 1 thrombocytopenia. 64 of these cases presented with relapse comprising 7 cases $(10.93 \%)$ of $P$. falciparum and $57(89.06 \%)$ of $P$. vivax infection. No evidence of intravascular haemolysis was seen. Conclusion: It was concluded that $P$. falciparum infected cases had higher parasitic index and lower platelet count as compared to $P$. vivax; mean platelet count being $86.25 \pm 53.9$ and $87.83 \pm 41.46$ respectively.

\section{BHL 41}

Global Hemostasis Assays for Monitoring Bypassing Agent Therapy in Hemophilic Patients with Inhibitors

\section{Puja Soni $^{\#}$, Bipin Kulkarni, S. Chandrakala, Shrimati Shetty}

Email ID for Correspondence: pujaas44@gmail.com National Institute of Immunohaematology (NIIH-ICMR)

Introduction and Background: Monitoring bypassing agent (BPA) therapy in hemophilia patients with inhibitors is presently carried out only on the basis of clinical responses. BPA is an expensive treatment protocol, so it's appropriate monitoring is need of the hour. Global hemostasis assays like Thromboelastography (TEG), Thrombin generation assay (TGA), and Clot waveform analysis have shown promising results for monitoring BPA, but none has been validated. There is a wide variability in response of the patients to the BPA. Into current study, parameters of two global hemostasis assays i.e. TEG and TGA will be compared with clinical responses to evaluate better monitoring assay for BPA. Patient/Material and Methods: The study group consisted of 20 bleeding episodes in 18 hemophilia A inhibitor $(>5 \mathrm{BU} / \mathrm{ml})$ positive patients. A total of 46 samples was evaluated for global hemostasis assays i.e. TEG and TGA and was correlated with clinical response to BPA. Assessment was made prior and two hours post infusion of bypassing agents. In vitro spiking experiment for TEG and TGA was also carried out by using different concentration of BPA to know the optimum concentration required for normal hemostatic response.Results: In vitro spiking experiment in both TEG and TGA showed a clear dose-dependent effect for increased hemostasis response. Conclusions: However being preliminary data, present study showed that investigating both TGA and TEG assay prior and post infusion of BPA will help in better monitoring of BPA. The sample size was less for the present study, should be verified with larger sample size. 


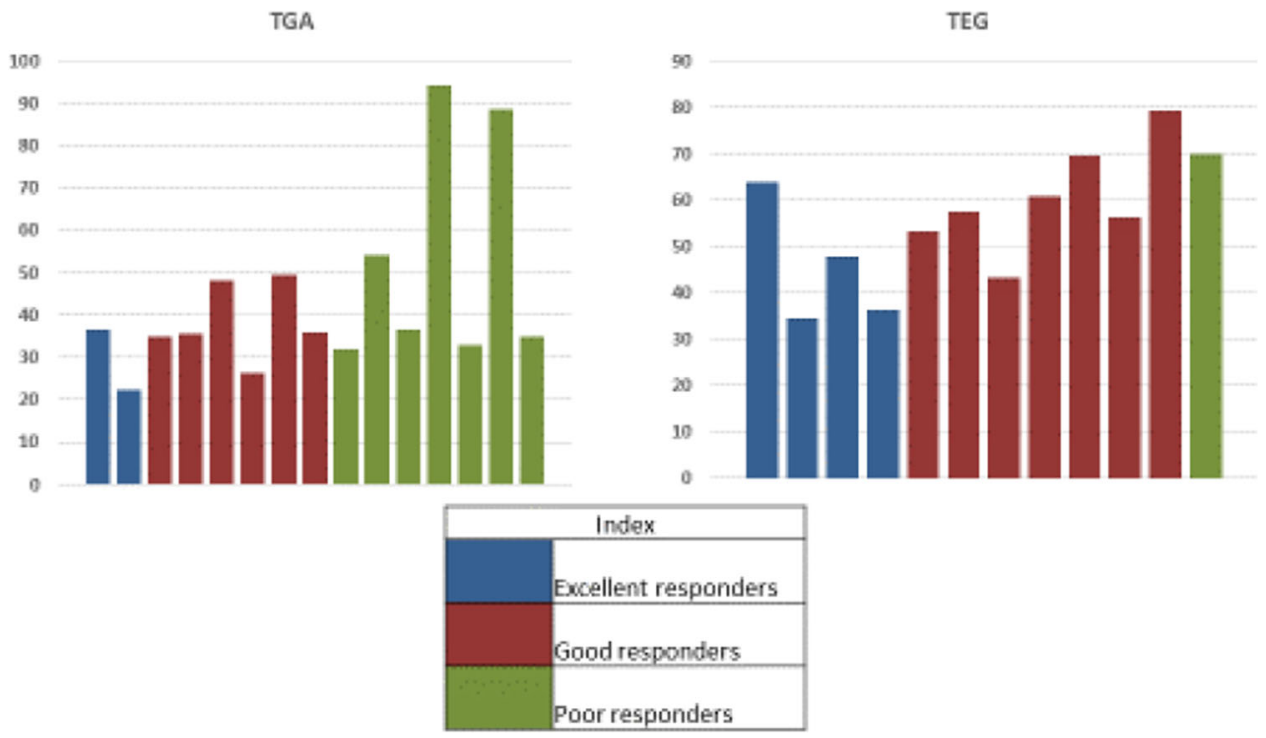

BHL 42

\section{Mast Cells in Tuberculosis: A Report of Two Cases}

\section{Poorva Singh", Mrinalini Kotru, Satendra Sharma, Nidhi Goyal,} Meera Sikka

Email ID for Correspondence: indiancordilerra@gmail.com University College of Medical Sciences, Delhi

Introduction and Background: Mast cells are inflammatory cells found in mucosal sites and bone marrow and are involved in innate immunity. They have been described in increased numbers in the bone marrow in allergic conditions and clonal proliferations like Chronic Myeloid Leukemia, Myelodysplastic Syndrome and Systemic Mastocytosis. In tuberculosis, mast cells have been described in the sites of primary infection. However, no literature exists on mast cells in bone marrow in patients with tuberculosis. Patient/Material and Methods: We report two cases of 40 year old men with tuberculosis. Complete clinical history, physical examination, CBC with peripheral smear examination, bone marrow aspirate examination and bone marrow iron was performed in both cases. 200 cell differential on peripheral smear and 500 cell differential on marrow were done in each case. Results: Case 1 presented with generalized lymphadenopathy and severe anemia with neutrophilia. FNA smears from the lymph nodes showed necrosis and epithelioid cell granulomas. His marrow was hypercellular with preponderance of myeloid precursors and increased mast cells $(10 \%)$. Case 2 was taking ATT for pulmonary tuberculosis for 1 year and presented with pancytopenia. He had a cellular marrow showing erythroid hyperplasia with megaloblastic change and increase in mast cells (20\%). In both cases, mast cells had normal morphology without any clustering. Both had adequate stainable marrow iron. There was no evidence of myelodysplasia, myelofibrosis, ringed sideroblasts, increase in blasts or abnormal localization of immature precursors (ALIP) in both cases. Conclusions: Both cases of tuberculosis showed increased mast cells in the marrow. This observation and its potential implication in the pathogenesis or diagnosis of tuberculosis need to be validated with further studies on a larger number of cases.
BHL 43

A Study of 22 Beta Globin Mutations Using Multiplex Reverse Dot Blot Strip PCR

Chetan Agarwal", Col Jyoti Kotwal, Col Bhushan Asthana, Lt Col Ankur Ahuja, Brig Kavita Sahai

Email ID for Correspondence: chetanpathologica@gmail.com Army Hospital Research and Referral, New Delhi

Introduction and Background: We studied the efficacy of newly introduced "multiplex PCR reverse dot blot strip method" for detecting 22 beta globin mutations in thalassemia patients to assess if it is an effective and rapid method for genetic screening of $\beta$-thalassemia in our population and compared it with the reference standard amplification refractory mutation system which is routinely done for five common mutations. Patient/Material and Methods: The analysis of mutations was done on diagnosed cases of $\beta$-thalassemia and their parents, spouse and sibling (total 50 cases) using multiplex PCR reverse dot blot strip method. The results were validated by comparing with reference standard, "amplification refractory mutation system". Results:

1. The "multiplex PCR reverse dot blot strip method" was found to be faster, reliable, less labour intensive, cost effective, self documented and easier method compared to reference standard, "Amplication refractory mutation system".

2. IVS $1.5(\mathrm{G}>\mathrm{C})$ is the most common mutation in Indian population. Other common mutations detected are Codon 8/9 [+G], Codon 41/42 [-TTCT] and three additional (uncommon) mutations viz., $\mathrm{Cap}+1[\mathrm{~A}>\mathrm{C}]$, Codon $30[\mathrm{G}>\mathrm{C}]$ and Codon 16 $[-\mathrm{C}]$. The multiplex PCR reverse dot blot strip method picked up these additional (uncommon) mutations which were missed by amplification refractory mutation system.

Conclusions: This tool may is a cost effective, rapid, easier and reliable method which can be utilized for a quick prenatal diagnosis of thalassemia in a busy hospital setting. 


\section{BHL 44}

Compound Heterozygous Hb Lepore Beta-Thalassemia-A Rare Phenomenon Causing Non transfusion Dependent Thalassemia

Neha Suman ${ }^{1 \#}$, Sarika Singh ${ }^{2}$, Sunita Sharma ${ }^{1}$, Deeksha Singh ${ }^{1}$, Aruna Chhikara ${ }^{1}$, Nupur ${ }^{3}$, Jagdish Chandra ${ }^{3}$

Email ID for Correspondence: drnehasuman@gmail.com ${ }^{1}$ Department of Pathology, Lady Hardinge Medical College, New Delhi, ${ }^{2}$ Department of Pathology, Maulana Azad Medical College, New Delhi, ${ }^{3}$ Department of Paediatrics, Lady Hardinge Medical College, New Delhi

Introduction: Haemoglobin $(\mathrm{Hb})$ Lepore is rare with very few cases reported in the literature. Hb Lepore is a structurally abnormal haemoglobin consisting of two $\alpha$-globin and two $\delta \beta$-globin chains $[\alpha 2$ $(\delta \beta) 2$ ]. It results from a fusion between a $\delta$ and a $\beta$ globin gene during meiosis. Compound heterozygosity for $\mathrm{Hb}$ Lepore and beta thalassemia ( $\beta$-thal) is even rarer and an unusual cause of non transfusion-dependent thalassemia. Case Summary: A 2 year male patient presented to the paediatric OPD with complaint of fever on and off with cough and coryza, easy fatiguability since last 3 months and history of pallor since 13 months. Patient was on hematinics since last 4 months. On examination, no hepatosplenomegaly was noted. Hematological investigation revealed mild anemia with microcytic hypochromic red cells. A comprehensive analysis of haemoglobin by high performance liquid chromatography (HPLC) was suggestive of compound heterozygous lepore beta thalassemia. HPLC was suggestive of $\beta$-thal trait in father and heterozygous Lepore $\mathrm{Hb}$ in mother while chromatogram was within normal limits in siblings. Family was screened for $\beta$-thal mutations and $\mathrm{Hb}$ Lepore deletions using $\mathrm{HBB}$ gene sequencing and Gap PCR respectively. Father was found to be a carrier of beta thalassemia and mother was a carrier of $\mathrm{Hb}$ Lepore deletion. The patient was found to be a compound heterozygous of the disorder Hb Lepore $\beta$-thalassemia. Conclusions: In Non transfusion dependent haemoglobinopathies-A possible diagnosis of Hb Lepore compound heterozygous Beta thalassemia should be considered. Family studies and patients' history and various laboratory results are helpful in establishing this diagnosis for proper clinical management and genetic counselling.

\section{BHL 45}

Molecular Basis of 10 Patients with Factor XI Deficiency from India: Identification of 4 Novel Mutations and a Common Mutation

\section{Nikesh Kawankar ${ }^{\#}$, Kanjaksha Ghosh, Shrimati Shetty}

Email ID for Correspondence: nikesh1005@gmail.com National Institute of Immunohaematology (ICMR) KEM Hospital, Parel, Mumbai

Introduction and Background: Factor XI (FXI) deficiency is an autosomal recessive disorder and the clinical manifestations are unpredictable. Factor XI (FXI) deficiency is characterized by decrease in the functional activity of FXI plasma protein. Plasma FXI, a zymogen homodimer (two identical polypeptide chains linked by disulfide bonds) is synthesized in the liver. During activation, the internal peptide bond is cleaved by factor XIIa, resulting in activated factor XIa, which activates factor IX. Patient/Material and Methods: We analyzed the molecular basis of 10 unrelated Indian patients with FXI deficiency by direct DNA sequencing. All 15 exons of F11 and the intron/exon boundaries were amplified using 14 pair of primers. The DNA sequencing was carried out using Big Dye DNA sequencing kits (Applied Biosystems, Foster City, CA, USA). In silico analysis of the novel mutations was carried out using prediction software SIFT, Provean, Polyphen 2, Mutation taster, Human splicing finder, MaxEnt score to predict the potential effect of mutations. Results: Eight mutations were detected in 10 patients with one common novel splice site mutation mutation i.e. IVS $3+4 A>G$ in 3 unrelated patients along with 3 other novel mutations (p.Asp556Ala, p.Leu465Ser, p.His214Pro). A wide range of clinical manifestations with no bleeding to hemarthrosis was seen in these patients. The bleeding manifestations were neither related to FXI levels nor the type of mutations. Conclusions: This report along with other similar data highlights the need to identify a laboratory predictor of severity in bleeding in FXI deficient cases.

\section{BHL 46}

Comparison of HbA1c Levels Between Beta Thalassemia Trait and Individuals with Normal Pattern on HPLC: A Pilot Study

\section{R. Madhurya ${ }^{\#}$, H. Anupama, H. B. Sridevi, T. Jaseem, K. S. Pooja}

Email ID for Correspondence: madhurya1198@gmail.com Kasturba Medical College, Mangalore

Introduction and Background: Hemoglobinopathies interfere with HbA1c analysis by altering RBC life span. Various studies were undertaken questioning its reliability for monitoring diabetics with hemoglobinopathies. In India $\beta$-Thalassemia Trait (BTT) is common with $3.2 \%$ prevalence. Whether there is any correlation between $\mathrm{HbA} 2$ (marker of BTT) and HbA1c is unknown. Hence aim of our study is to compare HbAlc of BTT with controls and correlate $\mathrm{HbA} 1 \mathrm{c}$ with $\mathrm{HbA} 2$ in entire population. Material and Methods: Study is done retrospectively including all individuals subjected to HPLC from January-June $2016(\mathrm{n}=155)$. Infants and hemoglobinopathies other than BTT are excluded. They are divided into three groups $1,2,3$ (HbA2 <3.3, 3.4-4.9, 5-9 \% respectively) based on HbA2. Group $1(n=65)$ have normal pattern on HPLC and are considered as controls. Groups 2, 3 ( $\mathrm{n}=38,52$ respectively) have BTT and are taken as cases. Further cases are analysed by dividing into groups A, B, C, D (1-20, 21-40, 41-60, >60 years respectively) based on age. Results: Age and hemoglobin fractions are comparable among three groups, except $\mathrm{HbF}$. Mean HbA1c of groups 1, 2, 3 are $4.3 \pm 1.6,4.9 \pm 1.6,5.06 \pm 0.93$ respectively with no significant difference between cases and controls. There is significant correlation of $\mathrm{HbA} 1 \mathrm{c}$ with age $(\mathrm{r}=0.437)$, $\mathrm{HbA} 2$ with $\mathrm{HbF}(\mathrm{r}=0.323)$. Upon analysis in cases and entire population, no significant correlation between $\mathrm{HbA} 2$ and $\mathrm{HbA} 1 \mathrm{c}$ could be derived. There are 30/155 individuals with hemolysis whose $\mathrm{HbA} 1 \mathrm{c}$ is $<3.8 \%$. Among them $63.4 \%$ belong to group 1 and $36.6 \%$ belong to groups 2,3 . On further investigation, the causes of hemolysis in group 1 were found to be hereditary spherocytosis, autoimmune-hemolytic anemia and drugs. Diabetics with BTT are $5 / 155$ whose HbA1c is $>6.5 \%$. Conclusions: Absence of statistical significance between $\mathrm{HbA} 1 \mathrm{c}$ of cases and controls, lack of correlation between $\mathrm{HbA} 1 \mathrm{c}$ and $\mathrm{HbA} 2$ suggests that presence of BTT alone does not affect HbA1c unless there is hemolysis. In diabetics HbA1c remains high inspite of coexisting BTT. Hence HbA1c can be reliable for monitoring glycemic status in diabetics with BTT. However, a prospective study on such population will be done to confirm this finding. 


\section{BHL 47}

\section{VCS Parameters as Early Indicators in Megaloblastic Anemia}

\section{T. Shruthi", Rajeshwari S. Handigund}

Email ID for Correspondence: mailshruthit@gmail.com Hi Tech Laboratory, KLES Dr. Prabhakar Kore Hospital and Medical Research Centre, Belagavi

Introduction and Background: Megaloblastic anemia is classified as a nuclear maturation defect, which is predominantly caused by vitamin B12 or folic acid deficiencies. Thus it affects all three blood cell lineages i.e., erythrocytes, leucocytes and platelets, unlike other anemias which involve only erythrocytes. The finding of hypersegmented neutrophils is considered as highly sensitive and specific and it can be found even in the absence of macrocytosis. In this study, we aim to analyse the CPD (Cell Population Data) of VCS parameters, (where "V" is Volume, "C" is Conductivity and " $\mathrm{S}$ " is Scatter) for neutrophil and monocyte in relation to the patients' B12 levels. Patient/Material and Methods: A cross sectional study has been undertaken where all the samples received for vitamin B12 assay have been taken and compared with the respective $\mathrm{V}, \mathrm{C}$ and $\mathrm{S}$ parameters of the neutrophil and monocyte along with conventional parameters like hemoglobin, MCV (Mean Corpuscular Volume), MPV (Mean Platelet Volume). A total of 212 samples of B12 were assessed, of which VCS data for 40 samples was not available. Results: The results were analysed by tabulating B12 values in three groups based on the reference range. Of the 172 samples, 66 were in group A (lower than normal range), 57 in group $\mathrm{B}$ (normal range) and 49 in group $\mathrm{C}$ (higher than normal range). $47 \%$ of samples in group ' $\mathrm{A}$ ' showed high mean neutrophilic volume, as compared to $28 \%$ in group 'B' and $30 \%$ in group C. The mean of conductivity and scatter of either neutrophil/monocyte and the mean monocyte volume have not shown significant changes. Conclusions: Mean neutrophil volume can be used as an early indicator in vitamin B12 deficiency. However further studies have to be undertaken to substantiate the results.

\section{BHL 48}

\section{Red Cell Pyruvate Kinase Deficiency in India: An Update}

\section{Prabhakar S. Kedar", Prashant Warang, Manisha Madkaikar}

Email ID for Correspondence: kedarps2002@yahoo.com National Institute of Immunohematology (ICMR) 13th Floor, KEM Hospital; Campus, Parel, Mumbai 400012

Introduction and Background: Pyruvate kinase deficiency (PKD) is one of the most common red cell enzymatic defects in the glycolysis pathways in humans and it is an autosomal recessive disorder causing chronic non-spherocytic haemolytic anaemia. PK deficiency severely affects RBC metabolism, causing adenosine triphosphate (ATP) depletion, which ultimately leads to hemolysis. Pathologic signs of PK deficiency are usually observed when enzyme activity is less than $25 \%$ normal activity and patients are generally homozygotes or compound heterozygotes with 2 different mutant alleles. So far approximately 600 cases have been described in the literature. According to the most recent database, around 223 mutations have been reported from different regions Clinical symptoms usually observed in true homozygotes and compound heterozygotes are variable, ranging from neonatal jaundice requiring exchange transfusions, to a fully compensated haemolytic anaemia. Patient/Material and Methods: Patients with hereditary non-spherocytic hemolytic anemia and/or neonatal jaundice of unexplained etiology were investigated. Three patients with severe haemolytic anaemia with a history of blood transfusions and hyperbilirubinemia were diagnosed as PK deficiency. All exons of the PKLR gene and their flanking sequences were amplified from the patient's genomic DNA using PCR. DEEP VIEW SWISS-PDB VIEWER and PYMOL bioinformatics software was used to evaluate the functional impact of the mutation found in this case. Results: The clinical, hematological, biochemical and molecular characterization of 3 cases of PK deficiency at the time of the study showed severe anemic and with jaundice, Hepatosplenomegaly and history of blood transfusion. PK activity was reduced less than $25 \%$ (3.10 to $6.49 \mathrm{IU} / \mathrm{g} \mathrm{dl})$ whereas their parents showed intermediate activity as compared to normal individuals. Molecular analysis was done in all the $3 \mathrm{PK}$ deficient cases. Five different PKLR mutations were identified among the 6 mutated alleles. Two splice site mutation (1268 $\mathrm{A} \rightarrow \mathrm{G}$, Ala $423 \rightarrow \mathrm{Glu}($ Novel), and IVS ds $\mathrm{A} \rightarrow \mathrm{T}+3$ and three missense mutations (890G $>$ A, 294Val $\rightarrow$ Met(Novel); $1492 \mathrm{C} \rightarrow \mathrm{T}, 498$ Arg $\rightarrow$ Cys; 1072 $\mathrm{G} \rightarrow \mathrm{A}, 358 \mathrm{Gly} \rightarrow \mathrm{Arg}$ ) were characterized. Parents were heterozygous for respective mutations. The 3 dimensional (3D) structure of human $\mathrm{PK}-\mathrm{R}$ was generated from atomic coordinates of the protein data bank entry 1LIU [PDB] using the program showed that novel mutations were located in the A domain of the PK subunit, a highly conserved region having the active site of the enzyme results in significantly reduced enzyme activity and responsible for severe hemolysis. Conclusions: Including present study, So far, we have diagnosed 33 cases of PK deficiency in the last 6 years by a systematic screening of patients with non-spherocytic haemolytic anaemia and neonatal jaundice. Hence, PK deficiency is not very rare in India. Collectively, the results provided us with a rationale for the observed enzyme deficiency and contribute to both a better understanding of the genotype-to-phenotype correlation in PK deficiency as well as the enzyme's structure and function.

\section{BHL 49}

OPG/RANK/RANKL Genes as a Surrogate Marker for Cardiac Abnormality in Thalassemia Major Patients

\section{Ravindra Kumar ${ }^{1 \#}$, Mable Misha Singh ${ }^{2}$, Sarita Agarwal ${ }^{2}$}

Email ID for Correspondence: ravindrachhabra@gmail.com ${ }^{1}$ Central Research Laboratory, Sri Aurobindo Medical College and Post Graduate Institute Indore, Madhya Pradesh, India; ${ }^{2}$ Department of Medical Genetics, Sanjay Gandhi Post Graduate Institute of Medical Sciences, Lucknow, India

Abstract: Objective: The aim of the study was to investigate the role of osteoprotegerin (OPG)/RANK/RANKL variants in left ventricular hypertrophy $(\mathrm{LVH})$ and diastolic dysfunction in patients with Beta Thalassemia Major (BTM). Materials and Method: one hundred and five beta thalassemia patient aged above ten years were enrolled for the study. Two dimensional and $\mathrm{M}$ mode Echocardiography analysis was done in all the patients and genotyping was done by PCR-RFLP for OPG [rs2073617 (950 T>C), rs2073618 (1181 G>C)], RANK $\left[\begin{array}{lllllll}(r s 1805034 & (+34694 & \mathrm{C}>\mathrm{T}), & \operatorname{rs} 12458117 & (+34901 & \mathrm{G}>\mathrm{A}) & \&\end{array}\right.$ rs75404003 (+35966insdelC)] and RANKL (rs2277438, rs9594782) variants. Serum OPG levels were estimated by enzyme linked immunosorbant assay. Results: Patients enrolled had a mean age of $16.36 \pm 5.08$ years. Variant of OPG rs2073618, RANK rs75404003 and RANKL rs9594782 was observed to be significantly associated with the presence of left ventricular hypertrophy with an odds ratio of $2.470,3.783$ and 2.148 respectively. RANK rs1805034 SNP has been found as a protective for diastolic dysfunction in thalassemia major patients Serum OPG levels were found to be significantly associated in patients with diastolic dysfunction $(\mathrm{p}=0.006)$. Conclusion: OPG rs2073618, RANK rs75404003 and RANKL rs9594782 snps can be used as surrogate marker for LVH. Patients with high serum OPG levels were at risk of diastolic dysfunction in BTM. 


\section{BHL 50}

\section{Molecular Spectrum of WAS Gene in Indian Patients with Wiskott Aldrich Syndrome}

\section{G. Sankari Devi", Sachin David, Eunice S. Edison, A. Abraham, B. George, V. Mathews, A. Srivastava}

Email ID for Correspondence: kgsankaridevi@yahoo.com Department of Hematology, Christian Medical College, Vellore 632004, Tamilnadu, India

Background: Wiskott-Aldrich syndrome (WAS), an X-linked disorder is characterized mainly by thrombocytopenia and immunodeficiency. The disease is caused by mutations in the WAS gene. More than 350 mutations have been identified in the WAS gene. Mutations are found throughout the WAS gene and consist of point mutations, insertions, deletions and splice site mutations. Aim: To document the distribution of mutations in WAS gene in Indian population. Methods: Diagnosis of WAS/XLT was based on low platelet count $\left(9000-95,000 / \mathrm{mm}^{3}\right)$ and presence of small platelets. Genomic DNA from the patients was screened for mutations in WAS gene by PCR followed by DNA sequencing. Mutations at or near the splice junction were analyzed by 'Splice Site Prediction program' (http://www. fruitfly.org/seq_tools/splice.html) to predict changes in RNA splicing. Results: Fifty four patients from 53 unrelated families were included in the study. The median age at first clinical symptoms of these patients was 2.5 years (range: 1-12). Disease causing mutation could be identified in all these patients. There were 39 unique mutations which included the following: nonsense $4(10.25 \%)$, missense 9 $(23 \%)$, frameshift $11(28.2 \%)$, indel $1(2.5 \%)$ large deletion 2 $(5.1 \%)$ and splice site mutation $12(30.7 \%)$. Eighteen $(18 / 54,33 \%)$ patients had 12 unique splice site mutations, $4(33.3 \%)$ were novel and eight $(66.6 \%)$ had already been reported. Of these splice site mutations that affect splicing, seventeen were due to single nucleotide changes and one with tetra nucleotide change. The occurrence of splice site mutations was centered on Exon 8 (44 \%) in our study. By using this molecular data we were able to offer genetic counselling for 7 families. Conclusion: This is the largest series of WAS gene mutations identified in patients with Wiskott-Aldrich Syndrome in India. Our study adds to the global mutation database of WAS and will be useful in offering genetic diagnosis and management of this condition.

\section{BHL 51}

\section{Role of Biomarkers in Management of Antibiotic Therapy}

\section{Gaurav Chhabra $^{1 \#}$, Raghavendra Linghiah ${ }^{2}$}

Email ID for Correspondence: gauravchhabra2001@gmail.com ${ }^{1}$ All India Institute of Medical Sciences, Bhubaneswar; ${ }^{2}$ AIIMS, New Delhi

Background: Antibiotics are the most successful chemotherapy that has helped the mankind to counter bacterial infections that were frequently fatal in the past. Antibiotic resistance has become a major healthcare problem worldwide. The injudicious use of antibiotics now threatens their effectiveness. A biomarker that can differentiate bacterial from non-bacterial infections is urgently needed to guide appropriate patient treatment and to preserve the effectiveness of existing antibiotics in a context of rapidly increasing antimicrobial resistance. Aim: To evaluate the usefulness of C-Reactive protein and total leukocyte count alone or coupled together as early diagnostic markers of bacterial infections among hospitalised patients. Material and Methods: Five hundred and twenty one patients presenting with signs of any local infection or sepsis were enrolled in the study and after detailed clinical evaluation. Blood samples were taken for various investigations such as complete haemogram, CRP, ESR and culture \& sensitivity examination. The results of culture sensitivity examination, CRP and haemogram analysis were compared and and receiver operating curve were drawn to evaluate the diagnostic efficacy of all the parameters collectively in differentiating between a bacterial and non bacterial infection. Results and Conclusion: The concentration of $\mathrm{C}$ reactive protein and total leukocyte count was found to be significantly higher in the patients who were culture positive as compare to the culture negative group. The area under the curve for CRP band TLC was 0.85 and 0.95 and we suggest an optimal cutoff range for both the parameters for differentiating between bacterial and non bacterial infection or inflammatory conditions. We conclude WBC count and $\mathrm{C}$ reactive protein together constitutes rapid and affordable tool for diagnosis of bacterial infections, before the culture results are available and could help in judicious use of antibiotics.

\section{BHL 52}

Correlation of Mean Platelet Volume and Plasma Glucose Regulation in Patients with Diabetes Mellitus Radhika Rai", Bhoomika Agarwal, Garima Goel, Rajnish Joshi,
Deepti Joshi, Neelkamal Kapoor

Email ID for Correspondence: drrairadhika@gmail.com All India Institute of Medical Sciences, Bhopal

Introduction and Background: Diabetes mellitus is a global pandemic characterized by hyperglycemia, metabolic abnormalities, and macro-microvascular complications. Platelets play an important role in pathophysiology of atherothrombotic diseases. Mean platelet volume (MPV) is a marker of platelet activity and increased platelet activation has been suggested to be involved in the pathogenesis of vascular complications in diabetic patients. Thus MPV can be used as a marker for vascular complications in these patients. In the present study we intend to find a correlation between MPV in diabetic patients so as to measure the risk of vascular complications in diabetics. Patient/Material and Methods: In this prospective study the platelet activity of 50 diabetic patients compared with 50 healthy age and sex matched controls. Demographic and other clinically relevant details of patients were recorded. Both the cases and controls underwent clinical examination for micro as well as macrovascular complications and subsequently blood samples were collected in EDTA vial for measuring $\mathrm{HbA1c}$ and MPV. The patients were divided into two groups based on their HbA1c values-Group A with regulated diabetes and $\mathrm{HbA} 1 \mathrm{c}$ value $\leq 6.5 \%$ and Group B with non regulated diabetes and $\mathrm{HbAlc}$ value $>6.5 \%$. The MPV levels amongst the two groups as well as diabetic and non diabetic subjects were correlated. Results: MPV were higher in diabetics than non diabetic subjects, $9.70 \pm 1.80 \mathrm{fl}$ versus $8.2 \pm 1.1 \mathrm{fl}$. The values MPV were also more in diabetic patients with vascular complication $(9.90 \pm 1.60 \mathrm{fl})$ than uncomplicated diabetes mellitus patients $(9.60 \pm 1.20 \mathrm{fl})$. Conclusions: Our results showed significantly higher MPV in diabetic patients than in the non diabetic controls. Higher value of MPV was also noted in diabetic patients with vascular complication than uncomplicated diabetics. Hence MPV can be used as a simple parameter to assess vascular complications in diabetic patients. 


\section{BHL 53}

In-Vitro Investigation to Modulate the Function of Activated Protein C (APC): Towards an Alternative Therapeutic Option for Hemophilia

\section{Darshana Mirgal ${ }^{\#}$, Vaishali Pujari, Kanjaksha Ghosh, Shrimati Shetty}

Email ID for Correspondence: mirgaldarshana@gmail.com National Institute of Immunohaematology

Introduction and Background: Haemophilia A and B are congenital bleeding disorders caused by insufficient thrombin generation due to deficiency in factor VIII and IX, respectively. In the presence of normal levels of endogenous anticoagulants, like protein $\mathrm{C}$ protein $\mathrm{S}$ and AT III, deficiency of factor VIII or IX results in an imbalance of the hemostatic system towards a bleeding phenotype. Deficiencies of any of the anticoagulant proteins or hypofibrinolytic factors in the anticoagulant pathways can result in increased thrombin generation which in turn results in the amelioration of clinical symptoms in severe hemophilia patients. Our earlier observational studies have shown that severe hemophilia patients who are deficient for any of the natural anticoagulants have a milder clinical phenotype. The present report is a step forward in this line showing partial silencing of $P R O C$ in an in vitro system using HepG2 cell lines. Patient/Material and Methods: Human HepG2 hepatic carcinoma cells were cultured and reverse transfected using various concentrations and different combinations of specific siRNA in presence of lipofectamine, to down regulate the $P R O C$ gene expression. After successful transfections of particular siRNA, RNA was isolated from the transfected cells and was converted to CDNA using reverse transcriptase followed by PCR amplification using real time PCR. PROC down regulation was determined using qRT-PCR by calculating fold change. Results: The 3 different siRNA's against exon3, exon 9 and exon 7 of protein $\mathrm{C}$ gene were used individually, 1st siRNA shows $35 \%$ and $75 \%$ silencing effect at the concentration of $10 \mathrm{pmol}$ and $12 \mathrm{pmol}$ respectively. Whereas 2nd siRNA shows $25 \%$ and $70 \%$ silencing effect at the concentration of 3 pmol and 6 pmol respectively and 3rd siRNA at concentration of $6 \mathrm{pmol}$ and $10 \mathrm{pmol}$ shows $50 \%$ and $80 \%$ silencing of gene respectively. When these siRNA's are used in different combinations it shows maximum silencing of gene at lower concentrations of specific siRNA. The combination of 1 st and 2nd siRNA showed $27 \%$ of gene silencing at $1.5 \mathrm{pmol}$ and $67 \%$ silencing at 3 pmol concentration. Another combination of 2 nd and 3rd siRNA showed $36 \%$ of gene silencing at 3 pmol and $59 \%$ silencing at $4.5 \mathrm{pmol}$ concentration. And combination of $1 \mathrm{st}$ and $3 \mathrm{rd}$ siRNA showed $34 \%$ of gene silencing at 1.5 pmol concentration and $58 \%$ silencing at 3 pmol concentration. This shows that siRNA targeting different exons of protein $\mathrm{C}$ in combinations gives higher silencing effect at lower concentration of siRNA than individually. Conclusions: These in-vitro findings show the feasibility of silencing $P R O C$ by a siRNA based approach as an alternate therapeutic approach for hemophilia.

\section{BHL 54}

Bone Marrow Aspirate Findings in Chronic Alcoholics

\section{Gunjan Mangla\#, Divya Bansal, Neha Garg, Mrinalini Kotru, Meera Sikka}

Email ID for Correspondence: manglagunjan@gmail.com University College of Medical Sciences, New Delhi
Introduction and Background: Chronic alcoholism is widely prevalent, however; haematological findings in these patients have not been extensively studied. We aim to study the bone marrow aspirate findings in chronic alcoholics. Patient/Material and Methods: Retrospective observational study. Complete blood count, peripheral smear and bone marrow aspirates of 72 chronic alcoholics were studied retrospectively from January 2013 to June 2016 ( $3.5 \mathrm{yrs}$ ). The slides were reviewed independently by 2 pathologists. The clinical history, complete blood counts, peripheral smear, bone marrow aspirate and bone marrow iron findings were recorded. Results: Out of 72 patients, 32 (44.44\%) patients had pancytopenia, $68(94.44 \%)$ had anaemia, and $53(73.61 \%)$ had thrombocytopenia on blood counts. Red cell morphology varied from normocytic normochromic $5(6.94 \%)$, microcytic hypochromic $4(5.56 \%)$, macrocytic $23(31.94 \%)$, to dimorphic anaemia $37(51.39 \%)$. Bone marrow aspirates were adequate in 56 of 72 cases. Sixteen $(28.57 \%)$ were normocellular, $38(67.86 \%)$ were hypercellular, and $2(3.57 \%)$ were hypocellular for age. Findings seen were erythroid hyperplasia $(75.00 \%)$, erythroid suppression $(5.35 \%)$, dyserythropoiesis (58.93\%), dysmyelopoiesis, increased plasma cells, increased histiocytes and haemophagocytosis. Bone marrow iron was available in 41 patients; iron stores were decreased in $2(4.88 \%)$, normal in 14 $(34.15 \%)$, increased in $25(60.97 \%)$. Seven $(17.07 \%)$ cases showed presence of ring sideroblasts. Eleven patients with increased iron stores showed presence of microcytes on peripheral smear. Conclusion: Chronic alcoholics show a variety of abnormalities in bone marrow aspirates, which closely mimic many haematological disorders. A specific history for alcoholism should always be taken in these circumstances.

\section{BHL 55}

\section{A Predication Model for Estimating Warfarin Dose in Indian} Patients

\section{Tejasvita Gaikwad ${ }^{\#}$, Kanjaksha Ghosh, S. Shetty}

Email ID for Correspondence: gtejasvita@yahoo.com National Institute of Immunohematology, 13th Floor, New Building, KEM Hospital, Parel, Mumbai, 400012

Introduction and Background: Warfarin, a derivative of coumarin, is a commonly prescribed oral anticoagulant all over the world. Although warfarin is indispensable for treatment of various clinical conditions, due to its narrow therapeutic index, high inter- and intraindividual variability in dosage and a high risk of bleeding or thrombosis due to over- or under coagulation, it has remained one of the most challenging medications to manage, despite over 60 years of experience with the drug. The main aim of this study was to generate warfarin dose prediction model by comprehensive analysis of warfarin pharmacokinetic, pharmcodynamic factors as well as non genetic and clinical factors. Patient/Material and Methods: Following a literature review, 21 genetic and non genetic risk factors were selected for investigation and screened in 300 patients who were prescribed warfarin for various clinical conditions. Results: Out of 21 risk factors analyzed, only 5 factors were found to be significantly associated with warfarin dose in univariate analysis. These 5 variables were entered in a stepwise multiple linear regression model. Based on the multiple regression analysis the following equation was generated to predicate daily warfarin dose in Indian patients, 


\section{Square root of dose $=2.61-0.41\left(\right.$ VKORC1-1639) $-0.21\left({ }^{*} 1 /{ }^{*} 2\right)-0.58$ \\ $\left({ }^{*} 1{ }^{*} 3\right)-0.86\left({ }^{*} 2 /{ }^{*} 3\right)-0.86\left({ }^{*} 3 /{ }^{*} 3\right)-0.002$ (Age in years) -0.08 (Vegetarian) \\ - For VKORC1-1639: '0' for GG, '1' for GA, '2' for AA \\ - For CYP2C9 $* 1 /{ }^{*} 2,{ }^{*} 1 /{ }^{*} 3,{ }^{*} 2 /{ }^{*} 3$ and ${ }^{*} 3 /{ }^{*} 3$, ' $O$ ' if genotype is absent, and ' 1 ' if genotype is present \\ - ' $O$ ' if patient is Non vegetarian, and ' 1 ' if patient is vegetarian.}

$\mathrm{R} 2$ of the multiple regression model was scored to $67 \%$ which indicates that, this predication model could explain nearly $67 \%$ of the dose variability. The association of predicated and calculated daily dose, was carried out using Passing-Bablok regression analysis which showed a good correlation between actual and calculated daily warfarin dose $(r=0.81)$. Conclusions: This prediction model can improve the management of this challenging therapy by minimising the severe manifestation of bleeding or thrombosis due to over or under anticoagulation and will give better quality of life in Indian patients with thrombosis and on warfarin therapy.

\section{BHL 56}

Genetic Testing is Mandatory to Identify All Congenital Antithrombin Deficiency Cases

\section{Rutuja Deshpande", Bipin Kulkarni, Kanjaksha Ghosh, Shrimati Shetty}

Email ID for Correspondence: rutujad1810@yahoo.in National Institute of Immunohaematology (NIIH)

Introduction and Background: Antithrombin (AT) is the primary inhibitor of thrombin and factor Xa. AT deficiency is an autosomal dominant disorder, its complete deficiency has lethal consequences and the heterozygous deficiency significantly increases (10- to 40-fold) the risk of thrombosis-recurrent venous thromboembolism (VTE), which includes both Deep Venous thrombosis (DVT) and pulmonary embolism and also arterial thrombosis. Recent evidences show that some SERPINC1 mutations responsible for functional abnormality of AT often show slightly decreased or even normal activity and cannot be detected by currently available laboratory assays. We are reporting 3 variants with normal and 1 variant with borderline AT activity. Patient/Material and Methods: 500 (arterial 125; venous 375) patients with thrombosis and normal AT levels were screened for the presence of mutations in SERPINC1 by direct DNA sequencing. AT activity was measured by chromogenic assay based on thrombin (IIa) and Xa inhibition. AT antigen was measured by ELISA. 100 normal healthy controls were sequenced for exon 5. Also 50 patients with borderline AT activity were sequenced for all seven exon and promoter region of SERPINC1 gene. Results: Total 11 out of 500 patients presented with p.Pro305His variation (rs549991084) where all patients presented with cerebral venous or arterial thrombosis. This variant is found to be deleterious in prediction softwares. 2 patients had recurrent thrombosis episodes. 3 out of 500 patients showed Arg293Gln variant (rs572313182) which was found to be benign. And 1 out of 500 patients, with recurrent DVT presented with novel variant His351Pro, was found to be deleterious in PolyPhen2 and polymorphic in Mutation Taster. None of the control presented above mentioned mutation. 1 out 50 borderline patients presented with splice site variant in promoter region. Controls were not screened for promoter region. Conclusions: Two variants were found to be deleterious in prediction softwares even though their AT activity was normal. Few patients had recurrent episodes of thrombosis. These mutations (except promoter variant) lie in thrombin binding region of SERPINC1 gene. Though the functional implications of this variant cannot be confirmed with the currently available techniques for AT estimation, the data strongly suggests that genetic screening for variants in SERPINC1 gene should be performed in all thrombosis cases.

\section{BHL 57}

Study of Dual Anti platelet Drugs Therapy 'Clopidogrel and Aspirin Effects' of Results Analysed from Multiplate Analyser [Roche] in Patients with Cardiovascular Events

\section{Ms. Enna Maria Lourenco ${ }^{\#}$, V. P. Antia}

Email ID for Correspondence: ennalou@ rediifmail.com Breach Candy Hospital Trust, Mumbai, India

Introduction and Background: Platelets are critical in haemostasis and the development of arterial thrombi. Damaged endothelium activates platelets which respond by adhering and aggregating. Their release of thromboxane A2 and adenosine diphosphate (ADP) amplifies and propagates the process by stimulating surrounding platelets. Dual therapy with aspirin and clopidogrel is currently recommended for the management of patients with non-ST segment elevation (NSTE) ACS, those with chronic stable coronary artery disease (CAD) at high risk of developing an MI, and those undergoing PCI with coronary stents. Aspirin: is active drug. Clopidogrel: is prodrug. Aim: To investigate dual antiplatelet drugs effect of Clopidogrel and Aspirin in cardiac disease patients and to identify patients who are either hyper- or hypo-responsive to antiplatelet medication. Patient/Material and Methods: 110 patients were tested with different history of cardiovascular events. Inclusion Criteria: Patients only on dual antiplatelet therapy daily ie $75 \mathrm{mg}$ clopidogrel \& $75 \mathrm{mg}-150 \mathrm{mg}$ Aspirin above 48 yrs to 84 yrs were investigated. Exclusion Criteria: NSAID or any anticoagulants or antiplatelet drugs. Blood Collected: In Hirudin tubes. All samples were kept at room temperature $\left(20-25{ }^{\circ} \mathrm{C}\right)$. Whole Blood samples were tested within $1 \mathrm{hr}$. The antiplatelet effect of clopidogrel and aspirin was assessed by measurement of platelet aggregation using multiplate electrode aggregometry [MEA], Multiplate analyser, Roche Diagnostic International. Aggregation measured with MEA is quantified as AU [aggregation unit in mins, an area under curve (AUC)]. CBC was analysed for all patients by Sysmex XN SERIES analyser. Patients platelets were between 125,000-450,000/cu mm. Normal values of ADP:59-119U \& Normal values of Aspirin [ASA]:76-139U. Electronic \& Liquid controls were used to ensure $\mathrm{QC}$ of impedence detection on site. Results: Normal patients [ $n=20] 18.1 \%$ were Normal for clopidogrel [ADP] \& Aspirin [ASA]. MI+CAD patients [n $=44] 37 \%$ were Responder to Clopidogrel [ADP] \& Aspirin 
[ASA]. Clopidogrel resistance patients ie nonresponder were $[\mathrm{n}=12] 10.9 \%$. Aspirin resistance patients ie nonresponder were $[\mathrm{n}=7] 6.3 \%$. Clopidogrel \& Aspirin resistance patients ie nonresponder $[\mathrm{n}=14] 12.7 \%$. Stent patients $[\mathrm{n}=8] 7.2 \%$ were responder to clopidogrel \& Aspirin. CABG patients [n=8] $7.2 \%$ were Responder. *(If $A D P<31 \mathrm{U}$ High bleeding risk in $C A B G$ patients. If $A D P>46 U$ High thrombotic risk in PCI. If ADP $<19 \mathrm{U}$ High bleeding risk in PCI. If $<40 \mathrm{U}$ inhibition of COX-1 by aspirin. $<30 U$ strong inhibition of $C O X-1$ by aspirin [strong responder to aspirin. For Normal control samples, patients were not on Clopidogrel and Aspirin drugs. CBC were normal. Platelets count was between 225,000-270,000/cu mm. Cholesterol and Triglycerides were normal. Conclusions: Platelet function test helps in identifying higher risk patients with poor compliance or drug resistance, who may benefit from therapy change. Clopidogrel resistance can be due to medication, lab, methodology, sub optional dosage, genetic polymorphisms [CYP2C19]. Poor absorption can contribute to a decreased response to clopidogrel and aspirin. Key Message: Patient with higher ADP test values shows incomplete or no platelet inhibition and have increased risk for arterial thromboembolism. Platelet function test should be used to measure before and after the institution of antiplatelet therapy to prove efficacy. Rapid turnaround time.

Keywords Dual antiplatelet drug therapy, Clopidogrel, Aspirin, Differential diagnosis

\section{BHL 58}

A Case of Somatic Mosaicism in a Severe HB Family Detected by Allele Specific PCR

\section{Sharda Shanbhag", Shrimati Shetty \\ Email ID for Correspondence: shardashanbhag@gmail.com National Institute of Immunohaematology}

Introduction: Approximately $30-40 \%$ of haemophilia cases are due to de-novo mutations. Origin of these mutations may be in a single germ cell or it might have taken place at different stages of embryogenesis resulting in either a somatic or germ line mosaic. Here, we report a case of somatic mosaicism detected by allelespecific PCR (ASP) in a severe Haemophilia-B family. Material and Methods: A family of two severe Haemophilia-B siblings was referred for carrier and antenatal diagnosis of their maternal aunt at 10th week of pregnancy. Linkage analysis by RFLP techniques revealed that the females were not informative. Hence, genetic study for $F 9$ was performed by direct sequencing. The affected brothers were found to be hemizygous for a known missense mutation, p.Glu54Ala; carrier test for their mother by targeted direct sequencing, revealed that she was homozygous for wild-type genotype (noncarrier). On suspicion of somatic mosaicism in the mother, we standardised further molecular genetic tests by ASP. Results: ASP was standardised in-house to confirm mother's carrier status and mosaicism in this family. The siblings had bands corresponding to the mutant genotype whereas, control samples exhibited the wild-type genotype, their mother, aunt and grandmother had both mutant and wild-type genotypes. Hence they were carriers. Conclusions: Postzygotically occurring somatic-mutations produce genetic mosaic (mutation occurs in some but not all cells). DNA sequencing is incapable of detecting somatic-mutations within cells as the ratio of the mutant to the wild-type allele can be quite small. In sporadic cases of haemophilia, the possibility of maternal somatic/germline mosaicism must always be considered. Presence of two types of cells in maternal circulation confirms mosaicism in this family. This finding has important implications in genetic diagnosis where a great percent of families still rely on gene tracking and presence of mosaic in the family can provide erroneous results.
BHL 59

\section{Myeloperoxidase Deficiency in Eosinophils Can Be Detected with ADVIA 2120}

\section{Sarwat Fatma ${ }^{\#}$, Mamta Soni}

Email ID for Correspondence: fatmasrwt@gmail.com Department of Haematology, Apollo Hospitals, Chennai

Introduction and Background: Myeloperoxidase deficiency (MPOD) is one of the most common inherited phagocyte defects, but it is rarely associated with clinical symptoms. Patient/Material and Methods: Our hospital have two types of hematological analyzer-Beckman coulter based on electrical impedance and Advia 2120 which differentiates leukocytes on the basis of cellular volume and their myeloperoxidase concentration. Here, we present a series of 3 cases that were submitted to our Hematology department for routine complete blood cell count. After processing in ADVIA 2120, monocytosis was observed in all three samples. Results: Because of flagging, peripheral blood smears of these cases were reviewed, which revealed eosinophilia and normal monocyte counts. Because of discordance between the analyzer report and peripheral blood smear review, the samples were reprocessed in Beckman Coulter, which revealed eosinophilia on histograms. On retrospective analysis of the scatter grams of ADVIA 2120, two populations of cells were observed due to different cell volumes of monocytes and eosinophils, and presence of MPO deficiency in the latter. Conclusions: Aim of this study is to emphasize the review of scatter grams by the pathologist as well as technician to promptly identify such cases.

\section{BHL 60}

Utility of Thromboelastography (TEG) to Predict Trauma Induced Disseminated Intravascular Coagulation (DIC)

Arulselvi Subramanian ${ }^{1 \#}$, Venencia Albert ${ }^{1}$, Renu Saxena ${ }^{2}$, Sanjeev Kumar Bhoi ${ }^{3}$, Deepak Agrawal ${ }^{4}$, Ravindra Mohan Pandey ${ }^{5}$, Mahesh Chandra Misra ${ }^{1}$

Email ID for Correspondence: arulselvi.jpnatc@gmail.com Departments of ${ }^{1}$ Lab Medicine, ${ }^{2}$ Hematology, ${ }^{3}$ Emergency Medicine, ${ }^{4}$ Neurosurgery and ${ }^{5}$ Biostatistics, Jai Prakash Narayan Apex Trauma Centre, AIIMS, New Delhi

Introduction and Background: Coagulation and fibrinolytic systems are activated early after injury. Thromboelastography (TEG) is a point of care device that graphically depicts the dynamic interaction of clotting factors and platelets indicating an overall clot quality within 30-60 mins. A TEM score (range, 0-4) was defined as the total number of parameters of these 4 that were deranged in any given case toward hypocoagulability. Objective: (1) To establish normal reference values for TEG in North Indian healthy volunteers. (2) To compare the ISTH criteria for the diagnosis of overt DIC with the thromboelastograph (TEG) score for DIC in severely injured patients. Patient/Material and Methods: Study design: Prospective cohort; Study Duration: 2 years. We prospectively collected citrated blood samples from 200 healthy volunteers $(1: 1$, males:females) \& 100 severe trauma patients (ISS $<9$ ); age group (18-50 years), admitted within 24 hrs of injury. Patients with prior h/o coagulation abnormalities were excluded from the study, sampling was done on admission, day 3 and day 5 of injury. Thromboelastography was performed on TEM-A automated thromboelastometer (Framar Biomedica, Rome, Italy); parameters analyzed were r-time, k-time, $\alpha$ angle, maximal amplitude (MA). DIC was diagnosed based on the (i) overt DIC (ISTH) score ( $\geq 5)$ \& (ii) TEM score (prolonged R and $\kappa$ 
times and shortened $\alpha$ angle and MA $(\geq 2)$. The $95 \%$ reference range was calculated as (mean-1.96 standard deviation [SD]) to (mean $+1.96 \mathrm{SD}$ ) using healthy volunteer data. Sensitivity, specificity and diagnostic accuracy of the TEM score was determined based on the patient data after applying the established TEG reference range. Results: Our reference values for $95 \%$ of 200 volunteers were r-time: 1.8-14.2 $\mathrm{min}$, k-time: $0.7-7.3 \mathrm{~min}, \alpha$-angle: $27.3^{\circ}-72.3^{\circ}$ and MA: $32.1-87.9 \mathrm{~mm}$. Out of the 100 trauma patients (94 male: 6 female), 16 were diagnosed as overt DIC based on ISTH criteria \& 10 based on the TEM score. 77 had non-overt DIC by the ISTH criteria and 10 by TEM score $(\mathrm{p}=0.03)$. High sensitivity and accuracy was observed for the diagnosis of overt DIC $(62.5 \%, 94 \%)$ compared to the nonovert DIC $(11.6 \%, 32 \%)$. Conclusions: Thromboelastography is of clinical significance for efficiently detecting Trauma associated coagulopathy with a diagnostic accuracy of $94 \%$. Results are obtained quickly and cost effective compared to the golden standard ISTH score method. However manufacturer's reference values for TEG may not be appropriate for different ethnicity, therefore we recommend the determination of normal TEG values by each laboratory for their target population.

\section{BHL 61}

\section{Alteration in Megakaryocytic Stem Cells Niche in Cirrhosis}

\section{Deepika Lal ${ }^{\#}$, Dhananjay Kumar, Anupama Patil,} Anupam Kumar, Chhagan Bihari

Email ID for Correspondence: deepika18021@gmail.com Institute of Liver and Biliary Sciences, D-1, Vasant kunj, New Delhi

Introduction: Hematopoietic stem cells (HSCs) remain in specific microenvironment known as arterial and sinusoidal niche. The HSCs in arterial niche are homeostatically in quiescent state, and tightly controlled by non-hematopoietic cells, whereas HSCs in sinusoidal niche are designated as potential migratory pool and controlled by hematopoietic cells e.g. megakaryocytes. Recently we have shown that in advanced liver disease, the arterial niche cells are significantly decreased. Aim: To study the bone marrow (BM) sinusoidal niche in liver disease. Method: We studied the histological and molecular changes in BM of cirrhosis patients $(n=40)$ and non-cirrhotic normal controls $(n=10)$. HSCs were evaluated by CD34+ staining; along the CD31 expressing sinusoid. Megakaryocytes PF4 was evaluated by immunohistochemistry and growth factors secreted by megakaryocytes: ELISA measured PF4 and FGF2 in BM plasma. Liver disease severity was assessed by Model of end stage liver disease (MELD). Results: Mean age in the study population was $48 \pm 12.4$ and control groups was $43 \pm 13.2$ years. Reactive CD34+ cells in cirrhotic patients were comparable in early cirrhosis, mildly low in cases with MELD: 10-20 and significantly reduced in advanced cirrhosis with MELD > $20 \quad(<0.001)$. PF4 expression in the megakaryocytes showed variable pattern in cirrhotic cases in comparison to control. Initial stages (MELD 6-10) it is comparable to control $(\mathrm{p}=0.87)$ and decreased in cases with MELD $(10-20)[\mathrm{p}=0.045]$ and again significantly low in cases with MELD $>20(\mathrm{p}=0.02)$. PF4 in BM plasma also had similar levels. It was higher in cases with MELD

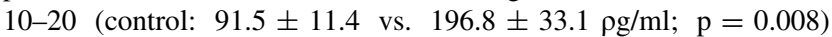
and significantly lower in patients with MELD $>20$ $(51.4 \pm 11.3 \mathrm{\rho g} / \mathrm{ml} ; \mathrm{p}<0.001)$. Whereas; FGF2 level was lower than the control in cirrhotics with MELD 10-20 $(\mathrm{p}=0.021)$ and MELD $>20(p=0.03)$. Conclusion: Sinusoidal stem cell compartment is controlled by megakaryocytes and secreted PF4 and FGF2, which get depleted in advanced cirrhosis along with HSCs reduction with severity of liver disease.

\section{BHL 62}

Sonoclot Signature Analysis Predicts Coagulopathy in Chronic Liver Disease

\section{Anupama G. Patil ${ }^{\#}$, Deepika Lal, Chhagan Bihari}

Email ID for Correspondence: itsagp@gmail.com Department of Clinical Hematology, Institute of Liver and Biliary Sciences, New Delhi

Introduction and Background: Patients with liver disease are frequently plagued with profound disturbances in their haemostatic system. The conventional tests are not clinically useful for predicting the risk of thrombosis or bleeding in such patients. We investigated whether the Sonoclot analyzer is useful for predicting coagulopathies in these patients. Also, we planned to correlate serum fibrinogen concentrations with peak amplitude (PA) from the Sonoclot signature analysis. Patient/Material and Methods: Clinical data (including history of bleeding and thrombosis) and laboratory data from 83 patients of four groups of liver disease, including cirrhosis, chronic hepatitis, acute-on-chronic liver failure (ACLF) and vascular pathology of liver were analyzed. All patients were subjected to Sonoclot analysis and correlated with routine coagulation parameters including platelet count, PT/INR and fibrinogen. Results: The Sonoclot signatures demonstrated more pronounced significant abnormalities in ACLF patients with maximal prolongation of the activated clotting time $($ ACT) (p-values $<0.0001$ ), lowest clot rate $(\mathrm{CR})$ levels (p-value $<0.0048$ ), lowest platelet function $(\mathrm{PF})$ values (p-values $<0.0004$ ), highest mean time to peak (TP) levels (p-values $<0.00971$ ) and lowest peak amplitude (PA) levels (p-values <0.044) when compared to the control group of chronic hepatitis. In patients with thrombosis, the clot rate (CR) was significantly elevated when compared to non-thromobotic patients $(\mathrm{p}<0.029)$. In all the groups, a significant correlation was obtained between the serum fibrinogen concentration and peak amplitude (PA) levels of Sonoclot signature (p-value $<0.05$ ). Conclusions: The CR levels on the Sonoclot signature can be used as a guide to predict thrombosis in these patients, although a definite cut-off needs to be determined for the same. Also, PA levels of Sonoclot correlate with serum fibrinogen concentration.

\section{BHL 63}

\section{Hemophagocytosis in Bone Marrow Aspirates: An Indicator of Hidden Pathologies}

\section{K. S. Sampath", S. Venkatesan, M. G. Manoj, R. Bharadwaj}

Email ID for Correspondence: dr.sampathks@gmail.com Armed Forces Medical College, Pune

Introduction and Background: Hemophagocytosis is the phenomenon of phagocytosis of erythrocytes, neutrophils, platelets, lymphocytes or other hematopoietic precursors by histiocytes or macrophages either in bone marrow, lymph node, liver or spleen. Hemophagocytosis in bone marrow aspirates is fairly common and can be observed in a spectrum of clinical conditions. This study was aimed at evaluating the clinicopathological profile of patients showing hemophagocytosis on bone marrow aspirates examined for varied clinical indications. Patient/Material and Methods: In this cross sectional descriptive study, we reviewed 10 bone marrow aspirates which were reported to be showing features of hemophagocytosis between July 2015 to July 2016. The clinicopathological profile in the form of Patient's age, sex, clinical diagnosis, peripheral blood features and bone marrow aspirate salient findings were compiled. Results: Out of the 10 bone marrow aspirates which showed hemophagocytosis, $08(80 \%)$ were from males and $02(20 \%)$ were from females. The mean age of the patients was 29 years. The 
commonest clinical diagnosis with the presence of hemophagocytisis was Pyrexia of unknown origin (05) followed by HIV positive status (03), Cytopenia (01) and one patient was a renal transplant recipient. The features of hemophagocytosis were documented and reported in $100 \%$ of the cases and the clinicians were advised to investigate the patients for the presence of Hemophagocytic Lympho Histiocytosis (HLH) by estimation of Serum Triglycerides, ferritin and plasma fibrinogen. Conclusions: Hemophagocytosis is a fairly common phenomenon however it should not be overlooked and always be documented and reported. Presence of hemophagocytosis may be the only clue to the underlying conditions like systemic infections or HLH. It is essential to suspect and diagnose HLH early as the condition can be life threatening. Diagnosis of HLH requires clinical and laboratory criteria to be met. This study therefore concludes that every case which shows hemophagocytosis on bone marrow needs to be reported and further work up be done to confirm the diagnosis of HLH, so that the patients can be timely and appropriately managed.

\section{BHL 64}

\section{Effect of Storage on Complete Blood Count Parameters Processed} in Beckman Coulter LH 750 Analyser

\section{Mamta Setia", Parul Arora, Tapasyapreeti Mukhopadhyay, Raghavendra Lingaiah, Subhadra Sharma, A. K. Mukhopadhyay}

Email ID for Correspondence: setiamamta@gmail.com Department of Laboratory Medicine, All India Institute of Medical Sciences, New Delhi

Introduction and Background: Stability of the various parameters of CBC (complete blood counts) depends on the storage practices followed at the lab. This study was conducted to identify the changes that occur in blood counts due to storage at various conditions and to standardize the retention period of the sample. Patient/Material and Methods: Random blood samples $(\mathrm{N}=100)$, processed in the BECKMAN COULTER LH 750 analyser within 6 h of collection were selected. Each specimen was further divided into two aliquots and stored at $4{ }^{\circ} \mathrm{C}$ and room temperature $\left(25 \pm 1{ }^{\circ} \mathrm{C}\right)$ separately. The stored samples were retested at the intervals of 24,48 and 72 hours. The mean absolute difference and mean percent change of all the parameters were compared with the initial run and statistically analysed using repeated measure ANOVA and FRIEDMAN test. Results: Total leukocyte count, Hemoglobin and $\mathrm{MCH}$ were relatively stable at both temperatures till 72 hours. RBC counts were stable till 24 hours at room temperature but increased in samples stored at $4{ }^{\circ} \mathrm{C}$. MCV, HCT, RDW and MPV were stable till 48 hours at $4{ }^{\circ} \mathrm{C}$, while MCHC descended over time. Platelets remained stable at $4{ }^{\circ} \mathrm{C}$ but altered at room temperature after 24 hours. Differential leukocyte counts showed significant changes in 24 hours under both storage conditions. Conclusions: Clinically reliable results can be obtained for most of the parameters of $\mathrm{CBC}$ in the samples stored at $4{ }^{\circ} \mathrm{C}$ till 48 hours. However differential count measured by VCS (Volume, conductivity and scatter) method as done in LH750 analyser are not reliable following 24 hours of storage.

\section{BHL 65}

Evalution of Platelet Parameters in Patients with Thrombocytosis in Tertiary Care Center

Umesh K. Tiwari", Rupali Awale, Ratnaprabha Maji, Subhadra Sharma, Asok K. Mukhopadhyay

Email ID for Correspondence: umesh_tiwari1987@yahoo.co.in All India Institute of Medical Sciences, New Delhi
Introduction and Background: Platelet count is one of the most commonly asked investigation in any disease condition. Increase in platelet count is termed as thrombocytosis. Normal count range from $1.5-4 \mathrm{lakh} / \mathrm{mm}^{3}$, above $4 \mathrm{lakh} / \mathrm{mm}^{3}$ is considered as thrombocytosis, it is either primary or reactive. Underlying causes leading to reactive thrombocytosis can be iron deficiency anaemia, infection or inflammatory condition. New hematology autoanalysers provide additional platelet parameter data like Mean platelet volume (MPV), platelet distribution width (PDW) and plateletcrit (PCT). Material and Methods: 200 patients admitted in hospital with various diagnosis were included in the study while 20 healthy volunteers were control group. $3 \mathrm{ml}$ peripheral venous blood was collected in EDTA vial and processed in LH750 Beckman coulter hemato-analyser. Detailed history of patient, examination finding, investigation and diagnosis were noted. Data collected and analysed. Results: In case of infection, inflammatory \& autoimmune causes, male patients showed predisposition to thrombocytosis whereas after operative intervention female patients showed increase in platelet count. Overall MPV, PCT \& PDW showed increased values. Conclusions: Platelet parameters are upcoming determinants available along with routine hemogram in new hematology autoanalysers and this information can be used to interpretate various conditions.

Keywords MPV: Mean platelet volume, PCT: Plateletcrit, PDW: Platelet distribution width thrombocytosis, Platelet indices

\section{BHL 66}

\section{Alpha Globin Gene Multiplication Accentuate the Severity of hHeterozygous $\beta$ Thalassaemia}

\section{K. Neelagandan", S. Eswari, S. Gopinath, Shaji R. Velayudhan, Eunice S. Edison \\ Email ID for Correspondence: neelagandankamalanathan@yahoo.com \\ Department of Haematology, Christian Medical College, Vellore}

Introduction: Heterozygous $\beta$ thalassaemia is clinically benign with mild degree of microcytic anaemia. The $\alpha$ globin gene multiplication increases the disease severity by altering the $\alpha / \beta$ globin chain ratio in heterozygous $\beta$ thalassaemia. We report a large series of cases of heterozygous $\beta$ thalassaemia with $\alpha$ globin gene multiplication and describe the clinical and haematological features of this condition. Aim: The aim of this study was to assess the prevalence of $\alpha$-globin gene multiplication in patients with severe heterozygous $\beta$ thalassaemia in Indian population. Methods: Based on haemoglobin analysis by HPLC and the clinical phenotypes, patients with severe heterozygous $\beta$ thalassaemia were included in the study. DNA was extracted, $\beta$ globin gene mutations were screened by Reverse Dot Blot (RDB) and DNA sequencing. The copy number of $\alpha$ globin genes was screened by PCR followed by capillary electrophoresis, and the type of multiplication, $\alpha \alpha \alpha^{3.7}$ and $\alpha \alpha \alpha^{4.2}$, was confirmed by specific PCRs. Results: One hundred and seven patients suspected to have severe heterozygous $\beta$ thalassaemia from 2006 till date were analysed. The haematological parameters are tabulated below. Out of these 107 cases, genetic analysis was performed for 33 patients (median age: 31 years). Out of 33 patients, 22 (66\%) had hepatosplenomegaly and $24(72 \%)$ had received at least one transfusion. The most common $(27 / 33 ; 81 \%) \beta$ globin gene defect observed was the splice site mutation, IVS I-5 (G>C). Analysis of $\alpha$ globin gene copy numbers revealed that $45.45 \%(15 / 33)$ had one extra $\alpha$ globin gene and $42.42 \%(14 / 33)$ had two extra copies of $\alpha$ globin genes and $12 \%(4 / 33)$ had extra $3 \alpha$-globin genes. The haemoglobin levels in the subjects with heterozygous $\beta$ thalassaemia and $\alpha$ globin multiplication were significantly lower than those with heterozygous $\beta$ thalassaemia without $\alpha$ globin gene multiplications $(\mathrm{p}=0.000)$ and 
$\mathrm{HbF}$ levels were significantly higher in the patients with $\alpha$ globin gene multiplications $(\mathrm{p}=0.000)$ than those with normal alpha globin genotype. Discussion: In heterozygous $\beta$ thalassaemia with additional $\alpha$ globin genes, the excess $\alpha$ globin chains causes a more pronounced degree of anaemia and phenotypic severity leading to thalassaemia intermedia phenotype than those with heterozygous $\beta$ thalassaemia without $\alpha$ globin multiplication.

Haematological parameters in heterozygous $\beta$ thalassaemia

\begin{tabular}{|c|c|c|c|c|}
\hline & $\begin{array}{l}\mathrm{Hb} \\
(\mathrm{g} / \mathrm{dL})\end{array}$ & $\begin{array}{l}\text { MCV } \\
\text { (fl) }\end{array}$ & $\begin{array}{l}\mathrm{HbF} \\
(\%)\end{array}$ & $\begin{array}{l}\mathrm{HbA}_{2} \\
(\%)\end{array}$ \\
\hline $\begin{array}{l}\text { Heterozygous } \beta \\
\text { thalassaemia } \\
(\mathrm{n}=2401)\end{array}$ & $\begin{array}{l}10.8 \\
(3-16)\end{array}$ & $\begin{array}{l}63.78 \\
(47-92.3)\end{array}$ & $\begin{array}{l}0.8 \\
(0-12)\end{array}$ & $\begin{array}{l}4.9 \\
(3.4-7.9)\end{array}$ \\
\hline $\begin{array}{l}\text { Severe } \\
\quad \text { heterozygous } \beta \\
\text { thalassaemia } \\
(\mathrm{n}=107)\end{array}$ & $\begin{array}{l}8.9 \\
(5.5-13.9)\end{array}$ & $\begin{array}{l}65.4 \\
(48.4-82.3)\end{array}$ & $3.5(1-12.9)$ & $\begin{array}{c}5 \\
(3.9-7.1)\end{array}$ \\
\hline $\begin{array}{l}\text { Heterozygous } \beta \\
\text { thalassaemia } \\
\text { with } \alpha \text { globin } \\
\text { multiplication } \\
(\mathrm{n}=33)\end{array}$ & $\begin{array}{l}8.3 \\
(5.7-11.3)\end{array}$ & $\begin{array}{l}65.3 \\
(55.5-73.1)\end{array}$ & $\begin{array}{l}3.6 \\
(1.2-10.4)\end{array}$ & $\begin{array}{l}5.1 \\
(3.96-7.5)\end{array}$ \\
\hline
\end{tabular}

Median values and ranges are shown

\section{BHL 67}

Clinico-hematological Profile of Visceral Leishmaniasis: Review from Tertiary Care Centre

Swasti Jain", Poojan Agarwal, Garima Baweja, Anjali Sharma, Vijay Kumar, Sadhna Marwah, A. S. Nigam, Gurdeep Buxi

Email ID for Correspondence: jain.swasti7@gmail.com Department of Pathology, PGIMER, Dr. RML Hospital, New Delhi

Introduction and Background: Visceral leishmaniasis (also called as Kala-azar) is a fatal zoonotic systemic disease caused by the protozoan complex Leishmania donovani and transmitted by the bite of Phleobotomine sandfly. Demonstration of amastigote forms of the parasite through bone marrow aspiration remains a vital investigation in disease diagnosis. In the present study we analyzed the clinicohematological profile of patients suspected of Visceral Leishmaniasis (VL). Patient/Material and Methods: In the present retrospective study, clinical details, complete hemogram with peripheral smear findings of cases reported as VL over a period of 5 years, was retrieved and analyzed. Bone marrow aspiration smears and biopsy slides were thoroughly reviewed and average Parasite Density (APD) graded on smears as (0)- 0 LD Bodies/1000 Fields, (1)+- 1-10 LD Bodies/1000 Fields, (2+)- 1-10 LD Bodies/100 Fields, (3+)- 1-10 LD Bodies/10 Fields, (4+)- 1-10 LD Bodies/Field, (5+)- 10-100 LD Bodies/Field and (6+)- >100 LD Bodies/Field. Hemophagocytosis (HPS) was graded as- (0)- Absent; $(1+)$ (Mild)- $<2$ histiocytes with HPS/Slide; (2+) (Moderate)- 2-5 histiocytes with HPS/Slide; (3+) (Severe) $->5$ histiocytes with HPS/Slide. Results: The study included 28 (12 pediatric and 16 adult) patients. Mean age of patients was 24.2 years (4-50 years) with fever being the most common presentation, followed by splenomegaly and then hepatomegaly. Cytopenias were observed in majority of patients and mean $\mathrm{Hb}$ at the time of bone marrow examination was $7.3 \mathrm{gm} \%$, TLC $-3800 / \mu 1$ and platelet-80,000/ $\mu$ l. Bone marrow examination revealed cellular aspirates with mean M:E-1.2:1 along with prominence of plasma cells and eosinophils. Correlation was studied between APD and features like hemophagocytosis and dyserythropoeisis. Conclusions: The knowledge of basic clinical parameters, morphological clues on peripheral smear, bone marrow aspirate and biopsy is important for diagnosis of VL. Hemophagocytosis and dyserythropoeisis are frequent observations in bone marrow aspirates of VL patients.

\section{BHL 68}

Are We Misleading the Would Be Parents with Haemoglobinopathy by CVS Analysis?

\section{Rajlaxmi Basu", Sila Chakrabarti, Siddhartha Sankar Ray}

Email ID for Correspondence: mampi_r@rediffmail.com Institute of Hematology and Transfusion Medicine, Medical College, Kolkata, 700073

Introduction and Background: Hemoglobin $E$ and the $\beta$ Thalssemia carrier, even diseased persons are common in South East Asia. As a result Hemoglobin $E / \beta$ Thalassemia is seen most commonly in this population. About half of these individuals behave like thalassemia major, requiring regular blood transfusions. Since inception of prenatal diagnosis in our institution we have stumbled upon a considerable number of patients with E- $\beta$ Thalassemia who did not require regular blood transfusion. These adults are in many cases successful parents without transfusion history. Here is the rub. CVS with E- $\beta$ thalassemia are advised to terminate despite of never requiring transfusion and even having a child who is transfusion independent. So before jumping to any conclusion the process has to be worked out in greater detail and policy restructure is the cry of the hour. Patient/ Material and Methods: The patients were all "at risk" couples who are either carrier or E- $\beta$ Thalassemic without requiring any transfusion. Prenatal diagnosis were done on CVS and if it is E- $\beta$ Thalassemia then a major ethical and social issue can arise whether to terminate the pregnancy specially where more intrusive molecular diagnosis are not introduced. Results: Among all these "at risk" couple number of E- $\beta$ Thalassemic non transfusion depended father/mother for the year 2011, 2012, 2013, 2014, 2015, and till today in 2016 were 3, 1, 2, 3, 9 and 4 respectively and their $\mathrm{Hb}, \mathrm{MCV}$, $\mathrm{MCH}$, MCHC, RDW, Ao, $\mathrm{A}_{2} \mathrm{HbF}$ values of $9.74 \pm 6.6$, $71.89 \pm 25.1, \quad 22.55 \pm 3.98, \quad 30.03 \pm 7.8, \quad 27.27 \pm 16.90$, $38.79 \pm 30.57,37.97 \pm 24.15,5.71 \pm 7.96$ respectively. Since 2011 number of children with E- $\beta$ Thalassemia without dependence are 1, 8,9 , and for 2015 was 1 respectively with $\mathrm{Hb}, \mathrm{MCV}, \mathrm{MCH}, \mathrm{MCHC}$, RDW, Ao, $\mathrm{A}_{2} \mathrm{HbF}$ values of $7.06 \pm 1.6,67.22 \pm 17.30$, $21.46 \pm 3.54, \quad 30.37 \pm 1.88, \quad 26.51 \pm 7.89, \quad 31.96 \pm 32.006$, $33.14 \pm 22.72$, and $25.96 \pm 21.75$ respectively. In 2014 only two families who fan the controversy massively with an independent E- $\beta$ Thalassemia and CVS followed the suit. More intrusive delve out method implementation is highly required. Conclusions: These results draws special attention to the issue of ethical brush up and of course it's a strategic issue whether to continue a pregnancy with E- $\beta$ Thalassemia or to terminate it, is a slippery way, but it is clear from our data that only CVS analysis for common $\beta$-mutation is not an efficient litmus test. 


\section{BHL 69}

Coexistence of Hereditary Spherocytosis and Beta Thalassaemia Trait: A Report of Two Cases

\section{Sonali Singh" ${ }^{\#}$ H. B. Sridevi, Anupama Hegde, B. Prashantha, Shrijeet Chakraborti, Pooja K. Suresh}

Email ID for Correspondence: singhsonali29@gmail.com Kasturba Medical College, Mangalore

Introduction and Background: The combination of hereditary spherocytosis (HS) with other hereditary disorders of RBCs such as sicklecell anaemia is well known, though rare. The co-existence of hereditary spherocytosis and $\beta$-thalassaemia trait (BTT) is extremely rare and only few cases have been reported so far in the literature. Patient: Patient 1 was an 18 year old female patient presented with fever and generalised weakness. On examination she was pale, icteric, and had hepatosplenomegaly. Patient 2 was a 52 year old female patient presented with a history of easy fatiguability since 9 to 10 years. On examination there was splenomegaly $15 \mathrm{~cm}$ below the left costal margin. Results: Peripheral smear of patient 1 and patient 2 showed pancytopenia with dimorphic RBC picture (microcytic hypochromic RBCs with presence of numerous uniform spherocytes). Haemoglobin and reticulocyte count were $4.4 \mathrm{gm} / \mathrm{dL}$ and $9.2 \%$ respectively in patient 1 and was $8.6 \mathrm{gm} / \mathrm{dL}$ and $8.4 \%$ respectively in patient 2. Mean corpuscular haemoglobin concentration were $35.1 \mathrm{~g} /$ $\mathrm{dL}$ and $31 \mathrm{~g} / \mathrm{dL}$ in patient 1 and 2 respectively. On further investigations of both cases, osmotic fragility test was increased. A quantitative HPLC on both samples showed BTT pattern with $3.8 \%$ of $\mathrm{HbA} 2$ and $2.9 \%$ of $\mathrm{HbF}$ in patient 1 and $4.8 \%$ of $\mathrm{HbA} 2$ and $2.6 \%$ of $\mathrm{HbF}$ in patient 2 . The patients had severe worsening anaemia and high transfusion dependency for which they underwent splenectomy and their spleen showed congestive splenomegaly. The cases were concluded as HS with BTT. Conclusions: From the literature, it is known that coexistence of HS and BTT is a rare phenomenon and when exists, presence of BTT generally silences the HS expression in most cases. In both our patients, we observed worsening of the clinical course where splenectomy was the ultimate modality of treatment to relieve the haemolytic component caused by HS. An awareness of such coexistence and varied clinical presentations is required for appropriate selection of treatment modality.

\section{BHL 70}

Correlation of Platelet Indices in Various Disease Groups with Thrombocytopenia

Tapasyapreeti Mukhopadhyay ${ }^{\#}$, Rupali Awale, Ratnaprabha Maji, Subhadra Sharma, A. K. Mukhopadhyay

Email ID for Correspondence: tapasya1810@gmail.com Department of Laboratory Medicine, All India Institute of Medical Sciences, New Delhi

Introduction and Background: Platelet parameters have been thought to be important in diagnosis of various diseases. They being mean platelet volume (MPV), pleteletcrit (PCT) and platelet distribution width (PDW), are readily available in the advanced hematological autoanalysers. This study was conducted to understand the relation of these indices in settings of thrombocytopenia. Our aim was to correlate these platelet parameters, in various disease groups presenting with thrombocytopenia. Patient/Material and Methods: This is a cross-sectional study conducted over a period of 2 months from April to May 2016. A total of 302 in-patients with various diagnosis and platelet count $<1 \mathrm{lakh} / \mathrm{mm}^{3}$, were included in the study. Control groups comprised of 28 healthy volunteers. $3 \mathrm{ml}$ of peripheral venous blood was received in $\mathrm{K}_{2}$ EDTA vial and analysed within 2-6 hours on Coulter Hematology LH750 auto analyser. Relation of platelet count with MPV, PCT, PDW and correlated with diagnosis. Results: Of the 302 blood specimens of thrombocytopenia analysed, 192/302 were males $(63.5 \%)$. Average age of patients was 37 years. Most causes were benign $(77.15 \%)$, rest being either malignant $(14.23 \%)$ or with unknown aetiology $(24.17 \%)$. MPV was found to be significantly higher in benign group than malignant $(\mathrm{p}<0.05)$. The cases were also grouped into pancytopenia (22.84\%), infections $(27.48 \%)$, chronic liver disease $(15.56 \%)$, haematological diseases $(12.91 \%)$, and others $(21.19 \%)$ for better understanding. Conclusions: Wherever facilities are available platelet parameters should be used as routine investigatory measures as they help in better evaluation in settings of thrombocytopenia.

Keywords Thrombocytopenia, Platelet indices, Mean platelet volume, Pleteletcrit, Platelet distribution width

\section{BHL 71}

Significance of Low HbA2 Levels and Correlation with Serum Iron Profile

\section{Pratyusha Gudapati ${ }^{\#}$, Chethan Manohar}

Email ID for Correspondence: prathyushagudapati@gmail.com Melaka Manipal Medical College

Introduction and Background: Haemoglobin $\mathrm{HbA}(\alpha 2 \beta 2)$ is the main haemoglobin component in postnatal life, accounting for $>96 \%$ of total $\mathrm{Hb}$, followed by $\mathrm{HbA} 2(\alpha 2 \delta 2)$ representing $2.5-3.5 \%$, and $\mathrm{HbF}$ ( $\alpha 2 \Upsilon 2$ ) constituting $<1 \%$. Decreased HbA2 levels can be detected in iron depletion, due to the preferential binding of $\beta$ to $\alpha$ chains, rather than $\delta$ chains, or to an inhibition of low iron levels on $\delta$ globin synthesis. Other causes of low HbA2 include $\alpha \beta$ thalassemia, $\mathrm{HbH}$ disease, $\mathrm{Hb}$ lepore, $\alpha$ chain variants etc. Material and Methods: Haemoglobin Electrophoresis reports were reviewed for a period of 2 years 6 months (Jan 2014 to June 2016) from archives at Hematology \& Clinical Pathology laboratory, Kasturba hospital, Manipal University, Manipal. Data from patient records with low $\mathrm{HbA} 2$ (defined as $<2.2$ ) was collected which included serum iron profile, RBC indices and peripheral smear findings and correlated. Haemoglobin electrophoresis is performed using Sebia capillary electrophoresis. Patients without serum iron profile were excluded from the study. Results: Total 127 patients were having HbA2 levels $<2.2$, out of which 26 cases were excluded in view of no serum iron profile. Out of remaining 101 cases, 58/101 had serum iron profile suggesting Iron deficiency anaemia with low iron, high TIBC and low ferritin. 4/101 patients had high $\mathrm{HbS}$ and high $\mathrm{Hbf}$ along with low $\mathrm{Hba} 2$ and diagnosed as sickle thalassemia. 2 cases of sickle cell trait and one case of sickle cell anaemia as well showed low Hba2. Also included in study were 2 cases of thalassemia intermedia, 3 cases of thalassemia major, 2 cases of $\alpha$ thalassemia minor and one case each of $\mathrm{HbH}$ disease and $\delta \beta$ thalassemia. 26/101 cases had normal iron profile and their RBC parameters were studied. Conclusions: Majority of cases with low $\mathrm{HbA} 2$ were having iron deficiency anaemia and other causes included various hemoglobinopathies with defective $\alpha$ and $\delta$ chain binding. 


\section{BHL 72}

\section{Is Marked Leucocytosis: Leukaemia or Leukaemoid Reaction?}

Chowdari Pavani", Rupali Awale, Ratnaprabha Maji, Subhadra Sharma, A. K. Mukhopadhyay

Email ID for Correspondence: drchowdaripavani@gmail.com Department of Laboratory Medicine, All India Institute of Medical Sciences, New Delhi

Introduction and Background: Leukaemoid reaction term was coined by Krumbhaar in 1926. It is defined as white blood cell count $>50 \times 10^{9} / \mathrm{L}$, with a cause outside the bonemarrow. Marked leukocytosis can be seen in both leukaemia as well as leukaemoid reaction. Several causes of leukaemoid reaction have been noted like infections (tuberculosis, sepsis), paraneoplastic syndromes (Carcinoma ovary, Carcinoma cervix), drug induced (G-CSF therapy, corticosteroids), miscellaneous (alcoholic hepatitis, multiple myeloma). It is due to increased cytokine production like Granulocyte colony stimulating factor, interleukin 3, interleukin 6 .

Case report:

\begin{tabular}{|c|c|c|c|}
\hline Case details & \multicolumn{2}{|l|}{ Case 1} & Case 2 \\
\hline Age/sex & \multicolumn{2}{|c|}{51 years/female } & 55 years/female \\
\hline Complaints of & \multicolumn{2}{|c|}{$\begin{array}{l}\text { Jaundice } \times 10 \text { days, } \\
\text { chest pain, shortness } \\
\text { of breath, } \\
\text { palpitations, } \\
\text { abdominal } \\
\text { pain } \times 4 \text { days }\end{array}$} & $\begin{array}{l}\text { High grade } \\
\quad \text { fever } \times 1 \text { week }\end{array}$ \\
\hline On examination & \multicolumn{2}{|l|}{ Splenomegaly } & $\begin{array}{l}\text { Abscess in right } \\
\text { nephrectomy site }\end{array}$ \\
\hline Hemoglobin $(\mathrm{gm} / \mathrm{dl})$ & \multicolumn{2}{|l|}{9.8} & 8 \\
\hline $\begin{array}{l}\text { Total leukocyte count } \\
\quad\left(\text { cells } / \mathrm{mm}^{3}\right)\end{array}$ & \multicolumn{2}{|l|}{123,000} & 150,000 \\
\hline \multirow{7}{*}{$\begin{array}{l}\text { Differential } \\
\text { Leukocyte count } \\
(\%)\end{array}$} & Neutrophils & 87 & 85 \\
\hline & Band forms & 10 & 13 \\
\hline & Lymphocytes & 2 & 2 \\
\hline & Monocytes & 1 & 0 \\
\hline & Eosinophils & 0 & 0 \\
\hline & Basophils & 0 & 0 \\
\hline & $\begin{array}{l}\text { Toxic } \\
\text { granules }\end{array}$ & Positive & Positive \\
\hline Platelets $\left(\mathrm{lakh} / \mathrm{mm}^{3}\right)$ & \multicolumn{2}{|l|}{184,000} & 100,000 \\
\hline LAP score & \multicolumn{2}{|c|}{360 (control-40) } & 283 (control-240) \\
\hline
\end{tabular}

Conclusion: In these two cases investigation revealed marked leukocytosis which could have mislead us to wrong diagnosis of leukaemia. Thus proper history, detailed clinical examination along with simple laboratory investigation like Leukocyte Alkaline Phosphatase score (LAP Score) can lead to proper diagnosis. On time initiation of treatment is necessary as leukaemoid reaction has increased morbidity and usually fatal outcome. Thus marked leukocytosis can masquerade acute leukaemia, so high index of suspicion is required for prompt diagnosis.

Keywords LAP Score, Leukemoid reaction, Leukemia
BHL 73

Relevance of Cell Population Data in Detecting Viral and Bacterial Infections-One Step Ahead!

Parul Arora $^{1 \#}$, Praveen Kumar ${ }^{1}$, Debasis Jash ${ }^{1}$, Raghavendra Lingaiah ${ }^{1}$, Arti Kapil ${ }^{2}$, Subhadra Sharma ${ }^{1}$, Asok K. Mukhopadhyay ${ }^{1}$

Email ID for Correspondence: parul6588@yahoo.co.in

${ }^{1}$ Department of Laboratory Medicine and ${ }^{2}$ Department of

Microbiology, All India Institute of Medical Sciences, New Delhi

Introduction and Background: Activated lymphocytes and neutrophils undergo morphologic alterations in infective conditions. Automated hematology analyzers can measure these morphological changes through the cell population data (CPD) such as volume, conductivity and scatter (VCS). We conducted this study to ascertain the usefulness of CPD as a better and rapid predictive marker of infections. Patient/Material and Methods: This observational study was conducted at a tertiary care hospital between January to July 2016. Complete hemogram along with cell population data obtained from LH750 (Beckman coulter, Fuellerton, CA) from 80 blood culture positive cases of bacterial sepsis and 156 cases with viral infections including hepatitis A-E, HIV were analyzed retrospectively and compared to 94 healthy subjects. Statistical analysis of data was done using SPSS v 16.0.

Results: Cell population data from cases of viral infections when compared to controls showed significant increase $(p \leq 0.05)$ in mean conductivity of neutrophils (MNC, $144.95 \pm 6.47$ vs. $136.14 \pm 11.99$ ), lymphocytes (MLC, $114.26 \pm 6.17$ vs. $106.15 \pm 4.49$ ) and monocytes (MMC, $120.03 \pm 13.81$ vs. $113.62 \pm 4.51)$. Mean neutrophil volume (MNV, $161.53 \pm 15.48$ vs. $149.10 \pm 5.74)$ and monocyte volume (MMV, $178.15 \pm 12.69$ vs. $168.53 \pm 5.48)$ was significantly increased in sepsis $(\mathrm{p}<0.05)$ while mean neutrophil scatter (MNS, $139.78 \pm 7.27$ vs. $145.37 \pm 4.64$ ) was significantly decreased as compared to healthy controls $(p \leq 0.05)$. Conclusions: CPD like volume, conductivity and scatter can be used as early markers for the prediction of viral and bacterial infections. Including these data in the routine flagging system of the analyzers can help in efficient screening and improvement in the laboratory work flow.

\section{BHL 74}

Deciphering the Molecular Mechanism of 3.7 Alpha Triplication Through Sequencing Analysis

Rudra Ray ${ }^{\#}$, Ranjini Chowdhury, Sujana Biswas, Soumita Choudhuri, Arnab Chattopadyay, Prof. Maitreyee Bhattacharya

Email ID for Correspondence: rudra.ray@gmail.com Institute of Hematology and Transfusion Medicine (IHTM), Calcutta Medical College

Introduction and Background: Alpha globin gene triplication results from misalignment and unequal crossover between the homologous X-, Y-, and Z-box segments of the $\alpha$-globin gene cluster. Generally, two types of triplicated alleles can be generated from an unequal crossover, $\alpha \alpha \alpha^{\text {anti3.7 }}$ and $\alpha \alpha \alpha^{\text {anti4.2. Triplication causes an }}$ extra copy of Alpha globin chain, that alters the balance of the Alpha and Non Alpha globin ratio in RBC. We have deciphered the DNA sequences of the entire alpha 3.7 triplicated gene product and analyzed its sequence homology to understand the behaviour of unequal crossover causing the triplication. Patient/Material and Methods: Patients with $\beta$-thalassemia trait, but behaving like beta thalassemia 
intermedia, were suspected for alpha globin gene triplication and screened by PCR analysis. The amplified triplicated gene products were then purified and subjected to DNA sequencing. Primer walking was done in order to sequence the entire triplicated gene of around 4000 bp. Results: The sequence of the entire 3.7 triplicated gene was searched for sequence homology with-(i) sequence of globin gene cluster consisting of normal alpha globin genes; (ii) sequence of globin gene cluster consisting of 3.7 deletions in alpha genes. The homology analysis demonstrates a striking similarity between the gene sequences of 3.7 deleted product and 3.7 triplicated product. The sequencing analysis also showed that the triplicated gene product is a fusion of alpha 1 and alpha 2 genes. Conclusions: The alpha 3.7 triplicated gene, a fusion of alpha 1 and alpha 2 gene, resides with an insertion of around $4 \mathrm{~kb}$ nucleotides in between the Alpha 1 and Alpha 2 genes of globin gene cluster. Moreover, the sequence identity comparison between the triplicated gene product with the $\mathrm{X} Y$ and $\mathrm{Z}$ boxes of the normal alpha globin cluster demonstrates the break point or initiation point from where the unequal crossing over starts.

\section{BHL 75}

Utility of the ISTH-SSC Bleeding Assessment Tool in Evaluating Inherited Bleeding Disorders in a Tertiary Care Hospital in South India

\section{P. L. Ambika\#, Debdatta Basu, Rakhee Kar}

Email ID for Correspondence: ambubotla@gmail.com Jawaharlal Institute of Postgraduate Medical Education \& Research (JIPMER), Puducherry

Introduction and Background: The International Society of Thrombosis \& Hemostasis-Bleeding Assessment Tool (ISTH-BAT) is a tool developed to accurately record bleeding symptoms in all hemorrhagic disorders. Many such tools have been developed; some being specific to certain bleeding disorders or for a distinct population such as children or women with menorrhagia. Material and Methods: This was a cross-sectional study over one and half years from March 2015 and Aug 2016 wherein 154 consecutive patients suspected to have inherited bleeding disorders referred to our coagulation lab and fifty healthy volunteers were enrolled and were administered ISTH-BAT. BAT score and screening tests were used to distinguish between primary and secondary hemostatic defects. Confirmatory tests were done in all secondary hemostatic defects and some tests for primary hemostatic defects are in progress. Results: The median age of the patients was 12 years (1 month-69 years) and included 92 males and 62 females. Ten cases were excluded which turned out to be acquired coagulopathies. Secondary hemostatic defects were seen in 68 patients which included Haemophilia A (55), Haemophilia B (5), Factor I deficiency (3), Prothrombin deficiency (2), Factor X deficiency (1) and Factor XIII deficiency (2). Primary hemostatic defects were suspected in 76 patients comprising platelet function disorders (1-BSS and 5-GT), vWD (9) and rest had normal screening coagulogram (61). The average BAT score of primary hemostatic defects, secondary hemostatic defects and patients with normal screening coagulogram are 7.38, 6.92 and 4.26 respectively compared to average of healthy volunteers (1.56). The patients with normal screening coagulogram predominantly had slight low normal levels of vWF:Ag indicating mild VWD for which further tests are ongoing. Conclusions: The pattern of bleeding and severity along with BAT score helps in discriminating between primary and secondary hemostatic defects and its use is recommended for systematic approach for the diagnosis of Inherited bleeding disorders.

\section{BHL 76}

Prevalence of HBH Disease Caused by Point Mutations in Indian Population

\section{A. Arun Kumar ${ }^{\#}$, K. Neelakandan, S. Easwari, R. V. Shaji, S. Eunice}

Email ID for Correspondence: arun000_kumar@yahoo.com Christian Medical College (CMC), Vellore

Introduction and Background: Haemoglobin $\mathrm{H}(\mathrm{HbH})$ disease is mostly caused by deletions of three $\alpha$-globin genes. Rarely, homozygosity of point mutations in $\alpha$ globin genes or compound heterozygosity for point mutations and deletions of two $\alpha$ globin genes can also cause $\mathrm{HbH}$ disease. The current study aims at addressing the clinical and genotypic heterogeneity of $\mathrm{HbH}$ disease in Indian population and to highlight the difference in the phenotypes in the patients with $\mathrm{HbH}$ disease caused by deletions and point mutations. Patient/Material and Methods: The study included 100 patients who were evaluated for their clinical phenotypes and haematological parameters at the Department of Hematology, CMC, Vellore and suspected to have alpha thalassaemia. Characterization of the deletions and the assay to calculate the copy number of $\alpha$ globin genes were done by multiplex gap PCR and gene dosage multiplex fluorescent PCR, respectively. Point mutations were screened by sequencing $\alpha 1$ and $\alpha 2$ globin genes. Results: Deletional $\mathrm{HbH}$ and point mutations causing $\mathrm{HbH}$ disease phenotype were identified in $28 \%$ and $22 \%$ of the patients, respectively. The genotypes that cause $\mathrm{HbH}$ disease are tabulated below.

The genotypes associated with $\mathrm{HbH}$ disease identified in Indian population

\begin{tabular}{|c|c|}
\hline Genotype & Number $(\%)$ \\
\hline Deletional $\mathrm{HbH}$ disease & $28(28 \%)$ \\
\hline$-\alpha^{3.7} /-\mathrm{SA}$ & $16(16 \%)$ \\
\hline$-\alpha^{4.2} /-\mathrm{SA}$ & $3(3 \%)$ \\
\hline$-\alpha^{3.7} /-\mathrm{SEA}$ & $7(7 \%)$ \\
\hline$-\alpha^{3.7} /-20.5$ & $2(2 \%)$ \\
\hline Non-deletional $\mathrm{HbH}$ disease $\left(\alpha \alpha^{\mathrm{T}} / \alpha \alpha^{\mathrm{T}}\right)$ & $22(22 \%)$ \\
\hline Homozygous Poly A (-AA) deletion $(\alpha 2)$ & $11(11 \%)$ \\
\hline Homozygous COD $104(\mathrm{G}>\mathrm{A})(\alpha 2)$ & $4(4 \%)$ \\
\hline Homozygous COD $122(\mathrm{~A}>\mathrm{T})(\alpha 2)$ & $1(1 \%)$ \\
\hline Homozygous CODON $130(\mathrm{G}-\mathrm{C})(\alpha 2)$ & $1(1 \%)$ \\
\hline $\begin{array}{l}\text { Compound heterozygous for Poly A (-AA) deletion } \\
\text { and a second mutation. [Codon } 142 \mathrm{~A}>\mathrm{C} \text {, Codon } \\
104 \mathrm{~T}>\mathrm{C} \text {, Codon } 22 \mathrm{C}>\mathrm{T} \text {, CODON } 122 \mathrm{~A}>\mathrm{T} \text { and } \\
\text { Codon } 65-75(-33 \mathrm{bp}) \text { del] }\end{array}$ & $5(5 \%)$ \\
\hline
\end{tabular}

Patients with point mutations were found to have more severe phenotype than those with deletions $(\mathrm{n}=28)$. They had lower $\mathrm{Hb}$ levels (mean: $7.1 \pm 1.55 \mathrm{gm} / \mathrm{dL}$ ) and RBC counts (mean: $4.06 \pm 0.75 \mathrm{million} / \mathrm{cu} \mathrm{mm}$ ), higher retic counts (mean: $7.4 \pm 3.57 \%$ ), and $37.5 \%$ of the patients were transfusion dependant. In contrast, the patients with deletions of three $\alpha$ globin genes had higher $\mathrm{Hb}$ (mean: $8.33 \pm 1.76 \mathrm{gm} / \mathrm{dL}$ ) and RBC counts (mean: $5.03 \pm 0.78 \mathrm{million} / \mathrm{cu} \mathrm{mm}$ ) and lower retic counts (mean: 
$3.57 \pm 1.46 \%$ ) and none of the patients were transfusion dependent. Conclusions: This is the first study analysing the clinical details of a large number of $\mathrm{HbH}$ patients with point mutations and comparing their phenotypes with the patients with deletions. The study also highlights that the point mutations causing $\mathrm{HbH}$ disease are not rare in the Indian population and it is important to screen for these mutations to delineate the molecular basis of the disease and for the clinical management of the patients with alpha thalassaemia.

\section{BHL 77}

Pure Red Cell Aplasia with Secondary Hemosiderosis: An Autopsy Case Report

\section{R. Keskar", T. Waghmare, P. Vaideeswar, D. Prabhat}

Email ID for Correspondence: reshmakeskar@gmail.com Seth G.S. Medical College and K.E.M. Hospital Parel, Mumbai

Introduction: Pure Red Cell Aplasia (PRCA) is an uncommon disorder characterised by severe anaemia, a reticulocyte count of less than $1 \%$, and the presence of less than $0.5 \%$ mature erythroblasts in the bone marrow. In 1922, Kaznelson recognized that this condition was a different entity from aplastic anaemia, which presents with pancytopenia. Case Report: A 27 year old male patient with a past history of dyspnoea on exertion since 2 yrs, dependent on blood transfusion for every 10 days since then. Patient was transferred to our hospital in poor general condition with same complains and fever with chills for 6 days. Within 1 hour of ward stay patient expired. In 2015 bone marrow aspiration done and was suggestive of dilute marrow with scanty cellularity showing myeloid preponderance and relative suppression of the erythroid series, based on which he was clinically labelled as PRCA. Hb was in the range of 3.8 to $7 \mathrm{gm} \%$. TLC and Platelets were in the normal range. Serum ferritin level markedly increased i.e. $7061 \mathrm{ng} / \mathrm{ml}$. PNH, MDS [Monosomy 5q, Monosomy 7q, Trisomy 8, Del (20q) and JAK2] and Parvovirus B19 DNA work up of the patient was negative. At Autopsy, lung revealed miliary tuberculosis. Liver, spleen markedly enlarged and showed brownish discolouration with hemosiderin deposition. Heart and pancreas also showed brownish discolouration. On histology tuberculous granulomas were seen in liver, spleen, kidney, bone marrow. Discussion: Although PRCA is a well defined clinical disorder, its clinical manifestation is heterogenous and its underlying pathogenesis varies. PRCA can evolve into aplastic anaemia, acute myelogenous leukemia which has high morbidity and mortality rates.

\section{BHL 78}

Antiphospholipid Antibody Positivity in Acute Immune Thrombocytopenia Patient: A Case Report

\section{Divya", S. Pahuja, S. Sharma, J. Chandra}

Email ID for Correspondence: its4div@yahoo.com

Department of Pathology, Lady Hardinge Medical College and Kalawati Saran Children's Hospital, New Delhi

Introduction: Antiphospholipid antibodies (APLA) are known to occur in cases of Immune thrombocytopenia (ITP). APLA are primarily associated with thrombosis. Few studies suggest that presence of APLA might increase the risk of thrombosis even in ITP patients. Case Report: We are presenting a case of 8 year old female child who came with petechiae and ecchymosis for 3 days. Patient gave history of cough and fever one week back which was relieved on medication. Examination revealed pinpoint rashes over face, bilateral axillae, trunk and both legs. There was no hepatosplenomegaly or lymphadenopathy. CBC findings revealed thrombocytopenia $(31,000 /$ $\mu \mathrm{l})$, normal haemoglobin $(11.6 \mathrm{~g} / \mathrm{dl})$ and WBC count $(7800 / \mu \mathrm{l})$. Bone marrow aspiration revealed normocellular particles showing normoblastic erythropoiesis. There was megakaryocytic hyperplasia with increase in number of both mature and immature forms. Other causes of thrombocytopenia like viral infections and SLE were ruled out. Thus a diagnosis of ITP was suggested. Prothrombin time was normal. However activated partial thromboplastin time was mildly increased (test-45s, control-38s)). Plasma samples from patient were analysed for Antiphospholipid antibodies (APLA). Lupus Anticoagulant (LAC) was tested to be positive. However Anti Cardiolipin antibody assay was negative. Patient was successfully treated with platelet infusions and IV immunoglobulin following which platelet counts returned back. At third month follow up, patient was asymptomatic with normal platelet count, negative LAC and negative anticardiolipin assay. During follow up, there was no history or clinical evidence of thrombosis. Discussion: APLA antibodies are found to be positive in ITP patients, with a frequency varying from 14 to $75 \%$. The role of APLA in ITP is controversial. However, many studies have suggested correlation of APLA with thrombosis among ITP patients. This case had a presentation of acute ITP with no thrombotic episodes, but showed LAC positivity.

\section{BHL 79}

\section{Multiplex Ligation Probe Amplification Versus Multiplex PCR} in the Diagnosis of Alpha Thalassemia

\section{Sumita Saluja", Neelakandhaan, Eshwari, E. Sindhvi, R. V. Shaji}

Email ID for Correspondence: drssaluja@gmail.com

Christian Medical College, Vellore and Safdarjung Hospitals, New Delhi

Introduction and Background: In alpha thalassemia it is always a challenge to diagnose copy number variations (deletions and duplications) of alpha genes. The conventional multiplex PCR has several limitations, like assessment of limited deletions, same size bands cannot be discriminated and is difficult for relative quantification of target sequences as the efficiency of primer pairs varies. Besides, multiplexing is not reliable for the diagnosis of heterozygous deletions. The detection of increased copy number is also difficult by PCR. MLPA is a multiplex PCR assay that utilizes up to 40 probes, each specific for a different DNA sequence to evaluate the relative copy number of each sequence. Each probe is composed of two halfprobes ( $5^{\prime}$ and $3^{\prime}$ half-probes), consisting of a target-specific sequence and a universal primer sequence allowing the simultaneous amplification of all probes. Additionally one or both half-probes contain a stuffer sequence permitting differentiation of the amplification products arising from the probes of same length. We compared the technique MLPA with multiplex PCR to identify copy number variations in alpha thalassemia. Patient/Material and Methods: MLPA was performed on twenty DNA samples on whom multiplex PCR was already attempted several times to categorise the mutation in alpha gene. MLPA was performed as per the manufacturer's protocol using HBA probe mix. The data was analysed using the coffalyser software. Results: Of the twenty DNA analysed, three were compound heterozygous for alpha thal deletions, $-20.5 /-\alpha 3.7$ (D) deletion, -SA/$\alpha 4.2,-\alpha 3.7 /-\alpha 4.2$ each. Homozygous $3.7 \mathrm{~kb}$ deletion was diagnosed in two cases and homozygous $4.2 \mathrm{~kb}$ in one case. Heterozygous $3.7 \mathrm{~kb}$ deletion and $4.2 \mathrm{~kb}$ deletion were identified in 3 cases each. Heterozygous SEA deletion was observed in one case. Heterozygous alpha duplication and triplications were identified in 3 and 4 cases respectively. Conclusions: MLPA is a simple, quick, reliable, non laborious technique for detecting known and unknown heterozygous and homozygous deletions and multiplication of alpha genes in 
comparison to multiplex PCR with good quality DNA as the most important requirement.

\section{BHL 80}

A Case Series Study on Variants of Haemoglobinopathies on Southern Part of Orissa Abstract for Poster Presentation

\section{Laxmi Triya $^{\#}$, S. K. Behera, D. P. Mishra, M. K. Patro}

Email ID for Correspondence: laxmidgp_2003@yahoo.co.in 2nd year, Department of Pathology, MKCG Medical College, Berhampur, Odisha

Background: Hemoglobinopathies are common genetic disorders of hemoglobin. Identification of these disorders is immensely important epidemiologically and can be prevented by population screening. Nearly $1200 \mathrm{Hb}$ variants have been isolated and unfortunately most of them are clinically asymptomatic. Aims and Objectives:

1. To determine the pattern of spectrum of hemoglobinopathies in the state of Orissa.

2. To report the cases of rare variants of hemoglobin found in the population of Southern Districts of Orissa and to observe the hematological parameters of the cases, having rare variants of hemoglobin.

Materials and Methods: A total of 1021 blood samples of microcytic hypochromic anaemia cases were analysed referred from different peripheral hospitals of southern part of Orissa state for a period of two years 6 months (2014-2016). 453 Cases came out with various hemoglobinopathies. About $2-3 \mathrm{ml}$ of intravenous blood samples were collected after obtaining informed consent from each individual. Haematological indices were measured using automated cell counter (SYSMEX-Xt 2000i) and HPLC (BIO-RAD VARIANT). Background data of each individual were recorded like age, sex, caste, place of origin and consanguinity etc. Haemoglobin electrophoresis were carried out on CAM in Tris-EDTA-borate buffer at PH-8.9 and sickling test were carried out simultaneously. The value more than $3.5 \%$ of A2 fraction of haemoglobin was taken as cut off point for beta-thalassemia trait and more than $10 \%$ as $\mathrm{HbE}$. Hb electrophoresis in acidic medium PH-6.2 was also carried out to confirm the presence of $\mathrm{HbD}$ or $\mathrm{HbE}$ band. Fetal haemoglobin estimation and hence confirmation were elicited by acid elusion test. Family studies were carried out for confirming the disease. Criteria-1: Inclusive-peripheral smear showing:

1. Blood samples with microcytic hypochromic anaemia.

2. normocytic normochromic anaemia with target cells.

3. Mentzer index $<13$.

Criteria-2: Exclusive-recent cases of blood transfusion are excluded. Results and analysis: Most common haemoglobinopathies observed out of 453 cases were sickle cell trait $(48 \%)$, sickle cell disease $(40 \%)$, sickle cell-b-thalassemia $(2.2 \%), \beta$-thalassemia trait $(4.8 \%)$, $\beta$-thalassemia major (3.3\%), HbE trait $(0.6 \%)$, HbE disease $(0.2 \%)$, alpha-thalessemia-sickle hetrerozygous $(0.2 \%)$. The percentage of paediatric population having sickle cell disease is more whereas sickle cell trait came to be higher for adult age group. Also paediatric age group outnumbered adult population for thalassemia major and trait. HbE homozygosity and heterozygousity is more evident in adult population. Conclusions: The heterogenous population is harbouring almost all major haemoglobinopathies in all the age groups belonging to coastal and south-western regions of Orissa. From this it is also concluded that some rare variants are found in the population. Hence screening procedure is needed for further detection of rare variants of haemoglobin among the population and genetic study of the cases are necessary for further evaluation.
Keywords Rare variants of haemoglobin, HPLC, Electrophoresis

\section{BHL 81}

Utility of VCS Parameters for Early Detection of Neonatal Sepsis

\author{
Tushar Tyagi", Anjali Kelkar, Preeti Doshi, N. S. Mani
}

Email ID for Correspondence: drtyagi.tushar@gmail.com Bharati Vidyapeeeth Medical College and Hospital, Pune

Introduction and Background: Neonatal sepsis comprises a major problem in India with septicemia and pneumonia accounting for $52 \%$ of deaths. Early detection of neonatal sepsis is challenging as clinical signs are subjective, and the gold standard, a positive blood culture, requires 1-3 days. VCS data are obtained simultaneously with the differential count without additional cost or sample requirement. The present study was undertaken in a tertiary-care teaching hospital; with the aim of evaluating VCS data and other laboratory parameters for diagnosis of neonatal sepsis. Patient/Material and Methods: Peripheral blood samples of 820 neonates were analysed over period of 18 months for CBC with band cells, eosinophils \%, CRP and culture. VCS parameters were obtained simultaneously. Other clinical and radiological data were collected for analysis. Inclusion criteria: Neonates aged 0-28 days admitted to the NICU in whom sepsis is suspected. Controls: Gestation-matched healthy controls with no clinical or laboratory suspicion of infection. The study population was divided into following groups: Proven sepsis + Clinical sepsis, No sepsis. A cut off value of 0.3615 based on CRP, MNV and VDW was taken to indicate presence or absence of sepsis; based on the previously published work by Celik et al. Results: Total number of 820 samples of neonates admitted in neonatal ICU of Bharati Hospital were analysed. All the three groups were analysed with respect to CBC values, $\mathrm{PBS}$, VCS parameters, CRP values, cultures. Sensitivity $76 \%$. Specificity $88 \%$. Positive predictive value: $77 \%$. Negative predictive value: $87 \%$. Conclusions: We analysed the utility of VCS parameters along with CRP for early diagnosis of neonatal sepsis. Our results indicate that the factor based on MNV, VDW and CRP with cut off of 0.3615 is a useful indicator of neonatal sepsis without incurring additional cost, with high specificity $(88 \%)$ and high negative predictive value $(87 \%)$.

\section{BHL 82}

Phenotypic Heterogeneity in Homozygous Sickle Cell Disease: Role of Co-inherited Alpha Thalassaemia

\section{S. Gopinath ${ }^{\#}$, A. Arun Kumar, K. Neelakandan, S. Easwari, R. V. Shaji, S. Eunice}

Email ID for Correspondence: srinivasagopi55@gmail.com Christian Medical College, Vellore

Introduction and Background: Homozygous $\mathrm{HbS}$ (SCD) shows significant difference in the phenotypes and the previous studies have shown that this is mostly due to co-inherited genetic factors that affect the levels of $\alpha$ and $\gamma$ globins. Differentiating SCD with sickle- $\beta$ thalassemia based on HPLC values is difficult in Indian patients due to the inherently high $\mathrm{HbF}$ levels. Although mean corpuscular volume (MCV) is a useful differentiation parameter, the patients with SCD can also present with microcytosis due to iron deficiency and coexisting $\alpha$ thalassaemia. This study aims to assess the role of $\alpha$ thalassaemia in determining the phenotype of patients with SCD. Patient/Material and Methods: Haemoglobin analysis was done using VARIANT II HPLC and $\beta$-globin gene mutations were detected by reverse dot blot or direct DNA sequencing. Characterization of the $\alpha$ 
globin gene deletions and their copy numbers were done by multiplex deletion PCR and gene dosage multiplex fluorescent PCR, respectively. Results: A total of 195 patients with homozygous HbS (SCD) diagnosed at the Department of Haematology, CMC, Vellore were included in the study. Their haematological parameters are tabulated below. The patients were categorised into two groups: $\mathrm{MCV}<75 \mathrm{fL}$ and MCV $>75 \mathrm{fL}$. In the patients with low MCV, $69 \%$ of them had co-existing $\alpha$ thalassemia, and these patients had a milder phenotype with higher hemoglobin levels and RBC counts compared to the patients without $\alpha$ thalassaemia (who had the most severe phenotype) and those with $\mathrm{MCV}>75 \mathrm{fL}$

Haematological parameters of SCD patients and their $\alpha$ globin genotypes

\begin{tabular}{lccc}
\hline Parameters & $\begin{array}{l}\text { MCV }>75 \mathrm{fL} \\
(\mathrm{n}=163)\end{array}$ & $\begin{array}{l}\text { MCV }<75 \mathrm{fL} \\
\text { with } \alpha \text { gene } \\
\text { deletion }(\mathrm{n}=22) \\
\text { Mean } \pm \mathrm{SD}\end{array}$ & $\begin{array}{l}\text { MCV }<75 \mathrm{fL} \\
\text { without } \alpha \text { gene } \\
\text { deletion }(\mathrm{n}=10) \\
\text { Mean } \pm \mathrm{SD}\end{array}$ \\
\hline $\mathrm{HbS}(\%)$ & $71.937 \pm 9.314$ & $75.842 \pm 5.914$ & $69.925 \pm 7.88$ \\
$\mathrm{HbF}(\%)$ & $20.349 \pm 7.148$ & $16.971 \pm 5.587$ & $23.92 \pm 7.618$ \\
$\mathrm{HbA} 2(\%)$ & $2.985 \pm 1.057$ & $3.135 \pm 0.997$ & $2.483 \pm 0.633$ \\
$\mathrm{Hb}(\mathrm{gm} / \mathrm{dL})$ & $9.78 \pm 1.824$ & $9.0 \pm 1.89$ & $5.77 \pm 1.375$ \\
$\mathrm{MCV}(\mathrm{fL})$ & $87.78 \pm 11.32$ & $70.0 \pm 4.86$ & $70.83 \pm 3.058$ \\
$\begin{array}{c}\mathrm{RBC} \text { count } \\
(\mathrm{millions} /\end{array}$ & $3.251 \pm 0.798$ & $4.14 \pm 0.606$ & $4.088 \pm 0.573$ \\
$\begin{array}{c}\text { cu mm }) \\
\text { Retic count } \\
(\%)\end{array}$ & $7.37 \pm 4.853$ & $4.05 \pm 2.55$ & $2.555 \pm 2.815$ \\
& & &
\end{tabular}

Conclusions: SCD can present with variable phenotypes. Low MCV observed in a large number of patients is due to co-inherited $\alpha$ thalassaemia and DNA analysis of $\alpha$ thalassaemia is helpful in the differential diagnosis of SCD and HbS- $\beta$ thalassaemia.

\section{BHL 83}

\section{Gelatinous Transformation of Bone Marrow in HIV Positive Patient}

\section{Gyanendra Singh", Sudeep Khera, D. Vedant, A. Purohit, S. Bhattacharya}

Email ID for Correspondence: gyanendra002@gmail.com All India Institute of Medical Science, Jodhpur, Rajasthan

Gelatinous transformation of bone marrow is a rare disease characterized by fat cell atrophy, focal loss of haematopoietic cells and deposition of extracellular gelatinous substance. We report a case of four-year old male child who presented with twenty-day history of fever and significant weight loss. On examination, he did not have lymphadenopathy or organomegaly. His routine haematological and biochemical parameters were within normal limits and no cause of fever could be identified even after complete preliminary workup which led to examination of bone marrow. Bone marrow aspirate smears revealed megaloblastic erythroid maturation whereas bone marrow biopsy revealed presence of focal collection of lymphocytes and histiocytes along with presence of many foci of fluid like gelatinous and pink hyaline material suggestive of gelatinous transformation of marrow. Such morphological picture led to investigations of HIV which were positive. The aim to present this case is to attract attention towards importance of morphology.

\section{BHL 84}

\section{Haemophagocytic Lymphohistiocytosis-A Case Report}

\section{Indu Choudhary ${ }^{\#}$, Kishore Khatri, Anand Raj Kalla}

Email ID for Correspondence: docindudbest@gmail.com Dr. S.N. Medical College, Jodhpur

Haemophagocytic lymphohistiocytosis (HLH) is a life-threatening condition of severe hyperinflammation caused by the uncontrolled proliferation of activated lymphocytes and histiocytes secreting high amounts of inflammatory cytokines. The hallmark of disease is low or absent NK cell and CD8+ T lymphocytes cytotoxicity. Two forms of syndrome-Familial hemophagocytic Lymphohistiocytosis (FHL) of infants is a rare autosomal recessive disorder demonstrating mutations in perforin gene in 30-50\% cases and other reactive hemophagocytic syndrome (RHS) at any age. Epstein-Barr virus, the pathogen that most commonly triggers Infection associated HPS (IA-HPS). HLH is fatal if left untreated. We report a case of HLH in an 8-year old male child who presented with high grade fever $\left(>103{ }^{\circ} \mathrm{C}\right)$, pancytopenia, hepatosplenomegaly along with skin rashes over scalp and face. Biochemical investigations showed very high serum ferritin level $(2830 \mu \mathrm{g} / \mathrm{l})$, hypertriglyceridaemia and hypofibrinogenamia. Examination of bone marrow aspirate and biopsy revealed all haematopoietic components with prominence of histiocytes and evidence of haemophagocytosis.

\section{BHL 85}

Utility of Automated RBC Parameters Generated by Advia 120 Hematology Analyzer as a Screening Tool in Hereditary Spherocytosis

\section{Sarah Arnestina ${ }^{1 \#}$, Naveen Kakkar ${ }^{1}$, M. Joseph John ${ }^{2}$}

Email ID for Correspondence: arnestina@gmail.com Departments of ${ }^{1}$ Pathology and ${ }^{2}$ Clinical Hematology, Hemato-oncology and Bone Marrow (Stem Cell) Transplantation, Christian Medical College and Hospital, Ludhiana, Punjab

Introduction and Background: Hereditary spherocytosis (HS) is the most common membrane disorder that causes hemolytic anemia. Its diagnosis is based on the presence of spherocytes in the peripheral blood smear and increased red cell osmotic fragility. Information from newer red cell automated parameters from some hematology analyzers can be used as a screening tool, thus aiding in the diagnosis of spherocytosis. Patient/Material and Methods: This study was done in 15 patients, clinically suspected to have Hereditary Spherocytosis. Blood samples collected in dipotassium EDTA were run on Advia 120 fully automated five part analyzer. All routine complete blood count data were recorded along with percentage of hyperchromic cells, flagging for hyperchromic RBCs, hemoglobin concentration variance (HC Var) and study of the RBC volume/ hemoglobin cytograms. Results: The mean hemoglobin in 15 patients with HS was $9.7 \pm 2.2 \mathrm{gm} / \mathrm{dl}$ with $\mathrm{MCHC}$ of $35 \pm 2.6 \mathrm{gm} / \mathrm{dl}$. All patients showed an increase in the percentage of hyperchromic cells ranging from 6.6-36.8 \% (mean: $16 \pm 9.5 \%$ ). Hyperchromic RBC flag was seen in all 15 patients while the HC Var flag was seen in 14/15 patients. Red cell cytogram in all patients showed a distinctive hyperchromic population. Osmotic fragility test was positive in all patients. Conclusions: Newer automated RBC parameters along with 
red cell cytograms generated by Advia 120 analyzer can be used as a rapid and cost effective screening tool in patients with suspected Hereditary Spherocytosis and can be a useful adjunct in the diagnosis.

\section{BHL 86}

\section{Spurious White Blood Cell (WBC) Count Due to Fibrin Clumps}

\section{Sunil, Neeti Arora ${ }^{1 \#}$, Naveen Kakkar', Amrith Mathew ${ }^{2}$, M. Joseph John ${ }^{2}$}

Email ID for Correspondence: neeti416@gmail.com Departments of ${ }^{1}$ Pathology and ${ }^{2}$ Clinical Hematology, Hemato-oncology and Bone marrow (Stem Cell) Transplantation, Christian Medical College and Hospital, Ludhiana, Punjab

Introduction and Background: Use of automated hematology analyzers for $\mathrm{CBC}$ reporting has improved the reliability of these results while reducing the turn around time significantly. It is important for operators of the analyzers and the end users to be aware of spurious results that may lead to inappropriate treatment in patients. Spurious WBC counts may be seen in laboratory practice and may cause diagnostic and therapeutic dilemma. Patient/Material and Methods: This study includes nine patients with varied clinical diagnoses whose blood samples were drawn in di-potassium EDTA for complete blood count (CBC). $\mathrm{CBC}$ was analysed using 3-part automated hematology analyser, Mindray BC 3000 plus. All automated haematological parameters were recorded. Leishman stained peripheral blood smears were also studied. Results: In all 9 patients, the WBC count reported initially did not correlate with the clinically expected value. Hence repeat samples were taken which showed lower WBC counts. Fibrin clumps were seen in the peripheral blood smear of all patients. Hemoglobin and platelet counts were not affected. All patients showed atypical WBC histogram pattern with a straight ascending limb as compared to a rising sloping pattern seen in normal WBC histograms. Blood smears showed fibrin clumps at the tail end of the smears. Conclusions: Presence of fibrin clumps in blood samples is an uncommon cause of spurious WBC count and may adversely affect diagnostic and therapeutic decisions. Careful evaluation of WBC histograms and examination of the peripheral blood smear may help in identification of this artefact.

Keywords Fibrin clumps, Hematology analyser, Spurious, WBC histogram

\section{BHL 87}

Phenotypic Expression of HbO Indonesia in Two Indian Families and Its Interaction with Sickle Hemoglobin (HbS)

Sidhwa Kainaz ${ }^{1 \#, ~ D a s ~ G u p t a ~ A m a r ', ~ N a d k a r n i ~ A n i t a ~}{ }^{2}$, Mehta Pallavi $^{2}$, Goriwale Manju ${ }^{2}$, Ramani Manisha ${ }^{1}$, Chaudhary Pradnya ${ }^{1}$, Mehrotra Vishal ${ }^{1}$, Colah Roshan ${ }^{2}$

Email ID for Correspondence: kainaz@srl.in

${ }^{1}$ Section of Hematology, SRL Limited, Mumbai, India; ${ }^{2}$ National Institute of Immunohematology, Mumbai, India

Background: Alpha globin chain variants are clinically significant since they directly influence the structure and function of the hemoglobin $(\mathrm{Hb})$ molecules they constitute, either in combination with normal beta globin chains or with variant beta chains, thereby altering the morbidity and mortality associated with the resultant hemoglobinopathies. We describe here two unrelated families from Madhya Pradesh who had a non-deletional alpha chain variant, $\mathrm{HbO}$ Indonesia [CD $116 \mathrm{G} \rightarrow \mathrm{A}]$. Members of one of the two families also had coinheritance of $\mathrm{HbS}$. Aims: To study the phenotype of $\mathrm{HbO}$
Indonesia and its interaction with $\mathrm{HbS}$. Material and Methods: $\mathrm{Hb}$ electrophoresis, High performance liquid chromatography (HPLC), Covalent reverse dot blot hybridization, Amplification refractory mutation system, Multiplex PCR and direct gene sequencing were used to identify and characterize the variant Hbs. Results: The abnormal hemoglobin moved with $\mathrm{HbS}$ in $\mathrm{Hb}$ electrophoresis at alkaline $\mathrm{pH}$ and gave an abnormal peak in HPLC with a retention time of 4.86-4.89 minutes. In two members of the family with coinheritance of $\mathrm{HbS}$ it produced small additional abnormal $\mathrm{Hb}$ peaks $(4.6 \%$ in heterozygous and $11.9 \%$ in homozygous member) in HPLC with a longer retention time (5.15-5.17 minutes) possibly resulting from a combination of $\mathrm{HbO}$ Indonesia alpha chain with $\mathrm{HbS}$ beta chain. Conclusions: It appears that depending on the zygosity of $\mathrm{HbS}, \mathrm{HbO}$ Indonesia would subtract a variable amount of $\mathrm{HbS}$ beta chain from the total pool thereby potentially reducing the clinical severity of $\mathrm{HbS}$ disease. HbO Indonesia per se does not cause anemia or alter the red cell indices.

\section{BHL 88}

Effect of Room Temperature and Refrigerated Storage on Automated Hematological Parameters

\section{J. Anu, Avinash Priyadarshi", Naveen Kakkar, M. Joseph John ${ }^{1}$}

Email ID for Correspondence: avinashpriyadarshi17@gmail.com CMC, Ludhiana

Introduction and Background: The complete blood count (CBC) is one of the most commonly ordered investigations in clinical practice. Delay in sample processing and ambient temperature may adversely affect $\mathrm{CBC}$ results. This study was done to assess the effect of room temperature and refrigerated storage on $\mathrm{CBC}$. Patient/Material and Methods: Five hundred blood samples sent for $\mathrm{CBC}$ were analysed using 3-part automated hematology analyser, Mindray BC 3000 plus. The samples were divided into two study groups: 24 hour room temperature storage $\left(20-24{ }^{\circ} \mathrm{C}\right)$ [250 samples], 24 hour refrigerated $\left(2-8{ }^{\circ} \mathrm{C}\right)$ storage [250 samples]. Samples were analysed within 30 minutes of collection, kept at room temperature and re-analyzed after 24 hour storage for the room temperature group and after refrigeration. Fifty random samples from the refrigerated group studied for extended storage till 48 hours. Manual differential count was also done. Results: All CBC parameters, except MPV showed good stability at refrigerated storage up to 24 hours $(r>0.9)$; while $\mathrm{Hb}, \mathrm{RBC}$ count, $\mathrm{MCH}$, WBC Count and platelets showed good stability at room temperature storage $(r>0.9)$. Mean corpuscular volume (MCV), MCHC, PCV and RDW and MPV showed statistically significant change $(\mathrm{p}<0.05)$ on room temperature storage. Both automated granulocytes and lymphocytes showed good stability at room temperature as well as after refrigerated storage. On extended refrigerated storage for 48 hours, both granulocytes and lymphocytes showed a statistically significant difference in the stability. Conclusions: Refrigeration of the samples at $2-8{ }^{\circ} \mathrm{C}$ for 24 hours can prolong the stability of most of the CBC parameters in case of an anticipated delay in processing. Selected parameters may also show stability till 48 hours.

Keywords Complete blood count, Refrigeration, Room temperature, Stability, Storage

\section{BHL 89}

Frequency and Types of Anaemia Among Female Students in Sakaka City

Abozer Y. Elderdery", Abdulaziz S. Alshaiba 
Email ID for Correspondence: abozer904@hotmai.com Faculty of Applied Medical Sciences, Aljouf University, Saudi Arabia

Introduction and Background: Aljouf is one of the biggest areas of Saudi Arabia, but little is known about the frequency and causes of anaemia in this population. This prospective cross-sectional study was aimed to detect the frequency and types of anaemia in healthy female university students at the Faculty of Applied Medical Sciences of Aljouf University. Patient/Material and Methods: This study was carried out among one hundred and ninety eight female university students in Sakaka city, Aljouf region between October and December 2015. Five $\mathrm{ml}$ of venous blood samples were collected from each participant into an EDTA container for full blood count (FBC), using a haematogyanalyzer and a plain container for ferritin levels, using a chemistry analyzer. Results: Approximately one third of our study population, sixty four participants $(32 \%)$ were found to be anaemic. Of anaemic cases, the frequency of mild microcytic $(10-11.7 \mathrm{~g} / \mathrm{dL})$, moderate microcytic $(8-10 \mathrm{~g} / \mathrm{dL})$ and severe microcytic $(\mathrm{Hb}<8 \mathrm{~g} /$ $\mathrm{dL})$ and normocytic anaemia $(\mathrm{Hb}<11.8 \mathrm{~g} / \mathrm{dL})$ was $24.4 \%, 3 \%$, $1.5 \%$ and $2.5 \%$ respectively. All these groups were diagnosed with iron deficiency anaemia (IDA) rather than other anaemia with microcytic picture, as their mean ferritin level was significantly lower, $6.9 \mathrm{ng} / \mathrm{ml}$ ( $\mathrm{p}$ value $<0.01$ ) than the control group $-96.3 \mathrm{ng} / \mathrm{ml}$. Macrocytic anaemia was observed in only one participant with high MCV level and normal ferritin level, $[\mathrm{MCV}$ level $=97 \mathrm{fl}$ and $\mathrm{Hb}=10 \mathrm{~g} / \mathrm{dl}$ and ferritin level $=120.5 \mathrm{ng} / \mathrm{ml}]$. Conclusions: The high frequency of IDA rather than other anaemias in the current study may be due to the nutritional habits and lifestyle of female university students in Sakaka city. Therefore, proper nutritional and educational programs are suggested as ways of avoiding the complications and risk of IDA in the future within university female students of Aljouf region, Saudi Arabia.

\section{Topic: Malignant Haematology—Clinical}

\section{MHC 1}

Management of Bleeding in Advanced Malignancy-A Case Series

\section{Rahul D. Arora}

Email ID for Correspondence: xerxes85@ymail.com Junior Resident (Academic), Depatment of Palliative Medicine, Tata Memorial Centre, Tata Memorial Hospital, Ernest Borges Marg, Parel, Mumbai 400012

Introduction and Background: Haemostatic Radiotherapy, Embolisation of feeding vessel, Component transfusion, packing with Vasoconstrictor agents and local installation of Haemocoagulase are modalities which can be employed to control Bleeding in Advanced Malignancy. Patient/Material and Methods: A 61 year Female, known case of moderately differentiated Carcinoma of the Colon, post NACT-RT, post Abdominoperineal Resection, post 5 cycles of adjuvant CAPOX presented with history of passage of blood in the urine along with passage of clots since the past 3 days. CECT abdomen was suggestive of heterogenous opacity in the Posterior wall of the Bladder, USG KUB done subsequently did not show presence of clots. Computed Tomogram with Angiography performed subsequently did not show any Bleeding from the Primary Tumour. A known case of Renal Cell Carcinoma with extension to the Urinary Bladder presented with gross haematuria along with presence of clots in the Bladder. He was started on continuous irrigation with a three way Catheter. Intravesical installation of Tranexamic Acid was initiated by installation of 10 vials of undiluted $10 \%$ Tranexamic Acid $(500 \mathrm{mg} / 5 \mathrm{ml})$ through the three way catheter which was clamped for
30 minutes. A known case of recurrent Squamous Cell Carcinoma of the Oral cavity presented with bleeding from maggot infested ulceroinfiltrative wound in the Floor of the Mouth. An Adrenaline soaked gauze (kept in situ for 15 minutes) was used to stop the Bleeding. A gauze soaked in Turpentine oil was kept in place for 10 minutes, followed by manual removal of Maggots and packing with a gauze soaked in Botropase solution. Results: Control of bleeding was achieved in each of the three cases by the above mentioned methods. Conclusions: Personalized Therapy directed at the underlying cause holds the key to mitigating the potential deleterious effect of Bleeding on survival.

\section{MHC 2}

\section{Does IPS Matter in the Management of Hodgkin Lymphoma}

\section{A. Praveen", G. Sadashivudu, Meherlakshmi, Ravishankar, Chaitanya, Madhav}

Email ID for Correspondence: Praveen.adusumilli@yahoo.com Nizams Institute of Medical Sciences

Background: The International Prognostic Score (IPS) is the most widely used risk stratification index for Hodgkin Lymphoma (HL). It is based on patients treated before 1992 and predicts 5-year freedom from progression (FFP) and overall survival (OS). The IPS has not been validated in terms of long term survival in which outcomes have improved compared with historic results. Material and Methods: By using the Tumour Registry Database of our institute, we identified all patients age $>16$ years newly diagnosed with advanced-stage HL (stage III to IV, or stage I to II with "B" symptoms or bulky disease $>10 \mathrm{~cm}$ ) from 2000 to 2011, treated with curative intent with doxorubicin, bleomycin, vinblastine, and dacarbazine (ABVD) regimen. Results: In all, 216 patients were identified. The median age at diagnosis was 34 years (range 16-75 years), and $71 \%$ of the patients were male. The most common histologic subtype was mixed cellularity HL, which was present in $70 \%$ of the patients. The most prevalent adverse prognostic factor was male sex; albumin $<4 \mathrm{~g} / \mathrm{dl}$ in $60 \%$ and haemoglobin $<10.5 \mathrm{~g} / \mathrm{dl}$ in $51 \%$ of patients. Ten-year FFP and OS were $78 \%$ and $90 \%$, respectively. The IPS was not prognostic for both FFP $(p=0.906)$ and OS $(p=0.087)$, with 10-year FFP ranging from $68 \%$ to $86 \%$ and 10-year OS ranging from $64 \%$ to $97 \%$. Male sex is the only prognostic factor that showed significance on univariate analysis. On multivariate analysis none of the IPS factors retained prognostic significance. Conclusions: The IPS doesn't retain its significance in the prognostication of advancedstage HL over long term. This improvement in outcome is due to the improved diagnostic accuracy \& staging, better treatment regimen, good supportive care and more frequent use of high dose chemotherapy \& autologous haematopoietic stem cell transplantation.

\section{MHC 3}

Bony Swellings in a Young Male Unusual Presentation of Multiple Myeloma

Shivam Kumar Verma\#, Satvik Khaddar, Rudra Prasad Sahu, Priyanka Singh, Amit Singh

Email ID for Correspondence: verma.verma.shivam@gmail.com Institute of Medical Sciences, Banaras Hindu University

Introduction and Background: Multiple myeloma represents a malignant proliferation of plasma cells derived from a single clone, primarily affecting the bone marrow and skeletal system. It is uncommon in young persons, accounting for only $2 \%$ in patients less 
than 40 years of age. Here we present a case of a young male presenting with multiple bony swellings as initial manifestation of Multiple Myeloma. Patient/Material and Methods: A 28 year male admitted with complaints of multiple diffuse painless swellings over anterior chest wall and face for last 4 months with severe generalized body pain and weight loss. On examination the swellings were hard nontender and fixed to the underlying bone. There was no lymphadenopathy or hepatosplenomegaly. On routine analysis patient had anemia, deranged creatinine and hypercalcemia therefore with background of severe bony pain and above mentioned parameters, diagnosis of multiple myeloma was kept. His Xray Skull revealed multiple lytic lesions. His bone marrow analysis and serum electrophoresis was done which confirmed the diagnosis. Results: Patient's Serum and Urine electrophoresis showed intense $\mathrm{M}$ peak and Bence-Jones proteinuria respectively with bone marrow showing $22 \%$ plasma cells. He was started on Bortezomib + lenalidomide + dexamethasone regimen and showed resolution of symptoms and clinical parameters with normalization of anemia, renal function, hypercalcemia and reduction of bony swellings. Conclusions: Multiple myeloma is rare in young and a higher proportion of them have only light-chain myeloma. Some very young patients, particularly those younger than 30 years, have multiple skeletal lesions with extramedullary spread and a small M-component with few bone marrow plasma cells. We have described an initial presentation of multiple myeloma in a young patient with multiple bony swellings. Few case reports have described myeloma in young patients with typical features and some have described myeloma in elderly population with bony swellings but both of these atypical findings together in a single patient has not been reported.

\section{MHC 4}

Clinical Profile \& Outcomes of Acute Myeloid Leukemia: An Institutional Experience from South India

\section{Venkatesh", T. Kannan, Ananth Pai, Shilpa, Praveena \\ Email ID for Correspondence: venky.mushinidvk@gmail.com Sri Venkateswara Institute of Medical Sciences, Tirupati}

Introduction and Background: Acute myeloid leukemia is a heterogenous clonal disorder of hematopoietic progenitor cells with different molecular genetic abnormalities, clinical characteristics and variable outcomes with currently available treatment. Aim of the study is to analyse the clinical profile \& outcomes of AML, an institutional experience where BMT facility is not available. Patient/ Material and Methods: Retrospective chart review of 51 patients diagnosed of AML with clinical \& therapeutic data. Results: 51 patients were analysed (males 30, females 21 ) with a male predominance. $90 \%(46 / 51)$ of patients were in the age group of $<60 \mathrm{yrs} \&$ nearly $40 \%$ (20/51) were 15-30 yrs which is significant. M2 was the most common FAB subtype with 23/51 (45\%) cases and most common presentation is fever. The observed average baseline parameters were: $\mathrm{Hb}$ of $6-8 \mathrm{gr} \%$ with $30 \%$ showed $<6 \mathrm{gr} \%$, TLC $>15,000$ in $90 \%$ of cases which confer poor outcomes $(60 \%$ cases $->50,000$ and $30 \%->1$ lakh). The average platelet count was $20,000-40,000$ (25\% cases-<20,000). Most patients had a baseline blasts $\%$ of $>70$. The average number of transfusions required during induction were in the range of (PRBCs-4-6, RDPs-20-30, SDPs-2-4 \& overall 25-35). Among 51 patients, approximately $75 \%(38 / 51)$ were treated with intensive chemotherapy and 13 were on BSC. Remission rate was found to be $42 \%(16 / 38)$ in patients who received induction chemotherapy while $>95 \%$ of them relapsed. As most of the patients were not on follow up/died, no followup data was found to calculate the survivals. Conclusions: AML is an aggressive malignancy with poor outcomes and high relapse rates, especially without BMT. Supportive care constitutes a major component of management. As most of the patients were presenting very late and in poor PS (bad parameters at baseline) early aggressive treatment with strong supportive care improve the cure rates and survival.

\section{MHC 5}

Frontline Imatinib in Treatment-Naïve Chronic Myeloid Leukemia: The Jewel in the Crown

Madhav Danthala", K. Siva Prasad, Sadashivudu Gundeti, Meher Lakshmi, Arigela Ravi Sankar, Maddali Lakshmi Srinivas

Email ID for Correspondence: docdanthala@hotmail.com Nizam's Institute of Medical Sciences

Introduction and Background: Imatinib continues to be the most frequently used oral TKI in the treatment of chronic myeloid leukemia (CML), despite the availability of alternatives. Material and Methods: In this retrospective analysis, data from 1367 of 1812 patients with CML treated with frontline Imatinib mesylate at a single institution between 2008 and 2014 is included. Results: At diagnosis, $98(7.1 \%)$ and $58(4.2 \%)$ were in accelerated phase (AP) and blast crisis (BC) respectively. After a median follow up of 43.7 months, $917(67 \%)$ patients achieved complete cytogenetic response (CCgR), $283(20.7 \%)$ achieved major molecular response (MR3), and 247 (18\%) achieved $4 \log$ reduction in $B C R A B L$ transcripts (MR4). Follow up assessments using real-time polymerase chain reaction (qPCR) and/or cytogenetic tests were not available in $270(19.7 \%)$ patients, despite inclusion in a sponsorship program. Adherence to treatment was poor in $222(16.2 \%)$ patients. In a fairly homogenous population of lower economic strata, on a free drug access program, the prime factors influencing adherence were low educational level, assumptions of "cure", recent bereavement, stigma of cancer diagnosis and repeated hospital visits. Transformation to AP/BC occurred in $42(3 \%)$ and $63(4.6 \%)$ of patients respectively. Testing for $B C R$ $A B L$ mutations was done in $163(11.9 \%)$ patients after failure of treatment or suboptimal response. Mutations were identified in 66 $(4.8 \%)$ patients. The subsequent treatments chosen were, dose escalation $279(20.4 \%)$, Nilotinib $34(2.4 \%)$, Dasatinib $20(1.4 \%)$ and hydroxyurea $15(1 \%)$. At a median follow up of 43.7 months (range 1.67-101.4), $1262(92.3 \%)$ were transformation free, 763 $(55.8 \%)$ failure free and $1104(80.7 \%)$ event free. There were no new or unexpected adverse events. Conclusions: The results of our study continue to support Imatinib as a safe and effective first line therapy for the long-term treatment of CML.

\section{MHC 6}

\section{Diagnosis of Lymphoplasmacytic Lymphoma}

\section{Amar Ranjan", Pranay Tanwar}

Email ID for Correspondence: dr.amarranjan@rediffmail.com DR. BRAI-RCH, All India Institute of Medical Sciences, New Delhi

Introduction and Background: Lymphoplasmacytic lymphoma (LPL) is a neoplasm of small B lymphocytes, plasmacytoid lymphocytes \& plasma cells, usually involve bone marrow (BM), sometimes lymphnodes \& spleen, which doesn't fulfill the criteria for any other B cell neoplasm that may have plasmacytic differentiation. Waldenstrom Macroglobulinemia (WM) is defined as LPL with BM involvement $(>10 \%$ infiltration) \& IgM monoclonal gammopathy (IgM-MG) in blood. [WM = LPL + BM involvement + IgM-MG]. It is a rare entity. Patient/Material and Methods: The case was retrieved from my hospital record. A 64 year male, presented with 
low back pain \& early fatigue 2-3 yrs. History of hypertension, diabetes mellitus with a healed $H$. zoster lesion in right T10 dermatome was present. There was bilateral axillary lymphadenopathy with no organomegaly, $\mathrm{Hb} 96 \mathrm{gm} / \mathrm{L} \&$ beta 2 microglobulin $2.49 \mathrm{mg} / \mathrm{L}(0.8-2.1)$. Occult blood in stool was present. BM aspirate smear showed $5 \%$ plasma cells. M band was seen on Protein electrophoresis (serum \& urine), immunofixation showed IgM lambda. There was raised twenty four hours urinary protein, serum IgM 1588 (40-230) \& IgG 1895 (70-1600). Skeletal survey showed patchy osteopenia in ribs. Results: Final diagnosis Waldenstrom Macroglobulinemia was made. Patient is on Chlorambucil + Prednisolone therapy. Discussion: The term "macroglobulinemia" (IgM-MG) refers to excess of monoclonal IgM. It includes MG of undetermined significance, Smoldering WM, WM, chronic lymphocytic leukemia, lymphoma variants and primary amyloidosis (2). There may be hyperviscosity syndrome (causing CNS signs and symptoms), neuropathy (IgM reactivity to myelin sheath antigen), cryoglobulinemia or paraprotein deposition. IgM deposition may occur in skin \& GIT to cause diarrhoea (1). Bone or kidneys involvement is uncommon. Tortuous retinal veins (sausage link) is characteristic. Asymptomatic WM is called SWM. Plasmacytic component will be CD138+, CD38+ and CD45- or dim. Conclusions: WM needs a careful exclusion from other variants of Non Hodgkin's Lymphoma.

Keywords Lymphoplasmacytic lymphoma, Waldenstrom Macroglobulinemia, IgM monoclonal gammopathy

\section{MHC 7}

Outcome of Granulocytic Sarcomas in Acute Myeloid Leukemia with Hypomethylating Agents

Ram V. Nampoothiri", Kamalkant Sahu, Ankur Jain, Aditya Jandial, Uday Yanamandra, Gaurav Prakash, Alka Khadwal, Deepesh Lad, Neelam Varma, Pankaj Malhotra, Subhash Varma

Email ID for Correspondence: ramvnampoothiri@gmail.com Post Graduate Institute of Medical Education And Research, Chandigarh

Introduction and Background: Granulocytic sarcomas (GS) in acute myeloid leukemia (AML) are uncommon but clinically significant entities. GS may be present in conjunction with bone marrow involvement or as an isolated entity. Treatment of GS consists of standard induction therapy of acute myeloid leukemia. Hypomethylating agents (HMA) are increasingly used in AML patients who are not candidates for standard therapy. The response of GS to HMA is not known. Patient/Material and Methods: We retrospectively studied all patients who presented with GS to our tertiary care centre in North India and were treated like AML. GS were confirmed by biopsy and immune-histochemistry (positive for Myeloperoxidase and $\mathrm{CD} 117 \pm$ other markers). Bone marrow examination was done for all patients. Cross-sectional imaging (CT or FDG PET) was done in all patients to look for other sites of involvement by GS. All patients were treated either with intensive induction chemotherapy or with HMA. Results: From Jan 2014 to Dec 2015, out of 121 AML patients, 10 patients had presented with GS. The median age of these 10 patients was 36.5 years with equal male and female ratio. Two patients had presented with GS on clinical examination while majority $(80 \%)$ were diagnosed on imaging. Spine and paraspinal areas were the most common sites of involvement $(40 \%)$. Three (30\%) patients harbored more than one site of GS. Sixty percent of patients $(n=6)$ with granulocytic sarcomas had concurrent bone marrow involvement. Seven patients received HMA alone as induction therapy because of elderly age and presence of comorbidities. Three patients were treated with intensive induction chemotherapy (Daunorubicin $\times 3$ days +7 days continuous cytarabine). After 4 cycles of HMA, $28 \%$ (2 out of 7) achieved remission while $33 \%$ (1 out of 3) patients achieved remission after intensive induction chemotherapy. The overall survival of patients with granulocytic sarcomas treated with HMA was comparable to patients receiving aggressive induction therapy at one year. Conclusions: AML patients with or without GS have poor prognosis. Imaging (CT, FDG-PET) picks up higher number of GS in patients with AML. Activity of HMA in GS appears to be similar to their activity in de novo AML.

\section{MHC 8}

Venous Thrombosis in Pediatric Patients with Acute Lymphoblastic Leukemia: Incidence, Risk Factors and Outcome

\author{
Akanksha Garg ${ }^{1 \#, ~ R a j e s h ~ K a s h y a p ~}{ }^{1}$, Heeralal ${ }^{2}$, \\ Soniya Nityanand ${ }^{1}$
}

Email ID for Correspondence: sadge85@gmail.com

${ }^{1}$ Department of Hematology, ${ }^{2}$ Department of Radiology, Sanjay

Gandhi Post Graduate Institute of Medical Sciences, Lucknow

Introduction and Background: Venous thrombosis is a frequent complication seen in Acute Lymphoblastic Leukemia (ALL). The incidence varies from 1 to $30 \%$. Central venous catheters and chemotherapy are identified as major risk factors. There is limited data on venous thrombosis in Indian pediatric patients with ALL. Patient/Material and Methods: Over a period of three years (2013-2016), 47 (36 males and 11 females) patients below the age of 18 years were diagnosed to have ALL and were treated at our centre. Nine of these $47(19.1 \%)$ had an episode of venous thromboembolism (VTE). All the symptomatic VTE were objectively diagnosed and confirmed by USG, CT Scan or MR imaging. The clinical and laboratory data were recorded from case files. All patients were treated on the BFM 95 protocol. All patients received low molecular weight heparin for treatment. Results: Nine ( 7 males and 2 females) patients with ALL had an episode of VTE. Seven of these 9 cases had B-ALL and remaining 2 had T-ALL. Three cases were high risk ALL and remaining were medium risk. The mean age of presentation was 14 (range 7-18) years. The incidence was higher in males (77\%). Three patients had cerebral venous thrombosis and it occurred during the induction phase of chemotherapy and was related to use of $\mathrm{L}$-asparaginase. Catheter induced thrombosis was seen in 3 cases $(2$ internal jugular vein and one femoral vein). One patient had thrombosis of left innominate vein at presentation. Other two patients had thrombosis of cephalic vein and common iliac vein respectively. All the patients were treated with low molecular weight heparin and one patient developed heparin induced thrombocytopenia (HIT) and was treated with fondaparinaux. No death occurred due to VTE. Conclusions: Incidence of venous thrombosis is high in acute lymphoblastic leukemia especially in young males and one should be vigilant for its detection.

\section{MHC 9}

Outcome of Pregnancy in Chronic Myeloid Leukemia Patients on Imatinib Therapy

\section{Rajesh Kashyap ${ }^{1 \#}$, Rohan Haldhar ${ }^{1}$, Pradeep Kumar ${ }^{1}$, Mandakini Pradhan ${ }^{2}$}

Email ID for Correspondence: rajkashyapmd@yahoo.co.in Department of ${ }^{1}$ Hematology and ${ }^{2}$ Maternal and Reproductive Health, Sanjay Gandhi Post Graduate Institute of Medical Sciences, Lucknow

Introduction and Background: CML accounts for $10 \%$ of all leukemia in the reproductive age group. Imatinib has revolutionised the 
treatment of CML. Management of pregnancy in CML patients is a big challenge because of the potential teratogenic effects of imatinib. We share our experience with pregnancy in $8 \mathrm{CML}$ patients who conceived while receiving imatinib. Patient/Material and Methods: Over a 10 years period (2004-2015), 8 CML patients had pregnancies. Patients were monitored for their haematological and obstetrical status. Clinical and laboratory data was recorded. Regular antenatal ultrasound was performed to detect any congenital anomalies. Imatinib was stopped in all the 8 patients and hydroxyurea was used to reduce the raised leukocyte counts. Results: Eight CML patients (age range 18-31 years) had pregnancy. 4 patients were primigravida, 2 were gravida 2 and one each gravida 3 and 4 . All patients were in chronic phase of disease at time of diagnosis of pregnancy. Three patients had achieved complete molecular remission, one major molecular remission and 4 complete hematological remissions. The period of gestation and exposure time to imatinib was 14.8 (range 4-31) weeks. 3 patients required hydroxurea to control their leucocyte counts. The antenatal period was uneventful in all patients. Pregnancy was terminated at 37 weeks in 7 cases and at 35 weeks in 1 case. Six patients had normal vaginal delivery and 2 cases underwent caesarean section for obstetrical indications. All the 8 cases delivered normal live healthy babies ( 5 males and 3 females). The average birth weight was 2.43 kilograms and no congenital abnormalities were observed. No progression of disease occurred during pregnancy. Conclusions: All our $8 \mathrm{CML}$ patients with pregnancy had a successful outcome. It's our recommendation that if pregnancy does occur it should be closely monitored and it may not be necessary to terminate it.

\section{MHC 10}

Hashimotos Thyroiditis and Thyroid Lymphoma: A Clear Association or a Causal Relationship

Varun Yadav", P. S. Ghalaut, Sudhir K. R. Atri, Mohini, Divya, Akhilesh, Nidhi

Email ID for Correspondence: varun_yadav325@yahoo.com Pt. BD Sharma UHS PGIMS Rohtak, Haryana 124001

Introduction and Background: Primary thyroid lymphoma, although rare, should always considered in the differential diagnosis of the rapidly increasing thyroid swelling. Thyroid lymphoma is always of Non Hodgkin B cell type and is about $2 \%$ of all extranodal lymphoma. Pre-existing Hashimotos thyroiditis is the known risk factor. The risk for thyroid lymphoma is 60 times higher than the patients without thyroiditis. Patient/Material and Methods: 51 year female known case of hypothyroidism for 8 years, now off treatment since 6 months came with complaint of midline neck swelling since 3 months. No history of fever, bleeding, and compressive symptoms. On examination there was midline neck swelling which was moving up on deglutition suggestive of enlarged thyroid gland. Pallor was present with no lymphadenopathy. Rest examination was within normal limit. Thyroid profile was suggestive of hypothyroidism with raised TSH. Anti TPO antibodies were also raised. USG neck showed features suggestive of thyroiditis. Patient was prescribed tablet thyroxine 100 microgram per day. Then FNAC neck swelling shows the cytological features of thyroid lymphoma. Core needle biopsy showed features of Non Hodgkin lymphoma with tumour cells diffusely positive for $\mathrm{CD} 20$. Bone marrow examination showed normal results. CECT neck, chest and abdomen showed homogenous enhancement in throid gland with no other lymphadenopathy. Results: Diagnosis of isolated thyroid lymphoma (stage 1E), a rare presentation was made. Patient was given 6 cycle RCHOP regimen 3 weekly and neck swelling completely regressed. Whole body PET CT was done later which shows no abnormal uptake. Conclusions: Lymphoma of thyroid gland often arises in background of Hashimoto thyroiditis. Most common type is Diffuse large B-cell lymphoma. Rapidly increasing size of thyroid gland suggest possibility of thyroid lymphoma. Thyroid lymphoma is highly sensitive to chemotherapy and radiotherapy. Surgery is not advised in these cases as it may lead to local spread.

\section{MHC 11}

Clinicohematological and Cytogenetic Profile of Myelodysplastic Syndrome with Special Reference to ICUS \& IDUS

\section{Mohammad Frayez ${ }^{\#}$, Vijai Tilak}

Email ID for Correspondence: drfrayezgsvm@gmail.com Banaras Hindu University, Varanasi

Introduction and Background: Minimal diagnostic criteria for myelodysplastic syndromes (MDS) include constant cytopenia recorded for at least 6 months, dysplasia, and exclusion of other causes of cytopenia and dysplasia. Two recently described 'premalignant' myeloid conditions are idiopathic cytopenia of undetermined significance (ICUS) and idiopathic dysplasia of uncertain significance (IDUS). In ICUS the dysplasia is mild and does not fulfill the WHO criteria for MDS but cytopenias can be severe. In IDUS the dysplasia is prominent but cytopenias, if detectable, are mild. For both groups of patients, a thorough hematologic follow up is recommended because of the potential of disease-manifestation and the unpredictable form and time of progression. Patient/Material and Methods: 141 patients of MDS, 4 cases of ICUS and 23 cases of IDUS seen from August 2008 to June 2016 at the Medicine and Pediatric department of S. S. Hospital, Banaras Hindu University, Varanasi are included in this study. Complete blood counts were performed in an automated cell counter. Bone marrow aspiration, trephine biopsy and chromosomal study were carried out. The dry film was stained using Leishman stain. Results: 81 cases were male and 60 cases were female. The mean age at presentation was 32 years (range 1 month76 years). A majority of the patients presented with pallor $(96.9 \%)$. Most common form was RCMD (41.8\%). Three patients had chromosomal abnormalities (del 20q, del 7q, del5q). 4 cases diagnosed as ICUS, 1 patient died during the follow up, cause unknown. Others had persistent cytopenia and did not progress to any other condition. 23 cases diagnosed as IDUS, 2 patients progressed to RCMD, 2 patients progressed to RAEB-1 \& 1 patient progressed to RAEB-2. All the other patients did not progress further and remained in IDUS during the follow up. Conclusions: MDS in India is a disease of pediatrics and young adults in contrast to Western culture where it is a disease of elderly. Hepatosplenomegaly is more common in children than adults. ICUS is a rarely diagnosed condition and can be fatal but due to paucity of cases, more study needs to be done to evaluate its fate. IDUS can progress to MDS or can remain clinically silent.

\section{MHC 12}

Imatinaib Induced Sweet Syndrome in a Patient with Chronic Myeloid Leukemia

Nidhi Yadav", P. S. Ghalaut, Sudhir K. R. Atri, Mohini, Varun Yadav, Anuj Chaudhary

Email ID for Correspondence: nidhiyadav642.ny@gmail.com Pt. BD Sharma UHS PGIMS Rohtak

Introduction and Background: Imatinib mesylate, a tyrosine kinase inhibitor is the drug of choice for Chronic Myeloid Leukemia patients. Adverse skin effects are very common and include nonspecific manifestations such as edema and maculopapular rashes or various types of eruptions e.g. lichenoid or psoriasiform lesions, acute 
generalized exanthematic pusutlosis and rarely Sweet syndrome. Sweet syndrome is an acute febrile neutrophillic dermatosis which is of 3 types: classical, malignancy related and drug induced. Main and more common drugs are Hydralazine, sulpha drugs, G-CSF are the main drugs that cause sweet syndrome. Patient/Material and Methods: 55 year male, known case of Chronic Myeloid leukemia since 1 year taking tablet Imatinib mesylate $400 \mathrm{mg}$ OD came with complaint of multiple painful edematous skin lesions in form of erythematous papules and nodules on hands, cheeks and neck associated with fever since 3 days. No history of bleeding, petechial spots and other systemic symptoms e.g. weight loss. On examination, the lesions are erythematous and scaly and tender on touch present on the forehead, and upper limbs. No organomegaly was there. Complete haemogram was done and it shows Haemoglobin 11.8, leukocytosis $(45,000)$ Peripheral blood smear showed blasts $(1 \%)$, promyelocytes $2 \%$ and metamyelocytes $10 \%$. Skin biopsy of the lesion showed neutrophillic infiltrate without evidence of leukocytoclastic vasculitis suggestive of Sweet syndrome. Results: The diagnosis of Sweet syndrome was entertained and it was thought to be due to imitinab although sometime CML can itself cause sweet syndrome. However possibility of imitinab is more in this case. Imatinib was withhold and patient was given treatment in form of oral corticosteroids (Tab. Prednisolone $40 \mathrm{mg}$ per day) and topical steroids and lesion subsided within a week. Conclusions: Sweet syndrome is rare dermatological condition. Haematological malignancy being the second most common cause and among it, Acute Myeloid Leukemia is most common, first being the idiopathic and last in this category is drug induced. CML is a rare risk factor and in CML, the most common reason is drug induced rather than CML per se. Hence we are reporting this case.

\section{MHC 13}

Non Hodgkin B Cell Lymphoma Presenting as Palatal Ulcer: A Rare Presentation

Akhilesh Kumar Singhal ${ }^{\#}$, Sudhir K. R. Atri, P. S. Ghalaut, Mohini, Varun Yadav, Divya, Dheeraj

Email ID for Correspondence: piyushsinghonly@gmail.com Pt, BD Sharma UHS PGIMS Rohtak

Introduction and Background: Non Hodgkin lymphoma consists of a diverse group of malignant neoplasms derived from the B cells, T cells, plasma cells and natural killers cells. Non Hodgkin lymphoma accounts for the $62 \%$ of all lymphoid malignancies out of which 25 to $40 \%$ are extranodal lymphomas. Palatal lymphoma is rare among the extranodal lymphoma and is almost always of T cell type. B cell palatal lymphoma is extremely rare. Patient/Material and Methods: 75 year old female patient presented with dry cough and running nose one year back. Patient was treated on the basis of deviated nasal septum for six months and was not relieved. After sometime the patient had painfulnon healing ulcer over the hard palate and fever on and off. There is no history of bleeding from nose, shortness of breath and other systemic symptoms. Patient took ENT consultation and treated on the basis of the granulomatous lesion and was not relieved. On examination she had pallor with no lymphadenopathy. Rest examination was within normal limit. Chest $\mathrm{X}$ ray was normal. Punch biopsy of the lesion shows cytological features suggestive of Non Hodgkin lymphoma, diffuse large B cell type with B cell diffusely positive for CD 20. Bone marrow aspiration and biopsy was normal. CECT PNS, neck, chest and abdomen shows no lymphadenopathy. Results: Diagnosis of isolated palatal lymphoma B cell type, a rare presentation was made. Patient was given 6 cycle of RCHOP three weekly. Patient responded well with complete regression of the palatal ulcer. Conclusions: Lymphoma of the palate is rare entity and out of which B cell type is extremely rare. This patient was B cell type responded well to RCHOP regimen. These lymphomas are sensitive to chemotherapy. Surgery is not needed in these cases.

\section{MHC 14}

\section{A Rare Case Presentation of T Cell Lymphoma in Intestine}

\section{Avishesh Kumar Singh"}

Email ID for Correspondence: avisheshsingh@yahoo.co.in Srimanakula Vinayagar Medical College and Hospital, Pudducherry

Introduction and Background: Intestinal lymphoma of T cell type is one of the rare entity seen. Intestinal lymphomas derived from intraepithelial $\mathrm{T}$ lymphocytes occur as 2 types Enteropathy associated T-cell lymphoma (EATL) and Monomorphic CD56+ (NCAM1) intestinal $\mathrm{T}$ cell lymphoma. Intestinal tumor of intraepithelial T-lymphocytes with large, polymorphic lymphoid cells, Negative for CD56 also known as Type-I EATL. More common in patients with celiac disease, Age $>50$ years and Mean age of presentation is 64 years with M:F-3:1 ratio. Patient/Material and Methods: A 41 year old female patient presented with chief complaint of abdominal pain and difficulty in passing stools for 1 month. Grossly received a segment of intestine (ileum, part of ascending colon and caecum with attached mesentric fat). A friable growth seen $40 \mathrm{~cm}$ away from ileocaecal junction $\mathrm{m} / \mathrm{s} 9 \times 5 \mathrm{~cm}$. On cut section, greywhite and yellowish areas with hemorrhages were seen. Results: It is a rare type of $\mathrm{T}$ cell lymphoma in gastrointestinal tract. Incidence occur less than $5 \%$ of total lymphomas and small intestine is the most common site of involvement. Immunohistochemically the tumor cells are $\mathrm{CD} 3+, \mathrm{CD} 4-\& \mathrm{CD} 20-$. The differential diagnosis includes maltoma, B cell lymphoma and other type of $\mathrm{T}$ cell lymphomas. Conclusions: $T$ cell lymphoma follow an aggressive course with a poor prognosis. Because of the incidence of multifocal involvement of the small intestine and frequent dissemination, complete remission is not achievable, many patients undergoing diagnostic Laparotomy have a high rate of post operative complications. This case satisfies the histology and ImmunoPhenotypical criteria for $\mathrm{T}$ cell lymphoma. Thus a better evaluation of real frequency of occurrence needs to be established for highly aggressive malignant tumor in the intestine.

\section{MHC 15}

Absence of CD66c Expression Excludes the Possibility of BCRABL1 Translocation in B Cell Precursor Acute Lymphoblastic Leukemia (BCPALL) Cases

\section{Shweta Kedia\#, Akshata Kawle, Nilesh Deshpande, P. G. Subramanian, Nikhil Patkar, Sitaram Ghogale, Y. Badrinath, Sumeet Gujral, Prashant Tembhare}

Email ID for Correspondence: skedia04@gmail.com Hematopathology Laboratory, Tata Memorial Centre, Mumbai, India

Introduction and Background: CD66c (CEACM6) is a myeloidlineage associated antigen expressed aberrantly by blasts in B-cell precursor acute lymphoblastic leukemia (BCPALL). A few studies have claimed that CD66c expression is strongly associated with BCRABL1 translocation in BCPALL and more recently, also shown to be associated with CRLF2-positive BCPALL. We studied the expression pattern of CD66c in a large cohort of 474 BCPALL cases and determined its association with BCRABL1 translocation in our patients. Material and Methods: Expression pattern of CD66c (Fluorochrome: PE, Clone: KOR-SA 3544, BC) was studied in the 
diagnostic samples from 474 BCPALL cases (age 1-67 years \& M:F 2.02). The diagnosis was made as per WHO-2008 classification and flow cytometric immunophenotyping (FCI) and recurrent translocations were studied by FISH and BCR-ABL1 translocation was studied using Abbott Mol LSI dual-fusion probes. Results: CD66c was positive in $56.3 \%$ diagnostic samples of BCPALL, followed by CD15$14.5 \%, \mathrm{CD} 13-11.9 \%$, CD33-5.6\% \& CD117-1.3\% samples. In 474 samples, its expression pattern was strong, intermediate, partial, weak and heterogeneous in $20.3 \%, 1.7 \%, 8.6 \%, 2.3 \%$ and $23.4 \%$ respectively. On cytogenetics study, BCR-ABL1 translocation was positive in $59(12.4 \%)$ cases. The sensitivity, specificity, positive predictive value and negative predictive value of CD66c expression in predicting BCR-ABL1 translocation was found to be $19.7 \%$ (95\% CI $14.88 \%-25.22 \%$ ), $95.2 \%$ (95\% CI $91.60 \%-97.59 \%$ ), $81.4 \%$ (95\% CI $69.09 \%-90.31 \%)$ and $52.8 \%$ (95\% CI $47.84 \%-$ $57.66 \%)$ respectively. On correlation study, spearman correlation was $r=0.19$ with $p$-value $<0.001$ suggesting weak but statistically significant positive correlation between CD66c expression and BCRABL translocation. Conclusions: CD66c is the most common myeloid antigen expressed aberrantly in BCPALL. CD66c expression has low sensitivity but very high specificity with high positive-predictive-value for BCR-ABL1 translocation in BCPALL. Thus, it can be reliably used to exclude the possibility of BCR-ABL1 translocation in resource limited settings and in cases where cytogenetics study is not available.

\section{MHC 16}

Flow Cytometric Immunophenotyping is a Highly Accurate and Far Superior Method for the Evaluation of Body Fluid Involvement by Hematolymphoid Neoplasms

\section{Manisha More", P. G. Subramanian, Nikhil Patkar, Gaurav Chatterjee, Y. Badrinath, Ashok Kumar, Sitaram Ghogale, Nilesh Deshpande, Sumeet Gujral, Prashant Tembhare}

Email ID for Correspondence: kedare11manisha@gmail.com Hematopathology Laboratory, Tata Memorial Centre, Mumbai

Introduction: Evaluation of body fluid involvement is very important in part diagnosis, staging and management of the hematolymphoid neoplasms (HLN). Traditionally, it is performed by microscopic analysis of cytomorphology. However, cytomorphology is not only subjective but also highly challenging in the paucicellular samples like CSF or vitreous fluid (VF) leading to misdiagnosis. Flow cytometric immunophenotyping (FCI) is a powerful tool used in the diagnosis, classification and monitoring of HLN involving peripheral blood and bone marrow aspirates. In the present study, we investigated the value of FCI in the evaluation of body fluid involvement by HLN. Methods: We studied 174 body fluid samples from the 164 patients of HLN. Diagnosis and classification of HLN were performed as WHO criteria. Cytomorphology was performed on the smears prepared through cytospin and stained with Wright' stain. FCI was performed using 8-10 color antibody panel on Navios software and data was analyzed using Kaluza-software 1.3 V. Results: We studied 174 body fluid samples including ascetic fluid (13.8\%), CSF (42.5\%), VF (1.7\%). Pericardial fluid (4.6\%), Plural fluid (35.6\%), synovial fluid (1.1\%), and Splenic aspirate in $0.6 \%$ from 164 patients of HLN (NHL-14.0\%, ALCL$1.8 \%$, AML-1.2 \%, BALL-15.2\%, Burkitt's lymphoma-3.7\%, BNH-NOS-1.2 \%, CLL-1.8\%, CML-1.2\%, DLBCL-17.1\%, FCL $-5.5 \%$, MCL-3.0 \%, NK/T-NHL- $0.6 \%$, PCNSL-6.1\%, granulocytic sarcoma-0.6\%, PMBCL-1.8 \%, MM-1.8 \%, SLL$0.6 \%$, T Cell LBL-6.7 \%, TALL- $15.2 \%$, and PTCL-0.6\%). Of 174 samples, $30 \%$ were involved and $44 \%$ were negative by both cytomorphology and FCI. However, $17 \%$ samples were involved by
FCI but were false-negative by cytomorphology and $9 \%$ were diagnosed as suspicious of involvement but were negative by FCI (false positive). In $1 \%$ samples, FCI was negative but cytomorphology was positive. Thus, sensitivity and specificity of cytomorphology was $64.3 \%$ and $82.2 \%$ when correlated with FCI. Conclusion: Flow cytometric immunophenotyping is a highly accurate and far superior method for the evaluation of body fluid involvement by hematolymphoid neoplasms and hence, FCI should be the method of choice.

\section{MHC 17}

Non Hodgkin Lymphoma in a Child with Unrecognised Celiac Disease

\section{Prashant Chhabra\#, Rimjhim Sonowal, Vineeta Gupta}

Email ID for Correspondence: prashantchabra@gmail.com; e-mail: vineetaguptabhu@gmail.com

Division of Hematology Oncology, Department of Pediatrics, Institute of Medical Sciences, BHU, Varanasi

Introduction and Background: Celiac disease (CD) is genetically determined autoimmune disorder resulting from allergy to gluten containing foods. Untreated celiac disease is associated with several haematological complications including lymphoma. Although risk of enteropathy associated lymphoma is several times higher than general population; most of the cases have been reported in adults. We present an unusual case of B cell rectal lymphoma in a child diagnosed simultaneously with celiac disease. Patient: Eight year old male child presented with fever and blood mixed stools for 6 months, increasing pallor for 2 months and swelling of feet for 15 days. Examination revealed pale and malnourished child with bilateral pitting pedal edema, ascites without hepatosplenomegaly. Provisional diagnosis of malabsorption syndrome possibly celiac disease was made. Results: On investigations, haemoglobin was $4.9 \mathrm{~g} / \mathrm{dl}$, serum iron $14 \mu \mathrm{g} / \mathrm{dl}$, TIBC $170 \mu \mathrm{g} / \mathrm{dl}$, saturation $8 \%$, ferritin $799.0 \mathrm{ng} / \mathrm{ml}$, total protein $5.1 \mathrm{~g} / \mathrm{dl}$ and albumin $2.8 \mathrm{~g} / \mathrm{dl}$. Raised serum anti TTG and villous atrophy on duodenal biopsy confirmed celiac disease. CT scan of lower pelvis revealed gross circumferential thickening of the rectum with infiltration of adjoining structures. Colonoscopic biopsy revealed B cell lymphoma. Final diagnosis was B cell lymphoma of rectum with celiac disease. Child was started on gluten free diet with gradually escalating chemotherapy in view of his poor nutritional status. He received 3 courses of modified COPADM and achieved complete remission. PET scan at end of chemotherapy was normal. Eighteen months later, child is in remission. Conclusions: Enteropathy associated lymphoma is a rare but potentially fatal manifestation of celiac disease which presents with non specific symptoms. This case signifies difficulties encountered in diagnosing this disorder, particularly when there is no preceding history of celiac disease. Early diagnosis is of utmost importance in order to start treatment before the effects of malnutrition increases the risk of complications from chemotherapy.

\section{MHC 18}

Synchronous Occurrence of Chronic Myelogenous Leukemia and Carcinoma Breast: A Rare Combination

\section{Praveena Voonna", T. Kannan, D. Bhargavi, M. Venkatesh, Ananth Pai, K. Shilpa}

Email ID for Correspondence: praveena.voonna@gmail.com Sri Venkateswara Institute of Medical Sciences, Tirupathi

Introduction and Background: Synchronous occurrence of chronic myelogenous leukemia (CML) and carcinoma breast is very rare. In 
view of the rarity of incidence with very few cases documented in literature, we hereby report a case of this rare combination. Case Summary: A 48 year old lady was evaluated for left breast lump of 2 months duration. Biopsy revealed Infiltrating duct cell carcinoma (IDCC), clinically staged as cT2N1M0-IIB (Triple negative). Metastatic work up showed coincidental finding of hyperleukocytosis $\left(145,000\right.$ cells $\left./ \mathrm{mm}^{3}\right)$ with significantly increased multilineage differentiation forms (myeloid progenitors and granulocyte-macrophage progenitors), thrombocytosis $\left(7.8\right.$ lakhs $\left./ \mathrm{mm}^{3}\right)$ and myeloblasts $(1 \%)$. Peripheral smear was suggestive of CML, which was further confirmed by bone marrow studies as CML-Chronic phase and BCRABL1 transcripts by RT PCR quantitative assay (Positive-37.7 international scale normalized copy number). She was finally diagnosed as synchronous CML and Carcinoma breast and was therapeutically approached with a curative intent addressing both the entities simultaneously. Patient was on Imatinib (CML) and neoadjuvant chemotherapy (Carcinoma breast) \& completed 2 months of therapy with a partial response. Discussion: Studies have shown that in CML, mutation at the stem level, that is, in ph chromosome, occurs around 6 years before the presentation of the disease, whereas in carcinoma breast it occurs many years prior to its presentation ${ }^{(1)}$. Conclusion: Synchronous primary cancers pose a variety of clinically important diagnostic and therapeutic problems. Treatment requires a careful coordination and assessment in a multidisciplinary team. In summary, our case of Carcinoma breast with CML is a rare presentation and it appears to be more of a coincidence than any association. Keywords Chronic myelogenous leukemia, Carcinoma breast, Synchronous malignancy. Reference: 1. Devita VT Jr, Hellman S, Rosenberg SA. Cancer principles and practice of Oncology. 7th ed. Philadelphia: Lippincott Williams and Wilkins; 2005. p. 1430.

\section{MHC 19}

An Open-Label, Randomised, Phase 3 Study of Daratumumab, Lenalidomide, and Dexamethasone (DRd) Versus Lenalidomide and Dexamethasone (Rd) in Relapsed or Refractory Multiple Myeloma (RRMM): POLLUX

\section{Naman H. Shah ${ }^{\#}$, Meletios A. Dimopoulos, Albert Oriol, Hareth Nahi, Jesus San-Miguel, Nizar J. Bahlis, Neil Rabin, Robert Z. Orlowski, Mieczyslaw Komarnicki, Kenshi Suzuki, Torben Plesner, Olga S. Samoilova, Sung-Soo Yoon, Dina Ben Yehuda, Paul G. Richardson, Hartmut Goldschmidt, Donna Reece, Nushmia Khokhar, Lisa O'Rourke, Christopher Chiu, Xiang Qin, Mary Guckert, Tahamtan Ahmadi, Philippe Moreau}

Email ID for Correspondence: nshah55@its.jnj.com Johnson \& Johnson Private Limited

Introduction and Background: We compared the efficacy and safety of daratumumab (D) in combination with Rd vs. Rd alone in patients with RRMM in a randomised, open-label, multicenter, phase 3 study (POLLUX; NCT02076009). Patient/Material and Methods: Patients with $\geq 1$ prior line of therapy were randomised (1:1) to R $25 \mathrm{mg}$ orally on Days $1-21$ of each 28 -day cycle and d $40 \mathrm{mg}$ weekly, with or without D $(16 \mathrm{mg} / \mathrm{kg}$ qw for 8 weeks, $\mathrm{q} 2 \mathrm{w}$ for 16 weeks, then $\mathrm{q} 4 \mathrm{w}$ until progression). The primary endpoint is PFS. Results: 569 patients were randomised. Patients received a median of 1 prior line of therapy (range $1-11$ ), $19 \%$ received $\geq 3$ prior lines of therapy. $86 \%$ received prior PI, $55 \%$ received prior IMiD (including $18 \%$ prior $\mathrm{R}$ ), and $44 \%$ received both PI and $\mathrm{IMiD} ; 27 \%$ were refractory to last line of prior therapy, $18 \%$ were PI refractory, and none were $\mathrm{R}$ refractory. After median follow-up of 13.5 months, D significantly improved median PFS (63\% reduction in the risk of progression/death) and TTP for DRd vs. Rd (Table 1). D significantly increased ORR (93\% vs. $76 \%$ ) and rates of VGPR or better (76\% vs. $44 \%$ ) and CR or better (43\% vs. $19 \%$ ) for DRd vs. Rd, respectively (all $P<0.0001$ ). The most common $(>30 \%)$ TEAEs (DRd/Rd) were neutropenia (59\%/43\%), diarrhoea $(43 \% / 25 \%)$, fatigue (35\%/28 \%), upper respiratory tract infection $(32 \% / 21 \%)$, and anaemia $(31 \% / 35 \%)$. Most common grade $3 / 4$ TEAEs $(>10 \%)$ were neutropenia $(52 \%)$ $37 \%)$, thrombocytopenia (13\%/14 \%), and anaemia (12\%/20\%). The rate of grade $3 / 4$ infections was $28 \%$ and $23 \%$, respectively. Discontinuation rates due to TEAEs were similar $(7 \% / 8 \%)$. D-associated infusion-related reactions (IRR; $48 \%$ of patients) mostly were grade $1 / 2$ (grade $3 / 4,5 \% / 0 \%$ ); most (92\% of IRRs) occurred during the first infusion. Conclusions: The combination of $\mathrm{D}$ and $\mathrm{Rd}$ potentially represents a new standard of care for patients with $\geq 1$ prior treatment.

Table 1

\begin{tabular}{lll}
\hline & DRd & Rd \\
\hline Median PFS, mo & NR & 18.4 \\
HR $(95 \%$ CI $)$ & $0.37(0.27,0.52)$ & \\
$P$ & $<0.0001$ & \\
Median TTP, mo & NR & \\
HR $(95 \%$ CI $)$ & $0.34(0.23,0.48)$ & \\
$P$ & $<0.0001$ & \\
\hline
\end{tabular}

\section{MHC 20}

The Prevalence of the Aberrant Antigenic Expression in Acute Leukemia in a Tertiary Care Hospital

B. Chandrasekhar ${ }^{\#}$, Varun Bafna, K. S. Nataraj,
B. Shobha, Swasti Sinha, Sharat Damodar

Email ID for Correspondence: chandunims2009@gmail.com Narayana Health City, Bangalore

Introduction and Background: Acute leukemia (AL) are a heterogeneous group of malignancies with varying clinical, morphologic, immunologic, and molecular features and display characteristic patterns of surface antigen expression. Immunophenotyping improves both accuracy and reproducibility of acute leukemia classification. However aberrant expression of CD markers has been observed in several cases of acute leukemia. Flow cytometry is a technique used to analyze multiple phenotypic and functional parameters simultaneously within a single cell or a population of cells. Patient/Material and Methods: This is a prospective case control observational study, involved a cohort of 151 de novo acute leukemia patients. All cases were confirmed by bone marrow aspiration sample and peripheral blood smear (PBS) based on the morphology, cytochemistry, immunephenotyping and cytogenetics. Results: In the present study 151 cases of Acute Leukemia were studied to find out the prevalence of aberrant antigen expression. Of these total, 74 cases are of AML, 72 cases of ALL and 5 cases of MPAL. AML cases were sub divided in to eight FAB groups. Out of 74 AML cases $45(60.8 \%)$ showed lymphoid associated markers while in ALL, 30 (41.6\%) cases showed myeloid associated antigens expression out of 72. In our 74 AML patients, CD7 is positive in 27 cases (36.5\%) and CD56 in 6 cases (8.1\%), CD5 in 2 cases $(2.7 \%)$, CD 19 in $2(2.7 \%)$ and CD15 in 4 (5.4\%). So CD7 is the most prevalent aberrant phenotype in our AML cases. In our ALL cases most common aberrancy is CD33 accounting for $30.5 \%$ of total ALL cases followed by CD13 in $20.8 \%$ of cases. The most common cytogenetics in AML cases is normal karyotype, followed by complex karyotype, then $\mathrm{t}(8 ; 21), \mathrm{t}(15 ; 17)$, Trisomy 8 and others which is 
comparable to the literature. In our ALL cases, the most common cytogenetics is normal karyotype, followed by complex, then Hyperdiploidy, $\mathrm{t}(9 ; 22)$, Monosomy 7 , Trisomy 8 and others. The least common one was Hypodiploidy. Conclusions: Aberrant phenotypes are present in a great majority of acute leukemia patients of south India. It is essential to prove that, aberrant markers whether portend a poor prognosis compared to acute leukemia with conventional immunophenotypes showing expression of lineage specific markers, and a poor response to drug therapies. Future studies will be directed to correlate of these markers with prognosis and therapeutic response as still it is controversial.

\section{MHC 21}

Efficacy and Safety of Ibrutinib in Indian Patients with Relapsed or Refractory Chronic Lymphocytic Leukaemia and Mantle Cell Lymphoma: Cases from a Named Patient Program

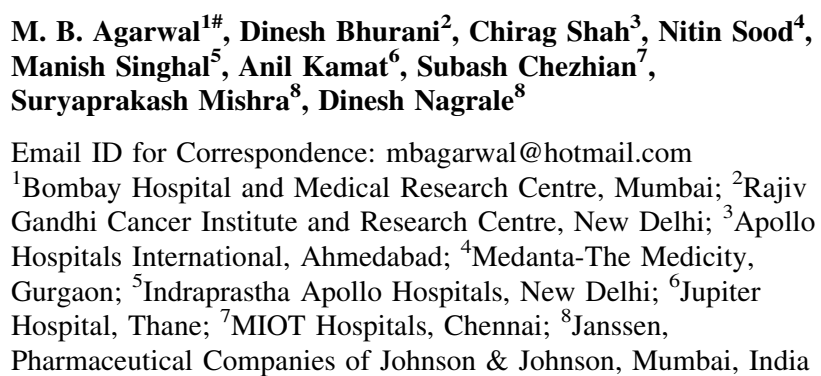

Introduction and Background: Ibrutinib, a selective inhibitor of Bruton's tyrosine kinase has demonstrated favorable clinical responses in global patient populations with refractory chronic lymphocytic leukaemia (CLL), mantle cell lymphoma (MCL) and CLL patients with chromosome 17 deletion (del17p)/TP53 mutation. However, ibrutinib treatment experience in Indian patients is unavailable. Named Patient Programs (NPPs) help provide controlled preapproval access to treatment in response to unsolicited requests by physicians on behalf of specific patients. We report the experience from a NPP describing the efficacy and safety of ibrutinib in Indian patients with relapsed/refractory CLL/MCL. Patient/Material and Methods: The 8 enrolled patients (refractory CLL: $\mathrm{n}=6$ including 4 patients with del17p; relapsed/refractory MCL: $n=2$ ) had median age of 55 years (range 52-60). Median number of prior therapies received by CLL patients was 3 while MCL patients received 4 prior therapies. All patients received once-daily dose of ibrutinib $(420 \mathrm{mg}$ : CLL, $560 \mathrm{mg}$ : MCL). Response assessments were conducted using clinical and laboratory investigations and safety (adverse events, AE) was monitored. Results: For CLL patients, median time on treatment at the time of analysis (July 2016) was 11 months (range 8-14); 5 patients continued treatment and 1 recommended for stem cell transplantation. In CLL patients, median (range) haemoglobin $(\mathrm{Hb})$ level and platelet count improved from baseline ( $\mathrm{Hb}, 9.8$ [7.2-11] to 12.0 [9.5-13.2] g/dL; platelet count, 150,000 [21,000-195,000] to 190,350 [130,000-394,000] cells/ $\mu \mathrm{L})$. Partial response (PR) was reported in 5 of $6(83 \%)$ CLL patients and 1 patient $(17 \%)$ achieved complete response (CR). Median time to response in CLL patients was 3 months (range $0.5-7$ ); 1 patient responded as early as 2 weeks (based on remission of symptoms: fever, lymphadenopathy and pleural effusion) of initiating ibrutinib. Of the 2 MCL patients, 1 achieved PR and 1 achieved CR within 4 and 2 months of treatment initiation, respectively. Haematuria and haematochezia occurred in 1 CLL patient that resolved upon interruption of treatment for 1 week without recurrence. Other AEs: infections (Herpes simplex virus infection and tonsillitis: $\mathrm{n}=1$, each), non-infective diarrhoea and skin lesion $(\mathrm{n}=1$, each) were managed effectively and mild skin depigmentation was observed in 1 patient. No serious AEs and no dose reductions or treatment discontinuation due to AEs were reported. Conclusions: In this first real-world experience in Indian patients, ibrutinib demonstrated therapeutic efficacy in MCL and CLL with/without del17p. Safety results in this study were consistent with the current known profile of ibrutinib.

\section{MHC 22}

Acute Lymphoblastic Leukemia Presenting as Pancytopenia: Clinical Characteristics and Treatment Outcome

\section{K. Rakul Nambiar ${ }^{\#}$, N. Geetha, Sreejith G. Nair, N. P. Prakash, T. M. Anoop}

Email ID for Correspondence: rakulnambiar@yahoo.com Department of Medical Oncology, Regional Cancer Centre, Trivandrum, Kerala 695011, India

Introduction and Background: Patients with acute lymphoblastic leukemia (ALL) presenting with pancytopenia pose a challenge to the treating hematooncologists in view of the complications they are prone to. There is scarcity of data on patients with ALL presenting with pancytopenia. This study is designed to assess incidence, clinic correlates and treatment outcome of patients with ALL presenting with pancytopenia. Patient/Material and Methods: This is a retrospective study of patients diagnosed as ALL in the Department of Medical Oncology at Regional Cancer Centre, Trivandrum during the period January 2008-December 2012. Patients presenting with pancytopenia and above 14 years of age are included in this study. Pancytopenia was defined as $\mathrm{Hb}<10 \mathrm{gm} \%$, TLC $<4500 / \mathrm{cmm}$, Platelet $<1 \mathrm{lakh} / \mathrm{cmm}$. Seventy patients met the above criteria and were the subjects of this study. Their clinical presentation, treatment details and survival are collected. Results: Among 819 patients diagnosed as ALL, 70 patients $(8.5 \%)$ presented with pancytopenia. There were 45 males and 25 females with a median age of 35 years (range 14-75 years). Main symptoms were fever, fatigue, bleeding and the median duration of symptoms was 4 weeks. Immunophenotype of these subjects were-B (31), pre B (19), pre T (13) and T (7). Two patients were Ph positive and myeloid co-expression was seen in 19 patients. Sixty two patients received curative treatment, 43 patients attained remission after induction chemotherapy, 16 patients died during induction and 28 patients completed the planned treatment. At a median follow up of 80 months, 22 patients (35\%) are alive. Conclusions: Pancytopenia is a rare presentation of ALL (8.5\%). There is a male predominance and myeloid co-expression is present in $27 \%$. Sepsis was the commonest cause of induction death which occurred in $20 \%$ of our cases. At a median follow up of 80 months, 22 patients (35\%) are alive.

\section{MHC 23}

Treatment of Invasive Fungal Infection in Hematology. Should We Fear Conventional Amphotericin B?

\section{Sreeraj", P. Mishra, G. Naveen, P. B. Govind, T. Seth, M. Mahapatra}

Email ID for Correspondence: sreerajfleming@gmail.com Department of Hematology, All India Institute of Medical Sciences

Introduction and Background: The mortality and morbidity due to Invasive fungal infection [IFI] is high in hematology patients. Amphotericin B deoxycholate (AmBD) is not preferred because of infusion-related adverse events [IRAE] and renal failure. Material and Methods: Non-interventional study of consecutive patients with IFI receiving $>7$ days of AmBD. A standardised infusion protocol 
containing age-adjusted doses of $0.9 \% \mathrm{NaCl}$, antihistamics, antipyretics, potassium, magnesium supplements through central lines, with thrice-weekly monitoring of electrolytes and renal functions was followed. End points were toxicity, treatment interruption and outcome. Results: 150 patients [mean age: 27.15 years] with AML [48 \%], ALL [27\%], aplastic anemia [21\%] were included. IFIs were proven [4\%], probable [34 \%] possible [51.3\%]. AmBD dose was $1 \mathrm{mg} / \mathrm{kg}$ in $81 \%, 1.5 \mathrm{mg} / \mathrm{kg}$ in $19 \%$. Total infusions were 3308 , with cumulative dose of $1145 \mathrm{mg} \pm 800 \mathrm{mg}$, for total of $22 \pm 15$ days. $43.3 \%$ responded to AmBD [ $25 \%$ with baseline IFI prior to chemo, $61 \%$ with IFI during chemo]. AmBD was stopped due to renal and electrolyte dysfunction in 10 [6.6\%] patients. 59 [39\%] patients died during treatment. 168 IRAE developed out of 3308 infusions in 48 patients [ $32 \%$ ]. Treatment was discontinued due to Grade-4 IRAE in $5 \%$. Incidence of IRAE was maximum at the start of the AmBD infusion, falling therafter over time, commonly fever and chills. The creatinine at treatment end was $0.896 \pm 0.61 \mathrm{mg} / \mathrm{dL}$ [range 0.1 to $3.3 \mathrm{mg} / \mathrm{dL}$ ]. There was a small rise in mean serum creatinine of $0.28 \mathrm{mg} / \mathrm{dl}$ because of a mean fall in GFR [37.8 $\mathrm{ml} /$ minute] during treatment $[\mathrm{p}=0.00]$. Doubling of creatinine from baseline developed in $28 \%$, of which $7.3 \%$ had creatinine $>2 \mathrm{mg} / \mathrm{dL}$ at end of therapy. $60 \%$ developed hypokalemia [mean $3.39 \pm 0.96 \mathrm{mEq} / \mathrm{L}]$. However both creatinine $[\mathrm{p}=0.58]$ and potassium [p $=0.12$ ] levels 30 days after stopping AmBD was similar to baseline. AmBD dose $>1 \mathrm{mg} / \mathrm{kg}$ [RR 2.05] and baseline fungal infection [RR 1.77] correlated with renal dysfunction. Conclusions: AmBD is associated with reversible renal dysfunction and manageable IRAE with efficacy comparable to liposomal preparations, provided appropriate supportive measures are strictly followed.

\section{MHC 24}

\section{5q deletion Myelodysplastic Syndrome}

\section{S. Kavya", Preethi S. Chari, Smith Chander, Sujay Prasad, Sundareshan}

Email ID for Correspondence: kavyasn10@gmail.com Anand Diagnostic Laboratory

Anemia is a common manifestation of various diseases, the causes of which are diverse. Detailed clinical history with relevant laboratory investigations plays a crucial role in appropriate disease diagnosis and management. Our patient was a 62 year old female who was referred to us for bone marrow examination as a part of evaluation of refractory anemia. Her peripheral blood counts showed reduced hemoglobin of $8 \mathrm{gm} / \mathrm{dl}$, white blood cell of 5590 cells $/ \mu 1$ and platelet count of 389,000 cells/ $\mu$ l. The peripheral blood smear revealed macrocytic red cells with anisocytosis. Bone marrow aspirate smears showed an increase in megakaryocytes, Pseudo Pelger Huet cells, erythrodysplasia in the form of mitosis and irregular nuclear borders. Trephine biopsy was remarkable for megakaryocyte hyperplasia that were seen distributed in the paratrabecular, intermediate and central regions of the marrow space. They appeared slightly smaller in size with most of them being mononuclear. Based on the above findings a diagnosis of myelodysplastic syndrome, $5 q$ deletion $(5 q-)$ was considered. Conventional karyotyping and GTG banding was done. Twenty metaphases were screened and fifteen karyotyped. The result was interpreted according to International System for Human Cytogenetic Nomenclature (ISCN 1995). There was interstitial deletion of long arm of chromosome 5 and reported as 46, XX, del (5) (q13q33), thus confirming our initial diagnosis. Fluorescent in situ hybridization indicated $5 \mathrm{q}-$ in $40 \%$ of analyzed cells.

\section{MHC 25}

Burkitt's Lymphoma in Adolescents and Adults: Clinical Characteristics and Treatment Outcome

\section{T. Sugeeth ${ }^{\#}$, N. Geetha, Sreejith G. Nair, N. P. Prakash, Lakshmi Haridas}

Email ID for Correspondence: sugeethmt@ymail.com Department of Medical Oncology, Regional Cancer Centre, Trivandrum, Kerala 695011, India

Introduction and Background: Burkitt's lymphoma (BL) is a highly aggressive B cell Non Hodgkin's lymphoma (NHL) associated with 3 distinct subtypes; endemic, sporadic and immunodeficiency associated. Sporadic BL accounts for only $1 \%$ of adult NHL. We present our experience with BL in adolescents and adults. Patient/Material and Methods: This is a retrospective study of 60 patients with BL treated in the Department of Medical Oncology at Regional Cancer Centre, Trivandrum, India during the period 2005-2014. Patients above 14 years of age were included and their clinical presentation, treatment details and outcome were studied. Results: Among 3811 cases of NHL treated during the study period, 60 cases were BL $(1.57 \%)$. There were 41 males and 19 females. The median age at presentation was 42 years (range 14-81 years). The main symptoms were lymphadenopathy, abdominal pain and abdominal mass. Two patients each had paraparesis, breast lump and jaw swelling and one patient had involvement of the cervix. B symptom was present in 39 patients. Thirteen patients had features of tumor lysis at presentation. The median duration of symptom was 4 weeks. Sixteen patients had Burkitt's leukemia. The Ann Arbor stage was I in 17, II in 16, III in 5, and IV in 22. Fifty five patients received combination chemotherapy which included R-HyperCVAD (28), HyperCVAD (7), RCHOP (7), CHOP (6), BFM (4), others (3). Thirty four patients attained remission, 13 patients had progressive disease and 8 patients died during chemotherapy. At a median follow up of 65 months, 33 patients $(60 \%)$ are alive. Conclusions: BL accounts for $1.57 \%$ of NHL above the age of 14 years and has male predominance. Intensive, short-duration combination chemotherapy is the preferred treatment in BL. In our study $60 \%$ of patients are alive at a median follow up of 65 months.

\section{MHC 26}

Mutation Profile in Indian Primary Myelofibrosis Patients and Its Clinical Implications

\section{Vinod R. Patil ${ }^{\#}$, S. Chandrakala, W. Nilesh, Farah Jijina}

Email ID for Correspondence: dr.vrp85@gmail.com Dr. J C Patel Department of Clinical Hematology, KEM Hospital, Mumbai

Introduction and Background: Primary myelofibrosis (PMF) is a myeloproliferative neoplasm (MPN) characterized by abnormal proliferation of megakaryocytes, bone marrow fibrosis, and extramedullary hematopoiesis. We did mutation profile of 40 newly diagnosed or follow up patients of PMF and tried to correlate it with initial clinical presentation of these patients. Patient/Material and Methods: This prospective study was done in department of clinical hematology of KEM hospital Mumbai from November 2015 to June 2016. We included either new patients or follow up patients of PMF. Mutation profile included JAK2 V617F, JAK2 Exon 12, CALR and MPL mutation. Three groups viz; JAK2 positive, CALR positive and 
all negative were studied and compared. Results: Out of 40 patients, 22 were males and 18 were females. Of these 27 were JAK2 positive $(67.5 \%), 10$ were CALR positive $(25 \%)$ and 3 were all negative $(7.5 \%)$. On clinical presentation, CALR positive patients were younger in age, had larger spleen, more reticulin fibrosis of marrow, low DIPSS score and had less chances of thrombosis as compared to JAK2 positive patients. Conclusions: CALR positive patients have better clinical parameters at presentation and have overall better prognosis as compared to JAK2 positive patients. Larger and more elaborative study is necessary to reemphasize abovesaid findings. Incidence of JAK2 and CALR mutations in Indian PMF patients is fairly similar to that in rest of the world.

\section{MHC 27}

A Retrospective Study of Transplant Related Mortality in Patients Undergoing Bone Marrow Transplantation at GCRI Ahmedabad: Extracting Positives from the Negative!

\section{Rahul S. Kulkarni", Asha S. Anand, Harsha P. Panchal, Apurva A. Patel, Sonia K. Parikh, Kamlesh M. Shah, Kinnari A. Patel, Kalpesh Prajapati, Gaurang Modi, Sandip A. Shah}

Email ID for Correspondence: r_kulkarni03@yahoo.co.in; dr.rsk08@gmail.com

Gujarat Cancer and Research Institute, Ahmedabad, India

Introduction and Background: Transplant Related mortality (TRM) is a major negative outcome in patients undergoing BMT. Over last few years there have been advances and research in the field of BMT in order to minimize TRM. There is paucity of Indian data on various causes of TRM and outcomes of BMT in resource limited settings. Analysis of TRM can help us to understand causes and trends in Indian setup and guide in taking essential steps in prevention of the same. Patient/Material and Methods: All patients who underwent BMT for both malignant and non malignant causes at GCRI since 1999 till feb 2016 were retrospectively evaluated and various causes of TRM and non TRM were analysed at 30 days, 100 days and 1 year. Results: TRM was higher in females than in males at 30 days (10.2 \% Females; $3.2 \%$ Males) and 100 days (12.8\% Females; $10.7 \%$ Males). 30 day TRM was $5.03 \%(8 / 159)$ in autologous BMT and $5.26 \%(7 / 133)$ in allogenic BMT. 100 day TRM was $8.17 \%$ for autologous BMT and $10.5 \%$ for allogenic BMT. Sepsis (Gram Negative) was the most common cause of both 30 day and 100 day TRM in autologous BMT $(37.5 \%$ and $46.15 \%$ respectively) and 30 day TRM in allogenic BMT (37.5\%) whereas acute GVHD was the most common cause of 100 day TRM in allo TRM $(50 \%)$. Highest 30 day and 100 day TRM for autologous BMT was seen in NHL mainly in heavily pretreated and elderly patients $\{7.14 \%$ and $14.2 \%$ respectively (excluding single cases of Ewings sarcoma and GCT) $\}$ and in MDS for allogeneic BMT (10\% and $40 \%$ respectively). Overall most common cause of death at end of 1 year was disease relapse in autologous BMT and GVHD in Allogenic BMT. 100 day TRM in MUD was $33.3 \%$ (1/3) and that in unrelated umbilical cord transplant was $0 \%(0 / 11)$. Conclusions: Newer antibiotics, antifungals and efficient blood component support has made BMT relatively safe and effective with high response rates even in resource limited settings. However sepsis and acute GVHD still remains the most common cause of 100 day TRM in autologous and allogeneic HSCT respectively. Early intensive and multispeciality care, reduced intensity conditioning regimens, and newer approaches for prevention and treatment of GVHD can further reduce TRM.

\section{MHC 28}

Extensive Bony Lytic Lesion in a Child with Acute Lymphoblastic Leukemia: A Case Report

Anil Sharma", Nishant Verma, Prof. Archana Kumar, Gitika, Bhvya

Email ID for Correspondence: anilmbbsbhu@gmail.com KGMU, Lucknow

Introduction and Background: Acute Lymphoblastic Leukemia (ALL) is the most common malignancy in children that comprises about $41 \%$ of the malignancies in children younger than 15 years old. Although ALL usually presents with fever, pallor, petechiae, ecchymosis in skin and mucus membranes, growing skeleton is an important site for proliferation of leukemic cells; therefore, during the course of disease, tenderness and multiple areas of bone destruction and repair due to infiltration of leukemic cells in the bone marrow may be seen. Patient/Material and Methods: A 30 months old girl presented in out-door department with fever, severe back pain, difficulty in sitting and walking due to pain. Examination revealed pallor and generalised bony tenderness. Complete blood count showed anaemia $(7.4 \mathrm{gm} / \mathrm{dL})$ and normal counts (TC-10,400 with $58 \%$ lymphocytes). A skeletal survey by radiography was done, which demonstrated extensive lytic lesions involving the skull, spine, visualized ribs, bilateral scapula, humerus, iliac bones, femur and tibia. Biopsy was done from one of the lytic lesions which revealed infiltration by blasts. These blasts stained positive for LCA, CD99, Tdt, CD19, and negative for S100 on immunohistochemistry (IHC). A bone marrow aspiration and flowcytometry was also done, which confirmed the diagnosis of CD10+, B-cell ALL. Child's Karyotype was 46, XX, inv(9) (q10[12]/46, XX[08]. Results: After obtaining a diagnosis of ALL (B-cell; standard risk) standard 3-drug induction chemotherapy (Vincristine, Dexamethasone, L-asparaginase) was started. Child tolerated the induction chemotherapy well and her symptoms and bony lesions showed a remarkable improvement. Child is now able to walk, bony pain has decreased and bony lytic lesions are resolving. Conclusions: In children presenting with extensive lytic lesions involving the entire skeleton ALL should be considered as a probable etiology. The diagnosis can be confirmed by performing IHC on the biopsy from lytic lesion or by performing a bone marrow aspiration. These bony lesions respond very well to treatment.

\section{MHC 29}

CNS Complications in Acute Leukemia: A Tertiary Care Centre Experience

\section{Shirali Agarwal ${ }^{\#}$, Prakash Gouda, S. Sirisha Rani, Lokesh Lingappa}

Email ID for Correspondence: shirali.agarwal@gmail.com Rainbow Children's Hospital, Hyderabad

Introduction and Background: Neurological complications either at diagnosis or while on chemotherapy can be minimized to improve overall survival in Acute Leukemia. This is one of the rare studies from India describing spectrum of neurological problems at a single centre. Patient/Material and Methods: It is a cross sectional observational study at Rainbow Children's Hospital held over a period of 4 year 9 months between May 2011 to Jan 2016. All admitted patients between age of 6 months to 15 years with neurological complications in leukemia were included. Results: Out of 296 
children diagnosed with Acute Leukemia, 36 i.e. $12 \%$ developed acute neurological complications. Male:Female ratio was 1.5:1. Median age of diagnosis was 52 months (14-186 months). 25 had B Cell ALL, 10 AML and 1 T-ALL. Seizures seen in 17 (47\%), walking difficulty $8(20.5 \%)$, headache $(11.7 \%)$, stroke (3), drowsiness (3), aphasia (2), facial palsy (2), and one each had abnormal movement and urinary retention. Cause of seizures were Methotrexate neurotoxicity (7), PRES-(1), SVT (3), idiopathic (2), 1 each had Ventriculitis, Varicella Encephalopathy, CNS Bleed, Leucoencephalopathy. Majority of methotrexate induced seizures happened during consolidation phase. Amongst 8 children with walking difficulty 5 had Vincristine induced neuropathy and one case each had MTX and Cytarbine induced Neuropathy and one had spinal compression due to tumor cells. Cause of headache was MTX induced chemical meningitis, sinus venous thrombosis, fungal sinusitis and thrombocytopenia induced parenchymal hemorrhages. 7 children developed MTX induced stroke. All recovered completely after folinic acid rescue except 1 who had mild residual hemiparesis. CT brain was done for 18 children out of which 13 were abnormal and 11 children underwent MRI brainwhich was abnormal in 10. MRA or MRV done for 2 children was abnormal. 2 out of 4 had abnormal Nerve conduction. 17 children required anticonvulsants, Neuropathy was treated with Pyridoxine and Gabapentin and PRES with supportive care. Out of 36 children, 4 died (2AML with relapse, 1 APML with CNS bleed and 1 ALL with hemorrhagic pancreatitis), 4 had mild residual disabilities 1 lost to follow up. Remaining (25) recovered completely. Conclusions: Acute Leukemia is associated with myriad of neurological complications. Incidence increases with cumulative exposure of chemotherapeutic drugs. The complications are usually reversible if diagnosed early and treated appropriately as reported in our study.

\section{MHC 30}

Long Term Outcome of Hairy Cell Leukemia from a Tertiary Care Centre in South India

\section{Archit Joshi" $^{\#}$ D. Manikandan, T. G. Sagar, Prasanth Ganesan \\ Email ID for Correspondence: archit_jsh@yahoo.co.in Cancer Institute, Chennai}

Introduction and Background: Hairy cell leukemia (HCL) is a rare chronic leukemia which usually presents with pancytopenia and splenomegaly. Purine nucleoside analogue (cladribine) produces remarkably high remission rates. Patient/Material and Methods: Data of all consecutive patients of HCL diagnosed and treated in Cancer Institute, Chennai between January 2001 and December 2015 was retrospectively obtained from medical records. Response to therapy and survival outcomes were analyzed. Results: Twelve patients were diagnosed with HCL and treated at our centre. Median age of presentation was 52.5 years. Male to female ratio was 5:1. Eight patients $(75 \%)$ had symptomatic splenomegaly at presentation. Median haemoglobin, total leucocyte count and platelets were $7.9 \mathrm{gm} / \mathrm{dl}, 4200$ cells $/ \mathrm{mm}^{3}, 83,000$ cells $/ \mathrm{mm}^{3}$ respectively. 9 patients received cladribine, 2 patients received interferon and 1 patient did not receive anti cancer therapy due to poor general condition. All treated patients $(\mathrm{n}=11)$ achieved complete remission. Median duration of response was 86 days. 3 patients $(33 \%)$ who received cladribine developed febrile neutropenia. Only $2 / 9$ patients receiving cladribine relapsed and were retreated with rituximab. One patient who received interferon, relapsed and, seven patients remain disease free since the first line of cladiribine, after a median follow up of 56 months (range: 22-147). Median PFS (progression free survival) and OS (overall survival) were 51 and 56 months respectively. The 5-year OS and 5-year PFS were $75 \%$ and $83.3 \%$ respectively.
Conclusions: HCL is a rare cancer with excellent outcomes with single agent cladribine. Cladribine can be safely delivered with appropriate precautions.

\section{MHC 31}

Enteropathy Associated T Cell Lymphoma (EATCL)—An Update of Our Experience

\section{Sumit Gupta\#, Tanuja Shet, Sridhar Epari, Sumeet Gujral, Hashmukh Jain, Manju Sengar, Hari Menon}

Email ID for Correspondence: agent.sumit88@gmail.com Department of Pathology and Adult Hematolymphoid Unit, Tata Memorial Hospital, Parel, Mumbai

Background: There are not many reports on EATCL in India. Our own study reported only 4 cases in 2009 . We wanted to recheck the incidence and disease pattern in Indian patients in present day. Material and Methods: A total of 16 cases labeled as EATCL were retrieved from archives in last 10 years. A wide immunohistochemistry and in situ hybridization panel for EATCL viz: CD4, CD8, EBERISH, CD56, TIA1 were done. Of these after workup In the 11 were confirmed as EATCL and 5 were reclassified as NK-T cell lymphoma ( 2 cases) and PTCLNOS (3 cases). Results: All patients with EATCL were $>25$ years of age and those reported $<25$ years were reclassified as NK/T cell lymphoma based on strong EBERISH staining. Of the 11 confirmed cases, 3 had history of celiac disease, one had lactose intolerance and others did not give history of celiac disease. Of the 11 cases confirmed as EATCL-10 affected the ileum predominantly and 1 involved duodenum. The two cases of jejunum involvement were reclassified as NK/T cell lymphoma. In 9 of these adjacent normal small intestine was available for analysis and showed enteropathy changes in 4 (besides the 3 with celiac disease one additional case). Histologically based on latest WHO classification 6 could be classified as EATCL type I (included 3 cases with history of celiac disease, one without history but with Enteropathy and one with lactose intolerance) and 5 as EATCL type II. EATCL type I usually presented with perforative peritonitis while none of EATCL type II had perforation. Only two patients presented with limited stage disease (stage II) while in others there was disseminated peritoneal disease (qualifying for Ann Arbor stage IV). Therapy details were available in 7 EATCL patients of whom 4 received CHOP and 3 gemcitabine (GDP or GCEOP) based therapy. Of these only three patients attained complete response and showed DFS of one to three years, after which they relapsed and succumbed to the disease, all other EATCL died without disease remission. Conclusion: Both EATCL type I and type II are seen in India and is seen in patients $>25$ years of age. NK/T cell lymphomas in $<25$ years mimic EATCL and should be recognized. The disease has poor prognosis however some patients do manage to receive CR.

\section{MHC 32}

\section{Endothelial Dysfunction in Diabetic Nephropathy}

\section{Nishu Bhardwaj ${ }^{\#}$, Satendra Sharma, O. P. Kalra, Meera Sikka,} Sonal Sharma

Email ID for Correspondence: nishu89.nb@gmail.com University College of Medical Sciences and GTB Hospital

Introduction and Background: Nitrous oxide (NO) plays an important role in the regulation of renal hemodynamics. Endothelial dysfunction and reduced NO generation are known to induce renal injury. Familial clustering seen in diabetic nephropathy (DN) points 
to a role of genetic factors in the pathogenesis of renal disease. Polymorphisms in the eNOS gene may alter its expression, thus affecting the production of NO. The present study was conducted to determine the role of eNOS G894T gene polymorphism in endothelial dysfunction and its association with the risk of developing DN. Material and Methods: The study comprised of three groups with 50 subjects each (group 1: DN cases; group 2: diabetics without nephropathy; group 3: controls). Venous blood was collected from all subjects for measurement of serum NO level by spectrophotometry and DNA extraction by phenol-chloroform extraction method followed by PCR-RFLP to determine the eNOS G/T polymorphism. Results: The frequencies of GT and TT genotypes were significantly higher in DN patients (34 \% and $26 \%$, respectively) than in diabetics without nephropathy (22\% and $4 \%$, respectively) and controls (24\% and $4 \%$, respectively) ( $\mathrm{p}<0.001)$. Serum NO level in subjects with GG genotype $(45.1 \pm 27.8 \mu \mathrm{M})$ was significantly higher than in those with TT genotype $(26.9 \pm 18.1 \mu \mathrm{M})(\mathrm{p}=0.029)$. The carriers of $\mathrm{T}$ allele had significantly lower levels of nitric oxide in their serum $(35.4 \pm 24.8 \mu \mathrm{M})$ as compared to subjects with only $\mathrm{G}$ allele $(45.1 \pm 27.8 \mu \mathrm{M}) \quad(\mathrm{p}=0.034)$, indicating that the eNOS G894T gene polymorphism may alter the production of NO. Conclusions: This study indicates a possible role of eNOS gene polymorphism in the pathogenesis of DN. The eNOS G894T gene polymorphism may contribute to the endothelial dysfunction in DN and may be a potential genetic marker for the risk of developing nephropathy in diabetic patients.

\section{MHC 33}

\section{After Haploidentical Hematopoietic Stem Cell Transplantation}

\section{Narendra Agrawal ${ }^{\#}$, Jyotsna Kapoor, Niharika Bhatia, Megha Gupta, Manish Sharma, Priyanka Verma, Rayaz Ahmed, Dinesh Bhurani}

Email ID for Correspondence: narendra_ag1@ rediffmail.com Rajiv Gandhi Cancer Institute and Research Centre, Delhi

Introduction and Background: Haplo-identical Hematopoietic Stem Cell Transplantation (HaHSCT) has evolved as standard therapy for patients who do not have HLA identical donor. Risk of CMV reactivation post HaHSCT is expected to be higher because of intense immune-suppression. Patient/Material and Methods: Records of all patients who underwent HaHSCT since October 2011 to April 2016 were reviewed. GvHD prophylaxis consisted of post-transplant Cyclophosphamide on day $+3,+4$ and Tacrolimus \& short course of MMF from day +5 onwards. Serial monitoring of CMV DNA on blood was done by weekly quantitative PCR starting after engraftment till day 100 and later as required. All CMV DNA copies more than $>500$ were treated preemptively with twice a day Gancyclovir till CMV antigenemia resolve. Results: A total of 42 patients with median age 34.5 years (9-63 years), female to male ratio of $1: 2.5$, were included. The majority $(73.8 \%, \mathrm{n}=31)$ received nonmyeloablative (NMA) conditioning regimen, using peripheral blood graft in $90.4 \%(\mathrm{n}=38)$ transplants. Forty one recipients were CMV sero positive. CMV reactivation occurred in $57.1 \%(\mathrm{n}=24)$ patients at median 35 days (20-56) post HaHSCT, out of which 7 had recurrent reactivation. CMV pneumonia developed in one patient (expired at day +30 ). Disease status prior to transplant, donor relationship, use of PBSC as a graft source, presence of acute/chronic GvHD, use of steroids pre and post-transplant were found to be significantly associated with CMV reactivation, while the sex, type of conditioning regimen, use of total body irradiation or ATG, use of second/third line regimens for GvHD remained insignificant. At median follow up of approximately 7.5 months (0.5-56.7 months), $45.2 \%(\mathrm{n}=19)$ of patients are alive and $35.7 \%(\mathrm{n}=15)$ patients had event free survival. Conclusions: HaHSCT patients are at high risk of CMV reactivation though the CMV disease and mortality is preventable with monitoring and pre-emptive therapy.

\section{MHC 34}

Impact of Cytogenetics on Outcomes in Pediatric Acute Lymphoblastic Leukemia-An Institutional Experience

\section{Rachana\#, G. Sadashivudu, Meher Lakshmi, Ravi Sankar, Stalin Bala}

Email ID for Correspondence: drrachanach@gmail.com Nizam's Institute of Medical Sciences

Introduction and Background: Cytogenetic analysis of leukemic blasts plays an important role in classification and prognosis in acute lymphoblastic Leukaemia (ALL). The purpose of this study is to determine the outcomes based on chromosomal abnormalities in children and adolescents with ALL. Patient/Material and Methods: A total of 220 patients with age less than 21 years and diagnosed as ALL at our institute between January 2011 to June 2016 were retrospectively analysed. Patients were sub grouped into five categories based on cytogenetic studies. Post induction responses and survivals were analysed for these groups. Results: The median age at presentation was 13 years (range 6 months21 years). The median hemoglobin, total leucocyte count and platelet counts were $8 \mathrm{gm} / \mathrm{dl} \quad(1.2-14 \mathrm{gm} / \mathrm{dl}), \quad 10,000 / \mathrm{mm}^{3}$ (500-4.2 Lac/ $\left./ \mathrm{mm}^{3}\right), 40,000 \mathrm{~mm}^{3}\left(6000-5.4 \mathrm{~L} / \mathrm{mm}^{3}\right)$ respectively. The five karyotype categories were normal diploid $[\mathrm{n}=74$ $(63 \%)], \mathrm{t}(9 ; 22)$ translocation $[\mathrm{n}=16(14 \%)]$, other unfavourable cytogenetics [which include complex cytogenetics, trisomy 8 , or $11 \mathrm{q} 23$ rearrangement, $\mathrm{n}=10(8.5 \%)]$, favourable cytogenetics [including high hyperdiploidy, $\mathrm{t}(12 ; 21), \mathrm{t}(1 ; 19), \mathrm{n}=10(8.5 \%)$ ] and miscellaneous $[n=7 \quad(6 \%)]$. Post induction absence of complete remissions and persistence of disease were higher in patients with $\mathrm{t}(9 ; 22)$ translocations and remaining 4 groups had similar rates complete remission and persistence disease. Response rates were similar in $\mathrm{B}(\mathrm{CR}-91 \%$, Persistent-9 \%) and $\mathrm{T}(\mathrm{CR}-$ $95 \%$, persistence-5 \%) lineages. Median EFS and OS of these 5 subgroups are tabulated in Table 1. EFS was significantly low for patients with $\mathrm{t}(9 ; 22)$ translocation $(4 \mathrm{~m}, \mathrm{p}=0.0049)$ (Table 1). Conclusions: Overall survival and Event free survival is better in those with favourable cytogenetics and worse with those with $t(9 ; 22)$ translocation and unfavorable cytogenetics. Cytogenetics have no impact on response to induction chemotherapy in ALL.

\section{Table 1}

\begin{tabular}{lcrrrrr}
\hline $\begin{array}{l}\text { Cytogenetic } \\
\text { group }\end{array}$ & $\begin{array}{l}\text { No. of } \\
\text { patients }\end{array}$ & CR & PR & $\begin{array}{l}\text { Persistent } \\
\text { disease }\end{array}$ & EFS & OS \\
\hline Normal & 74 & $59(79 \%)$ & $14(19 \%)$ & $2(2 \%)$ & 15 & 33 \\
$\mathrm{t}(9 ; 22)$ & 16 & $9(56 \%)$ & $2(13 \%)$ & $5(31 \%)$ & 4 & 6 \\
Unfavourble & 10 & $7(70 \%)$ & $2(20 \%)$ & $1(10 \%)$ & 9 & 19 \\
Favourable & 10 & $7(70 \%)$ & $2(20 \%)$ & $1(10 \%)$ & 13 & 48 \\
Miscellenous & 7 & $6(86 \%)$ & $1(14 \%)$ & $0(0 \%)$ & 9 & 11 \\
All & 117 & $87(74 \%)$ & $21(18 \%)$ & $9(8 \%)$ & 12 & 22 \\
\hline
\end{tabular}




\section{MHC 35}

Comparing Single-Agent Ibrutinib, Bendamustine Plus Rituximab (BR) and Ibrutinib Plus BR in Patients with Previously Treated Chronic Lymphocytic Leukemia/Small Lymphocytic Lymphoma (CLL/SLL): An Indirect Comparison of the RESONATE and HELIOS Trials

Peter Hillmen, Graeme Fraser, Jeffrey Jones, Simon Rule, Susan O'Brien, Marie-Sarah Dilhuydy, Ulrich Jaeger, Sebastian Grosicki, Florence Cymbalista, Steven Sun, Joi Ninomoto, Michelle Mahler, Mei Cheng, Joris Diels, Fong Clow, Mariya Salman, Danelle James, Angela Howes, S. Prudwidhar", Asher Chanan Khan

Email ID for Correspondence: ps10@ITS.JNJ.com Janssen Oncology, India

Background: In 2 large phase 3 trials including relapsed/refractory CLL/SLL patients, single-agent ibrutinib was superior to ofatumumab (RESONATE; Byrd. NEJM 2013) and ibrutinib+BR was superior to placebo+BR (HELIOS; Chanan-Khan. Lancet Oncol 2016). In the absence of head-to-head evaluations of single-agent ibrutinib vs. BR or single-agent ibrutinib vs. ibrutinib+BR, this indirect comparison from RESONATE and HELIOS provides a framework to establish relative treatment efficacy. Methods: In RESONATE, patients received oral ibrutinib $(420 \mathrm{mg} / \mathrm{d})$ or intravenous ofatumumab for up to 24 weeks. In HELIOS, patients received BR ( $\leq 6$ cycles) with either oral ibrutinib $(420 \mathrm{mg} / \mathrm{d})$ or placebo. In both studies, primary endpoint was progression-free survival (PFS); overall survival (OS) was secondary endpoint. Per protocol amendment in both studies, patients in comparator arms could switch to ibrutinib upon IRCconfirmed progression. This exploratory analysis was based on available data (median follow-up: RESONATE, 19 months; HELIOS, 17 months) using investigator assessments, but excluding del17p patients. Separate multivariate Cox proportional hazards models were constructed for PFS and OS including treatment and clinically relevant prognostic variables as covariates. Results: Both studies enrolled different populations making naïve comparison flawed. In adjusted analyses using the overall CLL/SLL population, PFS and OS were comparable for ibrutinib+BR vs. single-agent ibrutinib (hazard ratio [95\% CI]: 1.07 [0.65-1.76], $p=0.7808$ and 1.18 [0.61-2.29], $p=0.6281$, respectively), but were significantly improved for singleagent ibrutinib vs. BR $(0.15$ [0.09-0.23], $p<0.0001$ and 0.51 [0.27-0.96], $\mathrm{p}=0.0371$, respectively). Results for CLL patients only were similar. Conclusions: This adjusted indirect comparison suggests superiority of single-agent ibrutinib over BR for PFS and OS in relapsed/refractory CLL/SLL patients. Addition of BR to ibrutinib did not improve PFS or OS vs. single-agent ibrutinib. However, as median PFS was not reached in either ibrutinib arm, firm conclusions cannot be drawn. Overall, these findings suggest single-agent ibrutinib as an appropriate choice for previously treated CLL/SLL patients.

\section{MHC 36}

Global Mantle Cell Lymphoma (MCL) Named Patient Program (NPP) Experience in $>700$ Patients Treated with Ibrutinib

Simon Rule, Joris Diels, Nollaig Healy, Wafae Iraqi, Johan Aschan, Dinesh Nagrale\#, Mark Wildgust

Email ID for Correspondence: dnagrale@ITS.JNJ.COM Janssen Oncology, India

Background: A global NPP was opened in numerous countries allowing access to ibrutinib for relapsed/refractory MCL prior to approval. Methods: In an analysis of ibrutinib ordering/reordering in the global ibrutinib NPP, we estimated patient time-on-treatment using Kaplan-Meier analysis and Cox proportional hazard regression. Reordering data were censored at the date of last ibrutinib supply (resupplied every 1-3 months). Patients transferring to commercial ibrutinib after approval were censored at NPP closure in their country. Limited baseline information collected at NPP enrollment allowed additional exploration of time-on-treatment via multivariate analysis. Results: 715 MCL patients from 26 countries were analyzed (median age 70 years; $76.1 \%$ male) with $52.3 \%$ (95\% CI $43.5-60.4 \%$ ) remaining on-treatment at 12 months. This estimate was consistent with 12-month time-on-treatment (57.6\% [95\% CI 48.9-65.3\%]) and PFS (58.0\% [95\% CI 49.3-65.7\%]) rates observed in the phase 3 RAY study of ibrutinib for relapsed/refractory MCL (inclusion criteria were similar for the MCL NPP and RAY). Moreover, KaplanMeier curves for time-on-treatment for the global MCL NPP and RAY were not statistically different (MCL NPP vs. RAY: HR, 1.14 [95\% CI 0.83-1.54]). Timing of MCL diagnosis was the only independently significant variable in the multivariate analysis, with timeon-treatment being longer in patients diagnosed with MCL within the last 2 years. Of note, in this analysis, neither refractory disease, advanced disease, relapsed disease, nor response to the previous therapy, additionally impacted time-on-treatment. In total, 168 patients $(23.5 \%)$ discontinued treatment during the observation period, the most common reasons being death $(10.8 \%)$, disease progression $(7.3 \%)$, and AEs (1.3\%). Discussion: Although NPP data are based on physician declarations and are unmonitored, this analysis provides a real-world estimate of time-on-treatment, which can be considered a conservative proxy for PFS. Estimates were similar to RAY, suggesting that results observed in ibrutinib MCL clinical trials are reproducible in clinical practice.

\section{MHC 37}

Incidence of Complications Among Centrally Inserted Catheters in Haem-Onc Cases

Raghuveer Nanduri", Akshay Middinti, Vaishnavi Veerapaneni, K. V. Krishnamani, Anil Aribandi

Email ID for Correspondence: nanduriraghuveer@gmail.com American Oncology Institute

Introduction and Background: To study complications of centrally inserted catheters in cases of Haematological malignancies over 20 months period. Patient/Material and Methods: A retrospective study of 68 centrally inserted catheters and possible complications. These included 53 PICC lines, 6 Chemoports, 3 Hickman lines and 6 other central lines. The study group included male to female ratio 46:22 between 3 yrs to 79 yrs of age. Results: Main indications for line insertions were chemotherapy for AML (36.7 \%), ALL (32.3\%), HL $(10.2 \%)$, MM $(8.8 \%)$. Of PICC lines, 9 were removed suspecting infection (3 of them grew Escherichia coli, Staphylococcus hemolyticus and Pseudomonas aeruginosa) and one each for thrombosis, dislodgement and lumen fracture. Among 6 Chemoports, one was positive for Enterococcus gallinarum and Pseudomonas aeruginosa (Double infection) and one was removed due to leakage. Of 3 Hickman, one was insitu lasting 187 days and rest were removed post treatment. Conclusions: Incidence of line removal for all reasons was $14(20.5 \%)$. This is slightly lower compared to other studies. With asepsis, more infectious complications can be avoided and lines can be salvaged. Reference: Dr Arun K L et al. Experience with Hickman catheters and implantable ports. GCRI Journal. 2012; 14: 28-29. 


\section{MHC 38}

Role of Day 14 Interim Bone Marrow in Predicting Remission on Day 28

\author{
Shilpa Prabhu", Sharat Damodar, K. S. Nataraj \\ Email ID for Correspondence: shilpasgp@gmail.com \\ Narayana Hrudayalaya
}

Introduction and Background: AML is genetically heterogenous disease in which somatic mutations that disturb cellular growth, proliferation and differentiation accumulate in the hematopoietic progenitor cells. Management of AML typically involves day 14 marrow following induction chemotherapy with anthracyclines and cytarabine. The study aims to assess the value of day 14 marrow in predicting remission on Day 28. Patient/Material and Methods: A retrospective study (2012-April 2016) of AML patients undergoing routine induction chemotherapy were included in the study. Bone marrow examination was done on day 0 (day of diagnosis) day 14 (of induction chemotherapy) day 28 (recovery) and analyzed. Cytogenetics and mutation analysis were done at diagnosis and patients were risk stratified accordingly. Results: Study population comprised of 80 subjects, 48 were males and 32 females. Median age at presentation was 35 years. Among the 80 subjects, 55 (68.8\%) were denovo and $25(31.3 \%)$ were relapsed. The median blast percentage in bone marrow on day 0 , day 14 and at recovery were $68 \%, 3 \%$ and $4 \%$ respectively. The Fisher's exact test was employed to assess for associations between response to treatment at D14 and likelihood of recovering in CR. $20 \%$ of the patients had high risk cytogenetics at presentation. 45 $(56.25 \%)$ of the patients achieved remission on day 28 . Conclusions: The study showed that there was significant correlation between day 14 marrow blasts and day 28 remission. Status ( $p$ value $<0.0001$ ). However there was no significant correlation between day 5 peripheral blood blasts and remission status on day 28 .

\section{MHC 39}

ALK Negative Systemic Anaplastic Large Cell Lymphoma Presenting with Breast Mass and Paraparesis in an Adult Female-A Case Report

\section{Ritasman Baisya ${ }^{\#}$, Urmimala Bhattacharjee, Kristi Chattopadhyay, Tamoghna Biswas}

Email ID for Correspondence: ritasman91@gmail.com Medical College \& Hospital, Kolkata

Introduction and Background: Anaplastic large cell lymphoma (ALCL), a subtype of $\mathrm{T}$ cell lymphoproliferative disease, is a distinct clinicobiological entity with cutaneous and nodal involvement mostly. Frequency of this lymphoma is more in male pediatric population. In this case, ALCL was presented with diffuse systemic manifestions involving lung \& bone in an adult female and it was of $\mathrm{ALK}^{-}$type revealing more unfavourable outcome than $\mathrm{ALK}^{+}$ALCL. Patient/Material and Methods: Parbati Basu, a 24 year old female, presented with bilateral lower limb weakness with a lump on left breast. Weakness was of upper motor neuron type with acute tenderness at $\mathrm{T} 7 \mathrm{vertebral} \mathrm{level.} \mathrm{Cervical} \mathrm{and}$ axillary lymphadenopathy were present. 8 months back, she had history of superior vena cava obstruction for which treated outside and became symptomatically well. Results: Skiagram of dorsal vertebra revealed a wedge shaped collapse at T7 level. Contrast enhanced CT scan of chest, neck and whole abdomen revealed large mediastinal mass with calcific fous, numerous enhancing lung SOL, large enhancing mass in left breast, with paravertebral nodal mass insurating in neural foramina of dorsal vertebra causing cord compression. MRI dorsal spine showed posterior epidural soft tissue lesion extending from T6 to T8 level. Biopsy from breast mass and cervical lymph node were done and was suggestive of non-
Hodgkin lymphoma. Immunohistochemistry was positive for CD30, CD3, vimentin, EMA but negative for ALK 1, CD15, PAX 5. Patient was diagnosed to be a case of ALK negative systemic ALCL (ALK ${ }^{-}$ALCL). Conclusions: In new WHO classification, $\mathrm{ALK}^{-} \mathrm{ALCL}$ is a provisional entity. Though evidence of this lymphoma with extranodal involvement in adult female is rare in literature, this case revealed the widespread nature of this unfavourable lymphoma with diffuse presentation.

\section{MHC 40}

Unusual Presentation of Acute Myeloid Leukemia as Facial Nerve Palsy and Paraparesis-Report of 3 Cases

\section{P. Kumar\#, S. Bansal, P. K. Patra, S. Nityanand, R. Kashyap, K. Rahman, R. Gupta, A. Gupta, H. Lal}

Email ID for Correspondence: doctorpkgmu@gmail.com SGPGIMS, Lucknow, UP

Introduction and Background: Granulocytic sarcoma (GS) is an extramedullary tumor composed of immature myeloid cells. GS may occur at anywhere in the body and are associated with acute or chronic leukemia or myeloproliferative disorders. Presentation of acute myeloid leukemia (AML) as facial nerve palsy with paraparesis/paraplegia is very rarely reported. Here we report 3 cases of AML, all of them presented with facial nerve palsy with paraparesis/plegia. Material and Methods: 3 patients presented with a h/o fever, paraparesis with bladder bowel involvement and LMN type facial nerve palsy (paraparesis then right sided facial palsy in 2 cases and left sided facial palsy followed by paraplegia in 1 case). Examination revealed pallor, LMN type facial nerve palsy and sensory motor paraparesis with bladder bowel involvement. MRI head \& spine s/o chloromas at multiple sites and further investigations confirmed the diagnosis of AML. All of them treated with cranio-spinal irradiation and 2 patients received chemotherapy and 1 died because of sepsis after radiotherapy. Results: Both patients achieved remission and facial palsy improved after induction chemotherapy. Paraparesis improved in both cases but only one patient was able to walk with support. One patient relapsed within 1 year and died. Conclusions: Patients of AML with GS usually associated with overall poor prognosis but such patients can be salvaged only with prompt diagnosis and management in the form of radio-chemotherapy.

\section{MHC 41}

Bendamustine in B Cell NHL_Experience from India

A. Manuprasad", Prasanth Ganesan, Tenali G. Sagar, Trivadi S. Ganesan, Krishnarathinam Kannan, Venkatraman Radhakrishnan, Manikandan Dhanushkodi, Krishnarathinam Kannan

Email ID for Correspondence: drmanuprasad@gmail.com Cancer Institute (WIA), Adyar, Chennai

Introduction and Background: Bendamustine is one of the most common drugs used in low grade B cell NHL. There is limited data from India where the drug is available from 2009. Patient/Material and Methods: Toxicity profile and outcomes of patients with low grade B cell NHL who received bendamustine in our institute from 2010 to 2015 were analysed. Bendamustine $\left(70-100 \mathrm{mg} / \mathrm{m}^{2} / \mathrm{day}\right.$, D1 D2) was used as single agent, or in combination with rituximab/ vincristine/prednisolone as 4 weekly cycles (B, BR, BOP). Results: Over a 6-year period, 110 patients received bendamustine [median age-53 years (range 26-76 years), males $\mathrm{n}=68$ (61\%)]. Commonest histology was follicular lymphoma $[\mathrm{n}=55(50 \%)]$ followed by CLL/SLL $[\mathrm{n}=25(23 \%)]$, Mantle cell lymphoma $[\mathrm{n}=20$ 
(18\%)], Marginal zone lymphoma $[\mathrm{n}=7,(7 \%)]$, prolymphocytic Lymphoma $\mathrm{n}=2(2 \%)]$ and lymphoplasmacytic lymphoma $[\mathrm{n}=1$ $(1 \%)$ ]. Bendamustine-based therapy was used in the first line in 75 patients $(68 \%)$. The regimens used were: $\mathrm{BR}(\mathrm{n}=65,59 \%)$, Single agent $\mathrm{B}(\mathrm{n}=34,30 \%)$, or $\mathrm{BOP}(\mathrm{n}=11,10 \%)$. Commonest dosage used was $90 \mathrm{mg} / \mathrm{m}^{2} /$ day and median of 6 cycles were given. Skin toxicity was common $(\mathrm{n}=38,34 \%)$ including grade 3 in $6(5 \%)$. Overall response rate was $87 \%(\mathrm{CR}-50 \%, \mathrm{PR}-37 \%)$. Conclusions: Bendamustine based regimes show good efficacy in Indian patients with low grade B cell NHL. As reported earlier, there is a very high incidence of skin toxicity among Indian patients.

\section{MHC 42}

Depth of Disease Response Prior to Autologous Stem Cell Transplant in Myeloma: A Single Centre Analysis

\section{Amrit Mathew, Chepsy C. Philip, Sohan Singh, M. Joseph John \\ Email ID for Correspondence: mjosephjohn@gmail.com Department of Clinical Haematology, Haemato-Oncology and Bone Marrow (Stem Cell) Transplantation, Christian Medical College, Ludhiana 141 008, Punjab}

Background: Autologous Stem Cell Transplant (ASCT) is an effective therapy in multiple myeloma (MM). The importance of posttransplant depth of response is established. However, the role of depth of disease response before ASCT remains uncertain. We analyzed the clinical outcomes with ASCT of all patients with MM who have been autografted in our centre since 2009. Methods: We undertook a retrospective analysis of outcomes on patients with multiple myeloma who underwent an ASCT over a six year period from August 1st 2009 till July 31st 2015. Descriptive statistics were calculated for all variables. The probability of survival was estimated with productlimit method of Kaplan and Meier for overall survival Data analysis was performed using SPSS version 21.0 (Chicago, IL, USA), and statistical significance was set at $P<0.05$ (two-sided). Results: Fifteen patients underwent an ASCT during this period. IgG Myeloma was the commonest type in $11(66.7 \%)$ patients. Fourteen $(93.3 \%)$ patients achieved at least a partial response prior to ASCT. Plerixafor with GCSF was used for mobilization in $5(33.3 \%)$ patients. All patients received a myeloablative conditioning with Melphalan. Estimated median follow up was 35.0 months (95 \% CI 14.3-55.6). The two year overall survival (OS) and event free survival (EFS) were $71.1 \% \pm 14.7 \%$ and $60.5 \% \pm 16.0 \%$ respectively. The one-year overall survival (OS) were $100 \%$ and $87.5 \% \pm 11.7 \%$ respectively in patients who achieved a pre-transplant response of atleast a VGPR and only a PR respectively, $P=0.122$. Conclusions: We describe our experience with ASCT in MM. There is an interval from diagnosis to transplant which relates to attempts in deepening response. There was no significant difference in median OS in patients who achieved a very good PR or a partial response prior to ASCT.

\section{MHC 43}

Adult Acute Lymphoblastic Leukemia: A Cost Effective Strategy and Limitations of Intensification of Therapy in India

Punit Jain", Anu Korula, Prashant Deshpande, P. N. Nisham, Ansu Abu Alex, Aby Abraham, Alok Srivastava, Nancy Beryl Janet, Kavitha M. Lakshmi,

Poonkuzhali Balasubramanian, Biju George, Vikram Mathews

Email ID for Correspondence: punitjn@gmail.com

Christian Medical College, Vellore, India
Introduction and Background: There are substantial hurdles in intensifying chemotherapy regimens in the treatment of adult lymphoblastic leukemia (ALL) in developing countries. Patient/Material and Methods: Retrospectively, data from 507 consecutive adults ( $\geq 15$ years) with a diagnosis of ALL at our center were analyzed. Standard risk (SR) patients were treated with a modified GMALL regimen. High risk (HR) patients were offered intensified therapy with HCVAD regimen followed by an allogeneic stem cell transplant (allo-SCT) for those who had a HLA matched donor. HR patients who could not afford intensification, were treated similarly to SR patients. Results: A total of $344(67.8 \%)$ SR and 163 (32.2\%) HR patients were evaluated. Among $163 \mathrm{HR}$ patients, 53 (32.5\%) received intensification with HCVAD and an allo-SCT was performed in 18 $(33.9 \%)$ of these. Of the remaining $110(67.5 \%)$ HR patients 59 $(53.6 \%)$ opted to receive a modified GMALL regimen as was used for SR patients while 51 (46.3\%) opted for palliative chemotherapy. The rates of complete remission (CR), 5 year overall survival (OS) and event free survival (EFS) were $87 \%, 50 \%$ and $47 \%$ respectively. SR cohort showed a superior 5 year OS (61\% vs. $27 \%$; $\mathrm{P}<.001)$ and EFS $(47.3 \%$ vs. $23.6 \% ; \mathrm{P}<.001)$ than the HR cohort. Within HR subgroup, there was no statistically significant difference in OS or EFS between modified GMALL $(n=59)$ or HCVAD $( \pm$ HSCT $)(n=53)$ regimens. Conclusions: Intensifying therapy in the HR subset was associated with a significant increase in cost of treatment and early treatment related mortality with no significant survival advantage.

\section{MHC 44}

Impact of Kinase Domain Mutations on Second Line Nilotinib Therapy in Chronic Myeloid Leukemia-Experience from a Tertiary Care Center in North India

\section{Shinto Francis Thekkudan", Akhilesh Sharma, Anshul Gupta,} Soniya Nityanand

Email ID for Correspondence: meshinto@gmail.com Sanjay Gandhi Post Graduate Institute of Medical Sciences, Lucknow

Introduction and Background: Point mutations in BCR-ABL tyrosine kinase domain (KD) is the most frequent cause of resistance to TKIs in CML patients. The impact of $B C R-A B L \mathrm{KD}$ mutations occurring before and during second line Nilotinib therapy has been assessed previously only in few studies from western literature, with no Indian data available till date. Patient/Material and Methods: All patients of CML-CP aged $\geq 18$ years, on second line Nilotinib therapy, during the period from January 2013 to December 2015, were included in the study. Molecular response (BCR-ABL by RT-PCR IS) was assessed at 6 and 12 months. Patients with baseline T315I mutations were excluded from further analysis. Patients without baseline mutations were analyzed for mutations when they failed to achieve optimal response as per ELN guidelines. Primary objectives were to assess the molecular response as well as the prevalence, pattern and evolution of KD mutations before and during Nilotinib therapy. Results: Of the total 583 newly diagnosed CML patients presented from January 2013-December 2015, 72 (12.3\%) patients were started on Nilotinib, of which 11 patients were non-compliant/ lost to follow up and thus excluded from further analysis. Of the 61 patients included, 47 (77\%) patients had primary resistance to Imatinib. Mean duration of first line Imatinib therapy before switching over to Nilotinib was 42.5 months. 12 patients $(19.7 \%)$ had KD mutations at the time of switch over to Nilotinib. Optimal response as per ELN guidelines was achieved in $42 / 61(68.9 \%)$ patients at 6 months. Of the suboptimal responders (19/61-31.1\%), 9 patients had evolution of new mutations, majority being on the A loop of KD. Of the 47 patients who reached 1-year end point, $25(53.2 \%)$ patients 
achieved optimal response. Of the suboptimal responders at 1 year (22/47-46.8\%), 7 had evolution of new mutations, with 4 patients having dual mutations and 1 patient having triple mutations. 4 patients developed Grade III-IV hematologic toxicity. Conclusions: Evolving new mutations are a major cause of failure to Nilotinib therapy. Side effects are usually well tolerated. Large-scale studies with long-term follow up are required for assessing the durability of sustained molecular response with Nilotinib.

\section{MHC 45}

Ibrutinib (I) Plus Bendamustine and Rituximab (BR) in Previously Treated Chronic Lymphocytic Leukemia/Small Lymphocytic Lymphoma (CLL/SLL): a 2-Year Follow-Up of the HELIOS Study

Graeme Fraser, Paula Cramer, Fatih Demirkan,

Rodrigo Santucci Silva, Halyna Pylypenko, Sebastian Grosicki, Ann Janssens, Aleksander Pristupa, Jiri Mayer, Marie-Sarah Dilhuydy, Javier Loscertales, Andre Goy, Abraham Avigdor, Simon Rule, Charles Phelps, Michelle Mahler, Masha Salman, Angela Howes, Sriram Balasubramanian, Suryaprakash Mishra\#, Asher Chanan-Khan

Email ID for Correspondence: smishr35@ITS.JNJ.com Janssen Oncology, India

Background: The phase 3 HELIOS study evaluated I+BR vs. placebo (P)+BR in previously treated CLL/SLL patients. At first analysis (median follow-up: 17.0 months) progression-free survival (PFS) was significantly improved for I+BR vs. P+BR (HR [95\% CI]: 0.203 [0.15-0.28], $p<0.0001)$. Prior studies showed deepening responses with continued I treatment; long-term follow-up assessing durability and depth of response is important. Methods: 578 patients received BR ( $\leq 6$ cycles) and were randomized $1: 1$ to $\mathrm{I}(420 \mathrm{mg} / \mathrm{d})$ or $\mathrm{P}$ $(\mathrm{N}=289 / \mathrm{arm})$; patients with del17p ( $\geq 20 \%$ of cells) were excluded. Primary endpoint: PFS. Key secondary endpoints: overall survival (OS), overall response rate (ORR), minimum residual disease negative (MRD-ve) response rate. Results: Median follow-up was 25.4 months. I+BR continues to improve in PFS vs. P+BR (investigator-assessed median: not reached [NR] vs. 14.2 months; HR [95\% CI]: 0.199 [0.15-0.26], $p<0.0001 ; 2$-year rate: $74.8 \%$ vs. $20.9 \%$ ). Median OS was NR in either arm (HR [95 \% CI]: 0.670 [0.44-1.02], $p=0.0587 ; 2$-year rate: $86.2 \%$ vs. $81.5 \%) ; 142$ patients $(49.1 \%)$ on $\mathrm{P}+\mathrm{BR}$ with confirmed $\mathrm{PD}$ crossed over to receive I. Updated investigator-assessed ORR was $87.2 \%$ for I+BR vs. $66.1 \%$ for $\mathrm{P}+\mathrm{BR}(p<0.0001)$; updated CR/CRi rates were $33.9 \%$ vs. $7.2 \%$ (first analysis rates: $21.4 \%$ vs. $5.9 \%$ ). MRD-ve response rates (intent-to-treat population) were $18.0 \%(52 / 289)$ for I+BR and $4.8 \%$ $(14 / 289)$ for $\mathrm{P}+\mathrm{BR}(p<0.0001)$ (first analysis rates: $12.8 \%$ vs. $4.8 \%$ ). Though median PFS2 was unreached in both arms, PFS2 was significantly longer for I+BR vs. P+BR, despite crossover (HR [95\% $\mathrm{CI}]: 0.622$ [0.42-0.92], $p=0.0162$ ). Safety was consistent with first analysis. Conclusions: I+BR continues to demonstrate superiority vs. $\mathrm{P}+\mathrm{BR}$ with significantly longer PFS and higher ORR. Responses deepened further with continuous I therapy with CR/CRi and MRDve rates increasing over time. At every MRD level $(<0.01 \% / \geq 10 \%$ CLL), I+BR showed more sustained PFS than P+BR. These data confirm the importance of I in previously treated CLL patients.

\section{MHC 46}

Clinical Characteristics of Patients with Polycythemia Vera in a Tertiary Care Centre

\section{J. Preethi", Pankaj Malhotra, Jasmina Alhuwalia, Shano Naseem, Neelam Varma, Subhash Varma}

Email ID for Correspondence: preethijraman1@gmail.com PGIMER, Chandigarh

Introduction and Background: Polycythemia vera (PV) is an uncommon clonal disorder of stem cells. The literature regarding the thrombotic complications and patient characteristics of this disorder in the developing countries is scarce. The present study was undertaken retrospectively to look at the thrombotic complications of PV and various patient characteristics. Patient/Material and Methods: All the patients diagnosed to have PV according to Polycythemia Vera Study Group criteria from January 2005 to July 2016 were included. Their clinical characteristics, laboratory parameters, clinical complications such as thrombosis and myelofibrosis, treatment modalities, malignancies and deaths, if any were noted. Results: Out of the 71 patients seen during this period with erythrocytosis, 54 patients fulfilled the inclusion criteria. The median age at the time of diagnosis was 51.5 years. Thirteen patients developed thrombotic complications, $53 \%$ in abdominal vessels (6 patients). Six patients had bleeding complications, most common site being nose. Splenomegaly was present in $64 \%$. Bone marrow was available in 49 patients. Fibrosis was present in $28.5 \%$ patients. JAK 2 was negative in $1.8 \%$ of patients with PV. Eighty three percent of patients received hydroxyurea. Twenty percent developed hydroxyurea induced complications. None of these patients developed leukemia. Conclusions: PV is an uncommon disorder when compared with other hematological disorders in northern India. Most common site of thrombosis in patients with polycythemia vera is abdomen. One fifth of patients receiving hydroxyurea developed hydroxyurea induced complications. Very few patients with polycythemia vera do not harbour JAK 2 mutation.

\section{MHC 47}

Acute Promyelocytic Leukemia-Experiences from Rajiv Gandhi Cancer Institute and Research Centre

\section{Megha Gupta\#, Narendra Agrawal, Jyotsna Kapoor, Niharika Bhatia, Manish Sharma, Priyanka Verma, Mukul Aggarwal, Rayaz Ahmed, Dinesh Bhurani}

Email ID for Correspondence: megzgupta2214@gmail.com Rajiv Gandhi Cancer Institute \& Research Centre, Delhi

Introduction and Background: Acute Promyelocytic Leukemia treatment has evolved from chemotherapy only to now targeted therapies and so outcome from a deadly disease with very high mortality in 70 s to now cure in majority of patients. We here present our data of APML patients of last 7 years. Patient/Material and Methods: The case records of all patients diagnosed as APML and treated at our hospital from 2009-April, 2016 were reviewed. Patients in initial years were treated with chemo alone or ATRA with chemotherapeutic agents (anthracycline and cytarabine) till mid 2013 and thereafter with Arsenic + ATRA in low/intermediate risk and 
Arsenic + ATRA + anthracycline (APML 4 protocol) in high risk group till date. Results: 45 patients (26 males, 19 females) were diagnosed as APML during the study period. As per Sanz risk score, 12 were low risk, 13 intermediate and 20 were high risk patients. Treatment consisted of chemotherapy only in 3 patients; anthracycline + ATRA in 9 patients; Arsenic + ATRA in 21 and APML 4 protocol in 8 patients. 4 patients ( $3 \mathrm{HR}, 1 \mathrm{IR}$ ) with early death $(<48$ hour hospital stay) were excluded from further analysis. Differentiation syndrome developed in $19(46 \%)$ patients and was associated with higher TLC and clinical features of fever, weight gain, respiratory distress, renal derangement, effusions and response to treatment, but not mortality. Presentation in high risk Sanz group was associated with coagulopathy, infections, lower hemoglobin, deranged liver function tests and mortality. Overall survival is $88 \%$ (96\% for non high risk and $76 \%$ for high risk) amongst treated patients at median follow up of 28 months. Conclusions: APML in India more frequently presents as high risk group. Optimal therapy improves outcome in all risk groups.

\section{MHC 48}

Hasford Scoring in Patients of CML on Imatinib: A Sequential Follow Up

\section{Mrinalini Kotru\#, Neha Chopra Narang, Usha Rusia, Meera Sikka}

Email ID for Correspondence: mrinalini.kotru@gmail.com University College of Medical Sciences \& GTB Hospital

Introduction and Background: Hasford and Sokal scoring systems are available for classification of risk in CML patients. Hasford score has been used for patients treated with IFN-alpha. We studied the relevance of Hasford score to assess the outcome of CP-CML (Chronic phase CML) patients on Imatinib. Patient/Material and Methods: This longitudinal study, sequentially followed up $75 \mathrm{Ph}^{+}$ CML-CP patients, receiving 400-600 mg Imatinib over a period of 18 months. The Hasford score for all patients was calculated based on age, spleen size, basophils, eosinophils, blast percentage and platelet count at diagnosis, 6 months and 12 months. Score was determined using the online calculator available for Hasford scoring at http://www.pharmacoepi.de/cmlscore.html. Results: Three risk groups were defined based on the risk score: low risk $(\leq 780)$, intermediate risk (780-1480), high risk (>1480). It was intermediate in majority $(49.3 \%)$ of the patients at admission. A significant decrease was noted in Hasford score at 6 months and 12 months with $90.4 \%$ and $85.7 \%$ of the patients respectively showing low score. Conclusions: Imatinib downgraded the Hasford score. This indicated adequate response to therapy with Imatinib and predicted better overall progression free survival. Hence Hasford score can also be used to assess the outcome of CP-CML patients on Imatinib.

\section{MHC 49}

Analysis of 14 Patients of Chronic Myelomonocytic Leukemia: A Single Centre Study

\section{Jyotsna Kapoor", Megha Gupta, Narendra Agrawal, Niharika Bhatia, Manish Sharma, Priyanka Verma, Mukul Aggarwal, Rayaz Ahmed, Dinesh Bhurani}

Email ID for Correspondence: jkapoor022@gmail.com Rajiv Gandhi Cancer Institute \& Research Centre, Delhi

Introduction and Background: Chronic myelomonocytic leukemia (CMML) is a rare hematological malignancy classified under myelodysplastic/myeloproliferative (MDS/MPN) disorder. There are very few studies from India on CMML regarding patient demographics and treatment patterns. Patient/Material and Methods: We analyzed the records of patients diagnosed as CMML, at Rajiv Gandhi Cancer Institute and Research centre from Jan 2011 to Feb 2015, for their demographic, disease and treatment parameters. Results: A total of 20 patients were presented with CMML at our institute, of which 6 patients did not followed after their initial consultation. Rest 14 patients ( 7 patients in each of CMML-1 and CMML-2 categories) were eligible for the study. Median age of patients was $60.5 \mathrm{yr}$ (44-85 yr, 9 males and 5 females). Five patients were dysplastic type (MDS-CMML) and 9 were proliferative type (MP-CMML). Out of 14 patients, one expired before starting therapy; another one opted for alternative therapy so excluded from outcome analysis. Nine patients were started on chemotherapy with hypomethylating agent (decitabine as first line in all) and one was started on induction chemotherapy with $3+7$ regime followed by salvage with hypomethylating agent (azacytidine) while 2 received only hydroxyurea. Out of 10 patients who received chemo, 3 achieved CR, 1 optimal marrow response, 2 clinical benefit, 4 patients no response/ progression. Overall response rate was $60 \%$. Three patients went on to receive allogeneic stem cell transplant in CR (2 unrelated donor, 1 sibling donor). One patient rejected the graft and later on relapsed. Amongst chemo treated 10 patients, 4 patients are alive after a median follow up of 512 days. Median OS was 346 days. Conclusions: CMML is a rare disease with dismal outcome. Hypomethylating therapy and/or allogeneic stem cell transplant are feasible treatment option.

\section{MHC 50}

\section{Primary CNS Lymphoma. Our Experience}

\section{Abhishek Pathak ${ }^{\#}$, Amul Kapoor, Rajan kapoor,} S. N. Das

Email ID for Correspondence: drabhipat@gmail.com Army Hospital (Research and Referral)

Introduction and Background: Primary CNS lymphoma (PCNSL) is an uncommon variant of extranodal non-Hodgkin lymphoma (NHL) arising exclusively in the CNS, that is, the brain parenchyma, spinal cord, eyes, cranial nerves, and/or meninges. It is a highly aggressive malignancy. The standard chemotherapy regimens for treatment of systemic lymphoma (eg, CHOP), are either ineffective or too toxic. The definitive treatment of PCL is not known and there is a lot of institutional variation. Here we present our experience of managing 10 cases of PCNSL in immunocompetent patients, their initial presentation, diagnosis and their treatment. Patient/Material and Methods: !0 Patients who reported to our tertiary care centre were evaluated in detail. Detailed history, Clinical examination, MRI brain, CSF studies and other metastatic work up were carried out. On the basis of clinical and investigations patients were given International Extranodal Lymphoma Study Group (IELSG) score and divided into low, intermediate and high risk groups. Various treating modalities using chemotherapy (Methotrexate, cytosar, Rituximab) were given. Results: All the patients presented with headache and $60 \%$ had instability of gait and $40 \%$ had hemiparesis. On MRI, there was a single lesion in $50 \%$. On histopathology $90 \%$ were Diffuse large B cell lymphoma $80 \%$ of our patients fell in high risk score and $20 \%$ had intermediate risk. In our series we used high dose methotrexate $(8 \mathrm{gm} / \mathrm{sq} \mathrm{m})$ in 3 cases and they had complete remission. In another 4. Conclusions: There is no clear guideline in managemnt of PCNSL however methotrexate remains to be the backbone of treatment of primary CNS lymphoma. 


\section{MHC 51}

Encouraging Outcomes of Haploidentical Stem Cell Transplantation in Acute Leukemias: A Single Centre Experience

Mukul Aggarwal $^{\#}$, Jyotsna Kapoor, Niharika Bhatia, Narendra Agrawal, Rayaz Ahmed, Dinesh Bhurani

Email ID for Correspondence: mukulmamc@gmail.com Rajiv Gandhi Cancer Institute \& Research Centre, Delhi

Introduction and Background: Haploidentical Stem cell transplantation (Haplo SCT) is transplant option for patients with acute leukemia lacking an HLA matched donor. Patient/Material and Methods: The case records of all leukemia patients who underwent Haplo SCT at our centre from August, 2012 to April, 2016 were analyzed and included in the study. Results: 26 patients with acute leukemia $(\mathrm{AML}=16, \mathrm{ALL}=10)$ underwent Haplo SCT during the study period with non myeloablative $(n=20)$, myeloablative $(n=4)$ and reduced intensity conditioning regimen $(\mathrm{n}=2)$. All grafts (peripheral blood stem cells, $n=24$; bone marrow grafts, $n=2$ ) were $T$ cell replete. GvHD prophylaxis used was high dose cyclophosphamide on day $+3,+4$ and MMF from day +5 to +35 and tacrolimus day +5 onwards. At transplantation, there were 7 AML patients in CR1 (complete remission 1), 7 in more than or equal to CR2 and 2 refractory to treatment while 5 ALL patients in CR1 and 5 in more than or equal to CR2. Median follow up period is 6 months (1-21.6). Engraftment occurred in $84.6 \%(22 / 26)$ of patients. Cumulative incidence of acute GvHD (II-IV) was $42.3 \%$ and chronic limited GvHD was $34.6 \%$ at median of 158 days. Relapse Rate in AML and ALL was found to be $18.75 \%(3 / 16)$ and $50 \%(5 / 10)$ respectively. $37.5 \%(6 / 16)$ of the AML patients and $20 \%(2 / 10)$ of ALL patients had Non Relapse Mortality. Overall Survival (OS) at median 6 months in AML was $43.75 \%(7 / 16)$ and in ALL, it was $50 \%(5 /$ 10). Conclusions: Acceptable overall survival, low relapse rates and non relapse mortality rate and reasonable incidence of acute GvHD and chronic GvHD suggests that Haplo SCT is an effective and useful alternate in these high risk patients.

\section{MHC 52}

IKZF1 (IKAROS) Deletion Profile in B-Cell Acute Lymphoblastic Leukemia (B-ALL) and Its Clinical Correlation

\section{Sanjeev Kumar Gupta ${ }^{1 \#}$, Sameer Bakhshi ${ }^{2}$, Lalit Kumar ${ }^{2}$, Rajive Kumar}

Email ID for Correspondence: drskgupta1@gmail.com

${ }^{1}$ Laboratory Oncology Unit, ${ }^{2}$ Department of Medical Oncology, All India Institute of Medical Sciences (AIIMS), New Delhi

Introduction: IKZF1 (IKAROS) deletions correlate with poor outcome in B-ALL. We investigated for IKZFI deletion profile in our B-ALL patients and correlated it with clinical outcome. Methods: The genomic DNA from new, untreated B-ALL cases was prospectively analyzed for $I K Z F 1$ deletions using MLPA (SALSA MLPA P335 ALL-IKZF1 and P202 IKZF1 kits (MRC-Holland)). Fragment analysis was done on Genome Lab GeXP genetic analysis system and the data analyzed using Coffalyser software. The breakpoint-specific fluorescent multiplex PCR followed by capillary electrophoresis was performed in cases having IKZFI deletions using primers described by Caye et al. The outcome of induction chemotherapy and events including relapse or death were noted in all cases. Results: 162 B-ALL cases were analyzed with a median age of 8.5 years ( 2 months-67 years). The IKZF1 deletions were seen in 43 (26.5\%) cases. There were 47 deletions in 43 cases including complete deletions involving all the eight $I K Z F 1$ exons $(\mathrm{n}=14)$, dominant negative partial deletions involving exons $4-7(\Delta 4-7)(n=13)$ and other partial deletions $-\Delta 2-7$ (9), $\Delta 4-8(4), \Delta 2-3$ (3), $\Delta 2-8$ (2), and $\Delta 1$ (2). The breakpoint-specific fluorescent multiplex PCR helped in resolving the presence and type of deletion in three cases. The induction outcome was available in 137 cases. The cases with IKZF1 deletions had higher induction failure [11/32 (34.4\%) vs. 14/105 (13.3\%); p-value 0.009] compared to other cases. There were also more relapses [5/32 (15.6\%) vs. 8/105 (7.6\%); p-value 0.185] and deaths in this group [8/32 (25\%) vs. 20/105 (19.1\%); p-value 0.466]. Conclusions: Overall, IKZF1 deletions were detected in $43(26.5 \%)$ cases of B-ALL. The routine screening for IKZFI deletions at diagnosis can identify B-ALL cases with higher risk of therapy failure.

\section{MHC 53}

Impact of Modifiable and Non modifiable Factors on Quality of Life in Post Allogeneic Hematopoetic Stem Cell Transplant Recipients

\section{N. Suresh ${ }^{\#}$, Sharat Damodar, K. S. Nataraj, Sunil Bhat, B. Shobha}

Email ID for Correspondence: drsureshkaranth@gmail.com Department of Hematology, Narayana Hrudayalaya, Bangalore

Background: In this study, we report effect of modifiable factors like GVHD, CMV reactivation, infections etc, and non modifiable factors like sex, nature of disease, type of transplant, patient-donor sex match, belief in god etc, on overall quality of life [QOL] in patients post allogeneic stem cell transplantation. Patients and methods: Thirty four [34] patients who had undergone allogeneic stem cell transplantation at our centre from June 2014 to January 2016 were included in the study. FACT-BMT (Version4) questionnaire was used as assessment tool of QOL and individual scores were recorded on day of transplant and day 100 post transplant. The influence of monitored factors [sex, nature of disease, type of transplant, patientdonor sex match, Graft Versus Host Disease (GVHD), Cytomegalo Virus (CMV) reactivation, infections, belief in god] on QOL was determined using dispersion analysis. Results: Out of thirty four patients interviewed 22 were males, and 12 were females with an average age of 36.85 years. Age did not had any major effect on overall QOL in our study [p value 0.485 ], and similarly was gender [p value $=0.453]$. Presenting diagnoses were Acute Myeloid Leukemia [11], Chronic Myeloid Leukemia [10], Acute Lymphoblastic Leukemia [5], Aplastic Anemia [3] and Myelodysplastic Syndrome [5]. There was significant improvement in QOL post transplant in patients with Acute Myeloid Leukemia $[<0.005]$, and Chronic Myeloid Leukemia [0.014]. There was no significant improval in QOL post transplantation in patients with Acute Lymphoblastic Leukemia [0.066], Aplastic Anemia [0.109] and Myelodysplastic Syndrome [0.343]. Out of 34 patients, 29 [85.29\%] patients underwent Matched Sibling Donor transplant, 3 [8.82\%] underwent Haploidentical transplant and 2 [5.88 \%] patients received Matched Unrelated Donor transplant. There was significant improvement in QOL post transplant in patients post Matched Sibling Donor transplant [p value $<0.005$ ]. There was no significant improval in QOL in patients post Matched Unrelated Donor transplant [p value 0.180] and Haploidentical transplant [p value- 0.109$]$ transplants. $50 \%$ of patients had donor sex mismatch and remaining $50 \%$ had sex matched donor and in both groups there was statistically significant correlation with QOL [p value-0.001]. 28 out of 34 patients developed GVHD [82.35\%] and in which $60.7 \%$ [17/28] of patients developed gut GVHD, $14.3 \%$ [4/ $28]$ had skin and multi organ involvement each and $10.7 \%$ [3/28] had liver involvement. There was no statistically significant correlation of GVHD with QOL [p value-0.116]. Neither CMV infection [p value$0.341]$ nor presence of any other infection had any impact on overall QOL [p value-0.667] $94.1 \%$ patients believed in god and $5.9 \%$ 
patients were atheist. There was no significant correlation with QOL [p value-0.627]. Overall there was significant improvement in QOL [Day 100] post transplantation $[<0.05]$. Conclusions: Age and gender had no impact on overall QOL. Acute Myeloid Leukemia and Chronic Myeloid Leukemia contributed major chunk of disease burden in our study. Patient-Donor sex match and mismatched transplants had similar implications on overall QOL. Patients with Acute Myeloid Leukemia and Chronic Myeloid Leukemia had significant improvements in QOL post transplant. There was significant improvement in QOL post transplant in patients post Matched Sibling Donor transplantation. There was no significant improval in QOL in patients post MUD and Haplo transplants. Graft-versus-Host Disease had no significant effect on QOL. Infections including CMV reactivation had no bearing on overall QOL. Believer in god or atheist had no major differences in overall QOL. Overall there was significant improvement in QOL [Day 100] post allogeneic stem cell transplantation.

\section{MHC 54}

Monitoring of Minimal Residual Disease in Adult Acute Myeloid Leukemia-Clinical Correlation

Chinmayee Kakirde ${ }^{\#}$, Rohan Kodgule, Goutham Raval, Sanjay Talole, Shraddha Kadechkar, Gaurav Chaterjee, Sitaram Ghogale, Y. Badrinath, Nilesh Deshpande, Pratibha Amare-Kadam, Hari Menon, Manju Sengar, Navin Khattry, Hasmukh Jain, Bhausaheb Bagal, Prashant Tembhare, Sumeet Gujral, P. G. Subramanian, Nikhil Patkar

Email ID for Correspondence: radhika.kakirde@gmail.com ACTREC, Tata Memorial Centre

Introduction: Goal of chemotherapy in acute myeloid leukemia (AML) is to achieve morphological remission. However, not all patients achieve long term remission and subset of patients relapse. Detection of minimal residual disease (MRD) following chemotherapy, is highly predictive of outcome and allows treatment modifications if required. Here, we describe assessment of immunophenotypic MRD using a 10-colour two tube assay. Methods: 100 consecutive patients of adult AML over 14 month were observed. MRD testing was done at post induction (P.I.) and post 1st Cycle HiDAC (P.C.). Using conventional karyotyping and FISH, patients were classified into favourable, intermediate and poor cytogenetic risk. FLT3-ITD, NPM1 and CEBPA mutations were detected by fragment length analysis. Overall Survival was calculated from start of induction therapy to date of last follow up or death. Relapse Free Survival was calculated after 1 st remission till date of relapse or death or last follow up if in CR. Results of the MRD assays, cytogenetic and molecular risk groups were analyzed for their impact on OS and DFS. Results: Based on cytogenetics, $36.7 \%$ were favourable risk, $54.1 \%$ were intermediate and $9.2 \%$ were poor risk. FLT3-ITD, NPM1 and CEBPA mutations observed in $9 \%, 19 \%$ and $8 \%$ of patients respectively. The OS was $63 \%$ and RFS was $50 \%$ with a median follow up of 6 months. 24 had induction death and 17 had refractory disease. MRD positivity was seen in $35.7 \%$ in P.I. and $28.6 \%$ P.C. Favourable risk cytogenetics was predictive of better RFS not OS. FLT3-ITD positivity was associated with worse OS. Patients MRD at the end of induction was associated with worse OS \& RFS, post consolidation positive MRD status was strongly associated with inferior RFS. Conclusions: MRD serves as a useful guide for guiding post induction treatment strategies and should be incorporated into routine management algorithms.

\section{MHC 55}

Intra-arterial Steroid Therapy in Patients with Steroid Resistant/ Dependent Graft-Versus-Host Disease

M. Y. Shapira ${ }^{1}$, A. Klimov ${ }^{2}$, S. Vipul ${ }^{\#}$, P. D. Tsirigotis ${ }^{1}$, S. Grisariu ${ }^{1}$, B. Avni ${ }^{1}$, R. Or ${ }^{1}$, A. I. Bloom ${ }^{2}$

Email ID for Correspondence: dr.vipulsheth@gmail.com

${ }^{1}$ Department of Bone Marrow Transplantation, and ${ }^{2}$ Interventional Radiology Unit, Hadassah University Hospital, Hebrew University, Hadassah Medical School, Jerusalem, Israel

Introduction: Graft-versus-host disease (GVHD) is a major complication of allogeneic bone marrow transplantation (BMT), resulting in death in the majority of steroid-resistant patients. In the past 15 years, we revolutionized the use of interventional radiology procedures in the field of stem cell transplantation. This study was aimed to assess the efficacy of regional intra-arterial steroid (IAS) treatment in the largest published cohort of patients with resistant/dependent hepatic and/or gastrointestinal (GI) GVHD. In total, 113 patients with steroid resistant or dependent grade 3-4 hepatic $(\mathrm{n}=21)$, gastrointestinal (GI) $(n=62)$ GVHD or both $(n=30)$ were given intra-arterial treatment. Patients and Methods: Patients with liver GVHD received IAS in hepatic artery and patients with gastrointestinal GVHD received IAS in the superior and inferior mesenteric as well as the internal iliac arteries. Patients with pronounced upper GI symptoms also received IAS to the gastroduodenal artery. In total 148 procedures were carried out (range 1-3 per patient). Results: Partial responses were observed in 33 out of $51(65 \%)$ patients with hepatic GVHD, out of which $(55 \%)$ had complete response. Median time to complete response was 45 days (range 5 to 300 days). For GI GVHD complete responses were observed in 69 out of $92(75 \%)$ and partial responses were observed in 76 out of $92(83 \%)$. The median time to complete response was 20 days (range 3 to 200 days). The 1 year overall survival in these severely refractory patients was $25 \%$. As this is the largest published cohort of regional IAS therapy for GVHD, multivariate and univariate analysis was done in order to identify the patients with highest chance of response. Conclusions: Regional treatment of severe GVHD with IAS treatment is effective and safe. GI treatment is more effective than intrahepatic treatment. Early administration of isolated intra-arterial therapy in high-risk patients may further improve the outcome and reduce untoward effects of prolonged systemic immunosuppressive treatment. 

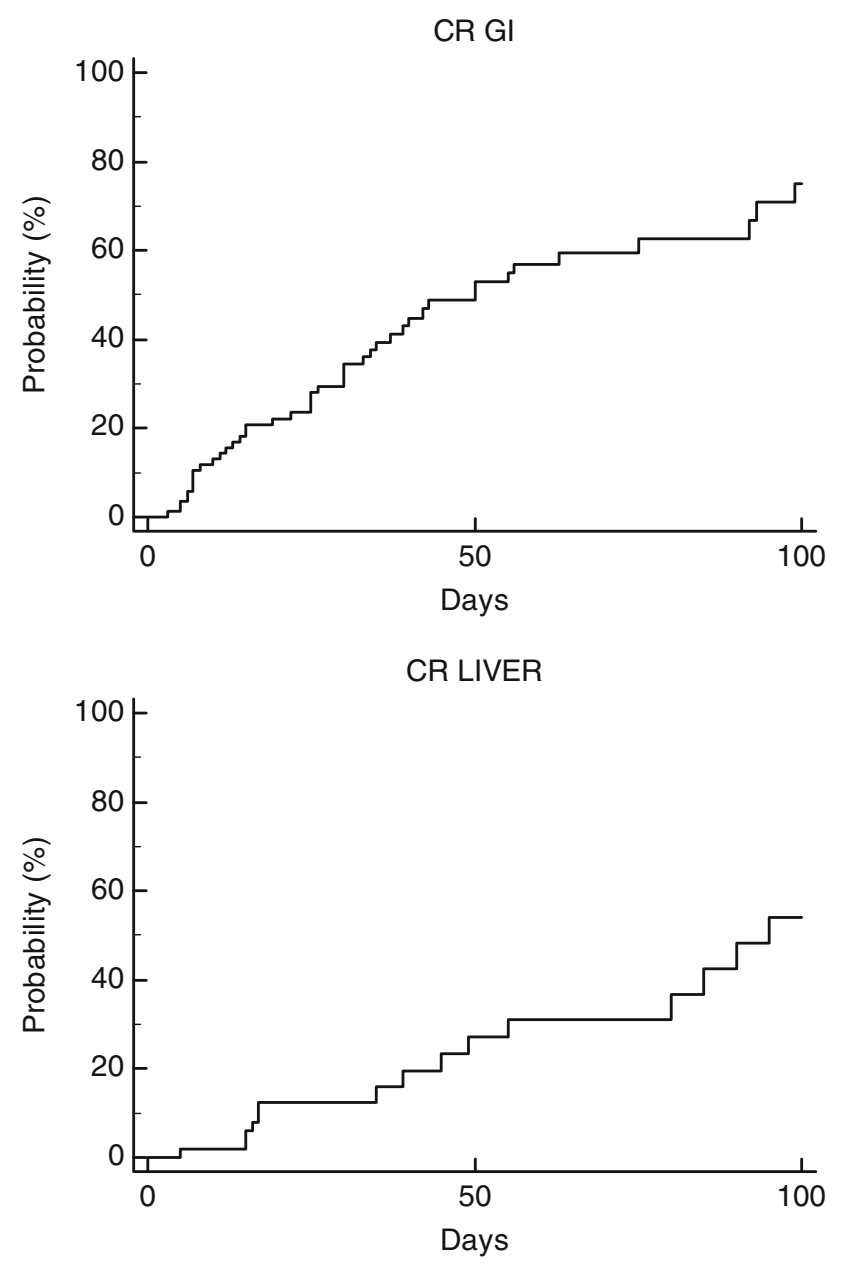

MHC 56

Polymyositis Rare Presentation of Chronic Graft Versus Host Disease Post Allogenic Bone Marrow Transplant

\section{Sharnangat Vijay", S. H. Advani, Ritu Jain, G. Bhat}

Email ID for Correspondence: drvijayvsh@gmail.com Jaslok Hospital and Research Centre

Introduction and Background: Chronic Graft Versus Host Disease (cGVHD), most common complication post Allogenic haematopoietic stem cell transplant (AlloHSCT), is main contributory factor for mortality and morbidity in transplant patients. Polymyositis and skin GVHD, are rare manifestation of cGVHD, led to functional impairment, disability and affect quality of life. Here we have 36 years male, he had skin and muscular GVHD post 9 months of AlloHSCT. He has been successfully treated with steroids and cyclosporine. Patient/Material and Methods: Thirty six year male diagnosed as chronic myeloid leukemia in chronic phase (CML CP) in November 2014 and was started on Imatinib, he had progression on Imatinib in form of extamedullary blast crisis. He had bilateral cervical lymphadenopathy, histopathological examination revealed $\mathrm{T}$ Lymphoblastic lymphoma. He was treated with HYPERCVD regimen and on remission was underwent AlloHSCT in Nov 2015. He had been treated with methotrexate and cyclosporine as GVHD prophylaxis, and cyclosporine was discontinued after 6 months'. He had grade 2 skin cGVHD after discontinuation of cyclosporin which was treated with steroids and had good response to it and was tapered. After about 15 days he had proximal muscle weakness along swollen, red, tender muscles of lower as well as upper limbs. CPKMM was high (1027), PETct showed disease activity, histopathological examination revealed mononuclear infiltrates. He was started on steroids and Imatinib as cGVHD prophylaxis, had complete resolution of cGVHD. Results: Polymyositis as clinical manifestation of cGVHD has been treated and has very good response to steroids and Imatinib in form of complete resolution of cGVHD. Conclusions: Polymyositis could be manifestation of cGVHD, should be considered in differentials following allogeneic HSCT, in patients having muscular weakness.

\section{MHC 57}

FIPILI-PDGFRA Fusion Gene Associated Hypereosinophilic Syndromes

R. M. Subbaiah", B. Poonkuzhali, Senthamizhselvi Anandan, N. A. Fouzia, Aby Abraham, Alok Srivastava, Vikram Mathews, Biju George

Email ID for Correspondence: drrms5@yahoo.co.in Christian Medical College, Vellore 632004

Introduction and Background: Hypereosinophilic syndromes (HES) are defined when absolute eosinophilic count $>1500$ /microlitre is present without an apparent cause and may be associated with end organ damage involving the lung, heart, skin, gastrointestinal tract or neural tissues. A proportion of cases had been consistently associated with the fusion gene transcript FIPILI-PDGFRA due to an interstitial deletion in the chromosome $4 \mathrm{q} 12$. Presence of this genetic abnormality marks the constitutional activation of tyrosine kinase. Patient/ Material and Methods: This is a retrospective analysis of 23 cases of FIPILI-PDGFRA fusion gene associated HES identified from 223 patients who were evaluated for hypereosinophilia over a 10 year period from 2007 to 2016. Data was obtained from individual medical records and institutional databases. Results: There were 21 males (91\%) and 2 females with a median age of 33 years (range 4-67). One third (8 patients) had constitutional symptoms (fever or significant weight loss). The mean total white cell count and eosinophil differential count were 56,400/cu mm (range: 9100-333,000) and $65 \%$ (range: $36-94 \%$ ) respectively. Thirteen patients $(56.5 \%)$ had splenomegaly on physical examination while evidence of end organ damage was identified in 10 patients [ 4 had $>1$ organ involvement). Organs involved included GI and heart in 3, lung in 5 and skin in 4. Corticosteroids were used as an initial therapy in 10 patients while hydroxyurea in 9 patients to stabilize the high total count. Thirteen patients received imatinib (50-100 mg/day) once FIP1LI-PDGFRA was reported as positive. Eleven patients showed evidence of response with normalization of blood counts and absence of eosinophilia in peripheral blood and were maintained on the same after achieving remission. Conclusions: In this case series we confirm that a proportion $(10 \%)$ of patients with HES are associated with FIPILIPDGFRA gene fusion transcript and show good response to Imatinib Mesylate. 


\section{MHC 58}

Pegfilgrastim-More Cost Effective and Equally Efficacious Option as Compared to Filgrastim in Autologous Stem Cell Transplant

Ritu Jain ${ }^{2 \#}$, Vipul Sheth ${ }^{1}$, Adwaita Gore ${ }^{1}$, Amit ghanekar ${ }^{1}$, Tapan Saikia ${ }^{1}$

Email ID for Correspondence: reebun@yahoo.com

${ }^{1}$ Stem Cell Transplant, Prince Aly Khan Hospital, Mumbai, ${ }^{2}$ Stem Cell Transplant, Jaslok Hospital, Mumbai

Introduction: American Society of Clinical Oncology guidelines recommend the use of growth factor after high-dose chemotherapy (HDC) and peripheral blood stem cell (PBSC) support. This retrospective study aims to compare the efficacy of Pegfilgrastim (PEG) compared with Filgrastim (FIL) after HDC. Material (or patients) and Methods: We collected data on 195 consecutive adult patients who received an autotransplant (Myeloma, lymphoma and others) between January 2004 and December 2014 at two tertiary care centres. The primary end point was the duration of neutropenia both in terms of days to reach an ANC $>0.5 \times 109 / 1$. Filgrastim was given to 110 patients and Pegfilgrastim was given to 85 patients. Results: Time to engraftment, defined as the time to reach an ANC of $0.5 \times 10^{9} / \mathrm{L}$ on 2 consecutive days after the day of auto-SCT, was 12.6 days with Filgrastim compared with 12.1 days with Pegfilgrastim $(p=0.126)$. When comparing the total days of severe neutropenia (WBC $<0.1 \times 109 / \mathrm{L}$ ), there were 5.5 days of severe neutropenia with Filgrastim compared with 5.8 days with Pegfilgrastim $(P=0.7)$. The duration of febrile neutropenia was an average of 5.3 days with Filgrastim and 4.6 days with Pegfilgrastim $(\mathrm{P}=0.029)$. The total number of antibiotic days was shorter for the patients who received Pegfilgrastim, being 11.08 days with Pegfilgrastim and 12.1 days with Filgrastim $(\mathrm{P}=0.184)$. The average cost savings per person in terms of number of days of hospitalization and number of days of total parental nutrition was $582 \mathrm{Rs},(\mathrm{p}=0.512)$ and $6003 \mathrm{Rs}(\mathrm{p}=0.018)$ respectively in favour of Pegfilgrastim arm. Conclusion: PEG is similar to FIL in haematological reconstitution; however it is more cost effective alternative after HDC and PBSC.

\begin{tabular}{lcll}
\hline & $\begin{array}{l}\text { Filgrastim } \\
\text { Mean } \\
\text { (SD) }\end{array}$ & Pegfilgrastim & Pean (SD) \\
& $8.7(4.8)$ & value \\
\hline $\begin{array}{l}\text { Days of WBC }<0.5 \times 10^{9} / \mathrm{L} \\
\text { (mean, range) }\end{array}$ & $14.5(6.1)$ & $13.7(5.03)$ & 0.4 \\
$\begin{array}{l}\text { Days to platelets platelet } \\
\text { engraftment (mean, range) }\end{array}$ & $12.6(4.2)$ & $12.1(4.4)$ & 0.126 \\
\begin{tabular}{l} 
Days to neutrophil engraftment \\
\hline
\end{tabular}
\end{tabular}

Comparison of engraftment kinetics in Pegfilgrastim versus Filgrastim arm.

\begin{tabular}{|c|c|c|c|}
\hline & $\begin{array}{l}\text { Filgrastim } \\
\text { Mean (SD) }\end{array}$ & $\begin{array}{l}\text { Pegfilgrastim } \\
\text { Mean (SD) }\end{array}$ & $\begin{array}{l}P \\
\text { value }\end{array}$ \\
\hline Duration of hospital stay (mean) & $19.6(6.9)$ & $18.4(6.6)$ & 0.123 \\
\hline $\begin{array}{l}\text { Documented Febrile neutropenia } \\
\text { (days) }\end{array}$ & $5.3(3.7)$ & $4.6(4.03)$ & 0.029 \\
\hline $\begin{array}{l}\text { Number of days of antibiotics } \\
\text { (mean) }\end{array}$ & $12.1(6.01)$ & $11.08(6.4)$ & 0.184 \\
\hline $\begin{array}{l}\text { Number of days of TPN used } \\
\text { (mean) }\end{array}$ & $7.6(5.9)$ & $4.08(4.9)$ & 0.005 \\
\hline
\end{tabular}

"Comparison of supportive care between two groups ."

\section{MHC 59}

Profile of Bacterial Infections in Patients Undergoing Allogeneic Stem Cell Transplantation

Shreyas Hamantgad ${ }^{\#}$, Anu Korula, Anup Devasia, N. A. Fouzia, P. N. Nisham, Aby Abraham, Alok Srivastava, Vikram Mathews, Biju George

Email ID for Correspondence: shreyashanmantgad@gmail.com Dept of Haematology, CMC Vellore

Introduction: Infections following hematopoietic stem cell transplantation (HSCT) are a major cause of transplant related mortality and morbidity. Study of the patterns of infections will help in making management decisions and improving outcomes. This study examines the distribution of bacterial infections in a large cohort of transplant recipients. Methods: We reviewed the records of patients who underwent allogenic stem cell transplantation at the Christian Medical College between 2005 and 2015. Data was sourced from individual medical records and databases in the department. 1170 patients (691 males and 479 females) with a median age of 17 years (range: 0.7 to 60 ) underwent HSCT during this period. This included 550 children (age $<15$ years). Donors were matched sibling donors in 1010 , matched unrelated donors in 116 and haplo-identical donors in 44. Indications for transplant included malignant disorders in 457 and non malignant diseases in 713. Results: All patients developed fever post HSCT. Documented bacterial infections (DBI) were seen in 317 episodes in 297 transplants $(25.3 \%) .93 \%$ of DBI occurred $<100$ days post HSCT. Sites of infection included Blood in $253(79.8 \%)$, Urine in 37, pus in 13, catheter in 5, ET aspirate in 7 and stool in 2. Infections were mainly Gram negative [77.5\%] than Gram positive [22.5\%]. Among patients who had GNB infection, carbapenem resistant organisms was seen in $20.5 \%$ while for gram positive infections, MRSA and VRE occurred in $16.5 \%$. When we compared data between 2005 to 2009 and 2010 to 2015 , the incidence of DBI had increased from 20.1 to $28.1 \%$ while the incidence of infection due to a resistant organism had increased from $18 \%$ to $29.7 \%$. Infection related mortality was $23 \%$ and $26.8 \%$ respectively but in those who had a resistant organism, the mortality rate was $42 \%$. Conclusions: Multidrug resistant bacterial infections are 
increasingly being seen in patients post HSCT. Newer strategies to prevent and treat these infections are urgently needed.

\section{MHC 60}

\section{Myelodysplastic Syndrome/Myeloproliferative Neoplasm} Unclassifiable: Rare Diagnosed Case Report

\section{Rohit Mangal ${ }^{\#}$, S. V. Suvarnakar, S. A. Deshpande}

Email ID for Correspondence: dr.rohitmangal@ymail.com Dr. Shankarrao Chavan Government Medical College, Vishnupuri, Nanded

Introduction and Background: MDS/MPN Unclassifiable is rare disease with mixed myelodysplastic and myeloproliferative features and cannot categorised into MDS OR MPN. WHO 2008 defined a MDS/MPN overlap category that includes (1) MDS/MPN Unclassifiable, (2) CMML, (3) JMML, (4) Atypical CML, BCR-ABL 1 negative (5) Refractory anaemia with ring sideroblasts and thrombocytosis. Patient/Material and Methods: 50 year serviceman vegetarian nonalcoholic came with $\mathrm{C} / \mathrm{o}$ generalised weakness since 1 month, Ecchymosis, petechiae since 15 days, swelling over right leg since 20 days, On FNAC from swelling over right leg we found atypical cells (? blasts cells) with morphology that is suspicious of haematological malignancy. Results: Peripheral smear-TLC: Increased, Blast cells and hypolobated myeloid cells are seen. DLC: Blast like cells-10\%, Myelocytes-30\%, Metamyelocytes-20\%, Band Forms-5\%, Mature polymorphs-20\%, Lymphocytes$10 \%$, Eosinophils-5 \%, Myeloid cells series shows hypolobulation with cytoplasmic vacuoles, Platelet depleted. BM Aspiration: Shows hypercellular marrow with marked paucity of differentiation myeloid series noted. Erythroid series are decreased, Megakaryocytes are not identified. Hypercellular marrow with myeloid preponderance \& $11 \%$ blasts. BM Biopsy-Hyperplastic bone marrow shows presence of Left shift of myeloid series, presence of pseudo pelger huet cells, blast cells. Erythroid series are decreased Megakaryocytes are not identified. Flow Cytometry CD $34+v e$, granulocytes are hypogranular shows asynchronous maturation. FISH-No abnormality detected. Conclusions: MDS/MPN Unclassifiable clinically can present AML or CML morphologically immunophenotyping like FISH, Cytogenetics are needed for confirmation of diagnosis and further management of patients.

\section{MHC 61}

Autologous Stem Cell Transplanatation in Hodgkin Disease: Outcomes and Survival

Jayastu Senapati ${ }^{\#}$ Anup J. Devasia, Anu Korula, N. A. Fouzia, P. N. Nisham, M. L. Kavitha, Aby Abraham, Alok Srivastava, Vikram Mathews, Biju George

Email ID for Correspondence: jsalwayswins@gmail.com; email: dranupjdevasia@gmail.com

Department of Clinical Haematology, Christian Medical College and Hospital, Vellore

Introduction and Background: Hodgkin disease is a relatively common lymphoproliferative disorder with a good therapeutic outcome. The standard of care for relapsed and progressive Hodgkin disease is salvage chemotherapy followed by Autologous stem cell transplantation (ASCT). We analysed the characteristics of patients with Hodgkin disease at our centre who underwent ASCT for disease progression or relapse after initial chemotherapy. Patient/Material and Methods: Consecutive patients with relapsed or progressive Hodgkins disease who underwent ASCT between January 1999 and January 2016 at our centre were included for this retrospective analysis. Disease characteristics at presentation, initial chemotherapy received, duration of initial remission, status at relapse or progression, cumulative salvage chemotherapies received, disease status at transplant, ASCT details and post transplantation disease status were studied. Results: Out of 65 patients who underwent ASCT for relapsed/progressive Hodgkin disease during this period, 62 patients ( 45 males and 17 females) with a median age of 26 years (range: 9-60) whose data were available were included for analysis. Majority of patients (90\%) received only ABVD chemotherapy as primary therapy (Median 6 cycles, range $4-12$ cycles). Fifteen patients $(24.2 \%)$ had primary progressive disease whereas 47 patients $(75.8 \%)$ had disease relapse after a median duration of 12 months (Range 3-101). Data on disease status at relapse were available for 52 patients (Early stage $=9$, Intermediate $=16$, advanced $=27$ ). The median lines of salvage chemotherapy received were 2 (range: $1-5$ ). All patients were conditioned with BEAM chemotherapy. The median CD34 cell dose was $4.84 \times 10^{6} / \mathrm{kg}$ (range: $1.86-40.1$ ). The median duration to neutrophil engraftment was 11 days (range: 7-26). Forty patients (64.5\%) underwent ASCT in remission, while $16(25.8 \%)$ were in partial remission and the rest had persistent disease. Twelve patients relapsed after ASCT at a median duration of 5 months (range: 1-19) while 5 patients died, with 3 in the peri-transplant period. Two patients amongst those who relapsed underwent Allogeneic stem cell transplantation. The Kaplan Meir estimates of EFS was $64.2 \pm 8 \%$ and OS was $82 \pm 8 \%$. Presence of peristsent disease at ASCT negatively affected EFS. Conclusions: Adequate salvage chemotherapy followed by ASCT offers good therapeutic outcome in progressive and Relapsed Hodgkins disease.

\section{MHC 62}

Cladribine as Salvage Therapy in Patients with Relapsed/ Refractory Langerhans Cell Histiocytosis

\section{Ajay Yadav", Bivas Biswas, Sameer Bakhshi}

Email ID for Correspondence: ajayalwar01@gmail.com Department of Medical Oncology, All India Institute of Medical Sciences (AIIMS), New Delhi

Introduction and Background: Langerhans cell histiocytosis is a heterogeneous disease. Data is minimal in literature regarding safety and efficacy of cladrabine in salvage setting. We evaluated the same in our relapsed refractory $\mathrm{LCH}$ patients. Patient/Material and Methods: Single institutional retrospective review of $\mathrm{LCH}$ patients treated with cladribine between March 2006 to March 2014. All patients had relapse or progression of $\mathrm{LCH}$ after 1 st line noncladribine based chemotherapy. Treatment response, toxicity and outcome data were analyzed. Progression-free survival (PFS) was calculated from the date of progression after 1st line treatment to the date of progression after 2 nd line salvage therapy. Results: Thirteen patients were included with median age of 4 years (range: 1-28); male: female ratio of 10:3. The site of disease at presentation was skin lesions in ten patients, bone lesions in eleven (multifocal in ten \& solitary in one), lung in four, liver in three, brain lesion in one and bone marrow involvement in one patient. All patients received prednisolone, 6-marcaptopurine, vinblastine and etoposide (two patients without etoposide) as their 1st line treatment and initial response was - partial response (PR) in ten, stable disease (SD) in one and progressive disease (PD) in two patients. Median time to progression before 2nd line treatment was 16.6 months (range: 5-30.9). Six patients received cladribine in 2nd-line setting and seven patients received it in 3rd-line setting and response was-complete response in five, PR in one, SD in two, PD in three and unknown in two patients. Three patients had grade $3 / 4$ febrile neutropenia and one 
patient had pneumonia with no toxicity related death. After median follow-up of 71.8 months (range: 6-122.2), 5-year PFS and overall survival was $33.3 \%$ and $92.3 \%$, respectively. Conclusions: Cladrabine was a highly effective therapy with manageable toxicity in patients with relapse/refractory $\mathrm{LCH}$, and it can induce a long term disease control.

\section{MHC 63}

\section{Clinical Profile and Outcome of Patients of AML/MDS on Hypomethylating Agents}

\section{Pritish Chandra Patra ${ }^{\#}$, R. De, P. Chakrabarti, T. K. Dolai, P. K. Mandal}

Email ID for Correspondence: drpritishpatra@gmail.com NRS Medical College and Hospital

Introduction and Background: Acute myeloid leukemia (AML) is an aggressive malignancy with poor prognosis. Older patients especially have poor survival because of a variety of host and disease related adverse prognostic risk factors, such as history of myelodysplastic syndromes (MDS), unfavorable karyotypes, poor performance status and comorbidities, which can limit treatment options. Very similar picture is seen in case of MDS also. So many older patients receive only palliative care. Treatment with standard induction chemotherapy is associated with higher rate of morbidity and mortality. Whereas treatment with hypomethylating agents usually experience better survival outcomes, lower rates of complications and mortality. So here we conducted a study using hypomethylating agents in patients of elderly AML/MDS. Patient/Material and Methods: All consecutive cases of MDS and elderly ( $>60 \mathrm{yr}$ ) AML and adult patients of AML with age $>50$ yrs with poor performance status were treated with hypomethylating agents (Decitabine/Azacytidine). Results: Total 12 patients of AML/MDS received Hypomethylating agents, 7 were male and 5 female. Those with AML were 11 and 1 with MDS. 3 were having secondary AML, 2 from MDS and 1 from Myelofibrisos. 8 patients were of age $>60$ and 4 were $<60.8$ were with ECOG1, 2 with ECOG2, 1 with ECOG3 and ECOG4 each. 9 were having normal cytogenetics and rest were with Trisomy 8, del (9)(q22), del (20q) each. Azacytidine was given to 12 patients and Decitabine to 3 patients. Among these 7 are still on treatment and 5 have died during the course of treatment. Of the patients died during treatment, 1 patient was having FLT3-ITD and 1 with del (9)(q22). Conclusions: Though the experience in different part of the world may show a better outcome, the result here we see may reflect some aggressive disease biology, late presentation or may represent the need of better supportive care.

\section{MHC 64}

Allogeneic Hematopoietic Stem Cell Transplantation for Patients with Acute Lymphoblastic Leukemia: Single Center 10-Years Experience

Abhijit Baheti", Kavita M. Lakshmi, Anup Devasia, P. N. Nisham, N. A. Fouzia, Anu Korula, Aby Abraham, Poonkuzhali Balasubramanian, Alok Srivastava, Biju George, Vikram Mathews

Email ID for Correspondence: baheti.abhijit@gmail.com Christian Medical College, Vellore

Introduction and Background: The objectives of this study was to study the clinical profile and long term treatment related outcomes of acute lymphoblastic leukemia (ALL) patients who underwent allogeneic stem cell transplant (allo-SCT) at our center over 10 years. Patient/Material and Methods: One hundred and eleven patients with ALL (CR1-53 and $\geq$ CR2-58) underwent HLA matched (sibling-77, unrelated-34) allo-SCT. Overall $93.7 \%$ patients received $\mathrm{Cy} / \mathrm{TBI}$ as conditioning regimen, $94.6 \%$ patients received PBSC and $96.4 \%$ patients received cyclosporine/methotrexate as GVHD prophylaxis. Fourty eight percent of patients underwent allo-SCT in CR1 and $52 \%$ in $\geq \mathrm{CR} 2$. Among patients in CR1, $68 \%$ patients had high risk karyotyping on presentation $(\mathrm{t}\{9,22\}-45.3 \%, \mathrm{t}\{4,11\}-5.7 \%$, Complex-17\%). Among patients who were $\geq$ CR2 group, $1.8 \%$ patients were transplanted with refractory disease, the rest were in hematological remission. Results: The 5 year KM estimate of OS and EFS of the whole cohort was $25.9 \% \pm 5.4 \%$ and $27 \% \pm 4.8 \%$ respectively. Day 100 transplant related mortality was $9.4 \%$ in CR 1 cohort and $19 \%$ in the $\geq \mathrm{CR} 2$ cohort (p-0.184). Rate of acute and chronic GVHD were comparable in both groups. Relapse rates after allo-SCT were significantly lower in CR1 patients ( $25 \%)$ than $\geq \mathrm{CR} 2$ patients $(46.3 \%)(\mathrm{p}=0.027)$. However OS for patients transplanted in CR1 $(29 \% \pm 9 \%)$ was comparable to that of patients transplanted in $\geq \mathrm{CR} 2(24 \% \pm 6 \%)(\mathrm{p} 0.235)$. EFS was also comparable in both groups $(33 \% \pm 7 \%$ and $22 \% \pm 6 \%$ respectively, $\mathrm{p}-0.179)$. Conclusions: Durable long term response are achieved in a proportion of cases with ALL post allo-SCT. However additional interventions need to be considered post allo-SCT to further improve clinical outcomes.

\section{MHC 65}

Drug-Induced Hypereosinophilia Related to RCHOP Therapy for NHL (DLBCL)

\section{Abhijit Chakraborty", Chinmoy K. Bose, Firoj H. Gharami,} Santu Das, Ashis Mukhopadhyay

Email ID for Correspondence: abhijit.drems@gmail.com Netaji Subhas Chandra Bose Cancer Research Institute, 16 A Park Lane, Kolkata 700016

Introduction and Background: Hypereosinophilic syndrome (HES) is a rare disease defined by organ damage directly attributable to hypereosinophilia of any type. The most commonly involved organ is the lung, followed by the skin. Patient/Material and Methods: We report a single-center cohort of patients with NHL (DLBCL). Of these patients, nine had been treated with RCHOP on standard treatment protocol (6 cycle chemotherapy). In a retrospective analysis, data on treatment response, frequency of relapses, adverse events reconstitution were evaluated. Heamotology and biochemistry tests abs analysis were performed after every cycle. Results: All patients had high disease activity before RTX treatment. We found 3 patients who developed severe drug-induced hypereosinophilia with gastrointestinal involvement as a consequence of RCHOP infusion. For the case 1 Full blood count revealed a new mild eosinophilia with eosinophil count of $1.9 \times 10^{9} / 1$. After the third infusion, the eosinophil count had risen to $7.0 \times 10^{9} / \mathrm{L}$. In case 2 , after the 6th infusion, the eosinophil count had risen to $6.4 \times 10^{9} / \mathrm{L}$. In case 3 also it occurred in follow up period after 6th infusion and $6.8 \times 10^{9} / \mathrm{L}$. However the finding was not associated with any severe toxicity or other malignancy. No alarming feature was also notice alongside this abnormality. Conclusions: Preemptive retreatment with RTX, combined with CHOP, resulted in a sustained treatment response. Hence clinical significant is not clear this stage. Strict post marketing vigilance for evaluating the use of RCHOP was warranted. 


\section{MHC 66}

Clinicopathological Analysis of 11 Patients with Light Chain Myeloma

\section{S. P. Verma ${ }^{\#}$, A. K. Tripathi, Nidhish Kumar, Sudheer Kumar, Suneel Kumar, Sweta Srivastava}

Email ID for Correspondence: drspkgmu@ @rediffmail.com; spverma1998@gmail.com

King George's Medical University, Lucknow, Uttar Pradesh

Introduction and Background: Light chain myeloma is relatively uncommon and is characterized by absence of or minimal heavy chain production. It has different clinical and laboratory features compared to usual myeloma. Prognosis also is reported to be bad compared to normal myeloma. No data is available on clinical and lab features of light chain myeloma from India. Patient/Material and Methods: Eleven patients of light chain myeloma were identified between Jan 2013 and July 2016 after retrospective analysis of our multiple myeloma records. All clinical data, laboratory data, treatment and available outcome data was analyzed. Results: Light chain myeloma constituted $15 \%$ of all myeloma cases. Median age was 55 years (43-68 years) and male to female ratio was 1.7:1. Average duration of symptoms was 6 month (range: 2 months- 2 years). Bone pain, symptomatic anemia, neurological deficit and infection were present in $73 \%, 63 \%, 18 \%$ and $18 \%$ respectively at the time of presentation. Renal dysfunction was present in $45 \%$ of these patients at presentation. Bone lesions and hyperuricemia was present in $90 \%$ and $18 \%$ respectively. None of the patients had hypercalcemia and hyperviscosity symptoms. LDH was elevated above normal in only $27 \%$ of cases. A/G ratio reversal was not prominent with mean $A / G$ ratio of $1.27(0.91-1.64)$. Only one patient had A. G ratio $\leq 1$. Median beta 2 micro globulin levels were $4.2 \mu \mathrm{g} / \mathrm{ml}$ and $45 \%$ of patients belonged to ISS-3 stage based on beta 2 microglobulin levels. $\mathrm{k}$ light chain was predominant light chain in $73 \%$ (8 of the 11) patients. Free $\mathrm{k}$ light chain median level was $10,500 \mathrm{mg} / \mathrm{L}$ (range $142 \mathrm{mg} / \mathrm{L}-15,100 \mathrm{mg} / \mathrm{L}$ ). BM examination showed mean plasma cell number of $32 \%$. Patients received weekly bortezomib based regimens i.e. CyBorD (Cyclophosphamide, Bortezomib and Dexamethasone), Bor D (Bortezomib, Dexamethasone), and Bor T D (Bortezomib, Thalidomide and Dexamethasone) in $45 \%(5 / 11), 36 \%$ $(4 / 11)$ and $19 \%(2 / 11)$ of cases respectively. Response data was available in 8 patients. Overall response rates were $75 \%$ with sCR, CR, VGPR, SD and PD of $12.5 \%$ (1), $37.5 \%$ (3), $25 \%$ (2), $12.5 \%$ (1) and $(12.5 \%)$ respectively at the end of 4 months of chemotherapy. At the Median follow up of 12 months OS was $100 \%$ and PFS was $87 \%$. Pneumonia, herpes zoster, peripheral neuropathy and pedal edema were seen in one patient each while diarrhoea and cytopenias were seen in 2 patients each during chemotherapy. Conclusions: Light chain myeloma has more renal failure, normal $\mathrm{A} / \mathrm{G}$ ratio, normal calcium and no hypervicosity compared to normal myeloma. Early responses on bortezomib based protocols are very good. Bone marrow examination and serum free light chain assay are important tools to identify patients with light chain myeloma.

\section{MHC 67}

Comparative Study of Bortezomib-CyclophosphamideDexamethasone (VCD) and Bortezomib-ThalidomideDexamethasone (VTD) Based Regimens in Patients of Multiple Myeloma-In a Tertiary Centre in West Bengal

U. Akshatha Nayak ${ }^{\#}$, Saikat Dutta, Kalantri Siddhesh Arun, Professor Maitreyee Bhattacharyya, Sambit Samanta, Siddhartha Roy
Email ID for Correspondence: anayakbejai@gmail.com Institute of Haematology And Transfusion Medicine, Medical College and Hospital, Kolkata

Introduction and Background: Multiple myeloma (MM) is a B cell malignancy characterized by clonal proliferation of plasma cells in the bone marrow, associated with an increased level of monoclonal protein in the blood and/or urine. Clinical features include increased risk of infection, pancytopenia, renal failure and bone disease. Although the disease remains incurable, outcomes have improved substantially in recent decades as a result of advances in treatment. Three-drug induction regimens have become the standard of care with two frequently used protocols are bortezomib, cyclophosphamide and dexamethasone (VCD) and bortezomib, thalidomide and dexamethasone (VTD). This study aims to study and compare the efficacy and treatment-related toxicity of the two induction regimens in multiple myeloma patients. Patient/Material and Methods: We retrospectively analysed patients of Multiple Myeloma from December 2014 to May 2016 who were upfront started on VCD or VTD. Results: Twenty six patients (Male-15 and female-11) with a median age of 47 years who had been randomly assigned to the two regimens VCD vs. VTD were analysed. Of these 14 belonged to the VCD arm and 12 belonged to the VTD arm. In the VCD arm, 2 patients belonged to ISS-1, 7 patients belonged to ISS-2 and 5 patients belonged to ISS-3. In the VTD arm, 6 patients belonged to ISS- 2 and 6 belonged to ISS-3. In the VCD arm response at 3 months were 10 patient-VGPR and 4 patients-PR and response at 6 months were 6 patients-CR and 7 patients-VGPR and 1-stable disease. In the VTD arm response at 3 months were 9-VGPR and 3-PR and response at 6 months 7-CR, 4-VGPR and 1-stable disease. Treatment related toxicity were more in VTD arm in the form of Peripheral neuropathy compared to VCD arm. Conclusions: A larger study is needed to analyse and satisfactorily compare the efficacy of these two regimens.

\section{MHC 68}

Extramedullary Chronic Myeloid Leukemia (CML): An Unusual Initial Presentation as Thigh Mass

\section{Prakash Singh Shekhawat", Dibakar Podder, Saurabh Jayant Bhave, Mammen Chandy}

Email ID for Correspondence: drprakashsinghshekhawat@gmail.com Tata Medical Center, Kolkata

Introduction and Background: Chronic Myeloid Leukemia (CML) is a myeloproliferative neoplasm, characterized by presence of Philadelphia $(\mathrm{Ph})$ chromosome. CML evolve through three distinct clinical phases, (a) chronic phase, (b) accelerated phase, (c) blast crisis. Extramedullary presentation in CML is well known in blast crisis. Chronic phase rarely present at extramedullary site. Patient/ Material and Methods: 36-year-old Bhutanese male presented to OPD with swelling and pain in left knee joint for 1 month and anemia for 1 month. There was a large echymotic patch on left thigh and a swelling in the left thigh which was tender. Lab investigations revealed following: $\mathrm{Hb}-7.8 \mathrm{gm} / \mathrm{dl}$, TLC $-342,300 / \mathrm{cu} \mathrm{mm}$, Platelet-5.49 L/cu mm. Differential: Neutrophil-35\%, lymphocyte$5 \%$, monocyte- $0 \%$, eosinophil-3\%, basophil-7\%, myelocytes/ metamyelocytes-50 \%, blasts-0 \% . FISH for BCR/ABL: $92 \%$ of cells are positive/200 interphase cells analysed. CT guided biopsy showed cores of skeletal muscle bundles infiltrated by eosinophils, immature myeloid cells, neutrophils and occasional plasma cells. Results: After all the investigations, patient was started on tab Imatinib $400 \mathrm{mg}$ od. The swelling significantly reduced after 15 days of treatment with tab Imatinib. Conclusions: Here we present a rare case report of a chronic myeloid leukemia-chronic phase presented at an 
extramedullary site (thigh mass). The swelling significantly reduced on imatinib on subsequent visit.

\section{MHC 69}

\section{A Rare Case of Triple Hit Lymphoma \\ Gumdal Vishesh", Prashant Kumbhaj, Prakash Chitalkar}

Email ID for Correspondence: g.vishesh@gmail.com Sri Aurobindo Institute of Medical Sciences

A 15 year old male presented with complaints of pain in the abdomen since 15 days, low grade fever which was intermittent since 14 days along with constipation since 2 days. Physical examination was unremarkable except for a hard mass palpable in the right hypochondriac region. There was no lymphadenopathy. On initial evaluation all the routine lab parameters were normal. USG abdomen was suggestive of a $10 \mathrm{~cm}$ mass involving the hepatic flexure. CT abdomen was suggestive of lymphoma of the colon involving the hepatic flexure. LDH was $2055 \mathrm{U} / \mathrm{ml}$. Trucut biopsy of the colonic mass confirmed lymphoma. Microscopy showed round cells in diffuse sheets, Cells were intermediate to large, Noncleaved nuclei, few cells show large nuclei with nucleoli. No starry sky pattern seen. Brisk mitosis seen. Conclusion: Triple hit lymphoma is rare type of high grade B cell Lymphoma usually occurring among adults. Here is a 15 year old male who presented to us with the same.

\section{MHC 70}

Cyclophosphamide Followed by Intravenous Busulfan (CY-BU) as Myeloablative Conditioning: Impact on Veno Occlusive Disease (VOD) and Transplant Outcomes, A Single Centre Experience

\section{Rajiv Kumar, Rajan Kapoor, Jasjit Singh, Tarun Verma,} Ajay Sharma, Velu Nair ${ }^{\#}$

Email ID for Correspondence: ankitabist9@gmail.com Department of Hematology Army Hospital R\&R, New Delhi

Introduction: Myeloablative conditioning (MAC) with busulfan followed by cyclophosphamide (BU-CY) is used for various malignant and non-malignant conditions during allogenic Hematopoeitic stem cell transplantation (HSCT). However it is associated with a significant risk of Veno-occlusive disease/Sinusoidal Obstruction Syndrome (VOD/ SOS) which also contributes to an increased 100 day mortality posttransplant. The standard protocol of MAC uses oral or IV Busulfan (BU) followed by IV Cyclophosphamide (CY). The reverse sequence of use of these drugs during conditioning as 'CY-BU' has been reported to be associated with a possible decreased incidence of VOD in few studies. Objectives: To test the possible effect on liver toxicity and clinical outcome of this approach, we started a clinical trial using CY-BU as a MAC regimen for HSCT from 2014 at our center. Methods: Seventeen patients who were at a higher risk of VOD due to previous chemotherapy exposure in acute leukemia/chronic myeloid leukemia (CML) in blast crisis or iron overloading in thalassemics due to repeated blood transfusions, received CY-BU conditioning for a matched sibling donor allogenic HCT. The conditioning regimen consisted of CY $60 \mathrm{mg} / \mathrm{m}^{2} / \mathrm{kg}$ on days -7 and -6 followed by IV BU $3.2 \mathrm{mg} / \mathrm{kg}$ as infusion over $3 \mathrm{~h}$ (total of 04 doses) on days -5 to -2 . As post-grafting immunosuppression, all patients received a short course of methotrexate and cyclosporin from day -1 . The historical control group consisted of 100 consecutive patients, treated in the same institution till 2014, using the same protocol of immunosuppression but with the conventional BU-CY conditioning regimen. Both groups were comparable demographically. Results: One patient $(5 \%)$ treated with CY-BU and eighteen (18\%) patients of the BU-CY group developed severe VOD before day+20 after HSCT, as per the modified Seattle criteria. While there was no mortality related to VOD in CY-BU group, there were four deaths $(4 \%)$ due to VOD in BU-CY group which was statistically significant $(\mathrm{p}<0.05)$. Serum bilirubin Alanine aminotransferase and aspartate aminotransferase serum levels were statistically higher in patients treated with BU-CY $(\mathrm{p}<0.05 \%)$. There was no engraftment failure in either group and the median time of neutrophil engraftment was 14 days in both the treated groups. D 100 mortality due to non-hepatic causes was similar in both groups. Conclusions: In our cohort of patients treated with CY-BU, significantly less hepatic toxicity was observed and neutrophil engraftment did not differ from that in patients treated with BU-CY. Though the number of patients are less, our results show that CY-BU' MAC is a feasible protocol which is well tolerated and is associated with a decreased incidence of severe VOD/SOS, especially in the high risk group of patients.

\section{MHC 71}

Spontaneous Chest Wall Hematoma-An Uncommon Presentation of Chronic Myeloid Leukemia

Saswata Saha", Aman Chaudhary, Sandesh M. Raykar, Siddharth Jain, Navneet Kumar Agrawal, Chandan Kumar, L. P. Meena, Prof. M. Rai

Email ID for Correspondence: saswataonline@gmail.com IMS, BHU

Introduction and Background: Spontaneous hematomas are most commonly caused by bleeding and clotting disorders followed by anticoagulant and antiplatelet drugs. Spontaneous hematoma secondary to malignancy is usually uncommon. CML is the haematological malignancy known for variability in presentation. Spontaneous hematomas are unusual presentation of CML with only few cases reported. Patient: A 63 years old male, presented with complaint of sudden painful, boggy swelling over the left chest wall few hours following trivial trauma. No history of drug intake, joint swelling following trauma or similar episodes in past. No history of similar illness in family members. On examination he was pale, the vital signs were within normal limits. The swelling was warm, firm, tender and overlying skin was reddish. He had a huge, firm, nontender splenomegaly. He had no petechiae, purpura, ecchymosis or other swelling over his body. Other system examinations were within normal limits. Investigations: Haemoglobin-7.6 g\%; TLC$474,300 / \mathrm{mm}^{3}$ with immature Neutrophil precursors, suggestive of CML. Liver and renal function tests were normal. PT/INR and APTT were not significantly raised. Sr. LDH was 859 and Sr. Uric Acid was 7. Chest $X$ ray showed homogenous opacity in the left lung field. USG of chest wall was suggestive of intramuscular hematoma extending upto lower scapular region. USG abdomen revealed gross splenomegaly $(22 \mathrm{~cm})$. Results: Bone marrow examination was suggestive of CML in chronic phase. Karyotyping revealed $\mathrm{Ph}$ ' chromosome in $100 \%$ cells. Chromosomal study showed BCR-ABL gene positive CML (ISNCN-52.45\%). Chest wall hematoma resolved spontaneously with conservative management within 3 wks. Patient was started on hydroxyurea for cytoreduction and later on imatinib was started. Conclusions: Few cases of spontaneous hematoma with CML have been reported till now. However, the exact mechanism of spontaneous hematoma in CML is not known. Further research is required to understand the exact mechanism and its management. 


\section{MHC 72}

Acute Central Nervous System Complications during Therapy in Acute Lymphoblastic Leukemia: Experience from Tertiary Care Centre of North India

\section{Pradeep Kumar, Anshul Gupta ${ }^{\#}$, Sanjeev Kumar Yadav,} Soniya Nityanand

Email ID for Correspondence: anshulhaemat@gmail.com Department of Haematology, Sanjay Gandhi Post Graduate Institute of Medical Sciences, Lucknow

Objective: To study the incidence, clinical features and outcome of acute neurological complications during therapy in newly diagnosed Acute Lymphoblastic Leukemia (ALL) patients treated on Berlin, Frankfurt, Munster (BFM)-ALL 95 protocol. Methods: It is a single institution retrospective study (July 2011 to November 2015) in which the data of 295 newly diagnosed cases of ALL who received treatment at our centre was analysed. We evaluated the incidence of acute neurological complications during therapy with focus on clinical features, putative risk factors for these complications and outcome of these patients. The patients who had CNS disease involvement at the time of presentation or developed CNS relapse at the time of neurological event and peripheral neuropathy were excluded from the analysis. Results: Twenty five $(8.4 \%)$ patients developed CNS complications during the ALL therapy in our cohort of 295 patients of which twenty one $(84 \%)$ patients developed neurological complications during induction phase of chemotherapy while $2(8 \%)$ developed during consolidation and $2(8 \%)$ during maintenance phase of chemotherapy. The most common clinical presentation at diagnosis was seizure present in 17 patients $(68 \%)$ while 6 patients $(24 \%)$ presented with headache with vomiting, 1 (4\%) each with hemi paresis and bilateral facial nerve palsy respectively. Cerebral sinus thrombosis was the most frequent cause for the neurological complication which was present in 7 (28\%) of our patients followed by intracranial hemorrhage present in $6(24 \%)$ patients. Thrombocytopenia (platelet count $<40,000 / \mu \mathrm{l}$ ), prolonged prothrombin time (INR >1.5), peripheral blood blast percent greater than $70 \%$ was significantly associated with the risk for fatal intracranial bleed $(\mathrm{P}$ value $<0.001)$. Of the 25 patients who developed neurological complication during therapy, $14(56 \%)$ succumbed due to their complications despite intensive care \& monitoring. The outcome was notably poor for the patients who developed intracranial hemorrhage with only 1 patient surviving the event. Conclusion: CNS complications are frequent events during ALL therapy and has dismal outcome despite intensive management. The early diagnosis of acute neurological complications during ALL therapy is a key to better management and it requires a strict collaboration between the haematologist, neurologist and neuro-radiologist.

\section{MHC 73}

Imatinib on Prevalence of Falciparum Malaria: A Case Control Study from a Tertiary Care Centre in West Bengal

\section{Asif Iqbal $^{\#}$, Arijit Nag, Jasashwi Chakraborty, Soumita Choudhuri, Rudra Ray, Maitreyee Bhattacharyya}

Email ID for Correspondence: driqbal85.ai@gmail.com IHTM, Kolkata

\footnotetext{
Abstract: Rising evidence of artemisin resistant malaria in Indian subcontinent and parts of South America has prompted researchers to search for alternative antimalarials. In the last few decades, few reports have emerged showing dependence of malarial parasites on RBC tyrosine kinases for its maturation. Subsequent in vitro tests have established that TKIs inhibit proliferation of Plasmodium
}

falciparum. The results were encouraging and later tests were done with Imatinib and other related TKIs. As there were no clinical data to support the hypothesis, we conducted a case control study with patients of CML-CP on fixed dose Imatinib as cases and data derived from a representative population as control. A standard questionnaire and rapid diagnostic testing for plasmodium antigens from the blood of cases attending CML clinic at IHTM were used as study tools. 191 CML patients on Imatinib $400 \mathrm{mg} / \mathrm{d}$ for a minimum period of 1 year were analysed. Out of total febrile episodes of 54 during the study period, one patient had vivax malaria after starting treatment with Imatinib, and no patient had falciparum malaria. RDT testing revealed four asymptomatic parasitemia of which two were falciparum infections. The prevalence of asymptomatic falciparum parasitemia was found to be $1.45 \%$ which was lower than the population control (2.8-5.7\%). When analysed, the difference was found to be statistically significant. To conclude, we support the hypothesis that Imatinib has protective effect against $P l$. falciparum infection.

Keywords Imatinib, TKI, Falciparum, Malaria

\section{Topic: Malignant Haematology_Laboratory}

\section{MHL 1}

Anti-thymocyte Globulin (ATG), Not Only Immunosuppressive But Also Antileukemic Drug

Rosy Dabas", Rachelle Lee, Maria Theresa Servito, Poonam Dharmani-Khan, Monica Modi, Tiffany van Slyke, Joanne Luider, Caylib Durand, Loree Larratt, Joseph Brandwein, Don Morris, Andrew Daly, Faisal M. Khan, Jan Storek

Email ID for Correspondence: rdabas@ucalgary.ca University of Calgary, Calgary, Canada

Introduction and Background: Allogenic hematopoietic cell transplantation (HCT) has become a critical part of treatment for patients with acute myeloid leukemia (AML). The success of HCT is however limited due to leukemia relapse and graft-versus-host disease (GVHD). Recent advances in HCT pioneered in Alberta, comes from the use of polyclonal anti-thymocyte globulin (ATG) which decreases GVHD without increasing relapse. The lack of increasing relapse is surprising because, so far, every immunosuppressive drug that decreased GVHD has also increased relapse. The reason why ATG decreases GVHD without increasing relapse is unclear. We hypothesize that this is because ATG has a direct anti-leukemic effect. In this study, we measured the cytotoxic activity of ATG against leukemic blasts in vitro at clinically relevant concentrations $(4.5 \mathrm{mg} / \mathrm{kg})$. Patient/Material and Methods: Blood or bone marrow from patients newly diagnosed with acute myeloid leukemia (AML, $n=31$ ) or acute lymphoid leukemia (ALL, $\mathrm{n}=5$ ), obtained before induction chemotherapy, was used as the source of leukemic cells. The cytotoxic activity of ATG was measured via complement-dependent cytotoxicity (CDC) and complement-independent cytotoxicity (CIC) using flow cytometry. Results: ATG induced death of leukemic blasts both via CDC and CIC in vitro. Median $0.3 \%, 2.8 \%, 12.6 \%$ and $42.2 \%$ blasts were killed with $1,10,25$ and $50 \mathrm{mg} / \mathrm{L}$ ATG, respectively, via CDC. Median $1.9 \%, 7.15 \%, 12.1 \%$ and $13.9 \%$ blasts were killed with 1, 10, 25 and $50 \mathrm{mg} / \mathrm{L}$ ATG via CIC. Conclusions: ATG at clinically relevant concentration kills primary leukemic cells in vitro. Some acute leukemias are highly sensitive and others relatively resistant to ATG. Whether this applies to in vivo needs to be determined. If yes, and given that the killing of leukemic cells is ATG concentration-dependent, conditioning regimens with high-dose ATG could be developed for patients with leukemia sensitive to ATG to maximize the concurrent anti-GvHD and anti-relapse effects. 


\section{MHL 2}

\section{Hematological and Bone Marrow Biopsy Evaluation in Non Hodgkin Lymphoma}

\section{Kanwalpreet Kaur ${ }^{\#}$, Nidhi Sharma, Karuna Gupta, Sandhya Gulati, Ajay Yadav}

Email ID for Correspondence: kanwalpreet.15@gmail.com SMS Medical College, Jaipur

Introduction and Background: International prognostic index for risk assessment in patients with Non-Hodgkin's lymphoma (NHL) included bone marrow evaluation as part of staging work up. Aim of this study is to analyze incidence and histological pattern of bone marrow involvement in diagnosed cases of NHL. Patient/Material and Methods: This is a retrospective study on 85 patients diagnosed with NHL. Bone marrow biopsy was performed under local anesthesia as a part of staging workup in cases of NHL. Complete blood counts $(\mathrm{CBC})$ were also noted in each case. Results: In this study, NHL cases comprised of low grade NHL $68.2 \%$, intermediate grade $28.2 \%$ and high grade $3.5 \%$. The age ranged from 10 to 75 years and M:F ratio was 1.07:1. $38.8 \%$ (33/85) cases showed bone marrow involvement. Majority of bone marrow involvement was seen in indolent lymphomas $(87.8 \%)$. Most common pattern of involvement was mixed (12/33) followed by diffuse (9/33), interstitial (8/33) and nodular (4/33). 87.9\% cases showed increase in reticulin which included reticulin grade $1(9 / 33)$, grade $2(14 / 33)$ and grade $3(6 / 33)$. Only $48.5 \%$ cases of bone marrow involved cases had CBC abnormalities while $51.5 \%$ were completely normal. CBC abnormalities included anemia (5/16), leucopenia (3/16), leukocytosis (8/16) and thrombocytopenia (9/16). Conclusions: Bone marrow biopsy is an essential part of staging work up of NHL affecting the prognosis and treatment.

\section{MHL 3}

Primary Extranodal Non-Hodgkin Lymphoma: A 2-Year Retrospective Analysis from a Tertiary Care Centre In Rajasthan

\section{Jayanti Mehta, Monil P. Thakrar ${ }^{\#}$, Matadeen Meena, Alka Mittal, Karuna Gupta \\ Email ID for Correspondence: monil.p.thakrar@gmail.com SMS Medical College and Attached Hospitals, Jaipur}

Introduction and Background: Primary extranodal lymphoma (pENL) refers to group of disorders arising from tissues other than lymph nodes and even from sites, which normally do not contain lymphoid tissue. The incidence of pENL is rising throughout the world. However, data regarding ENL as a group is limited. This study was undertaken to ascertain the prevalence, anatomical distribution, and histological subtypes of extranodal non-Hodgkin lymphoma (NHL) from a tertiary care institute in Rajasthan. Patient/Material and Methods: This was a cross-sectional study conducted in the Department of Pathology over a period of 2-years. Detailed clinical history, routine complete blood count, microbiological status was obtained from the medical records. Hematoxylin and eosin slides were reviewed and immunohistochemistry was performed using a panel of antibodies depending on the morphology. All cases were classified based upon morphologic and immunophenotypic criteria according to World Health Organization 2008 classification. Results: Primary extranodal NHL constituting $54.7 \%(70 / 128)$ of all NHL and the majority of patients were from higher age group with peak incidence seen in fifth and sixth decade of life. In the paediatric population, the most common site was GI tract $(45.5 \%)$ followed by skin $(18.2 \%)$ and the most common morphology was diffuse large B-cell lymphoma $(54.5 \%)$. Similarly in the adult population CNS was the most common site constituting $20.3 \%$, followed by head and neck $(18.6 \%)$ and diffuse large B-cell lymphoma was the most common morphological subtype comprising $64.4 \%$ of all adult extranodal lymphomas. Conclusions: Although the reported incidence of pENL is low in India compared to other parts of the world, the possibility of pENL should always be kept in mind even though it arises in an extranodal site.

\section{MHL 4}

Cytogenetic Study of Patients with Aplastic Anaemia

\section{Atreyee Dutta ${ }^{\#}$, Rajib De, Tuphan Kanti Dolai, Ajanta Halder}

Email ID for Correspondence: amimegh@gmail.com Vivekananda Institute of Medical Sciences, 99, Sarat Bose Road, Kolkata 700026

Introduction and Background: Aplastic anaemia (AA) is a rare clonal hematological complication with pancytopenia and hypo-cellular bone marrow. It can be classified broadly in two groups inherited/genetic \& acquired. The exact incidence of AA in India is still unknown. It is the first comprehensive study on cytogenetic abnormalities related with AA patients of eastern India. Our study was aimed to summarize the possible cytogenetic abnormalities related with AA patients in eastern India. Patient/Material and Methods: 51 peripheral venous blood and 19 bone marrow samples were collected with proper consent from 70 diagnosed AA patients for last one year. Then Human leukocyte culture followed by GTG-banding was done. Karyotyping was done according to the International System for Human Cytogenetics Nomenclature (ISCN). Between these 70 patients peripheral venous blood was collected from 49 morphologically normal ( $<50$ years) AA patients and Stress Cytogenetics was done to exclude the possibilities of Fanconi anaemia. Results: Out of 19 bone marrow cases of AA only one $(5.26 \%)$ patient showed (28 Y/M) multiple breaks in $44 \%$ cells and loss of $\mathrm{Y}$ chromosome 45, X-Y [3] with normal cell 46, XY [12]. Between 51 peripheral venous blood sample of AA patients cases only one $(1.96 \%)$ had structural chromosomal abnormality 45, XY, der(14:21) (p10:q10)[20]. Other 68 (97.14\%) patients showed normal karyotype irrespective of bone marrow or peripheral venous blood sample. Four $(8.16 \%)$ patients were found to be positive for stress cytogenetics thus suggestive for Fanconi anaemia. Conclusions: Routine Cytogenetic analysis of AA patient in not essential. Morphologically normal AA patient can be positive for stress cytogenetics. There was no significant difference between clinical and haematological profile of patients with normal versus abnormal cytogenetics. We have reported a unique chromosomal translocation through this study, never reported before related with AA. Further studies on a large sample are required to confirm the finding.

\section{MHL 5}

Aberrant Expression of CD Markers in Acute Lymphoblastic Leukemia: A Diagnostic Clue of Malignancy or Compounding Confusions

\section{Monika Gupta ${ }^{\#}$, Shalini Shah, Sumiti Gupta, Rajeev Sen}

Email ID for Correspondence: monikashashwat@hotmail.com Pt BDS, PGIMS, University of Health Sciences, Rohtak

Introduction and Background: Flow cytometric immunophenotyping is currently part of standard of care test for acute lymphoblastic leukemia (ALL). It helps to confirm the diagnosis, exclude neoplastic and non neoplastic mimics. Aberrant expression of $\mathrm{CD}$ markers is 
seen in several cases of acute leukemia due to their abnormal genetic programme, include abnormal or asynchronous expression of normal hematogone associated antigens and inappropriate expression of myeloid, T-lymphoid, and NK cell-associated antigens. In present study we evaluated the frequency of aberrant expression of $\mathrm{CD}$ markers in ALL. Patient/Material and Methods: Immunophenotyping was performed in 36 new cases of ALL patients on 8 Color Flowcytometer BD FACS Canto II using CD45/SSC gating. The CD panel used was CD45, CD34, sCD3, cyCD3, CD5, CD2, CD7, CD4, CD8, CD10, CD20, CD19, CD79a, Tdt, CD13, CD33, CD117, HLADR, MPO, and CD64. Results: There was slight male predominance with male to female ratio of 1.6:1. Average age of the patients was 24.2 years (range 2-70 years). On analysis of immunophenotypic expression, $32(88.9 \%)$ patients had B-ALL and $4(11.1 \%)$ patients had T-ALL. Seven $(19.4 \%)$ cases of B-ALL and one $(2.8 \%)$ case of T-ALL expressed aberrant markers. In B-ALL, most common aberrant marker was CD13 present in 5 cases, followed by CD33 in 2 cases. One case was positive for CD7. One case co- expressed CD13 and CD33. Infidelity marker seen in one case of T-ALL was CD79a. Conclusions: The presence of aberrancy helps to identify a neoplastic process. It has been demonstrated that several immunophenotypes of blast cells from cases of ALL do not exhibit the features of normal cellular differentiation but show the expression of aberrant markers. To conclude aberrant immunophenotypes are quiet common in ALL and have important implications for the diagnosis, monitoring and also establish baselines for subsequent detection of MRD.

\section{MHL 6}

\section{Isolated Lymphoid Blast Crisis in CNS in Juvenile CML}

\section{Deeksha Sikri ${ }^{\#}$, Sudarshan Choughale}

Email ID for Correspondence: deeksha.sikri@gmail.com Apollo Specialty Hospital, Chennai

Introduction and Background: Isolated blast crisis in CNS in patient of CML is a very rare occurrence. Here we present a case of a 5 year old female patient diagnosed with Juvenile CML, who after remission presented with a case of isolated CNS blast crisis of lymphoid type after one year. Patient/Material and Methods: We present a case of a 5 year old female patient who presented to us in May 2015 with complaints of tiredness. Complete blood count showed hyperleucocytosis with a total count of $191.4 \times 10^{9} \mathrm{cells} / \mathrm{cu} \mathrm{mm}$. On bone marrow aspiration and biopsy, the patient was diagnosed with Juvenile Chronic Myelogenous Leukaemia-chronic phase, which was confirmed on karyotyping, FISH analysis and RT-qPCR. The patient was started on Imatinib and went into clinical, haematological and cytogenetic remission. In June 2016, the patient was admitted again with complaints of headache, vomiting and diplopia. She was found to have right lateral rectus palsy and papilledema. Results: MRI Brain showed callosal dysgenesis with absent septum pellucidum and pericallosal lipoma. Cerebrospinal fluid showed a high leucocyte count with $97 \%$ atypical lymphoid cells with high $\mathrm{N}: \mathrm{C}$ ratio, scant cytoplasm, dense chromatin and prominent nucleoli. Xpert TB on CSF, CSF culture and Crytptococcal antigen were negative. Bone marrow aspiration showed normocellular marrow with no increase in blasts, eosinophils or basophils. On immunophenotyping of CSF by flow cytometry (8 colour 3 laser Beckman Coulter NAVIOS), $32 \%$ CD $45 \mathrm{dim}$ low side scatter population was gated. These cells were found to be brightly positive for CD 10 and CD 19 . HLA DR positivity was seen along with aberrant high expression of CD 33. All other myeloid and $\mathrm{T}$ cell markers were negative. Conclusions: In conclusion, the patient was diagnosed to have an isolated CNS Lymphoid blast crisis post treatment for Juvenile CML. She is now being treated with Dasatinib, ALL regimen A and Triple intrathecal chemotherapy.
MHL 7

Chronic Eosinophilic Leukemia with FIP-1 like 1-Platelet Derived Growth Factor Receptor Alpha Mutation (FIP 1L1-PDGRA)

\section{Ranjita Bansal", Karuna Gupta, Neetu Agrawal, Ajay Yadav, Kusum Mathur}

Email ID for Correspondence: ranjitabansal@gmail.com SMS Medical College, Jaipur, Rajasthan

Introduction and Background: Hypereosinophilic syndrome (HES) is a rare disorder that is characterized by persistent and marked eosinophilia combined with organ system dysfunction. HES has substantial clinical heterogeneity and can be fatal without treatment, especially in patients who present with a myelodysplastic variant of the disorder. Clonal eosinophilias belong to the group of myeloid malignancies. Karyotypically occult FIP1L1-platelet-derived growth factor receptor alpha and beta rearranged eosinophilic disorders respond to imatinib mesylate with almost $100 \%$ efficacy. FIP1L1PDGFRA fusion gene detectable in $5 \%$ to $15 \%$ of all cases with clonal HE. Patient/Material and Methods: Here we present a case of 38 year old male having 12 months history of progressive dyspnea, fever, cough, weight loss and pedal edema with recent worsening, examination reveal bilateral pneumonitis, right sided heart failure with hepato-splenomegaly and on further work up Peripheral blood showed leukocytosis with eoisinopihillia (65\% with TEC-14 $\times 10^{3} /$ $\mu \mathrm{l})$, Bone marrow biopsy showing myeloid hyperplasia with increased eosinophils and eosinophilic precursors, Lung biopsy shows mild destruction of lung parenchyma with thickening of septa, mild mononuclear cell infiltration \& patchy areas of fibrosis-favours UIP, elevated B12 and CPKMB with FIP1L1-PDGFRA rearrangementpositive. Results: Chronic eosinophilic leukemia with FIP-1 like 1-platelet derived growth factor receptor alpha mutation (FIP 1L1PDGRA) with Loffler's endocarditis with pneumonitis. Conclusions: Not all cases of hypereosinophilia are reactive and secondary to parasite infestation. Clinical suspicion of myloproliferative neoplasm with hypereosinophilia is essential for early diagnosis to limit morbidity and mortality associated with cardiac involvement. Imatinib is drug of choice in presence of PDGRFA/B positive mutation which suggest importance of mutation analysis.

\section{MHL 8}

Serum Transferrin Receptor in the Diagnosis of Iron Deficiency Anemia (IDA)

Abhishek Pathre, Ruchika Sodhi", Meera Sikka, Sunil Gomber, Satendra Sharma, Mrinalini Kotru

Email ID for Correspondence: ruchika.sodhi@gmail.com University College of Medical Sciences and GTB Hospital, Delhi

Introduction and Background: IDA constitutes a major public health problem in developing and developed countries. It adversely affects cognitive function, delays neurologic development and is associated with poor scholastic performance among children and adolescents. It is thus imperative to diagnose children with IDA and treat them early. Patient/Material and Methods: This cross sectional study was conducted in the Departments of Pathology and Pediatrics, UCMS and GTB Hospital, Delhi on 90 patients of age $\leq 12$ years. Iron profile and serum ferritin (SF) were done in all patients. Sixtytwo patients with IDA ( $\mathrm{SF}<16 \mu \mathrm{g} / \mathrm{L}$ and/or \% Transferrin saturation $(\%$ TS $)<16 \%$ ) were finally included. Thirty five non anemic children were studied as controls. Serum transferrin receptor (sTfR) levels were measured (ELISA) to study it's utility as a diagnostic test for IDA. Results: Mean \pm SD sTfR was significantly $(\mathrm{p}<0.001)$ 
higher in patients $(6.0 \pm 2.9 \mu \mathrm{g} / \mathrm{ml})$ as compared to controls $(1.2 \pm 0.6 \mu \mathrm{g} / \mathrm{ml})$. sTfR was elevated $(>1.8 \mu \mathrm{g} / \mathrm{ml})$ in $59(95.1 \%)$ patients and in $4(11.4 \%)$ controls. ROC curves were constructed. The AUC of sTfR was 0.985 indicating it's high clinical value as a diagnostic test for IDA. sTfR $\geq 2.25 \mu \mathrm{g} / \mathrm{ml}$ had a sensitivity and specificity of $90.3 \%$ and $94.1 \%$ respectively in diagnosis of IDA. Ratio of sTfR and log ferritin (sTfR-F index) was also significantly $(<0.001)$ higher in patients $(84.5 \pm 87.6)$ as compared to controls $(0.7 \pm 0.4)$. Conclusions: Raised receptor levels are a useful indicator of a functionally significant iron deficit. sTfR levels are not affected by an active acute phase reactant, thus it's more advantageous and specific than serum iron. Measurement of sTfR and sTfR-F index may be used as a non invasive tool for early diagnosis of IDA.

\section{MHL 9}

Retrospective Analysis of Hyponatremia and Syndrome of Inappropriate Anti-diuretic Harmone Secretion (SIADH) in Bone Marrow Transplant

Umakant A. Gavhane", B. G. S. Pillai, M. Tiwari, S. Pal, U. Gosavi, R. Mohite, A. Karmore, S. Vaykar, A. Kumari, P. Dalvi, D. Kawale, V. Bhat, P. Chavan, S. Mokashi, P. Poladia

Email ID for Correspondence: umakant_gavhane@ rediffmail.com Advanced Centre For Treatment Research \& Education In CancerTata Memorial Centre, Sector-22, Kharghar, Navi, Mumbai 410210, India

Introduction and Background: One of the common complication observed in oncology patients is electrolyte imbalance. Hyponatremia is common abnormality in hospitalized patients and is associated with increased morbidity and mortality. In patients of Bone Marrow Transplant (BMT), Syndrome of Inappropriate Anti-Diuretic Hormone secretion (SIADH) has been reported in the literature between 7-13\%. We undertook this study to evaluate the incidence of hyponatremia and SIADH in our patient's post-BMT. Patient/Material and Methods: We analysed 359 (252 male and 107 female, 69 paediatric and 290 adult, 160 allogenic and 199 autologous transplant) cases of peripheral blood BMT conducted between a period of June 2012 to may 2016 retrospectively. Serum electrolyte levels were assessed during the stay in the BMT unit and subsequently twice to thrice a week during their follow up outpatient department (OPD) visits. Hyponatremia was defined as serum sodium level $<136 \mathrm{mmol} /$ 1. In Hyponatremics further serum and urine osmolality were also assessed. Results: The incidence of hyponatremia was found to be $5.84 \%$ (21/359). Total number of patients with SIADH was $7 / 359$ $(1.95 \%)$. Incidence of Hyponatremia was $5.95 \%$ in men, $5.6 \%$ in women $(\mathrm{p}=0.8986) ; 8.75 \%$ in allogenic, $3.52 \%$ in autologous transplant $(\mathrm{p}=0.0610) ; 6.21 \%$ in adult and $4.34 \%$ in paediatric groups $(\mathrm{p}=0.7765)$ respectively. Serum sodium levels were $\leq 130 \mathrm{mmol} / \mathrm{l}$. In all Hyponatremics. Incidence of SIADH in allogenic and autologous BMT patients was $3.75 \%$ and $0.50 \%(\mathrm{p}=0.0479)$ respectively. Out of all 290 adult and 69 paediatric cases, SIADH was observed in $6.2 \%$ and $1.45 \%$ respectively $(p=1.0)$. Incidence of SIADH was $2.38 \%$ in men and $0.94 \%$ in women $(\mathrm{p}=0.6790)$. More than $70 \%$ of SIADH cases occurred within 100 days post transplant. Conclusions: Compared to other published studies incidence of hyponatremia and SIADH post BMT was observed to be lower in our patients. SIADH was more commonly seen in allogenic transplant patients than autologous transplant setting.
MHL 10

Picking Up Leukemia from Pancytopenia-An Art Every Pathologist Must Master

\section{Aditi Jain", Nidhi Sharma, Arpita jindal}

Email ID for Correspondence: aditijain0889@gmail.com SMS Medical College, Jaipur, Rajasthan

Introduction and Background: Acute leukemia is one of the common hematopoietic malignancy in adults. It usually presents with leukocytosis. However subleukemic leukemia/aleukemic leukemia can manifest as pancytopenia/bicytopenia. Approximately 8-12\% cases of ALL presents with pancytopenia and $2 \%$ with hypocellular marrow. Patient/Material and Methods: The study included all the cases diagnosed as acute leukemia (By PBF or Bone marrow aspirate/ biopsy wherever needed) in the OPD lab of our hospital in a year period. Results: A total of 226 patients aging between 13 yrs to 78 yrs were diagnosed as acute leukemia during our study period. $10 / 226$ cases $(4.44 \%)$ were having leukocytopenia (TLC $<4000$ cells $/ \mathrm{mm}^{3}$ ) and $26 / 226$ cases $(11.50 \%)$ cases were having leukocyte count within normal range $\left(4000-11,000\right.$ cells $\left./ \mathrm{mm}^{3}\right)$. 190/226 cases $(84.07 \%$ ) had leukocytosis. 8/226 cases $(3.54 \%$ ) presented with pancytopenia and 164/226 cases (72.6\%) had bicytopenia. Conclusions: Pancytopenia is the major hindrance to the diagnosis of acute leukemia, as majority patients presents with leukocytosis. Though pancytopenia/bicytopenia does not exclude the diagnosis of acute leukemia. Thus pathologist need to be vigilant about picking leukemia even amidst pancytopenia.

\section{MHL 11}

\section{ETP ALL, A Rare Entity}

\section{Shashi Bansal, Abhishek Purohit, Abhishek Kumar ${ }^{\#}$}

Email ID for Correspondence: dr.kr.abhishek07@gmail.com Bhagwan Mahaveer Cancer Hospital and Research Center, Jaipur

Introduction and Background: T-Cell acute lymphoblastic leukemia (T-ALL) is an aggressive hematologic tumor resulting from the malignant transformation of T-Cell progenitors. Among them early T-Cell precursor (ETP) leukemias have been recently recognized as a form of T-Cell acute lymphoblastic leukemia (T-ALL) with poor prognosis. Recently, genetic studies have shed new light on the biology of ETP-T ALLs, a distinct disease entity associated with poor prognosis and defined by a characteristic immunophenotype and a gene expression signature indicative of a very early arrest in T-Cell development. Here we are describing a case of this rare entity with characteristics immunophenotyping. Patient/Material and Methods: A 28 years female patient presented with complaint of bleeding per vagina, nausea \& vomiting. On investigation: Total leucocytes count was markedly reduced $\left(670 / \mathrm{mm}^{3}\right)$ with low $\mathrm{Hb}(8.2 \mathrm{gm} / \mathrm{dl})$ and low platelet $\left(78,000 / \mathrm{mm}^{3}\right)$. Peripheral blood film shows presence of $74 \%$ BLAST with lymphoid morphology. Flowcytometry was done on bone marrow using 6 colour flow cytometer using CD 45 vs. side scatter gating. Results: Gated blast population is positive for CD5 (Dim), CD7, CD10, CD34, cytoCD3. Aberrant antigen expressionCD13, CD33 and CD117. Rest of the other markers CD19, surface CD3, CD19, CD20, CD36, cytoMPO, HLA-DR \& CD79A were negative. Case is also double negative for CD4 \& CD8. On the basis of this impression, early T-Precursor ACUTE LYMPHOBLASTIC 
LEUKEMIA (ETP-ALL) was given. Conclusions: ETP and early immature T-ALL, associated with poor prognosis are characterized by a very early differentiation arrest and show unique genetic and transcriptional features that overlap both with T-ALL and AML. Further dissection of this heterogeneous leukemia group is needed to address the prognostic implication of specific immunophenotype, transcriptional signatures and genetic alterations.

\section{MHL 12}

Study of Platelet Function Defects in Cases of Chronic Myeloid Leukaemia

\section{Zaw Htet Min", S. Venkatesan, S. Sharma, R. Bharadwaj}

Email ID for Correspondence: hminzaw178@gmail.com Armed Forces Medical College, Pune

Introduction and Background: Chronic myeloid leukemia (CML) is the classic chronic myelo-proliferative disorder, characterized by the acquisition of an oncogenic BCR/ABL fusion protein. Aim of this present study is to carry out platelet function tests in cases of CML and to identify the presence of platelet function defects. Further to correlate the platelet function analysis with the clinical symptoms especially the bleeding manifestation in cases of CML. Patient/ Material and Methods: Laboratory and clinical data of all cases of CML, which were diagnosed and managed at a tertiary centre, from June 2015-June 2016 were analyzed. Patient's platelet rich plasma was subjected to platelet function tests with Chronolog Dual Channel Platelet Aggregometer. Platelet aggregation study was carried out with Arachidonic acid, Epinephrine, ADP and Ristocetin. Results: Out of 26 cases of CML, 4 cases were more than 60 years of age and above (mean age: 41 ), with 22 cases being males. Of the 26 cases, one presented with bleeding manifestation in the form of epistaxis and petechial haemorrhages. All patients had a platelet count of more than 1 lakh. Splenomegaly was present in all cases. Two cases showed aggregation defects with Arachidonic acid, while other two cases showed aggregation defects with Epinephrine. All the four cases had normal platelet counts and one patient with defect in aggregation with Epinephrine had bleeding manifestations. The remaining three patients with abnormal platelet function did not have any bleeding manifestations. Conclusion: The present study suggests that platelet function defects are seen in CML and may contribute to the bleeding manifestations in some of these cases.

\section{MHL 13}

PHA-Induced Peripheral Blood Cytogenetics and Molecular Analysis: A Valid Diagnostic and Follow Up modality for AMPL Treated with ATRA and or Arsenic Trioxide

\section{Shahid Baba", Aleem Jan, Z. A. Shah, Dilafroz, Niyaz Azad \\ Email ID for Correspondence: aleem_maleem@yahoo.co.in; aleemjan11@gmail.com \\ Sheri Kashmir Institute of Medical Science, Soura Srinagar J\&K 190011}

Introduction and Background: Acute promyelocyticleukemia (APML) is characterized by the reciprocal translocation $(15 ; 17)(\mathrm{q} 22 ; \mathrm{q} 12)$ resulting in the PML-RAR $\alpha$ fusion gene. A dual diagnostic and follow up approach was applied including cytogenetic demonstration of the $\mathrm{t}(15 ; 17)$ translocation and detection of PML-
RAR $\alpha$ chimeric transcripts by molecular means. Patient/Material and Methods: Conventional cytogenetics involving bone marrow is beset with high probability of poor metaphase index and was substituted with phytohemagglutinin (PHA) induced peripheral blood culture based cytogenetic analysis as diagnostic and follow up modality in APML patients. Both qualitative (RT-PCR) and quantitative (q-pcr) tests were simultaneously carried out to authenticate the modified cytogenetics. Patients samples were subjected to the said techniques to establish their baseline as well as follow-up status. Results: Initial cytogentics revealed 30 patients $(81 \%)$ positive for $\mathrm{t}(15 ; 17)$ where as $7(19 \%)$ had either cryptic translocation or were negative for $\mathrm{t}(15 ; 17)$. Two cases had chromosomes $16 \mathrm{q}$ deletion and no hallmark translocation $\mathrm{t}(15 ; 17)$. Q-PCR status for PML-RAR $\alpha$ was found to be positive for all patients. All the APML Patients were reassessed at the end of consolidation phase and during maintenance phase of chemotherapy where 6 patients had molecular relapse, wherein 4 also demonstrated cytogenetic relapse. Conclusions: It was found that PHA-induced peripheral blood cytogenetics along with molecular analysis could prove a reliable modality in the diagnosis and assessment of follow up response of APML patients.

Keywords APML, Cytogenetic response, ATRA, Arsenic tri oxide, Peripheral blood, Karyotying

\section{MHL 14}

CD19 Negative B-All: A Diagnostic Challenge in Flowcytometric Immunophenotyping

\section{Shashi Bansal, Akansha Jain", Upendra Sharma}

Email ID for Correspondence: akansha_552005@yahoo.com Bhagwan Mahaveer Cancer Hospital and Research Centre, Jaipur, Rajasthan

Introduction and Background: CD19 is a B-cell specific marker, expressed on all stages of B-lymphocytes including plasma cells. The analysis approach of Flow Cytometric Immunophenotyping (FCI) for the diagnosis and monitoring of B-cell acute lymphoblastic leukemia (B-ALL) is totally based on the CD19-based primary gating strategy and it would be challenging to study B-ALL without CD19 expression. Rarely a case may be assigned as B lineage even if CD19 negative. Patient/Material and Methods: We report a case 16 year old male presented with complaint of pain abdomen, fever, joint pain and hepatosplenomegaly. Bone marrow findings corroborated with blood film revealed $84 \%$ leukoblast giving an impression of acute leukemia. Results: Gated blast population is positive for CD34, TdT, HLADR, cytoCD22, cytoCD79a and dim heterogeneous CD10. The scatter parameters and antigen expression profile as studied by Flowcytometry of the sample reveals negativity for lineage specific markers of myeloid (cytoMPO), Monocytoid (CD64, CD11c, CD14), T-Lymphoid (CD1a, CD3, CD5, CD7 and even cytoCD3) and strong positivity of CD79a with positive CD22 and Dim heterogeneous positive $\mathrm{CD} 10$. In view of above Immunophenotypic profile, a possibility of Precursor B-Acute Lymphoblastic Leukemia is suggested. Negativity for CD19 in cases of B-ALL is quite unusual, however has been reported. Subsequently IHC was put on bone marrow biopsy which was positive for Tdt, CD34, CD10 (focal) and PAX-5 and immunonegative for CD20, CD3, CD117, MPO\&CD 68. Further, the patient was treated with standard ALL chemotherapeutic regimen and reached complete remission at one month and now in consolidation phase. Conclusions: Reports of CD19 negative B-ALL are extremely rare. However, Lack of awareness of negative CD19 expression in B-ALL can leads to incorrect immunophenotypic diagnosis. 


\section{MHL 15}

Unusual Presentations of Plasma Cell Neoplasms-Case Series from a Tertiary Care Centre

\section{Venkatesan Somasundaram", Rajat Bajaj, Sanjeevan Sharma, Reena Bharadwaj}

Email ID for Correspondence: 1tcolsvenkatesan@gmail.com Armed Forces Medical College, Pune

Introduction and Background: There have been few case reports and case series in the literature regarding variation in morphology, immunophenotype and treatment modalities of Plasma cell myeloma. The present study aims at enumerating unusual clinicohematological, laboratory, and prognostic profiles of five such cases of plasma cell neoplasm which had varied clinical outcomes. Patient/Material and Methods: In this study, laboratory and clinical data of all unusual cases of Plasma cell neoplasm, which were diagnosed and managed at a tertiary centre, from July 2015-May 2016 were analyzed. Results: Sixteen cases of plasma cell neoplasms were diagnosed based on the WHO (2008) criteria. Out of all the cases, five cases had unusual presentations. All the five cases were elderly (mean age: 62), with three males. Three cases presented with bony pains and fractures, one with lymphadenopathy and one with high grade fever. The peripheral blood of three patients had more than $20 \%$ plasma cells qualifying for a diagnosis of plasma cell leukemia. Fourth case showed pleomorphic plasma cells in bone marrow with severe eosinophilia (AEC: $10,250) / \mathrm{cu} \mathrm{mm}$ in the peripheral blood. The last case presented with multiple lymphadenopathy, serous effusions and hepatosplenomegaly. Bone marrow and lymph node examination revealed neoplastic plasma cells. In all cases, bone marrow aspirate, biopsy, Immunohistochemistry and serum protein electrophoresis with immunofixation confirmed the plasma cell neoplasm. The patients were started on pulse dexamethasone followed by induction therapy with three anti myeloma drugs. However two of them did not survive, whereas the remaining three cases showed complete response to therapy and continue to remain in remission. Conclusions: Plasma cell neoplasm spans a clinical spectrum from asymptomatic to aggressive forms. The diagnosis is based on a combination of pathological, radiological and clinical features. This case series outlines the variations in the clinical and laboratory features of plasma cell neoplasm including the rare forms namely plasma cell leukemia.

\section{MHL 16}

Post Chemotherapy Changes in Bone Marrow in Acute Leukemia with Emphasis on Detection of Residual Disease by Immunohistochemistry

\section{Pavithra Ayyanar ${ }^{\#}$, Debdatta Basu, Rakhee Kar, Biswajit Dubashi}

Email ID for Correspondence: pavithraayyanar@gmail.com JIPMER

Introduction and Background: The leading cause of treatment failure in acute leukemia is disease relapse, which necessitates proper evaluation of patients from the time of diagnosis till the completion of chemotherapy. This study was done to evaluate the histomorphological changes in post chemotherapy bone marrow in acute leukemias, monitor residual disease by immunohistochemistry (IHC) and correlate it with survival status. Patient/Material and Methods: 155 postinduction acute leukemia cases (AML-68, ALL-87), from January 2014 to December 2015 were included. Detailed histomorphological evaluation and identification of residual blasts by relevant IHC were done. NCCN (2014) response criteria was used while evaluating the marrow. Follow up ranged from 4 to 28 months to look for "sustained remission", "residual disease" or "relapse". Results: Majority of post-induction marrows were hypercellular in AML (55.9\%) and normocellular in ALL (56.3\%). Regenerating hematopoiesis was commonly noted in ALL (88.5\%). Serous atrophy and stromal edema were commonly associated with delayed recovery of counts. Cases with scattered positivity for blasts on IHC $(96 \%)$ showed sustained remission, whereas those with clustered positivity for blasts $(35.7 \%)$ showed either residual disease or relapse. Interestingly dyspoietic megakaryocytes showed positivity for CD 34 and CD79a. E-Cadherin highlighted early erythroid cells which were often confused with myeloblasts. Among the post-induction remission cases, majority $(88.9 \%)$ were in "sustained remission" whereas among the not in remission cases, $50 \%$ had "residual disease" and $10.5 \%$ showed "relapse". Majority of cases with sustained remission (85.7\%) were alive with median survival of 13 months whereas those with residual disease $(90 \%)$ and relapse $(62.5 \%)$ expired with median survival of 3 months and 12 months respectively $(\mathrm{p}=0.000)$. Conclusions: Histomorphological features of post-induction bone marrow augmented by IHC gives valuable information regarding response to chemotherapy and residual disease status. A statistically significant difference in the median survival of cases in the three groups was observed.

\section{MHL 17}

The Triple Negative (HLADR-/CD34-/CD11B-) Profile on Multiparametric Flow Cytometry Rapidly and Specifically Identifies Acute Promyelocytic Leukemia

\section{Khaliqur Rahman", Ruchi Gupta, Manoj Kumar Sarkar, Anshul Gupta, Soniya Nityanand}

Email ID for Correspondence: drkhaliq81@gmail.com Sanjay Gandhi Post Graduate Institute of Medical Sciences, Lucknow 226014, India

Introduction and Background: Diagnosis of acute promyelocytic leukemia (APL) traditionally relies on morphological identification of abnormal promyelocytes followed by confirmatory genetic analysis. But, the morphological diagnosis may be difficult in some situations owing either to morphological variants of APL or acute myeloid leukemias (AML) with hyper granular blasts. Multi parametric flow cytometric (MFC) provides objective evidence in the form of some characteristic immunophenotype and allows a rapid diagnosis of APL. The objective of this study was to analyze the expression pattern of different antigens targeted through a single tube eight color combination and to evaluate its utility in rapid diagnosis of APL. Patient/ Material and Methods: Bone marrow aspirate of 30 consecutive APL and 30 random non APL AML cases were evaluated using a single tube 8 antibody combination which included CD45-V500, HLADR-V450, CD64-FITC, CD33-PE, CD34-PerCP, CD13-PeCy7, CD117-APC, and CD11b-APC H7. APL diagnosis was confirmed by RT-PCR for PML-RARA transcript. Results: Immunophenotypic profile of APL was homogeneous CD33+ $(100 \%)$; heterogeneous CD13+ (100\%); CD117+ (83.3\%), dim CD64+ (76.6 \%); CD34$(83.3 \%)$, HLADR - $(93.3 \%)$ and CD11b- $(90 \%)$. Non APL-AML group differed significantly from APL group in expression pattern of CD34, CD11b and HLADR with the proportion of cases being negative for these three markers being $30 \%, 30 \%$ and $24 \%$ respectively. The specificity for single (HLADR) negative, double (HLADR/CD34) negative and triple (HLADR/CD34/CD11B) negative profile for diagnosing a case as APL was found to be $70 \%$, $76.6 \%$ and $93.3 \%$ respectively. MFC immunophenotyping could render a diagnosis of APL within $2 \mathrm{hrs}$ of receipt of sample. Conclusions: MFC evaluation using this single tube 8 color 
combination can rapidly identify APL cases. Addition of CD11b, to the well known dual negative (HLADR/CD34) profile increases the specificity of APL diagnosis from $76.6 \%$ to $93.3 \%$.

Keywords Acute promyelocytic leukemia, Flow Cytometry, Single tube eight color combination

\section{MHL 18}

Detection of Early T Cell Precursor Leukemia Subset by Flowcytometry in T Cell Acute Lymphoblastic Leukemias and Their Follow Up

Jasdeep Singh", Seema Tyagi, Tulika Seth,

H. P. Pati, M. Mahapatra, P. Mishra,

Renu Saxena

Email ID for Correspondence: jasdeep1077@ rediffmail.com Department of Hematology, AIIMS, New Delhi

Introduction and Background: Acute lymphoblastic leukemia (ALL) is a malignant clonal expansion of immature lymphoid cells of B-cell or T-cell origin. T-cell acute lymphoblastic leukemia (T-ALL) constitutes $15 \%$ of childhood cases and $25 \%$ of adult acute leukemia cases. In 2009, a new subtype of T-ALL, early T-cell precursor ALL (ETP-ALL) was identified based on gene expression profiling and immunophenotyping. Various studies have shown ETP-ALLs are associated with high risk of remission induction failure or early relapse with conventional therapies. We identified this subtype of T-ALL and looked for its response to therapy. Patient/Material and Methods: We evaluated 878 patients of suspected acute leukemias by flowcytometry and identified 48 patients of T-ALL from Jan 2014 to Jul 2016 in our prospective study. Baseline presentation and immunophenotyping data was recorded for these 48 patients. Thirty five patients of T-ALL were admitted for therapy, they were followed up for a minimum duration of six months. Results: We detected 08 cases of ETP-ALL based on expression of myeloid, stem cell markers, absence of CD1a and CD8 and weak expression of CD5 by flowcytometry of the confirmed 48 T-ALL patients (prevalence$16.67 \%)$. Response to steroids was similar in both ETP-ALL and T-ALL subsets, but response to induction therapy (66.7\% vs. $88.5 \%$ ), overall survival and event free survival was significantly lower in ETP-ALL subset at six months of median follow up. Both disease and therapy related mortality was higher in the ETP-ALL subset (75\% vs. $7.4 \%$ ). Conclusions: ETP-ALL is a high risk subset of T-ALL which can be identified by a comprehensive approach using morphology, cytochemistry and flowcytometry. We advocate CD1a and CD8, immunophenotypic markers must be put up in all cases of T-ALLs to identify this aggressive subset of T-ALL.

\section{MHL 19}

MYD88 and CXCR4 Mutation Profiling in Lymphoplasmcytic Lymphoma/Waldenstrom's Macroglobulinaemia: Implications for a Diagnostic Molecular Laboratory

Sushant Vinarkar ${ }^{1 \#}$, Neeraj Arora ${ }^{1}$, Sourav Sarma Chowdhury ${ }^{1}$, Poonam Santra ${ }^{1}$, Kallol Saha ${ }^{1}$, Biswajoy Pal $^{1}$, Mayur Parihar ${ }^{2}$, Anupam Chakrapani ${ }^{3}$, Saurabh Bhave ${ }^{3}$, Mammen Chandy ${ }^{3}$, Reena Nair ${ }^{3}$, Deepak K. Mishra ${ }^{1}$
Email ID for Correspondence: drsushantvinarkar@gmail.com; sushant.vinarkar@tmckolkata.com

${ }^{1}$ Department of Laboratory Haematology \& Molecular Genetics, Tata Medical Center, Kolkata; ${ }^{2}$ Department of Laboratory Haematology \& Cytogenetics, Tata Medical Center, Kolkata; ${ }^{3}$ Department of Clinical Haematology, Tata Medical Center, Kolkata

Introduction and Background: Recurrent mutations affecting Myeloid Differentiation Primary Response 88 (MYD 88) gene and Chemokine receptor (CXCR4) nowadays form the basis for diagnosis, risk stratification and use of inhibitors targeting these signalling pathways in Lymphomoplasmacytic lymphomas/ Waldenstrom macroglobulinaemia (LPL/WM). Patient/Material and Methods: The mutation status and clinicopathological significance of these mutations was sought in 25 patients, including suspected cases of LPL/WM $(n=22)$, activated B-cell like diffuse large B-cell lymphoma (ABC-DLBCL) $(n=2)$ and a case of ?CLL (CD5, CD19, CD20, CD23 positive, kappa restriction) with raised IgM paraprotein. MYD88 hotspot mutation L265 and exon 5 were screened by Allele-Specific Polymerase chain reaction (ASPCR) and Sanger Sequencing respectively where as CXCR4 hot spot mutations involving amino acids 295 to 352 were screened by Sanger Sequencing. Results: Of the total LPL/WM $(n=22)$ cases screened, (86.3\%, $\mathrm{n}=19)$ harbored MYD88 L265P mutation. This cohort had mean age 64.2 yrs, $\mathrm{M}: \mathrm{F}=2.5: 1$, median IPSSWM $2.8(\mathrm{n}=16)$ and raised $\mathrm{IgM}($ mean $=4025.5 \mathrm{mg} / \mathrm{dl}$, range $561.5-11,445 \mathrm{mg} / \mathrm{dl})$. All cases had anaemia $(\mathrm{Hb}:<11.5 \mathrm{gm} / \mathrm{dl})$ and BM involvement by lymphoplasmacytic cells. The three MYD88 mutation negative LPL cases included a case of LPL with $\operatorname{IgM}(3343 \mathrm{mg} / \mathrm{dl})$ and a case of LPL with $\operatorname{IgA}$ paraproteinemia. Both cases of ABC-DLBCL were positive where as ?CLL was negative for MYD88 mutation. Only one of the MYD 88 L265 positive LPL cases screened $(n=16)$ had a CXCR4 heterozygous non-sense mutation (p. Ser342). Conclusions: High frequency of MYD88 mutations in suspected LPL/WM cases with intermediate to high IPSS-WM score co-related well with literature illustrating the role of this mutation in establishing the diagnosis of LPL/WM. AS-PCR as a highly sensitive method for MYD88 L265P mutation analysis could be of great help for the recognition of LPL/WM in molecular labs. Further MYD88 L265P and CXCR4 WHIM like mutation can be used as potential prognostic markers and therapeutic targets in future as further co-relation studies are awaited.

\section{MHL 20}

Impact of Tumour Microenvironment and Double Hit Score Within Follicular Lymphoma in Predicting Outcome

\section{K. S. Vishrut ${ }^{1 \#}$, Ashim Das ${ }^{\text {a }}$, Amanjit Bal ${ }^{1}$, Gaurav Prakash ${ }^{2}$}

Email ID for Correspondence: vishrut.com@gmail.com

${ }^{1}$ Department of Histopathology \& ${ }^{b}$ Department of Internal Medicine, Postgraduate Institute of Medical Education and Research (PGIMER), Chandigarh

Introduction and Background: Follicular lymphoma (FL) is a mature B-cell Non-Hodgkin lymphoma with indolent course, however significant subset of FL patients have poorer responses to chemotherapy or transform to aggressive lymphomas. It is important to recognize the sources of clinical heterogeneity which may include variations in the tumour microenvironment or neoplastic 
cells. Patient/Material and Methods: Fifty four biopsy proven cases of follicular lymphoma were studied. The tumour microenvironment was studied by CD4, CD8, FOXP3, CD68 and CD163 and for double hit score $\mathrm{c}-\mathrm{Myc}$ and $\mathrm{Bcl} 2$ immunohistochemistry and FISH were used. Results: The median age was 58.0 years with a M:F ratio of $1.8: 1$. The majority cases $(95.2 \%)$ belonged to Ann Arbor stage III/IV and FLIPI high risk category $(72.1 \%)$. Predominant follicular growth pattern was noted in $64.8 \%$ cases with a more diffuse pattern in eight cases (14.8\%). Histologically, 29 (51\%) cases were grade 1 , and grade 2 and grade 3 cases comprised $27 \%$ (14) and $22 \%$ (11) respectively. There was an increase in CD4+, FOXP3+ T cells, and CD68+, CD163+ macrophages with increase in histological grade. CD8+ $\mathrm{T}$ cells showed an inverse relation with decrease in number in higher histological grades. The CD4/CD8 ratio and percentage of FOXP3+ $\mathrm{T}$ cells were also increased significantly with histological grade. BCL2 was positive in all cases, however, eight cases $(14.8 \%)$ showed lesser degree of BCL2 positivity (50-80\%). c-Myc expression was noted in 6 cases of FL (grade III) and had c-MYC translocation on FISH. Conclusions: CD4 $+\mathrm{T}$ cells and tumour-associated macrophages had a significant relation with histological grade. An increase in mean density of the immune cells except for CD8+ T cells was noted in higher FLIPI risk category cases. The findings of tumour microenvironment and c-Myc translocation can be structured into a specific patterns which can indicate higher histological grade and consequent histological transformation.

\section{MHL 21}

\section{CD3 Negative T Acute Lymphoblastic Leukemia: A Rare Entity}

\section{Abhishek Purohit, Shashi Bansal, Pragya Gupta\#, Upendra Sharma}

Email ID for Correspondence: pragya.gupta19@gmail.com Bhagwan Mahaveer Cancer Hospital and Research Centre

Introduction and Background: The distinction between Acute undifferentiated Leukemia (AUL) and early Acute Lymphoblastic leukemia is complex and affects patient outcome. AUL is acute leukemia with no more than one membrane marker of any given lineage. Precursor T ALL is a neoplasm of lymphoblast commited to $\mathrm{T}$ cell lineage in which lymphoblast are most often positive for $\mathrm{CD} 7$ and cytoCD3 but of these only cytoCD3 is considered lineage specific. However some cases of T ALL without CD3 in the cytoplasm has been reported. Patient/Material and Methods: A 22 year male patient with generalized weakness, fatique from 7 day was investigated and found to have markedly elevated Total Leucocyte count $(189,510 / \mathrm{cu} \mathrm{mm})$ with presence of $92 \%$ blasts in peripheral blood and bone marrow. 6 Color Flow cytometer using CD45 vs. side scatter gating. Results: On flow cytometric evaluation, 69.5 $\%$ blasts were positive for CD4, CD5, CD7, CD33, CD34, CD36 and HLA-DR and negative for specific lineage markers for myeloid (cyto MPO), monocytoid (CD64, CD11c, CD14), B lymphoid (CD10, CD19, CD22, CD20, and cytoCD79a) along with a negative cyCD3. As presence of positivity of three lineage positive markers of $\mathrm{T}$ cell lineage rules out the possibility of AUL, possibility of cytoCD3 negative Precursor $\mathrm{T}$ cell lymphoblastic leukemia was suggested. Conclusions: $\mathrm{CyCD} 3$ is a specific marker for Acute $\mathrm{T}$ cell Lymphoblastic leukemia and its negativity is quite unusual however cases with cytoCD3 negativity has been reported. In such cases positivity of other markers of the lineage are helpful.
MHL 22

Follicular Dendritic Cell Sarcoma: A Case Series from a Tertiary Care Hospital

Kripa Mariam Varghese", Deepak Burad, Mayank Gupta, R. V. Leena, Marie Therese Manipadam, Geeta Chacko, Anna Pulimood, Subhashini John, Raju Titus Chacko

Email ID for Correspondence: kripa24varghese@gmail.com Christian Medical College, Vellore

Introduction and Background: Follicular dendritic cell sarcoma was first described in the lymph nodes and subsequently in extra nodal sites. This rare entity is often mistaken for other spindle cell neoplasms while occurring in the extra nodal sites. We, at Christian Medical College, Vellore, studied 9 cases of follicular dendritic sarcomas reported over a period of 10 years, from 2006-2015. Patient/ Material and Methods: Cases of FDC sarcomas which occurred over a period of 10 years were retrieved from the hospital's electronic database and the clinical features, morphological pattern, immunohistochemistry and associated para-neoplastic syndromes were analysed. Results: Out of the nine cases studied the male: female ratio was 2:1 with a mean age of 39 years (20-55 years). The tumours were located in the lymph nodes (2), anterior mediastinum, parapharyngeal region, small intestine, rectum, oropharynx, liver and head of pancreas $(1$ each). These tumours ranged in size from $0.9 \mathrm{~cm}$ to $8.5 \mathrm{~cm}$. Microscopically these tumours predominantly had spindle cells and 3 cases with epithelioid cells. Common patterns seen were whorls and fascicles. Angiomatous pattern was seen in 2 cases. Mitotic activity ranged from 3-15/10 hpf with scattered atypical mitosis. Most of the tumours showed positivity for CD21 and CD23. Two of the cases showed recurrence of the tumour (small intestine to ovary and bladder wall and parapharyngeal mass to clivus). One case showed paraneoplastic pemiphigus. Conclusions: Follicular dendritic sarcoma, although rare, has to be kept in mind when encountering spindle cell/epithelioid neoplasms. The distinctive histologic patterns and accompanying lymphoid infiltrate should prompt the pathologist to ask for diagnostic immunohistochemical markers for follicular dendritic cell origin. Identification of these tumours is important because of its ability to recur and metastasize.

\section{MHL 23}

Chronic Neutrophilic Leukemia (CNL): A Case Report and Review of Literature

\section{Vipra Malik", Anshu Palta, Sanjay D'Cruz}

Email ID for Correspondence: vipramalik@yahoo.co.in Government Medical College and Hospital, Sector-32, Chandigarh

Introduction and Background: Chronic neutrophilic leukaemia (CNL) is classified as a myeloproliferative neoplasm (MPN) according to WHO classification, and is characterized by sustained neutrophilic leukocytosis, hepatosplenomegaly and bone marrow granulocytic hyperplasia without evidence of dysplasia, BCR-ABL1 or rearrangements of PDGFRA, PDGFRB or FGFR1. It is usually associated with a fatal clinical course. A case of 65 year old male is discussed who presented with persistent neutrophilia and moderate splenomegaly. Patient/Material and Methods: We evaluated a 65 year old male who presented with persistent neutrophilia and moderate splenomegaly for 5 months. Bone marrow examination was performed on clinical suspicion of Chronic Myeloid Leukemia. BCR/ABL1 evaluation was performed on bone marrow sample using RT-PCR. Blood cultures, 
urine cultures and fungal cultures were performed to rule out any focus of infection. Serum electrophoresis was performed to rule out any evidence of plasma cell dyscrasia. Results: Peripheral blood examination revealed WBC count of $73,500 / \mu \mathrm{L}$. Majority of these were mature neutrophils $(92 \%)$ with less than $10 \%$ immature granulocytes. Neutrophils showed presence of toxic granulation and cytoplasmic vacuolation. LAP score was markedly increased. Bone marrow examination revealed myeloid hyperplasia with predominance of neutrophilic granulocytes. No evidence of dysplasia was noted in any cell lineage. BCR/ABL1 transcript ratio was found to be $0 \%$ using RTPCR. Blood cultures, urine cultures and fungal cultures were negative. Bone marrow plasma cells were found to be $9 \%$. However, serum electrophoresis showed no evidence of monoclonal gammopathy. Conclusions: $\mathrm{CNL}$ is a rare, but distinct entity characterized by splenomegaly, sustained neutrophilia, raised NAP score and the absence of the $\mathrm{Ph}$ chromosome, with a fatal outcome. Recent discovery of specific oncogenic mutations in the colony-stimulating factor 3 receptor gene (CSF3R) in CNL patients may help in diagnosis and management.

\section{MHL 24}

Spectrum of Morphological Changes in Bone Marrow in Elderly Patients ( $\geq 60$ years age)

\section{Anshu Palta ${ }^{\#}$, Vipra Malik, Anita Tahlan, Monika Gupta}

Email ID for Correspondence: anshupalta@yahoo.co.in Government Medical College and Hospital, Sector-32, Chandigarh

Introduction and Background: Hematological derangements are a growing health concern in geriatric age group ( $\geq 60$ years). The associated morbidity and mortality necessitates the need for early and accurate diagnosis and appropriate therapy. Bone marrow examination is an essential tool in the diagnosis of hematologic disorders. Patient/Material and Methods: This is a retrospective observational study carried out in the Hematology section of the Department of Pathology, GMCH-32, Chandigarh. Patients aged $\geq 60$ years were evaluated over a period of 12 years and 3 months (January 2004March 2016). The detailed clinical data, slides of bone marrow aspirate and trephine biopsies done in all the cases were retrieved from archival material. Results: During this period, a total of 2982 Bone marrow aspirations along with Bilateral Trephine biopsies were performed and out of these, 660 cases were $\geq 60$ years old. Age of the patients ranged from 60 years to 95 years with male to female ratio of 1.9:1. The most common indication of bone marrow examination was cytopenias $(269,40.7 \%)$ followed by procedures carried out for staging of known cases of lymphoma $(118,17.8 \%)$, bony pains/ osteoporosis $(112,17 \%)$ and leucocytosis $(83,12.6 \%)$. After excluding the cases with known diagnosis, multiple myeloma was the most common diagnosis in 65 cases $(18.2 \%)$ followed by megaloblastic anemia $(56,15.6 \%)$, acute leukemia (54, 15.1\%), CLPD $(47,13.1 \%)$, metastasis $(36,10.1 \%)$, MPN $(25,7 \%)$, ITP (19, $5.3 \%)$, MDS $(16,4.5 \%)$ and others $(22,6.2 \%)$. The most common cause of cytopenias was found to be megaloblastic anemia in 54 cases $(28.3 \%)$ followed by acute leukemia $(34,17.8 \%)$, ITP $(19,9.9 \%)$, MDS (16, 8.4 \%), CLPD (14, $7.3 \%$ ) and metastasis (13, $6.8 \%)$. The most common cause of leucocytosis was diagnosed as CLPD in 33 cases $(42.9 \%)$ followed by MPN $(21,27.3 \%)$ and acute leukemia $(19,24.7 \%)$. Conclusions: Multiple myeloma is the most common cause of haematological derangements in elderly patients followed by megaloblastic anemia, acute leukemia, CLPD, metastasis, MPN, ITP and MDS.

\section{MHL 25}

Over-Expression of ERG Predicts Poor Outcome in Acute Myeloid Leukemia

Syed Khizer Hasan ${ }^{1 \#}$, Nikhil V. Patkar ${ }^{2}$, Devanand Vishwakarma ${ }^{1}$, Deepan Rajamanickam ${ }^{3}$, Anant Gokarn ${ }^{3}$, Arvind Sahu ${ }^{3}$ Prashant Tembhare ${ }^{2}$, Sadhna Kannan $^{1}$, Sumeet Gujral ${ }^{2}$, Bhausaheb Bagal ${ }^{3}$, Hasmukh Jain ${ }^{3}$, Hari Menon ${ }^{3}$, Manju Sengar ${ }^{3}$, Pratibha Kadam Amare ${ }^{4}$, P. G. Subramanian', Navin Khattry ${ }^{3}$

Email ID for Correspondence: shasan@actrec.gov.in

${ }^{1}$ ACTREC, Tata Memorial Centre, Navi Mumbai;

${ }^{2}$ Hematopathology Laboratory, Tata Memorial Centre, Mumbai;

${ }^{3}$ Adult Hematolymphoid Disease Management Group,

Department of Medical Oncology, Tata Memorial Centre, Mumbai;

${ }^{4}$ Laboratory of Cytogenetics, Tata Memorial Centre, Navi Mumbai

Introduction and Background: Assessment of minimal residual disease (MRD) is very useful for risk stratification and prediction of relapse in acute myeloid leukemia (AML) patients harbouring specific genetic markers such as fusion transcripts and gene alterations. However, for those patients where such markers are absent, it is crucial to identify molecular targets that are suitable to measure MRD. Patient/Material and Methods: We prospectively studied the prognostic impact of $B A A L C$ (Brain and acute leukemia, cytoplasmic) and $E T S$-related gene $(E R G)$ in 151 de novo acute myeloid leukemia (AML) including 72 cytogenetically normal AML (CN-AML) patients who were uniformly treated at Tata Memorial Centre Mumbai. BAALC and $E R G$ expression was determined by real-time reverse transcriptase-polymerase chain reaction in 369 bone marrow/ peripheral blood samples collected at baseline, post-induction and post-consolidation stages of the disease. Results: Receiver operating characteristics (ROC) and area under curve (AUC) were plotted to calculate the cut off value of $B A A L C$ and $E R G$ expression in distinguishing high expressers from low expressers. Compared to low expressers, high $B A A L C$ and $E R G$ expressers showed significantly inferior overall survival (BAALC: OS; median, 8.5 months vs. 2 years, $P<0.0001 ; \quad E R G$ : $\quad$ OS; $\quad$ median, 6.6 months vs. 2 years, $P<0.0001)$ and disease-free survival $(B A A L C$ : DFS; median, 7.4 months vs. 2 years, $P<0.0001 ; E R G$ : DFS; median, 6 months vs. 2 years, $P<0.0001)$. The kinetics of $B A A L C$ and $E R G$ expression showed a significantly shorter OS and DFS for patients where copy numbers of transcripts remains elevated (referred as group 1 patients) as compared to those where copy numbers remains below the cut-off during the complete course of treatment (group 2 patients). The estimated 2-year OS rates for group 1 patients was $4.3 \%$ as compared to $91 \%$ of group 2 ( $\mathrm{P}<0.0001)$ for both the transcripts. Multivariable analysis confirmed high $E R G$ expression as an independent risk factor. For high $E R G$ expressers the hazard ratio of an event for OS, and DFS was 4.6 and 7.6 respectively. Conclusions: We conclude that high $E R G$ expression predicts an adverse prognosis and may define an important risk factor in AML with or without normal cytogenetics. 


\section{MHL 26}

Hepatosplenic $\gamma \delta$ T-Cell Lymphoma with Amplification of 7q: A Case Report

Hemani Jain ${ }^{1 \#}$, Dhanlaxmi Shetty ${ }^{1}$, Hasmukh Jain ${ }^{2}$, Manju Sengar ${ }^{2}$, P. G. Subramanian ${ }^{3}$

Email ID for Correspondence: hemanijain7@gmail.com ${ }^{1}$ Department of Cancer Cytogenetics, ACTREC, Tata Memorial Centre, Kharghar, Navi Mumbai; ${ }^{2}$ Department of Medical Oncology, Tata Memorial Hospital, Parel, Mumbai; ${ }^{3}$ Department of

Hematopathology, ACTREC, Tata Memorial Centre, Kharghar, Navi Mumbai

Introduction and Background: Hepatosplenic T-cell lymphoma (HSTCL) is a rare, clinically aggressive malignancy predominantly affecting young men. This entity has been classified as a distinct clinico-pathological entity in the 2008 WHO classification. Isochromosome $7 \mathrm{q}$ with or without trisomy 8 is reported to be the primary cytogenetic abnormality in HSTCL. Recently, ring chromosome 7 has also been identified in HSTCL. The contribution of these aberrations to the pathogenesis of disease is still unknown. The disease shows a severe clinical course, resistance to therapy and poor prognosis. Material and Methods: Patient admitted at Tata Memorial Hospital was taken for the study. FISH analysis was performed using LSI 7q22, 7q35, CEP $7 \& 8$ (Vysis, Abott Molecular Inc.) on Bone marrow aspirate specimen. Result: Case Report: We present a case of a 24 year female patient who presented with anemia, thrombocytopenia and isolated hepatosplenomegaly with high levels of LDH. Morphology and immunophenotypic analysis were suggestive of hepatosplenic gamma delta T-cell lymphoma. FISH analysis revealed $8-10$ copies of $7 q 22$ region indicating amplification of $7 q$ sequences with loss of terminal $7 \mathrm{q}$ regions along with an additional abnormality of trisomy 8. Presently, the patient is on palliative treatment indicating that this would be a poor prognosis. Discussion and Conclusion: Isochromosome 7q typically occurs in HSTCL which results in loss of T-cell receptor $\gamma$ gene present on 7p15 and duplication of T-cell receptor $\beta$ gene present on 7q35. Trisomy 8 is an additional abnormality associated with disease progression. Our case revealed amplification of the $7 \mathrm{q} 22$ region, probably due to excess of oncogenes which leads to the disease progression and has a role in the pathogenesis of the disease. Further studies are required to narrow down the critical $7 \mathrm{q}$ regions to identify the targeted genes which will ultimately help in devising novel treatment regimens to such patients.

\section{MHL 27}

\section{Chronic Myeloproliferative Neoplasm: Report of Four Cases}

\section{Jyoti Verma $^{1 \#}$, Somanath Padhi ${ }^{1}$, Pritinanda Mishra ${ }^{1}$,} Susama Patra ${ }^{1}$, Sonali Mohapatra ${ }^{2}$, Prabodh Das ${ }^{3}$

Email ID for Correspondence: jyoti.verma2611@gmail.com Department of ${ }^{1}$ Pathology and Lab Medicine, ${ }^{2}$ Medical Oncology, All India Institute of Medical Sciences, Bubaneswar, Odisha; Department of ${ }^{3}$ Clinical Hematology, Institute of Medical Sciences and SUM Hospital, Bubaneswar, Odisha, India

Introduction and Background: Chronic myeloproliferative neoplasms (CMPNs) are a group of myeloid neoplasms with distinct molecular characteristics, overlapping bone marrow histology, and variable risk of transformation to acute leukemias. Patient: We describe the clinicopathological features, lab parameters, management and follow up data of four patients. The four patients included: 15 years male (Case 1), 22 years female (Case 2), 40 years female (Case 3 ) and 44 years male (Case 4). Case 1 presented with anorexia, fatigue, massive splenomegaly and co-existent subcutaneous tender nodules and was diagnosed to have chronic phase - CML (CML-CP, BCR-ABL +ve) with co-existent extra medullary myeloid cell tumor (myeloid sarcoma) (differentiated type) diagnosed by FNAC. Rest three patients presented with marked polycythemia resulting in neurological signs (Case 2), Budd-Chiari like symptoms (Case 4), and with co-existent giant uterine leiomyoma (Case 3 ). All three cases with polycythemia harboured JAK2-V617F mutation in the exon 14 diagnosed by RT-PCR analysis. The bone marrow histology, in correlation with JAK2-V617F and low serum erythropoietin levels, were consistent with the diagnosis of polycythemia vera as per WHO 2008 guidelines. All four patients are currently under routine follow up with Imatinib mesylate (Case 1), and hydroxyurea + venesection (cases 2, 3, 4). Conclusions: Accurate categorization of CMPNs with molecular techniques is essential for proper management in these patients. Myeloid sarcoma may also rarely co-exist with CMPNs such as chronic phase-CML; and requires close follow up.

Keywords Chronic myeloproliferative neoplasm, Myeloid sarcoma, Polycythemia vera, JAK2-V617F.

\section{MHL 28}

Bone Marrow Metastasis in a Young Child: An Unusual Presentation at an Unusual Age

\section{Seema Sharma", Ruchi Gupta}

Email ID for Correspondence: ssharma@sgpgi.ac.in Departments of Pathology and Clinical Hematology, SGPGIMS, Lucknow

Introduction: In children the tumors that account for the majority of metastases in bone marrow are neuroblastoma, rhabdomyosarcoma, Ewing's sarcoma, other primitive neuroectodermal tumors (PNET) and retinoblastoma. Here we present a rather unusual case of bone marrow metastasis in a young female child. Case: A 12 year old female child presented to the emergency department of our institute with complaints of generalized weakness and lethargy for three months and rapidly progressive breathlessness of 15 days duration. On examination she had tachypnea, tachycardia, severe pallor and right sided pleural effusion, mild hepatomegaly and splenomegaly. Her Hemoglobin was $8.3 \mathrm{~g} / \mathrm{dl}$, TLC $18 \times 10^{9} / \mathrm{L}$, platelet count $30 \times 10^{9} / \mathrm{L}$, and reticulocyte count $23.3 \%$. Peripheral blood smear showed numerous schistocytes, polychromasia, circulating nucleated red blood cells. Direct Coomb's test was negative. So a diagnosis of microangiopathic hemolytic anemia (MAHA) was made. Her bone marrow aspiration and biopsy showed presence of clusters of malignant cells, with signet ring morphology. On immunohistochemistry the tumor cells were positive for pan cytokeratin, CD7 and negative for CD 20. Tumour markers alfa-fetoprotein (AFP) and carcinoembryonic antigen (CEA) were negative. Abdominal ultrasound showed bilateral enlarged ovaries with solid and cystic components and maintained shape indicative of Krukenberg's tumor. A computed tomography of the thorax and abdomen was planned, however the patient died due to sudden cardiac arrhythmia and further investigations to ascertain the primary site could not be carried out. Conclusion: Disseminated malignancies have varied clinical presentations, and MAHA is one of the uncommonly known para-neoplastic complications of solid tumors. This case report aims to highlight MAHA as presenting feature in a very young girl. 


\section{MHL 29}

H Score and Extended Variables in the Diagnosis of Hemophagocytic Lymphohistiocytosis: A Pilot Study

Somanath Padhi ${ }^{1,2 \#}$, RajLaxmi Sarangi ${ }^{3}$, Renu G'Boy Varghese $^{2}$, Anita Ramdas ${ }^{2}$, Susama Patra ${ }^{1}$

Email ID for Correspondence: somanath.padhi@gmail.com Department of ${ }^{1}$ Pathology and Laboratory Medicine, All India Institute of Medical Sciences, Bhubaneswar, Odisha \& Departments of ${ }^{2}$ Pathology and ${ }^{3}$ Biochemistry, Pondicherry Institute of Medical Sciences, Pondicherry, India

Introduction and Background: Hemophagocytic lymphohistiocytosis (HLH), because of its severity and potential high mortality, requires timely diagnosis and early intervention with etoposide based therapy. However, the diagnostic criteria proposed for HLH are stringent and suffer from substantial limitations. Material and Methods: Seventeen patients (12 males, 7 females, age range: 6 months to 56 years) satisfied five of 6 tested HLH-2004 criteria. These were retrospectively analyzed and reclassified as i) definite, ii) likely, iii) negative for HLH by applying HLH probability score $(\mathrm{H}$ score, Fardet et al, 2014) using nine variables [underlying immunosuppression, fever, organomegaly, histiocytic hemophagocytosis in bone marrow aspirate, serum ferritin, triglyceride, and plasma fibrinogen]. Then additional variables such as low albumin, elevated lactate dehydrogenase, bilirubin, prothrombin time/INR, activated partial thromboplastin time, D-dimer; and low sodium [extended variables] were assessed in addition to above by using a 0 to 2 point scale. Results: Underlying infections/suspected sepsis/SIRS contributed 11/17 cases; four were malignancy associated (3 non Hodgkin lymphomas, 1 acute myeloid leukemia); and remainder one case each of systemic juvenile idiopathic arthritis and DRESS syndrome. Considering a $\mathrm{H}$ score of 169 as a cut off (93\% sensitivity, $86 \%$ specificity, Fardet et al), 9/17 were categorized as definite HLH (score range: 174-284), 4/17 as likely HLH (score: 150-169); and remainder 4 as negative for HLH (score range: 92-139). Extended variable score of $\geq 20$ correlated with a higher $\mathrm{H}$ score of $\geq 200$; a score of $\geq 15$ corresponded to $\mathrm{H}$ score: $\geq 150$; and a score of $<10$ to $\mathrm{H}$ score of $<140)$. Etoposide and/or steroids were used in $8 / 17$ cases $(H$ score: 162-284) of which six expired. Conclusions: H score and extended variables may be valuable for early diagnosis and initiation of therapy in HLH; and these require further validation in larger prospective studies.

\section{MHL 30}

NPM1 and FLT3 Gene Mutations in Acute Myeloid Leukemia- and Their Association with Clinico-hematological Features

\section{Hapreet Virk $^{2 \#}$, Shano Naseem ${ }^{1}$, Neelam Varma ${ }^{1}$,} Jogeshwar Binota ${ }^{1}$, Pankaj Malhotra ${ }^{2}$, Subhash Varma ${ }^{2}$

Email ID for Correspondence: harpreet.virk95@gmail.com Departments of ${ }^{1}$ Hematology and ${ }^{2}$ Internal Medicine, PGIMER, Chandigarh

Introduction: Acute myeloid leukemia (AML) develops through a complex multistep process that requires cooperation of different types of genetic alterations, which can be either balanced translocations or gene mutations. Mutations in nucleophospmin (NPM1) gene are the most frequent genetic change seen in patients with AML and are associated with a favorable prognosis. However, it has been demonstrated that the favourable prognostic implications of NPM1 mutation status are overridden by presence of FMS-like tyrosine kinase-internal tandem duplications (FLT3-ITD). Therefore, screening of patients for mutations in FLT3-ITD alongside NPM1 is required. Aim: To study the frequency of NPM1 and FLT3 gene mutations in patients with denovo AML and to compare the clinical and hematological profile of cases with and without these mutations. Materials and methods: NPM1 and FLT3-ITD/TKD gene mutations were tested in 87 consecutive cases of de-novo AML. Details of clinical and hematological parameters were noted. NPM1 mutation testing was done by RT-PCR and electrophoresis on $8 \%$ polyacrylamide gel. FLT3 mutation testing was done by restriction fragment length polymorphism (RFLP) with EcoRV digestion and electrophoresis on $3 \%$ agarose gel electrophoresis. Results: Out of 83 cases of AML tested, 21 were positive for NPM1 but negative for FLT3 mutation (NPM+ve/ FLT3-ve) $(25.3 \%) ; 12$ were negative for NPM1 but positive for FLT3 mutation (NPM-ve/FLT3+ve) $(14.5 \%)$ and 50 were negative for both mutations (NPM-ve/FLT3-ve) $(60.2 \%)$. Overall, median age was 35 years (1-89 years), with male:female ratio of 2:1. Patients in NPM1-ve/FLT3+ve group had a higher median age, TLC and blast percentage in peripheral blood at presentation. No significant difference was observed between other clinical, hematological and immunophenotypic parameters among the NPM1+ve/FLT3-ve, NPM1-ve/FLT3+ve and NPM1-ve/FLT3-ve groups. Conclusion: The overall frequency of NPM1 mutation seen in this cohort of patients was $25.3 \%$ and FLT3 was $14.5 \%$, comparable to previous reports. However, none of the cases in our study were positive for both mutations, which is in contrast to previous studies, as a concomitant FLT3 mutation has been reported in around $40 \%$ AML cases with mutated NPM1. This finding needs to be evaluated in a larger cohort of patients with mutated NPM1. Acknowledgment: This study was supported by 'Research grant from Postgraduate Institute of Medical Education and Research, Chandigarh'.

\section{MHL 31}

Monosomy 7 and Chromosome 7 Abnormalities in Acute Lymphoblastic Leukaemia (ALL)

\section{S. R. Arun\#, Manish K. Singh, Mr. Anil K. Yadav, D. K. Mishra, Neeraj Arora, Saurabh J. Bhave, Shekhar Krishnan, Arpita Bhattacharya, Reena Nair, Vaskar Saha, Mammen Chandy, Mayur Parihar}

Email ID for Correspondence: arunsr84@gmail.com Tata Medical Center

Introduction and Background: Chromosome 7 abnormalities especially deletion and monosomy, are associated with poor prognosis in myeloid neoplasms. There is little data on frequency of chromosome 7 abnormalities in ALL. We report chromosome 7 abnormalities in ALL. Patient/Material and Methods: Bone marrow findings, immunophenotype, Karyotyping and FISH results of ALL patients presenting at Tata Medical Center Kolkata between July 2011 and May 2016 were retrieved. FISH using Deletion probe for chromosome 7 was done in selected cases. Results: Of the 501 ALL patients 355 were pediatric $(<18$ years; $70.8 \%)$ and 146 were adult ( $\geq 18$ years; $29.2 \%)$. Chromosome 7 abnormalities were seen in $51(10.2 \%)$ patients, of whom 41 were precursor B cell ALL and were more common in adults $(19 \%$ vs. $6.5 \%)$. Monosomy 7 was the most common abnormality (43.1\%; 6 children and 16 adult) and was commonly seen as secondary abnormality in Ph+ve ALL. Monosomy 7 in association with complex karyotype and low hypodiploidy was present in $19.6 \%$ and $5.8 \%$ patients respectively. Structural abnormalities were seen in $56.9 \%$ cases. Short arm abnormalities were more common (3.5 times). Translocations were the most frequent structural abnormality. Conclusions: Chromosome 7 abnormalities constitute a significant proportion $(10 \%)$ of cytogenetic abnormalities in ALL and are more common in adult cases. Monosomy 7 is the 
most frequent cytogenetic abnormality in $\mathrm{Ph}+\mathrm{ve}$ and $\mathrm{Ph}$ negative cases. In contrast to myeloid malignancies, $7 p$ abnormalities are more predominant in ALL. Larger studies are required to comment on their prognostic impact.

\section{MHL 32}

\section{A Single Tube 4 Colour Flowcytometry-Impressionable} and Efficient Technique in Diagnosis of Acute Leukemia

\section{Siddartha Kasula ${ }^{\#}$, Faiq Ahmed, Rachna Khera, Manasi Mundada, N. Lavanya, Sudha S. Murthy, Senthil J. Rajappa}

Email ID for Correspondence: dr.siddartha1987@gmail.com Basavatarakam Indo-American Cancer Hospital and Research Institute

Introduction and Background: The classification of acute leukemia requires flow cytometry (FCM) data utilizing a large panel of antibodies for characterization of leukemic cells. An. However in resource limited settings, use of extensive panel of antibodies may not be possible. The aim of this study was to assess the usefulness of cocktail of lineage specific markers using single tube in defining/screening lineage of acute leukemias in conjunction with morphology and cytochemistry. Patient/ Material and Methods: We analyzed consecutive PB and BM samples of 181 patients for acute leukemia using 4 colors FCM and comparing lineage specific cytoplasmic markers in a single tube consisting of cyt.CD3/cyt.CD79a/cyt.MPO/CD45 vs. standard panel of markers using standard protocol. Results: A total of 181 patients were diagnosed as acute leukemia using standard multimarker FCM. $102(56 \%)$ patients were diagnosed as AML of which $58(32 \%)$ and $16(8.8 \%)$ patients were rendered a diagnosis of B-ALL and T-ALL respectively. 5 patients $(2.7 \%)$ were diagnosed mixed phenotypic acute leukemia (MPAL). Using cytoplasmic tube, diagnosis of AML was made in 76 cases, B-ALL in 37 cases and T-ALL in 15 cases. However in 33 cases $(18.2 \%)$ the neoplastic cells were negative for all 3 markers. Of these 33 cases 14 were AML M0, 10 were AML M5, 2 were AML M4 \& a single case of B-ALL. The other 6 cases showed more than one cytoplasmic marker positivity ( 2 cases of T-ALL and 4 cases of MPAL). Defining lineage using morphology alone was not definitive $(r=0.814$, $p=0.001$ ); but morphology and cytochemical stains were employed, the Pearson correlation ( $\mathrm{r}$ ) obtained was 0.855 . A good correlation was noted when morphology and cytochemistry were combined with cytoplasmic single tube FCM $(r=0.993, p=0.001)$. Conclusions: In a resource restricted set up; morphology and cytochemistry with a basic cytoplasmic single tube may best serve the purpose of diagnosing acute leukemia.

\section{MHL 33}

\section{Metabolic Uncoupler Sensitizes Acute Myeloid Leukemic Cells} to Arsenic Trioxide

\section{Nithya Balasundaram", Saravanan Ganesan, Hamenth Kumar Palani, Ansu Abu Alex, Sachin David, Anu Korula, Biju George, Poonkuzhali Balasubramanian, Vikram Mathews}

Email ID for Correspondence: nithyab@cmcvellore.ac.in Department of Haematology, Christian Medical College, Vellore

Introduction and Background: Arsenic trioxide (ATO) is used as a single agent frontline therapy for the treatment of acute promyelocytic leukemia (APL) with a 5 year survival rate of $80 \%$. Non-APL myeloid leukemic cells are generally resistant to ATO, extending the use of ATO in non-APL myeloid leukemia will be of greater importance due its minimal toxicity in comparison to the conventional chemotherapy used in the treatment of AML. The primary objective of the study is to evaluate the mechanisms of resistance to ATO in non-APL myeloid cells. Material and Methods: AML cell lines U937 and THP1 are used to study the ATO resistance in vitro. Inhibitory concentration 50 of ATO (IC50) was assessed by a MTT assay. Cells are treated with ATO and oxidative phosphorylation (OXPHOS) uncoupler FCCP for 48 hours, viability was measured by Annexin V-7AAD staining using FACS. Results: ATO has been reported to directly inhibit the glycolytic pathway; this effect is believed to contribute to its cytotoxic effect in APL. Hence, we studied the metabolic dependency of myeloid leukemic cells which promotes survival even in the presence of ATO. Towards that we assessed the sensitivity of the cells to OXPHOS uncoupler. FCCP treatment alone did not reduced the viability of these cells significantly, suggesting the ability of these cells to uncouple their metabolic pathway from OXPHOS to glycolysis when inhibited. However, when FCCP was combined with ATO it significantly restored the sensitivity to ATO. The data suggests that the non-APL myeloid cells could be sensitized to ATO by combining ATO with an OXPHOS uncoupler. Conclusions: Uncoupling the metabolic dependency of the myeloid cells could be potentially exploited to sensitize them to ATO. It is also important to note that a number of molecules that are FDA approved, used in the clinic also have OXPHOS uncoupling activity and could potentially be evaluated for their synergistic activity with ATO.

\section{MHL 34}

\section{Bone Marrow Micro-environment Mediated Drug Resistance} in Acute Myeloid Leukemia

Hamenth Kumar Palani", Saravanan Ganesan, Nithya Balasundaram, Ansu Abu Alex, Anu Korula, Biju George, Poonkuzhali Balasubramanian, Vikram Mathews

Email ID for Correspondence: hamenth@cmcvellore.ac.in Christian Medical College, Vellore

Introduction and Background: Accumulating evidence suggests that bone marrow stromal environment act as an asylum for leukemic cells and allows them to survive chemotherapy, eventually leading to disease recurrence. Our aim is to delineate the mechanism underlying stromal environment mediated drug resistance (EMDR) in AML cells, which could potentially lead to new targeted therapies for AML. Patient/ Material and Methods: In order to mimic the bone marrow microenvironment, we have developed an in-vitro stromal cell co-culture system, where the myeloid leukemic cell lines (HL-60, U937 \& K562) and primary AML cells was co-cultured with stromal cell line (HS-5) and exposed to different concentration of daunorubicin (DNR) \& Cytarabine (Ara-C). After 48 hours, the percentage of viability was calculated by Annexin V-7AAD staining using flow cytometry. Gene expression profiling was done using Agilent Human Whole Genome $8 \times 60 \mathrm{~K}$ Gene Expression Array (Genotypic technologies, Bengaluru). Results: co-culture experiments demonstrates that there is a significant stroma mediated protective effect on myeloid leukemic cell lines to DNR ( $p<0.001)$ and Ara-C $(p<0.01)$, the same phenomenon was observed in primary AML cells [DNR $\mathrm{p}<0.001$, Ara-C $\mathrm{p}<0.01$ $(n=26)]$. In order to decipher the molecular mediators of this resistance we did a microarray analysis of U937 cells co-cultured with HS-5 comparing control. We found that 1067 genes were differentially regulated ( $>2$ fold), out of which 699 genes were up regulated and 368 genes were down regulated. The functions significantly enriched for differentially regulated genes are cell proliferation, apoptosis, immune response, cell adhesion, cytokines and signalling pathways like PI3- 
Akt, JAK STAT, WNT signalling. Conclusions: Our preliminary results suggest there is a significant EMDR in AML. However the mechanisms underlying this effect should be explored further in greater detail. Elucidating the molecular mechanisms involved is likely to result in promising combination therapies to reduce chemotherapy resistance and relapse, and thereby improve survival in AML.

\section{MHL 35}

Clinico-pathological and Immunophenotypic Profile of Hairy Cell Leukemia: A Single Center Experience

Kotteeswari Kathirvel ${ }^{\#}$, Ansu Abu Alex, HarikrishnanBabu, Aby Abraham, Biju George, Alok Srivastava, Poonkuzhali Balasubramanian, Vikram Mathews

Email ID for Correspondence: kotteevasu@gmail.com Department of Haematology, Christian Medical College and Hospital, Vellore

Introduction and Background: Hairy cell leukemia (HCL) is a B-cell chronic lymphoproliferative disorder (B-CLPD) characterised by splenomegaly, pancytopenia and bone marrow involvement with fibrosis. HCL represents $2 \%$ of adult leukemia. The study describes the clinical, pathological and immunophenotypic profile of HCL cases diagnosed at our center. Patient/Material and Methods: We undertook a retrospective study of 63 patients diagnosed as HCL at our center from April 2006 to July 2016. The samples were stained for B-CLPD panel comprises of markers such as CD19, CD20, CD22, CD5, CD23, CD10, Kappa, Lambda, CD25, CD103, CD11c and CD123 followed by RBC lysis, wash and acquired in BD FACS Calibur flow cytometer. TRAP stain and immunohistochemistry (IHC) for DBA44 and Annexin A1 and BRAF mutations were done. Results: The median age of the cohort was 49 years (range: $30-71$ ) with male predominance $(93.6 \%$ males). The median WBC count was $2.5 \times 109 / \mathrm{L}$ (range: $0.5-40.9 \times 109 / \mathrm{L}$ ). The median percentage expression of CD11c was 91.2\% (11.8-99.6), CD103-73\% (1.5-99.3), CD25-65.5\% (3.28-95) and CD123 was $81 \%$ (7-99). One patient each were negative for CD11c $(n=63), C D 103$ $(n=62)$ and CD123 $(n=41)$. Three patients were negative for CD25 $(n=62)$ and among them 2 were relapsed. $81.6 \%$ had splenomegaly and $40 \%$ had hepatomegaly $(n=60) .42(n=51)$ patients were positive for TRAP, $34(n=43)$ were positive for DBA44 and $23(n=26)$ were positive for Annexin A1. BRAF mutations were positive for all 12 cases evaluated. Forty seven (74.6\%) of the 63 patients evaluated had taken treatment and 3 patients expired within a week during the course of treatment. One patient relapsed within a year and 3 patients beyond 3 years with a 5 year overall survival rate of $93.6 \%$. Conclusions: This is a descriptive single center experience of Hairy cell leukemia cases showing significant heterogeneity in clinicopathological and immunophenotypic profile.
MHL 36

Cytogenetic Profile of Adolescent, Adult and Elderly Precursor B Cell Acute Lymphoblastic Leukaemia (BCP-ALL)

Manish K. Singh", Mr. S. R. Arun, Mr. Anil K. Yadav, D. K. Mishra, Neeraj Arora, Saurabh J. Bhave, Shekhar Krishnan, Arpita Bhattacharya, Reena Nair, Vaskar Saha, Mammen Chandy, Mayur Parihar

Email ID for Correspondence: einstein24mks@gmail.com Tata Medical Center

Introduction and Background: Cytogenetic risk stratification in ALL has contributed to the optimization of therapy in childhood BCP ALL. There is little data on the cytogenetic profiles and their impact on therapy in adult BCP ALL with the exception of Ph positive ALL. We report of the cytogenetic profile of 178 BCP ALL patients aged more than 10 years. Patient/Material and Methods: Bone marrow findings, immunophenotype, Karyotyping and FISH results of ALL patients presenting at Tata Medical Center Kolkata between July 2011 and May 2016 were retrieved. Using FISH, BCR-ABL1 fusions and MLL gene rearrangements were investigated in all patients, ETV6RUNX1 in adolescent patients, and TP53 deletion in patients with complex karyotypes. Results: Of the 501 new ALL patients, 388 were diagnosed as BCP-ALL of which 178 were above 10 years. Karyotyping was successful in $84 \%$ of patients, of which $83 \%$ had abnormal cytogenetics that increased to $\sim 88 \%$ when coupled with FISH analysis. There were 63 adolescents (11-18 years), 73 young adults (19-40 years) and 42 older ( $>40$ years) patients. ETV6/ RUNX1 positivity was not seen in any of the patients. The $t(9 ; 22)$ was the most common cytogenetic abnormality (22.5\%) with an increasing frequency in older age groups. Monosomy 7 and $7 \mathrm{p}$ abnormalities (14.6\%) along with 9p abnormalities (16.3\%) were more frequent both as secondary abnormalities in Ph+ve ALL and as independent abnormalities in $\mathrm{Ph}-\mathrm{ve}$ ALL. The other abnormalities included chromosome 1q (11.2\%), del6q (3.9\%) and 14q32 (3.9\%) \& 8q24 $(1.7 \%)$ rearrangements. Complex karyotypes were seen in $18 \%$ of patients of which $44.4 \%$ had $17 \mathrm{p}$ abnormalities. Conclusions: We report one of the largest studies on cytogenetic Profile of Adult ALL from India with $\sim 88 \%$ being abnormal. $\mathrm{Ph}$ positivity, complex karyotype, $9 p$ and $-7 / 7 p$ abnormalities are more common after 40 years and increase with age. This data shall help in designing future adult ALL trials.

\section{MHL 37}

\section{ALK1 Positive Large B Cell Lymphoma: A Case Report}

\author{
Jai Wade", C. L. Pande, Vijay Kaul
}

Email ID for Correspondence: drzaiwade21@gmail.com Bhagwan Mahaveer Cancer Hospital \& Research Centre, Jaipur 
Introduction and Background: ALK1 positive large B cell lymphoma is a very rare mature B cell neoplasm, accounts for less than $1 \%$ of DLBCL. $<40$ cases have been reported so far. It comprises of monomorphic large immunoblast like B cells, which are CD20 \& PAX5 negative. Patient/Material and Methods: We report a case of 43 year old male who presented with complaint of right sided neck swelling. On cytology \& routine HPR, was initially diagnosed as metastatic undifferentiated neoplasm, which on IHC was proved to be ALK positive large B cell lymphoma. Results: On IHC, the tumor cells expressed LCA, MUM1, ALK1 (cytoplasmic granular), EMA, CK (dot like), IgA \& BOB1; immunonegative for CD20, CD3, PAX5, CD38, CD138 \& CD4. The B cell nature of neoplasm was expressed by $\operatorname{Ig}$ A \& BOB1, while lineage specific markers were negative. Conclusions: ALK1 positive large B cell lymphoma is an aggressive form of B cell lymphoma, due to CLTCALK gene arrangement in most patients. Recognition of this entity is important as most patients follow an aggressive disease course \& unlikely to respond favourably to current standard of care, as for CD20 positive DLBCL. These patients require novel treatment approaches \& are easily mistaken as having nonhaematopoietic malignancies, which results in delayed diagnosis $\&$ inappropriate therapy.

\section{MHL 38}

HLA-DR and CD34 Dual Negative Acute Myeloid Leukemia: A Distinct Subset of Leukemia or Just a Phenotype?

\section{Shivangi J. Harankhedkar", Ruchi Gupta, Khaliqur Rahman, Geeta Yadav, Manoj K. Sarkar, Tanvi Gupta, Anshul Gupta, Soniya Nityanand}

Email ID for Correspondence: dr.shivangih@gmail.com Sanjay Gandhi Postgraduate Institute of Medical Sciences, Lucknow

Introduction and Background: A dual negative (CD34 ${ }^{-}$/ $\mathrm{HLADR}^{-}$) profile, though a characteristic phenotype of acute promyelocytic leukemia (APL), has also been observed in other subtypes of acute myeloid leukaemia (AML). Morphologically these cases may mimic microogranular variant of APL, leading to a diagnostic dilemma. This study was aimed at analysing the clinico-pathological profile of dual negative non APL AML cases. Patient/Material and Methods: This was a retrospective analysis of all the AML cases diagnosed over a period of 42 months. Haematological parameters, MGG stained bone marrow smears were reviewed. Flow cytometric immunophenotyping (FCI) was done using stain-lyse-wash protocol in a 6 color antibody combination. Cytogenetic analysis was performed by conventional giemsa banding. Results: Out of 445 cases of AML diagnosed during this period, $62(13.9 \%)$ showed dual negativity for HLADR and CD34. Of these, 25 (40.3\%) were APL and $37(59.6 \%)$ were non APL. These non APL cases comprised of AML M1 $(\mathrm{n}=18)$, AML M2 $(\mathrm{n}=15)$, AML M5 $(\mathrm{n}=2)$ and a single case each of secondary AML and AML M0 subtypes. Nuclear cupping was evident in 18 (48\%) cases, Auer rods were seen in $16(43 \%)$ cases, cytoplasmic granularity was visible in $24(63 \%)$ cases. Nineteen $(51 \%)$ of these ${ }^{-}$cases resembled microgranular variant of APL, however RT-PCR did not show PML-RAR $\alpha$ fusion transcript. Aberrant expression of CD56 and CD7 was seen in 5 $(13.5 \%)$ and $2(5.4 \%)$ cases respectively. Karyotyping was performed in 27 cases, of which only $5(19 \%)$ showed abnormal karyotype. There was a single case of Philadelphia positive AML. Conclusions: A significant proportion of cases with CD34/HLADR dual negativity are non APL, and hence this profile does not always correspond to APL. Most of the patients with this profile have a normal karyotype. Larger clinical studies are required to ascertain their relevance as a prognostic marker in AML and treatment outcome.

Keywords Acute Myeloid Leukemia, CD34 ${ }^{-}, \mathrm{HLADR}^{-}$, Non APL

\section{MHL 39}

Clinico-pathological Profile of Multiple Myeloma Cases: Analysis at a Tertiary Care Centre

\section{K. V. Vinu Balraam", S. Venkatesan, Manish Sharma, Sanjeevan Sharma, Reena Bharadwaj}

Email ID for Correspondence: vbalraam@gmail.com Armed Forces Medical College, Pune

Introduction and Background: Plasma cell neoplasms are characterised by expansion of a clone of immunoglobulin secreting, heavy-chain class-switched, terminally differentiated B cells. They are bone marrow based neoplasms usually associated with secretion of monoclonal immunoglobulin and thus paraproteinemia. The aim was to explore the clinico-pathological profile of multiple myeloma cases managed at tertiary care centre. Patient/Material and Methods: In this study, we took out all the cases diagnosed as plasma cell neoplasm between Jan 2014-Aug 2016. Clinical sketch of the cases were retrieved from archives and correlated with the histopathological diagnosis. The clinico-pathological parameters accrued were age, sex, presenting complains, serum calcium and creatinine levels, blood haemoglobin concentration, skeletal survey, peripheral blood picture, bone marrow studies, serum protein electrophoresis (SPEP), immunofixation electrophoresis (IFEP) and serum free light chain ratio (SFLC). Results: 29 cases were included. 24 cases were male. Age group range was 41-85 years with a mean of 61 years. The most common presenting features were back ache and bony pain (14/29) followed by anaemia \& easy fatigability $(09 / 29)$ and pathological fractures (05/29). 09 of them had hypercalcemia, 18 were anaemic, 12 had deranged creatinine levels and 15 had lytic lesions in the bones. 22 had an $\mathrm{M}$ spike on SPEP and IFEP. SFLC ratios were deranged in the remaining 07 cases. 04 of them succumbed to the illness during this time period. Conclusions: Multiple myeloma is a complex and chronic illness presenting usually with subtle features like easy fatigability, anaemia or bony pains. Any elderly individual with low back ache or bony pain must definitely be evaluated and screened for this grave disease. Diagnosis of multiple myeloma requires a definite set of clinic-pathologico-radiological features as laid down by the IMWG guidelines so that the aim of initiating therapy before the setting in of organ damage is accomplished. 


\section{MHL 40}

Clinical, Morphological and Immunophenotypical Findings in Acute Lymphoblastic Leukemias: A Study From a Tertiary Care Hospital

\section{Aruna Chhikara" ${ }^{1 \#}$, Tanuja Bundela, Shailaja Shukla, Sunita Sharma, Jagdish Chandra ${ }^{1}$}

Email ID for Correspondence: sunrays.smile@gmail.com Department of Pathology and Paediatrics ${ }^{1}$, Lady Hardinge Medical College, New Delhi

Introduction: Acute leukemias comprise of heterogenous group of diseases charaterised by rapid and uncontrolled clonal expansion of progenitor cells of haematopoietic system. They are most common form of childhood neoplasia. Acute lymphoblastic leukemia (ALL) represents $75 \%$ of all such cases. This percentage is much lower in adults, in whom Acute myeloid leukemias (AML) are more common. The diagnosis of leukemia is based upon morphological and cytochemical investigations of bone marrow samples and/or peripheral blood. Immunophenotyping helps to subclassify acute lymphoblastic leukemias into subgroups with prognostic implications. Materials and Methods: 210 cases of acute lymphoblastic leukemias were included in study. Complete blood count along with peripheral blood smear and bone marrow aspirate smear examination was performed in all cases. Relevant cytochemistry was also performed. Immunophenotypic analysis was performed by Beckman Coulter Flow cytometer using monoclonal antibodies against various cell surface/cytoplasmic antigens was performed in 178 cases. Results: Out of 178 cases 164 were seen in children and 14 in adults. Among 164 cases in children 124 were CALLA positive B ALL while in adults 10 out of 14 were CALLA positive. T ALL comprised 21 cases in children and 3 cases in adults. Hepatosplenomegaly was seen in majority of cases in both children and adults. Most cases had total leucocyte count between 10,000 and 50,000/ $\mu 1$ and platelets $<1$ lakh. Haemoglobin levels were $<7.5 \mathrm{~g} / \mathrm{dl}$ in majority of them. CD19 and CD79a were $100 \%$ sensitive for B ALL while CCD3 was $100 \%$ sensitive for T ALL. Conclusion: B ALL was the most common acute lymphoid leukemia in both children and adults. Majority of cases presented with thrombocytopenia and moderate anaemia with leucocytosis. CD19 and CD79a were positive in all cases of BALL and CCD3 was positive in all cases of T ALL.

\section{MHL 41}

\section{A Retrospection of Primary-Extranodal Lymphoma 5-Year Study from a Tertiary Cancer Centre}

R. Archanadevi", Faiq Ahmed, Manasi Mundada, Rachna Khera, N. Lavanya, Sudha S. Murthy, Senthil J. Rajappa

Email ID for Correspondence: archanadevi30.ad@gmail.com Basavatarakam Indo-American Cancer Hospital and Research Institute

Introduction and Background: Primary extra nodal Lymphoma (PENL) constitutes diverse group of hematolymphoid malignancies. Diagnosis and characterisation of Extra nodal lymphomas (ENL) is on rise. There is limited data regarding ENL from India. This study is carried out to know the presenting site and subtypes of ENL as diagnosis and management require site specific strategies. Material and Methods: The study was conducted in the Department of Laboratory Medicine BIACH\&RI. The demographic details were retrieved from archives of pathology during a period of 5 years (2010-2015). H\&E slides and IHC slides were reviewed and all cases were classified based on WHO 2008 classification. Results: There were 127 cases of extranodal lymphomas reported over a period of 5 years. Male to female ratio was 2:1. Age ranged from 11 to 70 years. The commonest site of ENL in our study was GIT $(41.5 \%)$, followed by Head and neck region $(17.3 \%)$, soft tissue and bone $(17.63 \%)$ CNS $(4.72 \%)$, lung and pleural nodule $(3.14 \%)$, eye (3.14\%), Liver (3.14\%), kidney (1.57\%), adrenal (1.57\%) Female Genital Tract and breast (2.36\%), MGT (2.36\%), thyroid (1.57\%). Stomach was the commonest site in the GIT. Nasopharynx was the most frequent site in head and neck. The commonest histologic type was Diffuse Large B Cell Lymphoma (76.6 \%) followed by Extra nodal Marginal zone lymphoma (6.2\%), burkitt lymphoma (3.93\%), Anaplastic large cell lymphoma (3.14\%), Plasmablastic lymphoma $(3.93 \%)$, follicular lymphoma $(2.36 \%)$, Hodgkin lymphoma $(1.57 \%), \mathrm{NK} / \mathrm{T}$ cell lymphoma $(0.8 \%)$, Mantle cell lymphoma $(0.7 \%)$ and Small lymphocytic lymphoma $(0.7 \%)$. Conclusions: Prevalence of PENL is low in India compared to western population. The diagnosis of ENL is challenge due to rare morphologic mimics.

\section{MHL 42}

DNA Methylation Patterns of CDK Interacting Protein/Kinase Inhibitory Protein Family of Genes in Pediatric Acute Lymphoblastic Leukemia Patients: AIIMS Experience

\section{Nivedita Pathak ${ }^{1 \#}$, Rachna Seth ${ }^{1}$, Akhilesh Mishra ${ }^{2}$}

Email ID for Correspondence: nivisaiims@gmail.com All India Institute of Medical Sciences

Introduction and Background: Dysregulation of cell cycle by promoter methylation of cell cycle controlling genes is frequent event in Acute Lymphoblastic Leukemia (ALL) and is recognized as a critical event in the disease pathogenesis and progression. Cell cycle is negatively regulated by CDK interacting protein/Kinase inhibitory protein (Cip/Kip) family of genes. Epigenetic inactivation of these genes has been reported with discrepant frequencies. In India, the correlation of methylation status with treatment outcome has not been reported so far. In view of this, we aimed to investigate the methylation status of $\mathrm{CDK}$ interacting protein/Kinase inhibitory protein family of genes: p21 (CIP1) and p27 (KIP1) in our pediatric ALL patients and its correlation with response to treatment. Patient/Material and Methods: Bisulphite modification of the extracted total genomic DNA (from bone marrow/peripheral blood mononuclear cells) of pediatric ALL patients was followed by amplification, using methylation specific PCR. Results: A total of 65 pediatric patients (median age $9 \mathrm{yrs}$, range $1-17$ yrs; M:F 4:1; median TLC$17.4 \times 10^{9} / 1$, range $\left.1.1-715 \times 10^{9} / 1\right)$ were studied. Hypermethylation of p21 CIP1 (33\%) and p27 KIP1 (9\%) genes was observed in our patients. The frequency of hypermethylation was significantly associated with higher age group (age $>9$ years). Hypermethylation of p21 was also found to be predictor of poorer DFS \& OS. Conclusions: Frequency of hypermethylation of cell cycle controlling genes was found to be higher in our ALL patients as compared to mostly reported in various studies. This needs to be further explored in our Indian scenario with regard to prognosis.

\section{MHL 43}

A Case of Myelodysplastic Syndrome with Excess Blasts (MDSEB-1) Presenting with Basophilia

\section{Stuti Kumari", Deepa Rani, Prof. Vijai Tilak}

Email ID for Correspondence: stutikumari0791@gmail.com Institute of Medical Science, Banaras Hindu university (BHU), Varanasi 
Introduction and Background: Myelodysplastic syndrome (MDS) is an acquired clonal hematopoietic stem cell disorder characterized by cytopenias, dysplasia in one or more cell lines and ineffective haematopoiesis. MDS with basophilia is a rare condition and has yet to be classified under the World Health Organization classification. However, reports have described its prognostic significance and also as an independent risk factor for evolution to leukemia. Here we describe a case of MDS with basophilia which is rare. Patient: A 60 years old male was admitted to the hospital with complains of generalised body weakness, easy fatiguability and breathlessness on mild exertion for one month. Initial investigations showed features of pancytopenia with basophil $12 \%$ on complete blood count. Reticulocyte count was less than $0.5 \%$. After this, bone marrow aspiration and subsequently biopsy was done. Bone marrow imprints and clot section were also prepared. Marrow aspirate was sent for molecular study. Results: GBP showed features of pancytopenia with basophils $16 \%$. Bone marrow aspiration was diluted with blood and showed myeloid and erythroid series cells with features of dysmyelopoiesis and severe degree of dyserythropoiesis. Quadrinucleate and pentanucleate forms of erythroblasts were seen. Imprint smears showed erythroid hyperplasia with presence of blasts $(8 \%)$, quadrinucleate normoblasts and gigantoblasts along with cells of similar morphology as that of aspiration. Molecular study showed $7 \mathrm{q}$ deletion. Hence a diagnosis of MDS-EB-1 with basophilia was made. Because of presence of pentanucleate forms and gigantoblasts, initially bone marrow picture was mimicking congenital dyserythropoietic anaemia type III. The patient is still on follow up with little improvement in symptoms, frequent transfusion dependent and with persistent basophilia. Conclusion: MDS with basophilia is a rare condition which needs further studies about its prognostic significance. It also needs to be evaluated as an independent risk factor for leukemic transformation.

\section{MHL 44}

\section{Rare and Unusual Presentation of Acute Leukemia: A Tripod of Cases}

\section{Manjari Kishore", Vijay Kumar, Sadhana Marwah, A. S. Nigam}

Email ID for Correspondence: drmanjarik@gmail.com PGIMER, Dr. Ram Manohar Lohia Hospital, New Delhi

Introduction and Background: Acute Leukemia is one of the common haematological malignancies encountered with varied clinical and hematological presentation. Herein, we present a series of three cases of acute leukemia presenting with unusual clinical and other rare hematological findings. Case Report: Case 1: A 10 year old female presented with high grade fever and left hemiparesis for last 3 days before admission. No history of bleeding, rashes, double vision, lymphadenopathy or splenomegaly was noted. Peripheral smear showed pancytopenia. CT head showed a lesion in right temporo-parieto-occipital region, suggestive of infarction. Peripheral blood, bone marrow aspirate \& biopsy were suggestive of Acute promyelocytic leukemia (FAB subtype AML-M3). However, the patient succumbed after two months of follow up. The patient had presented with cerebral infarction before initiation of any treatment, which makes it a very rare presentation. Case 2: A 40 year old female presented with complaints of low grade intermittent fever for last 2 months, bone pain, decreased appetite and weight loss. Ultrasound of abdomen revealed hepatosplenomegaly and periportal lymphadenopathy. Peripheral smear showed $48 \%$ blasts. Flowcytometric analysis of peripheral blood revealed abnormal cluster with dim CD45 expression. Bright positivity was noted for CD10, CD19, tDT, HLA-DR along with moderate expression of CD22 \& CD34. The diagnosis of "Granular B cell Acute lymphoblastic leukemia with aberrant CD13 expression" was made. Case 3: An 18 year old male presented with multisystem involvement with osteolytic lesions in skull and skeletal survey (pelvis), hypercalcemia, decreased urinary output, hematuria, renal failure and skin lesions. Clinically, the differential diagnoses made were Multiple myeloma, Langerhans cell histiocytosis, T cell Acute lymphoblastic lymphoma. Peripheral smear showed leucocytosis with $30 \%$ of blast cells. Bone marrow aspiration \& biopsy showed almost complete replacement of normal hematopoietic elements by blasts. Flowcytometric analysis revealed an abnormal cluster showing moderate positivity for CD19 along with moderate to bright expression of CD22 and HLA-DR. The diagnosis of "B cell-Acute Lymphoblastic leukemia" was made. Conclusion: This case series brings about many rare presentations of acute leukemias. Moreover it also highlights the role of comprehensive work up to arrive at a definitive diagnosis so that optimal management can be offered to the patients.

\section{MHL 45}

\section{Biclonal Gammopathy with Multiple Myeloma: A Case Report}

\section{Shalini Trivedi ${ }^{\#}$}

Email ID for Correspondence: shaltriv@yahoo.com IGESI Hospital, Jhilmil, Delhi

Introduction and Background: Multiple myeloma is a neoplastic clonal disease characterised morphologically by plasma cell infiltration of the medullary space and extra osseous tissues. Multiple myeloma is characterised by presence of monoclonal protein in plasma and/or characterised by simultaneous appearance of two different $\mathrm{M}$ the urine. Multiple myeloma accounts for $10 \%$ of all haematological malignancies. Biclonal gammopathies are rarer; prevalence is approximately $1 \%$ of monoclonal gammopathies. Patient/Material and Methods: The patient, a 64 year old female, presented with lower backache of 3 months duration, anaemia, renal dysfunction, neurosensory involvement. Radiological features: X ray pelvis showed lytic lesion in left illium \& $\mathrm{X}$ ray lumbar spine showed lytic lesion in L 3 vertebrae. Results: Haematological \& Biochemical Results: Investigations revealed moderate anaemia: (Hemoglobin: 6.5 gm\%), Lymphocytosis with normal Total Leucocyte count (DLC showed lymphocytes $89 \%$ ), ESR was markedly elevated (ESR: $95 \mathrm{~mm}$ in first hour). Bio chemical Investigations revealed hyperproteinaemia with hyperglobulinaemia, hypercalcemia (Serum calcium: $13.1 \mathrm{mg} / \mathrm{dl}$ ), uraemia (Blood Urea: $67 \mathrm{mg} / \mathrm{dl}$ ) \& increased creatinine level of $3.7 \mathrm{mg} / \mathrm{dl}, \beta-2$ microglobulin: $6652 \mathrm{mg} / \mathrm{dl}$. Immunoogical tests: Serum Protein Electrophoresis: Showed two distinct peaks in Beta 1 \& Gamma region. Nephlometry results: IgG: $2400 \mathrm{mg} / \mathrm{dl}$,(Ref range 700-1600 mg/dl), IgA: $570 \mathrm{mg} / \mathrm{dl}$ (Ref range; $70-400 \mathrm{mg} / \mathrm{dl}$ ), IgM below $18.6 \mathrm{mg} / \mathrm{dl}$ (Female Ref Range: 40-230 mg/dl), Free Kappa, serum; $15.90 \mathrm{mg} / \mathrm{dl}$ (Ref Range: $3.3-19.4 \mathrm{mg} / \mathrm{dl}$ ), Free lambda, Serum: $33.7 \mathrm{mg} / \mathrm{dl}$ (Ref range: $5.71-26.3 \mathrm{mg} / \mathrm{dl}$ ). Immunofixation electrophoresis showed the presence of IgG lambda \& IgA lambda. Urine: BJP was Positive. Histopathological Tests: Bone Marrow Aspiration showed hyper cellularity with a preponderance of plasma cells immature, mature \& bi nucleate forms: It is a rare case of multiple myeloma with biclonal gammopathy. Biclonal gammopathies account for $1 \%$ of all multiple myelomas. IgG \& IgA biclonal gammopathy account for $53 \%$ of all cases of biclonal gammopathy. As per our experience \& literature that the survival in patients with monoclonal \& biclonal gammopathy was not different. Bone marrow Biopsy demonstrated a diffuse infiltrate of plasma cells (mature, binuleate, immature and typical) up to $35 \%$. Feature suggestive of plasma cell dyscrasias. Conclusions:

(1) It is a rare case of multiple myeloma with biclonal gammopathy. 
(2) Biclonal gammopathies account for $1 \%$ of all multiple myelomas.

(3) IgG \& IgA biclonal gammopathy account for $53 \%$ of all cases of biclonal gammopathy.

(4) As per our experience \& literature that the survival in patients with monoclonal \& biclonal gammopathy was not different.

\section{MHL 46}

AML with Gene Mutation-A Correlation with Haematological and Immunophenotypic Characteristics: Our Experience with 126 Cases at a Tertiary Care Cancer Center

Rachna Khera ${ }^{\#}$, Faiq Ahmed, Manasi C. Mundada, Sudha S. Murthy, Sandhya G. Devi, N. Lavanya, Senthil J. Rajappa, Krishna Mohan Mallavarapu, Pavan Kumar, A. Santa

Email ID for Correspondence: rachnakhera@yahoo.com, wadhwa.rachna@gmail.com

Basavatarakam Indo American Cancer Hospital and Research Institute, Hyderabad, Telangana, India

Introduction and Background: Molecular genetic analysis of FLT3, NPM1, CEBPA, is already standard of care in patients with Acute Myeloid Leukemia (AML) and represent the most frequent genetic alterations and important diagnostic and prognostic indicators. Patient/Material and Methods: Morphological, haematological and immunophenotypic characteristics of NPM1 and FLT3 mutations in 126 patients of de novo AML including adults and children were studied. Apart from French American British (FAB) method for classification, blasts were assessed for cup like morphology as per strict definition for cuplike nuclei, $\geq 10 \%$ blasts with nuclear invaginations $\geq 25 \%$ of the nuclear area. Results: FLT3 mutation in $31 / 126(25 \%)$ and NPM1 mutation was found in 17/126 (13.4\%) of the AML patients. $6(5 \%)$ samples were positive for both NPM1 and FLT3/ITD mutations. Adult patients had significantly higher frequency of FLT3 and NPM1 mutation than children. FLT3/ITD mutation was associated with higher white blood count $(\mathrm{P}=0.008)$ and higher blast count in the peripheral blood $(P=0.003)$. Cup like morphology of the blasts was seen in $81 \%$ of the cases positive for FLT3 and/or NPM1 mutation. Immunophenotypically, NPM1 mutation was associated with the lack of CD34 $(P=0.009)$ while FLT3/ ITD mutation was positively associated with the expression of CD117 and CD7 $(P=0.04)$. CD34 and CD14 were found to be most important markers for diagnosis in NPM1 cases while CD117 was found to be predictive for FLT3 mutations by regression analysis. Conclusions: The results suggest that presence of distinct morphology, haematological and immunophenotypic characteristics together may serve as important indicators and surrogate for NPM1 and FLT3/ ITD mutations. Further, comprehensive studies on the biological effects of NPM1 and FLT3/ITD mutations and their interactions with other genetic alterations are needed to gain insight into the molecular mechanism of these mutations involved in the pathogenesis of AML.

\section{MHL 47 \\ Correlation of Expression of Aberrant Immunophenotypic Markers in T-ALL with Its Morphology-A Pilot Study}

\section{Neha Garg", Dilip Kumar, Rajesh Pathak, Mrinalini Kotru, Meera Sikka}

Email ID for Correspondence: gargdoc118@gmail.com UCMS and GTBH with Max Saket, New Delhi
Introduction and Background: Aberrant expression of immumophenotypic markers is commonly found in patients of acute leukemia. T-ALL also shows aberrant markers like CD13, CD33, CD117, CD10 and CD79a. Morphologically, T-ALL has been categorized into L1, L2 and L3 subtypes. Till now, no study has been done to correlate these markers with morphological features of T-ALL. This study aimed to correlate the expression of these aberrant immunophenotypic markers with morphology in T-ALL. Patient/ Material and Methods: All the cases of T-ALL diagnosed by flow cytometry over a period of $2 \frac{1}{2}$ year were taken out from the records of Hematology section of department of pathology of UCMS and GTBH and MAX hospital, Saket. Their peripheral blood smear was screened to correlate the morphology of blasts with the expression of aberrant markers. Results: A total of 40 cases of T-ALL were identified during $2 \frac{1}{2}$ year period of our study. Morphological correlation was available for 23 cases. Aberrant expression of CD10 was present in $6(26.0 \%)$ cases, CD79a in $9(39.13 \%)$ cases, CD117 in 5 $(21.73 \%)$ cases and myeloid antigen CD 33 in $5(21.73 \%)$ cases. CD117 and CD33 positive cases showed $\mathrm{L}_{2}$ morphology with presence of convolutions while cases with expression of CD10 and CD79a had $\mathrm{L}_{1}$ morphology with absence of convolutions. Conclusions: There seems to be an association of aberrant markers with $\mathrm{L}_{1}$ and $\mathrm{L}_{2}$ morphology. However, this needs to be tested for statistical significance on a larger sample size.

\section{MHL 48}

Immunophenotype Characterization of Patients with Acute Promyelocytic Leukemia: A Singe Centre Experience

Faranaz Khamruddin", Ansu Abu Alex, Kotteeswari Kathirvel, Hari Krishnan Babu, Aby Abraham, Alok Srivastava, Biju George, Poonkuzhali Balasubramanian, Vikram Mathews

Email ID for Correspondence: faranaz13@gmail.com Department of Haematology, Christian Medical College, Vellore, India

Introduction and Background: Acute promyelocytic leukemia (APL) is a sub type of acute myeloid leukemia (AML) characterized by a reciprocal translocation between chromosome 15 and 17 $[t(15 ; 17)]$. We undertook a retrospective study to immunophenotypically (IPT) characterize and correlate clinical outcomes of patients with APL diagnosed in our center. Patient/Material and Methods: Clinical data from 122 APL patients (diagnosed between 2011 and 2016) was analyzed. For IPT CD13, CD33, CD34, HLA-DR, CD117, CD56, CD64, CD19, CD11b antibodies were used and was done according to standard protocols. The data was acquired using FACS Calibur (BD, Mansfield, MA, USA) and analyzed by CELLQuest Pro software (BD). RT-PCR was performed according to standard protocol as a part of diagnosis. Results: The median age was 31 years (2-60 years) of which $86(70.5 \%)$ were females and $36(29.5 \%)$ were males. All the patients were positive for RT-PCR (PML-RARA) of which $68(55.7 \%)$ patients were bcr1, $5(4.1 \%)$ were bcr2 and 49 $(40.2 \%)$ were bcr3 isoforms respectively. The median total WBC count was $7.3 \times 10^{9} / \mathrm{L}(4.0-180.7)$. The median blast percentage by IPT was $86 \%$ (17-97). The median expression of CD13 was $68.4 \%$ (9.2-99.3), CD33 was 92.2\% (1.2-99.8), CD34 was $0.9 \%$ (0.04-97.7) and HLA-DR was $3.2 \%[(0.1-65)$. Five (4\%) cases had co-expression of CD34 and HLA-DR $(>20 \%)]$ at diagnosis. The median CD56 was $0.8 \%$ [0.02-72; only 10 patients had aberrant expression of CD56 $(>20 \%)]$. The median CD117 was $30 \%$ $(0.08-85.2 \%)$, CD64 was $24 \%(0.2-93.9 \%), \mathrm{CD} 11 \mathrm{~b}$ was $1.5 \%$ (0.1-80.7\%) and CD19 was 0.3 [0.03-33.6\%; only one case each had aberrant expression of CD19 and CD11b (>20\%)] respectively. 
Early deaths accounted for 15 patients $(12.3 \%)$ and 19 patients $(15.6 \%)$ relapsed during the study. The overall survival (OS) was $87.7 \%$ and the event free survival (EFS) was $72.1 \%$. Conclusions: This study demonstrates that there is a significant heterogeneity in the IPT of APL patients at diagnosis. However, this did not correlate with clinical outcomes or PML-RARA isoforms.

\section{MHL 49}

\section{Congenital Leukaemia in a 3 Days Old Child-A Rare Case Report}

\section{Jyoti Shukla, Sandip Kumar, Rishila Majumder" \\ Email ID for Correspondence: wrishila@gmail.com Institute of Medical Sciences (Banaras Hindu University)}

Introduction and Background: Congenital leukaemia (CL) is an extremely uncommon disease with an incidence of approximately $0.8 \%$ of all childhood leukaemias. It develops in utero and is usually diagnosed within one month of life. It is most commonly of myeloid lineage with very poor prognosis. Here we present a case of congenital leukaemia in a 3 days old male child presenting with hepatosplenomegaly. Materials and Methods: A 3 days old male child presented with hepatosplenomegaly. Haematological examinations revealed marked leukocytosis and thrombocytopenia. Peripheral blood and bone marrow smears showed differential blast count of $55 \%$ and $90 \%$ respectively. A diagnosis of Acute Megakaryoblastic Leukaemia (AML-M7) was made based on morphology coupled with cytochemical stain. Conclusion: Here we are reporting a case of congenital leukaemia of AML-M7 subtype which is not associated with Down syndrome.

\section{MHL 50}

Patterns of Bone Marrow Involvement in Chronic Lymphoproliferative Disorders Along with Correlation with the Symptomatology: A Review of 60 Cases

\section{S. Anusha\# ${ }^{\#}$ Richa Gupta \\ Email ID for Correspondence: anushasbhatt@gmail.com Maulana Azad Medical College, New Delhi}

Introduction and Background: Chronic lymphoproliferative disorders are staged based on their infiltration into the bone marrow. The symptoms with which the patient presents also depends on the extent of bone marrow involvement and the hematopoietic reserve. Patient/ Material and Methods: This study is a retrospective study which included the cases between January 2012 to December 2012 and November 2013 to August 2016. 60 formalin fixed and paraffin embedded bone marrow biopsies were studied with relevant clinical details, retrieved from the record. Results: Of the 60 cases, 35 $(58.3 \%)$ were males and $25(41.7 \%)$ females with a median age of $50 \mathrm{yrs}$ ( 9 to $75 \mathrm{yrs})$. The study included 37 cases of Non-Hodgkin's lymphoma (61.7\%), 14 of chronic lymphocytic leukemia (23.3\%), 3 of Hodgkin's lymphoma (5\%), 2 of Hairy cell leukemia and plasmablastic lymphoma, and 1 case each of Anaplastic Large cell lymphoma and Peripheral T-cell lymphoma. Majority presented with fever $(38.3 \%)$, splenomegaly $(45 \%)$, and lymphadenopathy $(36.7 \%) .4$ patients had concomitant history of tuberculosis, 3 of HIV infection, 2 of malaria and 1 of hepatitis $\mathrm{C}$. The mean $\mathrm{Hb}$ was 8.3 $\mathrm{g} / \mathrm{dL}$ (2.6 to 12.4$)$ with predominantly normocytic normochromic blood picture. Leucocytosis was noted in most with a mean of 57,460/ cu mm (250 to 466,730), with 9 cases showing atypical cells in peripheral blood. Thrombocytopenia was noted in majority cases with a mean of $78,000 / \mathrm{cu} \mathrm{mm} \mathrm{(4000} \mathrm{to} 607,000)$. BM examination revealed diffuse involvement in 27 cases (45\%), nodular in 12 $(20 \%)$, interstitial in $6(10 \%)$ and mixed in 15 cases $(25 \%)$. Cases with diffuse involvement had high tumor burden with reduced hematopoietic reserves. Conclusions: The extent of bone marrow involvement along with the symptomatology serves as an indicator of tumor burden.

\section{MHL 51}

A Cost-Effective, High Sensitivity 10-Color Single-Tube FlowCytometry (FC) Based B-Cell Precursor Acute Lymphoblastic Leukemia (BCPALL) Minimal Residual Disease (MRD) Assay

\section{Gaurav Chatterjee ${ }^{\#}$, Nilesh deshpande, Nikhil Patkar,} Y. Asma Bibi

Email ID for Correspondence: gauravchatnobel@gmail.com Hematopathology Laboratory, Tata Memorial Hospital, Mumbai

Introduction and Background: Minimal residual disease (MRD) monitoring is widely used for monitoring treatment-effectiveness and risk-stratification of ALL. Hence, the methodology for MRD assessment needs to be fast, highly sensitive, affordable and most importantly, widely applicable. Studies have shown that flow-cytometrybased MRD (FC-MRD) technique cannot reach beyond the $0.01 \%$ sensitivity and PCR-based MRD-technique is more sensitive. We present a study of a cost-effective highly-sensitive 10-color single tube FC-MRD assay. Material and Methods: We studied 102 cases of BCPALL in Hematopathology-Laboratory, Tata Memorial Centre, Mumbai. FC-immunophenotyping was performed on Navios flow-cytometer using bulk-lysis-and-stain method and data was analyzed with Kaluza-software. MRD was monitored in 163 bone-marrow samples at post-induction (PI, day 29-35), post-consolidation (PC, day-90) and subsequent follow-up time-points (SFU) using 10-color FC-MRD-assay. 10-color MRD-panel included CD123, CD20, CD58, CD86, CD25, CD19, CD10, CD34, CD45 and CD38. An additional four-color nuclear-dye (SYTO-13)-tube was used for calculation of MRD in total nucleated-cells. Samples with a cluster of $\geq 20$ events expressing $\geq 2$ leukemia-associated immunophenotypes (LAIPs) were called MRDpositive. Results: Of 102 cases studied, 67 were childhood-BCPALL (0-14 years) and 35 were adult-BCPALL (>14-64 years). In FC-MRD assay, high number of events were acquired with median-events $2,113,000$ (range 506,000 to $3,010,000$ ). Of 163 , MRD was positive in $84(55 \%)$ samples (median, $0.135 \%$ and range, $0.0007 \%-47.5 \%$ ). MRD-positive samples with MRD levels $<0.001 \%, 0.001-<0.01 \%$, $0.01-<0.1 \%, 0.1-<1.0 \%, 1.0-<10 \%$ and $>10 \%$ were respectively $2.4 \%, 7.1 \%, 34.5 \%, 27.4 \%, 14.3 \%$, and $14.3 \%$. Of $163 \mathrm{MRD}$ samples, PI-MRD were 102 (62.6\%), PC-MRD were 38 (23.9\%) and SFU were $22(13.5 \%)$. Of 102 diagnostic-samples, <2-LAIPs were seen in only $1 \%$ and $>6$-LAIPS in $2 \%$ (median-LAIPs, $4 \&$ range, $1-8)$. In MRD samples, four had <2LAIP (2.4\%) and labelled as "suspicious". Of 84 MRD-positive, samples with 2-LAIPS were only $7.1 \%$. Furthermore, in 10 samples with MRD-positive $\leq 0.01 \%$ and $>1.5$-million events, the results were compared between time-gated initial 500,000-events, 1,000,000 events and all acquired-events. Amongst them, five samples found negative in initial 500,000-events and three in initial 1,000,000-events (since number of MRD-events were $<20$-events) highlighting importance of acquisition of $>1.5$-million cells to increase the sensitivity of FC-MRD-assay. Cost-per-assay was 25 USD. Conclusions: We established a cost-effective, 10-color, single-tube FC-MRD-assay with high sensitivity of 1-in- $10^{5}$ MRD detection and applicability in $>97 \%$ samples. Hence, this assay could be highly useful in resource-limited settings. Our study also showed 
that the acquisition of less than one-million events can reduce the sensitivity of FC-MRD-assay.

\section{MHL 52}

Application of Next Generation Sequencing (NGS) in Diagnosis and Prognostication of Hematological Malignancies

\section{K. Kelkar\#, V. Ramanan, S. Ranade, T. Ragte-Wathare, N. Gogate, P. Gangodkar, K. Patil, M. Agarwal, K. Khatod, N. Phadke}

Email ID for Correspondence: ketki.kelkar@genepathdx.com GenePathDx, Pune

Introduction and Background: We have developed and validated Next generation sequencing (NGS) based assays for diagnosis and prognostication of hematological malignancies. Our ability to simultaneously multiplex our in-house developed assays, sequence at ultra-high depths, turn-around results rapidly, interpret unambiguously and provide these services at affordable rates makes it extremely helpful to the hematologist and ultimately to the patient. Some of the assays that we are using routinely include NGS based $B C R$ $A B L$ fusion-transcript analysis for diagnosis of drug resistance in chronic myeloid leukemia (CML), multiplexed $J A K 2, M P L$ and $C A L R$ targeted deep sequencing in Philadelphia negative chronic myeloproliferative neoplasms, and FLT3, NPM1 and WT1 mutations in Acute Myeloid Leukemia (AML). We present sample cases of each type and their comparative performance vis-à-vis conventional molecular test methods. Patients/Material and Methods: Peripheral blood/bone marrow samples from patients with chronic myeloproliferative neoplasms (CML, ET, PV and PMF) and AML patients were collected in EDTA vacutainers, and processed after custom library preparation on an Illumina MiSeq NGS platform. Orthogonal verification was carried out by real-time PCR or capillary sequencing as appropriate. Results: A variety of drug resistance causing mutations were detected in the TK domain and SH2-SH3 domains of the BCR-ABL fusion transcript in CML patients. CALR (Type 1, type 2 and type 2 like) mutations were detected in ET and PMF patients, $J A K 2$ exon 14 mutations in several PV, ET and PMF cases, and FLT3 (ITD and TKD) mutations were detected in AML patients. Conclusions: We have developed highly multiplexed Next Generation Sequencing (NGS) assays for hematological malignancies with increased detection sensitivity to as low as $1 \%$, and with rapid turnaround times. The NGS data are unambiguous and easy to interpret. Furthermore, the results are quantitative, thus providing an indication of the relative abundance of the mutation.

\section{MHL 53}

\section{NUDT15 c.415C > T Variant Explains 6-MP Toxicity in Patients with Acute Lymphoblastic Leukemia}

Stallon Ilangeswaran", Ezhilpavai Mohanan, P. N. Nisham, G. Amirthavani, Biju George, Alok Srivastava, Vikram Mathews, Poonkuzhali Balasubramanian

Email ID for Correspondence: stallonillang@cmcvellore.ac.in Department of Haematology, Christian Medical College, Vellore

Introduction and Background: Incorporation of thiopurines in maintenance chemotherapy has improved treatment outcomes in ALL. Thiopurines have narrow therapeutic indices owing to severe toxicity, which is moderately explained by the presence of thiopurine methyl transferase (TPMT) genetic variants (TPMT $* 2 * 3 \mathrm{~A} * 3 \mathrm{~B}$ or $* 3 \mathrm{C})$. However, rarity of TPMT polymorphisms in Indian population
(Desire et al, 2009) fails to explain thiopurine-induced toxicity, suggesting that additional factors may contribute to the variability in thiopurine metabolism. Recent reports have suggested a coding variant $($ c. $415 \mathrm{C}>\mathrm{T}(* 3))$ in NUDT15 gene to be a determinant of thiopurine toxicity in the Asian population. NUDT15 dephosphorylates the thiopurine active metabolites thereby reducing cytotoxic effects of thiopurines and the NUDT15*3 variant shows reduced or loss of enzyme activity. We screened for NUDT15 polymorphism in ALL patients on 6-MP maintenance therapy with myelosuppression, for whom TPMT polymorphism status was requested. Patient/Material and Methods: Twenty-eight ALL patients with the median age of 15 yrs (2-57 yrs) who underwent 6-MP maintenance therapy were included in this study. Genomic DNA isolated from peripheral blood was used for screening NUDT15 polymorphism by PCR followed by Sanger sequencing. Results: NUDT15*3 variant was seen in 15 (10 heterozygous and 5 homozygous variant) of the 28 patients. One of these patients had TPMT $* 3 \mathrm{C}$ heterozygous genotype and was not positive for NUDT15 variant. Twenty two of these 28 patients had 6-MP dose reduction. Conclusions: TPMT polymorphisms in Asian population is rare and NUDT15*3 variant frequency alone explains $22 \%$ of variability in mercaptopurine intolerance in childhood ALL (Yang et al. J. Clin. Oncol, 2015). In addition to TPMT polymorphism, a screening panel including NUDT15 c.415C $>\mathrm{T}$, as well as other polymorphisms in known genes involved in 6-MP metabolism would be necessary to understand the genetic basis of 6-MP intolerance in patients with ALL for personalizing 6-MP therapy.

\section{MHL 54}

Frequency of CEPBA Mutations in Normal Karyotype AML-A Single Centre Study

\section{Sathya ${ }^{\#}$, A. Arun Kumar, Ajay Abraham,} SavithaVaratharajan, Vivi M. Srivastava, Sreeja Karathedath, Anu Korula, P. N. Nisham, Biju George, Alok Srivastava, Vikram Mathews, Poonkuzhali Balasubramanian

Email ID for Correspondence: smileesathya@gmail.com Christian Medical College and Hospital, Vellore

Introduction and Background: Mutations in the CEBPA gene, described in 10-15\% ofall AML patients with normal karyotype (AML-NK) is associated with good prognosis. However, recent data have shown that a favourable prognosis in CEBPA-mutated AML is restricted only to those patients who are presenting with CEBPA double mutations and do not carry cytogenetic aberrations or an FLT3 mutation. We prospectively evaluated the CEBPA mutation spectrum in NK-AML and compared the immunophenotype, cytogenetic as well as other molecular aberrations seen in this group of patients. Patient/Material and Methods: Among the AML patients evaluated in the department of Haematology, Christian Medical College, Vellore between June 2009 and June 2016, patients with normal karyotype (AML-NK) were included in the study. DNA was extracted from bone marrow/peripheral blood mononucleated cells (BM or PBMNC) at diagnosis. Mutations in CEBPA, NPM1 and FLT3 was screened using established protocols. The type of the mutation was confirmed by directDNA sequencing. Results: Of a total of 633 AML patients diagnosed during the study period with cytogenetic results, 257 had AML-NK (40.6 \%) out of which DNA sample was available for 235 patients. There were 140 males $(59.5 \%)$ and 95 females $(40.5 \%)$. The median age of the patients was 40 years (range 6-79 years). Mutations in CEBPA genewere identified in 31 of the $235(13.2 \%)$ NK-AML patients; $23(8.9 \%)$ had mutation in a single allele, while 8 patients $(3.4 \%)$ had a double allelic mutation. A total of 37 different mutations were identified in 29 patients. Majority of 
the mutations were $\mathrm{N}$-terminal $(30.7 \%)$ or $\mathrm{C}$-terminal mutations $(61.5 \%)$. With respect to the distribution, $48.6 \%$ of the mutations were in-frame deletions/insertions while $37.9 \%$ mutations were frameshift deletions/insertions. Point mutations were seen in a smaller fraction of patients $(13.5 \%)$. Most of the N-terminal mutations $(84 \%)$ were frame shift mutations resulting in activation of a smaller p30 isoform while $75 \%$ of the C-terminal mutations were in-frame insertions/deletions/duplications. In addition, two common polymorphisms, c.584_589 DUP ACCCGC and c.690 (G>T) were identified in $16(6.8 \%)$ and $7(3 \%)$ patients respectively. Of the patients with CEBPA mutations, $24.1 \%, 20.7 \%$ and $10.3 \%$ had a co-existing FLT3, NPM1 and both FLT3 and NPM1 mutations respectively which is consistent with the earlier reports. There was a trend to higher blast count in CEBPA mutated (mean $71.1 \%$ ) vs. wild type patients $(58.5 \%) \quad(\mathrm{p}=0.052)$. Conclusions: The frequency of CEBPA mutations and its interaction with other established molecular markers (FLT3\& NPM1) observed in our study was consistent with the earlier reports from other populations.

\section{MHL 55}

MPL W515 Mutations in JAK2 V617F Negative Patients with Primary Myelofibrosis and Essential Thrombocythemia

\section{Mukinkumar ${ }^{1 \#}$, Shano Naseem, Neelam Varma, Jogeshwar Binota, Pankaj Malhotra ${ }^{1}$, Subhash Varma ${ }^{1}$}

Email ID for Correspondence: mukin2005@yahoo.co.in, mukin.kumar@gmail.com

Departments of Hematology and ${ }^{1}$ Internal Medicine,

Post Graduate Institute of Medical Education and Research, Chandigarh

Introduction and Background: The $M P L$ gene encodes the thrombopoietin receptor. Activating mutations of MPL exon 10 have been described in patients with primary myelofibrosis (PMF) and essential thrombocythemia (ET). The most common $M P L$ mutations are W515L (tryptophan-to-leucine substitution) and W515K (tryptophan-to-lysine substitution). MPL mutations testing aids in the diagnosis of JAK2 V617F negative patients by establishing clonality and meeting one of major diagnostic criterion of World Health Organization classification of ET and PMF. Aim: To study the frequency and clinico-hematological features of $M P L \mathrm{~W} 515 \mathrm{~K} / \mathrm{L}$ mutations in JAK2 V617F negative PMF and ET patients. Patient/Material and Methods: $M P L$ W515K/ $\mathrm{L}$ mutation mutations were tested in 31 consecutive cases of JAK2 V617F negative PMF and ET. Details of clinical and hematological parameters were noted. MPL W515K/L mutation testing was done by amplification refractory mutation system-polymerase chain reaction (ARMS-PCR) and electrophoresis on $3 \%$ agarose gel. Results: Out of the 31 cases of JAK2 V617F negative PMF $(\mathrm{n}=22,71 \%)$ and ET $(\mathrm{n}=9,29 \%), M P L$ W515L mutation was identified in $6(19.4)$ patients, MPL W515K was not seen in any patient. All 6 patients were of PMF, none of the $9 \mathrm{ET}$ patients tested were positive for MPL mutation. MPL positivity was thus seen in $27.3 \%(6 / 22)$ PMF patients. On comparing PMF patients with and without MPL W515 mutation, no significant difference was found in median age (54 versus 56 years), sex distribution (male predominance was seen in both groups), median total leucocyte $\left(13.5\right.$ versus $\left.14.2 \times 10^{9} / \mathrm{L}\right)$ and platelet counts $(114$ versus $148 \times 10^{9} / \mathrm{L}$ ); however, splenomegaly was seen at a higher frequency in MPL W515 positive patients $(83.3 \%$ versus $50 \%)$ and lower median hemoglobin values in MPL W515 negative patients (8.7 versus $10.3 \mathrm{~g} / \mathrm{dL}$ ). Conclusion: In this cohort, $M P L$ W515L mutation was found at a frequency of $27.3 \%(6 / 22)$ in $J A K 2$ V617F negative PMF patients. None of the $9 \mathrm{ET}$ patients tested were positive for MPL mutation. No significant clinical differences were observed between $M P L$ W515L positive versus negative patients with PMF, except in splenomegaly being more frequent in positive cases and median hemoglobin count being lower in negative cases. However, the statistical power of this analysis is limited by the small number of patients in our cohort and future studies with larger number of patients are required which will further delineate the phenotypic effects of these mutations. Small number of patients in our cohort and future studies with larger number of patients are required which will further delineate the phenotypic effects of these mutations.

\section{MHL 56}

Primary Extranodal Non Hodgkin Lymphoma-A Retrospective Study from a Tertiary Care Center in Eastern India

Aditi Kundoo $^{1 \#}$, Radhamohan Gharei ${ }^{1}$, Pritinanda Mishra ${ }^{1}$, Somanath Padhi ${ }^{1}$, Saroj Kumar DasMajumdar ${ }^{2}$, Susama Patra ${ }^{1}$

Email ID for Correspondence: aditi.kundoo@gmail.com Department of ${ }^{1}$ Pathology and Lab Medicine and ${ }^{2}$ Radiation Oncology, All India Institute of Medical Sciences, Bhubaneswar, Odisha, India

Introduction: Primary extra nodal non Hodgkin lymphomas (pENL) are distinct group of non-Hodgkin lymphomas with diverse histomorphology and unpredictable biology. This study was undertaken to ascertain the anatomic distribution and histopathological subtypes of pENL. Materials and Methods: Thirty four cases of pENL over a period of two and half years (2014-2016 till date) were retrieved from the departmental archives and analyzed as per 2008-WHO classification. Results: Thirty four cases included 19 males and 15 females (mean age; 40 years, range; 24 to 78 years). The most common anatomical sites were head and neck followed by skin, gastrointestinal tract, brain, bone; and one case each from breast, ovary and retroperitoneum. Tonsil was the commonest site of involvement in head and neck. Three most common histological types were diffuse large B cell lymphoma $(\mathrm{n}=12)$, extra nodal marginal zone lymphoma $(\mathrm{n}=6$; three associated with $H$. pylori), and cutaneous $\mathrm{T}$ cell lymphomas $(n=6$; one with associated hemophagocytic lymphohistiocytosis). Conclusion: Accurate categorization of non-Hodgkin lymphomas at extranodal sites is essential for proper patient management and understanding the biology of the disease.

\section{MHL 57}

Transformation of Chronic Myeloid Leukemia into Chronic Lymphocytic Leukemia-An Extremely Rare Entity

\section{Sandip Kumar, Nivedita Mahto ${ }^{\#}$, N. Kumar, Shashikant Patnae,} Jyoti Shukla

Email ID for Correspondence: mahtonivedita@gmail.com IMS, Banaras Hindu University, Varanasi, U.P., India

Introduction and Background: Chronic Myeloid Leukemia is the most common Chronic leukemia of elderly patients in india with an incidence of $0.8-2.2 / 100,000$ population in male and $0.6-1.6 / 100,000$ population in female. Transformation of CML into myeloblastic or lymphoblastic form in the terminal stage is common but transformation into Chronic Lymphocytic Leukemia is extremely rare. Till now only three cases has been reported in the literature. Patient/Material and Methods: 50 years old female was diagnosed as CML who developed CLL after 18 months of Imatinib Mesylate therapy. Results: Transformation of CML patient into CLL, diagnosis of which was based on examination of peripheral blood smear, bone marrow aspiration and biopsy including immunohistochemistry, cytogenetics, immunophenotyping and polymerase chain reaction. Conclusion: Here we are presenting this case because of extreme rarity. 


\section{MHL 58}

Variable Busulfan Pharmacokinetics Among Different Generic Busulfan-Need for Therapeutic Drug Monitoring

Rajesh Nagarajan", Shareen Stella Backia Royan, Ezhilpavai Mohanan, Anup Devasia, Anu Korula, N. A. Fouzia, P. N. Nisham, Aby Abraham, Biju George, Alok Srivastava, Vikram Mathews, Poonkuzhali Balasubramanian

Email ID for Correspondence: rajeshdr.1990@gmail.com Christian Medical College, Vellore

Introduction and Background: Therapeutic drug monitoring and targeted dose adjustment of intravenous busulfan improves the hematopoietic stem cell transplantation outcome. However, several generic versions of busulfan are used by different transplant centres due to availability and cost of original formulation (Busulfex, Otsuka Pharmaceuticals). There are limited reports on the pharmacokinetics (PK) of generic busulfan in comparison with the original formulation. We have previously demonstrated the differential PK behaviour between different generic busulfan, viz. Bucelon (CelonLabs, India) and Buslera (Biem Pharmaceuticals, Turkey). Here we prospectively compared the PK of busulfan between the generic and original formulation, Busilvex. Patient/Material and Methods: Two hundred and three patients receiving i.v Busulfan (Bucelon $n=142$; Buslera $\mathrm{n}=50$; Bufatas $\mathrm{n}=2$; Busilvex $\mathrm{n}=9$ ) prior to HSCT was included in the analysis. Plasma Busulfan levels were measured using established LC-MS/MS protocol after the 1st dose and further doses were adjusted to achieve target levels (cumulative total AUC of 22,000 $\mu$ moles min). Results: We compared the Busulfan day 1 AUC separately in Q24H and Q6H dosing schedule since the subsequent doses were adjusted. The AUC of Bucelon was significantly low when compared to other generic version Buslera or original version Busilvex (Median Q24H day 1 AUC-3932 vs. 5690 vs. 7002 respectively $\mathrm{P}=0.0001$ ). As there were only 2 patients receiving Busilvex in Q6H arm, we compared the AUC between Bucelon and Buslera; the median day 1 AUC of Bucelon was lower compared to patients receiving Buslera (Median Q6H day 1 AUC-635 vs. 853, $\mathrm{P}=0.02$ ). Conclusions: Among the generic versions, the dose adjustment with Buslera was never beyond $30 \%$ while with Bucelon, some patients required upto $66 \%$ dose increase to achieve target levels. This study strongly recommends the need for therapeutic monitoring of iv Busulfan prior to HSCT owing to the degree of variation observed in each formulation in achieving the target AUC.

\section{MHL 59}

c-Kit Mutations in Core Binding Factor Leukemias: A Retrospective Study

K. Vinodhini ${ }^{\#}$, A. Arun Kumar, A. Senthamizhselvi, V. Savitha, Ajay Abraham, Sreeja Karathedath, Vivi M. Srivastava, Anu Korula, P. N. Nisham, Biju George, Alok Srivastava, Vikram Mathews, Poonkuzhali Balasubramanian

Email ID for Correspondence: vinodhinisibi@gmail.com Christian Medical College

Introduction and Background: Chromosomal abnormalities targeting the core binding factors $(\mathrm{CBF})$ namely $\mathrm{t}(8 ; 21)(\mathrm{q} 22 ; \mathrm{q} 22)$ and inv(16)(p13;q22) fall under favourable cytogenetic risk group and are associated with good survival and remission rates. However, mutations in the Tyrosine Kinase Receptor genes including c-Kit, c-FMS or H-RAS have been shown to have an adverse effect on treatment outcome in CBF AML patients. C-kit mutations have been reported in $12 \%-45 \%$ of $\mathrm{CBF}$ AML and occur frequently within exon 17 (encoding the activation loop in kinase domainTKD), and in exon 8 (encoding the extracellular portion of KIT receptor-ITD). In this study, we aimed to evaluate the frequency and distribution of c-KIT mutations in core binding factor AML. Patient/Material and Methods: Among the AML patients evaluated in the department of Haematology, CMC, Vellore between May 2009 and June 2016, patients with $\mathrm{t}(8 ; 21)(\mathrm{q} 22 ; \mathrm{q} 22)$ [AMLETO fusion transcript] or inv(16)(p13;q22) [CBFB-MHY11 fusion transcript] were included in the study. Mutations in exons 8 and 17 of c-KIT gene were assessed and confirmed by direct DNA sequencing. Results: Of the 633 AML patients diagnosed during the study period, 93 had CBF leukemia $(14.7 \%)$. There were 65 males $(70.2 \%)$ and 28 females $(29.8 \%)$. The median age of the patients was 31 years (range 3-76 years). Out of the 94 patients, $66(70.9 \%)$ had $t(8 ; 21)$ and $27(29.1 \%)$ had inv(16) or $t(16 ; 16)$ (one missing). C-KIT mutations were identified in 15 patients (16\%; 11 with AML-ETO transcripts and 4 with CBFB-MHY11 transcripts). The D816V was the most common mutation in this study $(33 \%)$ followed by D816H (20\%). Other mutations identified in the study include D816Y, N822K, D419E, D419del and D419-R420delinsTG. A common polymorphism in exon 17 (rs1008658) was also identified in 17 patients. Unlike previous reports, there was no significant difference in bone marrow blast percentage between c-KIT wild type and mutated patients. Conclusion: Molecular characterization of $\mathrm{t}(8 ; 21)$ and inv (16) patients using c-KIT mutations can help to identify a subset of CBFL patients with higher-risk disease. However, further analysis of other prognostic parameters like relapse following CR and survival rates are required to ascertain its significance.

\section{MHL 60}

Evidence Based Morphological Correlation of Immunophenotypic Aberrancies in Acute Leukemias

\section{Mamta Yargop", Mrunali Thakkar, V. P. Antia \\ Email ID for Correspondence: mvyargop@yahoo.com Jaslok Hospital and Research Centre}

Introduction and Background: Immunophenotypic aberrancies are defined as abnormal, asynchronous or inappropriate patterns of myeloid, T-lymphoid, B-lymphoid or NK cell-antigen expression on neoplastic cells that are different from those seen for that particular type of neoplasm. Expression of myeloid antigens in ALL and lymphoid antigens in AML is common, the expression of which is generally partial or dim and have important implications for the diagnosis and monitoring of acute leukemias. Material and Methods: Immunophenotype of leukemias in 71 unique, untreated patient specimens of variable ages and sex from year 2015 were selected for the retrospective study, comprised of 4 groups: AMLs and ALLs without aberrancies, AMLs with lymphoid and ALLs with myeloid aberrancies. Flow cytometry data was used to identify cases of aberrant phenotypes in ALL and AML. Specimen processing, antibody staining, and data analysis were performed as per the protocol. Briefly, blood or bone marrow samples were subjected to a panel of variable flurochrome tagged antibodies (CD13, CD33, C117, AMPO, CD19, CD10, CD22, CD34, HLADR, CD3, CD4, CD5, CD7, CD8, TDT, CD20, CD45) staining in all specimens followed by RBC lysis in BD FACS LYSE $(1: 10)$ for 10 minutes. The samples were then washed with PBS and resuspended in $0.5 \mathrm{ml}$. PBS. Six-color flow cytometry was performed using BD FACSCanto II flow cytometer, and data was collected using FACS Diva software. Data analysis was performed by using multiparameter cluster analysis. Results: $25 \%$ of both ALL and AML cases examined showed expression of at least 1 myeloid and lymphoid antigen respectively. The most frequently 
expressed antigen was CD13, followed by CD33 in B ALLs and CD19 in AMLs. The ALL cases which expressed 2 myeloid antigens, did not fulfill WHO criteria for biphenotypic leukemia. All cases of ALLs associated with myeloid antigens, microscopically the blasts exhibited the features of myeloblasts and AML blasts associated with lymphoid antigens, exhibited the features of lymphoblasts, whereas the other 2 grps of ALLs and AMLs without aberrancies did not show any morphological changes. Conclusion: Morphologic distinction of leukemic blasts in ALLs and AMLs can be difficult and at times deceptive, as the presence of aberrant antigen may impart the respective morphological features to the blasts. Above study highlights the fact that Myeloid aberrancies on lymphoblasts, morphologically resemble myeloblasts and vice versa. However the lymphoblasts and myeloblasts without aberrancies purely share the morphological criteria of their innate phenotype. We demonstrated significant immunophenotype-morphology correlations. The presence of aberrancies strongly supports the continued use of flow cytometry in the diagnosis and monitoring of acute leukemias.

\section{MHL 61}

Acute Megakaryoblastic Leukemia Versus Transient Abnormal Myelopoeisis: Diagnostic Conundrum and Therapeutic Implication

\section{Prashant Deshpande", Gajanan Padmawar, Alka Ekbote, Venkatesh Ekbote}

Email ID for Correspondence: drpradesh100@gmail.com Marathwada Medical and Research Institute (MMRI), Kamalnayan Bajaj Hospital, Aurangabad

Introduction and Background: Acute megakaryoblastic leukemia (AMKL), a rare infantile acute myeloid leukemia (AML) shares many morphological \& immunophenotypic features with transient abnormal myelopoiesis and myeloid leukemia associated with Down syndrome (TAM/ML-DS), a self-resolving disease seen in infants with Down syndrome \& less frequently in phenotypically normal infants harboring a low level mosaicism for trisomy 21. Differentiating AMKL from TAM in the Down mosaics is essential since it has prognostic \& therapeutic implications. Patient/Material and Methods: A 10 month old male infant presented with fever, easy bruisability \& hepatosplenomegaly. There were no stigmata of Down syndrome. CBC revealed thrombocytopenia \& elevated WBC counts with abnormal cells/blasts. A complete work-up for acute leukemia was carried out. Results: Peripheral blood (PB) and bone marrow (BM) aspirate examination revealed presence of blasts $(>20 \%)$ with characteristic basophilic cytoplasm with blebs. Flow cytometric immunophenotyping of these cells revealed megakaryoblastic differentiation (CD33+/CD36+/CD41+/CD61+/CD34 ${ }^{\mathrm{dim} / \mathrm{CD} 64^{-}}$). BM karyotyping showed high hyperdiploidy, including +21 and del1p32 abnormality. Presence of megakaryoblastic blasts and +21 raised a possibility of TAM. Hence, we analyzed the leukemic BM sample for presence of GATA1 exon 2 mutation and no GATA1 exon 2 mutation was identified. Patient was thus diagnosed with AMKL. Patient was given induction chemotherapy with Cytarabine-Idarubicin-Etoposide doses tailored to account for presence of trisomy 21 , as leukemic clones with additional copies of chromosome 21 are more sensitive to ara-C. He achieved complete morphological \& cytogenetic remission. Post-induction PB and BM cytogenetic studies did not show any chromosomal abnormalities including that of chromosome 21, thus ruling out possibility of TAM/ML-DS. Conclusions: Multimodality approach, including flow cytometry, cytogenetics and molecular diagnostics, differentiated AMKL with +21 from TAM/ML-DS in our patient. This approach also proved useful in guiding therapy and limiting toxicity to the patient.
MHL 62

\section{Dual Pathology—A Rarity}

Dia Mansukhani", Balkrishna Padate, Shweta Gupta, Kainaz Sidhwa, Shanaz Khodaiji

Email ID for Correspondence: preeti.mansukhani@gmail.com P. D. Hinduja National Hospital \& Medical Research Centre, Mumbai

Introduction and Background: Coexistence of Multiple Myeloma (MM) with Acute Myeloid Leukemia (AML) is a rare association with only isolated case reports in literature, more so in chemotherapynaive patients. Given the rarity of the disease, there has been no established treatment and hence, prognosis remains extremely poor. The major challenges faced in diagnosing such cases arises from the possibility of myeloma alone with a plasmablastic morphology that mimics AML or reactive polyclonal plasmacytosis [showing Auer rod like inclusions] occurring in a bonafide case of AML alone. We report a case of true dual pathologies. Patient/Material and Methods: A 36 year old male with no comorbidities presented with pelvic pain in April 2013. Radiological and laboratory work-up including histology confirmed the diagnosis of plasmacytoma. He received 22 fractions of radiotherapy to complete $44 \mathrm{~Gy}$ of radiotherapy. He was asymptomatic for three years. In April 2016 he presented with pain in bilateral shoulders and upper back. Complete blood count showed $\mathrm{Hb}$ : $14 \mathrm{gm} / \mathrm{dL}$, WBC: $3.96 \times 10^{9} / \mathrm{L}$ and platelet: $258 \times 10^{9} / \mathrm{L}$. Peripheral smear showed few blasts with Auer rods. Flow cytometric immunophenotyping of peripheral blood and bone marrow aspirate showed $5 \%$ blasts with myeloid phenotype. Molecular genetics on the bone marrow aspirate sample showed translocation $(8 ; 21)$ in $48 \%$ cells. Serum free light chain assay revealed kappa light chain of $2665 \mathrm{mg} / \mathrm{l}$ and lambda light chain of $24.9 \mathrm{mg} / \mathrm{l}$ and kappa/lambda: 107. Serum protein electrophoresis did not show an M band. There were no predominant light chains on immunofixation electrophoresis. Whole body PET-CT scan was done which showed multiple new metabolically active osteolytic lesions in calvarium, left clavicle, right scapula, sternum, multiple cervico-dorso-lumbar vertebrae, multiple bilateral ribs, right iliac bone left acetabulum and bilateral femur. CT guided biopsy was done from sternum which showed sheets of plasma cells on microscopy. On immunohistochemistry they were positive for CD138 and kappa light chain, however negative for lambda light chain, CD117 and CD34. Results: Hence a diagnosis of acute myeloid leukemia with multiple myeloma was made. Conclusions: Concurrent diagnosis of AML and multiple myeloma is extremely unusual, with less than 20 published case reports in medical literature, and only five published cases since 1991. Simultaneous AML with MM was diagnosed in a 36 years old chemotherapy naïve man. His therapy poses a challenge however chemotherapy and an allogenic marrow stem cell transplant are the proposed course of treatment for this patient.

\section{MHL 63}

Standarization and Optimization of BCR-ABL Quantitative PCR Assay for Clinical Use

Poonam Santra ${ }^{\#}$, Sushant Vinarkar, Neeraj Arora, Biswajoy Pal, Kallol Saha, Sourav Sharma Chowdhury, Mayur Parihar, Deepak Kumar Mishra

Email ID for Correspondence: poonam.santra@tmckolkata.com Tata Medical Center, Kolkata, 156

Introduction and Background: Molecular monitoring of $B C R-A B L 1$ by $R Q-P C R$ is the recommended to assess optimal response to therapy in CML. The standardization of RQ-PCR BCR-ABL1 has evolved 
over time and at present concept of MMR $\left(\mathrm{MR}^{4.0}\right)$ and Deep Molecular Response $\left(\mathrm{MR}^{4.5}, \mathrm{MR}^{5.0}\right)$ are introduced in CML response evaluation. These definitions of Molecular response are based on the number of control gene (ABL1) copies. The multiple platforms, reagents and methodology for RQ-PCR are cause for variation in BCR-ABL1 ratio reported by different labs. International scale (IS) has been established to have inter laboratory agreement for BCRABL1 quantification. Aims and Objectives: Here we highlight the steps involved in the standardization of RQ-PCR BCR-ABL1 assay and reporting by using the International Scale at our tertiary care centre. Material and Methods: Steps involved in RQ PCR BCRABL assay are sample collection (storage and handling), RNA extraction, cDNA synthesis, assay set-up (use of target specific primers/probes, probe master mix, calibration standards/plasmids controls), analysis and reporting in IS scale. The different steps in standardization and optimization will be displayed and discussed in the poster. Results: On RQ PCR assay, our mean ABL1 copy numbers improved from 5000 to 26,000 which is important for reporting in $\mathrm{IS} \%$ ratio $\left(\mathrm{MR}^{4.0}\right)$. Subsequently, we have participated in UKNEQAS BCR-ABL Quantitation scheme and our IS\% ratio is comparable with the different participating labs and is satisfactory. Conclusions: The standardization of RQ-PCR BCR-ABL1 assay is challenging and involves modification in the different steps till the desired results (i.e. ABL1 copy numbers) is achieved as well as the International scale reporting using lab specific conversion factor is obtained. We in our laboratory standardized a protocol.

\section{MHL 64}

\section{MCV as an Early Predictor of Acute Leukemia in Febrile} Children

\section{Garima $^{\#}$, Sheesham Agarwal, Kapil Garg \\ Email ID for Correspondence: garimasultania07@gmail.com SPMCHI, SMS Medical College, Jaipur}

Introduction and Background: Mean corpuscular volume (MCV) is a parameter which is mainly evaluated for the classification of anaemia. Despite wide prevalence of iron deficiency anaemia in children in our country, most children with ALL show macrocytosis. Our aim was to compare the MCV of children with ALL, presenting with fever to that of febrile children due to other etiologies, and determine the usefulness of MCV as a criteria for early prediction of ALL in children. Patient/Material and Methods: In this retrospective case control study, we recorded MCV of 57 patients presenting with fever, which were later diagnosed as ALL, and compared with an age matched control population of children presenting with fever due to other etiologies. Percentage of patients having macrocytosis were compared in both groups and the significance level calculated. Macrocytosis was defined as MCV $>$ (Age in years $\times 0.6+84$ ). Results: Out of 57 children suffering from acute leukaemia presenting with fever, 20 had macrocytosis ( $35 \%$ ), while in control group, out of 57 children, none had macrocytosis. Mean MCV in the ALL group was $84.9 \mathrm{fL}$ (range $62-124 \mathrm{fL}$; $\mathrm{SD} \pm 11.12$ ), while that in control group was $71.9 \mathrm{fL}$ (range 46.1-88.6 fL; $\mathrm{SD} \pm 8.6$ ). The difference was significant $(p<0.05)$. Conclusions: Our study shows that in the presence of macrocytosis in a febrile child, diagnosis of acute leukemia should strongly be considered. This is of further significance in our country where most of the children should rather have microcytosis due to prevalent iron deficiency anaemia.

\section{MHL 65}

Bone Marrow Biopsy in an Adult Male-A Morphological Challenge

Kunal Sehgal, Abhishek Dashora, Sushant Vinarkar, Shanaz Khodaiji ${ }^{\#}$, Shyam Rathi

Email ID for Correspondence: skhodaiji@hotmail.com P. D. Hinduja National Hospital \& Medical Research Centre, Mumbai

Introduction and Background: Even in this era of IHC, role of bone marrow morphology cannot be undermined. A good morphological diagnosis is prerequisite to doing an IHC panel in order to economize and provide accurate and timely diagnosis. Patient/Material and Methods: The bone marrow of a 52 years old male patient was sent to us for examination with history of refractory anemia and hepatosplenomegaly. The CBC picture showed low hemoglobin $(5 \mathrm{~g} / \mathrm{dL})$ with high MCV (139 fL). Bone marrow aspirate examination showed a hypercellular marrow with megaloblastic erythoid hyperplasia. The M:E ratio was 1:3. Bone marrow biopsy showed megaloblastic erythoid hyperplasia along with presence of a large number of spindle-shaped cells in bundles posing a diagnostic dilemma. The initial differential diagnosis considered was fibroblastic/histiocytic tumours, or a rarer possibility of infiltration by spindle cell malignancy. However, reticulin stain did not show increased fibrosis and CD68 did not highlight an increase in histiocytes. At this point a further detailed clinical history was obtained from the clinician which suggested that patient had liver cirrhosis and mild portal hypertension and complaints of abdominal pain, which required hospital admission a couple of times in recent past. GI scopy showed picture of pan-gastritis. Patient was also recently diagnosed as Hepatitis B positive with very low viral DNA load, not sufficient to cause cirrhosis. Results: With this background and after reviewing the morphology, we did a more specific IHC panel, which clinched our final diagnosis of this rare condition. Overall features were consistent with Indolent systemic Mastocytosis. Conclusions: Rare conditions can be easily missed or mis-labelled, hence cognizance of these conditions with the help of good morphology and appropriate IHC is essential to provide an accurate diagnosis for the patient.

\section{MHL 66}

\section{Spectrum of Mutations in Myeloproliferative Neoplasms}

Nikhil Rabade", Rohan Kodgule, Goutham Raval, Shruti Chaudhary, Swapnali Joshi, Kanchan Kulkarni, Pratibha Amare, Prashant Tembhare, Sumeet Gujral, P. G. Subramanian, Nikhil Patkar

Email ID for Correspondence: nikhilrabade@gmail.com; nvpatkar@gmail.com

Tata Memorial Hospital, Mumbai

Introduction and Background: Classical BCR-ABL1 negative myeloproliferative neoplasms (MPN) comprise polycythemia vera (PV), essential thrombocythemia (ET) and primary myelofibrosis (PMF). Upto $95 \%$ PV and 50-60 \% ET and PMF patients harbour the JAK2V617F mutation. Mutations affecting codon W515 within exon 10 of thrombopoietin receptor, $M P L$, are identified in upto $10 \%$ ET and PMF patients. Recently, high frequencies of mutations in exon 9 of calreticulin gene have been described in JAK2/MPL unmutated ET and PMF. Patient/Material and Methods: Ninety 
cases, diagnosed and classified based on WHO 2008 criteria were included in our study. JAK2V617F and MPL mutations were detected by high sensitivity allele specific polymerase chain reaction (PCR) using fluorescent labelled primers followed by capillary electrophoresis on ABI3500 genetic analyser. CALR mutations were assessed using fluorescent labelled primers and the amplicons were subjected to capillary electrophoresis and fragment length analysis. Results: We studied 90 MPN cases with a median age was 49 years (range 17-73 years) including 54 males and 36 females (M:F 1.8:1). There were 7 cases of PV, 25 cases of ET and 45 of PMF. JAK2, MPL and $C A L R$ mutations were mutually exclusive of each other. $J A K 2 V 617 F$ and MPL mutations were present in $53.3 \%$ (48 of 90) and $4.4 \%$ (4 of 90) of all MPNs. All PV cases were $J A K 2 V 617 F$ positive. CALR was mutated in $53.3 \%$ (11 of 21) and $30 \%$ (3 of 19) of $J A K 2 / M P L$ negative PMF and ET cases respectively. Nine types of CALR indels ( 7 deletions and 2 insertions) were detected. Type I and type II CALR mutations were most common, with a frequency of $34.8 \%$ (8 of 23) and $26.1 \%$ (6 of 23) respectively. Conclusions: We report frequencies of JAK2V617F, MPL and CALR mutations at a tertiary cancer centre. The identification of CALR mutations has changed the diagnostic algorithm of MPNs. These mutations carry diagnostic as well as prognostic relevance.

\section{MHL 67}

\section{Acute Leukemias of Mixed Phenotype-An Enigma} for the Pathologist

\section{G. Vijaya Lakshmi ${ }^{\#}$, J. Sree Rekha, Rakhee Kar, Sajini Elizabeth Jacob, Debdatta Basu, Biswajit Dubashi}

Email ID for Correspondence: dr.vijjynaani@gmail.com Departments of Pathology and Medical Oncology, JIPMER, Puducherry

Introduction and Background: Rare cases of acute leukemia show positivity for a variety of myeloid and lymphoid markers. As per WHO 2008, these come under acute leukemias of ambiguous lineage, which are further subtyped into acute undifferentiated leukemia (AUL) and Mixed phenotype acute leukemia (MPAL). It also includes leukemia arising in a background of CML/MDS with a heterogenous immunoprofile. Diagnosis and classification of these leukemias relies on morphology, immunophenotype and their distinction from expression of aberrant markers. It is important to categorize these leukemias as they have worse clinical outcome when compared with AML or ALL alone. Patient/Material and Methods: This study is a record based analysis of acute leukemia with mixed immunophenotype diagnosed on bone marrow aspiration and biopsy with immnohistochemistry (IHC) from January 2013 to August 2016 in our hospital. Results: We have ten cases which fall into this category. The median age of presentation was 23 years. All the patients had generalised lymphadenopathy and hepatosplenomegaly. Nine patients were diagnosed as MPAL-myeloid/B cell type. All these patients were treated with BFM-98 protocol for ALL. Follow up was available for five cases, out of which 1 case showed relapse after bone marrow transplantation, other four cases were in remission. The remaining case was that of a $B C R-A B L$ positive $C M L$ with a mixed phenotypic blast crisis (myeloid/B cell type). Conclusions: Acute leukemia of mixed phenotype are a challenge to the pathologist, as it needs a high index of suspicion and a systematic approach for reaching the diagnosis, and to distinguish these from Acute leukemia with aberrant immunophenotype.
MHL 68

Minimal Residual Disease Monitoring by 8-Color Flow Cytometry in Precursor B-ALL: An Experience from a Tertiary Care Center

Sambhunath Banerjee ${ }^{1 \#}$, Subhajit Brahma ${ }^{1}$, Neeraj Arora ${ }^{1}$, Mayur Parihar ${ }^{1,3}$, Nishit Gupta ${ }^{1}$, Asish Rath ${ }^{1}$, Saurabh Bhave ${ }^{2}$, Arpita Bhattacharya ${ }^{2}$,

Shekhar Krishnan ${ }^{2}$, Reena Nair ${ }^{2}$, Mammen Chandy ${ }^{2}$, Vaskar Saha $^{2}$, Deepak K. Mishra ${ }^{1}$

Email ID for Correspondence: sambhunath.banerjee@tmckolkata.com Department of Laboratory Haematology ${ }^{1}$, Clinical Haematology ${ }^{2}$ and Cytogenetics ${ }^{3}$, Tata Medical Center, Kolkata, India

Introduction and Background: Monitoring of flow cytometricminimal residual disease (FC-MRD) has become routine clinical practice in frontline treatment of virtually all precursor B-Cell Acute lymphoblastic leukaemias (B-ALL). MRD diagnostics has proven to be the strongest prognostic factor, allowing for risk group stratification and treatment modification. Single tube 8 colour MRD analysis is sensitive $\left(\leq 10^{-4}\right)$, broadly applicable, accurate, reliable, fast and affordable technique. Patient/Material and Methods: We evaluated 67 cases which were diagnosed with precursor B-ALL from Jan 2016 to August 2016. Subsequently, the bone marrows performed at different stages of therapy were analysed with single tube 8 colour MRD panel on BD FACS CANTO II (Diva v 6.0) and Kaluza (v 1.3). In every case attempt was made to acquire $1,000,000$ events. Syto 41 dye was used to correct the MRD value. Cytomorphological and cytogenetic (karyotyping and FISH) assessment was also done in parallel. Results: The age range of patients included in the study was 1 year to 68 years. Of the 67 cases analysed, $36(54 \%)$ showed a negative MRD $(<0.01 \%)$. Positive MRD $(>0.01)$ was seen in 31 (46\% cases). Out of these 31 MRD positive cases $15(48.4 \%)$ were children aged $1-10$ years, $8(25.8 \%)$ were adolescents (11-18 years of age), 7 $(22.6 \%)$ were young adults (19-40 years of age) and $1(3.2 \%)$ elderly ( $>40$ years of age). The frequency of CD45 under-expression was significantly higher in adult patients while aberrant CD20 expression was lower when compared to children and adolescents. In $7 / 31$ cases $(22.5 \%)$ had intermediate to high risk cytogenetics, while $24(77.5 \%)$ had good risk cytogenetics. In 22 out of the 31 FC-MRD positive cases $(70.96 \%)$, the cyto-morphology was consistent with morphological complete remission (Marrow blasts $<5 \%$ ). Conclusions: Eight colour single tube FC-MRD is faster and economical in comparison to other available methods for MRD monitoring in precursor B-ALL cases. It offers high specificity and sensitivity even in small samples.

\section{MHL 69}

Ischemic Stroke as the Presenting Manifestation of Polycythemia Vera-A Case Report

\section{Radhika Kapil ${ }^{\#}$, Suvarna Inumella, Rohan Bhise}

Email ID for Correspondence: drradhikapath@gmail.com KLES Dr. Prabhakar Kore Hospital and Medical Research Centre

Introduction and Background: Patients with Polycythemia Vera are at high risk for vaso-occlusive events including cerebral ischemia. Although unusual, acute ischemic stroke may be an initial presentation of Polycythemia Vera. Patient/Material and Methods: We 
present the case of a 70 year old female patient who presented to the emergency department of our centre with acute onset left sided hemiparesis. She denied history of vomiting, blurring of vision, loss of consciousness, seizures. There was no sensory involvement. Her blood pressure was $170 / 80 \mathrm{mmHg}$. No significant past history. Results: Haematological investigations revealed a hyper viscous state with PCV of $56.2 \%$. MRI was consistent with sub-acute infarct in the right parietal region. Colour Doppler showed atheromatous plaque in the right carotid bulb extending into the internal carotid artery. The bone marrow examination revealed hypercellular marrow with increased erythroid and megakaryocytic precursors. A diagnosis of Myeloproliferative neoplasm, most consistent with Polycythemia Vera was made and Bone Marrow sample was analysed for JAK-2 mutation which was detected in the sample, confirming the diagnosis of Polycythemia Vera. BCR-ABL mutation was also tested for and was not detected in the sample. The patient underwent therapeutic phlebotomies with clinical recovery and is being followed up regularly for her haematocrit levels. Conclusions: Although unusual, acute ischemic stroke may be an initial presentation of Polycythemia Vera. All clinicians involved in the care of stroke patients should be aware of the association of Polycythemia Vera and ischemic stroke.

\section{MHL 70}

Myeloid and Lymphoid Neoplasms with Eosinophilia and FGFR1 Abnormality-Report of Two Rare Cases

\section{Sathiya Devi", J. Sree Rekha, Rakhee Kar, Debdatta Basu}

Email ID for Correspondence: sathiya2907@gmail.com JIPMER, Pondicherry

Introduction and Background: Haematological malignancies associated with PDGFR and FGFR1 abnormalities are rare and may manifest in several ways-as acute myeloid leukaemia or myeloproliferative neoplasm (MPN) or even lymphoblastic lymphoma, and usually have eosinophilia as a prominent feature. These malignancies have several chromosomal abnormalities which lead to aberrant tyrosine kinase activity. Currently there is no targeted therapy for FGFR1 abnormality and development of a targeted therapy will improve the overall prognosis of these patients. Here we report two cases with FGFR1 abnormality. Patients/case details: Two adult males aged 23 and 25 years presented with multiple lymph nodes and splenomegaly. Lymph node biopsy showed morphological and immunohistochemical features of precursor $\mathrm{T}$ lymphoblastic lymphoma in both the patients. In addition, the lymph node showed increase in eosinophils in one and few megakaryocytes and myeloid cells in the other. The peripheral smear showed leucocytosis and moderate eosinophilia. Bone marrow done did not show lymphoma infiltration, but was hypercellular with eosinophilia in both, and mast cell hyperplasia in one. A possibility of co-existing MPN was considered. BCR-ABL was done in one case and was negative. In view of these findings on the marrow and lymph node, mutational analysis for PDGFR $\alpha$, PDGFR $\beta$ \& FGFR 1 was advised. PDGFR $\alpha$ was negative in both the cases, however, cytogenetics showed FGFR1 abnormality with $\mathrm{t}(1 ; 8)$ in one and $\mathrm{t}(8 ; 13)$ in the other. Conclusions: Young individuals presenting with generalised lymphadenopathy, showing evidence of lymphoblastic lymphoma, and with marrow eosinophilia or features resembling MPN should raise a suspicion of abnormalities in PDGFR $\alpha$, PDGFR $\beta$ and FGFR1 and these should be evaluated in order to diagnose this rare entity.

Keywords Lymphoblastic lymphoma, PDGFR $\alpha$, PDGFR $\beta$, FGFR1
MHL 71

A Simple Four Colour Antibody Panel to Differentiate the Neoplastic Nature of Plasma Cells by Flow Cytometry

Parshw Singh", Khaliqur Rahman, Ruchi Gupta, Manoj Kumar Sarkar, Navkirti Mittal, Soniya Nityanand

Email ID for Correspondence: parshw.singh@gmail.com Sanjay Gandhi Post Graduate Institute of Medical Sciences, Lucknow 226014, India

Introduction and Background: Flow cytometric evaluation of plasma cells is a challenging issue owing to their variable scatter properties, high auto-fluorescence, cluster-forming tendency, antigenic loss on storage, and their scant number in marrow samples owing to hemodilution and random distribution. Moreover, there is lack of consensus for the informative antibodies to be used. CD56 and CD200 are aberrantly expressed on plasma cells in cases with MM, but are not seen on normal plasma cells. The aim of this study was to evaluate the utility of a simple 4 color single tube combination to define the neoplastic nature of plasma cells by flow cytometry (FCM). Patient/Material and Methods: EDTA anti-coagulated bone marrow samples from 23 newly diagnosed MM patients and 8 reactive plasmacytosis cases were evaluated. The four colour panel included following antibody-fluorochrome conjugate: CD138-FITC, CD200-PE, CD38-PerCP/APC H7 and CD56-APC. Samples were acquired within 12 hrs of receipt of sample. Plasma cells were gated by CD138/CD38 dual positive phenotype. A minimum of 100 plasma cells were acquired. Expression pattern of CD56 and CD200 was then evaluated. Results: Median percentage (range) of plasma cells in MM patients as assessed by FCM was $6.5 \%$ (0.6-34.1\%). This value was significantly lower than that assessed on morphological examination of bone marrow [55 \% (6-90\%)]. CD56 and CD200 positivity was noted in $19 / 23(82.6 \%)$ and 20/23 (86.9 \%) cases respectively. Either of these antigens was positive in $95.6 \%(22 /$ 23) of MM cases. The expression pattern was dim in two cases each. In the remaining cases, the expression was moderate to bright. None of the reactive plasmacytosis cases expressed these antigens. Conclusions: CD56 and CD200 expression differentiates a neoplastic plasma cell proliferation from the reactive plasma cells. A simple 4 colour panel including these two antigens can be helpful in defining the clonal nature of plasma cells through FCM, even if the number is scant.

Keywords Plasma cell Neoplasm, Flow Cytometry, CD56, CD200

\section{MHL 72}

Acute Myeloid Leukemia (AML) with Variant RARA Translocation-Report of Three Cases with PLZF-RARA Fusion

Yogita Rohil ${ }^{\#}$, Nikhil Rabade, Rohan Kodgule, Goutham Raval, Shruti Chaudhary, Swapnali Joshi, Kanchan Kulkarni, Pratibha Amare, Prashant Tembhare, Sumeet Gujral, P. G. Subramanian, Nikhil Patkar

Email ID for Correspondence: nvpatkar@gmail.com; email: yogitarohila@gmail.com

Tata Memorial Centre, Mumbai

Introduction and Background: Acute promyelocytic leukemia (APL) is caused by reciprocal translocation $\mathrm{t}(15 ; 17)(\mathrm{q} 22 ; \mathrm{q} 12)$. Cases lacking PML-RARA fusion are termed AML with variant RARA translocation by the 2016 WHO update on hematolymphoid tumors. RARA can fuse with one of 7 partner genes, including promyelocytic leukemia zinc finger (PLZF) and nucleophosmin (NPM). Patient/ 
Material and Methods: We present 3 cases of AML with variant RARA translocation. Flow cytometric immunophenotyping by lysestain-wash method was analyzed on Navios (Beckman Coulter) flow cytometer using Kaluza analysis software. FISH for PML-RARA fusion and RARA variant detection was done using a dual fusion probe and RARA breakapart probe. Reverse transcriptase PCR was used for RARA fusion transcript identification. Real time quantitative PCR was used for quantifying PLZF-RARA. Results: Case 1: 15 year old male, with intermittent fever, weakness and abdominal pain. Bone marrow examination revealed myeloperoxidase positive abnormal promyelocytes with round to oval, regular nuclei, dense cytoplasmic granularity and absence of Auer rods. Immunophenotyping revealed cells with high side scatter, dim CD45, heterogeneous CD13, homogeneous CD33 and lack of CD34, HLADR, CD56 expression. Case 2: 45 year old male with fever and easy fatigability for 2 months. Morphological features similar to case 1. Case 3: 37 year old male patient with intermittent fever, easy fatigability, dyspnea for 2 weeks. Peripheral blood smears revealed abnormal promyelocytes with regular nuclei, scanty cytoplasmic granularity, Auer rods and pelgeroid neutrophils. FISH detected variant RARA translocation. All three cases lacked PML-RARA but harboured PLZF-RARA fusion. Cases 1 and 3 received arsenic trioxide but remained positive for PLZF-RARA post induction. Case 1 later received idarubicin followed by consolidation with high dose cytosine arabinocide. Conclusions: PLZF-RARA fusion accounts for $\sim 0.8 \%$ cases of APL. Differences in morphological and immunophenotypic features of PLZF-RARA can help distinguish from classical APL. Differentiation from classical APL is essential as patients with PLZF-RARA are resistant to ATRA/Arsenic trioxide.

\section{MHL 73}

\section{Gastrointestinal Involvement in Acute Myeloid Leukemia-A Report of 2 Cases at Autopsy}

\section{Pooja Keshan", Tejaswini Waghmare, Daksha Prabhat \\ Email ID for Correspondence: keshan.pooja@gmail.com Department of Pathology, Seth G.S Medical College and KEM Hospital, Mumbai}

Introduction: Gastrointestinal manifestations of leukemia occur in up to 25 per cent of patients at autopsy. They are more common in acute than chronic leukemia. Its presence varies according to the type of leukemia and has been decreasing over time due to chemotherapy. We present two cases of acute myeloid leukemia (AML) with involvement of the gastrointestinal tract. Case Report: (1) A $30 \mathrm{yr}$ male presented with fever, chills with oral ulceration for 15 days followed by altered sensorium. Peripheral smear examination revealed features of AML-M3. He deteriorated within 5 hours of ward stay. A complete autopsy revealed longitudinal ulcers in the oesophagus and small intestine. Stomach and large intestine showed multiple tiny nodules. Microscopy showed infiltration of atypical haematolymphoid cells in the lamina propria throughout the gastrointestinal tract. Lung, liver, spleen and bone marrow also revealed infiltration by leukemic cells. (2) A $30 \mathrm{yr}$ male presented with grade 4 dyspnoea on exertion and generalised weakness since 7 days, had pallor and hepatosplenomegaly on examination. Diagnosed as AMLM6 on peripheral smear examination. ECG showed evidence of right bundle branch block. A complete autopsy revealed multiple necrotic ulcers only in the mucosal aspect of small intestine. Microscopy revealed infiltration of intestine by leukemic cells. In addition the SA node of heart, liver, spleen and bone marrow also showed infiltration by leukemic cells. Conclusion: Gross leukemic lesions are most common in stomach, ileum and proximal colon and include nodules, plaques, diffuse infiltrates, polyps and a convoluted brain like appearance of mucosal folds. The infiltration of gastrointestinal tract by leukemic cells is usually asymptomatic and is often revealed during autopsy. In such patients, the prognosis is usually grave leading to death within few weeks.

\section{MHL 74}

Leucoerythroblastic Reaction: An Indicator of Bone Marrow Metastasis

\section{Sagar Kulat ${ }^{\#}$, Tejaswini Waghmare, Daksha Prabhat}

Email ID for Correspondence: dr.sagarkulat@gmail.com Department of Pathology, Seth GS Medical College and K.E.M Hospital, Mumbai

Introduction: Leucoerythroblastic reaction (LER) is defined as the presence of immature red cells and immature white cells of the myeloid series in peripheral blood. Literature has revealed various causes for LER, which include haemolytic anaemia, infections, megaloblastic anaemia, liver diseases and others. Here, we present a case of breast carcinoma with bone marrow metastasis revealing LER on peripheral blood smear (PBS). Patient/Material and Methods: A 29 year old female with 30 weeks gestation presented with vomiting \& breathlessness of 4 days duration, transferred from a peripheral hospital in an unconscious state on ventilatory support. On routine antenatal visit, lump in the right breast was detected which on FNAC revealed carcinoma breast. PBS revealed a leucoerythroblastic picture. DLC showed polymorphs $68 \%$, lymphocytes $24 \%$, eosinophils $01 \%$, myelocytes $04 \%$, metamyelocytes $03 \%$ \& nucleated RBC's 20 per 100 WBC's counted. Total WBC count was 16,300 per cumm. CT scan of brain revealed multiple lytic lesions in skull vault. Bone marrow aspiration revealed infiltration of marrow by carcinoma. Patient succumbed on 4th day of admission. At autopsy right breast showed a lump measuring $6 \times 5 \times 3 \mathrm{cms}$, irregular, grey white in colour, firm to hard in consistency with foci of haemorrhage. Pleura, mesentery \& peritoneum revealed whitish plaques. Results: Histology confirmed carcinoma of the breast with metastasis in liver, ovary \& bone marrow. Conclusion: LER on PBS can be a valuable indicator of bone marrow metastasis in cases of malignancy, therefore further workup is required.

\section{MHL 75}

Diagnostic Value of Bone Marrow Aspiration and Biopsy in Routine Hematology Practice

\section{Mangilal Choudhary ${ }^{\#}$, Deepika Mishra, Neetu Agarwal}

Email ID for Correspondence: drmlnadol@gmail.com Department of Pathology, SMS Medical College, Jaipur, Rajasthan

Introduction and Background: Bone marrow examination is an important diagnostic tool to evaluate various disorder including both neoplastic and non-neoplastic hematological diseases. The two most important techniques used for the diagnosis are bone marrow aspiration and bone marrow trephine biopsy which are complementary to each other. Aim and objectives: To compare the diagnostic value of bone marrow aspiration and biopsy. Patient/Material and Methods: A total of 50 cases with both bone marrow aspiration and biopsy were included in the study. All the aspirate smears were routinely stained by Jenner Giemsa while the trephine biopsy sections were stained by routine Hematoxylin and Eosin stain. All the smears and sections were reviewed and the findings on BMA and BMB. Results: In the present study, the main indications for bone marrow examination were categorized. Out of 50 cases studied. A strong positive correlation between BMA and BMB was noted. However, it was found that in the cases of 
aplastic anaemia, different phases of myeloproliferative neoplasm (MPN), multiple myeloma, tubercular granulomas and hemato-lymphoid neoplasm, involvement of the marrow was detected better in bone marrow biopsies. Conclusions: The study concludes that preparations of aspirate and trephine biopsy are easy, rapid and complementary to each other in majority of the lesions. The advantage of both the procedures done together enabled us to study the cytomorphology of the cells along with the pattern of distribution of the cells depending on the cases, hence help in making the diagnosis accurately.

\section{MHL 76}

Leukemic Presentation of Mantle Cell Lymphoma: Report of Six Cases and Their Diagnostic Dilemmas

\section{K. Ganga Bhavani", A. R. Ranjith, J. Sree Rekha, Rakhee Kar,} Debdatta Basu

Email ID for Correspondence: gangakundeti308@gmail.com JIPMER, Pondicherry

Introduction and Background: Mantle cell lymphoma (MCL) is a B cell Non Hodgkin Lymphoma (NHL) with characteristic cytogenetic abnormality $\mathrm{t}(11 ; 14) \mathrm{q}(13 ; 32)$ and overexpression of cyclin D1. These lymphomas are usually widespread at diagnosis with extensive bone marrow involvement. Rarely MCL may present as a leukemia mimicking Chronic Lymphocytic Leukemia, Prolymphocytic leukemia or even acute leukemia (Blastoid variant of MCL). We report six cases of Mantle cell lymphoma with a primary leukemic presentation. Patient/Material and Methods: It is a record-based study of 21 cases of MCL diagnosed over a five year period. Amongst these, six cases showed a leukemic presentation on peripheral smear examination and were later confirmed by bone marrow examination and immunohistochemistry. Three cases had a lymph node biopsy done. Results: The mean age of presentation was 54 years with male preponderance. Except for one case which presented with necrotizing fascitis, all the other cases presented with generalised lymphadenopathy and splenomegaly. Peripheral blood showed leucocytosis (40 to $\left.799 \times 10^{9} / 1\right)$ with circulating abnormal lymphoid cells of heterogenous morphology ranging from medium sized monomorphic lymphoid cells to large cells with prominent nucleoli resembling blasts. The pattern of bone marrow infiltration was predominantly nodular and interstitial. Immunohistochemistry on the marrow showed positivity for CD20, CD5, cyclin D1 and negativity for CD23 and TdT. Lymph node biopsy done in three cases showed classical MCL. Conclusions: Mantle cell leukemia needs to be differentiated from other leukemic states, both chronic and acute. Early diagnosis plays a vital role owing to the aggressive clinical course of this disease.

Keywords Mantle cell lymphoma, Mantle cell leukaemia, Cyclin D1

\section{MHL 77}

\section{A Case of Novel BCR3 Type PML/RARA Fusion Transcript} in Acute Promyelocytic Leukemia

\author{
Anam Fatima Shaikh ${ }^{\#}$, Goutham Raval, Rohan Kodgule, \\ Nikhil Rabade, Shruti Chaudhary, Swapnali Joshi, \\ Kanchan Kulkarni, Russel Mascerhans, Pratibha Amare, \\ Prashant Tembhare, Sumeet Gujral, P. G. Subramanian, \\ Nikhil Patkar
}

Email ID for Correspondence: anamshaikh4@gmail.com Tata Memorial Hospital, Mumbai

Introduction and Background: Acute Promyelocytic Leukemia (APL) is characterized by $P M L / R A R A$ gene fusion arising out of translocation between chromosome 15 and 17. There are three main types of APL depending upon the breakpoint in PML gene namely; long, variant and short type. In addition, atypical breakpoints occur in a small fraction of APL patients and are rare. The current case report is on an APL patient with short fusion type transcript but having a unique breakpoint. Patient/Material and Methods: The peripheral blood smear of a 35 year old male showed $29 \%$ of abnormal promyelocytes with bi-lobed nuclei with maturing myeloid series cells (myelocytes, metamyelocytes and neutrophils) which are not seen in classical APL cases. FISH using PML/RARA dual fusion probe revealed PML/RARA fusion in $93 \%$ of cells. Flow immunophenotypic analysis on abnormal promyelocytes showed high side scatter along with moderate CD45, CD64, heterogeneous CD13, CD15, bright CD33 expression and CD117 in a subset of the cells. CD34 and HLADR expression was classically absent. The patient is currently on ATO induction. Results: PCR reaction for PML/RARA transcript detection with BCR 3 primers revealed an abnormal band size of around $480 \mathrm{KDa}$ on agarose gel. Subsequent Sanger sequencing of patient cDNA showed an extremely rare fusion sequence involving exon 4 of PML and exon 3 of RARA with additional 40 nucleotides (originating from RARA intron 2) in between. Cloning of PCR product into a vector backbone and sequence analysis using BCR3 primers revealed similar results, thereby confirming previous result. The same in house vector was used for preparing standards for real time PCR. The patient was tested positive for PML/RARA transcript on baseline sample by real time PCR. Conclusions: The current report is on a novel APL case showing unusual breakpoints and in our knowledge is never reported before.

\section{MHL 78}

Detection of Additional Mutations in t(8;21) Positive AML Cases

Vaibhavi Vengurlekar ${ }^{\#}$, Rohan Kodgule, Goutham Raval, Nikhil Rabade, Shruti Chaudhary, Swapnali Joshi, Kanchan Kulkarni, Russel Mascerhans, Pratibha Amare, Prashant Tembhare, Sumeet Gujral, P. G. Subramanian, Nikhil Patkar

Email ID for Correspondence: vaibhavivengurlekar@gmail.com Tata Memorial Hospital, Mumbai

Introduction and Background: Acute myeloid leukemia (AML) is an aggressive heterogeneous disease of myeloid lineage. AML $t(8 ; 21)$ is grouped under AML with recurrent genetic abnormalities by the World Health Organization 2008, and is part of core binding factor (CBF) AML. Even though $\mathrm{t}(8 ; 21)$ is considered to be favorable risk, about $30 \%$ of the patients relapse after standard chemotherapy. It has been found that $\mathrm{t}(8 ; 21)$ itself is not sufficient for developing leukemia and additional mutations are required for the same. Hence the frequency of these additional mutations should be analyzed and there correlation with the clinical outcome should be established. Mutations in $C$-kit are known to occur in cases of CBF-AML. Recently recurring ZBTB7A mutations were observed in AML with $\mathrm{t}(8 ; 21)$. Here we analyse frequency of FLT3-ITD, NPM1, CEBPA, ZBTB7A and C-kit mutations in AML with $\mathrm{t}(8 ; 21)$. Patient/Material and Methods: 10 cases of AML with $\mathrm{t}(8 ; 21)$ mutation detected by Fluorescent in situ hybridization (FISH) were selected for this study. DNA was extracted from the suitable blood/bone marrow samples. Mutations in FLT3ITD, NPM1 (exon 12) and CEBPA (ZIP and TADI domain) were detected using fluorescently labelled primer based polymerase chain reaction (PCR) followed by capillary electrophoresis. PCR was carried out for ZBTB7A (exon 2) and C-Kit mutation (Exon 8 and 17). Agarose gel electrophoresis was carried out followed by Sanger sequencing of the PCR products \& results were analysed. Results: 
Mean age among the study group was 20 years. Male to Female ratio was $4: 1$. Only one case was positive for FLT3-ITD. Two cases were found to have ZBTB7a mutation. $C$-Kit mutation was detected in 3 cases. No mutations were detected in NPM1 and CEBPA. Conclusions: $C$-Kit and ZBTB7a seem to be associated with AML with $\mathrm{t}(8 ; 21)$. Clinical correlation of these mutations will help in prognostic stratification of favourable risk group.

\section{MHL 79}

FLT3-ITD Positive Acute Myeloid Leukemia (AML)— Clinicopathological Correlation

\section{Aboli Dilipkumar Saraf ${ }^{\#}$, Nikhil Rabade, Rohan Kodgule, Gautham Raval, Shruti Chaudhary, Swapnali Joshi, Kanchan Kulkarni, Russel Mascerhans, Pratibha Amare, Prashant Tembhare, Sumeet Gujral, P. G. Subramanian, Nikhil Patkar}

Email ID for Correspondence: sarafaboli @yahoo.co.in Tata Memorial Hospital, Mumbai

Introduction and Background: Acute myeloid leukaemia (AML) is clinically and biologically heterogenous disease. Cytogenetically normal AML patients (40-50\% of AML patients) belong to the intermediate prognostic subgroup and yet show heterogeneity in their outcomes. FLT3ITD mutations are known to be associated with poor outcome. A high allelic ratio has worse prognosis even amongst FLT3-ITD positive cases. Patient/ Material and Methods: 98 cases of AML diagnosed based on WHO 2008 criteria. Flow cytometric immunophenotyping performed at baseline and post induction on a Navios (Beckman Coulter) flow cytometer using the Kaluza analysis software. Fluorescence in situ hybridization (FISH) was used to detect presence of RUNX1-RUNX1T1, CBFB-MYH11, MLL translocation, $5 \mathrm{q}$ deletion, $7 \mathrm{q}$ deletion and trisomy 8 . Genomic DNA extracted from samples was amplified by a multiplexed polymerase chain reaction (PCR) using fluorescently labelled primers and the amplicons were subjected to capillary electrophoresis. The results were compared with positive and negative controls. Results: We studied 98 FLT3-ITD positive AML cases with median age 36 (range 3-70 years) including 59 males and 39 females (M:F ratio-1.5). AML-M1 was commonest FAB subtype. Aberrant expression of CD56, CD7 and CD19 was seen in $11.2 \%, 25.5 \%$ and $9.1 \%$ respectively. Majority of our patients $(87 \%)$ showed no cytogenetic abnormalities by FISH. High allelic ratio was seen in $54 \%$ cases. Multiple FLT3 mutations were detected in 20 cases. Only 3 of these 20 cases showed MRD as well as FLT3 positivity post induction. MRD by high sensitivity flow cytometry was positive in 24 patients, amongst these 13 cases had high allelic ratio at baseline and 16 patients showed FLT3-ITD positivity post induction. Conclusions: We found no association between presence of multiple mutations and MRD positivity in our cohort. Persistence of FLT3-ITD mutations in MRD negative samples suggests presence of these mutations in non-leukemic clones.

\section{MHL 80}

Frequency and Pattern of Leukemia Associated Aberrant Immunophenotype (LAIP) in B-Acute Lymphoblastic Leukemia (B-ALL): An Experience from a South East Asian High-End Referral Laboratory

Shivani Sharma ${ }^{1 \#}$, Narendra Kumar ${ }^{1}$, Anil Kumar, Arvind Kumar, Reena Mittal, Aurobinda Samal, Raman Arora, Bhavna Khandpur, Lata Kini, Sambit K. Mohanty

Email ID for Correspondence: shivani.sharma@corediagnostics.in CORE Diagnostics, Gurgaon, Haryana
Introduction and Background: Immunophenotypic aberrancy in antigen expression in B-acute lymphoblastic leukemia (B-ALL) is critical for diagnosis and classification of B-ALL and detection of minimal residual disease (MRD). Here in we sought to determine the frequency and pattern of LAIP in B-lineage leukemic lymphoblasts. Patient/Material and Methods: This is a retrospective study, which included 83 consecutive and pre-treatment B-ALL patients, where flow cytometric immunophenotyping was performed either on peripheral blood or bone marrow samples on BD FACS Canto II instrument and analyzed on DIVA version 8 software. The criteria to determine aberrancy were asynchronous antigen expression, lineage infidelity, and absence of lineage-specific antigen. Results: The male to female ratio was 1.07:1 and the age ranged from 1 year to 60 years. The most frequent aberrancies were either uniform or spectrum of expression of terminal deoxynucleotidyl transferase and CD34 (dual positive $=47.6 \%$; either positive $=20.9 \%$; dual negative $=$ $10.4 \%)$, under expression of CD45 [negative (22.8\%), dim $(69.8 \%)$ and moderate $(2.4 \%)$, overexpression of CD10 [bright $(54.2 \%)$, moderate $(31.3 \%)$, partial $\operatorname{dim}(2.4 \%)$, dim $(0.3 \%)]$, and under expression of CD38 (75.9\%). Asynchronous co-expression of CD34 and CD20 was also frequently observed $(75.9 \%)$. Of the 83 cases, $84.3 \%$ and $36.1 \%$ cases exhibited CD33 and CD13 (myeloid-associated antigens) aberrant expression. Various $\mathrm{T}$ cell-associated antigens, including CD4, CD7, and CD5 were expressed in $1.2 \%$, $13.2 \%$, and $4.8 \%$ of cases. CD117 expression was seen in $3.4 \%$ of cases. Conclusions: Antigenic aberrancies observed in B-ALL helps in diagnosis, appropriate classification, detection of MRD by distinguishing leukemic blasts from hematogones.

\section{MHL 81}

\section{Hypoplastic Acute Myeloid Leukemia}

\section{Rabish Kumar", Babita Khangar, Meeta Singh,} Richa Gupta

Email ID for Correspondence: me9650@gmail.com MAMC

Introduction and Background: Hypocellular variants of acute myeloid leukemia are rare with few cases reported till date. They occur in old patients and often develop secondary to radiation or chemotherapy. Patient/Material and Methods: Case 1: A 65-yearold male presented with fever, generalized weakness, and easy fatigability for the past 3-months. On examination, patient had pallor and hepatomegaly. Routine CBC examination showed pancytopenia with HB of $7.2 \mathrm{gm} / \mathrm{dl}$, TLC of $2240 / \mathrm{mm}^{3}$ and platelet count of $74,000 /$ $\mathrm{mm}^{3}$. The marrow aspirate smears had few hypocellular particles with scattered blasts in the background. These blasts had high $\mathrm{N}: \mathrm{C}$ ratio, scant to moderate amount of cytoplasm, opened-up chromatin and conspicuous nucleoli. Normal hematopoietic elements were diminished. Flow cytometry was also performed on marrow aspirate which revealed $36.3 \%$ identified as distinct cluster with dim CD45 and low side scatter (Blasts). These blasts were positive for myeloperoxidase (MPO) and CD34. Hence a diagnosis of hypoplastic AML was made. Case 2: A 70-year-old female presented with shortness of breath, generalised weakness and weight loss for last 1.5 -month. On examination patient had pallor, hepatomegaly. Routine $\mathrm{CBC}$ examination showed HB of $9.1 \mathrm{gm} / \mathrm{dl}$, TLC of $2520 / \mathrm{mm}^{3}$ and Platelet count of $90,000 / \mathrm{mm}^{3}$. Peripheral smear showed normocytic normochromic Red blood cells and differential showed $79 \%$ blasts, $2 \%$ myelocytes, $3 \%$ metamyeloctes, $10 \%$ polymorphs, $1 \%$ lymphocytes, $4 \%$ monocytes, $1 \%$ eosinophils. Blasts were MPO positive. Bone marrow aspirate smear showed hypocellular particles with scattered atypical cells in the background with high $\mathrm{N}: \mathrm{C}$ ratio, opened up nuclear chromation, inconspicuous to $1-2$ prominent nucleoli. These 
atypical cells comprise $55 \%$ of marrow nucleated cells. Bone marrow biopsy was also hypocellular with infiltration by sheets of atypical cells. Few erythroid, myeloid precurssors and megakaryocytes were identified. Blasts showed focal expression of CD34 and anti MPO. Flow cytometry was also performed on peripheral smear, and blasts showed CD64, CD33, CD13CD34, CD117, HLA-DR POSITIVITY. Hence a diagnosis of Hypoplastic AML was made. Conclusion: Hypoplastic variant of AML is very rare and seen in elderly patients. Hypoplastic AML should be differentiated from Aplastic anemia and Hypoplastic myelodysplastic syndrome. A careful examination of the smear and bone marrow to enumerate the number of blasts is very essential in such cases.

\section{MHL 82}

Non Secretory Plasma Cell Leukaemia with Unusual Morphology and Abberant Expression of CD23

\section{Sneha Goswami", Richa Gupta, Radhika A. Garg}

Email ID for Correspondence: sneha.g01@gmail.com Maulana Azad Medical College and Associated Hospitals, New Delhi

Introduction and Background: Plasma cell leukaemia (PCL) is a rare and aggressive variant of multiple myeloma with poor prognosis. Its incidence is believed to be less than 1 case/million. In rare PCL cases there is absence of detectable secretion of $\mathrm{M}$ component, known as non secretory plasma cell leukaemia. Non secretory PCL accounts for $1 \%$ of PCL with very few cases reported. Nonsecretory plasma cells are difficult to diagnose due to absence of routine serological markers. Immuno-phenotyping plays a role inaccurately identifying the leukemic cells. Patient/Material and Methods: A 56-year-old lady presented with shortness of breath, generalised weakness and facial puffiness since 2 months. Physical examination revealed bony tenderness in the back with elevated blood pressure $(160 / 100 \mathrm{mmHg})$. CBC revealed bicytopenia with leucocytosis ( $\mathrm{Hb}: 7.7 \mathrm{~g} / \mathrm{dl}$, TLC: 53,000/ $\mu \mathrm{l}$ and platelets: $98,000 / \mu \mathrm{l}$ ) RFT were deranged (B. Urea: $77 \mathrm{mg} / \mathrm{dl}$; S. Creatinine: $5.4 \mathrm{mg} / \mathrm{dl}$ ). Radiograph of dorso-lumbar spine revealed compression collapse of D11 vertebral body. Peripheral smear showed $52 \%$ atypical lymphoid cells with high N/C ratio, fine chromatin, inconspicuous nucleoli and scant cytoplasm. Some of the cells showed cytoplasmic projections. Bone marrow aspirate and biopsy showed infiltration by similar cells comprising atypical lymphoid cells. A flow cytometric evaluation of these cells was done on peripheral smear. The cells showed low side scatter and absence of CD45. These atypical cells were also negative for CD19, CD10, CD20, FMC7, CD5, surface kappa and lambda. CD23 was moderately expressed. CD 4, CD8 and CD3 were also negative. All cytoplasmic markers were negative (CD79a, MPO, CD3). A plasma cell tube was added and the cells showed bright expression of CD38 and CD138 along with expression of CD56. Consequently a diagnosis of plasma cell leukemia with CD23 expression was made. A serum protein electrophoresis (SPE), urine protein electrophoresis (UPE) and immunofixation electrophoresis (IFE) was performed but showed no monoclonal proteins. Conclusions: Based on flow cytometry a diagnosis of primary non-secretory plasma cell leukemia with aberrant expression of CD56 and CD23 was made. CD23 expression in these tumors is associated with a $\mathrm{t}(11 ; 14)(\mathrm{q} 13 ; \mathrm{q} 32)$ translocation. The case is presented for its rarity and atypical presentation.

\section{MHL 83}

Acute Promyelocytic Leukaemia in the Setting of Myelofibrosis Secondary to Polycythaemia Vera-A Challenging Case

Pankhi Dutta ${ }^{\#}$, Sameer Tulpule, Jaya Vyas
Email ID for Correspondence: pankhidutta@gmail.com Kokilaben Dhirubhai Ambani Hospital

Introduction and Background: Acute promyelocytic leukaemia (APL) arising in the setting of myelofibrosis (MF) secondary to polycythemia vera (PV) is rare and pose few unique challenges, both diagnostically as well as therapeutically. We present such a case. Patient: A 69 year old male presented with epistaxis to the Emergency department. Complete blood count and peripheral smear revealed-

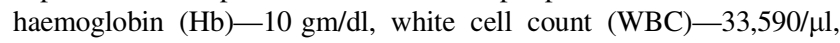
platelets $(\mathrm{Pl})-19,000 / \mu \mathrm{l}$ with $40 \%$ neutrophils $(\mathrm{N}), 10 \%$ metamyelocytes $24 \%$ myelocytes $5 \%$ lymphocytes $9 \%$ basophils (B), $08 \%$ blasts (BL). There were circulating megakaryocyte fragments \& 5 nucleated red cells per 105 nucleated cells. Coagulation profile showed a mildly deranged prothrombin time. Patient history and older reports revealed that the patient was a known case of MF secondary to PV diagnosed 5 years earlier. He was positive for Jak 2 V617F mutation. Earlier reports did not mention circulating blasts. Acceleration/transformation to acute leukaemia was thought of and a bone marrow (BM) examination done. The epistaxis was managed locally and patient transfused platelets. BM aspiration smears were paucicelluar and showed few scattered degenerated and granular cells. The Pl count did not improve in spite of repeated transfusions and epistaxis was relentless. After 4 days of admission, $\mathrm{Hb}$ was $8.8 \mathrm{gm} / \mathrm{dl}$, WBC-17,690/ $\mu 1$ \& $\mathrm{Pl}-15,000 / \mu \mathrm{l}$. The BL increased to $24 \%$ with a $\mathrm{N}$ count of $46 \%$. Few hypergranular cells with cleaved nuclei were seen. On searching actively, a rare Faggot cell was noted. Coagulation profile was deranged and fibrinogen level was $92 \mathrm{mg} / \mathrm{dl}$. FISH for PML; RARa was positive, confirming a diagnosis of APL. The trephine biopsy showed sheets of immature cells which were MPO and CD117 positive but were negative for CD34. Diffuse reticulin fibrosis was present (grade 3). Flowcytometric findings on peripheral blood were consistent with APL. All Trans Retinoic Acid (ATRA) and Arsenic (As) was started as per the UKMRC AML17 Trial protocol. He developed differentiation syndrome with breathlessness, edema and WBC counts of 100,000/ul necessitating addition of Dexamethasone and discontinuation of ATRA and As for a few days. He was also given pulsed mitoxantrone and cytarabine to bring down his counts. The patient improved and became PML; RARa negative (by PCR) at the end of consolidation. However, he continued to have a slightly raised counts with left shift and an 1-2\% blasts in the peripheral smear. The challenging features in this case was rarity of abnormal promyelocytes and presence of a large number of mature $\mathrm{N}$ in the PS alongwith the overwhelming features of a myeloproliferative neoplasm. The bone marrow fibrosis led to a paucicellular BM aspirate. On the management front, the presence of JAK2 mutation might put such a patient at higher risk of differentiation syndrome while the underlying BM fibrosis can make recovery of pancytopenia delayed.

\section{MHL 84 \\ Paediatric Chronic Myelogenous Leukemia with Myeloid Blast Crisis at Presentation: A Rare Case Report}

\section{Kanwaljeet Singh", Sudha Sazawal, Sunita Chikkara, Rekha Chaubey, Pravas Mishra, Tulika Seth, Renu Saxena}

Email ID for Correspondence: kanwaljeet2009@gmail.com Departments of Hematology, AIIMS New Delhi, 110029, India

Introduction and Background: In paediatric age group CML-BC is a rare haematological disorder. Approximately only $5 \%$ of children present in the accelerated phase CML (CML-AP) or in blast crisis (CML-BC). Blast crisis is the final phase in the evolution of CML and behaves like an acute leukemia and has poor prognosis in paediatric cases. We present a case of four year old male child with constitutional symptoms and hepatosplenomegaly who was diagnosed as 
CML in blast crisis with complex karyotype. This is first paediatric CML case from India with blast crisis at presentation and complex karyotype. Patient/Material and Methods: A four year old child who was referred to AIIMS, New Delhi as Acute Leukemia with complaints of pain abdomen, fever and loss of appetite. Workup showed hepatosplenomegaly, $\mathrm{Hb}-8.8 \mathrm{gm} / \mathrm{dl}$, TLC $-18,900 / \mu \mathrm{L}$ and platelet count-142,000/ $\mu \mathrm{L}$ with DLC of $32 \%$ blasts with left shift and basophilia in peripheral blood. Considering the possibilities of acute leukemia \& CML-BC, bone marrow aspirate and biopsy, IPT, cytogenetics, RT-PCR (BCR-ABL) was done. Results: Bone marrow aspirate revealed blast count-30\%, myelocytes \& metamyelocytes-33\%, and basophils-02\%. Bone marrow biopsy revealed Grade 1-2 fibrosis. IPT showed for positivity for CD34, HLA-DR, MPO and negativity for T and B CD markers. Karyotyping showed complex karyotype-trisomy $8, \mathrm{t}(9 ; 22)$ and isochromosome $17 \mathrm{q}$, band $17 \mathrm{q} 10$. RT-PCR for BCR-ABL showed positivity for p210 fusion transcript. Real time PCR (RQ-PCR) revealed BCR/ABL transcript ratio $94.09 \%$. Final diagnosis of CML-BC was made. Conclusions: The case shows the importance of molecular techniques in the diagnosis and risk stratification, which helped in the targeted therapy of child.

\section{MHL 85}

Detection of CALR Mutation by Allele Specific PCR Versus Sanger Sequencing in PMF and ET Patients-AIIMS Experience

\section{Sudha Sazawal ${ }^{\#}$, Rekha Chaubey, Sunita Chikkara, Kanwaljeet Singh, Dinesh Chandra, Pravas Mishra, Manoranjan Mahapatra, Tulika Seth, Renu Saxena}

Email ID for Correspondence: sudha_sazawal@hotmail.com Departments of Hematology, AIIMS New Delhi 110029, India

Introduction and Background: In myeloid proliferative neoplasms mutations in CALR exon 9 have been reported to be the key markers of molecular diagnosis which have widely been analyzed by Sanger sequencing. We report a rapid and convenient method, allele specific PCR for screening CALR mutations in primary myelofibrosis (PMF) and essential thrombocythemia (ET) cases. Patient/Material and Methods: A DNA was extracted from peripheral blood of $150 \mathrm{PMF}$ and 55 ET cases. All the samples were screened for the JAK2 mutation by ARMS-PCR. Patients who were found negative for JAK2 mutation were screened for CALR mutation by Sanger sequencing and confirmed by Allele Specific PCR (ASO-PCR). Results: Out of 150 PMF cases (median age 49.0 years, range 23-83 years, $\mathrm{M}: \mathrm{F}:: 2.6: 1)$, JAK2V617F was negative in 65 cases. Type I CALR mutation was found in 16/65 (24.6\%) PMF cases. Out of 55 ET cases (median age 55 years, range $30-75$ years, M:F::1.6:1) JAK2V617F was found negative in 22 cases. CALR mutation was found positive in $3 / 22(13.6 \%)$ ET patients. None of the patients showed co-existence of JAK2 and CALR mutations. Type II mutation was not seen in any of these patients. In PMF patients CALR mutated status was significantly associated with younger age $(\mathrm{p}=0.005)$ and larger spleen size $(\mathrm{p}=0.003)$. Conclusion: There was $100 \%$ concordance in the results of both techniques. In contrast to Sanger sequencing which is time-consuming and cannot be performed in every laboratory, allele specific PCR is rapid, economical, sensitive and cost-effective method for the detection of CALR mutations. Therefore, this can be used routinely for the detection of CALR mutations especially in Indian laboratories that do not have sequencing facility.

\section{MHL 86}

Light Chain Deposition Disease: A Rare Disease with a Very Rare Presentation

Priyanka Singh ${ }^{\#}$, Shubham Prasad, S. S. Chakrabarty, Prof. I. S. Gambhir

Email ID for Correspondence: pushpal14hy@gmail.com Department of General Medicine, Institute of Medical Sciences, Banaras Hindu University, Varanasi

Introduction: Most common manifestation of light chain deposition disease [LCDD] is renal dysfunction. Here is the case report of a 45 year old male presenting only with episodic moderate headache who was finally diagnosed as full blown LCDD. The most unusual point in this case was symptomatic extra renal LCDD that too of lamda type which is very rare. Case Report: 45 year old, non hypertensive, non diabetic male presented with on and off moderate headache for 1 year. No h/o fever, generalised weakness or body swelling, weight loss, bony pain. Clinical examination including fundoscopy revealed no abnormality with stable vitals. On investigation: Hb-14.7 gm/dl, TLC-10,630 [N63, L24], Plt-2 lac, Tp/Alb7.8/5.2, SGOT/SGPT-40/53, ALP-86, LDH-458, Ca/Po4-10.9/4, Uric acid-8.1, Creatinine/urea-1/30, Urine routine-protein traces with 2-3 pus cells. His routine examination was almost normal. CT brain showed focal punched out lesion on left side of frontal bone s/o metastasis or plasmacytoma (pt. did not give consent for biopsy). Following this serum electrophoresis was done which had no M peak. Urine bence jones protein was positive. On immunofixation serum free lambda chain $-2650 \mathrm{mg} / \mathrm{dl}$, kappa chain $-16.8 \mathrm{mg} / \mathrm{dl}$. Bone marrow examination had $20 \%$ plasma cells. Serum $\beta$-2-microglobulin-2624 ng/ml. Thus ultimately he was diagnosed as multiple myeloma with LCDD. He was treated conservatively with bortezomib, dexamethasone and lenalidomide. He became asymptomatic within 4-6 weeks. Bone marrow examination was repeated after 16 injections of bortezomib which showed $<1 \%$ plasma cells and repeat CT brain showed no lesion. Conclusions: LCDD is a rare condition characterized by the deposition of monoclonal proteins mainly in the kidneys and most commonly presents with renal failure. In this case diagnosis was difficult because patient presented with non specific complain like headache with absolutely normal renal parameters, thus with rare presentation of a rare disease.

\section{MHL 87}

Molecular Response to Imatinib and Its Correlation with mRNA Expression Levels of Imatinib Influx Transporter (OCT1) in Chronic Myeloid Leukaemia Patients: AIIMS Experience

Sunita Chhikara", Sudha Sazawal, Rekha Chaubey, Pravas Mishra, Manoranjan Mahapatra, Renu Saxena

Email ID for Correspondence: sunita1409@gmail.com Department of Hematology, All India Institute of Medical Sciences, New Delhi, India

Introduction and Background: Imatinib mesylate is approved for the treatment of Chronic Myeloid Leukemia (CML). About $20 \%$ of patients with CML do not respond to treatment with Imatinib either initially or because of acquired resistance. In addition to mutated BCR-ABL1 kinase, the organic cation transporter1 (OCT1, encoded 
by SLC22A1) has been considered to contribute to Imatinib resistance in patients with chronic myeloid leukemia (CML). To date, only a few studies have been reported on involvement of influx transporters in development of Imatinib resistance. Therefore this study was aimed to determine the expression level of Imatinib uptake transporter (OCT1) in CML patients and to correlate this level with molecular response. Patient/Material and Methods: One hundred fifty eight patients on Imatinib were considered for gene expression analysis study for OCT1 gene. Patients were classified into two groups; responders and non-responders. Total RNA was extracted from peripheral blood, cDNAs was synthesized and Real Time Polymerase Chain Reaction (RQ-PCR) was performed. Results: A total of $158 \mathrm{CML}$ patients were analyzed. There were $98(62 \%)$ male and $60(37 \%)$ female patients (M:F::1.6:1). Median age at the time of presentation was 35 years with age ranging from 18-70 years. High OCT1 expression was present in $81(51.8 \%)$ patients and low OCT1 expression was in 77 $(48.7 \%)$ patients. Low Sokal risk score group have a significantly high OCT1 expression $(\mathrm{P}<0.048)$. The rate of molecular response was higher in those with high OCT1 expression than in those with low OCT1 expression $(\mathrm{P}<0.05)$. Moreover, responders group had significantly higher OCT1 gene expression as compared to non responders $(\mathrm{P}<0.018)$. Both event-free survival and median overall survival were significantly shorter in patients with low OCT1 expressions when compared to the patients with high OCT1 expression $(\mathrm{P}=0.03$ and $\mathrm{P}=0.05)$. Conclusions: Our findings demonstrated that the mRNA expression level of OCT1 was significantly correlated with molecular response in CML patients. Based on these findings, present study believes that the pre-therapeutic higher expression of OCT1 may help to predict response to imatinib therapy in CML patients.

\section{MHL 88}

Clinical and Molecular Profile of Low, Intermediate and High Risk MDS Patients: AIIMS Experience

Rekha Chaubey ${ }^{\#}$, Sudha Sazawal, Sunita Chhikara, Kanwaljeet Singh, Manoranjan Mahapatra, Renu Saxena

Email ID for Correspondence: drrekhacgupta@gmail.com Departments of Hematology, AIIMS New Delhi 110029, India

Introduction: Accurate diagnosis of MDS is difficult, and its classification requires evaluation of cytopenias, bone marrow morphology, blast percentage, and cytogenetics. These factors, as well as patient performance status and red blood cell transfusion dependence, can be used to predict prognosis in MDS. IPSS scoring system has been widely used for the risk stratification of MDS patients. We have compared the molecular and clinical parameters of patients within low, intermediate and high risk groups and analysed the association in these groups. Patient/Material Method: Cytogenetics was done, PCR and RT-PCR was used for the mutation screening of JAK2, IDH2, hTERT, RAS and FLT3 genes. MSP-PCR was used to study the methylation status of p15INK4b, SOCS-1, calcitonin and FHIT genes in a series of 51 MDS patients. Results: Of the 51 patients (median age 49.0 years range: 9-84 years, M:F::1:1.5), 7 (14\%) were high risk of which 4 transformed to leukemia. Out of 7 cases, six patients were transfusion dependent. Two patients were positive for RAS mutations and 3 were positive for JAK2 mutation. None of the patient was positive for IDH2, hTERT and FLT3 mutations. Gene hypermethylation for SOCS-1 was present in all the 7 cases, for FHIT in 2 cases, for CALCA in 7 and for P15 in 3 cases. Out of 19 (37\%) intermediate risk patients 4 transformed to acute leukemia. hTERT mutation was present in 3 cases and RAS in 2 patients. 16 patients were transfusion dependent. Gene hypermethylation for SOCS-1 was present in 14, for FHIT in 11, for CALCA in 16 and for P15 in 10. Of
$25(49 \%)$ low risk cases one transformed to leukemia and 21 patients were transfusion dependent. hTERT mutation was present in 3 patients. Gene hypermethylation for SOCS-1 was present in 7, for FHIT in 15, for CALCA in 8 and for P15 in 8 . Conclusion: These results suggest that the molecular mutations, especially gene methylation differentially present in different IPSS risk groups. Molecular factors along with other risk factors might enhance the power of risk assessment of the MDS patients but independent validation of several other, larger data sets should be necessary.

\section{MHL 89}

Incidence of Leukemia in Ajmer Region (Rajasthan)

Geeta Pachori, Priya Jangid ${ }^{\#}$, Rashmi Sharma, Soniya Sanaadhya

Email ID for Correspondence: priyaparijangid@gmail.com J.L.N. Medical College, Ajmer

Introduction and Background: The prevalence of leukemia is known to vary through out the India. An attempt was made to find out pattern of leukemia and its distribution through out the Ajmer region, That can play a vital role in understanding the occurrence and outcome of the disease. Patient/Material and Methods: The samples from 45,391 patients were collected in haematology department of, J.L.N. Medical College Ajmer during last 2 year \& 7 months from Jan. 2014 to July 2016. Patients were from wards \& OPD of medical college hospital \& other hospitals of Ajmer region. Clinically suspected patient for anaemia, hepatomegaly, lymphadenopathy \& weakness were sent for CBC, P.B.F. \& bone marrow examination \& other routine investigation. Special stain like PAS, peroxidase, sudan black, alkaline phosphatise were also done. Results: The most common leukemia was C.M.L. 47 (35\%), followed by A.L.L. 43 (32\%), A.M.L. $31(23 \%)$ \& least number of cases were C.L.L. $13(9.7 \%)$ out of 134 leukemia cases. Acute leukemia were more diagnosed in young adult, while chronic leukemia were more in middle $\&$ old age group. Leukemia was found more in male patient (male:female ratio is 73:61). Conclusions: Even leukemia can be inheritated or sporadic. Specific cases need to be diagnosed by genetic \& molecular study. This will assist in specific treatment $\&$ timely management of disease \& complication.

\section{MHL 90}

Clinicohematological Presentation of Myelodysplatic Syndrome (MDS) in Last 3 Years

\section{Usha Rani", Richa Gupta}

Email ID for Correspondence: u.narwal@gmail.com Maulana Azad Medical College and Associated Hospitals

Introduction and Background: Myelodysplastic syndromes (MDS) are clonal haematopoietic stem cell disorders characterised by ineffective and dyspoetic haematopoiesis. Few studies have been done in context to clinicohematological presentation in MDS. Few data of prevalence and incidence in India is present however in US population 14,000 people per year diagnosed with MDS. Materials and Method: A Retrospective study of 19 patients with myelodysplastic syndrome in past 3 yrs were retrieved and correlated with clinicohematological presentation. Results: out of 19 cases $10(52 \%)$ belonged to $>40$ yrs age group, 5 patient $(26 \%)-<18$ to $40 \mathrm{yrs}$ and 4 to the pediatric group ( $21 \%$ ). MDS most commonly presented with pancytopenia (54\%), other presenting with (29\%) bicytopenia, $(27 \%)$ refractory anaemia and $(20 \%)$ fever. PRIMARY MDS$75 \%$ case reported primary MDS after ruling out any secondary 
cause like infectious disease like HIV leishmaniasis, history of chemotherapy or radiation. $14 \%$ attributed to high grade MDS and $86 \%$ attributed to low grade MDS. On peripheral smear majority of cases show NCNC to macrocytic RBC picture. On bone marrow aspiration most commonly affected was myeloid lineage (71\%), then erythroid (57\%) and $14 \%$ show dysmegakaryopoesis. Majority cases show two lineage dysplasia. Secondary MDS-5 cases with secondary causes were reported, HIV (2), alcoholic liver disease (2) and leishmaniasis. Alcoholic cases show pancytopenia and on bone marrow aspiration show erythroid hyperplasia with features of dyserythropoiesis. HIV cases show pancytopenia with dysplastic changes in all the lineage in BM aspiration. Conclusion: MDS can present with wide range of clinical and haematological features so MDS should be kept in mind while dealing with refractory anaemia, bicytopia and pancytopenia.

\section{MHL 91}

Non-haematopoietic Malignancies Metastasing to the Bone Marrow: A 6-Year Study

\section{G. Smeeta ${ }^{\#}$, Ritesh Sachdev, Rashi Sharma, Shalini Goel, Bhawna Jha, Tushar Sahni}

Email ID for Correspondence: drsmeeta@gmail.com Department of Pathology \& Laboratory Medicine, Medanta-The Medicity

Introduction: Bone marrow is one of the important sites of metastasis of solid tumors. Bone marrow metastasis can be presented as cytopenias and may be misdiagnosed as a primary haematopoietic disorder. Its detection has both therapeutic and prognostic significance. Immunohistochemistry is a useful adjunct to morphology in reaching a definitive diagnosis even in case with unknown primary and cases without any suspicion of solid tumor, presented as hematological problems or any non-specific symptoms. The study was undertaken to assess the involvement of bone marrow with non-haematopoietic malignancies and its significance in establishing primary diagnosis in clinically unsuspected cases. Materials and Methods: This is a retrospective study which included record review of the departmental archives for the last six years (January 2010 to December 2015) in Medanta, the Medicity. Sixty cases were studied; which included clinically diagnosed nonhaematopoietic malignancy for staging $(n=45)$ and clinically unsuspected malignancy with incidentally detected bone marrow metastasis $(n=15)$. Results: Bone marrow metastases of solid tumors were identified in 60 cases (Male: 40 and female: 20) which included $75 \%$ of clinically diagnosed non-haematopoietic malignancy for staging $(\mathrm{n}=45)$ and $25 \%$ of clinically unsuspected malignancy with incidentally detected bone marrow metastasis $(\mathrm{n}=15)$. Out of 15 cases, in 12 cases a definitive diagnosis could be made by correlating clinicoradiological findings with morphology and immunohistochemistry. In 3 cases the diagnosis of primary site was not possible. Out of 10 paediatric cases, the most common tumor was neuroblastoma and Ewing's sarcoma (90\%). In 50 adults, the most common solid tumor metastasizing to bone marrow in male was prostate $(20 \%)$ and in female was breast $(20 \%)$. Conclusion: Bone marrow aspiration and biopsy with immunohistochemistry is an easy, rapid, cost effective and gold standard method of detection of bone marrow metastasis for staging and monitoring of prognosis in solid non hematopoietic tumors. Moreover it is also helpful in detecting unknown primary and also in diagnosing case of unsuspected malignancies.
MHL 92

Relative Importance of CD Markers in the Differential Diagnosis of T-ALL with Myeloid Antigen Expression

\section{Amar Das Gupta\#, Kainaz Sidhwa, Manisha Ramani, Vidya Powar and Santosh Parab}

Email ID for Correspondence: adasgupta@srl.in Sections of Hematology \& Flow Cytometry, SRL Limited, Mumbai

Introduction and Background: Expression of myeloid antigens by blast cells is seen in 20-30\% cases of T-ALL. Early T Precursor (ETP) ALL which is a subset of these cases shares a well defined immunophenotypic profile with the common myeloid and T lymphoid progenitor cell (CD5-ve/dim+ve; myeloid and/or stem cell marker+ve, CD1a-ve and CD8-ve) and has a poor clinical outcome. We present here the immunophenotypic profile of T-ALL cases and highlight the relative importance of $\mathrm{CD}$ markers that allow us to distinguish among the subtypes of T-ALL with myeloid antigens expression, namely ETP ALL, non-ETP T-ALL and Mixed Phenotype AL (T\&MY). Patient/Material and Methods: Blood and/or bone marrow samples from 82 cases of T-ALL seen during an 8 month period (January to August, 2016) were examined morphologically, cytochemically and immunophenotypically using a multicolour reagent panel consisting of antibodies against CD1a, CD2, CD3 (surface and cytoplasmic), CD4, CD5, CD7, CD8, CD10, CD11b, CD13, CD14, CD16, CD19, CD20, CD22, CD33, CD34, CD45, CD56, CD64, CD79a (cytoplasmic), CD117, HLA-DR and MPO (cytochemical and cytoplasmic). Results: The mean age of ETP patients (14 cases) was higher 35.7 years (12-95 years) than that of non-ETP group (68 cases) 16.5 (3-67 years). Males dominated in both the groups although a stronger male bias was seen in non-ETP group (5:1 in non-ETP vs. 2:1 in ETP). As required for diagnosis, one or more myeloid markers were present in all cases of ETP while myeloid markers were seen only in 7/68 non-ETP cases. Distinction between ETP cases and the non-ETP group with myeloid markers was largely based on the expression of CD34 and HLA-DR and absence of CD8 in the former, and expression of CD5 and CD1a in the latter. Blast cells in the single case of MPAL (T\&My) had both cMPO and cCD3. Conclusions: Unlike the earlier reports of ETP seen predominantly in the pediatric age, our cases are mostly adults. Male sex bias is more marked in non-ETP ALL. CD1a, CD5, CD8, CD34 and HLA-DR are important for distinguishing between ETP and non-ETP ALL with myeloid antigen expression.

\section{MHL 93}

Winds of Change: Diagnostic Challenges in Non-CML Myeloproliferative Neoplasms in Conforming to Who (2008) Criteria

Krishti Chakraborty ${ }^{1 \#}$, Parul Jain ${ }^{2}$, Senjuti Dasgupta ${ }^{3}$, Debdas Bose ${ }^{3}$, Abantika Konar ${ }^{4}$, Nirmal Kumar Bhattacharya ${ }^{4}$, Pranab Kumar Biswas ${ }^{5}$, Uma Banerjee ${ }^{6}$

Email ID for Correspondence: ckrishti9@gmail.com

${ }^{1}$ Post Graduate Trainee, ${ }^{2}$ Demonstrator, ${ }^{3}$ Assistant Professor,

${ }^{4}$ Associate Professor, ${ }^{5}$ Professor, ${ }^{6}$ Professor and Head, Department of Pathology, Medical College and Hospital, Kolkata

Introduction and Background: In myeloproliferative neoplasms (MPN), controversy remains in usefulness and reproducibility of bone marrow (BM) features mainly in discriminating Essential 
thrombocythaemia and pre-fibrotic/early Progressive myelofibrosis (PMF) and prodromal Polycythaemia vera (PV) due to lack of standardization of distinctive bone marrow features precluding correct morphological pattern recognition. Distinction between WHO (2008) - defined ET and pre-PMF is important due to poor prognosis of pre-PMF. Patient/Material and Methods: Over a period of one year, 42 non-CML cases were evaluated using bone marrow aspirate, imprint, biopsy samples alongwith clinical profiles of patients. Correlation was drawn with diagnostic criteria as laid down by the WHO (2008). Molecular analysis of selected cases was done. Results were compiled. Results: out of 42 non-CML MPNs, 11 cases were polycythaemia vera, 08 cases were essential thrombocythaemia, 23 cases were diagnosed as progressive myelofibrosis out of which 10 were pre-fibrotic PMF. In $26(68 \%)$ cases, accurate diagnosis of bone marrow morphology matched with the WHO (2008) criteria. In the remaining $32 \%$ of cases molecular testing was warranted as morphology did not match with the diagnostic criteria. Conclusions: Clinical evidence supports the need for changing the diagnostic criteria of 2008 updated classification of non-CML MPN particularly Polycythaemia vera. Hence bone marrow morphology was upgraded to a major diagnostic criteria. Regarding ET, there is a need to differentiate true ET and early PMF due to different epidemiology and clinical outcomes. Thus molecular diagnostic criteria gains utmost importance in cases of inconclusive marrow picture. However molecular negative cases require proper defining criteria as they are the major pitfalls and challenges to the present guidelines.

\section{MHL 94}

\section{Multiple Myeloma-A Case Report}

\section{Roshan Choudhary ${ }^{\#}$, A. R. Kalla, Kishore Khatri}

Email ID for Correspondence: roshan_choudhary1111@yahoo.com Dr S.N Medical Collage, Jodhpur

Multiple myeloma is the neoplastic plasma cell dyscrasia that is characterised by clonal proliferation of malignant plasma cells in the bone marrow microenvironment, monoclonal protein in the blood or urine, lytic bone lesions, M-proteins in serum and renal insufficiency. The hallmark features of multiple myeloma are highlighted by the acronym CRAB-calcium elevation, renal insufficiency, anemia and bone diseases. Myeloma accounts for $1 \%$ of all malignancies and $10-20 \%$ of all hematologic malignancies. The median age of myeloma is 65 years. Here is a case of a 35 year old female patient who presented with history of bone pains and weakness. Clinically, the patient was anaemic. Plain radiography revealed multiple punched out lytic lesions in skull and vertebral column. Biochemical investigations showed high serum calcium levels. M protein band was detected in serum protein electrophoresis. Peripheral smear examination showed pancytopenia and rouleaux formation. Bone marrow aspiration smears were hypocellular with hypercellular imprints. Erythropoiesis was normoblastic, myelopoiesis was suppressed and well represented. Among the abnormal cells, $65 \%$ of the nucleated cells appeared to be plasma cells, binucleate, multinucleate forms and some intermediate forms were seen. Iron stain-reduced.

\section{MHL 95}

BRAFV600E Negative Classical Hairy Cell Leukemia: A Rare Case Report

Asish Rath ${ }^{1 \#}$, Poonam Santra ${ }^{2}$, Sourav Sarma ${ }^{2}$, Sushant Vinarkar ${ }^{1}$, Nishit Gupta ${ }^{1}$, Reena Nair ${ }^{3}$, Mammen Chandy ${ }^{3}$, Mayur Parihar ${ }^{1}$, Neeraj Arora ${ }^{1}$, D. K. Mishra ${ }^{1}$

Email ID for Correspondence: asishdoct@gmail.com Department of Laboratory Hematology ${ }^{1}$, Molecular Genetics ${ }^{2}$ and Clinical Hematology ${ }^{3}$, Tata Medical Center, Kolkata, India

Introduction and Background: Hairy Cell Leukemia (HCL) is a rare B-cell neoplasm with classical morphology and immunophenotype of B lymphocytes with fine hair-like microvilli involving peripheral blood (PB) and bone marrow (BM). In recent years, a significant progress has been made in understanding molecular biology of HCL. The discovery of the disease defining, acquired mutation $B R A F \mathrm{~V} 600 \mathrm{E}$ in exon 15 has been a breakthrough in diagnosis and therapy of HCL seen almost in $100 \%$ of these lymphomas. We describe a unique case with classical morphological and immunophenotypic features but negative for this disease defining BRAFV600E mutation. Case History: A 61 year male was referred to our centre with fever and right inguinal lymphadenopathy since 6 weeks. On routine $\mathrm{CBC}$, there was anemia and leucopenia with predominantly neutrophils $(71 \%)$ and lymphocytes $(21 \%)$ in PB. The patient was diagnosed with CD20 positive, CD5 negative Lymphoproliferative disorder elsewhere. A repeat Bone marrow aspirate showed-39 \% abnormal lymphoid cells with hairy cytoplasmic projections and biopsy showed-dense interstitial lymphoid cell infiltrate with fried egg appearance and increased reticulin. The FCM of aspirate revealed classical immunophenotype of HCL (bright expression of CD20, CD22, CD11c, CD103, CD25, CD123 and negative for CD5, CD23, CD10, CD38 with no light chain restriction). IHC for Annexin A1 was negative in the lymphoid infiltrate in biopsy. In view of these morphological and immunophenotypic profile $B R A F \mathrm{~V} 600 \mathrm{E}$ mutation Allele specific PCR was done on bone marrow sample and was found to be negative. BRAFV600E negativity was also confirmed by Sanger Sequencing assay. Conclusions: $B R A F \mathrm{~V} 600 \mathrm{E}$ acquired mutation in B cell lymphoproliferative disorder is shown by numerous studies in literature as defining feature of Classical HCL. We report here that HCL can be BRAFV600E negative.

\section{MHL 96}

Clinicopathological Correlations of the $\operatorname{dic}(9 ; 12)(p 11 \sim 12 ;$ p11 13)

V. T. Manoj Moni", A. Palle, N. A. Fouzia, A. J. Devasia, P. N. Nisham, A. Korula, A. Abraham, B. George, V. Mathews, B. Poonkuzhali, A. Srivastava, V. M. Srivastava

Email ID for Correspondence: mnjmoni@yahoo.co.in Christian Medical College, Vellore

Introduction and Background: The dicentric $(9 ; 12)$ is associated with acute lymphoblastic leukemia (ALL) and a favourable prognosis. This unbalanced rearrangement results in the formation of a chimeric 
fusion gene involving the PAX5 gene, a regulator of B cell development, on chromosome 9p13 and the ETV6 gene on 12p13; the resulting PAX5/ETV6 fusion gene encodes a protein which is a repressor of transcription. The $\operatorname{dic}(9 ; 12)$ is reported to be associated with ETV6-RUNX1 fusion which results from the cryptic t(12;21). Patient/Material and Methods: G-banded karyotypes of ALL with the $\operatorname{dic}(9 ; 12)$ seen in the Department of Haematology, CMC, Vellore from January 2003-December 2015 were correlated with blood and bone marrow findings. Results: There were 16 patients with the $\operatorname{dic}(9 ; 12)$ who accounted for $1.3 \%$ of the 1199 ALL seen during the period of study. Twelve were children (up to 18 years), with a median age of 16.5 years (range 2-49 years). Fourteen were males (88\%). The median haemoglobin was $7 \mathrm{~g} / \mathrm{dl}$ (range 4-14.4 g/dl), median WBC count, $2.9 \times 10^{9} / \mathrm{L}$ (range $8-47 \times 10^{9} / \mathrm{L}$ ) and median platelet count, $73 \times 10^{9} / \mathrm{L}$ (range $7-21 \times 10^{9} / \mathrm{L}$ ). Bone marrow blast percentage varied from $69-100 \%$; there was no morphological evidence of disease in one patient who had received treatment. Immunophenotype was available in 14 patients all of whom had pre B ALL. RTPCR was negative for the ETV6/RUNX1 fusion gene in all 10 patients studied. One or more additional abnormalities were seen in 11 patients $(69 \%)$. These included trisomy $8(25 \%)$ and abnormalities of chromosomes $7 \mathrm{p}$ and 17 (three each). Of the eight with complex karyotypes, one was an adult with the $t(9 ; 22)$ and with gain of the derivative chromosome 22 ( $\mathrm{Ph}$ chromosome) and trisomy 21. Conclusions: The frequency $(1.3 \%)$ of the $\operatorname{dic}(9 ; 12)$ in our ALL is similar to what has been reported in the literature. We did not find the associated ETV6/RUNX1 fusion. We had four adults with this abnormality. The association with the $t(9 ; 22)$ is also an unusual finding, with only one other patient being reported till date.

\section{MHL 97}

\section{Trisomy 8 in Acute Myeloid Leukemia}

S. Yuvarani", A. Palle, N. A. Fouzia, A. J. Devasia, P. N. Nisham, A. Korula, A. Abraham, B. George, V. Mathews, B. Poonkuzhali, A. Srivastava, V. M. Srivastava

Email ID for Correspondence: cytogen@cmcvellore.ac.in Christian Medical College, Vellore

Introduction and Background: Trisomy 8 is the most frequent cytogenetic aberration in acute myeloid leukemia (AML), occurring in $10 \%$ to $15 \%$ of all AML. When trisomy 8 occurs as the sole abnormality it is associated with an intermediate to poor prognosis. Trisomy 8 may also be associated with additional abnormalities. In the recurrent genetic abnormalities (RGA) associated with AML, trisomy 8 is a frequent secondary change. Patient/Material and Methods: All patients with AML and trisomy 8 seen in the Department of Haematology, Christian Medical College, Vellore from January 2003 to December 2015 were included in the study. G-banded karyotypes were reported according to the International System for Human Cytogenetics Nomenclature (ISCN). Cytogenetic, blood and bone marrow findings were correlated. Results: Trisomy 8 was seen in 235/2487 AML (9.4\%). The majority $(61 \%, 144 / 235)$ were males. There were 206 adults $(88 \%$ ) and 29 children (median age 39, range $1-76$ years). The median haemoglobin was $8.1 \mathrm{~g} / \mathrm{dl}$ (range $2.3-15.1 \mathrm{~g} / \mathrm{dl}$ ), median WBC $4 \times 10^{9} / \mathrm{L}$ (range $0.3-537 \times 10^{9} / \mathrm{L}$ ), and median platelet count, $33 \times 10^{9} / \mathrm{L}$ (range $2-400 \times 10^{9} / \mathrm{L}$ ). The morphological subtypes of AML with trisomy 8 were: AML M1-21; AML M2-41; AML M3-39; AML M4-13; AML M4E0-2; AML M5-
15; AML M6-12; AML M7-3; MDS-AML-4; AML with myelodysplasia-related changes-22; AML not further classified-62. The trisomy 8 was solitary in $29 \%$ of patients $(68 / 235)$. Trisomy 8 was seen in association with the RGA $\mathrm{t}(15 ; 17), \mathrm{t}(8 ; 21)$ and inversion 16 in 39 $(23 \%), 7(4 \%)$, and $9(5 \%)$ of patients respectively. The other RGA seen were the inversion $3, \mathrm{t}(9 ; 11)$ and $\mathrm{t}(6 ; 11)$ in $0.6 \%-3 \%$ of patients. Other commonly seen abnormalities were trisomies 21 (23\%), 4, 19, and $22(11-12 \%)$ and monosomies 5, 7 and 17 and the deletion 5q (5-10\%). Complex karyotypes with three or more abnormalities were seen in $31 \%$ of patients, most of whom had AML not further classified (42\%), AML-M2 (16\%) and AML with myelodysplasia-related changes $(15 \%)$. Conclusions: The frequency $(9.4 \%)$ of trisomy 8 in AML is similar to the literature. In the majority $(71 \%)$ of our patients, trisomy 8 was seen in association with other abnormalities, notably the $\mathrm{t}(15 ; 17)$ and trisomy $21(23 \%$ each). The median age of our patients is lower than what has been reported ( 39 vs. 47-64 years) reflecting the overall lower median age of AML in our country.

\section{MHL 98}

Cytogenetic Study of the $t(6 ; 11)(q 27 ; q 23)$ in AML

J. Esther Banu", A. Palle, N. A. Fouzia, A. J. Devasia, P. N. Nisham, A. Korula, A. Abraham, B. George, V. Mathews, B. Poonkuzhali, A. Srivastava, V. M. Srivastava

Email ID for Correspondence: esther6114@yahoo.com Christian Medical College, Vellore

Introduction and Background: The MLL (Mixed-Lineage Leukemia or Myeloid-Lymphoid Leukemia) or KMT2A (lysine(K)specific methyltransferase $2 \mathrm{~A}$ ) gene is involved in translocations with several partner chromosomes in acute myeloid leukemia (AML). The $\mathrm{t}(6 ; 11)(\mathrm{q} 27 ; \mathrm{q} 23)$ is one such translocation that is typically associated with AML with monocytic differentiation. This translocation results in the formation of the KMT2A/MLL T4 (MLL/AF6) fusion gene and is associated with a poor prognosis. Patient/Material and Methods: G-banded karyotypes of the patients with the $\mathrm{t}(6 ; 11)$ seen in the Department of Haematology, CMC, Vellore, were reported according to the International System for Human Cytogenetics. The period of study was from January 2003 to December 2015. Cytogenetic findings were correlated with blood and bone marrow findings. Results: The $\mathrm{t}(6 ; 11)$ accounted for 11/2487 (0.4\%) patients with AML. There were 10 adults and 1 child ranging from 6-65 years of age (median age 32); six were males $(55 \%)$. The median hemoglobin was $9.4 \mathrm{~g} / \mathrm{dl}$ (range 6.5-14.2 $\mathrm{g} / \mathrm{dl}$ ), median WBC count, $67.5 \times 10^{9} / \mathrm{L}$ (range $3.4-168 \times 10^{9} / \mathrm{L}$ ) and median platelet count, $16 \times 10^{9} / \mathrm{L}$ (range $8-157 \times 10^{9} / \mathrm{L}$ ). All 11 patients had AML with monocytic differentiation (AML M4:4, AML M5:7). Four patients (36\%) had a solitary $t(6 ; 11)$. The remaining seven had one or more additional abnormalities. These included gain of the derivative (abnormal) chromosome 6 (27\%); trisomies $8(36 \%)$ and $19(27 \%)$ and tetrasomy $21(18 \%)$ with a complex karyotype in $36 \%$. Conclusions: The frequency $(0.4 \%)$ of the $\mathrm{t}(6 ; 11)$ in AML and the morphological findings are similar to the literature. The median age of our patients is slightly lower than what has been reported ( 32 years vs. 40 years). It is important to be aware of the morphological features associated with this translocation because it is a subtle abnormality which may be difficult to detect. 


\section{MHL 99}

Acute Lymphoblastic Leukemia with $\mathbf{t}(9 ; 22)$ and High Hyperdiploidy

B. Bexy Bensega ${ }^{\#}$, A. Palle, N. A. Fouzia, A. J. Devasia,

P. N. Nisham, A. Korula, A. Abraham, B. George, V. Mathews,

B. Poonkuzhali, A. Srivastava, V. M. Srivastava

Email ID for Correspondence: bexahbenz@gmail.com

Christian Medical College, Vellore

Introduction and Background: High hyperdiploidy (HHy) with a modal chromosome number of $51 \sim 65$ is associated with a favourable prognosis in acute lymphoblastic leukemia (ALL). The $t(9 ; 22)$ is another ALL-associated abnormality seen in $\sim 3$ of childhood ALL and up to $30 \%$ of adult ALL with a poor prognosis. This translocation results in the formation of the $B C R-A B L 1$ fusion gene which encodes a constitutively activated tyrosine kinase. Several secondary changes may be seen in $t(9 ; 22)$ ALL, notably an additional Philadelphia $(\mathrm{Ph})$ chromosome, trisomies 8,19 and 21 and the isochromosome $17 \mathrm{q}$. HHy with trisomy 2 is another secondary change associated with $\mathrm{t}(9 ; 22)$ ALL. This is an important category of ALL and is associated with a poor prognosis unlike other high hyperdiploid-ALL. Patient/Material and Methods: The study group consisted of all patients with $t(9 ; 22)$ ALL and high hyperdiploidy seen in the Department of Haematology between January 2003 and December 2015. G-banded karyotypes were reported in accordance with the International system for Human Cytogenetics Nomenclature (ISCN) and correlated with blood and bone marrow findings. Results: Among the 1199 ALL seen during the period of study, 11 patients $(0.9 \%)$ had the $t(9 ; 22)$ and $\mathrm{HHy}$. The patients ranged from $7-50$ years of age (median age 39 years). Nine were adults $(82 \%)$ and eight were males $(73 \%)$. The median haemoglobin was $8 \mathrm{~g} / \mathrm{dl}$ $\left(4-12 \mathrm{~g} / \mathrm{dl}\right.$ ), the median WBC count, $17 \times 10^{9} / \mathrm{L}$ (range $2-328 \times 10^{9} / \mathrm{L}$ ) and the median platelet count, $22 \times 10^{9} / \mathrm{L}$ (range $5-113 \times 10^{9} / \mathrm{L}$ ). The percentage of blasts in the marrow varied from 49-99 \%. The modal chromosome number was 51 65; one patient had a second clone with near-triploidy. The additional $\mathrm{Ph}$ chromosome was seen in 8/11 (72\%). Other chromosomal gains associated with the $\mathrm{t}(9 ; 22)$ such as trisomies 8,19 and 21 , were seen in $5 / 11$ (45\%), 3/11 (27\%) and 8/11 (72\%) respectively. Trisomy 2 was present in $6 / 11(54 \%)$. Other secondary changes included an additional $\mathrm{Ph}$ chromosome (72\%), trisomies 8, 19 and 21 in $45 \%, 27 \%$ and $72 \%$ respectively. Conclusions: The frequency of ALL with the $\mathrm{t}(9 ; 22)$ and high hyperdiploidy was $0.9 \%$. Trisomy 2 was seen in $54 \%$ of $\mathrm{t}(9 ; 22)$ ALL with HHy. T most common associated abnormalities were gain of the $\mathrm{Ph}$ chromosome and trisomy $21(72 \%$ each). It is important to look for $\mathrm{t} t(9 ; 22)$ in HHy-ALL because of its adverse prognosis.

\section{MHL 100}

High Abundance of Circulating Megakaryocytic Cells in Chronic Myeloid Leukemia in Indian Patients. Revisiting George Minot to Re-interpret Megakaryocytic Maturation

\section{Mona Anand, Biswadip Hazarika, Lalit Kumar, Rajive Kumar, Anita Chopra\#}

Email ID for Correspondence: chopraanita2005@gmail.com IRCH, All India Institute of Medical Sciences, New Delhi

Abstract: Circulating megakaryocytic cells abound in chronic myeloid leukemia (CML) seen in India and uniquely provide a setting for observing megakaryocytic maturation in the peripheral blood, a milieu not native to megakaryocytes. Peripheral blood megakaryocytic cells were studied in 324 cases of CML (235 chronic, 65 accelerated and 24 blastic phases). Two maturation themes were evident. Megakaryocytic blasts, especially in some cases of blast crisis, precociously make a foray into platelet formation and end up producing huge agranular or poorly granular cytoplasmic lobulated masses that break off and come to lie in the circulation. This evidence of unsuccessful effort may exist, in a considerably attenuated form in chronic phase, alongside of the second major theme of megakaryocytic maturation centered around the familiar micromegakaryocyte, characteristic of the chronic phase. This cell is regarded as dysplastic, but produces morphologically normal platelets. The possibility that this occurs via a hitherto unstudied alternative path of platelet maturation that plays out in the peripheral blood, and the contrasting disorderly premature attempt of blasts to form platelets, represent exciting maturation processes that need further study. Our observations fortuitously constitute a revisit of the insightful exposition on the subject by George Minot nearly a century ago.

\section{MHL 101}

Proposed Minimal Panel of Antibodies for Cost-Effectiveness and Accuracy in Acute Leukemias Immunophenotyping: Prospective Study at a Tertiary Care Center

Neha Singh", Hara Prasad P. Pati, Seema Tyagi, Roopam Deka, Rahul Sharma, Renu Saxena

Email ID for Correspondence: drnehasingh123@gmail.com All India Institute of Medical Sciences, New Delhi 110029, India

Introduction: Flowcytometry has an essential role in the diagnosis and classification of acute leukemias. However, there exists a great degree of inter-laboratory variability on issues like panel selection, antibody combinations, gating strategies, fluorochromes, and clonal selection. Aim: The primary aim of this study was to derive a minimal panel of antibodies and evaluate its diagnostic usefulness in acute leukemias by flowcytometry by using the detailed immunophenotype of different lineage-specific or non-specific markers. Materials and methods: This prospective observational study involved 400 newly diagnosed cases of acute leukemias. Bone marrow aspirate samples were subjected to morphological evaluation, cytogenetics and flow cytometric Immunophenotyping. Results: A minimal panel of eight antibodies comprising of CD45/CD34/CD19/MPO/cytoCD3/CD64/ CD117/CD79a was derived by applying different permutations and combinations with a diagnostic yield of $97.5 \%$. The minimal panel was further validated by testing in an independent cohort of patients with similar demographic characteristics, where it showed a high diagnostic yield of $98 \%$ in comparison with the screening panels proposed by other recently published studies. Conclusion: It may be concluded that the diagnostic performance of the eight antibody panel is better than most other panels used across the different laboratories in terms of yield, number of antibodies used and the scientific approach used to derive and validate the results and so henceforth may be applied in any setting with limited resources for better diagnostic accuracy.

Keywords Immunophenotyping, Acute leukemia

\section{MHL 102}

Prognostic Significance of RHAMM (CD 168) in Pediatric Acute Leukemias-A Study Done to Correlate Clinical Outcome, Postinduction, End of Treatment and Minimal Residual Disease Assessment

Rithika Rajendran", C. N. Sai Shalini, Febe R. Suman, Jerusha S. Jacob, Julius X. Scott, M. S. Latha 
Email ID for Correspondence: dr.rithikarajendran@gmail.com Sri Ramachandra Medical College and Research Institute, Chennai, India

Introduction: Hyaluronan (HA) of the extracellular matrix environment (ECM) plays an active role in tumor cell proliferation and invasion. Receptor for hyaluronan-mediated motility (RHAMM) and CD44 are HA receptors which have been implicated in promoting malignancy by inducing cell signaling pathways. Though few studies have been done on RHAMM expression in AML, there is a lacuna in the study of the expression of this protein in pediatric leukemias. Materials and Methods: Patients with acute leukemia of pediatric age group who were diagnosed, treated and followed up in our centre were enrolled. The bone marrow biopsies (BMB) done prior to treatment were subjected to RHAMM immunohistochemical (IHC) staining (54 BMBs namely ALL-45, AML-9). The blast counts at diagnosis and at the end of induction phase of chemotherapy as well as end of chemotherapy were counted. The minimal residual disease (MRD) assessment and the follow up details of the patients were collected. RHAMM positivity was correlated with initial blast count, post induction blast count, MRD and patient follow up. Results: There was no correlation between the initial blast count and the percentage of blasts which showed RHAMM expression. The positive correlation between percentage of blasts which expressed RHAMM and the number of post induction blasts is moderate in AML (0.74) and mild in ALL (0.48). There was statistically significant difference in the expression of RHAMM between the two MRD risk groups ( $p$ value 0.012 ) with a negative prognostic effect of RHAMM expression. A negative prognostic effect of RHAMM expression was noted when patient survival is considered. Conclusion: This study shows that AML blasts show more RHAMM (CD 168) positivity than ALL indicating the aggressive nature of the leukemic type. In acute leukemias, patients with high percentage of RHAMM positive blasts had more post induction blasts, blasts in MRD and poorer prognosis.

\section{MHL 103}

Congenital Mixed Phenotype Acute Leukaemia-A Case Report

\section{Meena Swati ${ }^{\#}$, S. Sharma ${ }^{1}$, P. Prakhar ${ }^{1}$, D. Patra $^{2}$, S. Kumar ${ }^{2}$ \\ Email ID for Correspondence: Swatiballot@hotmail.com Department of ${ }^{1}$ Pathology and ${ }^{2}$ Paediatrics, Lady Hardinge Medical College \& Associated Hospitals, New Delhi}

Introduction: Congenital leukemia and Mixed phenotype acute leukemia are individually rare disorder, comprising 4.3-8.6 per million live births and $2.4-3.7 \%$ of all childhood leukemia respectively. Combining these two constitute an extremely rare condition. Diagnosis of congenital leukemia requires clinical presentation within 30 days of birth, proliferation of immature white blood cells, infiltration of cells into extrahematopoietic tissue and absence of congenital leukemia stimulator. Herein, we report a case of 27 days old infant, presented, with complaints of fever black coloured stool, generalized macular rash and abdominal distension since day 7 of birth. Physical examination showed hepatosplenomegaly and multiple generalised reddish macular patches. Blood examination revealed marked leukocytosis (TLC$\left.4.89 \times 10^{5} / \mu \mathrm{l}\right)$, thrombocytopenia $(28,000 / \mu \mathrm{l})$ and normocytic anemia $(\mathrm{Hb}-8.3 \mathrm{gm} / \mathrm{dl})$. Peripheral smear and bone marrow smear showed dimorphic population of blasts comprising of $73 \%$ and $83 \%$ of all nucleated cells respectively, of which small blasts showed block PAS positivity, while larger blasts showed granular NSE positivity. Flow cytometry performed on peripheral blood showed two different blasts on SSC vs. CD45 gating. Blasts with high side scatter and moderate CD 45 positivity constitute $47 \%$ and were positive for CD15, CD33, CD14, CD7, CD13, tdt, CD11c and weak CD79a. Blasts with low side scatter and dim CD45 positivity constitute $42 \%$ and were positive for cCD79a, CD34, CD19, HLA-DR, CD7, Tdt and negative for CD10. Cytogenetic studies showed $\mathrm{t}(4 ; 11)(\mathrm{q} 21 ; 23)$ translocation, Based on this a diagnosis of congenital mixed phenotype acute leukemiaMyeloid (M5)/ B lymphoid was given. Next day patient developed intracranial bleed and expired soon after. Conclusion: Congenital mixed phenotype acute leukaemia is an extremely rare condition with an aggressive course and poor prognosis. This case is being presented because of its extreme rarity.

\section{Topic: Transfusion Medicine}

\section{TM 1}

RHD Positive Variants in Moroccan Blood Donors Serologically D Negative: Identification of 6 Novel Intronic Mutations

\section{Elwafi Mariam $^{\#}$, H. Elhousse ${ }^{1,2}$, N. Zaid ${ }^{3}$, S. Zouine ${ }^{1,2}$, N. Nourichafi ${ }^{4}$, M. Benajiba ${ }^{5}$, N. Habti ${ }^{1,2}$}

Email ID for Correspondence: mariam.benabdeljalil@gmail.com ${ }^{1}$ Laboratory of Biotechnology and Experimental Medicine, Faculty of Medicine and Pharmacy Casablanca, Hassan II University of Casablanca, Morocco; ${ }^{2}$ Laboratory of Hematology, Cellular and Genetic Engineering, Faculty of Medicine and Pharmacy Casablanca, Hassan II University of Casablanca, Morocco; ${ }^{3}$ Montreal Heart Institute, McGill University; ${ }^{4}$ Regional Blood Transfusion Center in Casablanca, Morocco; ${ }^{5}$ National Blood Transfusion and Hematology Center, Morocco

Background: Blood group genotyping is increasingly utilized in transfusion and obstetric medicine. In Caucasians, the presence of antigen $\mathrm{D}$ is predicted, if two or more RHD specific polymorphism are detected. In the presence of non-functional RHD, this prediction must fail giving false positive results. Excluding RHD pseudogene $(R H D \psi)$ and $C d e S$ frequent in individuals of African origin, most of these variants have not been determined in Morocco. Methods: Among a total of $544 \mathrm{D}$ negative blood donors, 65 blood samples RhC and/or $\mathrm{RhE}$ positive were tested by serologic tests. Samples serologically D negative were screened by PCR using sequence specific priming, multiplex PCR and next generation sequencing. For novel mutations, the Alamut software was used to predict their possible effect on splicing. Results: Seventeen samples $(26.1 \%)$ previously documented as $\mathrm{RhD}$ negative had been missed by routine serology. The complete deletion of the RHD gene is observed in $81.2 \%$ of blood donors serologically D negative $C$ and/or E positive. Sequencing reveals 9/48 RHD positive samples. Five new variants were detected, each one were caused by more than 2 intronic mutations. Overall, 6 novel mutations were characterized far from conserved splice sites. The bioinformatics results suggest that 3 mutations affect splicing. Two samples were $C d e s$ allele and 2 variants were not confirmed. Conclusion: This study is the first to describe RHD variants caused by intronic variations far from the splice sites. Further investigations in RHD gene in Maghrebian population will allow improving RHD genotyping strategy.

\section{TM 2}

Implementation of Upgraded Technology in the Management of Thalassemic Patients-An Excellent Response Covering 14 Districts of North Karnataka

\section{Shreedevi S. Bobati ${ }^{1 \#}$, Mr. Shrikant V. Viragi}

Email ID for Correspondence: drshree14@gmail.com Assistant Professor/Junior Consultant ${ }^{1}$, Department of Transfusion Medicine (Blood Bank) KLE University's JN Medical College \& 
KLEs Dr. Prabhakar Kore Hospital, Medical Research Centre Belagavi, Karnataka 90010

Introduction and Background: Thalassemia is one among the few diseases which requires lifelong treatment and medication. This again calls for a tedious management of the documents for those suffering from it and for their family members too. Making the best use of technology, with a dedicated software called ThalCare meant specifically for systematic management of medical information and records for thalassemia patients. Patient/Material and Methods: This is a prospective study which includes all the registered thalassemia patients from 14 districts of North Karnataka. The thalacare tool tracks transfusions by scheduling for transfusion, estimates the volume of blood that should be transfused based upon the pre transfusion haemoglobin $(\mathrm{Hb})$ and weight, tracks the variation of haemoglobin with time graphically. Also tracks chelation, lab investigations, complication management, vaccination, growth and other relevant issues. The packed RBCs are 3 times saline washed and issued to the patients free of cost. Results: Total 164 patients are registered (male-104\& female-60), age ranges from 9 months to 25 years, 150 patients are diagnosed as thalassemia major, 7 as thalassemia minor, 5 as thalassemia intermediate and 2 cases of AT- $\alpha$ thalassemia, Hb-E thalassemia respectively. Number of transfusions varied from 1-290, average volume of blood transfused was $264.51 \mathrm{ml}$. The average initial $\mathrm{Hb}$ varied from $8.05 \mathrm{gm} \%$ to final $\mathrm{Hb}$ of $12.38 \mathrm{gm} \%$ and 151 patients had normal levels, 4 with low and 8 with intermediate $\mathrm{Hb}$ levels at the time of discharge. The ferratin levels were high in 133 patients with remaining in normal range. None of the samples were positive for allo or autoantibodies. Serology status for TTD showed 3 positive cases for HBV with one patient being positive for both HIV and HBV. The periodical check of the transfusion therapy was effective in all the cases except 4 cases. Conclusions: Thalcare helps improve outcome of the treatment regimens involving multi-disciplinary experts, optimal utilisation of staff, enables delivery of high quality end to end thalassemia management and enhances patient engagement and adherence.

\section{TM 3}

To optimise the rationale of Use of Platelet Concentrate at Tertiary Care Hospital

\section{Arshid Sidique $^{\#}$, Aleem Jan, Irfan Kan, Naira Firdous}

Email ID for Correspondence: aleem_maleem@yahoo.co.in Sheri Kashmir Institute of Medical Science,

Soura Srinagar J\&K 190011

Introduction and Background: Platelet transfusion in the form of platelet concentrate is traditionally given to bleeding thrombocytopenic patients, patients with haematological malignancies and cancer patients on chemo-radio protocols. The study was done to assess, audit and optimize rational use of platelet concentrate. One year study was carried out at skims hospital, which is a deemed university and also a tertiary care super speciality hospital of north India. The study was conducted on inpatients as well as day care ward patients. Patient/Material and Methods: The dates for platelet preparations was obtained from departmental records. Patients information was obtained from requisition forms and platelets issue record register. Cross checks were done to ensure correct data entry. Results: Requisition received: 1722, Appropriate: 158 (92 \%), Inappropriate: $136(8 \%)$. Details of inappropriate requests: haemophilia (8\%), Vonwillibrand's disease (2\%), ITP (7\%), TTP (2\%), Requisition with count 750,000 (19\%), Requisition above 10 bags (27\%). Main user specialities were clinical haematology (65\%) requisition with $9.7 \%$ inappropriate requisition and medical oncology (24\%) requisition, $3 \%$ inappropriate requisition. AML was the most common diagnosis. Conclusions: Overall percentage of inappropriate requests was $7.8 \%$. Hospitals must do regular audit of blood component usage and educate all clinicians especially new resident staff about its appropriate and rational use. Further all user departments must have periodic interactions with Transfusion medicine and there should be incorporation of posting of postgraduate and postdoctoral scholars both in discipline of Medicine and surgery in Blood Banks.

\section{TM 4}

\section{Limitations of Using Only Haemoglobin Levels for Screening Blood Donors}

\section{Preeti Chavan", Vivek Bhat, Minal Poojary, Shashank Ojha, Sanjay Pal, Babu Pillai}

Email ID for Correspondence: pchavan@actrec.gov.in ACTREC-Tata Memorial Centre, Kharghar, Navi Mumbai 410210

Introduction and Background: Blood and aphaeresis donors are screened for fitness based on their haemoglobin value. However, donors with acceptable haemoglobin levels may have low mean corpuscular volume (MCV) with high total Red Blood Cell (RBC) Count. The cause for these low levels of MCV may be iron deficiency or haemoglobinopathy. Aim of this study was to assess the low MCV values, total RBC count and its correlation with haemoglobin levels in blood, aphaeresis donors found fit-for-donation. Patient/Material and Methods: Pre-donation blood samples from 751 (M:558; F:193) blood, aphaeresis donors found fit-for-donation were processed for Complete Blood Count (CBC) on Advia2120i, Siemens Healthcare. Serum Ferritin levels of donors with MCV levels below reference range (83-101 fL) were analysed on Architect 1000i, Abbott Healthcare. Serum Ferritin levels < reference range (M: 21.8-274.6 ng/ml; F: 4.63-204 ng/ml) were defined as iron deficiency. Mentzer index (MCV/RBC count) was calculated for all samples. Mentzer index $<13$ was considered suggestive of haemoglobinopathy, $>13$ was suggestive of iron deficiency anemia. Results: Of 751 samples analysed 27 (M:19; F:8) samples (3.6 \%) showed MCV levels $<80$ fl. Of these, $58 \%(11 / 19)$ male donors and $50 \%(4 / 8)$ female donors had Mentzer index $<13$ suggesting haemoglobinopathy. The rest (M: 8; F: 4) had Mentzer index $>13$ suggesting iron deficiency anemia. Three male donors showed low serum ferritin levels, of these two had Mentzer index $<13$ suggesting coexistence of hemoglobinopathy and iron deficiency anemia. RBC count was $>$ reference range in all donors with low $\mathrm{MCV}$ count. Reference range for total RBC count was M: $4.5-5.5 \times 10^{12} / \mathrm{L} ; \mathrm{F}: 3.8-4.8 \times 10^{12} / \mathrm{L}$. Conclusions: Screening blood, aphaeresis donors for haemoglobin alone is not a reliable method for selecting healthy individuals fit-fordonation. CBC count with emphasis on MCV and RBC count should be done while screening to select donors to help exclude individuals with hemoglobinopathy or iron deficiency anemia, but higher haemoglobin levels due to high RBC count. Detailed study with further investigation for iron deficiency and haemoglobinopathies is desired.

\section{TM 5}

Practice of Transfusing D Positive Platelets in D Negative Recipients-A Reappraisal (POINT-R)

Ravi C. Dara ${ }^{\#}$, Aseem K. Tiwari, Dinesh Arora, Geet Aggrawal, Deviprasad Acharya, Vimarsh Raina

Email ID for Correspondence: rcdara@gmail.com Department of Transfusion Medicine, Medanta, The Medicity, Gurgaon

Introduction: Many centres provide D-mismatched platelet transfusions. Platelet concentrates may contain enough red cells leading to 
formation of anti-D antibody in D negative recipient. In resource poor settings Random Donor Platelet Concentrate (RDPC) is used often and carries higher risk of allo-immunization. At our institution platelets are transfused across the $\mathrm{ABO}$ and $\mathrm{Rh}$ blood group barrier without any RhIG immune-prophylaxis. In this study we retrospectively appraised allo-immunization after D positive platelet transfusion in D negative patients. Materials and methods: Single centre five year data was collected from Hospital Information System (HIS) for D negative patients who received D positive platelet transfusions. Naive patients who had neither pre-existing antibody nor received $\mathrm{D}$ positive platelets transfusion before admission were included. Those who had repeat antibody screen at-least 28 days after D positive platelet transfusion were considered. Results: 332 D negative patients who received 1996 D positive platelet transfusions were analysed. Median follow up was 7 weeks (range $=4-90)$. Anti-D was detected in three $(0.9 \%) \mathrm{D}$ negative patients at 5,8 , and 24 weeks after receiving $\mathrm{D}$ positive platelet transfusions. Two of these patients received RDPCs and one received both RDPCs and SDPCs. Conclusion: D positive platelet transfusion to D negative patients seems an acceptable practice as only $0.9 \%$ patients developed anti-D antibody.
BIORAD Asia) was used for antibody screening. In antibody positive cases, 11 cell panel was used for antibody identification and antibody titer was done by conventional tube method. In alloantibody positive cases husband's blood group along with $\mathrm{Rh}$, cell and other relevant antigen phenotyping of parents was done. The medical history and detailed obstetric history of these women was recorded. Results: The overall red cell alloimmunisation rate was $1.05 \%$ (21/2000). Dual antibody was present in 4 pregnant women, so total 25 antibodies were detected in 21 pregnant women. Most common antibody was anti-D, which was detected in $71.42 \%(15 / 21)$ of alloimmunised women. Other antibody found included anti-D+C $19.04 \%(4 / 21)$, anti-E $4.76 \%(1 / 21)$ and unspecified $4.76 \%(1 / 21)$. Titer levels of anti-D antibody ranged from Negative to $1: 512$ by tube method. Conclusions: However, In this study, we reported that anti-D still contributed to $76 \%(19 / 25)$ of all the alloantibodies formed (alone or in combination). We need to focus on universal and adequate anti-D immunoprophylaxis programs in antenatal services. Moreover, all pregnant women should be screened for anti-D and other red cell antibodies irrespective of Rhesus type.

332 (Received $1996 \mathrm{D}$ positive platelets) ( $D$ negative patients)

\begin{tabular}{|c|c|c|c|c|c|c|c|}
\hline & & \multicolumn{2}{|c|}{ Group I } & \multicolumn{2}{|c|}{ Group II } & \multicolumn{2}{|c|}{ Group III } \\
\hline Patients & & \multicolumn{2}{|c|}{$269(81 \%)$} & \multicolumn{2}{|c|}{$25(7.5 \%)$} & \multicolumn{2}{|c|}{$38(11.5 \%)$} \\
\hline Mean \pm SD & & \multicolumn{2}{|c|}{$5.2 \pm 4.7$} & \multicolumn{2}{|c|}{$1.6 \pm 0.9$} & \multicolumn{2}{|c|}{$19.4 \pm 32.1$} \\
\hline Median & & \multicolumn{2}{|l|}{4} & \multicolumn{2}{|l|}{1} & \multicolumn{2}{|c|}{11} \\
\hline Range & & \multicolumn{2}{|l|}{$1-59$} & \multicolumn{2}{|c|}{$1-5$} & \multicolumn{2}{|c|}{$2-231$} \\
\hline \multicolumn{2}{|c|}{ Platelets transfused } & \multicolumn{2}{|c|}{1278} & \multicolumn{2}{|c|}{39} & \multicolumn{2}{|c|}{679} \\
\hline & D posi & & Both* & D positive & Both & D positive & Both \\
\hline Patients & 192 & $57.8 \%)$ & $77(23.2 \%)$ & $23(6.9 \%)$ & $2(0.6 \%)$ & $13(4 \%)$ & $25(7.5 \%)$ \\
\hline Mean \pm SD & $4.2 \pm$ & & $7.8 \pm 7.4$ & $1.5 \pm 0.67$ & $3.5 \pm 2.1$ & $9.1 \pm 6.8$ & $24.6 \pm 38.4$ \\
\hline Median & 4 & & 5 & 1 & 3.5 & 7 & 15 \\
\hline Range & $1-17$ & & $2-59$ & $1-4$ & $2-5$ & $2-26$ & $2-231$ \\
\hline $\begin{array}{l}\text { Platelets } \\
\text { transfused }\end{array}$ & 731 & & 547 & 33 & 6 & 108 & 571 \\
\hline
\end{tabular}

* Both-means patients receiving both $\mathrm{Rh}$ positive and negative platelets; *-Indicates index cases which developed anti $D$ at five, eight and 24 weeks after $D$ positive platelet transfusion.

\section{TM 6}

\section{Study on Magnitude of Red Cell alloimmunization and Type of Alloantibody Among Pregnant Women}

\section{Rit i Yadav", Sangeeta Pahuja, Manisha Kumar}

Email ID for Correspondence: riti0022@gmail.com Department of Pathology, Lady Hardinge Medical College and Smt. Sucheta Kriplani Hospital, New Delhi

Introduction and Background: Red cell alloimmunization during pregnancy remains the major cause of hemolytic disease of the fetus and newborn. There is limited data available on magnitude of red cell alloimmunization among pregnant women in India. We, therefore, studied the magnitude of red cell alloimmunization and type of alloantibody among pregnant women. Patient/Material and Methods: A total of 2000 pregnant women attending antenatal clinics, irrespective of their gestational age, were typed for $\mathrm{ABO}$ and $\mathrm{D}$ antigens and screened for alloantibodies. A commercially available 3 cell panel (ID Diacell I, II, III; BIORAD ID microtyping system,

\section{TM 7}

Therapeutic Plasma Exchange (TPE) in Recurrent FSGS Post Renal Transplantation: A Case Report

Ashok Pal ${ }^{\#}$, Sunita Bundas, Rachna N. Saxena, Parmendar Pachori, Sarita Sharma

Email ID for Correspondence: dr.palashok@gmail.com Department of IHBT SMS Medical College and Hospital, Jaipur

Introduction and Background: Focal segmental glomerulosclerosis (FSGS) has been shown to recur after renal allograft in 20-30\% of the cases. It usually presents in the early post operative period with reemergence of proteinuria and progressive graft dysfunction period. This differentiates it from denovo FSGS which usually presents after 12 months of transplantation. Aim: To study the efficacy of early and intensive Therapeutic plasma exchange in obtaining remission in these cases. Patient/Material and Methods: TPE was performed for this patient on three consecutive days and then five more procedures on alternative days using HAEMONETICS MCS+. On each occasion 
$2500 \mathrm{ml}$ plasma was extracted with $100 \%$ replacement. Replacement was done with $5 \%$ albumin and 2 units of group compatible plasma at the end. Along with plasmapheresis 2 doses of Rituximab $(500 \mathrm{mg})$ each at 2 weeks interval were also given. Results: Laboratory investigations after the eight procedures showed a drop in proteinuria from $4+$ to $2+$ and serum creatinine from $2.8 \mathrm{mg} / \mathrm{dl}$ to $1.6 \mathrm{mg} / \mathrm{dl}$. The patient was then discharged with advice for regular follow up. Conclusions: TPE along with rituximab therapy is an effective approach for obtaining remission in recurrent FSGS post renal transplantation even after a delay of one month in diagnosis and start of therapy.

\section{TM 8}

Evaluating Collection Efficiency (CE2) of Automated Interphase Controlled Apheresis (AIM) System for Peripheral Blood Stem Cell/Heamatopoietic Progenitor Cell (HPC-A) Collection by Apheresis-A Single Center Experience

\section{A. Sivaramakrishnan ${ }^{\#}$, V. Lakshman, T. Raja, Jose M. Easow, Revathiraj}

Email ID for Correspondence: docshivstanlean@gmail.com Apollo Cancer Institute

Background: Mobilised peripheral blood stem cells are commonly used for hematopoietic stem cell transplantation due to the rapid engraftment of graft and lower microbial contamination rates. Most PBSC collections yielded super doses of CD 34+cells. However some collections failed to achieve target yield and needed large volume of blood to be processed. So predicting yield based on Collection Efficiency (CE2) and CD34+ cell count before the procedure will optimize the volume of blood to be processed, and reduce the donor exposure to anticoagulant. The target yield and blood volume to be processed is predicted by using the following formula: Total Blood Volume Processed $=$ Target Yield $\times$ Recipient Weight $\times 100 /$ CE $2 \times$ Pre Procedure CD $34+$ cell Count. Patients and DonorsAssessed Variables: Retrospective evaluation of 102 harvests from autologous and allogeneic donors was analysed from 2015 to 2016. Results:

1. Our study shows that the mean CE2 for the allogeneic donations was $56.88 \%$ and for autologous donations was $46.22 \%$.

2. Average volume of processed blood for allogeneic donors was $7990 \mathrm{ml}$ in 156 minutes comparatively for autologous donors processed blood was $13,444 \mathrm{ml}$ in 211 minutes.

3. Mean yield was 14.58 and $3.15 \times 10^{6} / \mathrm{kg}$ recipient weight for allogeneic and autologous donors respectively.

4. There is statistically significant strong correlation found between CE2 and Yield.

5. Donor Age, Recipient weight, Total blood volume processed, Inlet speed, access and return flow rates have significant correlation with CE2.

Conclusion: To accomplish target yield, assessment of Collection Efficiency of the Apheresis system is a useful tool. With appropriate CE2 we can achieve target yield with minimal required blood volume and less anticoagulant infusion to the donor or patient from whom PBSC is collected. The CE2 reflects optimal performance of the cell separator under standard conditions.

Keywords Collection efficiency (CE2), Peripheral blood stem cell, Yield, CD34+ cells

\section{TM 9}

To Evaluate the Effectiveness of Surgical Diagnosis in the Estimation of Blood Transfusion Requirements and Use of Blood Typing Only Policy for Increasing Blood Usage After Cross-Matching

Sankalp Sharma\#, Dhananjay Prasad,

Kamlesh Janghel, Ajay Yadav, Santosh Patidar, Kamlesh Sahu, Bhupendra

Email ID for Correspondence: sunray2077@gmail.com AIIMS Raipur

Introduction and Background: The tools for optimizing blood transfusion for the pre surgical patients are Maximum Allowable Surgical Blood Loss (MSBOS) and Standard Blood Ordering Schedule (SBOS). These are prone to inaccuracies due to patients with diverse clinical diagnosis, undergoing the same surgical procedure. Patient/ Material and Methods: This study involves a 250 bedded multi-speciality hospital. We did a retrospective data compilation after the segregation of pre-surgical diagnoses, towards cross matching $(\mathrm{CM})$ of blood units against type only patients. Aim of this study was to observe $\mathrm{RBC}$ requirements and haemoglobin thresholds for transfusion in various surgical diagnosis; a sub-group analysis evaluated the effectiveness of blood typing only (BTY) policy and cost of blood transfusion in the surgical management of a patient (Rs 1050/Red Cell Unit). Results: In a two year observation period with educational interventions; surgical procedures requiring anaesthesia $(n=195)$ were integrated under 16 categories. An overall cross match to transfusion ratio was 1.6 with average blood usage after CM 73.06 \%. BTY was performed on $57.0 \%$ of requisitions with $41.4 \%$ of BTY converted to CM blood. An average pre-transfusion haemoglobin was $8.7 \mathrm{gm} / \mathrm{dl}$ (SD: 2.4); BTY reduced the number of $\mathrm{CM}$ of blood units by $50 \%$ (P value $<0.05$ ). The blood usage in the $\mathrm{CM}$ group showed a significantly higher blood usage due to BTY ( $p$ value $<0.05$ ). An average erythrocyte use per patient for various clinical diagnosis was 0.5 units; (SD 0.28; Correlation Coefficient of -0.49 with BTY) with the average expenses per patient on blood unit at Rs 525/respectively. Blood usage was $30 \%$ or less for ovarian cyst, nodular goitre and colloid goitre. Conclusions: A segregation of surgical diagnosis for calculating the transfusion requirements minimises inter-individual variation of a surgical procedure, increases blood usage, provides evidence for using type and reserve policy and save costs of transfusion per patient.

\section{TM 10}

\section{Platelet Rransfusion in Dengue Patients}

\section{Rajendra Chaudhary ${ }^{\#}$, R. Katharia}

Email ID for Correspondence: rkcsgpgi@gmail.com

Sanjay Gandhi Postgraduate Institute of Medical Sciences, Lucknow

Introduction and Background: Thrombocytopenia is common in acute dengue infection. Although severe bleeding and coagulopathy may occur in dengue infection, the cause is multifactorial and is not caused by thrombocytopenia alone. Despite this, platelet transfusion was frequently given for severe thrombocytopenia to adults with acute dengue infection. Appropriate use of blood components is required to ensure their availability for needy patients as well as to avoid necessary risk of transfusion transmitted infection. Patient/ Material and Methods: The study was conducted on 264 
serologically confirmed dengue patientsduring a dengue epidemic. Haematological investigation and platelets transfusion requirements were obtained and analysed. Results: A total of 204 (77.2 \%) patients were transfused either with random donor platelets or single donor platelets. Out of 204 patient transfused, $89(43.6 \%)$ patients has platelet count $<20,000 / \mu$ Lwhere as $115(56.4 \% 0$ patients were transfused at a platelet count $>20,000 / \mu \mathrm{L}$. Among 115 patients transfused, 85 patients had hemorrhagic manifestations such as petechiae, ecchymossis, and gum bleed which necessitate the use of platelet transfusion. Of these 85 patients transfused, 81 had platelet count between $20,000-50,000 / \mu \mathrm{L}$ where as 4 patients who were transfused at platelet count $>50,000 / \mu \mathrm{L}$ in the presence of bleeding. However, $30(14.7 \%)$ patients received inappropriate platelet transfusion without any bleeding manifestation just on the basis of platelet count $(20,000 / \mu \mathrm{L})$. Conclusions: The study concludes that thrombocytopenia is common finding in dengue infection. The transfusion should not be based alone on platelet count in patient with uncomplicated dengue. This approach will help to avoid unnecessary transfusions.

\section{TM 11}

Prevalence and Specificity of RBC Alloantibodies in Antenatal Women

\author{
Asim Israr Khan ${ }^{1 \#, ~ S y e e d u l ~ H a s a n ~ A r i f ~}{ }^{2}$, Taiba Khan ${ }^{3}$, \\ Saima Khan ${ }^{1}$, Suhailur Rehman ${ }^{4}$ \\ Email ID for Correspondence: asimisrarkhan@gmail.com \\ ${ }^{1}$ Junior Resident, ${ }^{2}$ Professor, ${ }^{4}$ Senior Resident, Department of \\ Pathology, Jawaharlal Nehru Medical College, Aligarh Muslim \\ University, Aligarh; ${ }^{3}$ Junior Resident, Department of Pathology, \\ GSVM, Kanpur
}

Introduction and Background: Maternal alloimmunization is defined as the presence of irregular red blood cell alloantibodies in the blood of a pregnant woman that can theoretically cause haemolytic disease of the fetus and newborn. Alloimmunisation in pregnancy is an immune disorder due to genetic differences between maternal and fetal red blood cell antigens. This study was conducted to determine the prevalence and specificity of RBC alloantibodies in pregnant women attending antenatal clinic at a tertiary care hospital of north India. Patient/Material and Methods: Pregnant women visiting the hospital between November 2014 and April 2016, were randomly selected and screened for irregular antibodies using a commercial 3 cell antibody screening panel. On those samples found to be positive on screen antibody identification was performed using a commercial 11 cell panel. Results: Screening was performed on 750 women, of these, $48(6.4 \%)$ were $\mathrm{Rh}(\mathrm{D})$ negative. The majority $(32.8 \%)$ of the pregnant women were primigravidae. On screening $13(1.73 \%)$ women were found positive for red cell alloantibodies. Anti-D was the most frequent antibody identified followed by anti-E. The other clinically significant antibodies found were anti-C, anti$\mathrm{c}$, anti-K and anti-Jka. The study revealed significant correlation between positive antibody screen and bad obstetric history. Conclusions: This study showed that the prevalence of red cell alloimmunisation among pregnant women at this centre corroborated with data reported from other centres in India. Alloimmunization among $\mathrm{Rh}(\mathrm{D})$ positive women was low as compared to $\mathrm{Rh}(\mathrm{D})$ negative women but it included clinically significant antibodies. Thus, red cell alloantibody screening should be made available for all the antenatal women, including $\mathrm{Rh}(\mathrm{D})$ positive, so as to reduce possible complications of alloimmunisation.

\section{TM 12}

Analysis of Donor Deferral Pattern in a Regional Blood Transfusion Centre in Delhi

\section{Wonchibeni T. Murry ${ }^{\#}$, Preeti Diwakar, Priyanka Gogoi, Bharat Singh}

Email ID for Correspondence: wonchimury@gmail.com University College of Medical Sciences and Guru Teg Bahadur Hospital, Delhi

Introduction and Background: Blood banking is an important part of a hospital and health care services. Requirement of blood and blood components is particularly high in India. Donor registration and screening is mandatory for ensuring safe blood supply. Donor deferral can be temporary or permanent. The donor deferral criteria have been adopted from the developed countries, however sufficient "in-house" data and its scientific validation are still required to test the applicability of these criteria in Indian blood donors. Hence there is a need to evaluate the donor deferral pattern for better applicability in Indian scenario, to decrease the donor deferral rate. Aims and Objectives: To evaluate the causes of deferral in donors. To suggest the modifiable parameters in selection criteria. Patient/Material and Methods: The study is a retrospective audit of the records of deferred donors from January 2014 to July 2015 in Regional Blood Transfusion Centre, Guru Teg Bahadur Hospital and UCMS, Delhi. Results: In nineteen months (Jan 2014 to July 2015) a total of 57,278 prospective donors were screened for blood donation. There were 4232 deferral cases constituting almost $7.3 \%$ of total screening. The common causes of deferral were anaemia, patients on medication or who had alcohol intake, patients with infection and allergic symptoms, chronic medical illnesses and presentation for subsequent donation before recommended time gap. Conclusions: Rationalize and revalidate deferral criteria based on studies done in Indian population. Educate, motivate and treat donors deferred due to haemoglobin $<12.5 \mathrm{gm} / \mathrm{dl}$, so that they can be regular repeat donors. Create awareness about deferral criteria among prospective blood donors.

\section{TM 13}

Transfusion Support in Glanzmann's Thrombasthenia: A Case Report

\section{Nippun Prinja ${ }^{\#}$, Rachna Narain, Sunita Bundas, Amit Sharma, Parmendra Pachori}

Email ID for Correspondence: gmchprinja@yahoo.com SMS Medical College and Hospital

Introduction and Background: We describe post operative management of 41 year old female with Glanzmann's Thrombasthenia (GT) who came to hospital for elective hysterectomy. GT is rare autosomal recessive platelet disorder manifested by lack of glycoprotein II B-III A complex in the platelet membrane and characterized by prolonged bleeding time and severe hemorrhagic mucocutaneous diathesis. Patient/Material and Methods: Case report: 41 years old patient $75 \mathrm{~kg}$ female, with one child, came to hospital for elective hysterectomy. As an infant she had multiple episodes of epistaxis and frequent bruising. She was diagnosed with GT at the age of 3. She had required frequent admissions for blood and platelet transfusions because of menorrhagia. 1st caesarean section was uneventful with prolonged hospital stay. Hysterectomy was performed with pre- 
operative cover of six random donor platelet units. Blood loss during surgery was 500-600. Three units each of packed cells and platelets were transfused. Post operatively the patient continued to ooze from the abdominal drain site and passed clots per vaginum. Incision site was normal and there was no intra abdominal free fluid on ultrasonography. The total blood loss was estimated $1800 \mathrm{ml}$. She was then administered two units of apheresis plasma, $500 \mathrm{ml}$ each and two packed cell. She was continuously monitored in the ICU. Over 24 hours she stopped passing clots and oozing from surgical site markedly reduced. Haemostasis was achieved by giving two more plasma apheresis along with cryoprecipitate. The patient was discharged on the third post operative day from ICU. Discussion: GT is uncommon condition. Risks of post operative bleeding and haemorrhage are high and its clinical course is difficult to monitor in these patients. Platelet transfusions, plasma apheresis and cryoprecipitate help achieve haemostasis and better platelet efficacy. Conclusions: Our patient was managed with multidisciplinary approach that comprised achieving good surgical haemostasis, postoperative transfusion support and intensive monitoring.

\section{TM 14}

The Critical Role of Plasmapheresis in ABO-Incompatible Renal Transplantation

\section{S. Sangwan", R. M. Jaiswal, H. B. Saxena, S. Sharma}

Email ID for Correspondence: sangwansavi44@gmail.com Mahatma Gandhi Institute of Medical Sciences and Technology

Introduction and Background: In India, patients without a compatible blood group donor are usually excluded from renal transplantation. $\mathrm{ABO}$ incompatible live donor renal transplantation has come up as a solution. But, ABO-incompatible kidney transplants require the anti- $\mathrm{ABO}$ titer to be reduced to below 1:16 before transplantation. This can be achieved by preoperative plasma exchange or cascade plasmapheresis (CP). Patient/Material and Methods: We report a case of a 20 year old male patient with end stage renal disease. The patient's blood group was $\mathrm{O}+\mathrm{ve}$, and no compatible donor was found in the family. His father was $\mathrm{B}+\mathrm{ve}$ and was willing to donate. The cascade plasmapheresis was performed with an apheresis equipment Com.Tec (Fresenius Kabi, Germany) to lower the anti-A and anti-B antibody titer in the recipient. An Antihumanglobulin (AHG) titer was performed for anti-A and anti-B antibody following the departmental standard operating procedure. Results: A total of 4 plasmapheresis procedures were performed preoperatively to maintain the antibody titers at or below the desired levels. The baseline anti-A and anti-B antibody titers were 1:256 and 1:512 respectively which came down to $1: 8$ and $1: 16$ at the end of 4 th cycle of plasmapheresis. During the post-operative follow up the anti-A and anti-B antibody titres have been maimtained at 1:8 and 1:4 respectively. The patient continues to have normal renal function with a creatinine of approximately $1.0 \mathrm{mg} / \mathrm{dl}$ and has had no episodes of rejection. Our case could be the first reported case from Rajasthan in which we used a cascade plasmapheresis procedure as a part of preconditioning protocol. Conclusions: Cascade plasmapheresis offers a cost-effective and efficient way to decrease blood group antibody titer and helps in successful transplantation.

Keywords Cascade Plasmapheresis (CP), ABO incompatible, Renal transplant, Titer

\section{TM 15}

Abstract has been retracted.

\section{TM 16}

Analysis of Donor Deferral Characteristics in 101 Voluntary Blood Donation (VBD) Camps Conducted by Tertiary Care Hospital of North-Western India

\section{Ishan Joshi", Rachna Narain, Sunita Bundas,} Amit Sharma, Parmendra Pachori

Email ID for Correspondence: dr.ishanj13@gmail.com SMS Medical College, Jaipur

Introduction and Background: The deferral of blood donors leads to loss of precious blood units available for transfusion purpose. However blood donors deferral, either temporarily or permanently is an important step in improving the safety of blood transfusion. Aims \& Objectives: To find the incidence and causes of deferral in NorthWestern India and apply relevant findings to modify recruitment strategy. Patient/Material and Methods: A retrospective study was conducted at blood bank, SMS Hospital by Deptt. Of Immunohaematology \& Transfusion Medicine, SMS Hospital, Jaipur (Rajasthan). Donor deferral records of 101 Voluntary Blood Donation Camps held from 1 April 2016 to 28 august 2016 were analysed. National guidelines were used for selection and deferral of VBD. Results: Of total 6208 donations in 101 VBD camps 5225 (84.2\%) donors were found fit. 983 VBD $(15.8 \%)$ were deferred during the study period. Majority of them $(92.7 \%)$ being deferred for temporary reasons. Most common reasons for deferral were low $\mathrm{Hb}$ (Haemoglobin) $(39.7 \%)$, underweight $(12.7 \%)$, History of medication use $(11.4 \%)$, High blood pressure $(4.8 \%)$, History of jaundice/hepatitis $(1.3 \%)$. Permanent Deferral accounted for $7.3 \%$ with uncontrolled hypertension $(4.8 \%)$ being the most common cause in this category. Conclusions: It is important to analyze the rate and causes of VBD deferral in our region \& compare them with national \& international level to guide the recruitment and retention efforts and also to improve donor \& recipient safety.

\section{TM 17}

Analysis of Blood Transfusion Services in Tertiary Care Hospital of North Western India

\section{Kamal Sharma\#, Sunita Bundas, Rachna Narain, Parmendra Pachori}

Email ID for Correspondence: kamalsharma@gmail.com SMS Medical College

Introduction and Background: Transfusion Medicine has emerged as a distinct specialty which has improved and reconstructed BTS (blood transfusion services). Efficient Clinical practices \& proper usage of blood \& blood components is demand of hour. It involves complex laboratory technologies, community interface \& patient care. Patient/Material and Methods: Retrospective analysis of past 4 years was done from our records at Blood Bank, Department of Immunohaematology \& Transfusion Medicine, SMS Hospital, Jaipur in terms of Total Donation, Total Supply, Individual Component Supply, SDP Supply \&VBD Camps Organized in Year 2011, 2012, 2013, 2014, 2015. To analyze blood transfusion services \& proper usage of all Components (Packed cell, Platelet, FFP) with quality care. To analyze platelet services in form of Single Donor Platelet (SDP) to combat crisis like DENGUE. To analyze increasing trend of Voluntary Blood Donation (VBD) camps. To prove how effectively blood transfusion services (BTS) is managed by single blood bank of North Western India. Results: In year 2012, 59,103 blood donations (both voluntary \& replacement) were done \& total supply including all components was 93,473.57004 packed cell, 13,566 FFP, 9925 PLC 
\& 2011 PRP were supplied. In year 2013, 56,986 blood donations (both voluntary \& replacement) were done \& total supply including all components was 90,340.56526 packed cell, 14,447 FFP, 12,730 PLC \& 2058 PRP were supplied. In year 2014, 62,101 blood donations (both voluntary \& replacement) were done \& total supply including all components was 105,735.61880 packed cell, 17,441 FFP, 14,232 PLC \& 4086 PRP were supplied. In year 2015 (till 15 DEC 2015), 60,471 blood donations (both voluntary \& replacement) were done \& total supply including all components was 109,929.58351 packed cell, 18,836 FFP, 15,608 PLC \& 5461 PRP were supplied. SDP donation in year 2011, 2012, 2013, 2014, 2015 were 199, 318, 667, 632, 1111. Voluntary Blood Donation (VBD) CAMPS organized in year 2011, 2012, 2013, 2014, 2015 were 202, 232, 238, 243, 281 respectively. Conclusions: Audit of total donations, total supply of all components, supply of individual components, SDP supply \& Increasing trend of VBD camps in past 5 years has proved that how effectively \& efficiently a Single Blood Bank has managed Transfusion Service both in quality as well as in quantity in North Western India and has proved Transfusion Medicine as the backbone of Medical Sciences.

\section{TM 18}

Clinical Profile of Dengue Infection at a Tertiary Care Hospital in North India

\section{R. Katharia\#, R. Chaudhary}

Email ID for Correspondence: rk.kats@gmail.com Department of Transfusion Medicine, Sanjay Gandhi Postgraduate Institute of Medical Sciences, Lucknow, Uttar Pradesh, India

Background: The dengue infection is the most important arboviral infection occurring worldwide which is transmitted to humans by bite of Aedes mosquitoes. Early clinical recognition of dengue infection and anticipatory treatment for those who develop DHF or DSS can save lives. Therefore a descriptive study was planned to gather information regarding the demographic details and clinical features of the patient with dengue infection. Methods: A total of 350 patients were identified as probable cases by clinical suspicion. The patients who were sero positive for dengue were followed up for clinical profile. Results: Of the 264 cases, $141(53.4 \%)$ were dengue fever, $106(40.6 \%)$ had dengue hemorrhagic fever and $16(6.0 \%)$ were dengue shock syndrome. The most common presentation was fever $(93.6 \%)$ along with rashes $(50.0 \%)$, headache $(35.2 \%)$, myalgia $(49 \%)$ and abdominal pain (19.6\%). Bleeding manifestations were observed in $48 \%$ of all the patients. The most common bleeding manifestation was ecchymosis which was seen in $86.6 \%$ of all the bleeding patients followed by epistaxis $(35.4 \%)$. Elevated levels of SGPT and SGOT were observed in $52.3 \%$ of all cases with dengue infection where as thrombocytopenia and leucopenia was seen in $92.8 \%$ and $23.8 \%$ of all patients. Conclusions: Community awareness, early diagnosis and management and vector control measures need to be strengthened in order to reduce the increasing number of dengue cases. Significant differences in the clinical presentation of dengue virus (DENV) infection, indicative of a variation in disease severity from dengue fever (DF) to dengue hemorrhagic fever (DHF)/dengue shock syndrome (DSS).

\section{TM 19}

Study of Fresh Frozen Plasma Transfusion in Severe Isolated Traumatic Brain Injury Patients with Acute Trauma Induced Coagulopathy (ATIC) and Its Influence on Immediate Outcome

Venencia Albert ${ }^{\#}$, S. Arulselvi, Deepak Agrawal, H. P. Pati, A. K. Mukhopadhyay, Sanjeev Kumar Bhoi, S. Dutta Gupta, D. N. Rao, M. C. Misra

Email ID for Correspondence: albertvenencia@gmail.com Jai Prakash Narayan Apex Trauma Centre, All India Institute of Medical Sciences

Introduction and Background: Fresh Frozen plasma (FFP) is a valuable therapeutic option for management of coagulopathy and bleeding in traumatic brain injury patients. However there is conflicting information about the efficacy of FFP transfusion following severe TBI. We aimed to study the effect of early coagulopathy and FFP transfusion on mortality in severe isolated traumatic brain injury patients (iSBTI). Patient/Material and Methods: Data was prospectively collected on isolated severe TBI patients (GCS $\leq 8$; within $12 \mathrm{hrs}$ of injury) at a Level I trauma centre between Aug 2014 and Jan 2016. Patients were stratified by conventional coagulation tests as ATIC (PT $\geq 16.7 \mathrm{~s}$, INR $\geq 1.27 \&$ aPTT $\geq 28.8 \mathrm{~s}$ ) and No ATIC. Clinical and transfusion details were extracted from the blood bank records and patients files. Study outcome was defined as the in-hospital mortality. Univariate analysis followed by multivariate logistic regression analysis was done to compare study groups. Results: 120 iSTBI patients [ave age $35.3 \pm 12.6$ yrs; $88 \%$ male] included. ATIC was observed in $42 \%$ (50) patients. Overall $28 \%$ (34) received FFP. $46 \%$ (28) ATIC patients received FFP vs. $15 \%$ (11) No ATIC $(\mathrm{p}<0.0001)$. Overall Mortality rate was $30 \%$ (36) of which 61 $\%$ (22) had ATIC. FFP transfusion was more in non survivors compared to survivors of No ATIC group $(2.7 \pm 1.1$ vs. $0.5 \pm 0.2 ; \mathrm{p} 0.006)$, which was not significant different in the ATIC group. FFP transfusion was an independent predictor for mortality in iSTBI patients who did not develop ATIC OR 12.7 (95 \% CI 1.39-116.1; p 0.02). Conclusions: We report that although FFP transfusion has a role in correction of coagulopathy in TBI patients, it has detrimental effects on patients who do not have coagulation abnormalities following brain injury, and therefore the practice of early and aggressive use of FFP transfusion should be reevaluated and advised only when indicated by prolonged coagulation assays.

\section{TM 20}

Blood Donation-Donor's Perspective

Suresh K. R. Lakhara\#, Sanjay Prakash, Vandana Chhabra, Bhagchand Regar

Email ID for Correspondence: drlakharask12@gmail.com R.N.T. Medical College, Udaipur

Introduction and Background: There is a need to strengthen blood donor management in order to reduce the demand supply gap. Efforts are needed in this direction at all levels including educating and motivating more individuals to be involved in voluntary blood donation and converting them to retention donors. In order to develop 
rational and evidence based systems to be address blood shortage, we need to assess the current donor services and improve them to encourage more future donations. A study was planned to observe the current blood donation practice in order to assess the blood donation experience and reasons and barriers for blood donation. Material and Methods: Donors were monitored for their perceptions and satisfaction levels regarding the donation experience through a pre-designed questionnaire. Results: It was found that the motivating factors included altruism, need to help friend or relative or sometimes even the small gift that was being handed out. Most donors had heard about blood donation from relatives in need or news. Factors that influenced blood donation experience were found to be the attitude of the person at the reception, phlebotomy and counseling areas, staff professionalism and appreciation for the act of blood donation. They also reported that the infrastructure including reception, donation and refreshment area also play an important role in the overall experience. $99 \%$ donors reported a good experience. Many of them (84\%) said they wanted to be regular blood donors. Limitations of the study were small sample size and it was time intensive. Conclusions: It was concluded that donor satisfaction affects donor retentions and such studies help to improve quality of blood donation services.

\section{TM 21}

Prevalence of Weak D Among Antenatal Cases: Implications and Future Strategies: A Hospital Based Study

\section{Shikha Dawar", Priyanka Gogoi, Preeti Diwaker, Pankaj Aggrawal, Bharat Singh}

Email ID for Correspondence: drshikhadawar@gmail.com Regional Blood Transfusion Centre (East), Guru Teg Bahadur Hospital, Dilshad Garden, Delhi

Introduction and Background: $\mathrm{Rh}(\mathrm{D})$ antigen possess greater immunogenicity after A \& B antigens. Five principal antigens in (Rh) system are D, C, E, c, e. D antigen is a protein of 416 amino acids and alterations in these amino acid sequence results in "Weak D" expression. This assumes greater significance in antenatal cases who are candidates for repeated blood transfusion and also, it serves as a guide for administration of $\mathrm{Rh}$ immunoglobulin in postpartum period. Literature search revealed only few studies on weak D antigen from India, according to which prevalence of weak D ranges from $0.15 \%$ to $1 \%$. However, no such study has been done in antenatal cases. Patient/Material and Methods: Study was conducted in RBTC(E), GTB Hospital over a period of 4 months. 70 EDTA blood sample of antenatal cases which turned out to be $\mathrm{Rh}$ negative on blood grouping by column agglutination, were included in the study. Weak D testing was done on every case by Indirect Agglutination Test using ID gel card containing Anti-D (IgG). Results: Cases were considered as Rh D positive if the reaction was $4+$ whereas reaction of $3+$ to $1+$ was considered to be weak D positive. Out of the 70 samples studied, 3 turned out to be weak D positive. Conclusions: Prevalence of weak D in the present study came out to be $4.2 \%$ which is quite significant compared to the previous data. The result of present study suggest that weak $\mathrm{D}$ testing should be mandatory in all $\mathrm{Rh}$ negative antenatal cases. However, further studies on larger and different groups of population are required to validate this data.

\section{TM 22}

An Analysis of Transfusion Support in Acute Promyelocytic Leukemia (APL): A Single Institutional Experience

\section{Rizwan Javed ${ }^{\#}$, Sabita Basu, Saurabhjayant Bhave, Mammen Chandy}

Email ID for Correspondence: drrizwan39@gmail.com TATA Medical Center, Kolkata

Introduction: Acute promyelocytic leukemia (APL) is a distinct subtype of acute myelogenous leukemia (AML) which is potentially curable and transfusion support plays a cornerstone in it's management. The treatment and cure of patients with APL depends on the effective use of all-trans retinoic acid (ATRA) therapy and appropriate transfusion therapy. Aim: To study and analyze the number of red cells, platelets, plasma and cryoprecipitates transfused during the treatment of APL. Method: We retrospectively retrieved transfusion records of all diagnosed cases of APL who underwent treatment and transfusion at our hospital from May 2011 to August 2016. Confirmation of the PML-RAR $\alpha$ transcripts was obtained in all the cases by RT-PCR. The patterns of blood product usage in different age categories and in patients with DIC complication were studied. Results: There were 53 diagnosed APL patients [29 F: $24 \mathrm{M}$ ], median age 30.5 (4-66 years). Red cells were transfused to 50 (94.3\%) patients, median 4 (range 1-12) units; Random donor platelets (RDP) to 43 $(86.7 \%)$ patients, median 14 (1-74) units; Single donor platelets (SDP) to 29 (54.7\%) patients, median 0.5 (1-11.5) units; fresh frozen plasma (FFP) to $34(64.2 \%)$ patients, median $2(1-72)$ units and cryoprecipitate to $28(52.8 \%)$ patients, median $2.5(1-47)$ units. There was $2(3.7 \%)$ patient who did not require blood or blood products, $3(5.7 \%)$ did not require red cell transfusions, $7(13.2 \%)$ platelet transfusions, $19(35.8 \%)$ plasma transfusions and 25 $(47.1 \%)$ did not require cryoprecipitate. Conclusion: Our review found that the majority of cases $(>85 \%)$ required red cells and platelet transfusion but only around $60 \%$ of the patients required Plasma and cryoprecipitate were used predominantly for treating coagulopathy.

\section{TM 23}

Seroprevalence and Trends in Transfusion Transmitted Infections Among 706853 Blood Donors in a Tertiary Care Hospital in Jaipur

\section{Sarita Sharma ${ }^{1 \#,}$ Sunita Bundas ${ }^{2}$, Rachna N. Saxena ${ }^{3}$, Parmendra Pachori $^{4}$, Ashokpal ${ }^{5}$}

Email ID for Correspondence: sharmaji03116@gmail.com; e-mail: drsunitabundas@yahoo.in;

e-mail: bloodbanksms@gmail.com; e-mail: dr.p.pachori@gmail.com; e-mail: dr.palashok@gmail.com

${ }^{1}$ 2nd year Resident, MD IHBT, ${ }^{2}$ Professor \& Head,

${ }^{3}$ Professor, ${ }^{4}$ Assistant Professor, ${ }^{5} 1$ st Year resident, MD IHBT; SMS Medical College and Hospital, Jaipur

Introduction: Transfusion Transmitted Infections (TTI) posses a great threat for the health and safety of patients. In order to provide safe and adequate quantity of blood and blood components, Govt. Of India made pretransfusion screening and testing mandatory in the National Blood 
Policy (2002). Aims and Objectives: The aim of this study is to assess the trends and prevalence of TTI's among voluntary and replacement donors in the Department of Immunohematology \& Transfusion Medicine at SMS Medical College \& Hospital, Jaipur (Rajasthan) during the period from 2002 to 2015. Material and Method: A retrospective review of donor's record covering the period between 2002 to 2015 is carried out at Blood Bank, SMS Hospital, Jaipur. All samples were screened for HIV, HBV, HCV, Syphilis \& Malarial parasite. Method used is ELISA (for HIV, HBV, \& HCV; RPR for Syphilis and Peripheral blood film examination till 2013 and Rapid test kit for malaria from 2014 onwards). Result: Prevalence of TTI in total donors is $3.02 \%$. Prevalence of Hep B $(1.83 \%)$ was highest followed by Syphilis $(0.75 \%)$, HCV $(0.28 \%)$ HIV $(0.15 \%)$ and Malaria $0.01 \%$. Discussion and Conclusion: It is important to analyze the incidence, prevalence and causes of TTI in our region \& compare them with national \& international level. It is well within the reach to reduce the incidence of TTI's in Indian scenario with the implementation of strict donor criteria and use of sensitive screening tests.

\section{TM 24}

Use and Abuse of FFP-An Audit of Appropriate and Inappropriate Use of Fresh Frozen Plasma

\section{Mansi Gulati ${ }^{\#}$ P. S. Mulay, Santosh Pawar, S. A. Deshpande \\ Email ID for Correspondence: Drgulatimansi@gmail.com Dr Shankarrao Chavan Government Medical College}

Background: Blood transfusion is life saving in patients. There is no substitute for human blood, so it should be utilized judiciously. There is shortage of blood products in most of the developing countries. According to WHO report, India faces a blood shortage of 3 million units. Despite being a country with a population of 1.2 billion, only 9 million units are collected annually, while the need is for 12 million units. Thus, appropriate use of blood components is required to ensure their availability for the needy patients and avoid unnecessary risk of transfusion-transmitted diseases. Material and Methods: One year retrospective study was conducted in Dr. S.C.G.M.C. and hospital blood bank to evaluate usage of fresh frozen plasma according to indications and to reduce its inappropriate usage. Based on the guidelines published by college of American pathologist, national health and medical research council, NACO and Australian society for blood transfusion, FFP usage was categorized into appropriate and inappropriate. Pre and post transfusion INR/PT were recorded and the effect of FFP was studied in patients who received FFP. Results: During the study, 1278 units of FFP were transfused to 316 patients. Out of 316 requests made, $148(46.84 \%)$ requests were appropriate and $168(53.16 \%)$ were inappropriate. Pregnant female with active labour suffering from severe anemia with shock was the most common reason for inappropriate use of FFP. Out of 148 appropriate requests, 100 patients were compared by evaluating pre and post transfusion PT/INR by using fully automated coagulometer. Conclusions: Inappropriate use of FFP not only increases the treatment costs, but also causes loss of productive power and exposes the patient to unnecessary side effects of transfusion. Inappropriate FFP transfusion should be prevented by means of education and awareness programme by establishing the hospital transfusion guidelines.

Keywords Appropriate, Inappropriate, Fresh frozen plasma (FFP)

\section{TM 25}

Safety and Efficacy of Granulocyte Transfusion in Hematopoetic Stem Cell Transplant Patients-A Single Centre Experience

\section{Minal Poojary ${ }^{\#}$, Deepa Susan, Shashank Ojha, Navin Khattry, Ms. Sarika Parab, Ms. Pallavi Rane, Rajadhyaksha}

Email ID for Correspondence: drminalkhairnar@gmail.com; mpoojary@actrec.gov.in

Tata Memorial Centre, Advanced Centre for Treatment, Research and Education in Cancer (ACTREC)

Introduction and Background: Inspite of modern antimicrobials and antifungal drugs, life threatening bacterial \& fungal infections continue to be a consequence of severe neutropenia in Hematopoetic Stem Cell Transplantation (HSCT). Neutrophils play an integral role in host defense against bacterial \& opportunistic fungal pathogens. Neutrophil production by the marrow and an adequate number of blood and tissue neutrophils are the key predictors of recovery from infections. Hence, granulocyte transfusion therapy is a possible way to bridge a gap between marrow suppression and neutrophil recovery. Patient/Material and Methods: We conducted a retrospective analysis to determine safety and efficacy of granulocyte transfusion in 14 HSCT patients with severe neutropenia related infections unresponsive to appropriate antimicrobial agents. Granulocytes were donated by healthy donors stimulated by subcutaneous $5 \mu \mathrm{g} / \mathrm{kg}$ G-CSF plus $8 \mathrm{mg}$ dexamethasone orally on Cobe spectra cell separator machine. The response was analysed in terms of clinical response and survival of patient at 42 days after first granulocyte transfusion. Results: Patients received a mean of 2.92 granulocyte transfusions (range, $1-7$ ) and a mean dose of $3.59 \times 10^{8} / \mathrm{kg}$ granulocytes (range, 0.22 to $11.6 \times 10^{8} / \mathrm{kg}$ ). Out of total 42 granulocyte transfusions, 24 had higher granulocyte dose $\left(>2.8 \times 10^{8 /} \mathrm{kg}\right)$ per transfusion (equivalent to $>2 \times 10^{10} / \mathrm{bag}$ for an average $70 \mathrm{~kg}$ patient) and $18 \mathrm{had}$ lower granulocyte dose $\left(\leq 2.8 \times 10^{8 /} \mathrm{kg}\right)$. 1 hour and 24 hour post transfusion ANC increments of patients are significantly higher for higher granulocyte dose than lower dose by Mann-Whitney test. 9 patients $(64.28 \%)$ responded favourably to the granulocyte transfusions and 10 patients $(71.42 \%)$ survived at 42 days after first granulocyte transfusions from 14 patients during that particular neutropenic episode. Granulocyte transfusions were well tolerated with adverse reactions seen in only 1 patient. Conclusions: We conclude that granulocyte transfusion therapy may be clinically useful in HSCT patients with neutropenia related infections.

\section{TM 26}

Rare Phenotypic Expressions of Common Beta Thalassaemia Mutations

\section{Soumita Choudhuri ${ }^{\#}$, Rudra Ray, Malay Ghosh, Maitreyee Bhattacharya}

Email ID for Correspondence: schoudhuri09@gmail.com Institute of Haematology and Transfusion Medicine

Introduction and Background: Beta thalassemia screening includes a complete hemogram with RBC indices, followed by HPLC analysis. But a significant number of population may show low $\mathrm{MCV}, \mathrm{MCH}$ with normal $\mathrm{Hb}(\mathrm{A} 2)$ value in HPLC analysis, the causes of which is 
often not investigated. To investigate the underlying genetic abnormality that leads to low MCV, MCH and normal Hb (A2) level. Patient/Material and Methods: Initially individuals were screened by measuring hematological indices by cell counter, followed by HPLC analysis. The genetic analysis, for the individuals with low $\mathrm{MCV}, \mathrm{MCH}$ indices and normal $\mathrm{Hb}$ (A2) level was done by screening for common beta mutations by ARMS PCR followed by multiplex gap PCR analysis to detect deletions in alpha gene. Results: 17,876 individuals were screened under this study; among which 2694 had low MCV ( $<80.00 \mathrm{fL})$, MCH $(<25 \mathrm{\rho g}$ ) with normal ( $\mathrm{Hb}) \mathrm{A} 2$ values. Among those 1883 individuals, excluding the individuals with iron deficiency anemia determined by ferritin test, were further studied for mutation analysis. ARMS PCR revealed 13 individuals carrying beta mutations; among which IVS $1-5(\mathrm{G} \rightarrow \mathrm{C})$ mutations were the most common (11 individuals) followed by Cap site and CD 15 mutations. These were further checked for the presence of alpha gene mutations; and none of those 13 individuals were detected to have alpha gene deletions. Conclusions: IVS $1-5(\mathrm{G} \rightarrow \mathrm{C})$ and $\mathrm{CD} 15$ mutations were found to behave like 'silent' beta mutation. Sequence analysis is necessary to further confirm this finding and to elucidate the mechanism behind.

\section{TM 27}

Retrospective Evaluation of Adverse Transfusion Reactions Following Blood Product Transfusion from a Tertiary Care Hospital

Lokesh Sharma\#, Sonam alha, D. R. Arya, N. L. Mahawar, Arun Bharti, Shailendra Vashishtha

Email ID for Correspondence: lokeshsharma04@gmail.com Sardar Patel Medical College

Introduction and Background: Blood transfusion is a life-saving procedure, which can occasionally be unsafe and result in a spectrum of adverse events. The aim of the study was to determine the frequency and type of transfusion reactions (TRs) occurring in patients, reported to the blood bank at a tertiary care centre and make blood transfusion process as safe as possible. Material and Methods: A retrospective study was conducted at the Department of Immunohaematology and Blood Transfusion, Sardar Patel Medical College and AG Hospitals, Bikaner. All acute transfusion reactions reported to the department from 1 Jan 2014 to 31 December 2015 were evaluated. All the adverse events related to transfusion of blood and blood products reported to the department were recorded and analyzed as per Departmental Standard operating procedures. Results: During the study period a total 76,629 units of blood and components were issued by our blood bank and total of 207 $(0.27 \%)$ adverse reactions were reported to blood bank. The most frequent were febrile non-haemolytic transfusion reactions $(54.1 \%)$ followed by allergic reactions (36.2\%), and non-specific reactions $(9.7 \%)$. Conclusions: Each transfusion has to be monitored carefully with prompt recognition and treatment of acute transfusion reactions to decrease transfusion-related morbidity and mortality. Reporting can be improoved by proper hemovigilance system for monitoring blood transfusion safety and contribute to evidencebased transfusion medicine.

\section{TM 28}

Laboratory Monitoring of Plasma Transfusion Therapy: Experience from Tertiary Care Hospital in South India

\section{Manish Raturi", Shamee Shastry, B. Poornima Baliga}

Email ID for Correspondence: manishnsr@gmail.com Kasturba Medical College, Manipal, Manipal University, India

Introduction and Background: Conventional coagulation laboratory tests are most often used to predict risk of bleeding in various clinical situations. The present study aims to observe the effect of plasma based on these test results in the recipients. Patient/Material and Methods: We did prospective analysis of all the requests for plasma in a tertiary care referral hospital. Patients' demographics and laboratory parameters like PT, APTT, INR and platelet values within 12 hours preceding the requests and 24 hours post transfusion were observed to assess the level of correction. Patients were further categorized into three cohorts based on their pre-transfusion INR; mild elevation (INR <1.5), moderate elevation (INR 1.5-3) and severe elevation (INR $>3$ ). The mean change in INR values against the pretransfusion INR values per unit of plasma transfused was also calculated. The magnitude of improvement in INR was determined using the formula by Holland and Brooks. Therefore, changes in laboratory parameters were used to evaluate the therapeutic efficacy in patients. Data was analyzed using IBM SPSS Statistics 20. Results: Among 2082 episodes, 4991 units of plasma were transfused at an average of 5 units per patient. There was statistically significant change observed in both PT \& APTT values $(\mathrm{p}<0.001)$. The mean change in INR following plasma transfusion was $8.9 \%$ of pre-transfusion INR and thus considered as statistically significant change. Clinical improvement following plasma transfusion was better in the cohort of patients with pre-transfusion INR value ( $>3.0)$. Conclusions: Plasma transfusion is ineffective in patients with mildly abnormal pre-transfusion coagulation parameters. 